\title{
Polarity in GaN and ZnO: Theory, measurement, growth, and devices
}

Cite as: Appl. Phys. Rev. 3, 041303 (2016); https://doi.org/10.1063/1.4963919

Submitted: 25 March 2016 . Accepted: 24 August 2016 . Published Online: 21 November 2016

Jesús Zúñiga-Pérez, Vincent Consonni, Liverios Lymperakis, Xiang Kong, Achim Trampert $(\mathbb{D}$, Sergio Fernández-Garrido, Oliver Brandt (iD, Hubert Renevier, Stacia Keller, Karine Hestroffer, Markus R. Wagner (D), Juan Sebastián Reparaz, Fatih Akyol, Siddharth Rajan, Stéphanie Rennesson, Tomás Palacios, and Guy Feuillet
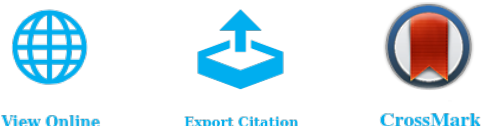

View Online

\section{ARTICLES YOU MAY BE INTERESTED IN}

Growth far from equilibrium: Examples from III-V semiconductors

Applied Physics Reviews 3, 040801 (2016); https://doi.org/10.1063/1.4944801

A comprehensive review of $\mathrm{ZnO}$ materials and devices

Journal of Applied Physics 98, 041301 (2005); https://doi.org/10.1063/1.1992666

Metal oxide semiconductor thin-film transistors for flexible electronics

Applied Physics Reviews 3, 021303 (2016); https://doi.org/10.1063/1.4953034

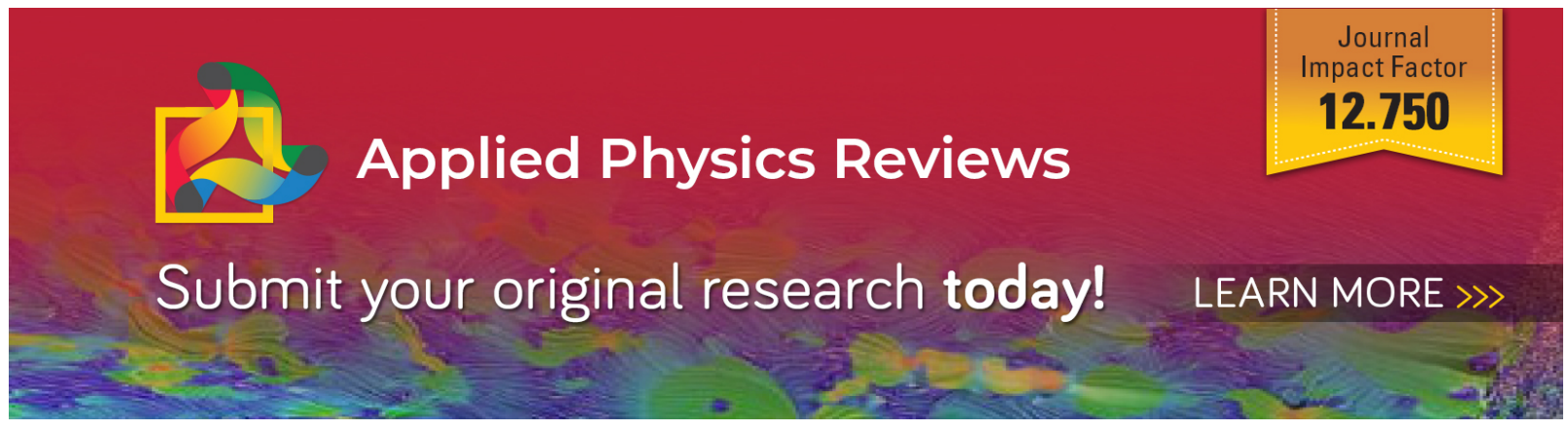




\title{
Polarity in GaN and ZnO: Theory, measurement, growth, and devices
}

\author{
Jesús Zúñiga-Pérez, ${ }^{1, a)}$ Vincent Consonni, ${ }^{2, a)}$ Liverios Lymperakis, ${ }^{3}$ Xiang Kong ${ }^{4}$ \\ Achim Trampert, ${ }^{4}$ Sergio Fernández-Garrido, ${ }^{4}$ Oliver Brandt, ${ }^{4}$ Hubert Renevier, ${ }^{2}$ \\ Stacia Keller, ${ }^{5}$ Karine Hestroffer, ${ }^{5}$ Markus R. Wagner, ${ }^{6}$ Juan Sebastián Reparaz, ${ }^{6}$ \\ Fatih Akyol, ${ }^{7}$ Siddharth Rajan, ${ }^{7}$ Stéphanie Rennesson, ${ }^{8}$ Tomás Palacios, ${ }^{8}$ \\ and Guy Feuillet ${ }^{9, a)}$ \\ ${ }^{1}$ CRHEA, CNRS, F-06560 Valbonne, France \\ ${ }^{2}$ Université Grenoble Alpes, CNRS, LMGP, F-38016 Grenoble, France \\ ${ }^{3}$ Computational Materials Design Department, Max-Planck-Institut für Eisenforschung, Max-Planck.Str. 1, \\ 40237 Düsseldorf, Germany \\ ${ }^{4}$ Paul-Drude-Institut für Festkörperelektronik, Hausvogteiplatz 5-7, 10117 Berlin, Germany \\ ${ }^{5}$ Electrical and Computer Engineering Department, University of California Santa Barbara, Santa Barbara, \\ California 93106, USA \\ ${ }^{6}$ Institute of Solid State Physics, Technische Universität Berlin, Hardenbergstr. 36, 10623 Berlin, Germany \\ ${ }^{7}$ Electrical and Computer Engineering Department, The Ohio State University, Columbus, Ohio 43210, USA \\ ${ }^{8}$ Microsystems Technology Laboratory, Department of Electrical Engineering and Computer Science, \\ Massachusetts Institute of Technology, Cambridge, Massachusetts 02139, USA \\ ${ }^{9}$ Université Grenoble Alpes, CEA, LETI, F-38054 Grenoble, France
}

(Received 25 March 2016; accepted 24 August 2016; published online 15 November 2016)

\begin{abstract}
The polar nature of the wurtzite crystalline structure of $\mathrm{GaN}$ and $\mathrm{ZnO}$ results in the existence of a spontaneous electric polarization within these materials and their associated alloys (Ga, $\mathrm{Al}, \mathrm{In}) \mathrm{N}$ and $(\mathrm{Zn}, \mathrm{Mg}, \mathrm{Cd}) \mathrm{O}$. The polarity has also important consequences on the stability of the different crystallographic surfaces, and this becomes especially important when considering epitaxial growth. Furthermore, the internal polarization fields may adversely affect the properties of optoelectronic devices but is also used as a potential advantage for advanced electronic devices. In this article, polarity-related issues in $\mathrm{GaN}$ and $\mathrm{ZnO}$ are reviewed, going from theoretical considerations to electronic and optoelectronic devices, through thin film, and nanostructure growth. The necessary theoretical background is first introduced and the stability of the cation and anion polarity surfaces is discussed. For assessing the polarity, one has to make use of specific characterization methods, which are described in detail. Subsequently, the nucleation and growth mechanisms of thin films and nanostructures, including nanowires, are presented, reviewing the specific growth conditions that allow controlling the polarity of such objects. Eventually, the demonstrated and/or expected effects of polarity on the properties and performances of optoelectronic and electronic devices are reported. The present review is intended to yield an indepth view of some of the hot topics related to polarity in $\mathrm{GaN}$ and $\mathrm{ZnO}$, a fast growing subject over the last decade. Published by AIP Publishing. [http://dx.doi.org/10.1063/1.4963919]
\end{abstract}

\section{TABLE OF CONTENTS}

I. INTRODUCTION

II. THEORETICAL AND FUNDAMENTAL

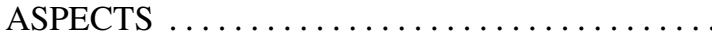

A. Methodological aspects ...............

B. Spontaneous polarization ...............

C. Surface polarity $\ldots \ldots \ldots \ldots \ldots \ldots \ldots \ldots$

D. Principles of semiconductor surface reconstructions.................... 6

E. Non-polar surfaces................. 7

F. Polar surfaces ..................... 10

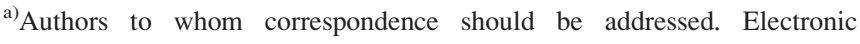
addresses: jesus.zuniga.perez@crhea.cnrs.fr; vincent.consonni@grenobleinp.fr; and guy.feuillet@cea.fr.
G. Semipolar surfaces .................

III. ASSESSING POLARITY AND

SPONTANEOUS POLARIZATION

A. Sensitive chemical etching ............ 15

B. Transmission electron microscopy ........ 16

1. Convergent beam electron diffraction (CBED) . . . . . . . . . . . . . . . . 16

2. Electron energy loss spectroscopy (EELS) $\ldots \ldots \ldots \ldots \ldots \ldots \ldots \ldots$

3. High angle annular dark-field (HAADF)and annular bright-field (ABF)-STEM...

C. Low-energy electron diffraction ...........

D. Angle-resolved x-ray photoelectron diffraction (ARXPD) $\ldots \ldots \ldots \ldots \ldots \ldots \ldots \ldots, 20$

E. Resonant X-ray diffraction in synchrotron... 21 1. Theoretical aspects ............... 21 
2. Experimental determination...........

F. Coaxial impact collision ion scattering spectroscopy (CAICISS)...............

G. Scanning probe microscopy (SPM) $\ldots \ldots \ldots$ 1. Kelvin probe microscopy (KPM) ...... 2. Piezoelectric force microscopy (PFM) ...

H. Experimental determination of the spontaneous polarization of $\mathrm{GaN}$ and $\mathrm{ZnO}$. .

IV. CONTROLLING THE POLARITY:

NUCLEATION AND GROWTH............

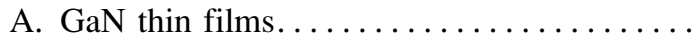

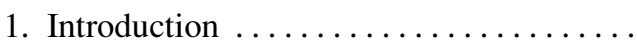

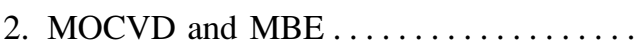

3. Growth on different substrates ........

4. Oxygen and carbon impurity incorporation....................

5. Doping with $\mathrm{Si}, \mathrm{Mg}$, and $\mathrm{Fe} \ldots \ldots \ldots$

6. Ternary alloys $\ldots \ldots \ldots \ldots \ldots \ldots . . . \ldots$

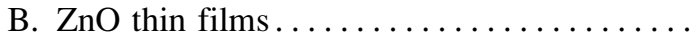

1. Introduction $\ldots \ldots \ldots \ldots \ldots \ldots \ldots \ldots$

2. Homoepitaxially grown $\mathrm{ZnO}$ thin films..

3. Heteroepitaxially grown $\mathrm{ZnO}$ thin films .

C. GaN nanowires and nanorods ...........

1. MOCVD (MOVPE) .................

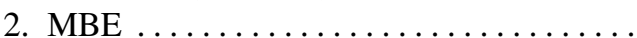

D. $\mathrm{ZnO}$ nanostructures and nanowires $\ldots \ldots \ldots$

1. $\mathrm{ZnO}$ nanostructures with different shapes by physical vapor deposition..........

2. $\mathrm{ZnO}$ nanowires by chemical vapor deposition .......................

3. $\mathrm{ZnO}$ nanostructures by solution deposition techniques ...............

V. ELECTRO-OPTICAL PROPERTIES AND DEVICES

A. $\mathrm{ZnO}$ electro-optical properties. ...........

1. Luminescence and absorption ..........

2. Dielectric function and optical reflectivity.....................

3. Electrical properties................

B. N-polar GaN optoelectronic devices: LEDs and solar cells ...................

1. Effects of polarization on optoelectronic devices: LEDs and solar cells ..........

2. Experimental work on $\mathrm{N}$-polar $\mathrm{GaN}$ LEDs ..........................

C. GaN-based high electron mobility

transistors .......................

1. Basics of $(\mathrm{Al}, \mathrm{Ga}) \mathrm{N} / \mathrm{GaN}$ HEMTs ......

2. Improving transistor performance through polarization and carrier confinement .....................

3. Normally off HEMTs ..............

4. N-face GaN-HEMTs ...............

VI. OUTLOOK

\section{INTRODUCTION}

The most studied and utilized wide band gap (WBG) semiconductors, i.e., $\mathrm{GaN}, \mathrm{ZnO}$, and the associated materials
FIG. 1. Ball and stick models of the (a) wurtzite and (b) zincblende GaN and $\mathrm{ZnO}$ structures. $a_{w}$ and $c$ are the wurtzite lattice constants and $a_{z}$ is the zincblende lattice constant. The wurtzite structure is composed of two interpenetrating hexagonal close packed (hcp) sublattices, each of which consists of one type of atom. The two sublattices are displaced with respect to each other along the threefold [0001]-axis by the amount of $u$.

families [( $\mathrm{Al}, \mathrm{Ga}, \mathrm{In}) \mathrm{N}$, which will be referred to as III-nitrides, and $(\mathrm{Zn}, \mathrm{Mg}, \mathrm{Cd}) \mathrm{O}],{ }^{1-8}$ are, for most of them, materials with a hexagonal wurtzite-type crystalline structure. In the vast majority of cases, these materials are grown along the $\langle 0001\rangle$ direction, the so-called $c$-axis: as of today, electronic and optoelectronic industrial devices based on WBG heterostructures are grown solely along this high symmetry/low index orientation. The reasons are partially related to the use of affordable foreign substrates, which impose the growth direction, and, above all, to the good stability and morphology of these surfaces in diverse growth environments. Growth orientations other than $\langle 0001\rangle$, i.e., inclined at a given angle to the c-axis, can also be stabilized and have widely been investigated in recent years: favoring growth along these orientations mainly implies departing from the usual growth conditions associated with the $c$-axis-oriented growth and changing the nature and/or crystalline orientation of the substrates. This is, for instance, the case when considering the growth of semi-polar or nonpolar orientations, which are envisaged to circumvent polarization issues. Employing growth conditions further away from the $c$-axis-related ones is also at the basis of nanostructure formation with different shapes, in particular nanowires (NWs) grown along the $c$-axis. Most importantly, the $c$-direction has two possible senses that, in the crystals considered here, are not equivalent. This has important consequences, which will be at the heart of the current review article.

Let us dwell upon the atomic positions of the wurtzite crystallographic structure. This structure is composed of two hexagonal closed packed (hcp) sublattices, one made up of anions $(\mathrm{N}$ or $\mathrm{O}$ ) and the other occupied by cations ( $\mathrm{Ga}$ or $\mathrm{Zn})$. The cation sublattice is shifted with respect to the anion sublattice along the $c$-axis by an amount $u \cdot c$ ( $u$ being a reduced coordinate, and $\mathrm{c}$ being the lattice parameter in the $\langle 0001\rangle$ direction). In the non-distorted hcp structure, the $c / a$ ratio is equal to $\sqrt{8 / 3}$ and $u$ equals $3 / 8$. In most wide band gap semiconductors such as $\mathrm{ZnO}$ and $\mathrm{GaN}$, however, $u$ departs from the $3 / 8$ value. Besides, as for zinc-blende, the wurtzite structure is non-centrosymmetric (see Section II B), but, contrary to it, wurtzite exhibits a spontaneous polarization (i.e., a nonzero dipole moment per unit volume) due to its polar character. As shown in Figs. 1(a) and 2(f), planes of cations alternate with planes of anions along the $c$ direction.

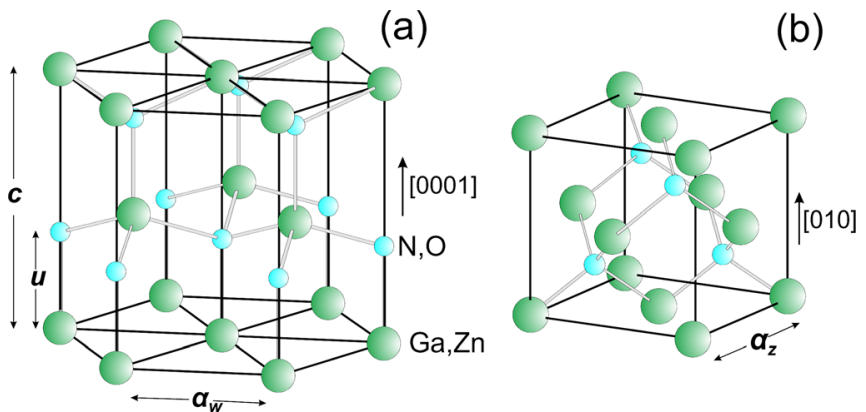


(a) Type 1

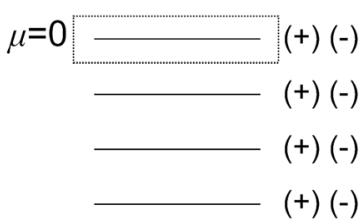

(d)

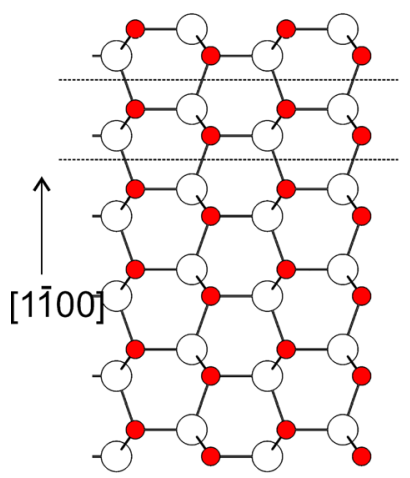

(b) Type 2

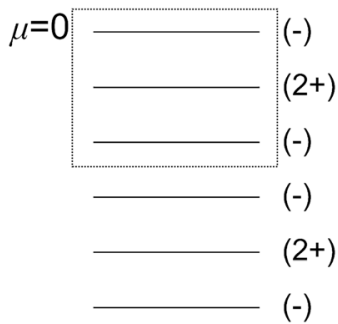

$(-)$

(e)

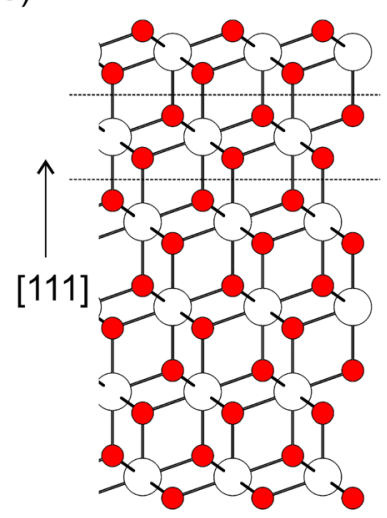

(c)

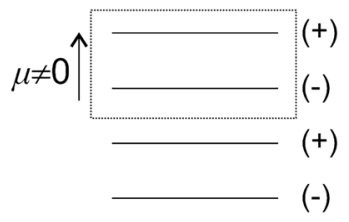

$(+)$

$(-)$

$(-)$

(f)

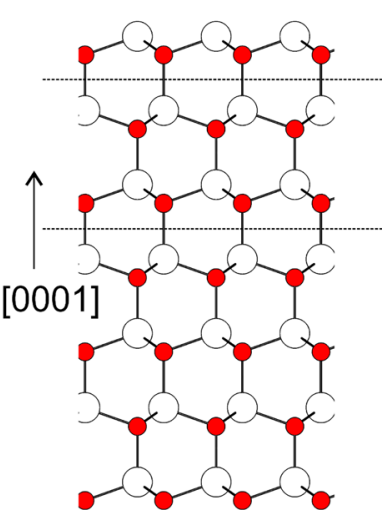

FIG. 2. (a)-(c) Schematic representation of the distribution of charges on planes parallel to the surface. The horizontal lines enclose the repeat units, and $\mu$ is the dipole moment in these units perpendicular to the surface. In (a), the planes contain an equal number of anions and cations and correspond to type 1 surfaces. The situation in (b) corresponds to type 2 surfaces: The planes are charged but have no net dipole moment in the repeat unit perpendicular to the surface, while in (c) the dipole moment is finite and corresponds to type 3 surfaces. Adapted with permission from P. W. Tasker, J. Phys. C: Solid State Phys. 12, 4977 (1979). Copyright 1979 Institute of Physics Publishing. Ball and stick models of (d) type 1 (1100) GaN, (e) type 2 (111) $\mathrm{CaF}_{2}$, and (f) type 3 (0001) GaN surfaces. Open (filled) balls correspond to cations (anions), respectively.
For defining the polarity, one usually refers to the cationanion bond that is colinear to the $c$-axis of the wurtzite cell. By convention, the vector going from the metallic cation and pointing toward the anion defines the [0001] direction, the positive sense of the $c$-axis. A structure is said to be metal polar when its growth direction is [0001]. Reciprocally, a structure is said to be anion polar when its growth direction is $[000 \overline{1}]$.

One may expect that the structural differences between these two orientations have important effects on their chemical reactivity, especially for the growth of two-dimensional (2D) thin films and one-dimensional (1D) nanostructures including NWs. Similarly, different surface electronic structures can be anticipated, because of different surface states, hence leading to different surface band bendings and different surface accumulation layers: this may strongly affect, for instance, the nature of metallic contacts thereon, the optical reflectivity and, possibly, the properties of electronic devices fabricated on the two opposite polar surfaces. As another example, the different chemical terminations of these two surfaces might lead to different impurity incorporation as growth proceeds: this could help finding solutions to the long lasting problems related to doping in these WBG materials, in particular, p-type doping in $\mathrm{ZnO}$. In a similar way, polarity selection could be a solution to improve the limited solubility of some cations in ternary alloys (e.g., Indium in (In,Ga)N alloys) or, conversely, to reduce incorporation of undesired impurities. Furthermore, when grown along either [0001] or [0001] directions, heterostructures based on these materials are subject to internal electric fields that arise from spontaneous and/or piezoelectric polarization discontinuities. Taking into account that opposite polarity heterostructures present opposite spontaneous polarization contributions, new possibilities open up in terms of band gap engineering for designing more efficient electronic and/or optoelectronic devices.

Knowing the potential advantages that controlling polarity would yield, the question now arises whether these two opposite polarities can easily and equally be obtainable or not. If one refers to simple electrostatic energy considerations, both surfaces are non-equilibrium ones and should be facetted instead of planar. But charge compensation effects have to be taken into account, which, together with surface relaxation, suggest that flat polar surfaces can actually be stabilized. Indeed, the flurry of research on the epitaxy of these materials has proven that thin films grown along the $c$-axis can exhibit smooth surface morphologies when proper growth conditions are used. Obtaining opposite polarities does not only depend on the growth conditions and environment (vacuum, hydrogen-rich, etc) but also the substrate itself may be chosen so as to favor the cation or anion polarity. This holds for thin films as well as for NWs. On the one hand, the easiest way consists in performing a homoepitaxial growth on top of substrates of the one or the other polarity, although substrates of the WBG materials considered here are not available in large enough dimensions and at affordable prices. Still, homoepitaxial growth has certainly helped in determining the right growth conditions for controlling the polarity of thin films as well as of nanostructures, the growth being unperturbed by the presence of numerous structural defects. On the other hand, when growing thin films or nanostructures on foreign substrates, growth initiation is the corner stone for defining and maintaining the polarity. If not tackled properly, the two polarities may coexist with the formation of inversion domain boundaries (IDBs) that disrupt the structural perfection. Alternately, different methods can be used to reverse the polarity at different stages during growth, for instance, through the insertion of 
adequate interlayers or dopants/impurities. As a result, a number of methods exist and can be implemented in order to control the polarity of thin films as well as of nanostructures. Conversely, the effects associated with the polarity of the structure may control the shape of nanostructures, as clearly evidenced for $\mathrm{ZnO}$, for which a whole zoology of different types of low dimensionality objects has been synthesized, including nanobelts, nanorings, nanospirals, or nanocombs, for instance.

Controlling polar surfaces might as well address a need to improve existing devices, whose performances might be further enhanced. Indeed, in some WBG related applications, the presence of spontaneous and/or piezoelectric polarization discontinuities may raise problems owing to the preferred $c$-orientation. In quantum well (QW) heterostructures, for instance, the resulting internal electrostatic field pushes electron and holes apart on either side of the well, thus reducing the emission efficiency and decreasing the emission energy (the so-called quantum confined Stark effect). Potential barriers at the interfaces also limit electron and hole injection into the QW: this negative effect may, in principle, be taken care of either by using non-polar or semipolar materials, but also by simply reversing the polarity, namely, from Ga polar to $\mathrm{N}$ polar in the case of $\mathrm{GaN}$ heterostructures. Interestingly, internal fields in these materials can be used as an advantage instead of a drawback. Typically, the strong polarization difference between $\mathrm{GaN}$ and $(\mathrm{Al}, \mathrm{Ga}) \mathrm{N}$ barriers, or between $\mathrm{ZnO}$ and $(\mathrm{Zn}, \mathrm{Mg}) \mathrm{O}$ barriers, can induce the formation of a high density and high mobility $2 \mathrm{D}$ electron gas (2DEG) at the interface, with charges being most probably provided by surface states. When formed due to the presence of internal electrostatic fields, 2DEGs offer a strong advantage with respect to carrier injection through doping modulation, as utilized in (Al,Ga)As/GaAs high-electron mobility transistors (HEMTs), because of less impurity-induced carrier scattering. Following the fast improvement in material quality, this effect was used for designing HEMT devices for RF and power transistors with rapidly progressing performances. Further, reversing the polarity of HEMT structures from cation to anion was theoretically shown to provide advantages in terms of back barrier isolation, ohmic contacts, and transconductance. In the case of $\mathrm{GaN}, \mathrm{N}$-polar devices could allow obtaining normally off transistors with strong advantages in terms of circuit simplicity and energy consumption.

Still, there are a number of open questions related to the fundamental understanding of polarity, its measurement, and characterization, and especially to the quantitative comparison between the optoelectronic properties/performances of opposite polarities, that need to be addressed, and this for both, thin films and nanostructures. This is, all the more, necessary knowing the expected or demonstrated advantages associated with polarity control in these WBG materials. With this in mind, the current review is intended to provide the reader with the present state-of-the-art in terms of polarity characterization and control, including thin films and NWs. Gathering results obtained in $\mathrm{GaN}$ and $\mathrm{ZnO}$ is intended to provide valuable comparisons and bring further insight into polarity-related phenomena. It is also the purpose of this review to give the status of research on the optoelectronic and electronic devices, where polarity is expected to play a major role for improving device performances.

Accordingly, the present review article is organized as follows. The first section deals with theoretical considerations on the compared stability and structure of the different polar, non-polar, and semipolar surfaces of the WBG GaN (and related materials) and $\mathrm{ZnO}$. Beyond defining polarity, one has to know how the polarity can be assessed, and the different methods are presented and compared in the second section. The third section provides an exhaustive review of the methods that are actually implemented to control the polarity of thin films as well as of nanostructures and discusses the consequences that polarity has on the growth of these structures. Finally, in the last section, the focus is devoted to the electro-optical properties and devices, for which polarity control may open up new possibilities in terms of device efficiencies and functionalities.

\section{THEORETICAL AND FUNDAMENTAL ASPECTS}

$\mathrm{GaN}$ and $\mathrm{ZnO}$ preferentially crystallize within the hexagonal wurtzite structure and the technologically preferred growth surface is the polar (0001) plane (see Fig. 1). However, the stability, reconstructions, and relaxation mechanisms of polar surfaces constitute a long standing enigma in semiconducting research. ${ }^{9-12}$ Based on electrostatic considerations, the surface energy of cleaved unreconstructed polar planes diverges. Thus, one may expect that polar planes should be prone to faceting. However, as discussed in the following, epitaxially grown polar planes of III-Nitrides, which are widely employed in the optoelectronic industry, can be stable and give smooth surface morphologies.

$A b$ initio calculations constitute a well-established tool, which can be applied in understanding, explaining, and/or guiding experiments and can provide information that is not directly or straightforwardly accessible by the latter. Density functional theory (DFT) is a well-established method to study the quantum mechanics of many-body systems and constitutes the working horse in atomistic investigations in solid state physics and in semiconductor physics and technology. ${ }^{13,14}$ Thus, in Section II A, a brief overview of the methods and terminology used to investigate the surfaces and growth from first principles is given. Next, the concept of spontaneous polarization (Section II B) and surface polarity and its effect on the surface energies and stability are discussed (Section II C). Motivated by the instability of the unreconstructed polar surfaces, the general guiding principles, in terms of the electron counting rule (ECR), for tetrahedrally coordinated compound semiconductor reconstructions are discussed in Section II D. The ECR constitutes the key aspect in Sections II E-II G, where a review on the surface reconstructions of non-polar, polar, and semipolar surfaces, respectively, is given: early as well as recent first principles calculations on these surfaces are discussed and the role of the ECR on the relaxation and reconstruction mechanisms of these surfaces is elucidated. The focus is both on the intrinsic surfaces, i.e., surfaces without foreign adsorbates as well as on surfaces in the presence of hydrogen. The former allows us to investigate the intrinsic properties of these surfaces and highlights the charge 
compensation mechanisms. Furthermore, they are the relevant surfaces for molecular beam epitaxy (MBE). However, hydrogen is present in the technologically important hydride vapor phase epitaxy (HVPE) and metalorganic chemical vapor deposition (MOCVD) growths as well as in atmospheric or aqueous environments, which are relevant for $\mathrm{ZnO}$ surfaces.

When appropriate, a comparative analysis with the conventional III-V semiconductor GaAs is given. GaAs surfaces such as the non-polar (110) and the polar (111) planes constitute model systems for surface relaxation, rehybridization, and reconstruction of tetrahedrally bonded compound semiconductors. Furthermore, this comparison allows us to highlight the similarities and differences of the more ionic III-Nitrides and $\mathrm{ZnO}$ with the more covalently bonded GaAs.

Although the stability of the polar III-Nitrides and $\mathrm{ZnO}$ surfaces can be explained in terms of charge compensation, the latter cannot account for the growth of smooth IIINitrides surface morphologies at growth temperatures well below $50 \%$ of the corresponding melting temperature. Hence, emphasis is made on the peculiarities of III-Nitrides surfaces, i.e., the cases where the ECR is not applicable. In contrast to the ECR, III-Nitrides planes stabilize metallic cation surfaces for growth under metal-rich conditions. This arises from the very different binding and cohesive properties and atomic radii of the constituents of the binary III-Nitrides and has important consequences on the surface thermodynamics and kinetics, which is discussed in detail. The adatom kinetics on the facets of novel nanostructures such as NWs is further discussed and the effects on the growth mechanisms are elucidated.

\section{A. Methodological aspects}

Key quantities in the $a b$ initio investigation of surfaces and growth are forces and total energies as well as the electronic structure (i.e., eigenvalues) of the system under consideration. DFT constitutes the most widely applied method in condensed matter and, more specifically, in semiconductor materials. ${ }^{13,14}$ Surfaces can be regarded as defects that destroy the periodicity of the bulk crystal structure. They can be created by cleaving and removing half of the crystal. However, a semi-infinite system in the direction normal to the surface cannot be directly studied by DFT, since it requires the diagonalization of a Hamiltonian consisting of an infinite number of matrix elements. Nevertheless, many of the surface related phenomena are short ranged in the above mentioned direction and geometries, which are small enough to be described by DFT and the current available computational power and are suitable to model surfaces, can be constructed.

Within the supercell approach, where periodic boundary conditions are applied in all three directions, surfaces can be studied using a slab geometry. DFT calculations on slab geometries are a widely used approach to theoretically study surfaces in condensed matter. In order to create a slab, we cut the semiinfinite crystal with a free surface at a particular lattice plane parallel to the surface and remove the rest. Within the slab approach, the system can be assumed as a cluster in the directions perpendicular to the surface. This is achieved by using a vacuum region in between the slab and its periodic images.
Obviously, the slab has two surfaces. The "top" side corresponds to the surface of interest, while special care must be taken for the treatment of the bottom surface of the slab. For example, in the case of wurtzite crystal structure, (hkil) surfaces with nonzero $l$ Miller index can be modelled by slabs consisting of inequivalent top and bottom surfaces, i.e., (0001) and $(000 \overline{1})$ or $(11 \overline{2} 2)$ and $(11 \overline{2} \overline{2})$. See, for example, in Fig. 2(f) a ball and stick model of a slab bounded by (0001) (upper side) and (0001) (lower side) planes. In this case, the different polarities of the two sides introduce an electric field in the vacuum region, and dangling bond states lying at different energetic positions in the fundamental band gap result in charge transfer from one side of the slab to the other. ${ }^{15}$ However, even in the case of non-polar surfaces, such as the $(1 \overline{1} 00)$ or $(11 \overline{2} 0)$, where both sides of the slab are bounded by equivalent surfaces (see in Fig. 2(d) a ball and stick model of a slab bounded by (1100) planes), surface states appear in pairs, and, due to the finite thickness of the slab, the tails of these states interact each other. Thus, the degeneracy of the states is lifted and the eigenenergies are split, an effect which is most pronounced at the $\Gamma$ point of the Brillouin zone (BZ).

A common approach to overcome the above mentioned problems is to passivate the dangling bonds of the bottom surface by fictitious partially charged hydrogens (pseudohydrogens) and avoid charge accumulation. The passivation is done to comply with the ECR (for more details in the ECR, see Section IID). According to the ECR, in the case of IIINitrides, each group-III cation contributes $3 / 4 \mathrm{e}^{-}$to a bond, whereas each group- $\mathrm{V}$ anion contributes $5 / 4 \mathrm{e}^{-}$. Hence, the $\mathrm{N}$ atoms at the bottom surface should be saturated by pseudohydrogens providing $3 / 4 \mathrm{e}^{-}$and the metal atoms by pseudohydrogens providing $5 / 4 \mathrm{e}^{-}$.

The equilibrium state of a surface is the one with the minimum excess Gibbs energy $(\Delta G)$

$$
\Delta G=E_{\mathrm{tot}}-T \Delta S-\sum_{i}^{n_{\mathrm{sp}}} n_{i} \mu_{i}
$$

where $E_{\text {tot }}$ is the computed total energy, $T$ is the temperature, $\Delta S$ is the entropy of the surface atoms, $n_{\mathrm{sp}}$ is the number of species, and $n_{i}$ and $\mu_{i}$ are the number of particles and the chemical potential of the $i$ th species, respectively. The chemical potential $\mu_{i}$ describes the change in the free energy if the number of particles of the $i$ th species changes by $d n_{i}$ under constant temperature, pressure, and the population of the other particles

$$
\mu_{i}=\left(\frac{\partial G}{\partial n_{i}}\right)_{T, p, n_{j \neq i}}
$$

The key quantity associated with the chemical potentials is the partition function $Z$, which for any species in any phase can be derived by first principles calculations and depends on partial pressure $p$ and temperature $T$ (see Ref. 16 and references therein). Thus, the concept of chemical potential allows us to efficiently describe realistic systems. Furthermore, it can be experimentally controlled by varying the partial pressures in epitaxial growth, and the link between theory and experiment is straightforwardly established. 


\section{B. Spontaneous polarization}

Crystals with a unique polar direction, i.e., a direction of which the two ends are not related by any symmetry element of the point group,${ }^{17}$ are pyroelectric, and exhibit a spontaneous polarization $P_{\mathrm{SP}}$ in equilibrium (at zero strain). Ten polar classes exist $(1,2,3,4,5, \mathrm{~m}, \mathrm{~mm} 2,3 \mathrm{~m}, \mathrm{~mm}$, and $6 \mathrm{~mm}),{ }^{830}$ which results in wurtzite $(6 \mathrm{~mm})$ being the highest-symmetry structure compatible with an spontaneous polarization. ${ }^{18}$ The zinc-blende structure (point group $\overline{4} 3 \mathrm{~m}$ ) lacks inversion symmetry and is piezoelectric but cannot sustain a spontaneous polarization since it has four symmetry-equivalent polar axes whose contributions cancel each other. Indeed, all unique directions are polar but only some polar directions are unique. The direction of $P_{\mathrm{SP}}$ is determined by the orientation of the unique polar axis, ${ }^{19}$ which for group III-Nitrides and $\mathrm{ZnO}$ is the $\langle 0001\rangle$-axis. Bernardini $e t a l .^{20}$ predicted by ab initio calculations that the III-Nitrides in the wurtzite phase are characterized by large values of $P_{\mathrm{SP}}$ and that the magnitude of this effect sensitively depends on the structural parameters (i.e., the degree of deviation from the ideal wurtzite structure), in particular, the deviation of the internal cell parameter $u$ from its ideal value of $3 / 8 .{ }^{21}$ More specifically for GaN, AIN, InN, and $\mathrm{ZnO}$, they reported that $\mathrm{P}_{S P}$ has a value of -0.029 , $-0.081,-0.032$, and $-0.074 \mathrm{C} / \mathrm{m}^{2}$, respectively.

However, $P_{\mathrm{sp}}$ itself is ill-defined for an infinite bulk crystal. On the other hand for a finite crystal, the surface and the bulk regions cannot be easily decoupled and bulk definition of polarization is inaccessible. Furthermore, any attempt to calculate the polarization via integration over a unit cell is appropriate only in the extreme case where the periodic charge density of a bulk crystal can be partitioned with no ambiguity into localized contributions. Moreover, calculation of the polarization field as the cell average of a microscopic polarization defined by the differential form of the Gauss' law is defined up to a constant field vector, i.e., it does not allow for a unique definition of the former.

The microscopic treatment of the polarization relies on the transformation between a reference state and the investigated system, ${ }^{22,23}$ which means that only polarization differences are accessible, experimentally as well as theoretically. For $P_{\mathrm{sp}}$ of wurtzite materials, the natural choice for such a reference is the zinc-blende phase. ${ }^{24-28}$ This phase has the same next neighbor configuration and a similar bond-length as wurtzite ${ }^{24-26}$ but, as already stated, a vanishing $P_{\mathrm{sp}}$ because of its higher symmetry.

Two conceptual frameworks are used to theoretically determine $P_{\mathrm{sp}}$ as the difference between the wurtzite and zinc-blende, namely, the electrostatic approach ${ }^{29}$ and the Berry phase method. ${ }^{22,23}$ Both DFT methods solve the reference problem by considering wurtzite/zinc-blende heterostructures, enabling one to determine $P_{\mathrm{sp}}$ as a difference. This approach has been implemented for various wurtzite semiconductors: $\mathrm{BeO},{ }^{24-26} \mathrm{SiC},{ }^{29}$ and $\mathrm{ZnO},{ }^{30}$ as well as the III-Nitrides. ${ }^{21,27,28,31}$ However, the accuracy of DFT is limited, as the value of $u$ predicted by this technique depends sensitively on the choice of the exchangecorrelation functional. Slight differences in $u$ result in large changes of $P_{\mathrm{sp}}$, with published values for GaN ranging from -0.014 to $-0.074 \mathrm{C} / \mathrm{m}^{2}{ }^{21,27,28}$

\section{Surface polarity}

Tasker proposed a classification scheme of the surface stability of ionic or partly ionic materials based on classical electrostatics, which considers the charge and dipole moment in the repeat unit cell perpendicular to the surface. ${ }^{11}$ The aforementioned classification scheme is shown in Fig. 2: Type 1 and type 2 surfaces do not possess a finite dipole moment perpendicular to the surface unit cell. Hence, these surfaces should have modest surface energies. However, type 3 surfaces have a finite dipole moment normal to the surface in all the repeated unit cells throughout the material. Thus, according to classical electrostatics, these surfaces should have diverging surface energy.

A different classification scheme based on frozen bulk termination was proposed by Goniakowski and collaborators. ${ }^{32}$ According to this approach, a frozen bulk termination is polar, if it cannot be obtained by exclusively piling up dipole-free bulk unit cells. According to the aforementioned classification schemes, the $\mathrm{ZnO}$ and III-Nitrides $\{0001\}$ surfaces are of type 3 and polar, while (1100) and (1120) surfaces are of type 1 and non-polar. Nevertheless, the frozen bulk termination scheme indicated that the polarity of a surface cannot be determined by the orientation of the surface alone (i.e., by the corresponding $(h k l)$ or $(h k i l)$ Miller indices) but also depends on the actual termination. Type 2 surfaces can be regarded as polar or non-polar depending on the actual frozen bulk termination. For example, the rutile $\mathrm{TiO}_{2}(110)$ surfaces, which are of type 2, can be regarded as non-polar, if they are terminated by $\mathrm{O}$, or polar, if they are terminated by $\mathrm{Ti}$.

\section{Principles of semiconductor surface reconstructions}

Irrespective of the way a surface is prepared, i.e., by cleavage or by growth, the atomic geometry at the surface always deviates from the ideal frozen bulk one. The surface undergoes surface relaxation and/or reconstruction to achieve the lowest free energy structure kinetically accessible under the corresponding thermodynamic conditions, i.e., the lowest values of the chemical potentials [see Eq. (1)]. A surface is considered as reconstructed, if the symmetry parallel to the surface is lower than that of the bulk. However, if the symmetry of the surface is the same as that of bulk, then the surface is said to be relaxed. ${ }^{10}$

For covalent or partly ionic semiconductor surfaces, such as $\mathrm{ZnO}$ and III-V materials (including III-Nitrides), the minimum free energy criterion can be met by the following guiding principles (for a detailed description, see Refs. 10 and 33): A surface (i) minimizes the number of dangling bonds, either by forming new bonds and/or by saturating the remaining bonds, (ii) tends to compensate charges, and (iii) tends to be semiconducting (as opposed to a metallic surface). These requirements can be achieved if the surface obeys the ECR, i.e., bonding and non-bonding surface states below the Fermi level are completely filled, whereas antibonding and non-bonding surface states above the Fermi 
level are empty. This can be achieved via rehybridization, charge transfer, conversion of the dangling bonds into nonbonding electronic states, which may be filled by a lone pair of electrons or be completely empty, and/or change of surface stoichiometry.

Based on the aforementioned criterion for charge neutrality at the surface, and taking into account the effective atomic charges in covalent or partly ionic semiconductors, Harrison investigated the stability of polar GaAs surfaces. ${ }^{9}$ In a III-V semiconductor, the energy levels of the dangling bonds in the unreconstructed and unrelaxed surface can be estimated from the corresponding energies of the $s$ and $p$ atomic states: As shown in Fig. 3(a) these states on each atom form $s p^{3}$ hybrids, with energies $\varepsilon_{h}$, which combine each other to form the bonding and antibonding orbitals. Linear combinations of the former form the valence band (VB), while of the latter forms the conduction band $(\mathrm{CB})$. Furthermore, the energy levels of the metallic cation dangling bonds lie near the $\mathrm{CB}$, while that of the anion dangling bonds are near the VB. The occupation of the dangling bond states is estimated by the valency in the (a)
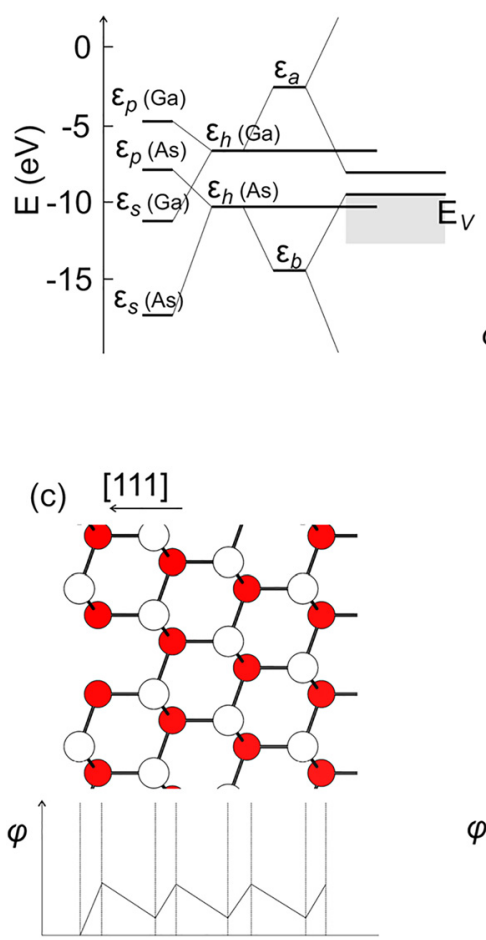

(b)

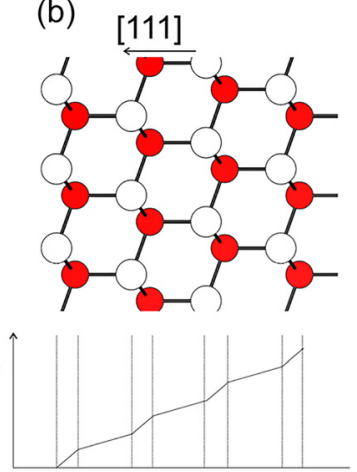

(d) $\stackrel{[111]}{\longleftarrow}$

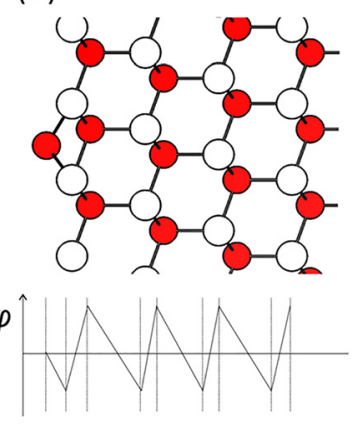

FIG. 3. (a) Energy levels diagram for a GaAs surface. $\varepsilon_{p}(\mathrm{Ga}), \varepsilon_{s}(\mathrm{Ga})$, $\varepsilon_{p}(\mathrm{As})$, and $\varepsilon_{s}(\mathrm{As})$ are the energies of the $p$ and $s \mathrm{Ga}$ and As atomic states, respectively. $\varepsilon_{h}(\mathrm{Ga})$ and $\varepsilon_{h}(\mathrm{As})$ denote the $\mathrm{Ga}$ and As $s p^{3}$ hybrids, respectively. $\varepsilon_{b}$ and $\varepsilon_{a}$ are the corresponding bonding and antibonding combinations of these $s p^{3}$ hybrids. The broadening of the bonding (antibonding) states forms the valence (conduction) bands, respectively. $E_{V}$ denotes the valence band maximum. The Ga dangling bond energies are in the conduction band, while the As dangling bond hybrids are mixed with the valence band. (b)-(d) Schematic representations (in side view) of the GaAs (111) surface. In (b), the cleaved surface is shown. In (c), one in four surface As atoms has been removed and in (d) one Ga atom in every four surface unit cells has been added in a bridging position over three As atoms. Below the ball and stick models, the potential $\varphi$ which results from the integration of the Poisson's equation normal to the surface taking the potential zero outside is plotted. Open (filled) balls denote As (Ga) atoms, respectively. Adapted with permission from W. A. Harrison, J. Vac. Sci. Technol. 16, 1492 (1979). Copyright 1979 American Vacuum Society. bulk, where each cation redistributes some of its valence charge to the anions. Harrison assumed for GaAs that each bond contributes $1-\alpha_{p}$ and $1+\alpha_{p}$ electrons to the metallic cations and anions, respectively, where $\alpha_{p}$ equals $1 / 2$.

Based on these effective charges, the integration of the Poisson's equation from the vacuum to the bulk is schematically shown in Figs. 3(b)-3(d) for the cleaved (111)As, the $2 \times 2$ As vacancy, and $2 \times 2$ Ga adatom surfaces, respectively. As can be clearly seen, the as-cleaved (111) As surface has a finite average potential gradient, and hence the surface energy diverges. However, if the stoichiometry at the surface is altered by removing an As surface atom from or adding a Ga adatom to every 4 surface unit cells, which result in an equal number of anion and cation dangling bonds, charge neutrality is achieved and the surface energy does not diverge. The $2 \times 2$ As vacancy and Ga adatom reconstructions were predicted by DFT calculations to be the energetically most favorable surface reconstructions for a wide range of $\mathrm{Ga}$ and As chemical potentials, from Ga-rich to As-rich conditions. ${ }^{34}$ Furthermore, as discussed later, the $2 \times 2 \mathrm{Ga}$ vacancy and $2 \times 2 \mathrm{Ga}$ adatom surfaces are the energetically most favorable GaN (0001) surface structures for $\mathrm{N}$-rich to moderate Ga-rich conditions. In general, and, in contrast to non-polar surfaces, in order to achieve charge neutrality of intrinsic polar surfaces, i.e., without foreign adsorbates, in the absence of an external electric field, the surface stoichiometry has to deviate from the stoichiometry of the frozen bulk termination. In Sections II E-II G, we discuss the aforementioned reconstruction guiding principles by first considering the non-polar III-Nitrides and $\mathrm{ZnO}$ surfaces.

\section{E. Non-polar surfaces}

The majority of III-Nitrides based devices are grown on the (0001) plane of the wurtzite structure. However, as indicated in the Introduction, the built-in spontaneous and piezoelectric polarization fields associated with the polar $\langle 0001\rangle$-axis have a substantial influence on the optical and electronic properties of group III-Nitrides heterostructures: in particular, the quantum confined Stark effect produces a shift in the wavelength of emission lines and reduces the quantum efficiency due to poor electron-hole overlap. ${ }^{35}$ Thus, the growth of non-polar $a-(11 \overline{2} 0)$ and $m-(1 \overline{1} 00)$ plane surfaces has attracted considerable interest as alternative growth directions to avoid polarization in wurtzite GaN epilayers.

In Fig. 4(b), a schematic top view of the GaN (1100) surface is shown. It consists of equal numbers of three fold coordinated $\mathrm{Ga}$ and $\mathrm{N}$ atoms that form an array of Ga-N dimers along the $\langle 0001\rangle$ direction. Each surface unit cell has one $\mathrm{Ga}$ and one $\mathrm{N}$ triply coordinated surface atoms. Thus, charge neutrality can be obtained without changes in stoichiometry or reconstruction. Nevertheless, the surface relaxes. In general, surface relaxation on heteropolar covalent or ionic semiconductors is driven by quantum mechanical hybridization effects and by classical Coulomb attraction between anions and cations. In order to reduce the hybridization energy, the cations tend to move downward and establish planar $s p^{2}$-like bonds with their three nearest neighbors. In contrast, the anions tend to move upwards and to establish 

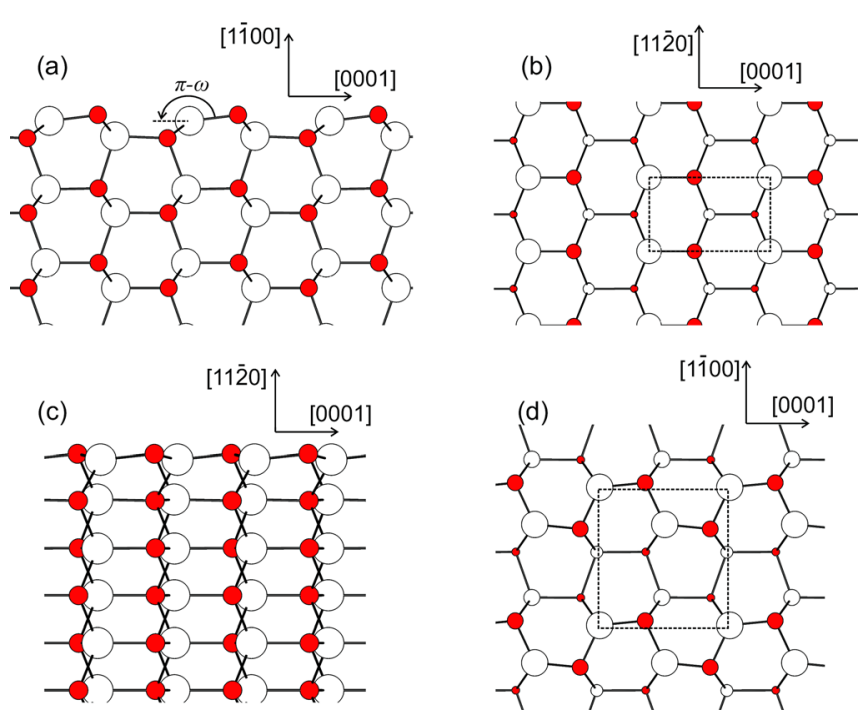

FIG. 4. (a) Side and (b) top view of the relaxed (1100) GaN surface. (c) Side and (d) top view of the relaxed $(11 \overline{2} 0) \mathrm{GaN}$ surface. The rectangles in (b) and (d) denote the $1 \times 1$ surface unit cell. Open (filled) balls denote $\mathrm{Ga}$ (N) atoms, respectively. In (b) and (d), larger (smaller) balls denote first (second) layer atoms, respectively. In (a), the surface cation-anion bond rotation $\pi-\omega$ is shown.

a $p^{3}$-like configuration. The re-hybridization results in a rotation of the dimer (see Fig. 4(a)). This rotation is accompanied by a lowering of the energy of the occupied anion dangling bond and by an increase of the energy of the cation empty state. Finally, the relevant cation-anion displacement costs electrostatic energy, and, in order to minimize it, the anion-cation distance tends to shorten.

Depending on the ionicity and the symmetry of the system, one of the aforementioned relaxation mechanisms might dominate over the others. For more covalent systems, the quantum mechanical re-hybridization effects dominate, giving rise to relatively large bond rotation angles, while the anion-cation bond length is nearly preserved. In contrast, for more ionic systems, the anion-cation Coulomb attractions dominate the relaxation, resulting in a relatively small buckling angle $\omega$ and relatively large bond contraction. Since the III-Nitrides group is fairly ionic, it is expected that the $m$-plane GaN surface relaxation should be mainly governed by the ionic Coulomb attractions, the bond-angle rotation to be relatively small (i.e., compared with the bond-angle rotation in the equivalent (110) GaAs surfaces), and the surface bonds to contract.

Indeed, for the GaAs (110) surface, Alves et al. ${ }^{36}$ reported, based on DFT calculations, relatively large bondrotation angle $\left(\omega \approx 30^{\circ}\right)$ and almost negligible bond contraction $(\approx 1 \%)$. Regarding the electronic structure, they found surface states in the fundamental bandgap that arise from the empty cation-derived state and the occupied anion-derived dangling bond state. Upon relaxation, the empty state moves upwards and the occupied state shifts to lower energies, leading to a reduction of surface energy. The more ionic character of GaN as compared with GaAs, results on the other hand in smaller buckling angles, in the range of $7.5^{\circ}-11.5^{\circ}$, and larger bond contractions, in the range of $5.3 \%-7.5 \% .^{37,38}$ However, similar to the GaAs (110) surfaces, the metal atoms move downwards and relax to an $s p^{2}$ configuration, while the $\mathrm{N}$ atoms remain close to their original positions, tending to adopt a more $p^{3}$-like configuration. In general, the non-polar surfaces of III-Nitrides group exhibit quantitative differences compared with their GaAs counterparts that arise from the more ionic character of the former.

Although the relaxation mechanism of the cleaved GaN $m$-plane surface is well established, its electronic structure, and, in particular, the presence of an unoccupied surface state within the fundamental bandgap have highly been debated. DFT calculations within the local density approximation (LDA) predict that both the occupied and unoccupied surface states shifted out of the fundamental band gap. ${ }^{37}$ However, the LDA calculated bulk band gap is underestimated $\left(E_{g}=1.9 \mathrm{eV}\right)$. More recent calculations based on modified pseudopotentials, which correctly reproduce the experimental bulk band gap, find the unoccupied surface state to be in the band gap in the whole BZ. ${ }^{39,40}$ In contrast, scanning tunneling microscopy (STM) experiments suggest the absence of surface states in the band gap. ${ }^{41,42}$ In order to clarify this controversial issue, DFT calculations were combined with STM experiments on freshly cleaved GaN (11 100$)$ surfaces, and it was shown that the minimum of the empty surface state at $\Gamma$ is $0.6 \pm 0.2 \mathrm{eV}$ below the bulk $\mathrm{CB}$ minimum (CBM), i.e., within the fundamental band gap. ${ }^{43}$ However, this state has a steep dispersion minimum with very low density-of-states (DOS) near the $\Gamma$ point, as compared with the bulk states, and an extremely flat area with high DOS at the edge of the BZ (see Fig. 5). Thus, conventional STM mapping modes failed to probe the physically most relevant minimum of the unoccupied surface state. This electronic structure is universal for all non-polar III-Nitrides surfaces, i.e., for both $m$ - and $a$-plane GaN, AlN, and $\mathrm{InN}$.

The structure of the $(11 \overline{2} 0)$ surface corresponds to a chain of threefold-coordinated $\mathrm{Ga}$ and $\mathrm{N}$ atoms along the

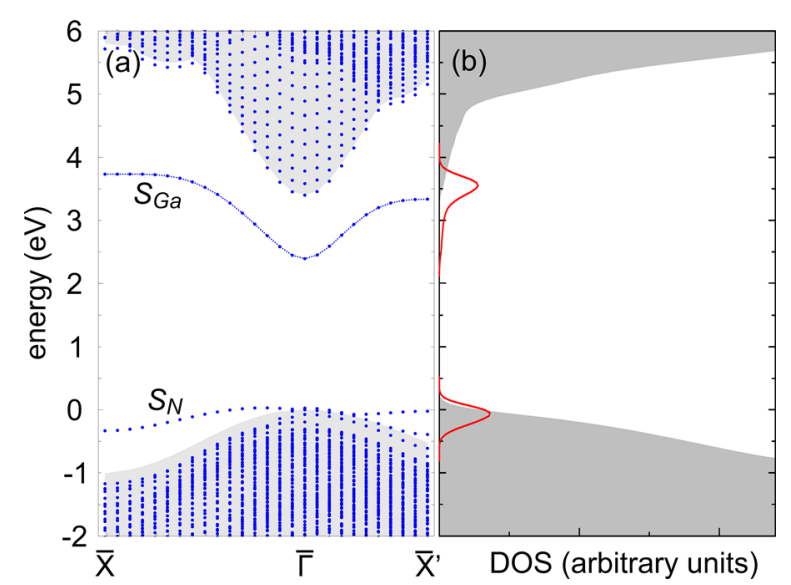

FIG. 5. (a) Calculated band structure and (b) DOS of the relaxed GaN (1100) surface along high symmetry lines of the surface Brillouin zone. $S_{G a}$ $\left(S_{N}\right)$ indicates the unoccupied (occupied) surface state, respectively. In (b), the red solid curves indicate the DOS arising from the surface states, i.e., the $S_{G a}$ and $S_{N}$ states. In (a) and (b), the grey shaded areas denote the projected bulk band structure and DOS, respectively. In both cases, the top of the bulk valence band is set to 0 and the bulk conduction band has been rigidly shifted to meet the experimental band gap of GaN. Adapted with permission from Appl. Phys. Lett. 103, 152101 (2013). Copyright 2013 AIP Publishing LLC. 
$\langle 0001\rangle$ direction (see Fig. 4(d)). Each surface unit cell has two Ga and two $\mathrm{N}$ triply coordinated surface atoms. The same relaxation mechanisms, as described for the $m$-plane, apply for the $a$-plane: Charge neutrality can be obtained without changes in stoichiometry or reconstruction. However, the triply coordinated surface atoms on the $a$-plane have one back bond, i.e., one bond with atoms in the deeper layer and two bonds in the surface plane, as opposed to the two back bonds and one bond in the surface plane of atoms on the $m$-plane surface. While on the $m$-plane surface the inplane displacements of the atoms from the frozen bulk positions are restricted to the $\langle 0001\rangle$ direction, i.e., parallel to the dimers chain, on the $a$-plane surface the atoms show considerable displacements also in the direction perpendicular to $\langle 0001\rangle$ (i.e., $\langle 1 \overline{1} 00\rangle$ ).

The aforementioned differences in the geometry of $\mathrm{m}$ and $a$-plane surfaces influence both the energetics of the clean surfaces, i.e., stoichiometric surfaces, as well as the adatom kinetics and have important consequences on the growth of GaN NWs. ${ }^{37,44}$ The surface energy of stoichiometric $m$-plane was calculated to be $1.95 \mathrm{eV}$ per dimer, while that of $a$-plane to be $1.75 \mathrm{eV}$ per dimer, i.e., the energy per surface dimer is higher on the $m$-plane surface. However, the density of surface dimers is higher on the $a$-plane surface and the surface energy per unit area is lower on the $m$-plane surface, $118 \mathrm{meV} / \AA^{2}$ on the $(1 \overline{1} 00)$ versus $123 \mathrm{meV} / \AA^{2}$ on the $(11 \overline{2} 0)$. The surface energies of non-polar $\mathrm{GaN}$ surfaces are approximately twice as large as for the GaAs (110) surface, which is $54 \mathrm{meV} / \AA^{2}{ }^{37}$ This is attributed to differences in both geometry and chemistry of these materials: the density of dangling bonds on the unreconstructed $\mathrm{GaN}$ surface is larger and the Ga-N bonds are stronger than the Ga-As bonds.

GaN NW growth and characterization experiments provide strong evidences that the side facets of the $c$-axis NWs consist of $m$-plane surfaces (see Ref. 45 and Section IV C). This is in agreement with the surface energetics of $m$ - and $a$-plane surfaces. Recent DFT calculations revealed a strong anisotropy in the diffusion barriers along and perpendicular to the $c$-axis: On the $m$-plane surface, $G$ a adatoms experience a considerably larger barrier for diffusion along the $c$-axis than for diffusion along the $a$-axis. ${ }^{44}$ At typical NW growth temperatures, this corresponds to two orders of magnitude larger lateral than axial diffusion lengths. This was explained in terms of the atomic geometry of these surfaces: At the stable position, a Ga adatom sits almost on top of the $\mathrm{N}$ surface atoms and creates a strong covalent bond. For diffusion along the $c$-axis, this bonds has to be broken and replaced by weaker metallic $\mathrm{Ga}-\mathrm{Ga}$ bond at the transition points. However, for diffusion along the $a$-axis, the next nearest $\mathrm{N}$ surface atom is only $\approx 3.2 \AA$ away. Thus, for diffusion along this direction, the breaking of the $\mathrm{Ga}-\mathrm{N}$ bond at the stable position is, to a large extent, compensated by the formation of a second Ga-N bond at the transition point. The anisotropy in the energetic barriers for diffusion on the $a$-plane surface is reversed: Ga adatoms experience lower barriers for axial than for lateral diffusion.

The diffusion induced-growth mechanism of NWs, i.e., growth of a NW by material transfer from the side facets to the top facet, is less pronounced on $m$-plane side facets.
Thus, only Ga adatoms adsorbing in close proximity to the top of the NW are able to reach the top before getting desorbed or incorporated into the side facets. As a consequence, only a small fraction of Ga adatoms reaching the NW contributes to its axial growth. Nevertheless, desorption-readsorption to a neighboring NW cannot be excluded, in particular, in dense NW arrays. This may substantially increase the effective axial diffusion length of the $\mathrm{Ga}$ adatoms.

The aforementioned stoichiometric $m$ - and $a$-plane $\mathrm{GaN}$ surfaces can be produced by cleavage or cracking. Furthermore, they are the energetically most favorable surfaces under N-rich to moderate Ga-rich MBE growth conditions. However, for growth under Ga-rich conditions, non-stoichiometric surfaces become energetically more favorable. The first studies on non-stoichiometric non-polar GaN surfaces were reported by Northrup and Neugebauer. ${ }^{37}$ Two different non-stoichiometric configurations were studied that formed by replacing the surface $\mathrm{Ga}$ or $\mathrm{N}$ atoms by $\mathrm{N}$ or $\mathrm{Ga}$ atoms and are called N-N-dimer and Ga-Ga dimer (GD), respectively. Although the formation of the N-N dimer configuration might be expected to be favorable for large values of the $\mathrm{N}$ chemical potential, i.e., under $\mathrm{N}$-rich conditions, calculations revealed that the N-N dimer surface is higher in energy than the others.

The metallic GD surface becomes energetically favorable under more Ga-rich conditions. Lee et al. combined STM experiments with DFT calculations and revealed the stability of more metallic adlayers on the $m$-plane GaN surfaces under Ga-rich conditions having either the $1 \times 1$ reconstruction with 4 extra $\mathrm{Ga}$ atoms per surface unit cell or a " $4 \times 5$ " reconstruction where, on top of the $21 \mathrm{Ga}-\mathrm{N}$ dimers of the surface unit cell, a compressed Ga adlayer consisting of 45 atoms is placed. ${ }^{46}$ These calculations provide strong evidence that contrary to the general principles for semiconductor surface reconstructions (see Section IID), metallic surfaces are thermodynamically allowed and favored in the Ga-rich growth regime. The tendency to stabilize metallic surfaces under metal-rich conditions is a universal characteristic of all III-Nitrides surfaces and, as discussed in Section II F, it has important consequences on the adatom kinetics as well as implications on the growth of these surfaces.

As has already been mentioned, for plasma-assisted MBE (PAMBE) growth, it is sufficient to consider the intrinsic surfaces. However, in other growth techniques commonly employed in industry for III-Nitrides semiconductors, such as MOCVD or HVPE, $\mathrm{NH}_{3}$ is present and hence hydrogen is present in high concentrations. Northrup et al. have investigated the thermodynamics of $\mathrm{H}$ and $\mathrm{NH}_{2}$ at $\mathrm{GaN}$ (1100) surfaces. ${ }^{47}$ They found that if only molecular hydrogen is considered, then $\mathrm{H}$ atoms passivate both surface cation and anion dangling bonds. Saturation of only cations or only anions is against the ECR, since it results in a hole occupying the anion dangling bond or in an electron occupying the cation dangling bond, respectively. However, the structure where both dangling bonds are saturated by $\mathrm{H}$ atoms obeys the ECR and removes the surface states from the fundamental band gap. This structure is thermodynamically stable at temperatures as high as $\approx 1200 \mathrm{~K}$. However, at typical growth conditions, entropic effects significantly decrease the 
hydrogen chemical potential. Thus, at higher temperatures, $\mathrm{H}$ atoms are only weakly bound at the surface or are thermodynamically unstable. Nevertheless, if the presence of $\mathrm{NH}_{3}$ is considered, then the $\mathrm{NH}_{3}$ molecules dissociate into an $\mathrm{NH}_{2}$ and a $\mathrm{H}$ bonding at the $\mathrm{Ga}$ and $\mathrm{N}$ surface atoms, respectively. The resulting surface obeys the ECR. Still, as with the saturation of the surface with hydrogen only atoms, at typical MOCVD growth conditions, the entropic effects dominate, and this surface is thermodynamically unfavorable with respect to the clean surface.

The cleaved non-polar $\mathrm{ZnO}$ surfaces show a behaviour similar to III-Nitrides surfaces, typical for rather ionic compounds with a strong covalent contribution. The calculated buckling angles for $\mathrm{ZnO} m$-plane surfaces are $10.7^{\circ}-11.7^{\circ}$ and the corresponding bond contraction in the range of $6 \%-7.5 \% .^{38,48-51}$ Although earlier ab initio calculations using the $\mathrm{LDA}^{52}$ or the $\mathrm{B} 3 \mathrm{LYP}^{53}$ functionals as well as Hatree-Fock ${ }^{54}$ calculations reported buckling angles as small as $2^{\circ}-5^{\circ}$. These apparently small angles are attributed to convergence problems arising either from insufficiently thick slabs or not sufficiently dense sampling of the BZ. ${ }^{48}$ The LDA calculated surface energies for the $m$-plane are $72-74 \mathrm{meV} / \AA^{2}$, while the $a$-plane has $4-5 \mathrm{meV} / \AA^{2}$ higher energies. $^{48,49}$

Total energy calculations on the intrinsic $\mathrm{ZnO} m$-plane surfaces revealed that the stoichiometric surface is the energetically most favorable one for almost the whole range of thermodynamically allowed values of $\mathrm{O}$ chemical potential, i.e., under conditions where $\mathrm{ZnO}$ is stable against the competing phases of oxygen molecules and Zn bulk. ${ }^{49}$ Only at the limit of $\mathrm{Zn}$-rich conditions, a surface, where $1 / 4$ of the $O$ surface atoms were removed, has lower energy. The electronic structure of both $\mathrm{ZnO}$ non-polar surfaces was evaluated by B3LYP hybrid functionals. ${ }^{51}$ The authors calculated the band gap of the $(1 \overline{1} 00)$ surface to be $0.24 \mathrm{eV}$ smaller than the bulk band gap, while the $(11 \overline{2} 0)$ surface has essentially the same band gap as the bulk structure.

The band gap of the (1100) $\mathrm{ZnO}$ surface was reported to take the bulk value upon saturation of both surface dangling bonds with hydrogen atoms. ${ }^{55}$ Nevertheless, the binding energy of the two hydrogen atoms to the surface is computed to be $0.62 \mathrm{eV}$ relative to an isolated gas phase $\mathrm{H}_{2}$ molecule. This indicates that the two atoms are only weakly bounded to the surface, and hence, at high temperatures, the hydrogen saturated surface might be thermodynamically unstable. A more recent work by Wang et al. indicated that exposure of the bare $(1 \overline{1} 00)$ surface to atomic hydrogen at room temperature results in a metallic surface consisting of hydroxyl groups and unsaturated $\mathrm{Zn}$ surface atoms. ${ }^{56}$ However, if the same surface is exposed to hydrogen atoms at a lower temperature (i.e., $200 \mathrm{~K}$ ), both cation and anion surface atoms are saturated by hydrogens and the surface is semiconducting. The hydrogen induced metallicity of the $m$-plane $\mathrm{ZnO}$ surface at room temperature is in contrast to the guiding principles for surface relaxation and reconstruction. The authors speculate that hydrogen atoms saturating the $\mathrm{Zn}$ dangling bonds at low temperatures diffuse into the bulk upon heating.

\section{F. Polar surfaces}

The common growth direction of wurtzite $\mathrm{GaN}$ is normal to the hexagonal $\{0001\}$ basal plane. In the $\{0001\}$ basal plane, the atoms are arranged in bilayers consisting of two closely spaced hexagonal layers, one with metallic cations and the other with anions. Thus, the bilayers should be either Ga-or N-faced [see Fig. 2(f)]. The term Ga-face (or (0001) surface) indicates $\mathrm{Ga}$ on the top position of the $\{0001\}$ bilayer. Similarly, N-face (or $(000 \overline{1})$ surface) indicates $\mathrm{N}$ on the bottom position of the $\{0001\}$ bilayer.

As has been discussed in Section II D, in order to achieve charge neutrality, the stoichiometry on the polar surfaces should deviate from the stoichiometry of the corresponding bulk termination. Indeed, previous DFT calculations on the (0001) GaN surfaces revealed that $2 \times 2$ surface reconstructions are the energetically most favorable one for a wide range of III/V ratios ${ }^{57}$ (see Fig. 6): For growth under N-rich conditions, the $2 \times 2 \mathrm{~N}$ adatom on the hollow site $\left(\mathrm{N}_{\mathrm{H} 3}\right)$ is the energetically most favorable one, while the $2 \times 2$ Ga vacancy $\left(\mathrm{V}_{\mathrm{Ga}}\right)$ structure is only slightly higher in energy. Going toward more Ga-rich growth conditions, the $2 \times 2 \mathrm{Ga}$ adatom $\left(\mathrm{Ga}_{\mathrm{T} 4}\right)$ configuration is favored. The electronic structure of the aforementioned reconstructions was studied by DFT as well as by in situ X-ray photoelectron spectroscopy (XPS) and ultraviolet photoelectron spectroscopy (UPS): ${ }^{15}$ These surfaces are semiconducting and are characterized by high densities of surface states near the VB edge, occupied intragap states, and unoccupied states $\approx 0.5-0.8 \mathrm{eV}$ below the CBM. The origin of the unoccupied surface states is the low coordinated Ga surface atoms. Thus, Fermi-level pinning in $n$-type $\mathrm{GaN}$ at about $0.5-0.8 \mathrm{eV}$ below the CBM is an inherent characteristic of the intrinsic (0001) GaN surfaces originating from the dangling bonds of low coordinated $\mathrm{Ga}$ surface atoms.

In MBE growth under Ga-rich conditions, a pseudo" $1 \times 1$ " reconstruction was observed. ${ }^{58}$ The term pseudo" $1 \times 1$ " relates to the fact that RHEED showed a " $1 \times 1$ " pattern, while low-energy electron diffraction (LEED) measurements suggested an incommensurate surface structure. The " $1 \times 1$ " GaN (0001) surface is the most Ga-rich reconstruction. Modelling of Auger spectral intensities indicated that it contains between 2 and 3 monolayer (ML) of Ga above the last GaN bilayer, while STM images revealed the height of the " $1 \times 1$ " Ga layer to be $3.8 \AA$, indicating $\approx 2 \mathrm{ML}$ of Ga atoms. ${ }^{58,59}$ Since the melting point of bulk Ga $\left(29.8^{\circ} \mathrm{C}\right)$ is very close to room temperature, it is reasonable to assume a fluid character of this Ga-rich surface.

Based on the first principle calculations, it was confirmed that, for growth under metal rich conditions, a surface with a total Ga coverage of $2.33 \mathrm{ML}$ is the energetically most favorable. ${ }^{57}$ This surface was modelled with a $\sqrt{3}$ $\times \sqrt{3}$ unit cell with two Ga layers atop the bare (0001) GaN surface. The bottom layer is coherent (i.e., pseudomorphic) to the Ga-polar surface and contains $3 \mathrm{Ga}$ atoms per $\sqrt{3}$ $\times \sqrt{3}$ surface cell and the top layer $4 \mathrm{Ga}$ atoms (see Figs. 6(d) and 6(e)). The lateral spacing of the $\mathrm{Ga}$ atoms in the overlayer is $\approx 2.75 \AA$ and is very close to the first nearestneighbors distance in bulk $\alpha$-Ga. Though the $\sqrt{3} \times \sqrt{3}$ 

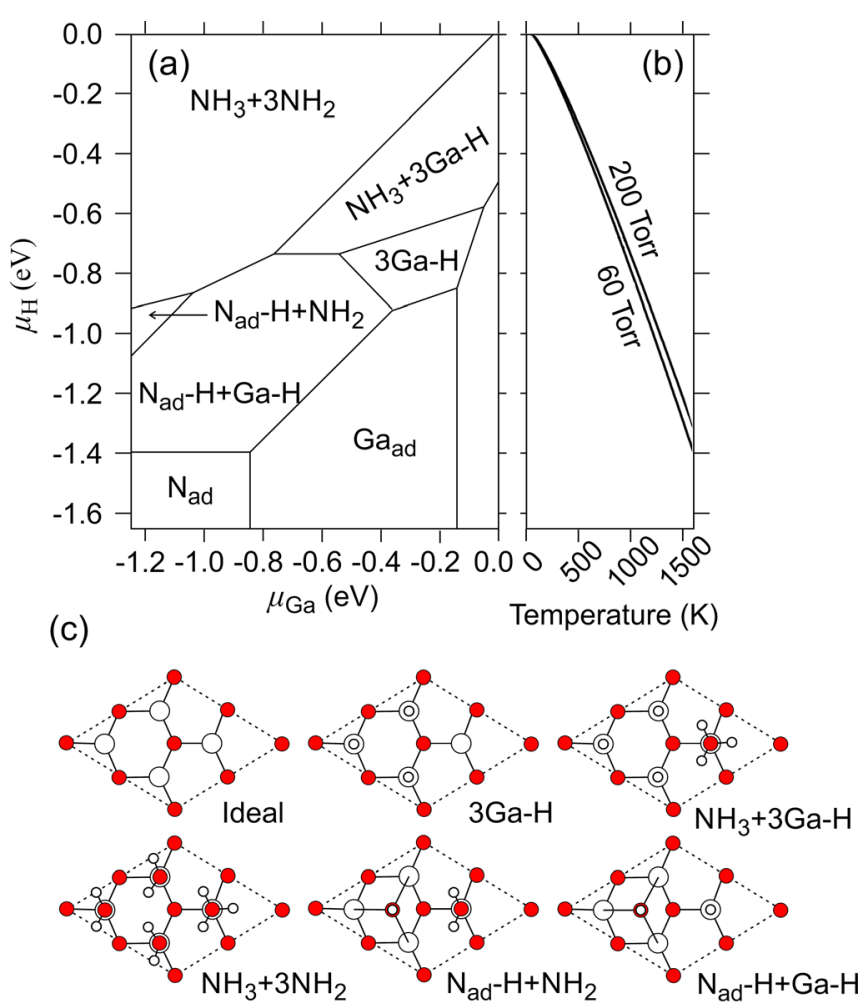

(d)

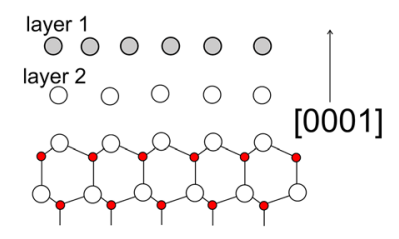

(e)

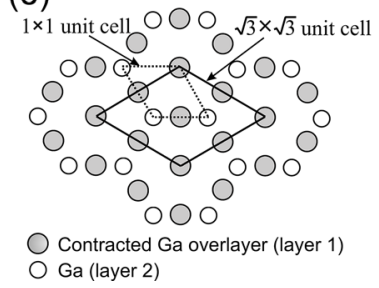

FIG. 6. (a) Phase diagram for the GaN (0001) surface in the presence of $\mathrm{H}$, as a function of $\mathrm{Ga}\left(\mu_{\mathrm{Ga}}\right)$ and $\mathrm{H}\left(\mu_{\mathrm{H}}\right)$ chemical potentials. $\mu_{\mathrm{H}}=0\left(\mu_{\mathrm{Ga}}=0\right)$ corresponds to $\mathrm{H}_{2}$ molecules (Ga bulk) at $T=0$, respectively. (b) Temperature dependence of $\mu_{\mathrm{H}}$ for two different pressures. Note that for extreme $\mathrm{H}$ poor conditions, i.e., $\mu_{\mathrm{H}}<-1.4 \mathrm{eV}$, the diagram denotes the energetically most stable structures for MBE relevant growth conditions. (c) Schematic representation (in top view) of the ideal and hydrogenated prevalent $2 \times 2$ reconstructions for $\mathrm{GaN}$ (0001) surfaces. Large open circles represent $\mathrm{Ga}$ atoms, solid red circles $\mathrm{N}$, and small open circles H. Adapted with permission from C. G. Van de Walle and J. Neugebauer, Phys. Rev. Lett. 88, 066103 (2002). Copyright 2002 American Physical Society. (d) and (e) Side and top view schematic representations of the $\sqrt{3} \times \sqrt{3}$ laterally contracted Ga bilayer structure. In (d), large gray and open (small and filled) balls denote $\mathrm{Ga}(\mathrm{N})$ atoms, respectively. In (e), only the Ga bilayer is shown.

reconstruction may not be the one observed in RHEED patterns, it constitutes an excellent model to simulate a $\mathrm{Ga}$ bilayer structure, where the interatomic distances of the $\mathrm{Ga}$ atoms in the topmost layer resemble those of liquid Ga.

Both polar and non-polar III-Nitrides surfaces obey the ECR for growth under N-rich to moderate metal-rich growth conditions. However, under metal-rich conditions, these surfaces exhibit a unique behaviour, without any analogue among other compound semiconductors, by stabilizing an excess metal surface. This peculiarity was explained in terms of the relatively small lattice constant of III-Nitrides, i.e., the lattice constant of $\mathrm{GaN}$ is $\approx 20 \%$ smaller than the lattice constant of GaAs, as well as the unique bond strength of $\mathrm{N}_{2}$ molecules: ${ }^{60}$ due to the smaller radius of $\mathrm{N}$ atoms, $\mathrm{Ga}$ atoms in $\mathrm{GaN}$ have approximately the same distance as in $\mathrm{Ga}$ bulk. Thus, metallic Ga-Ga bonds can straightforwardly form on the surface without the need of sizeable relaxation. Furthermore, there is a strong asymmetry of the binding properties of the end constituents of the binary III-Nitrides: The cohesive energy of bulk Ga is $2.81 \mathrm{eV} /$ atom, comparable to that of GaAs, which is $2.96 \mathrm{eV} /$ atom. Additionally, the $\mathrm{N}-\mathrm{N}$ bond in the $\mathrm{N}_{2}$ molecule is one of the strongest in nature with a binding energy as large as $5.0 \mathrm{eV} /$ atom. Therefore, more energy is required to transfer $\mathrm{N}$ atoms from their reservoir to the surface than to transfer Ga atoms to the surface.

The tendency of III-Nitrides surfaces to stabilize excess metal adlayers during growth under metal-rich conditions has important consequences, both on surface energetics as well as on adatom kinetics. The topmost layer of the Ga bilayer is expected to be liquid at typical growth conditions and, thus, feels only weakly the surface underneath. Thus, the surface energy can be minimized by minimizing the surface area. Since faceting increases the surface area, it is expected that it is unfavorable for growth under Ga-rich conditions. ${ }^{61}$ Furthermore, it was shown by DFT calculations that the presence of a metallic adlayer offers an efficient channel for below surface diffusion: ${ }^{62}$ It was shown that $\mathrm{N}$ atoms prefer to adsorb and sit below the top surface layer. Then, the diffusion of the $\mathrm{N}$ atom from one stable site to the next stable site is enhanced by the presence of the metallic adlayer, which largely compensates for the bonds that have to be broken (see Fig. 7). This mechanism results in considerably smaller diffusion barriers and allows to achieve smooth surface morphologies even at the relatively low MBE growth temperatures, i.e., at growth temperatures below $50 \%$ of the melting temperature.

The reconstructions on the $\mathrm{N}$ polar $(000 \overline{1})$ surface follow the same trends of the metal-polar III-Nitrides surfaces: For growth under N-rich to moderate metal-rich conditions, the semiconducting $2 \times 2$ reconstruction with a $\mathrm{Ga}$ adatom on the hollow site is the energetically most favorable one. For growth under metal-rich conditions, the surface is covered with one ML of Ga, where the Ga atoms sit on top of the $\mathrm{N}$ surface atoms. ${ }^{63}$

The theoretical investigations on hydrogen adsorption on $\mathrm{GaN}\{0001\}$ surfaces indicate the tendency of hydrogen to bind to GaN surfaces. Early works, as well as more recent studies, revealed that under hydrogen-rich conditions, and neglecting the presence of $\mathrm{NH}_{3}$, these surfaces are covered by $75 \%$ hydrogen. ${ }^{64-69}$ Furthermore, it was shown that, if $\mathrm{NH}_{3}$ is considered, then it dissociates to $\mathrm{H}$ and $\mathrm{NH}_{x}$ radicals. ${ }^{64,70}$ In all the cases, the lowest energy surfaces obey the ECR and hence are semiconducting. However, these works were restricted to rather limited number of selected surface structures and/or did not consider the effect of temperature and partial pressures on the surface energetics.

Based on the DFT calculations, Van de Walle and Neugebauer derived the surface phase diagram of Ga-polar GaN surfaces, ${ }^{71}$ including both temperature and partial pressure effects (see Fig. 6). They performed DFT calculations on a large number of different surface morphologies with $1 \times 1,2 \times 2$, and $\sqrt{3} \times \sqrt{3}$ periodicity. They found that, although no a priori assumptions had been made, all the 


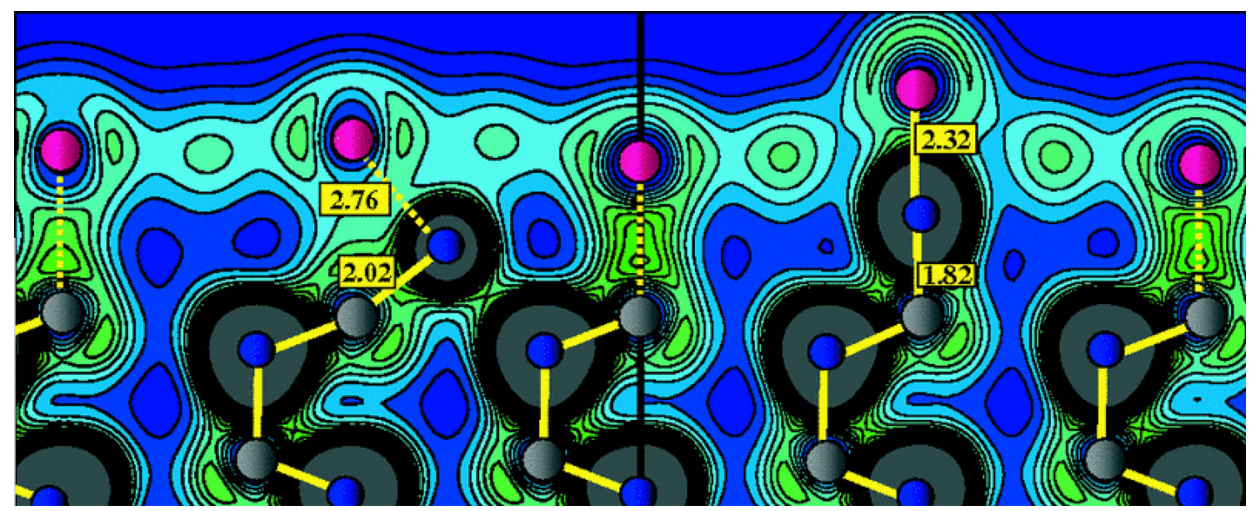

FIG. 7. Cross-sectional view of the valence charge density for a nitrogen adatom below a metallic adlayer at the (0001) GaN surface. Left (right): The N atoms is at the stable (barrier configuration for subsurface diffusion) subsurface site, respectively. Grey and dark grey (online color: blue) filled circles denote Ga and $\mathrm{N}$ atoms, respectively. The balls in the top layer (inline color: purple) denote In atoms. The numbers give bond lengths in $\AA$. Adapted with permission from Neugebauer et al., Phys. Rev. Lett. 90, 056101 (2003). Copyright 2003 American Physical Society.

lowest energy structures obey the ECR and that in a hydrogen-rich atmosphere, they exclusively exhibit $2 \times 2$ reconstructions. More specifically, at $T=0 \mathrm{~K}$ and for N-rich to moderate metal-rich conditions, one $\mathrm{NH}_{3}$ and three $\mathrm{NH}_{2}$ molecules are bounded to the four Ga surface atoms. At the limit of metal-rich conditions, a $\mathrm{NH}_{3}$ molecule is bounded to a $\mathrm{Ga}$ surface atom, and three $\mathrm{H}$ atoms saturate the remaining Ga dangling bonds. However, similar to the $m$-plane GaN surfaces, at typical growth conditions, entropy effects dominate and the $\mathrm{H}$ atoms are only weakly bounded to the surface.

Regarding the N-polar GaN surfaces, early results indicated that, under hydrogen-rich conditions, $3 / 4$ monolayer of $\mathrm{H}$ is adsorbed on the ideally cleaved surface. ${ }^{65}$ More recently, DFT calculations, where more surface structures were considered, confirmed and highlighted the strong affinity of hydrogen on the N-polar GaN surfaces: ${ }^{72}$ At typical MOCVD conditions, a $2 \times 2$ surface reconstruction with three hydrogens saturating three anion dangling bonds is the energetically most favorable one. At low hydrogen partial pressures, as those that correspond to high vacuum MBE conditions, a transition to $2 \times 2 \mathrm{Ga}$ adatom (for N-rich) or $1 \times 1 \mathrm{Ga}$ adlayer (for moderate metal rich-conditions) surfaces takes place.

The mechanisms of charge compensation and stabilization of polar $\mathrm{ZnO}$ surfaces have also been a matter of intense debate and controversy. In order to explain the stability of polar $\mathrm{ZnO}$ surfaces, early theoretical works investigated the fractional charge transfer of half an electron per surface unit cell from the O-polar $(000 \overline{1})$ to the Zn-polar (0001) surface. ${ }^{73}$ Although the charge transfer is in agreement with electrostatic arguments associated with the ionic model, the corresponding surface metallic states have not been observed yet. The cleavage energy of the polar $\mathrm{ZnO}$ surfaces was found to depend strongly on the slab thickness, due to the charge transferred from one side of the slab to the other. Nevertheless, assuming that the central bulk-like slab layers behave like a dielectricum of a capacitor, the cleavage energy was calculated to be $3.41 \mathrm{~J} / \mathrm{m}^{2}{ }^{74}$

A second scenario providing charge neutrality at the surfaces is to change the stoichiometry. This can be achieved by removing $1 / 4$ of the anions (cations) or by adding $1 / 4$ of cations (anions) to the $\mathrm{O}-(\mathrm{Zn}-)$ polar surface, respectively. Removing one in every four $\mathrm{Zn}$ atoms from the as-cleaved (0001) surface completely depletes the surface state. In contrast, adding one $\mathrm{O}$ atom in every 4 surface unit cells takes up the electrons from the surface state and results in a semiconducting surface. Indeed, DFT calculations revealed that, under O-rich conditions, the surface energy of the intrinsic (0001) $\mathrm{ZnO}$ surfaces is minimum, if $1 / 4 \mathrm{ML}$ of $\mathrm{Zn}$ atoms are removed or 1/4 ML of $\mathrm{O}$ atoms are adsorbed on the as-cleaved Zn-polar surface. ${ }^{74}$ The aforementioned reconstructions are similar to the $2 \times 2 \mathrm{Ga}$ vacancy and $\mathrm{N}$ adatom reconstructions of the (0001) GaN surfaces. Nevertheless, in contrast to $\mathrm{GaN}$, the formation of $\mathrm{Zn}$ adlayers on the $\mathrm{Zn}$ polar $\mathrm{ZnO}$ surface was found to be energetically unfavorable even under extreme $\mathrm{Zn}$-rich conditions. ${ }^{75}$

Motivated by the presence of nanosized triangular islands and pits revealed by STM on the (0001) ZnO surface, Dulub et al. investigated the energetics of triangularly shaped reconstructions. ${ }^{74,76}$ These reconstructions consist of triangular one-layer-deep pits. Such a pit of side length $n$ has exactly the same stoichiometry and number of cleaved bonds as found for $n$ isolated $\mathrm{Zn}$ vacancies at the surface. The authors found that it is energetically more favorable to accommodate $n \mathrm{Zn}$ vacancies in the form of triangular pits rather than in the form of single vacancies. They calculated that, for each single $\mathrm{Zn}$ vacancy incorporated into a triangular pit, the energy of the system is reduced by $300 \mathrm{meV}$. The preferential accommodation of $\mathrm{Zn}$ vacancies into triangular pits is attributed to the lower Madelung energy of the latter.

Another mechanism of charge compensation at the polar $\mathrm{ZnO}$ surfaces is the adsorption of hydrogen either in the form of $\mathrm{H}$ adatoms or in the form of hydroxyl ad-groups. Early DFT calculations indicated that under hydrogen-poor conditions, the surface phase diagram is dominated by triangular reconstructions. Moving towards more hydrogen-rich conditions, hydroxyl groups adsorb at the $\mathrm{H} 3$ sites of the surface, i.e., at three fold hollow sites with no $\mathrm{Zn}$ atoms beneath. $^{74,76}$ The typical hydroxyl coverage is $1 / 2 \mathrm{ML}$, which results in a semiconducting surface: the ideal (0001) $\mathrm{ZnO}$ surface is metallic due to the $\mathrm{Zn} 4 s$ surface state, which 
is occupied by half of an electron. The adsorption of $1 / 2 \mathrm{ML}$ of $\mathrm{OH}$ on the aforementioned surface forms a low lying acceptor level that depletes the Zn $4 s$ surface state. Nevertheless, even at the hydrogen-rich limit of the thermodynamically allowed range of hydrogen chemical potential, adsorption of atomic $\mathrm{H}$ on the $\mathrm{Zn}$-terminated surface is thermodynamically unstable. More recent calculations highlighted the importance of the vibrational entropic contributions from the surface atoms to the surface free energy ${ }^{77}$ In GaN, only entropic contributions to the chemical potentials of the gas phases are considered important. ${ }^{71,78}$ However, for the $\mathrm{ZnO}$ (0001) surfaces, a large number of different reconstructions have very similar stoichiometries, and their surface energies lie within a rather narrow range of $20-30 \mathrm{meV}$. Due to the high vibrational entropy of surface atoms in adatom structures at $\mathrm{ZnO}$ surfaces, differences in the vibrational entropy dominate the free energy differences. More specifically, it was shown that at low hydrogen partial pressures and low temperatures, the surface phase diagram is dominated by triangular reconstructions. However, as the temperature is raised, entropic contributions lower the energy of the $2 \times 2 \mathrm{O}$ adatom reconstruction, which eventually prevails (see Fig. 8).

The aforementioned calculations investigate, however, the surface reconstructions under conditions of thermodynamic equilibrium, i.e., the maximum values for the $\mathrm{O}$ and $\mathrm{H}$ chemical potentials are those of $\mathrm{H}_{2}$ and $\mathrm{O}_{2}$ molecules, respectively. Furthermore, thermodynamically metastable structures were not considered. However, kinetic limitations may be important and have to be considered. These limitations may arise from the requirement of massive rearrangements of atoms at the surface to reach the thermodynamically stable structure. Furthermore, adsorption/desorption kinetics or the use of a plasma source may drive the hydrogen chemical potential to values higher than that of a $\mathrm{H}_{2}$ molecule. Valtiner et $a l .{ }^{79}$ constructed a metastable phase diagram for (0001) $\mathrm{ZnO}$ surfaces that includes kinetically stabilized surface reconstructions and goes beyond the thermodynamic limit, which allowed them to explain the experimental observation of a "1 $1 \times 1$ " surface reconstruction. ${ }^{80}$ They found that under an activated $\mathrm{H}_{2}$ atmosphere, which is achieved if a hot filament is used to dissociate the $\mathrm{H}_{2}$ molecules, simultaneous passivation of the $\mathrm{O}$ dangling bonds at the step edges and of the $\mathrm{Zn}$ surface dangling bonds, within a triangular reconstruction, takes place.

Based on electrostatic considerations, charge compensation on the $(000 \overline{1}) \mathrm{ZnO}$ surfaces can be achieved by the removal of $1 / 4 \mathrm{ML}$ of $\mathrm{O}$ atoms. Indeed, early theoretical works indicated that a $2 \times 2$ reconstruction with an $\mathrm{O}$ vacancy is the energetically most favorable intrinsic $(000 \overline{1}) \mathrm{ZnO}$ surface for a wide range of $\mathrm{O}$ chemical potentials, i.e., for $\mathrm{O}_{2}$ partial pressure less than $10^{-5} \mathrm{mbar}$ at $T=800 \mathrm{~K} .{ }^{81}$ Furthermore, a $1 \times 3$ reconstruction with $33 \%$ oxygen vacancies at the surfaces, i.e., where every third oxygen atom in the $[1 \overline{1} 00]$ direction has been removed, was also considered. ${ }^{82}$ The presence of this reconstruction was corroborated by the agreement between experimentally and theoretically determined $\mathrm{CO}$ binding energies. However, this surface is electrostatically not stable and slight changes of the stoichiometry or the adsorption of one $\mathrm{H}$ atom are required to achieve charge neutrality. Nevertheless, a

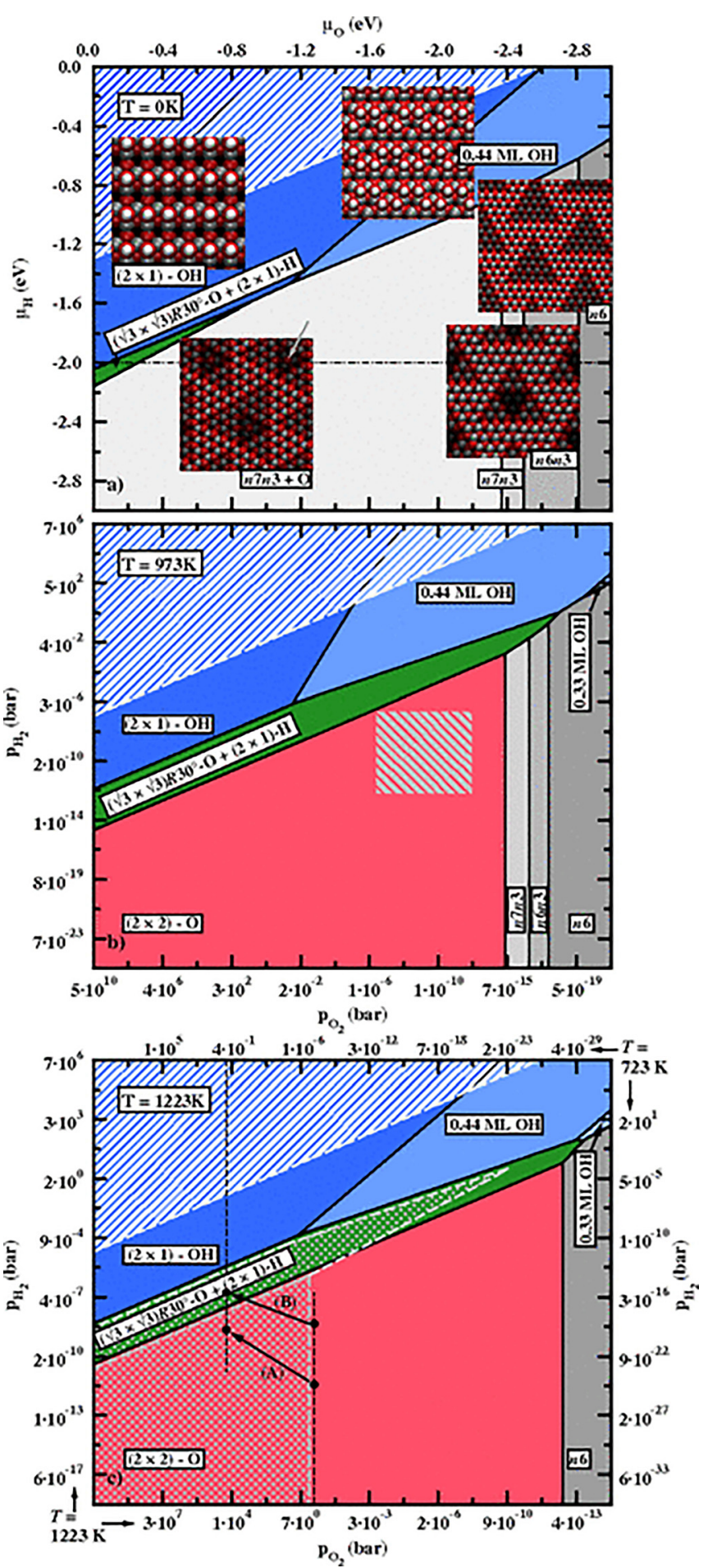

FIG. 8. Phase diagram for the $\mathrm{ZnO}$ (0001) surface in equilibrium with a humid oxygen atmosphere for different temperatures. In the hashed area in the upper left corners, the compensating phase of water is thermodynamically favorable and would condensate on the surface. (a) $T=0 \mathrm{~K}$. The geometries of selected stable surface structures, in top view, are shown as insets. The gray arrow in the representation of the $n 3 n 7+\mathrm{O}$ reconstruction indicates the oxygen atom. (b) $T=973 \mathrm{~K}$. (c) $T=1223 \mathrm{~K}$, the geometry of the $2 \times 2-\mathrm{O}$ in top view is shown as inset. Adapted with permission from Valtiner et al., Phys. Rev. Lett. 103, 065502 (2009). Copyright 2009 American Physical Society.

theoretical confirmation of the stability of the $1 \times 3$ reconstruction with $33 \%$ oxygen vacancies is still lacking. ${ }^{83}$

More recent DFT works on larger $\mathrm{ZnO}(000 \overline{1})$ surface unit cells revealed that at larger values of $\mathrm{O}$ chemical 
potential, a $5 \times 5$ reconstruction prevails. ${ }^{84}$ This reconstruction consists of a single layer deep hexagonal hole formed by removing $11 \mathrm{O}$ atoms and $7 \mathrm{Zn}$ atoms from the topmost layer. In the topmost surface layer, half of the $\mathrm{O}$ atoms in the $5 \times 5$ surface cell are arranged in a hexagon following the regular wurtzite $\mathrm{ZnO}$ stacking sequence along $000 \overline{1}$ and the other half in a hexagon following the zinc-blende stacking sequence. However, the stoichiometry of this reconstruction does not allow for charge compensation at the surface and the adsorption of $\mathrm{H}$ atoms to form hydroxyl groups is necessary. Indeed, the authors revealed that for $\mathrm{H}_{2}$ partial pressure as low as $\approx 10^{-25} \mathrm{mbar}$ at $T=450^{\circ} \mathrm{C}$, it is energetically favorable to form $\mathrm{OH}$ groups. The coverage of $\mathrm{OH}$ is increased with the hydrogen chemical potential. The very different reconstruction pattern of the $(000 \overline{1})$ with respect to the (0001) $\mathrm{ZnO}$ surfaces is explained in terms of the higher bonding flexibility of the 3 -fold coordinated surface $\mathrm{Zn}$ atoms as compared with the $\mathrm{O}$ atoms.

It is worth mentioning here that slabs exposing only one type of non-polar surface can be constructed. Thus, absolute surface energies can be calculated. On the other hand, due to the low symmetry of wurtzite crystal, i.e., the presence of a single polar axis, a slab or a cluster bounded only by one type of wurtzite polar or semipolar surfaces cannot be constructed. Hence, absolute surface energies for polar or semipolar planes are fundamentally ill-defined. However, the higher symmetry of zinc-blende structure allows the construction of tetrahedral clusters or infinitely long triangular wedges, bounded only by one of the polar (111) or ( $\overline{1} \overline{1} \overline{1})$ surfaces. ${ }^{85}$ Dreyer et al. followed a rather pragmatic approach and invoked the similarities between the wurtzite $(0001)$ and $(000 \overline{1})$ and the zincblende (111) and ( $\overline{1} \overline{1} \overline{1})$ surfaces, respectively. ${ }^{86}$ Using absolute surface energies for the hydrogenated bottom site of the polar slabs and the energies of the corresponding zinc-blende surfaces, they calculated absolute energies of the technologically most relevant polar GaN surfaces. Their calculations revealed that the Ga-polar surfaces have lower energies than the $\mathrm{N}$-polar ones for all the experimentally relevant growth conditions.

\section{G. Semipolar surfaces}

Apart from the polar and non-polar surfaces, semipolar ones have attracted considerable interest for the growth of III-Nitrides heterostructures. Semipolar planes are (hkil) planes with a nonzero $h, k$, or $i$ and nonzero $l$ Miller index (see Fig. 9). Semipolar growth is expected to reduce or even eliminate the problems associated with the polarization fields: calculations by Romanov et al. on the elastic strains in anisotropic mismatched III-Nitrides layers and the corresponding strain-induced polarization in (In, Ga)N/GaN heterostructures predicted that the polarization discontinuity at the interface is considerably reduced for the semipolar $(11 \overline{2} 2)$ as compared with the polar (0001) interface. ${ }^{87}$ This finding was further confirmed by Northrup, ${ }^{88}$ who employed updated values for the piezoelectric coefficients for $\mathrm{GaN}$ and $\mathrm{InN}$ and included the effect of bowing in the calculation of the spontaneous polarization. He found that the polarization discontinuity at $\mathrm{In}_{0.3} \mathrm{Ga}_{0.7} \mathrm{~N} / \mathrm{GaN}$ heterostructure can be reduced from more than $0.04 \mathrm{C} / \mathrm{m}^{2}$ for the polar interface to about $-0.002 \mathrm{C} / \mathrm{m}^{2}$ for the semipolar $(11 \overline{2} 2)$ interface (see Fig. 10).

The energetics and electronic structure of the semipolar $(11 \overline{2} 2)$ and (1101) GaN and InN surfaces as well as that of $\{11 \overline{2} 2\}$ AlN surfaces were addressed by first principles calculations. ${ }^{89-91} \mathrm{GaN}$ and AlN semipolar surfaces follow the general trends for surface reconstructions on III-Nitrides surfaces: The $(11 \overline{2} 2)$ surfaces stabilize semiconducting reconstructions for $\mathrm{N}$-rich to moderate metal-rich growth conditions, while a transition from semiconducting to metallic reconstructions occurs at growth under extreme metalrich conditions. In contrast, the $(11 \overline{2} \overline{2})$ AlN surfaces favour semiconducting reconstructions only under extreme N-rich conditions. ${ }^{91}$ However, metallic $\mathrm{InN}(11 \overline{2} 2)$ reconstructions in the form of In adlayers were calculated to be the energetically most favorable one even under extreme N-rich conditions. The authors attributed this finding to the rather narrow thermodynamically allowed range of the In chemical potential. As with polar and non-polar III-Nitrides surfaces, hydrogen-rich conditions stabilize ECR reconstructions at semipolar surfaces where the $\mathrm{N}$ dangling bonds are saturated by $\mathrm{H}$ atoms and the $\mathrm{Ga}$ dangling bonds are saturated by $\mathrm{H}$ atoms or $\mathrm{NH}_{x}(x=1,2)$ molecules. ${ }^{92,93}$

\section{ASSESSING POLARITY AND SPONTANEOUS POLARIZATION}

Different techniques to assess the polarity of thin films as well as of individual NWs and NW ensembles are presented, including chemical etching, TEM-, x-ray diffraction (XRD)-, and AFM-based experiments. The basic principles of each of these methods, together with their assets and limitations, are introduced and illustrated through representative (a)

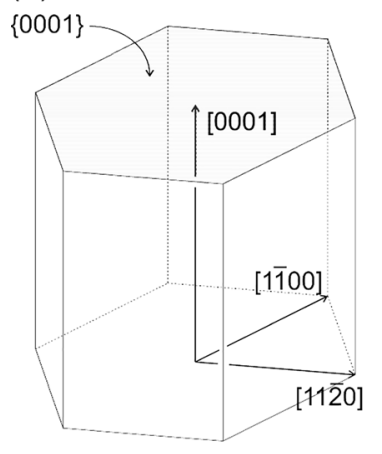

(b)

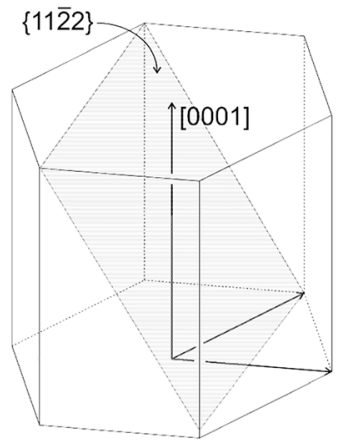

(c)

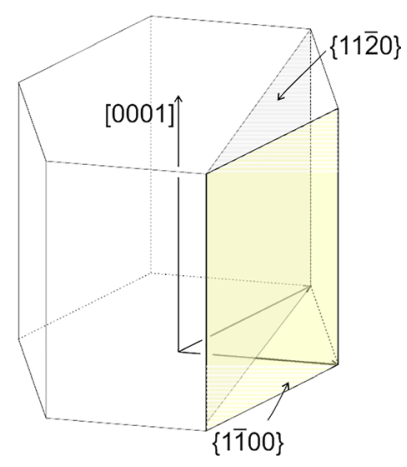

FIG. 9. Schematic representation of selected low index planes in the wurtzite crystal. The shaded areas denote in (a) the polar $\{0001\}$, in (b) the semipolar $\{11 \overline{2} 2\}$, and in (c) the non-polar $\{1 \overline{1} 00\}$ and $\{11 \overline{2} 0\}$ planes. 


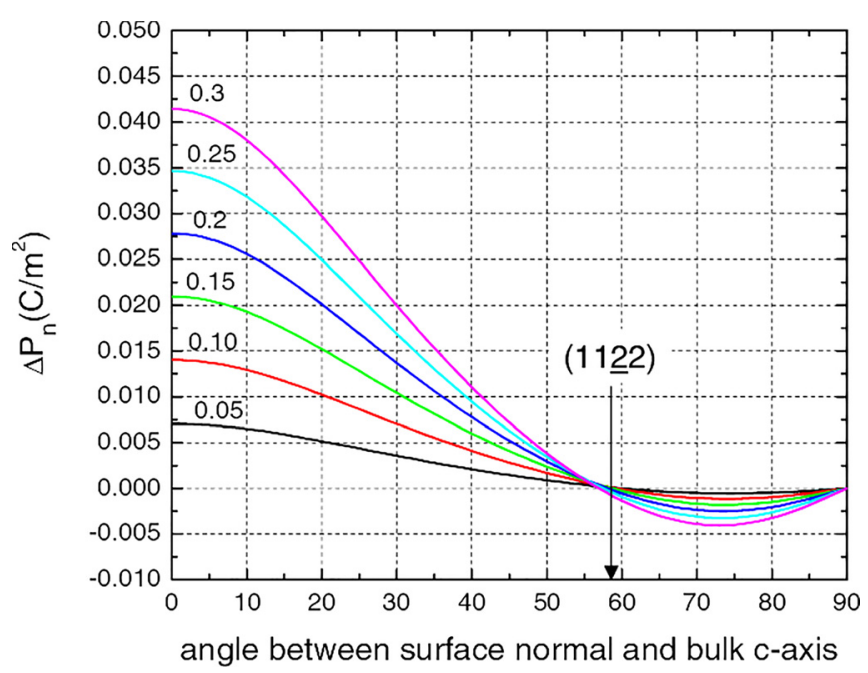

FIG. 10. Polarization discontinuity in the normal component at an interface between $\mathrm{GaN}$ and $\operatorname{In}_{x} \mathrm{Ga}_{1-x} \mathrm{~N}$ in $\operatorname{In}_{x} \mathrm{Ga}_{1-x} \mathrm{~N} / \mathrm{GaN}$ heterostructures. The arrow indicates the angle between the (112 2$)$ surface normal and the bulk [0001]axis. Each curve corresponds to the In content $x$ as indicated. Adapted with permission from Appl. Phys. Lett. 95, 133107 (2009). Copyright 2009 AIP Publishing LLC.

examples. It is worth noticing that some of these techniques are designed for measuring the polarity on the microscopic scale, while others asses the polarity on the macroscopic scale. Furthermore, most of the polarity assessment techniques presented here are also able to provide information on other aspects of the investigated materials.

\section{A. Sensitive chemical etching}

Wet chemical etching is certainly the most straightforward way for determining the polarity of $\mathrm{GaN}$ and $\mathrm{ZnO}$ thin films and NWs. The principle is based on the fact that the etching behavior is strongly dependent upon the chemical nature of the surface. Both the resulting etching rate, etched surface shape, and roughness are determined by the polarity, which can therefore be unambiguously identified.

In the case of $\mathrm{Zn}$ - and $\mathrm{O}$-polar $\mathrm{ZnO}$ surfaces, nitric acid $\left(\mathrm{HNO}_{3}\right)^{94}$ and hydrochloric acid ${ }^{95-99}(\mathrm{HCl})$ were usually found to induce a strong disparity in the etching behavior as well as acetic acid, ${ }^{98}$ sulfuric acid, ${ }^{99}$ and $\mathrm{CP} 4,{ }^{100}$ as shown in Figs. 11(a)-11(d). In contrast, hot phosphoric acid $\left(\mathrm{H}_{3} \mathrm{PO}_{4}\right)$ up to $220^{\circ} \mathrm{C},{ }^{99,101-103}$ sodium oxide $(\mathrm{NaOH}),{ }^{104-106}$ and potassium hydroxide $(\mathrm{KOH})^{99,104-113}$ were shown to induce a strong disparity in the etching behavior in the case of Ga- and N-polar GaN surfaces, as presented in Figs. $11(\mathrm{e})-11(\mathrm{k})$. It was revealed that the etching rate is much slower (i.e., one order of magnitude) on $\mathrm{Zn}$-polar $\mathrm{ZnO}$ and Ga-polar GaN surfaces than on O-polar $\mathrm{ZnO}$ and $\mathrm{N}$-polar GaN surfaces. ${ }^{95-99,104-113}$ Stepped hexagonal pits exhibiting basal plane terraces are created on $\mathrm{Zn}$-polar $\mathrm{ZnO}$ and $\mathrm{Ga}-$ polar GaN surfaces, typically where holes or dislocations intersecting the surface occur, as revealed in Figs. 11(a) and 11(g). In contrast, more uniformly distributed hexagonal pyramids are formed on O-polar $\mathrm{ZnO}$ and $\mathrm{N}$-polar $\mathrm{GaN}$ surfaces, as depicted in Figs. 11(b), 11(c), and 11(e): the angle of the side facets of the pyramids with the basal plane is around $61^{\circ}$, revealing that the $\{10 \overline{11}\}$ side facets are involved as the slowest etching plane due to their low coordination number (see Figs. 11(d) and 11(f)).

The wet chemical etching mechanisms operating on both $\mathrm{Zn}$ - and O-polarity as well as Ga- and N-polarity when using acid etchants were accounted for in the framework of the dangling bond model, as initially proposed by Mariano
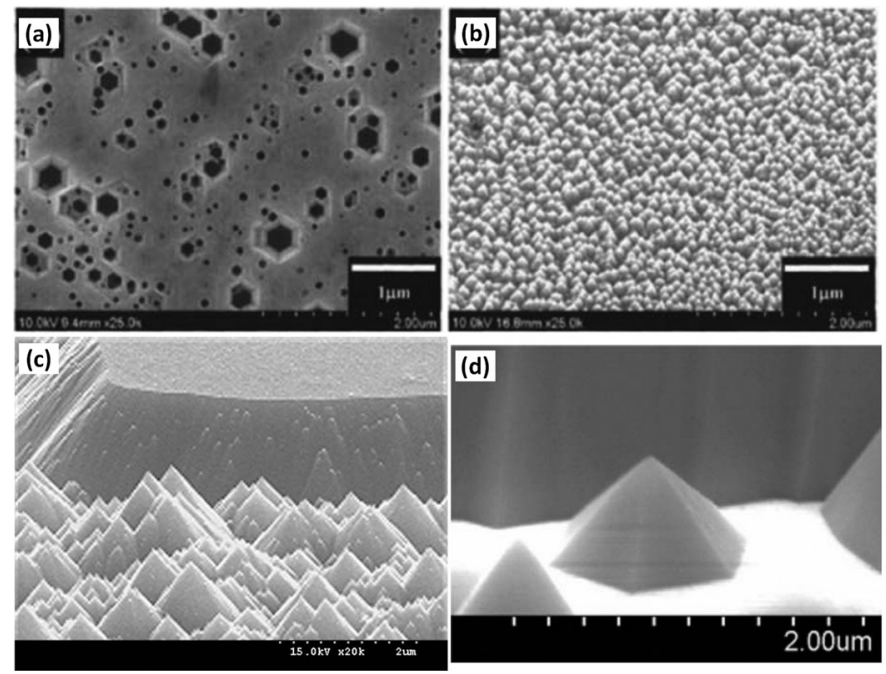

(d)

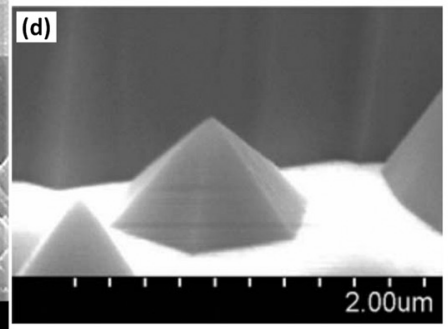

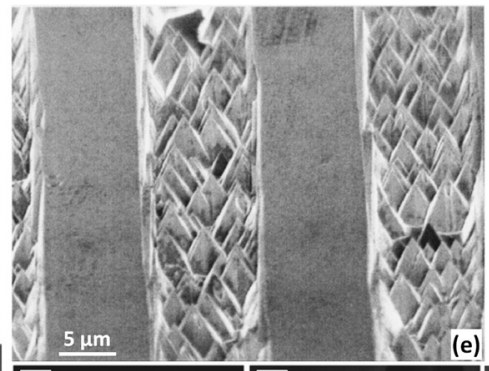
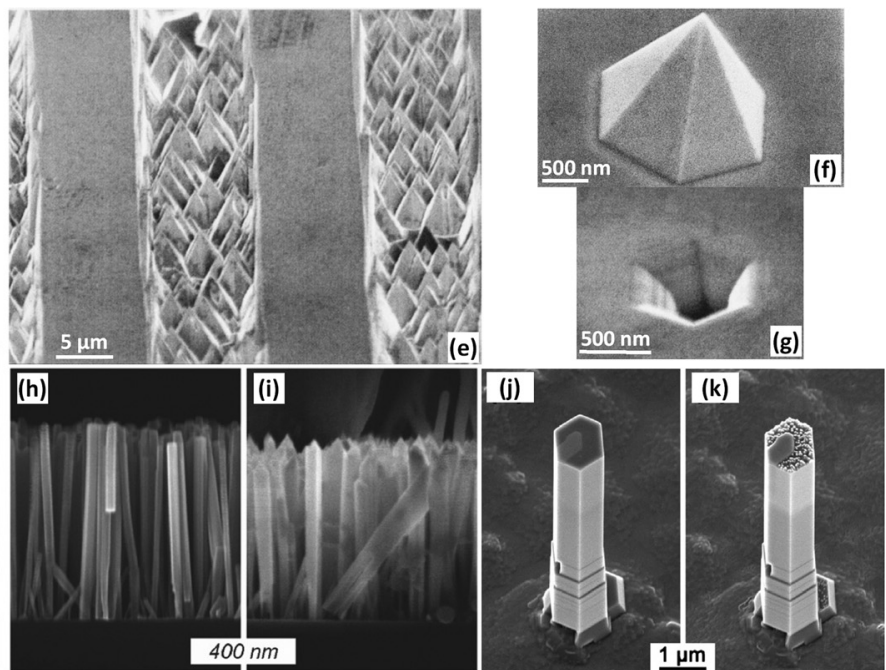

FIG. 11. Top-view SEM images of (a) the etched $\mathrm{Zn}$-polar $\mathrm{ZnO}$ thin film and of (b) the etched O-polar $\mathrm{ZnO}$ thin film grown by MBE on c-sapphire with a MgO buffer layer using a $12 \mathrm{mM} \mathrm{HCl}$ solution. Reprinted with permission from Appl. Phys. Lett. 87, 141904 (2005). Copyright 2005 AIP Publishing LLC. Crosssectional SEM image of (c) the etched O-polar ZnO thin film grown by radio-frequency magnetron sputtering on c-sapphire using $1 \% \mathrm{HCl}$ solution with (d) a high magnification SEM image showing the formation of hexagonal pyramids with $\{10 \overline{11}\}$ side facets. Reprinted with permissions from Han et al., J. Electrochem. Soc. 157, D60 (2010). Copyright 2010 The Electrochemical Society. SEM image of (e) the etched Ga- and N-polar GaN thin film grown by $\mathrm{MBE}$ on templated c-sapphire with an Al buffer layer or not, respectively, using a $2 \mathrm{M} \mathrm{KOH}$ solution at $90^{\circ} \mathrm{C}$. High magnification SEM images revealing the formation of (f) hexagonal pyramids on the N-polar GaN thin film and of (g) stepped hexagonal pits on the Ga-polar GaN thin film. Reprinted with permission from J. Appl. Phys. 94, 650 (2003). Copyright 2003 AIP Publishing LLC. Cross-sectional SEM image of N-polar GaN NWs grown by MBE on Si(111) (h) before and (i) after etching using a saturated $\mathrm{KOH}$ solution at room temperature. Reprinted with permission from Hestroffer et al., Phys. Rev. B 84, 245302 (2011). Copyright 2011 American Physical Society. SEM image of GaN NWs with mixed polarities grown by MOVPE on c-sapphire (j) before and (k) after etching using KOH solution. Reprinted with permission from Appl. Phys. Lett. 98, 011914 (2011). Copyright 2011 AIP Publishing LLC. 
and Hanneman as regards polar $\mathrm{ZnO}$ surfaces. ${ }^{94}$ Basically, the surface atoms on the perfect polar planes are bonded with three nearby atoms in the bulk, while the underlying atom is only bonded with one atom in the bulk, forming a double layer (see Fig. 2(f)). As discussed in Section II F, in the case of O-polar $\mathrm{ZnO}$ or N-polar GaN surfaces, the dangling bonds of the $\mathrm{O}$ or $\mathrm{N}$ surface atoms have a partial negative charge and strongly interact with positively charged hydronium ions released from acid etchants, respectively, resulting in a high etching rate. In contrast, in the case of $\mathrm{Zn}$ polar $\mathrm{ZnO}$ or Ga-polar GaN surfaces, the dangling bonds of the $\mathrm{Zn}$ or Ga surface atoms have a partial positive charge and, thus, the interaction with positively hydronium ions released from acid etchants is inhibited owing to charge repulsion, resulting in a very low etching rate. However, it was found from STM that small triangular holes (width $<5 \mathrm{~nm}$ ) with single height, O-terminated step edges are formed on top of the $\mathrm{Zn}$-polar $\mathrm{ZnO}$ surfaces. ${ }^{76}$ These holes favor the interaction of hydronium ions with the $\mathrm{O}$ atoms located underneath the $\mathrm{Zn}$ surface atoms and thus form in turn hexagonal pits with basal plane terraces. A similar feature was pointed out on top of Ga-polar GaN surfaces through the interaction of hydronium ions with $\mathrm{N}$ atoms located underneath the Ga surface atoms. ${ }^{99}$

A distinct wet chemical etching mechanism of both $\mathrm{Ga}-$ and N-polarity, when using $\mathrm{KOH}$, was emphasized by $\mathrm{Li}$ et al. from XPS. ${ }^{108,109} \mathrm{KOH}$ is here expected to act as a catalyst to form gallium oxide through the release of hydroxide $\left(\mathrm{OH}^{-}\right)$ions adsorbing on the surface and reacting with $\mathrm{Ga}$ atoms. It also acts as a solvent to dissolve the resulting gallium oxide surface layer. The process can be described as follows: ${ }^{108,109}$

$$
2 \mathrm{GaN}+3 \mathrm{H}_{2} \mathrm{O} \stackrel{\mathrm{KOH}}{\longrightarrow} \mathrm{Ga}_{2} \mathrm{O}_{3}+2 \mathrm{NH}_{3} .
$$

In the case of N-polar GaN surfaces, only one single negatively charged dangling bond from the $\mathrm{N}$ surface atom occurs. $\mathrm{OH}^{-}$ions released from $\mathrm{KOH}$ can thus attack the $\mathrm{Ga}-\mathrm{N}$ bond below and be adsorbed to form gallium oxide, which is subsequently dissolved by $\mathrm{KOH}$. There is no dependence on the surface termination layer for the present wet chemical etching mechanism of N-polar GaN surfaces. In contrast, in the case of Ga-polar GaN surfaces, once the Ga surface atoms are removed, $\mathrm{OH}^{-}$ions could not attack the $\mathrm{Ga}-\mathrm{N}$ bond below due to its large charge repulsion with the triple negatively charged dangling bonds from the $\mathrm{N}$ surface atoms.

Furthermore, the etching rate was found to be dependent upon the etchant nature and concentration, as well as the temperature used..$^{99,101-113}$ Typically, in the case of polar $\mathrm{GaN}$ surfaces, increasing the $\mathrm{KOH}$ concentration increases the etching rate by releasing more $\mathrm{OH}^{-}$ions. Also, raising the temperature results in a higher etching rate by forming $\mathrm{OH}^{-}$ions with a larger kinetic energy. The etching rate was also revealed to be higher with hot $\mathrm{H}_{3} \mathrm{PO}_{4}$ than with $\mathrm{KOH}$ : the activation energies of 3.2 and $2.97 \mathrm{kcal} / \mathrm{mol}$ were reported for etching with $\mathrm{H}_{3} \mathrm{PO}_{4}$ and $\mathrm{KOH}$, respectively, indicating that the etching rate is diffusion-limited. ${ }^{99,114}$ The evolution of the hexagonal pyramids following the wet chemical etching of N-polar $\mathrm{GaN}$ surfaces using $\mathrm{KOH}$ involves their formation, growth, dissociation, and isolation. ${ }^{99}$ In contrast, the evolution of the hexagonal pits from the wet chemical etching of Ga-polar GaN surfaces using $\mathrm{H}_{3} \mathrm{PO}_{4}$ involves their formation, lateral widening, and merging. ${ }^{99}$ The use of more diluted $\mathrm{HCl}$ and acetic acid was also found to form rings with rounded shapes as well as sharp edges and corners on O-polar $\mathrm{ZnO}$ single crystals. ${ }^{95,98}$

The wet chemical etching using $\mathrm{H}_{3} \mathrm{PO}_{4}$ or $\mathrm{KOH}$ was used to determine the polarity of GaN single crystals, ${ }^{104,106}$ thin films deposited by plasma-assisted MBE (PAMBE), 107,110,111 MOCVD, ${ }^{108,109,112,113}$ and HVPE, ${ }^{108}$ as well as of GaN NWs grown by PAMBE ${ }^{15-117}$ and MOCVD, ${ }^{118,119}$ respectively. Similarly, the wet chemical etching using $\mathrm{HCl}$ was employed to investigate the polarity of $\mathrm{ZnO}$ single crystals ${ }^{94,95,98}$ and thin films deposited by PAMBE. ${ }^{96,97}$

\section{B. Transmission electron microscopy}

Transmission electron microscopy (TEM) techniques are able to determine the absolute polarity of wurtzite $\mathrm{GaN}$ and $\mathrm{ZnO}$ crystals with high spatial resolution and sensitivity and were used to analyze thin films, planar multi-layers, anti-phase domains, or low-dimensional semiconductor systems like quantum dots and NWs. The TEM techniques basically utilize electron diffraction, spectroscopic, or imaging information for the nanoanalysis, including convergent-beam electron diffraction (CBED), energy dispersive x-ray spectroscopy (EDS), and electron energy-loss spectroscopy (EELS), as well as high-resolution Z-contrast scanning transmission electron microscopy (STEM). TEM measurements have the advantage of combining information about the local crystal polarity with other structural properties like surface morphology or extended defects.

\section{Convergent beam electron diffraction (CBED)}

CBED is a well-established and widely used method to determine the polarity of non-centrosymmetric crystals. Initially, the method was applied to $\mathrm{GaN}$ and $\mathrm{ZnO}$ epitaxial thin films, ${ }^{120-124}$ and for the definition of inversion domain boundaries (IDBs). ${ }^{125}$ Fundamentally, the CBED method uses the breakdown of Friedel's law caused by the contribution of thickness-dependent multi-beam scattering processes, i.e., $\mathrm{I}_{\mathrm{g}}(\mathrm{t}) \neq \mathrm{I}_{\bar{g}}(\mathrm{t}) .{ }^{126}$ Thus, the intensity distributions $\mathrm{I}_{\mathrm{g}}(\mathrm{t})$, $\mathrm{I}_{\overline{\mathrm{g}}}(\mathrm{t})$, within the $\mathrm{g}$ and $\overline{\mathrm{g}}$ diffraction discs, are different and this difference can be attributed to the $\mathrm{Ga}(\mathrm{Zn})$ and $\mathrm{N}(\mathrm{O})$ distribution within the unit cell. Conventionally, the CBED pattern is taken along a $\langle 1 \overline{1} 00\rangle$ zone axis of the wurtzite crystal lattice because, on the one hand it contains the (0002) and $(000 \overline{2})$ reflections, which are sensitive to the crystal polarity, and on the other hand the CBED discs are well separated in practice (Fig. 12(a)). The exact zone axis orientation is achieved if the intensity fringes (i.e., the so-called Kossel-Möllenstedt fringes) ${ }^{127}$ within the central (0000) disc display a symmetric arrangement. Because of the strong thickness dependency of the scattered intensities $\mathrm{I}_{0002}(\mathrm{t})$ and $\mathrm{I}_{000 \overline{2}}(\mathrm{t})$, the CBED patterns have to be simulated for various sample thicknesses using dynamic electron-diffraction theory, ${ }^{128}$ until the asymmetrical intensity distributions within 


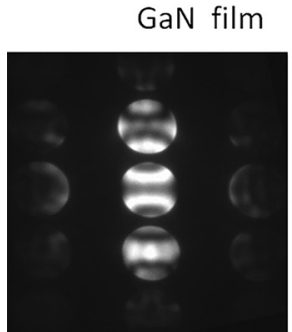

$\operatorname{CBED}(1-100)$

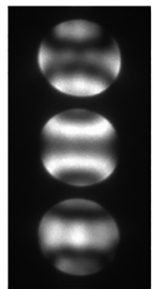

Exp.

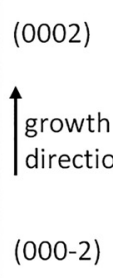

$(000-2)$

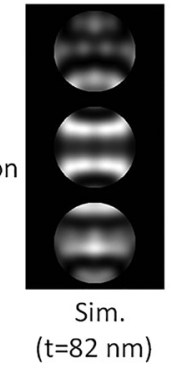

GaN nanowire
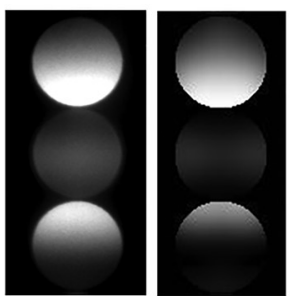

Sim.

$(\mathrm{t}=28 \mathrm{~nm})$
(0002)

(0000)

$(000-2)$
FIG. 12. Experimental [10̄̄o] CBED patterns and simulations of GaN film (a) and GaN NW (b) grown by MBE. The patterns were recorded with a Jeol 3010 microscope operating at $300 \mathrm{kV}$. The convergence semi-angle was set to $3.5 \mathrm{mrad}$. the $(0002)$ and $(000 \overline{2})$ diffraction discs match between experiment and calculation, and, thus the polarity is identified. To confirm the result of the polarity determination, $1 \overline{1} 00$ CBED patterns should be recorded for different thicknesses attainable at different sample regions and fitted by simulations.

Fig. 12(a) shows a CBED pattern along the [1100] zone axis of a GaN film deposited on AlN buffered Si(111) substrate. The distinct variation of the Kossel-Möllenstedt fringes inside the diffraction discs allows clear assignment of a given calculated pattern, including their definite Miller indices and, thus, the determination of the polarity. The simulations do not include the effect of temperature on the atomic scattering factors or the influence of absorption of incident electrons. However, it is expected that these contributions do not modify the polarity result, although a systematic investigation remains to be done for $\mathrm{GaN}$ and $\mathrm{ZnO}$, in particular, for larger sample thicknesses. ${ }^{129}$ A drawback limiting a precise applicability of the CBED method consists in the necessity of relative large probe volumes, i.e., sample thicknesses and generating dynamic diffraction. For this reason, the technique becomes very challenging for GaN or $\mathrm{ZnO}$ objects with very small dimensions, as, for instance, NWs with diameters far below $50 \mathrm{~nm}$. Fig. 12(b) shows the CBED pattern of a GaN NW with radius of about $14 \mathrm{~nm}$. It is obvious that the missing dynamic contribution inside the diffraction discs limits the significance of the polarity in the obtained result and requires supplementary methods of investigation.

\section{Electron energy loss spectroscopy (EELS)}

Channeling-enhanced microanalysis, including EELS in a TEM, represents an alternative technique to the electron diffraction method offering both a higher spatial resolution and a minor dependency on the sample thickness. The microanalysis technique originates from the approach of Taftø, ${ }^{130}$ who measured a pronounced difference of the characteristic $\mathrm{x}$-ray emission intensities between $\mathrm{g}_{111}$ and $\mathrm{g}_{\overline{1} \overline{1} \overline{1}}$ Bragg conditions for the polar direction in GaAs. This energydispersive $\mathrm{x}$-ray microanalysis method was also applied successfully to polarity determination in GaN films. ${ }^{131}$ In the related EELS experiment, the core-loss edges are used instead of the x-ray emission. ${ }^{132}$ As an example, Fig. 13 presents the results of two EELS spectra taken from a thin GaN NW that is tilted into (0002) and (000 $\overline{2})$ two-beam Bragg condition, respectively. ${ }^{133}$ The spectra were acquired in the diffraction mode with a collective semi-angle of about $3.5 \mathrm{mrad}$. The background of the $\mathrm{N} K$-edge was subtracted and the spectra were normalized at the Ga $L$-edges. It is evident that the $\mathrm{N} K$-edge peak collected at the (0002) orientation has higher intensity compared with the $(000 \overline{2})$ case. This result can be explained on the basis of the theory of fast electrons propagating in a thin crystal as a superposition of Bloch waves. ${ }^{134}$ In case of the noted two-beam Bragg condition, the electron distribution is highly localized at special atom planes as a consequence of the interference between the Bloch waves. The thickness-averaged electron current intensity is given as ${ }^{135,136}$

$$
\begin{aligned}
I(\vec{r}) & =\frac{1}{t} \int_{0}^{t}|\psi(z)|^{2} d z \\
& =1-\frac{1-\cos \left(2 \pi t / \xi_{g}\right)}{2 \pi t / \xi_{g}} \sin \left(2 \pi \vec{g} \cdot \vec{r}+\phi_{g}\right) \\
& =1-A(t) B(\vec{r}),
\end{aligned}
$$

where $\psi(z)$ is the electron wavefunction inside the crystal, $z$ is the electron beam propagation depth, and $t$ is the thickness of the crystal. The parameter $\xi_{g}$ denotes the two-beam extinction distance. The atomic position inside the unit cell is given by $\vec{r}$, and $\phi_{g}$ describes the phase shift due to the absence of the center of symmetry. ${ }^{137}$ The component

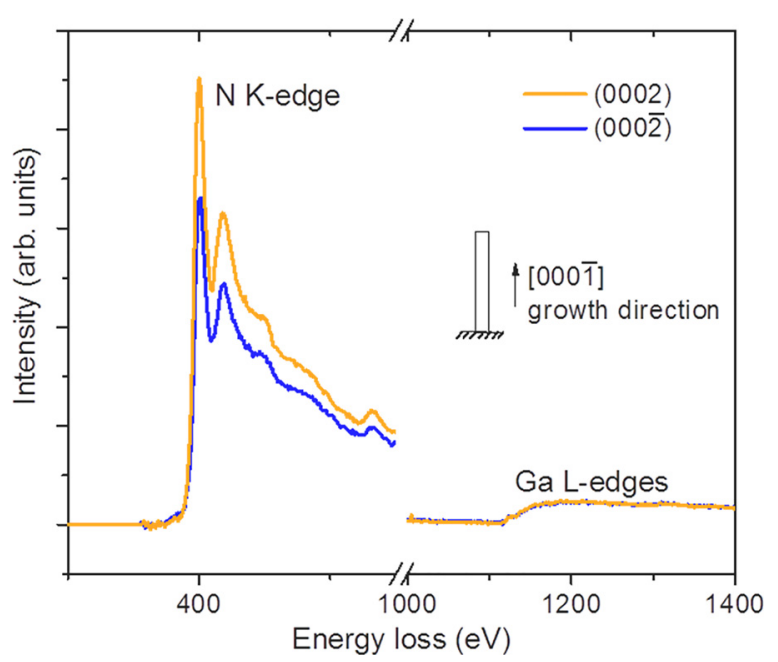

FIG. 13. A pair of EEL spectra of GaN NWs acquired at (0002) and $(000 \overline{2})$ Bragg conditions. The background of the $\mathrm{N} K$-edge is subtracted and the spectra are normalized to the Ga $L$-edges. The spectra were recorded with a Jeol 3010 microscope equipped with the Gatan Enfina spectrometer. 
$B(\vec{r})=\sin \left(2 \pi \vec{g} \cdot \vec{r}+\phi_{g}\right)$ indicates that the wavefunction oscillates within the unit cell reflecting the difference of electron intensity at different atomic planes (see Fig. 14). The thickness-averaged intensity at the $\mathrm{N}$ atomic plane has maximum intensity for the $\mathrm{g}=0002$ Bragg condition (dashed line in Fig. 14) and minimum intensity for the $\bar{g}=000 \overline{2}$ Bragg condition, while the intensities at the Ga atomic planes are roughly equal. For this reason, the larger thickness-averaged intensity $I(\vec{r})$ is expected to correspond to a higher intensity of the $\mathrm{N} K$ - edge in the EELS spectrum, consistent with the observation of higher intensity experimentally measured for the (0002) compared with $(000 \overline{2})$ diffraction condition (cf. Fig. 13). The component $A(t)$ of the thickness-averaged intensity is only associated with sample thickness $t$ and, thus, merely influences the magnitude of the measured EELS signal difference. This means that it does not affect the relative intensity at the atomic planes and, thus, the polarity determination. The absolute maximum of $A(t)$ is found for a thickness of about half of the extinction distance (Fig. 15), which makes the method most suitable for sample thicknesses of about $29 \mathrm{~nm}$ for $\mathrm{GaN}\left(\xi_{0002}=57 \mathrm{~nm}\right)$ and $27 \mathrm{~nm}$ for $\mathrm{ZnO}\left(\xi_{0002}=54.5 \mathrm{~nm}\right){ }^{138}$ Additionally, it must be noticed that a small deviation $s_{g}$ from the exact twobeam condition cannot experimentally be avoided, often due to sample bending and beam convergent effects. The orientation of the electron beam with respect to the crystal is quantified by the parameter $w=s_{g} \cdot \xi_{g}{ }^{133}$ The variation of the thickness-averaged intensity as a function of the parameter $w$ for $\mathrm{g}=0002$ and $\bar{g}=000 \overline{2}$ conditions is shown in Fig. 16 , revealing no remarkable change in the intensity as a function of beam deviation. In summary, it can be stated that the channeling-enhanced EELS has to be considered complementary to the CBED method.

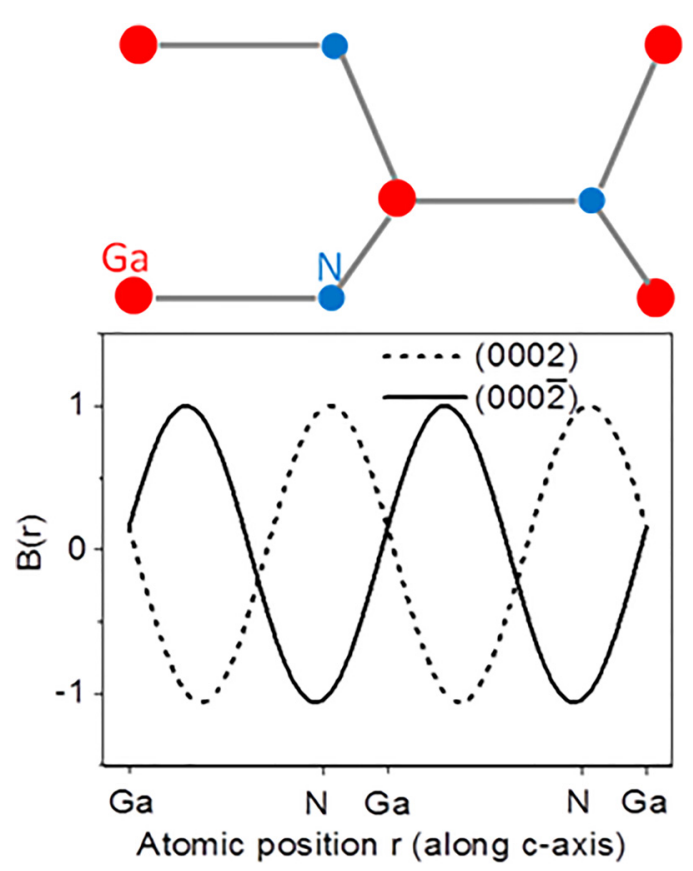

FIG. 14. Plot of $B(r)$ calculated for a GaN crystal and the corresponding unit cell.

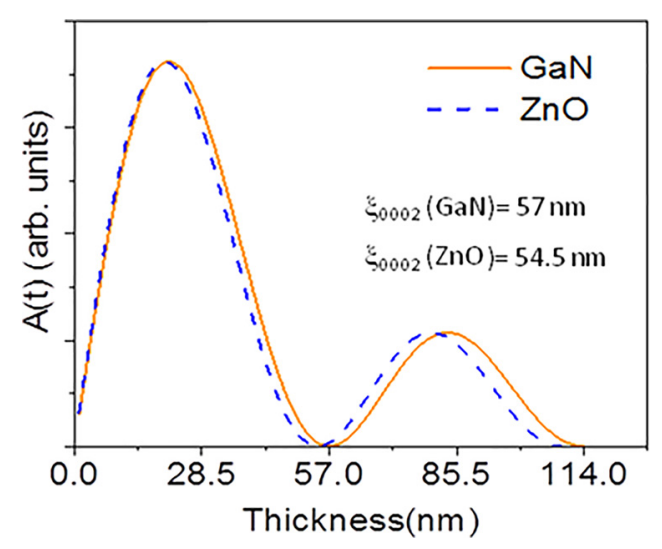

FIG. 15. Plot of $A(t)$ for $\mathrm{GaN}$ and $\mathrm{ZnO}$ crystals.

\section{High angle annular dark-field (HAADF)- and annular bright-field (ABF)-STEM}

The methodology for determining the polarity with ultimate spatial resolution is based on a direct imaging of the individual $\mathrm{Ga}-\mathrm{N}$ and $\mathrm{Zn}-\mathrm{O}$ atom columns, respectively. In addition, it has the eminent advantage to allow the visualization of polarity changes at hetero-interfaces or anti-phase boundaries and to be less affected by the presence of stacking faults (SFs), nano-twins, or other structural defects. ${ }^{139}$ Modern aberration corrected STEMs are able to reach subAngstrom resolution and display sufficient chemical sensitivity by using a high angle annular dark-field detector (HAADF) for Z-contrast imaging, allowing a direct discrimination of the different elements during observation. ${ }^{140}$ In the $[11 \overline{2} 0]$ orientation of $\mathrm{GaN}$ and $\mathrm{ZnO}$, the smallest projected spacing between $\mathrm{Ga}(\mathrm{Zn})$ and $\mathrm{N}(\mathrm{O})$ is $0.11 \mathrm{~nm}(0.12 \mathrm{~nm})$, resolvable by probe $\mathrm{C}_{\mathrm{s}}$-corrected HAADF imaging. In this way, Rouvière et al. determined the polarity of GaN quantum dots embedded in an AlN matrix. ${ }^{141}$ They demonstrated that, in crystals with large differences in atomic numbers including a low Z-number element (such as $\mathrm{GaN}$ ), it is experimentally easier to locate the "tunnel" position of the structure rather than the $\mathrm{N}$ atom column directly. The "tunnel" position indirectly represents the position of $\mathrm{N}$ columns and, thus, indicates the crystal polarity on the local scale. Recently, a new type of STEM imaging was re-introduced by Findlay et al. to detect light atoms. ${ }^{142}$ It consists of adapting the camera

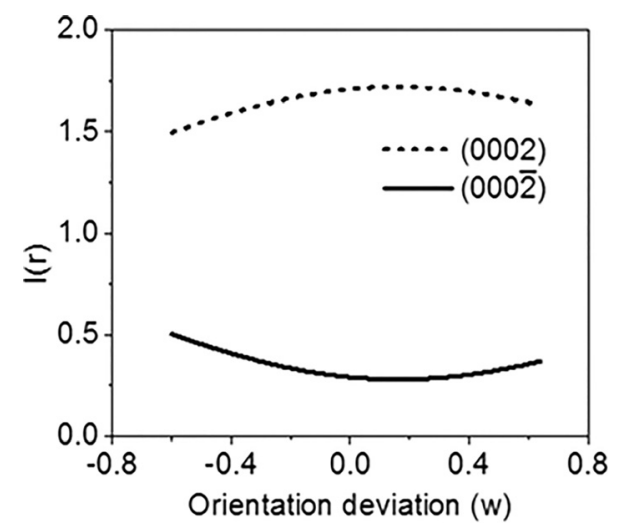

FIG. 16. Variation of the thickness-averaged electron current intensity $I(r)$ in dependency of the deviation from the exact Bragg condition. 
length and convergence angle so that the rim of the transmitted beam ("bright-field") reaches the annular detector-producing an annular bright-field (ABF) image. This $\mathrm{ABF}$ imaging is very contrast sensitive to light elements, as demonstrated by images of a $\mathrm{ZnO} \mathrm{NW}$ grown along the [0001] direction (Fig. 17(a)). ${ }^{143}$ The HAADF-STEM image in Fig. 17(b) gives rise to bright spots at the $\mathrm{Zn}$ column position only, whereas the $\mathrm{ABF}$ image clearly locates both the $\mathrm{Zn}$ and O position (Figs. 17(c)-17(e)). The intensity difference across the $\mathrm{Zn}-\mathrm{O}$ dumbbells as well as the projected distance perfectly correspond to theory (Fig. 17(f)). Therefore, $\mathrm{Zn}$ and O atom positions are unambiguously identified and, thus, the polarity as well. However, the drawback of this imaging mode lies in the requirement of high convergence angle to resolve the atom columns in $\mathrm{ZnO}$ and, furthermore, in the fact that the $\mathrm{ABF}$ contrast is thickness and defocus dependent-similar to phase contrast in high-resolution TEM.

Nevertheless, these kinds of ABF images allow not only a direct polarity determination on the level of unit cells but also the simultaneous observation of individual atom positions and their bond lengths. In objects with small dimensions, such as NWs, the STEM method can be additionally used in combination with electron holography as a basis for polarization measurements across phase boundaries ${ }^{144}$ or to correlate polarity and crystal structure with optoelectronic and transport properties. ${ }^{145}$

\section{Low-energy electron diffraction}

Low-energy electron diffraction (LEED) is a nondestructive surface sensitive technique that requires an ultrahigh vacuum (UHV) environment (i.e., $<10^{-9}$ Torr). In LEED, a collimated beam of electrons, with a well-defined energy in the range of $20-500 \mathrm{eV}$, impinges on the sample at a given angle. The effective diameter of the electron beam is typically about $1 \mathrm{~mm}$. The impinging electrons are scattered both elastically and inelastically by the atoms contained in the upper 1-10 atomic layers of the sample, ${ }^{146}$ hence LEED measurements are very sensitive to surface properties and can be negatively affected by sample contamination. The elastic scattering of electrons results in a diffraction pattern that can be monitored on a luminescent screen. The relative position of the diffracted spots reflects the periodicity of the surface but does not contain information on the specific atomic arrangement. The latter, however, can be retrieved from the quantitative analysis of the intensity of the diffracted spots as a function of the angle of incidence and/or the electron beam energy. ${ }^{146,147}$ Because modifying and controlling the incidence angle are difficult from an experimental point of view, it is preferable to analyze the intensity as a function of the electron beam energy at a given angle (usually at normal incidence). These measurements are known as LEED spectra or $\mathrm{I}-\mathrm{V}$ profiles. The analysis of I-V profiles is not straightforward because the strong interaction between low-energy electrons and matter results in not single but multiple scattering events. ${ }^{146,147}$ Consequently, in order to retrieve the information about the atomic arrangement, the experimental I-V profiles must be compared with the theoretical simulations performed within the framework of the dynamical theory of diffraction. ${ }^{146,147}$ More details on LEED experiments and their underlying theoretical background can be found elsewhere. ${ }^{146-148}$

The sensitivity of LEED I-V profiles to the specific atomic arrangement facilitates the assessment of the polarity in non-centrosymmetric crystals. As an example, Fig. 18 shows the experimental I-V profiles of the $(1,0)$ and $(2,0)$ diffracted spots measured by Romanyuk et al. in freestanding N- and Ga-polar GaN thin films ${ }^{149}$ as well as in N-polar GaN NW ensembles. ${ }^{150}$ The ensemble is homogeneous in height and consists of $730 \mathrm{~nm}$ long vertically aligned $\left( \pm 0.3^{\circ}\right) \mathrm{NWs}$ with an average diameter of $85 \mathrm{~nm}$ and an areal density of $\approx 5 \times 10^{9} \mathrm{~cm}^{-2}$. As can be observed in the

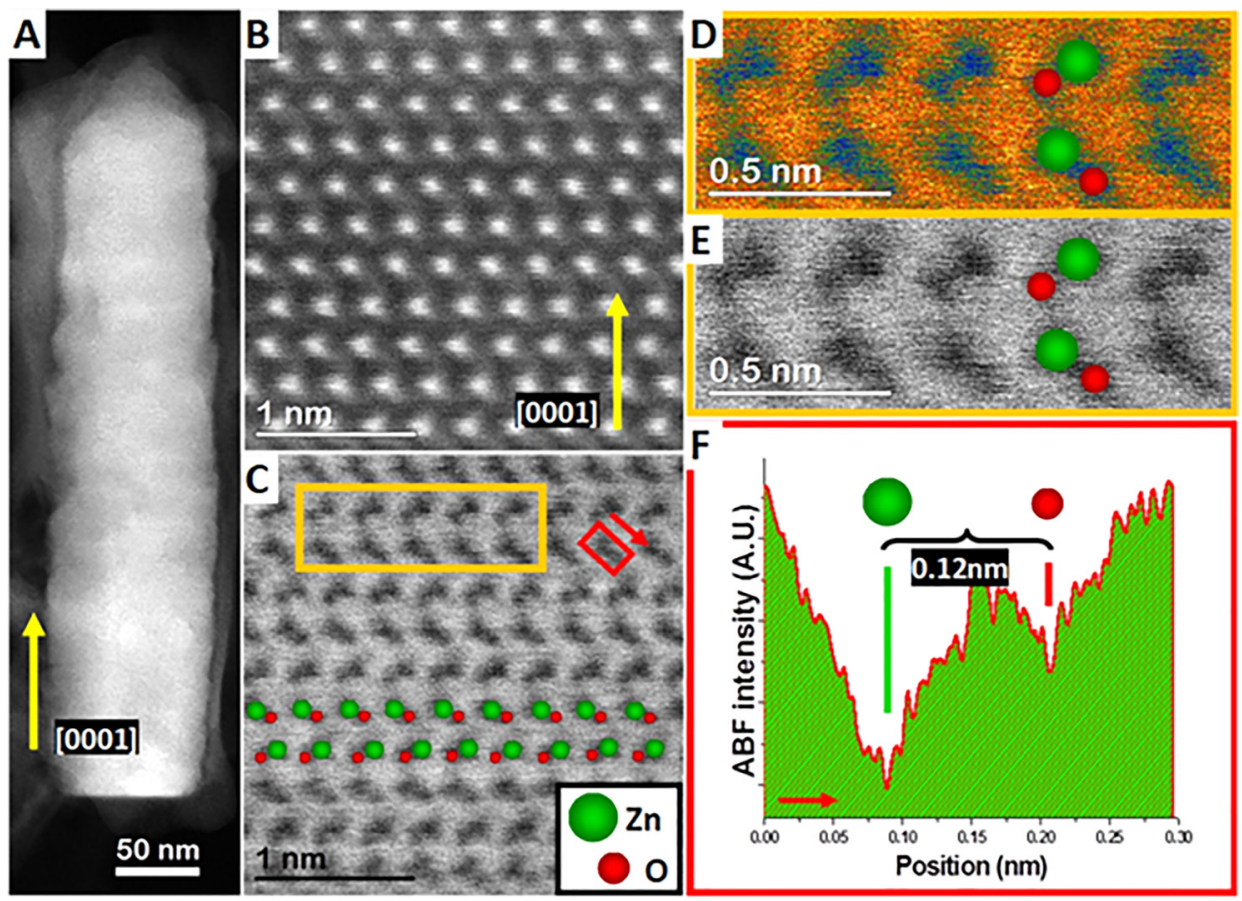

FIG. 17. (a) HAADF-STEM image of a $\mathrm{ZnO} \mathrm{NW}$, (b) atomic resolution Cscorrected HAADF-STEM image, and (c) corresponding atomic resolution Cs-corrected ABF-STEM. (d) and (e) Magnified details of the part marked by the orange rectangle in (c). (f) $\mathrm{ABF}$ intensity profile across the $\mathrm{Zn}-\mathrm{O}$ pair. Adapted with permission from de la Mata et al., Nano Lett. 12, 2579 (2012). Copyright 2012 American Chemical Society. 

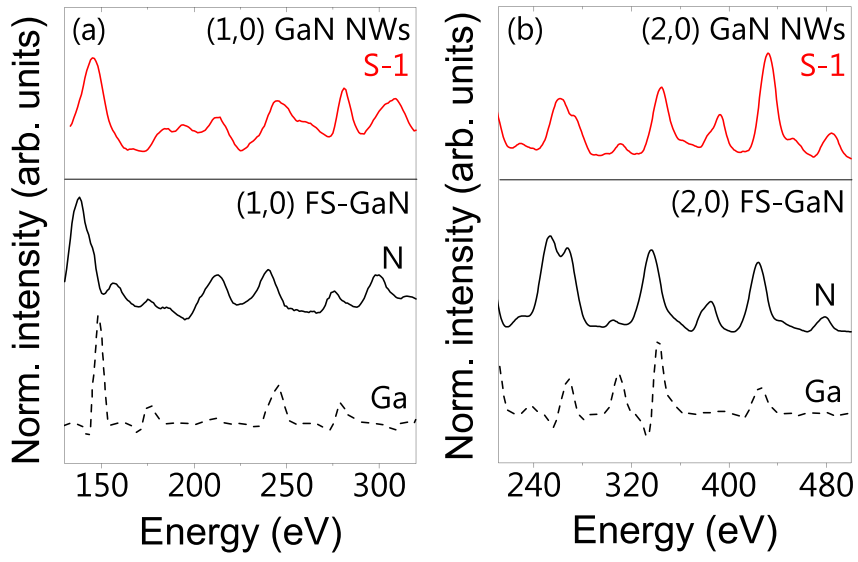

FIG. 18. LEED I-V profiles of the $(1,0)$ (a) and $(2,0)$ (b) diffracted spots measured in N-polar GaN NW ensembles (top) and in N- and Ga-polar freestanding GaN layers (bottom). The latter is vertically shifted. Reprinted with permission from Appl. Phys. Lett. 106, 021602 (2015). Copyright 2015 AIP Publishing LLC.

figure, for both diffracted spots, the I-V profiles of the Gaand N-polar layers are clearly different. Therefore, the polarity of GaN layers can be elucidated by comparing the I-V profiles with the theoretical calculations ${ }^{149,151}$ or experimental profiles obtained from reference samples. The comparison of the $\mathrm{I}-\mathrm{V}$ profiles measured in $\mathrm{N}$-polar layers and $\mathrm{NW}$ ensembles indicates that this technique is also suitable to investigate the polarity of such a kind of nanostructures. As shown in Fig. 18, the profiles exhibit the same peaks. The only difference is a small shift of a few $\mathrm{eV}$ in the position of the diffraction peak maxima. The latter effect was attributed to either variations in the inner potential or differences in the actual values of the lattice parameters. ${ }^{150}$ However, because LEED patterns are very sensitive to the angle of incidence of the electron beam, ${ }^{146,147}$ this technique is not suitable to investigate the polarity of NW ensembles with a broad outof-plane orientation distribution. ${ }^{150}$

\section{Angle-resolved x-ray photoelectron diffraction (ARXPD)}

As LEED, angle-resolved x-ray photoelectron diffraction (ARXPD) is a non-destructive surface sensitive technique that requires UHV conditions. In XPD, the sample is irradiated with $\mathrm{X}$-ray photons having energies above $1 \mathrm{keV}$. These measurements do not require synchrotron radiation but can be performed using, for example, the $\mathrm{MgK} \alpha, \mathrm{CuK} \alpha$, or $\mathrm{AlK} \alpha$ radiation produced by standard laboratory x-ray tubes. ${ }^{152}$ As a result of the x-ray radiation, core-level electrons with welldefined kinetic energies are emitted by the different types of atoms present in the sample. ${ }^{152,153}$ Consequently, XPD spectra are characteristic of the constituent elements of the sample. Photo-emitted electrons propagate through the sample before escaping into the vacuum. In their way through the sample, the electrons can be inelastically and elastically scattered. Inelastic scattering limits the depth sensitivity of XPD to a few atomic layers. ${ }^{153}$ Therefore, as in the case of LEED, surface contamination may deteriorate the quality of the measurements. In crystalline samples, the forward elastic scattering by neighboring atoms results in an angular dependence of the distribution of photo-emitted electrons. ${ }^{152,153}$ This angular dependence is mainly determined by the specific atomic arrangement, as well as by the electron scattering crosssection of the constituent elements. In ARXPD, the intensity of photo-emitted electrons is analyzed as a function of the azimuth and polar detection angles. ARXPD measurements can thus be used to retrieve crystallographic information, including the polarity of non-centrosymmetric crystals. ${ }^{150,153-158} \mathrm{~A}$ detailed description on XPD can be found elsewhere. ${ }^{152,153}$

In wurtzite crystals, the angular distribution of the photo-emission intensity is not the same for anion- and cation-polar layers, because of the different relative positions of the cation and anion sublattices. ${ }^{150,155-157}$ Due to the forward scattering of electrons, significant differences can be observed when measuring the intensity along those directions that intercept neighboring anions and cations. The polarity of both $\mathrm{ZnO}$ and $\mathrm{GaN}$ layers was investigated using ARXPD by different groups. ${ }^{150,155-157,159}$ Williams et al. ${ }^{156}$ analyzed the ARXPD intensity of $\mathrm{Zn}$ - and O-polar $\mathrm{ZnO}$ layers as a function of all azimuth and polar detection angles ( $\varphi$ and $\theta$, respectively) to indentify the most suitable conditions to assess the polarity. Their results demonstrate that it is not necessary to measure the entire ARXPD pattern to elucidate the polarity. For instance, when analyzing the photo-emission intensity from the $\mathrm{Zn} 2 \mathrm{p}_{3 / 2}$ core-level, it is sufficient to measure the peak intensity $I_{\varphi, \theta}$ at the angular coordinates $\left(\varphi=0^{\circ}, \theta=32^{\circ}\right)$ and $\left(\varphi=30^{\circ}, \theta=32^{\circ}\right)$, where $\varphi=0^{\circ}$ corresponds to the $[11 \overline{2} 0]$ azimuth and $\theta$ is measured with respect to the surface normal. The $I_{0,32} / I_{30,32}$ ratio amounts to 0.6 and 0.9 for $\mathrm{Zn}$ - and O-polar layers, respectively, and thus indicates the polarity. For GaN, SeelmannEggebert et al. ${ }^{155}$ investigated the complete angular dependence of the Ga $3 d$ and $\mathrm{N} 1 s$ photo-electron emission intensities for $\mathrm{Ga}$ - and $\mathrm{N}$-polar layers. For both core-level emissions, they observed significant differences that can be used to discriminate between the two polar orientations. As in the case of $\mathrm{ZnO}$, it is not necessary to measure the entire XPD pattern to distinguish between $\mathrm{Ga}$ - and N-polar layers. ${ }^{157}$ Romanyuk et al. ${ }^{157}$ investigated the emission from the Ga $3 d$ and N $1 s$ core-levels along the [1010] and $[11 \overline{2} 0]$ azimuths as a function of the polar angle. They found that the $\mathrm{N} 1 s$ emission is more suitable than that of $\mathrm{Ga} 3 d$ for polarity determination because of the larger electron scattering cross-section of Ga atoms. For both azimuths, they observed pronounced differences between the polar plots of Ga- and N-polar layers. The analysis of the measurements performed along the $[10 \overline{1} 0]$ azimuth was found to be simpler. In order to assess the polarity, they concluded that measuring the $I_{30,20} / I_{30,25}$ ratio is sufficient. The value of the $I_{30,20} / I_{30,25}$ ratio is either larger or smaller than one depending on whether the GaN layer is $\mathrm{Ga}$ or $\mathrm{N}$ polar, respectively.

ARXPD was also used to determine the polarity of $\mathrm{GaN}$ NW ensembles as those described in Section IV C 2 and in Ref. 150. Figure 19 shows the photo-emission intensity from the N $1 s$ core-level measured for Ga- and N-polar free-standing GaN layers as well as for two different N-polar GaN NW ensembles, labeled as S-1 and S-2. As can be seen in the figure, the angular distributions of the XPD intensity in N-polar 


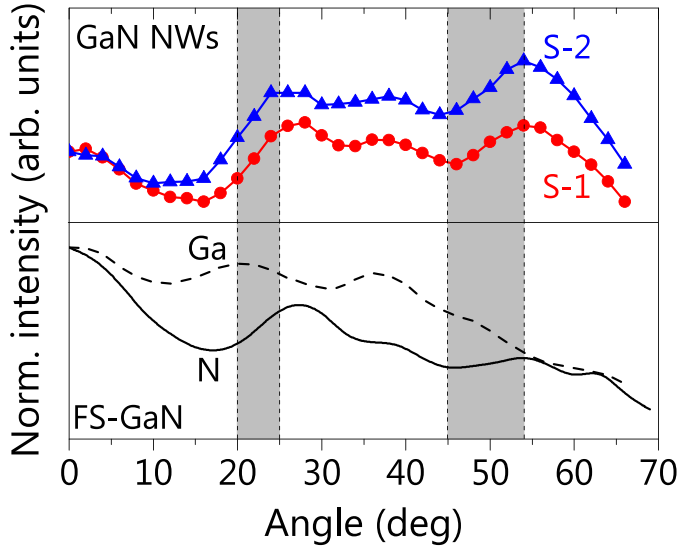

FIG. 19. Normalized angular distribution of photoemitted electrons for GaN NWs prepared on AlN buffered $6 \mathrm{H}-\mathrm{SiC}$ (0001) (sample S-1) and bare $\mathrm{Si}(111)$ (sample S-2). As a reference, the normalized angular distributions measured in free-standing Ga- and N-polar layers are shown below. The polar angle $\theta$ is measured with respect to the surface normal. Reprinted with permission from Appl. Phys. Lett. 106, 021602 (2015). Copyright 2015 AIP Publishing LLC.

layers and NW ensembles are very similar. Therefore, the polarity of GaN NWs can also be determined by evaluating the $I_{30,20} / I_{30,25}$ ratio. ${ }^{150}$ The main difference between the angular distributions measured in NW ensembles and thin films is the higher intensity observed for the NW ensembles at larger polar angles. For thin films, the intensity tends to decrease with increasing values of $\theta$ because the effective thickness of the film that contributes to photo-electron emission decreases with the emission angle. ${ }^{152,153}$ For NWs, however, this phenomenon is partly compensated by photoelectron emission from NW edges. ${ }^{150}$

\section{E. Resonant x-ray diffraction in synchrotron}

Resonant elastic x-ray diffraction (XRD), most often using synchrotron radiation, can be used to unambiguously determine the $\mathrm{GaN}$ and $\mathrm{ZnO}$ crystallographic polarity at a macroscopic scale. This approach is based on the breakdown of the Friedel's law (similar to the CBED technique: see Section III B), which states that the $\mathrm{x}$-ray diffracted intensity $I(h k l)=I(\bar{h} \bar{k} \bar{l})$. When the $\mathrm{x}$-ray beam energy is tuned close to an absorption edge, the anomalous scattering factor becomes nonnegligible (i.e., resonant scattering). In the case of a non-centrosymmetric crystal structure, a disparity in the diffracted intensity of Bijvoet's pairs of reflections ${ }^{160}$ is observed due to the abrupt variation of the imaginary term ( $f^{\prime \prime}$, which is proportional to the absorption coefficient). Note that Friedel's pairs refer to reflections with Miller indices $h \mathrm{kl}$ and $\bar{h} \bar{k} \bar{l}$, while Bijvoet's pairs more generally refer to pairs of reflections which are symmetry-related (including the additional inversion center) but have different intensities (i.e., Friedel's law does not hold).

\section{Theoretical aspects}

As discussed in Section IIB, the wurtzite structure (point group $6 \mathrm{~mm}$ ) lacks a center of symmetry. Friedel's law implies that the set of equivalent diffracted intensities is given by the Laue point group $\left(\frac{6}{m} \mathrm{~mm}\right)$. However, in the case of a non-centrosymmetric structure, Friedel's law breaks down when the scattering factor of one kind of atoms is complex, namely, it has a nonzero imaginary component (i.e., the photoelectric absorption is significant). ${ }^{161}$ Then, the set of equivalent diffracted intensities obeys the wurtzite crystal point group (i.e., $6 \mathrm{~mm}$ ). For instance, 6-fold symmetry equivalent reflections $\{11 \overline{2} 2\}$ have the same diffracted intensity, which is different from that obtained with the 6-fold symmetry equivalent $\{11 \overline{2} \overline{2}\}$ reflections. Two reflections, one from the first group and another from the second group, are named a Bijvoet's pair. The GaN and $\mathrm{ZnO}$ wurtzite cell contains 4 atoms (2 anions, 2 metallic cations) on the Wykcoff position $2 b$, the coordinates of which are $\frac{1}{3}, \frac{2}{3}, z ; \frac{2}{3}$, $\frac{1}{3}, \frac{1}{2}+z$ (origin on $6_{3}$ screw axis). In the following, $z$ is equal to 0 (resp. $u$ ) for the metallic cations (resp. anions). Note that this definition implies that the metallic cation-anion vector has the same orientation as the $c$-axis. Thus, a diffraction experiment with a positive $l$ concludes to a metallic cationpolar orientation. Hereafter, the diffracted intensities difference for a Bijvoet's pair of reflections is calculated. The example of $\mathrm{GaN}$ is taken, but the same arguments hold for $\mathrm{ZnO}^{162}$ The atomic scattering factors are supposed to be complex

$$
f(Q, E)=f^{0}(Q)+f^{\prime}(E)+i f^{\prime \prime}(E),
$$

where $Q$ is the modulus of the scattering vector $Q, E$ is the $\mathrm{x}$-ray photon energy, $f^{0}$ is the Thomson atomic scattering factor, and $f^{\prime}$ (respectively, $f^{\prime \prime}$ ) is the real (resp. imaginary) parts of the anomalous scattering factor. The structure factor writes

$$
\begin{aligned}
F(h k l)= & f_{G a} e^{2 i \pi\left(\frac{1}{3} h+\frac{2}{3} k\right)}+f_{G a} e^{2 i \pi\left(\frac{2}{3} h+\frac{1}{3} k+\frac{1}{2} l\right)}+f_{N} e^{2 i \pi\left(\frac{1}{3} h+\frac{2}{3} k+u l\right)} \\
& +f_{N} e^{2 i \pi\left(\frac{2}{3} h+\frac{1}{3} k+\left(\frac{1}{2}+u\right) l\right)}
\end{aligned}
$$

The diffracted intensity is proportional to the structure factor squared modulus

$$
\begin{aligned}
I(h k l)= & F(h k l) F(h k l)^{*} \\
= & 2\left[f_{N} f_{N}^{*}+f_{G a} f_{G a}^{*}+f_{G a} f_{N}^{*} e^{2 i \pi(u l)}+f_{N} f_{G a}^{*} \times e^{-2 i \pi(u l)}\right] \\
& \times\left[1+\cos \left(2 \pi\left(\frac{1}{3}(h-k)+\frac{1}{2} l\right)\right)\right] .
\end{aligned}
$$

And the diffracted intensity difference $I\{h k l\}-I\{h k \bar{l}\}$ of a Bijvoet's pair is given by

$$
\begin{aligned}
I(h k l)-I(h k \vec{l})= & 8\left[1+\cos \left(2 \pi\left(\frac{1}{3}(h-k)+\frac{1}{2} l\right)\right)\right] \\
& \times\left[\left(f_{G a}^{0}+f_{G a}^{\prime}\right) f_{N}^{\prime \prime}-\left(f_{N}^{0}+f_{N}^{\prime}\right) f_{G a}^{\prime \prime}\right] \\
& \times \sin (2 \pi u l) .
\end{aligned}
$$

In the case of an ideal wurtzite crystal structure $(u=3 / 8)$, Equation (8) shows that the intensity difference cancels out when $l=4 \mathrm{n}$ ( $\mathrm{n}$ is an integer). All the other rules are the conditions limiting the possible reflections according to the space group $\mathrm{P}_{3} m c$. Also, Equation (8) clearly shows that the intensity difference cancels out if $f_{G a}^{\prime \prime}$ and $f_{N}^{\prime \prime}$ approach 
zero. Besides, it is known that $f^{\prime \prime}$ (as well as $f^{\prime}$ ) undergoes an abrupt variation when the X-ray beam energy is scanned across an absorption edge ${ }^{163,164}$ (i.e., resonant scattering). Therefore, one expects the intensity difference to increase for X-ray beam energies above the edge. At the Ga K-edge, the $\mathrm{N}$ anomalous scattering factors stay constant and are very small in comparison with $f_{G a}^{\prime}$ and $f_{G a}^{\prime \prime}$. Then, for $h h l$ reflections, for instance, the intensity difference can be approximated as follows:

$$
I(h h l)-I(h h \bar{l}) \simeq-8[(1+\cos (\pi l))] \times f_{N}^{0} f_{G a}^{\prime \prime} \sin (2 \pi u l) .
$$

Equation (9) shows that the intensity difference is proportional to $f_{G a}^{\prime \prime}$ and significantly increases above the Ga K-edge. It can be checked that $I(0002)-I(000 \overline{2})<0$ and $I(0004)$ $-I(000 \overline{4})=0$.

As mentioned above, three effects thus modify the intensity level at and above the absorption edge: fluorescence yield (levels up the intensity), absorption (levels down the intensity), and the noncentrosymmetry, as in the case of polar wurtzite structure (levels up or down the intensity depending on the chosen Bijvoet reflection). The fluorescence signal may not be negligible, especially in the case of weak reflections and/or poor crystallinity. Nowadays, by using a $2 \mathrm{D}$ pixel detector, it is possible to simultaneously measure both the diffracted and fluorescence signals. Absorption attenuation depends on the linear absorption along the x-ray path, the incidence and exit angles, and the equivalent film thickness, which is the actual film thickness times the film density. The latter can be obtained by measuring the critical angle by x-ray reflectometry. ${ }^{165}$ In the case of a single layer, one possible experimental way to eliminate the absorption effect is to measure a Bijvoet's pair of reflections, to correct the diffraction data for the fluorescence signal, and to calculate the diffracted intensity ratio. However, the present procedure is time-consuming due to the sample preparation and also when compared with the measurement of only one reflection.

By using monochromatic synchrotron radiation, the experiment is quite easy to perform and fast (about $2 \mathrm{~h}$ or less per sample, depending on the x-ray source brilliance and the available amount of material) and the data analysis is user-friendly. Provided that fluorescence signal and absorption attenuation can be properly estimated, the measurement of one Bijvoet reflection is sufficient. This method is fairly suitable for studying thin textured films, for which the fluorescence background and the absorption attenuation are small or negligible (i.e., an equivalent thickness less than few hundreds of $\mathrm{nm}$ ). Besides $\mathrm{x}$-ray is non-destructive, it probes the sample in depth and analyzes a surface area going from $\mathrm{mm}^{2}$ down to hundreds of $\mu \mathrm{m}^{2}$ (or even less using synchrotron radiation).

\section{Experimental determination}

X-ray anomalous scattering was applied for the first time in the late 1930s to determine the polarity of bulk cubic ZnS with (111) facets. ${ }^{166,167}$ Later on, several groups measured the absolute polar direction of bulk single crystals, ${ }^{168-171}$ including $\mathrm{ZnO} .{ }^{172,173}$ More recently, by using the continuous spectrum of a conventional x-ray tube, Tampo et al. applied anomalous scattering at the Zn K-edge to determine the polarity of (0001) $\mathrm{ZnO}$ thin films grown by $\mathrm{MBE}$ on $\mathrm{MgO}$ buffers of variable thicknesses, evidencing the gradual transition change of polarity. ${ }^{174}$ In an extensive study, Shelton et al. measured the polar orientation and polarity fraction of unipolar and mixed polar wurtzite crystals, including (0001) $\mathrm{ZnO}$ thin films grown on $\mathrm{MgO}$ buffers and GaN lateral polar structures. ${ }^{175}$ By using polychromatic synchrotron radiation, Meyer et al. ${ }^{176}$ determined the polarity of a (Ga,In)P epilayer grown on a (001) GaAs substrate.

Recently, resonant XRD using synchrotron radiation was used to unambiguously determine the polarity of GaN NWs grown by PAMBE on bare $\mathrm{Si}(111) .{ }^{177} \mathrm{~A}$ reference sample was grown by PAMBE on a 2-in. Si(111) substrate. GaN NWs were grown directly on $\mathrm{Si}$ at a temperature of $800^{\circ} \mathrm{C}$ in the usual N-rich conditions. As-grown NWs were $730 \mathrm{~nm}$ long and had a diameter of $30 \mathrm{~nm}$ on average, and a density of about 200 NWs $\mu \mathrm{m}^{-2}$. For being able to measure a Bijvoet's pair of reflections $((11 \overline{2} 2)$ and $(11 \overline{2} \overline{2})$, for instance), an upended sample was prepared. For that, a piece of the as-grown sample, with the $\mathrm{Si}(111)$ substrate etched, was stuck upside down onto a sapphire lump in order to let the x-ray beam go through. XRD experiments with monochromatic synchrotron radiation were carried out at the beamline BM02/D2AM at the European Synchrotron Radiation Facility. Figure 20 shows the diffracted intensity variations as a function of the $\mathrm{x}$-ray beam energy, across the Ga K-edge (10 $367 \mathrm{eV}$ ), of a $\{11 \overline{2} 2\}$ Bijvoet's pair of reflections, one measured with the as-grown and the other with up-ended NW samples. The spectra were corrected for the Ga fluorescence and diffuse scattering signals. Equation (4) was used to fit the background-subtracted spectra by taking into account the $\mathrm{x}$-ray beam absorption through a $150 \mathrm{~nm}$ thick equivalent GaN layer. Only a scale factor and a linear detector efficiency were fitted. The $(11 \overline{2} \overline{2})$ and $(11 \overline{2} 2)$ were unambiguously assigned to the as-grown and

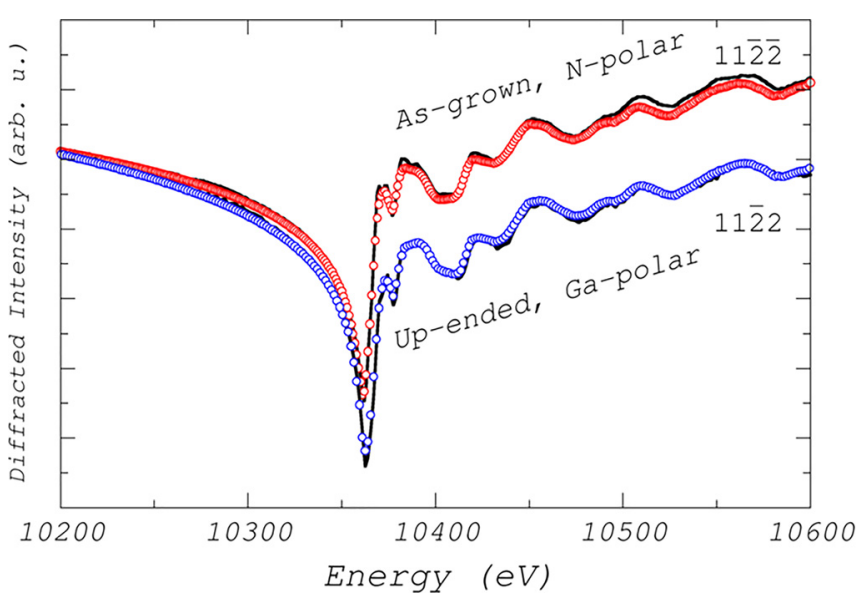

FIG. 20. Experimental (solid lines) and calculated (open circles) $11 \overline{2} \overline{2}$ and $11 \overline{2} 2$ diffracted intensity variation as a function of the $\mathrm{x}$-ray beam energy across the Ga K-edge for as-grown and up-ended NWs, respectively. Asgrown and up-ended NWs are N-polar and Ga polar, respectively. 
up-ended samples, respectively, allowing to identify the asgrown NWs as N-polar and the up-ended NWs as Ga-polar.

\section{F. Coaxial impact collision ion scattering spectroscopy (CAICISS)}

Regarding impact-collision ion scattering spectroscopy (ICISS) as developed in the early 1980s, the scattering angle of the recorded ions and/or neutral particles is close to but not exactly $180^{\circ}$. Still, this geometry enabled to obtain quantitative information on the crystalline structure, as the influence of shadowing and focusing effects is enhanced with respect to conventional ISS. About ten years later, Aono et al. developed a new spectrometer, in which the necessary pulsed ion source and the time-of-flight energy analyzer were arranged coaxially giving rise to CAICISS, i.e., the scattering angle was exactly $180^{\circ}$, further enhancing shadowing effects and minimizing the signal due to multiple scattering processes. ${ }^{178,179}$ As schematically represented in Fig. 21 , by measuring the intensity of particles scattered by atom $\mathrm{B}$ as a function of the incidence-detecting angle $\alpha$ with respect to the surface plane, it is possible to determine both the bond orientation and the bond length between neighboring atoms. The importance of shadowing and focusing effects is also illustrated clearly in the figure: on the one hand, the intensity dip reflects how the atom closer to the surface shadows atoms deeper in the material; on the other hand, the intensity enhancement at each side of the intensity dip reflects the concentration of scattered ions trajectories at the edges of the shadowing cones. Furthermore, it should be noted that, compared with other closely related and more popular techniques, such as Rutherford Backscattering (RBS), the use of relatively low energy particles ( $\mathrm{keV}$ in CAICISS, compared with $\mathrm{MeV}$ in RBS) leads to shadowing cones of several angstroms in lateral size, instead of tenths of angstrom, enhancing the scattering cross-section and the sensitivity to atoms close to the surface. ${ }^{180}$

In order to identify the polarity of a thin film thanks to CAICISS, the signal intensity as a function of the incident angle must be compared either with the simulated spectra or with the CAICISS signal variation measured on a reference sample of known polarity. Since the first numerical simulations of CAICISS spectra for wurtzite structures were developed in parallel to the initial measurements, ${ }^{181}$ the earliest polarity determinations were conducted by comparing
CAICISS spectra measured on $\mathrm{ZnO}$ thin films and $\mathrm{ZnO}$ single crystals of known polarity, ${ }^{182}$ as shown in Figs. 22(a)-22(c). Interestingly, since the crystal structure and lattice constants of $\mathrm{GaN}$ and $\mathrm{ZnO}$ are similar, the incidence angle dependence of the CAICISS signal obtained on these materials is also very similar and only depends on the observed polarity (i.e., the Ga- and $\mathrm{Zn}$-polar orientations give similar CAICISS spectra, which are different from the $\mathrm{O}$ - and $\mathrm{N}$-polarities signals). This enabled to determine the polarity of $\mathrm{GaN}$ thin films based on the spectra acquired on $\mathrm{ZnO}$ single crystals. ${ }^{183}$

Sonoda et al. employed CAICISS to determine the polarity of MOCVD and MBE-grown GaN thin films by comparison to simulated spectra ${ }^{181,184}$ (Fig. 22(d)). They concluded that the MOCVD-grown thin films were Ga-polar, while the MBE ones were N-polar (Fig. 22(e)). As shown in Figs. 22(e) and 22(f), the CAICISS intensity signal is much larger for the metallic cations ( $\mathrm{Ga}$ or $\mathrm{Zn}$ ) than for the $\mathrm{N}$ or $\mathrm{O}$ anions, due to larger scattering cross-sections. Thus, only their signal is most often recorded. Besides, these numerical simulations enabled to identify the origin of each of the features observed in the CAICISS spectra and to associate them to precise shadow cones. ${ }^{184}$

Two aspects are worth mentioning: first, it should be stressed that CAICISS allows to determine the polarity rather than the surface termination, with O-polar $\mathrm{ZnO}$ giving a very different signal compared with a Zn-polar orientation terminated by $\mathrm{O}$ atoms. ${ }^{185}$ Second, although, in principle, it is possible to analyze samples with mixed polarity, just by comparing the measured spectra with structural models, in which the volume ratio between the two polarities is varied, ${ }^{186}$ the approach does not allow to quantify precisely the exact ratio, requiring complementary techniques to address this issue.

\section{G. Scanning probe microscopy (SPM)}

The term scanning probe microscopy (SPM) covers a wide range of techniques that share the same working principle: the movement of a tip above a surface, either in contact with it or not, and the simultaneous monitoring of some specific tip-surface interaction. Among this large palette, which includes STM and atomic force microscopy (AFM), two of them have been used to analyze the polarity of $\mathrm{GaN}$ and $\mathrm{ZnO}$ : Kelvin probe force microscopy (KPFM, or KPM, or KFM), eventually assisted by some external illumination (photoassisted KPM), and piezoresponse force microscopy
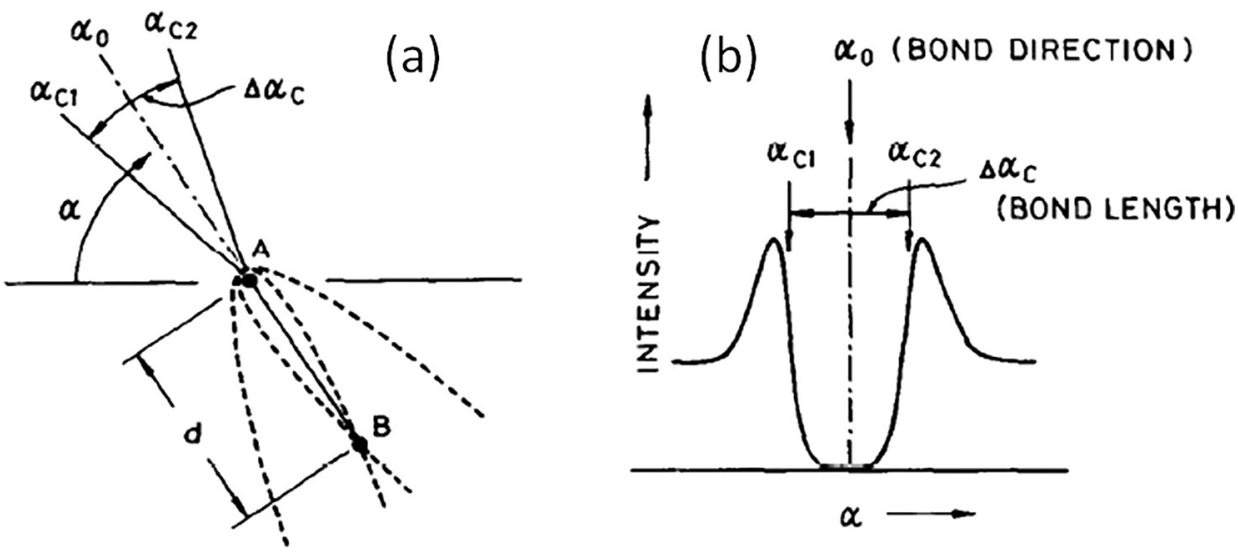

FIG. 21. Schematic representation illustrating the physical magnitudes measured in CAICISS and that are sufficient to extract the bond direction and the bond length between neighboring atoms. Reprinted with permission from Aono et al., Nucl. Instrum. Methods Phys. Res., Sect. B 37, 264 (1989). Copyright 1989 Elsevier. 

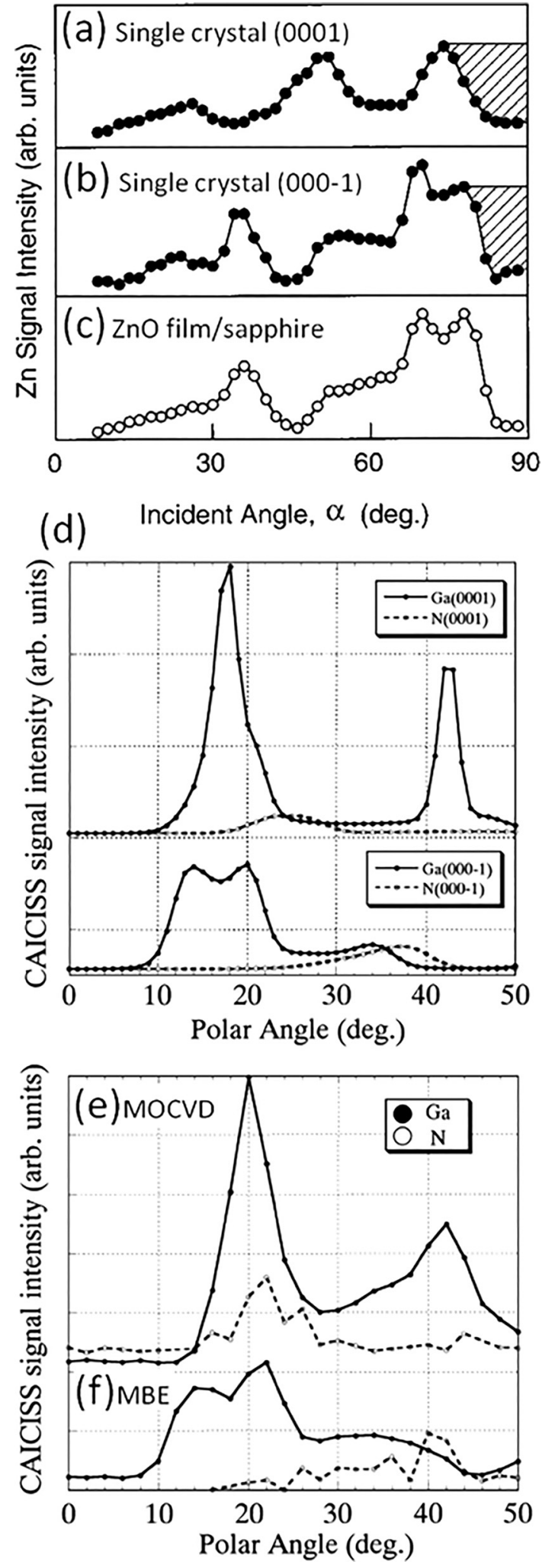

FIG. 22. Incident angle dependence of the $\mathrm{Zn}$ CAICISS signal intensity along the [11-20] azimuth obtained on (a) Zn-polar ZnO single crystal, (b) O-polar $\mathrm{ZnO}$ single crystal, and (c) $\mathrm{ZnO}$ thin film grown on sapphire. Reprinted with permission from Appl. Phys. Lett. 72, 824 (1998). Copyright 1998 AIP Publishing LLC. (d) Simulated incident angle dependences of Ga and N CAICISS signals on Ga-polar GaN (top) and Npolar GaN (bottom). Incident angle dependence of the Ga and N CAICISS signals on (e), an MOCVD-grown thin film, and (f) on an MBE-grown thin film. Reprinted with permission from Sonoda et al., Jpn. J. Appl. Phys., Part 2 38, L1219 (1999). Copyright 1999 The Japan Society of Applied Physics.
(PFM). Both techniques are non-destructive and do not require vacuum conditions, but while KPM is a contactless technique PFM requires physical contact between the tip and the sample surface.

\section{Kelvin probe microscopy (KPM)}

KPM provides the contact potential difference (CPD) between the metallic tip scanned above the sample and the semiconductor surface, the CPD being simply given by the work function difference. ${ }^{187}$ Since the work function of the semiconductor surface is sensitive to the actual band bending and, in turn, this depends on the polarization charges at its surface, KPM enables to identify and map the polarity of the underlying surface with a high spatial resolution, typically in the order of magnitude of $100 \mathrm{~nm}$ or even better, ${ }^{188}$ as illustrated in Fig. 23 for GaN thin films and NWs. It should be noted that the band bending is very sensitive to any adsorbate or contamination screening the local electric fields. Thus, in addition to the polarity and to the doping level of the semiconductor, the measured CPD between any of the two polar $\mathrm{ZnO}$ or $\mathrm{GaN}$ surfaces and a known metallic material might display a large variety of values. ${ }^{189,190}$ Besides measurements artifacts, such as, for example, a cross-talk between morphology and electrostatic signal, ${ }^{191}$ the measurement conditions might influence the CPD values, given that adsorbed water or the formation of a surface oxide, in the case of GaN, might modify the semiconductor work function. Thus, it is extremely important to have some samples of well-known polarity that can be used as internal reference (i.e., measured under the same experimental conditions of humidity, illumination, etc.) before and after each KPM measurement. This is particularly important when studying NWs, given that, due to a very abrupt topography, the tip can easily be damaged and, thus, its workfunction modified. ${ }^{192,193}$

The semiconductor work function can be modified by shining light into it, which leads to a change of the band bending known as surface photovoltage. ${ }^{194}$ Even if belowband gap illumination might also induce such an effect, by inducing trapping and detrapping of carriers in deep-levels, most studies have focused on the surface photovoltage generated by means of above-band gap illumination ${ }^{192,193,195}$ and, in particular, the assessment of polarity thanks to the time-dependent behavior of the surface photovoltage.

\section{Piezoelectric force microscopy (PFM)}

PFM is based on the reverse piezoelectric effect, i.e., the generation of a mechanical deformation by applying an electric field. The measurement principle is simple: the tip in contact with the semiconductor surface measures the surface displacement due to the application of an external electric field, which can be applied either between a bottom and a top electrode both deposited on the material or between a bottom electrode and the tip itself. ${ }^{196}$ The polarity of the wurtzite materials is then determined by determining the relative phase between the modulation voltage applied to the material and the material deformation, which just depends on the sign of the piezoelectric coefficient $\left(d_{33}\right.$ for $\{0001\}$ oriented thin films and NWs). This results in a deformation 

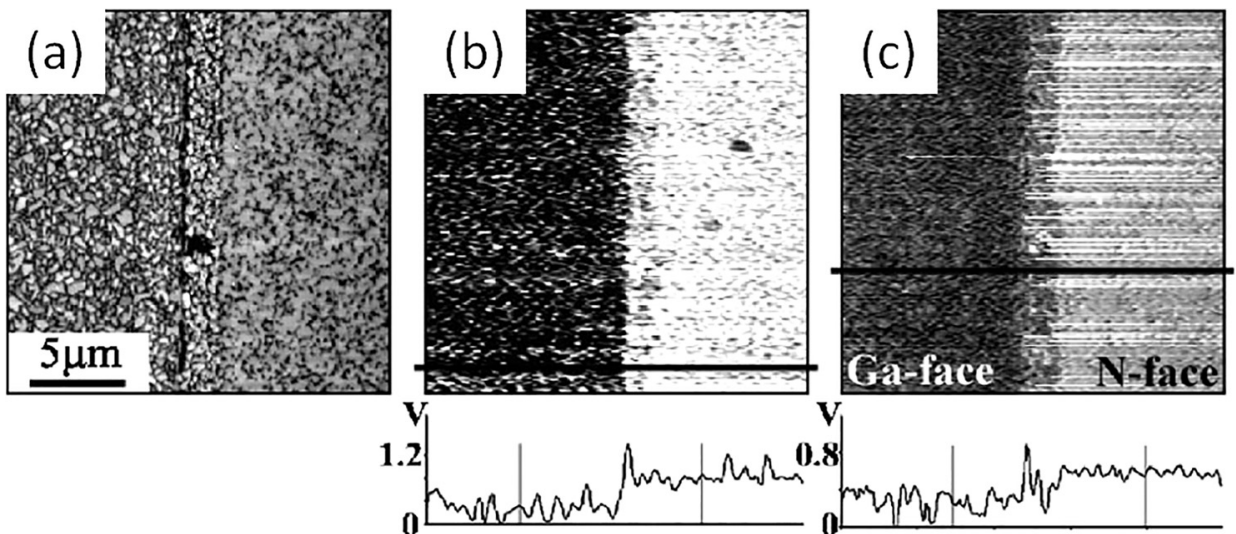

V
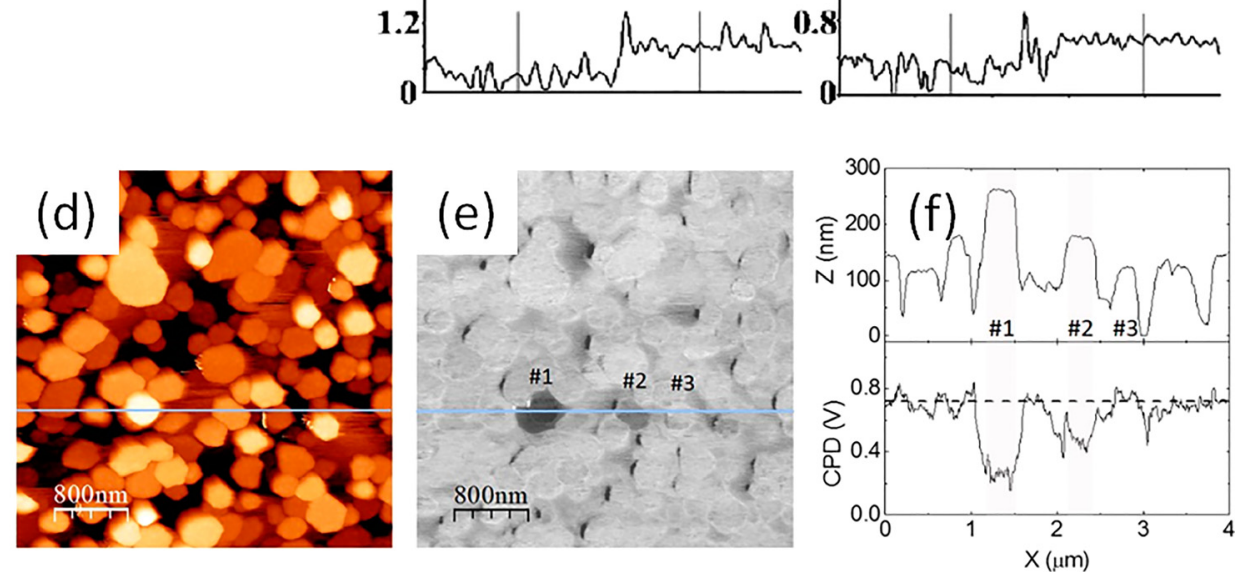

FIG. 23. (a) Topography of a $20 \times 20$ $\mu \mathrm{m}^{2}$ area. (b) KPM (with line profile) of a GaN-lateral polarity heterostructure prior to the surface treatment and (c) KPM of the same area after surface treatment. Reprinted with permission from Appl. Phys. Lett. 86, 112115 (2005). Copyright 2005 AIP Publishing LLC. (d) AFM topography $\left(4 \times 4 \mu \mathrm{m}^{2}\right.$, color scale from 0 to $250 \mathrm{~nm}$ ) and (e) corresponding KPM map (color scale from 0 to $1 \mathrm{~V}$ ). (f) Height (top) and CPD (bottom) profiles along the marked lines in (d) and (e). The dashed line marks the mean CPD value of the NW ensemble. Reprinted with permission from Minj et al., Nano Lett. 15, 6770 (2015). Copyright 2015 American Chemical Society. being in-phase for the metal cation polarity and out-of-phase for the anion polarity. ${ }^{193,196,197}$ While the contact between the tip and the surface might induce a faster tip degradation than in non-contact measurements, especially for NW ensembles, it allows, on the other hand, for a high spatial resolution. For instance, it enables to address the presence of inversion domains within single nanoobjects, ${ }^{193}$ as illustrated in Figs. 24(a)-24(d). Indeed, PFM provides enhanced spatial resolution (i.e., compared with KPM) combined with reliable statistics (i.e., compared with TEM studies). ${ }^{198}$ Furthermore, its application to more insulating materials such as AIN (see Figs. 24(e)-24(f)) is straightforward. ${ }^{198}$

\section{H. Experimental determination of the spontaneous polarization of GaN and ZnO}

The experimental determination of the spontaneous polarization $P_{\mathrm{sp}}$ of pyroelectric materials, such as $\mathrm{GaN}$ and $\mathrm{ZnO}$, is even more challenging than its computation, as discussed in Section II B. First of all, and as explained before, it is principally not possible to measure (or calculate) the $P_{\mathrm{sp}}$ of bulk materials. Second, the polarization difference accessible from optical experiments on wurtzite heterostructures arises from the difference of the total polarization, i.e., including the piezoelectric polarization $P_{\mathrm{pz}}$ between the constituent materials.

Because of these fundamental difficulties, very few attempts were made to determine $P_{\mathrm{sp}}$ on the basis of experiments. Jerphagnon and Newkirk ${ }^{199}$ employed the proportionality between the second-order nonlinear susceptibility and $P_{\text {sp }}$, following from the Landau-Khalatnikov equations (a time-dependent extension of the Ginzburg-Landau theory of phase transitions in ferroelectrics). By experimentally determining the second harmonic coefficients, a value of
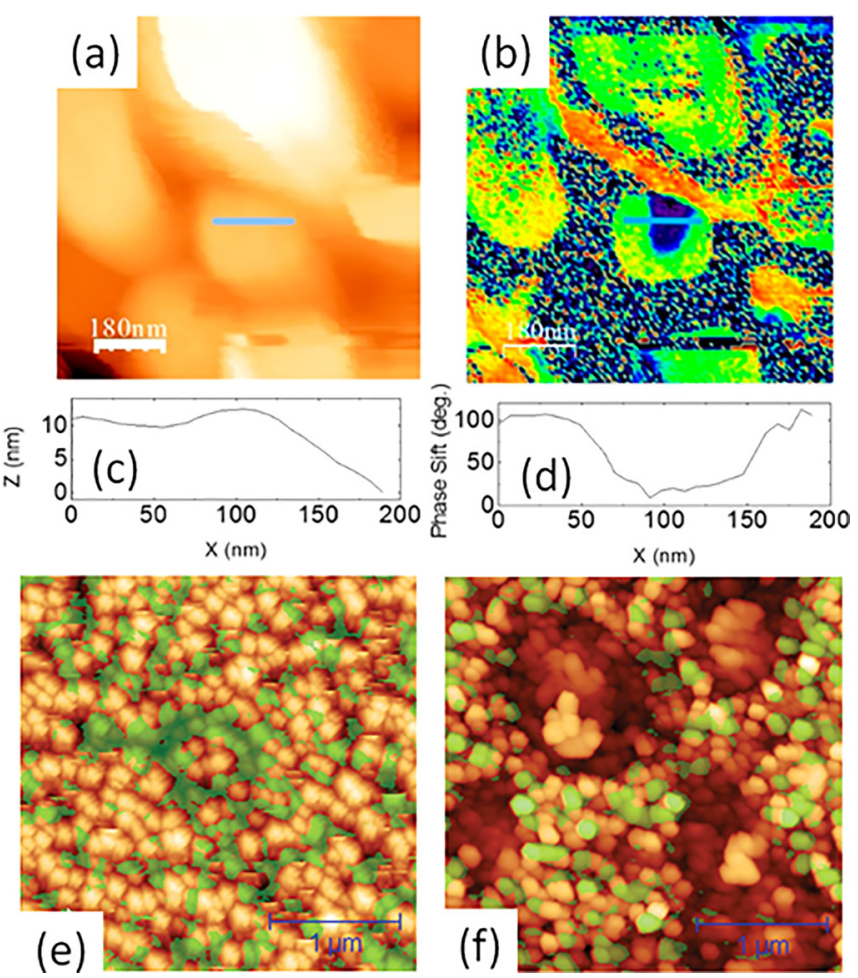

FIG. 24. (a) Topography (color scale from 0-220 nm), (b) corresponding PFM phase image (color scale from $0^{\circ}$ to $180^{\circ}$ ), (c) topography and (d) phase shift profiles along the marked lines in (a) and (b). Reprinted with permission from Minj et al., Nano Lett. 15, 6770 (2015). Copyright 2015 American Chemical Society. PFM images of AlN films topography (in a brown color scale) and the PFM phase overlay (green $=$ Al-polar, unmarked $=\mathrm{N}$-polar) corresponding to $(\mathrm{e})$ very $\mathrm{N}$-rich conditions (V/III flux ration $=1.7$ ) and (f) Al-rich conditions (V/III flux ratio=0.8). The z-scale for images (e) and (f) is $150 \mathrm{~nm}$ and $45 \mathrm{~nm}$, respectively. Reprinted with permission from J. Appl. Phys. 110, 053506 (2011). Copyright 2011 AIP Publishing LLC. 
$(-0.07 \pm 0.015) \mathrm{C} / \mathrm{m}^{2}$ was inferred for $P_{\mathrm{sp}}$ of $\mathrm{ZnO}$. More than 20 year later, Dal Corso et al. ${ }^{200}$ determined the $P_{\mathrm{sp}}$ of $\mathrm{ZnO}$ from first principles and noted with considerable surprise that their calculated value of $-0.05 \mathrm{C} / \mathrm{m}^{2}$ was close to the earlier indirect estimate. They commented on this fact stating that "the embarrassing point is that wurtzite-structure materials are not ferroelectric, their polarization is not reversible, and no kind of Landau expansion is meaningfully devisable."

More recently, Yan et al. ${ }^{201}$ proceeded along very similar lines to determine the $P_{\mathrm{sp}}$ of GaN. Employing the Ginzburg-Landau-Devonshire theory of phase transitions in ferroelectrics, these authors derived an approximate expression connecting $P_{\mathrm{sp}}$ to the high-pressure phase transition from the wurtzite to the rocksalt structure. Using available experimental data for the quantities entering this expression, a value of $-0.022 \mathrm{C} / \mathrm{m}^{2}$ was deduced for $P_{\mathrm{sp}}$ of $\mathrm{GaN}$. As in the case of $\mathrm{ZnO}$, the agreement of this result with the most recent values obtained by ab initio methods is most surprising, given the fact that the underlying theory should not be even applicable to a pyroelectric material.

The so far only direct determination of the $P_{\mathrm{sp}}$ of any wurtzite semiconductor was recently performed by Lähnemann et al. ${ }^{31}$ These authors studied GaN microcrystals obtained by pendeoepitaxial overgrowth of spontaneously formed $\mathrm{GaN}$ NWs (cf. Fig. 25). High-resolution TEM revealed that these microcrystals contain basal plane stacking faults (SFs) of the intrinsic $\mathrm{I}_{1}$ and $\mathrm{I}_{2}$ as well as the extrinsic $\mathrm{E}$ types. These SFs constitute a local deviation from the hexagonal wurtzite (0001) stacking sequence to the cubic zinc blende (111) stacking sequence $^{202}$ with a structurally well-defined thickness. These samples were, thus, a natural implementation of the theoretical approach to determine $P_{\mathrm{sp}}$ by defining the zinc blende structure as a reference phase with vanishing polarization (see Section II B) ${ }^{24-26}$ What proved to be crucial for the subsequent analysis was the fact that the microcrystals contained all three types of SFs in abundance.

The authors investigated these GaN microcrystals by both $\mu$-photoluminescence (PL) and cathodoluminescence (CL) spectroscopy. The SFs act as perfect zinc blende-like QWs in a wurtzite matrix, ${ }^{203-209}$ in the sense that strain or fluctuations in composition and thickness do not exist. The radiative decay of excitons bound to these SF QWs gives rise to lines in PL and CL spectra with an energy characteristic for the given SF (cf. Fig. 25). Additional lines due to the electronic coupling of states in SFs in close vicinity of each other complicated the analysis, which the authors solved by investigating several dozens of microcrystals. The energies related to isolated SFs emerged in the statistical analysis of the spectral positions of the emission lines.

Using DFT, the authors showed that $P_{\mathrm{sp}}$ in the wurtzite matrix induces an electrostatic field in the zincblende insertion. Poisson-Schrödinger calculations demonstrated that the change in the emission energy $\Delta E$ with the thickness of the zinc blende insertion is governed by the quantum-confined Stark effect resulting from this field. In other words, the polarization sheet charges at the interface between the zinc blende and wurtzite modifications essentially constitute a plate capacitor, for which the addition of a slab of dielectric
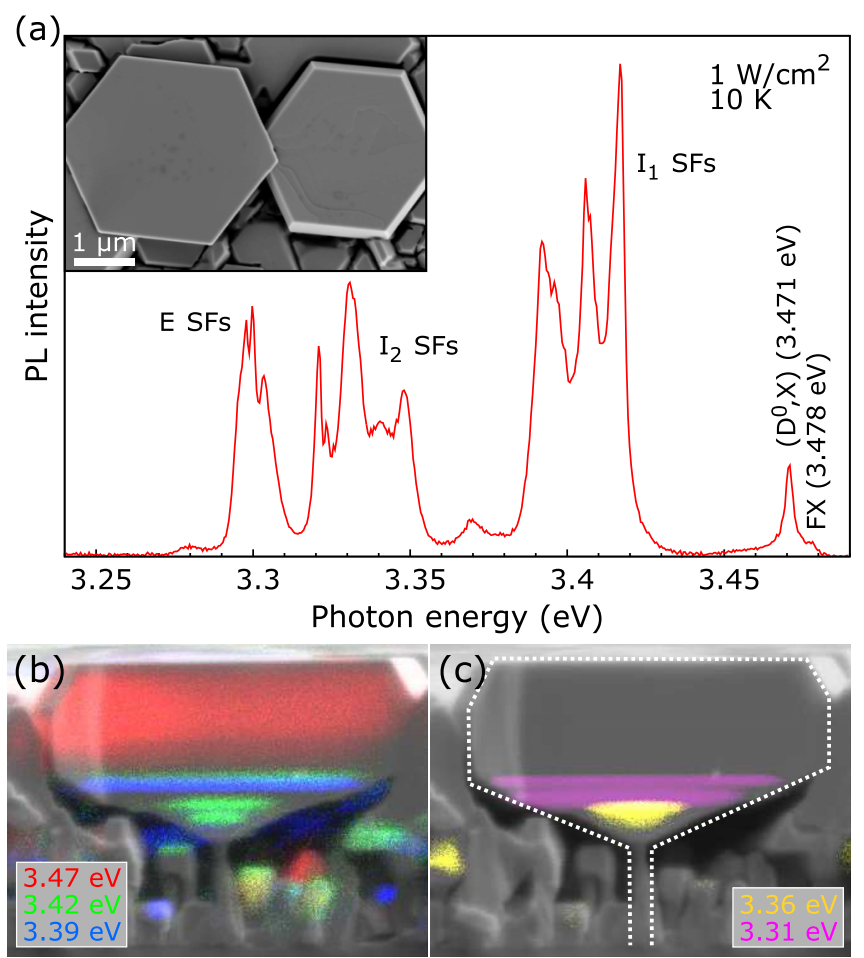

FIG. 25. (a) $\mu$-PL spectrum of a single GaN microcrystal detached from the film and dispersed onto a $\mathrm{Si}$ substrate. At high energies, emission from bound $\left[\left(\mathrm{D}^{0}, \mathrm{X}\right)\right]$ and free $(\mathrm{FX})$ excitons is observed at the positions expected for strain-free $\mathrm{GaN}$. The spectrum is dominated by lower energy lines with a high-energy onset at about $3.42,3.35$, and $3.31 \mathrm{eV}$, which is associated with emission from $\mathrm{I}_{1}, \mathrm{I}_{2}$, and E SFs, respectively. The lines below each of these high-energy onsets are due to SFs separated by only a few wurtzite bilayers, resulting in an electronic coupling between the respective bound states that lowers their energy. The inset shows a top-view scanning electron micrograph of such microcrystals. Monochromatic CL imaging on the cross section of such a crystal, as depicted in (b) and (c), shows that the near-band edge luminescence originates from the upper part of the microcrystals, while the remaining lines originate from the (lower) region of lateral expansion from a single nanowire to a microcrystal. All the low-energy lines appear as stripes running along the basal plane, confirming their common origin. Reprinted with permission from Lähnemann et al., Phys. Rev. B 86, 081302 (2012). Copyright 2012 American Physical Society.

of the width $\Delta d$ results in the potential difference $\Delta V$ equal to the experimental value of $\Delta E$.

This insight led to the key idea of the study of Lähnemann et al., ${ }^{31}$ namely, to rely on the differences in the emission energies rather than on their absolute values. This idea worked since the characteristic emission from all three SFs was observed and permitted a very simple and essentially parameter-free determination of the $P_{\mathrm{sp}}$ in the framework of the plate capacitor model. With the differences in the emission energies $\Delta E_{I_{1} \rightarrow I_{2}}=70 \pm 15 \mathrm{meV}$ and $\Delta E_{I_{2} \rightarrow E}=$ $60 \pm 18 \mathrm{meV}$, the mean energy difference for adding a bilayer to the $\mathrm{QW}$ was found to be $\overline{\Delta E}=65 \pm 23 \mathrm{meV}$. The magnitude of the $P_{\mathrm{sp}}$ is then given by

$$
\sigma=\left|P_{s p}\right|=\frac{\overline{\Delta E} \epsilon \epsilon_{0}}{\Delta d}=0.021 \pm 0.007 \mathrm{C} / \mathrm{m}^{2},
$$

with $\Delta d$, the difference in thickness between each of the SFs, being a single (111) bilayer (or molecular monolayer) of zinc blende $\mathrm{GaN}$, i.e., $0.259 \mathrm{~nm}$. $\epsilon=9.5$ is the relative 
permittivity of $\mathrm{GaN}$ and $\epsilon_{0}$ is the permittivity of free space. A detailed analysis of the transition energy differences using both a type I and a type II band alignment changed this value only slightly, and Lähnemann et al. ${ }^{31}$ finally recommended to use a value of $P_{s p}=-0.022 \pm 0.007 \mathrm{C} / \mathrm{m}^{2}$ for the spontaneous polarization of $\mathrm{GaN}$, which is quite close to the value of $-0.018 \mathrm{C} / \mathrm{m}^{2}$ they obtained in the same work by DFT employing a projector-augmented wave method. Note that the recommended value is equal to the estimate of $P_{\mathrm{sp}}$ by Yan $e t a l .{ }^{201}$ as discussed above. While the exact agreement is certainly fortuitous, it is remarkable that this very indirect estimate is close to that determined by more suitable means just as for the cases of $\mathrm{BeO}^{26}$ and $\mathrm{ZnO}^{200}$

\section{CONTROLLING THE POLARITY: NUCLEATION AND GROWTH}

In the present section, the polarity and its effects on the nucleation, growth, and doping mechanisms of thin films as well as nanostructures and NWs are presented in detail. GaN (and its compounds) thin films grown by MOCVD and $\mathrm{MBE}, \mathrm{ZnO}$ (and its compounds) thin films grown by CVD, MOCVD, MBE, and pulsed laser deposition (PLD), as well as GaN NWs and nanorods grown by MOCVD and MBE, and $\mathrm{ZnO}$ nanostructures (with different shapes) and NWs grown by vapor phase and solution deposition techniques are comprehensively addressed. It is worth noticing that the analysis of the polarity of NW ensembles is a much more demanding task than that of thin films because all NWs are not necessarily identical. Therefore, in order to conclusively assess their polarity, it is necessary to investigate a statistically meaningful number of NWs. In that respect, it is important to keep in mind the assets and limitations of the different techniques used to measure polarity, as presented in Section III, especially when comparing the results reported by different groups. In many cases, microscopic and macroscopic polarity measurements are not always equivalent, but complementary, because they provide information on either individual NWs or the majority of them.

\section{A. GaN thin films}

\section{Introduction}

As bulk GaN substrates have been available only in recent years and are still expensive, GaN has historically been grown on foreign substrates, such as sapphire, silicon carbide $(\mathrm{SiC})$, and $\mathrm{Si}$, naming only the most technologically relevant ones. Among those, only $\mathrm{SiC}(4 \mathrm{H}-\mathrm{SiC}$ and $6 \mathrm{H}-\mathrm{SiC})$ is polar as well, and $\mathrm{Ga}$ - and $\mathrm{N}$-polar GaN films are obtained on $\mathrm{Si}$ - and $\mathrm{C}$-polar $\mathrm{SiC}$, respectively. Despite the similar polarity of $\mathrm{SiC}$ and $\mathrm{GaN}$, the different chemical nature of substrate and epitaxial layer requires a careful optimization of the initial growth steps to transfer the polarity from substrate to epitaxial layer since, for instance, under certain conditions Ga-polar GaN growth can be commenced also on C-polar SiC. ${ }^{210}$ The conditions under which the growth is initiated are even more critical when the epitaxial process is conducted on a non-polar substrate such as sapphire, historically the most widely used substrate. The details of the growth processes on $\mathrm{SiC}$, sapphire, and $\mathrm{Si}$ substrates are thoroughly discussed hereafter.

As far as the methods for epitaxial growth are concerned, historically first $\mathrm{HVPE}^{211}$ and MOCVD were explored. ${ }^{212}$ Due to the significantly higher growth rates, today HVPE is primarily used for the fabrication of thick GaN films and bulk GaN substrates, including epitaxial lateral growth techniques for threading dislocation reduction. ${ }^{213}$ MOCVD is the most widely used growth technique for $(\mathrm{Al}, \mathrm{Ga}, \mathrm{In}) \mathrm{N} / \mathrm{GaN}$ devices and the majority of commercially available GaN-based devices are fabricated by MOCVD. In addition, MBE, utilizing either a nitrogen plasma or ammonia as nitrogen source, is used for the fabrication of $(\mathrm{Al}, \mathrm{Ga}, \mathrm{In}) \mathrm{N}$ heterostructures. The present section focuses on MOCVD and PAMBE as growth techniques, by both of which not only Ga-polar but also high quality N-polar Nitrides heterostructures were demonstrated in the past years.

Due to difficulties in the growth of high quality N-polar GaN films, previously most of investigations focused on the development of Ga-polar GaN, and the majority of today's devices are based on (0001) (Al,Ga,In) N heterostructures. While Ga-polar GaN films were smooth and contained threading dislocation densities in the lower $10^{8} \mathrm{~cm}^{-2}$ when deposited under optimized conditions, $\mathrm{N}$-polar GaN layers were typically rough originating from the formation of large hexagonal hillocks and contained significantly higher threading dislocation densities. ${ }^{214,215}$ Thereby, the characteristic hillocks did not only form when the films were grown on sapphire substrates but also on N-polar GaN bulk crystals, ${ }^{216} \mathrm{C}$-face $\mathrm{SiC}^{210,217}$ and when $\mathrm{N}$-polar films were realized through polarity inversion caused by heavy Mg-doping of Ga-polar GaN:Mg layers. ${ }^{218-221}$ The hillock formation was originally associated with the existence of inversion domains. ${ }^{222}$ The first smooth N-polar GaN films were demonstrated by MBE on C-polar $\mathrm{SiC}$ in $2000 .{ }^{223}$ At the same time, improvements in the surface quality of MOCVD grown films were observed for films deposited on misoriented N-polar GaN bulk crystals, ${ }^{224,225}$ which had been obtained by high pressure growth. ${ }^{226}$ One report on the MOCVD growth of smooth N-polar GaN films on sapphire substrates was published in $2006 .{ }^{227}$ Later, high quality $\mathrm{N}$-polar $(\mathrm{Al}, \mathrm{Ga}, \mathrm{In}) \mathrm{N}$ epitaxial films were demonstrated on misoriented sapphire, ${ }^{228} \mathrm{SiC},{ }^{229,230}$ and $\mathrm{Si}^{231}$ substrates opening the way for the utilization of MOCVD grown N-polar $(\mathrm{Al}, \mathrm{Ga}, \mathrm{In}) \mathrm{N}$ heterostructures for device applications. Excellent device performance was demonstrated, in particular, for $\mathrm{N}$ polar transistors, ${ }^{232,233}$ cf. Section V C.

Generally, the present section focuses on recent results, given that early reviews on the polarity of GaN were published by Hellmann in $1998,{ }^{234}$ Stutzmann et al. in $2001,{ }^{215}$ and Sumiya and Fuke in $2004 .{ }^{235}$ As the Ga- polar (Al,Ga,In)N epitaxy process has been widely discussed in the literature, this section emphasizes the growth of $\mathrm{N}$-polar $(\mathrm{Al}, \mathrm{Ga}, \mathrm{In}) \mathrm{N}$ layers and provides a comparison between the specific growth conditions of $\mathrm{N}$ - and Ga-polar (Al,Ga,In)N films.

\section{MOCVD and MBE}

In the MOCVD process, ammonia and metal-organic Ga compounds, typically trimethylgallium (TMGa) or 
triethylgallium (TEGa), are transported in $\mathrm{H}_{2}$ or $\mathrm{N}_{2}$ carrier gas and react on a heated substrate under formation of $\mathrm{GaN}$ following the Brutto reaction:

$$
\mathrm{Ga}\left(\mathrm{CH}_{3}\right)_{3}+\mathrm{NH}_{3} \rightarrow \mathrm{GaN}+3 \mathrm{CH}_{4} .
$$

The epitaxy process is typically conducted at pressures between 70 and 760 Torr and temperatures above $900^{\circ} \mathrm{C}$ in excess of $\mathrm{NH}_{3}$, a regime where the $\mathrm{GaN}$ growth rate is limited by the gas phase transport of Ga-species to step and kink positions on the surface of the growing crystal. An exception are the nucleation layers, which are deposited at significantly lower temperatures, typically between 550 and $650{ }^{\circ} \mathrm{C}$ for Ga-polar GaN, where the growth rate can be limited by the abstraction of methyl groups from the metal precursor molecules, and/or by the $\mathrm{NH}_{3}$ decomposition, which is kinetically activated at temperatures below $750^{\circ} \mathrm{C} .{ }^{236}$ For the growth of alloys with $\mathrm{Al}$ and $\mathrm{In}$, typically trimethylaluminum (TMAl) and trimethylindium (TMIn) are used as precursors.

In MBE, Ga atoms are vaporized from an ultra-pure liquid $\mathrm{Ga}$ source in a high vacuum environment and ballistically reach the surface of a heated substrate on which they condense with active nitrogen species to form GaN. The active nitrogen species are provided either through thermal dissociation of $\mathrm{NH}_{3}$ molecules on the heated substrate (i.e., ammonia-MBE) or through cracking of $\mathrm{N}_{2}$ molecules by a radio-frequency (rf) wave at $13.56 \mathrm{MHz}$ (i.e., rf-PAMBE). In both cases, the pressure during growth is in the order of $10^{-5}$ Torr leading to atom mean free paths of about $10 \mathrm{~m}$. GaN growth by PAMBE is typically performed around $700{ }^{\circ} \mathrm{C}$ using slightly Ga-rich conditions, ${ }^{237}$ as discussed in more detail in Subsection IV A 3 a. Ammonia-MBE operates at higher temperatures and under highly $\mathrm{N}$-rich conditions closer to the MOCVD environment. ${ }^{237,238}$

\section{Growth on different substrates}

a. Bulk GaN. As for other semiconductors, the surface processes during GaN epitaxy are strongly influenced by the nature of the crystal surface. Whereas the surface of Ga-polar (0001) GaN is terminated with Ga-atoms, that of N-polar $(000 \overline{1}) \mathrm{GaN}$ is composed of N-atoms, both of which have one dangling bond when considering a perfect crystal in vacuum (see Section II). In the growth ambient, atoms and molecules can adsorb on (or desorb from) the surface, altering its properties. ${ }^{239}$ The N-polar GaN surface has a particularly high affinity for the adsorption of hydrogen, and the hydrogen-terminated surface was found to be more stable compared with any clean surface. ${ }^{72}$

Under typical MOCVD growth conditions, the growth rate is limited by the transport of Ga-species to step and kink positions on the surface. On a Ga-polar GaN surface, only relatively weak delocalized metallic bonds form between Ga-species and Ga-surface atoms, enabling easy desorption (and subsequent re-condensation) ${ }^{240,241}$ and movement to step and kink positions, resulting in step-flow growth. In contrast, on the N-polar GaN surface, significantly stronger Ga$\mathrm{N}$ bonds form with the $\mathrm{N}$-terrace atoms, resulting in a higher activation energy for desorption, thus hampering the transport of adsorbed species to steps and kinks and increasing the probability of island nucleation on the terraces. Diffusion barriers of $0.4 \mathrm{eV}$ and $1.4 \mathrm{eV}$ for $\mathrm{Ga}$ and $\mathrm{N}$ atom diffusion on Ga-terminated (0001) GaN surfaces, respectively, were predicted in theoretical calculation. ${ }^{242}$ To compensate for the higher diffusion barrier, the MOCVD process for $\mathrm{N}$-polar $\mathrm{GaN}$ is often conducted at higher temperatures and using reduced $\mathrm{NH}_{3}$ flows, the latter to lower the probability of Ga-N- cluster formation on the terraces, which can act as seeds for hexagonal islands. From a thermodynamic point of view, the preferred growth conditions for $\mathrm{N}$-polar GaN films are characterized by a low supersaturation, as discussed in Ref. 243.

The probability for island nucleation on the terraces can be further suppressed when the terrace length between surface steps is reduced by introducing crystal misorientation. A significant reduction in the density of hexagonal hillocks from about $1 \times 10^{5} \mathrm{~cm}^{-2}$ to about $1 \times 10^{3} \mathrm{~cm}^{-2}$ was reported by Zauner et al., when GaN films were deposited on N-polar GaN substrates misoriented by $4^{\circ}$ toward the $\langle 10 \overline{1} 0\rangle \mathrm{m}$ direction of the GaN crystal, compared with on-axis substrates. ${ }^{224,225}$ In the following studies, a misorientation angle of $2^{\circ}$ was found to be sufficient to eliminate the formation of hexagonal surface features, though the process was more robust when higher misorientation angles of $3^{\circ}$ or $4^{\circ}$ were used (Fig. 26). ${ }^{228}$ The preferred GaN misorientation direction was toward the $m$-direction of the GaN crystal with steps parallel to the $\langle 11 \overline{2} 0\rangle a$-direction, resulting in a more regular surface morphology compared with the orthogonal direction. In addition, the surfaces maintained a low roughness, also when the N-polar GaN layers were grown under higher supersaturation conditions with higher $\mathrm{NH}_{3}$ flows and/or at lower deposition temperatures, for example (see Subsection IV A $3 \mathrm{c}$ ). More recently, smooth films were demonstrated also using misorientation angles below $1^{\circ} .{ }^{244,245}$

The surface mobility of adsorbed species can be further enhanced through the use of surfactants such as indium, ${ }^{246,247}$ magnesium, ${ }^{244}$ and hydrogen. ${ }^{248,249}$

In difference to Ga-polar GaN films, which typically exhibit $0.26 \mathrm{~nm}$ high surface steps, corresponding to the height of one Ga-N monolayer (Fig. 27), ${ }^{250,251}$ two monolayer high steps or their multiples were observed on N-polar GaN surfaces. The existence of double steps was related to the circumstance that, in hexagonal crystals, three out of six step edges have one dangling bond, whereas the remaining three steps have two dangling bonds per edge atom, namely, the so-called A and B type steps following the terminology of Xie et al. ${ }^{25,252} \mathrm{~A}$ and B type steps alternate with each other (Figs. 28 and 29). Due to their two dangling bonds, type A steps propagate faster, growing out until bounded by slower type B steps, resulting in two monolayer high steps. ${ }^{253} \mathrm{~A}$ detailed investigation of the impact of the growth conditions on the step structure of Ga- and N-polar GaN layers was reported in Ref. 254. The surface morphology of MOCVD grown N-polar GaN films is further discussed in Subsections IV A 3 b-IV A 3 d.

Comparing the growth rates of GaN films on co-loaded Ga- and N-polar GaN-on-sapphire base layers, the latter ones misoriented by $4^{\circ}$ toward the $m$-direction of the GaN crystal, 


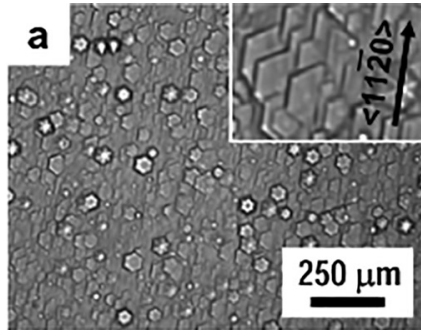

$0.5^{\circ}$ toward A

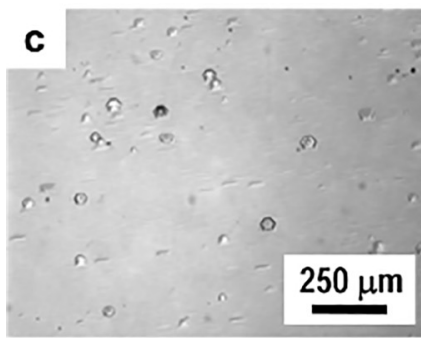

$1^{\circ}$ toward A

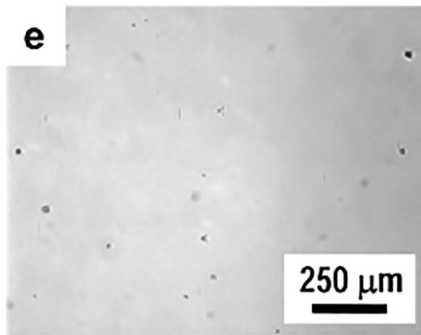

$2^{\circ}$ toward A

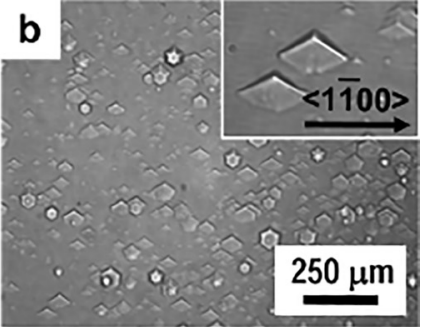

$0.5^{\circ}$ toward $\mathrm{M}$

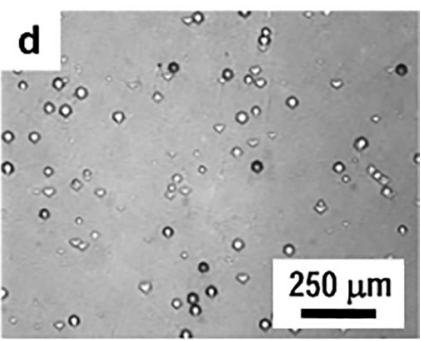

$1^{\circ}$ toward $M$

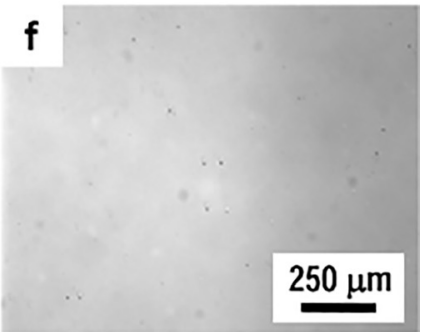

$2^{\circ}$ toward $M$
FIG. 26. Optical micrographs of $0.8 \mu \mathrm{m}$ thick $\mathrm{GaN}$ films grown on (0001) sapphire substrates with misorientation angles of (a) $0.5^{\circ}$, (c) $1^{\circ}$, (e) $2^{\circ}$ toward the $a$-plane, and (b) $0.5^{\circ}$, (d) $1^{\circ}$, (f) $2^{\circ}$ toward the $m$-sapphire plane. The insets in (a) and (b) are enlarged 3 fold. Reprinted with permission from J. Appl. Phys. 102, 083546 (2007). Copyright 2007 AIP Publishing LLC.

little differences were observed. The situation, however, is entirely different when Ga- and N-polar surfaces co-exist, also together with other crystal orientations such as $a$ - or $m$ plane $\mathrm{GaN}$, on a patterned surface, for example. Among those, the growth rate on the N-polar plane is typically the slowest one in the MOCVD process. ${ }^{255,256}$

No such dramatic adjustments of the growth process are required when depositing N-polar GaN films by PAMBE. For both $\mathrm{N}$ - and Ga-polar surfaces, $\mathrm{N}$-rich growth regimes lead to rougher surfaces because of reduced $\mathrm{Ga}$ adatom mobility. ${ }^{242,257-262}$ Ga-rich regimes are therefore preferred but require a precise control of the Ga adlayer in order to avoid unwanted accumulation of $\mathrm{Ga}$ droplets on the surface. On Ga-polar GaN surfaces, up to $\sim 2.5$ monolayers (ML) of Ga can wet the surface, forming an auto-regulated bilayer ${ }^{61,263}$ that serves as an auto-surfactant. ${ }^{57,62}$ On N-polar GaN surfaces, Ga metal accumulation occurs after the deposition of only $\sim 1 \mathrm{ML}$ of $\mathrm{Ga}^{242}$ The smoothest films are therefore achieved with a Ga excess of $\sim 2.5 \mathrm{ML}$ on Ga-polar surfaces and of $\sim 1 \mathrm{ML}$ on N-polar ones. As the surface is covered with $\mathrm{Ga}$ in both cases, the surface mobility of reactant species is largely independent upon the surface polarity. For this reason, no significant adjustments of the growth process are required when depositing N-polar GaN films by MBE, and
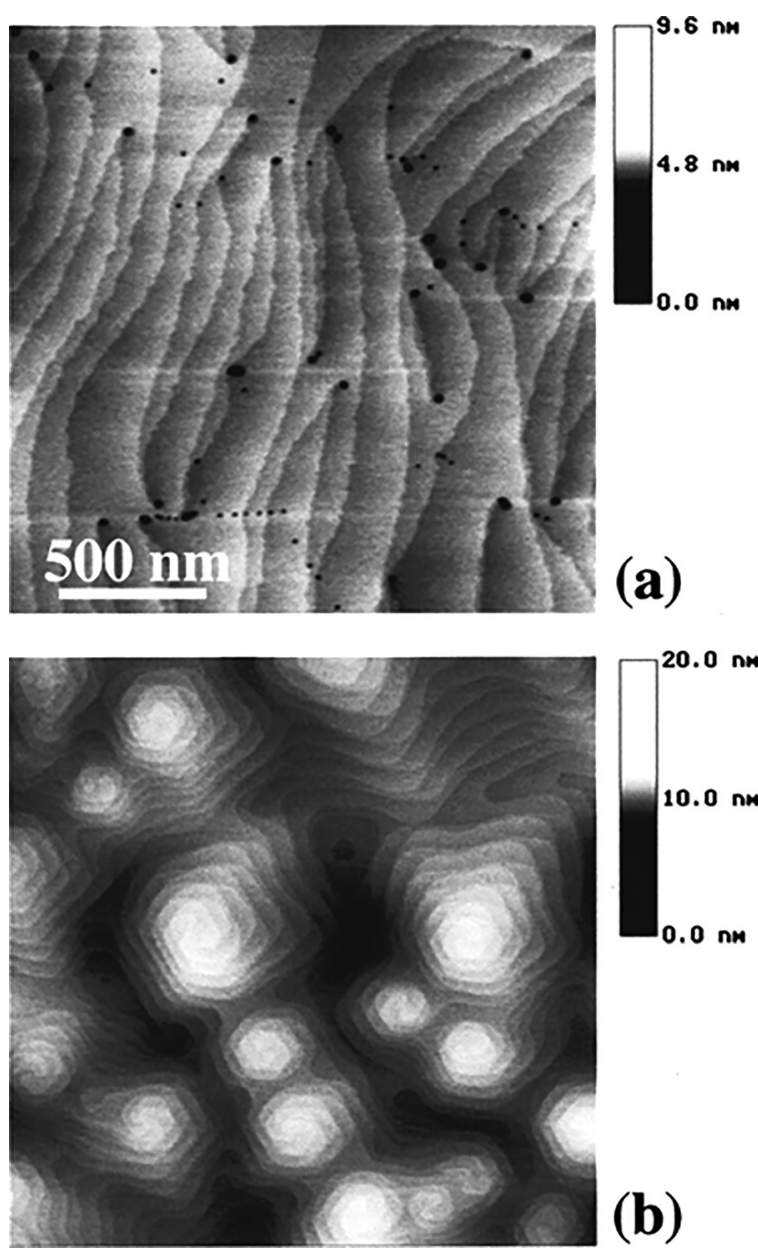

FIG. 27. AFM images of representative morphologies of Ga-polar GaN films grown by (a) MOCVD and (b) MBE on c-plane sapphire substrates. Reprinted with permission from J. Appl. Phys. 85, 6470 (1999). Copyright 1999 AIP Publishing LLC.

smooth N-polar GaN layers were demonstrated using standard on-axis substrates.

$\mathrm{N}$-polar GaN films can also be obtained through polarity inversion by heavy Mg-doping of Ga-polar GaN:Mg layers,

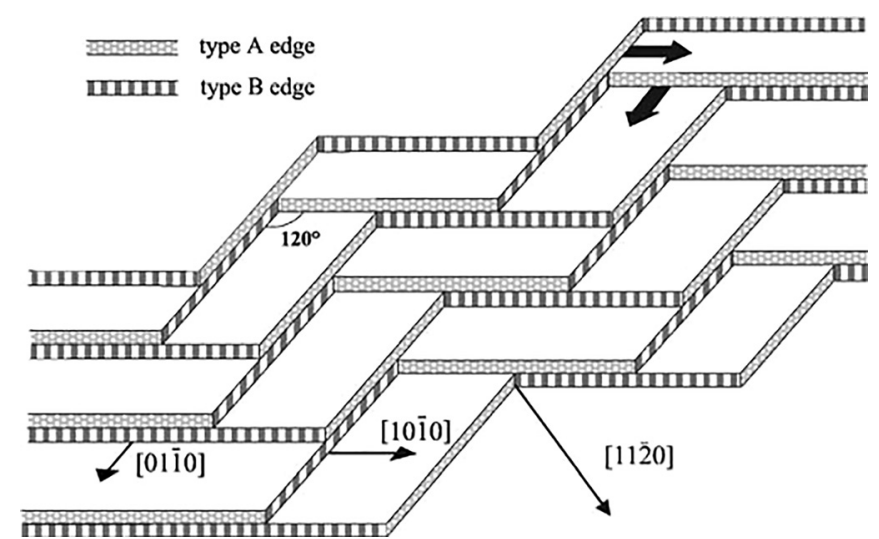

FIG. 28. Schematic presentation of the misorientation induced steps towards the $1120_{\mathrm{GaN}}$ direction. The broad arrows indicate the direction in which the fast type A edge can freely propagate over the underlying terrace. The step directions towards $[10 \overline{1} 0]$ and [0110] correspond to steps parallel to $\langle\overline{1} 2 \overline{1} 0\rangle$ and $\langle 2 \overline{1} \overline{1} 0\rangle$, respectively (Figure 29). Reprinted with permission from Zauner et al., J. Cryst. Growth 240, 14 (2002). Copyright 2002 Elsevier. 


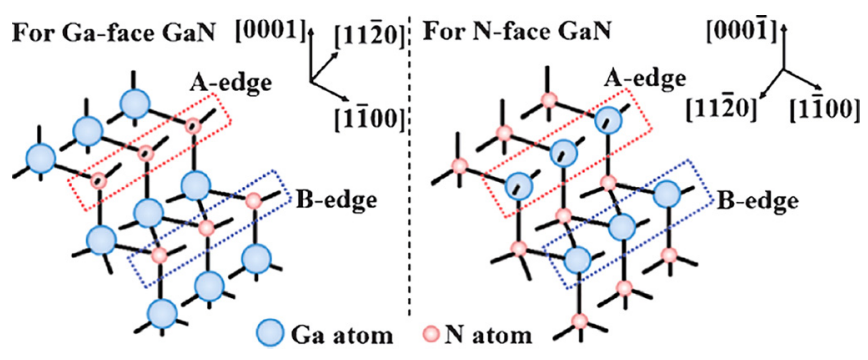

FIG. 29. Different arrangements of atoms and dangling bonds at the steps of Ga- and N-polar (face) GaN. Reprinted with permission from Lin et al., Appl. Phys. Express 6, 035503 (2013). Copyright 2013 The Japan Society of Applied Physics.

using the following different growth methods: MOCVD, ${ }^{220,221}$ PAMBE, ${ }^{218,219,264}$ as well as ammonia-MBE. ${ }^{265,266}$ Using MOCVD on typical on-axis Ga-polar GaN, the characteristic hillocks were again observed. ${ }^{220}$ Smooth N-polar $(\mathrm{Al}, \mathrm{Ga}) \mathrm{N}$ films were obtained when the technique was used on misoriented Si substrates, as discussed in more detail in Subsection IV A $3 \mathrm{~d}$.

Conversely, Ga-polar GaN films can be achieved from $\mathrm{N}$-polar GaN using MBE through the formation of $\mathrm{Mg}_{\mathrm{x}} \mathrm{N}_{\mathrm{y}}$, which is obtained by exposing the surface simultaneously to $\mathrm{Mg}$ and $\mathrm{NH}_{3}{ }^{265}$ or active $\mathrm{N}$ from $\mathrm{N}_{2}{ }^{267}$ while interrupting the growth at a reduced temperature, or alternatively, through insertion of an $\mathrm{AlN} / \mathrm{AlO}_{\mathrm{x}}$ interlayer. ${ }^{268}$ Using MOCVD, conversion from $\mathrm{N}$ - to Ga-polarity was demonstrated by inserting AlN interlayers. ${ }^{269}$

b. SiC. Since $4 \mathrm{H}-$ and $6 \mathrm{H}-\mathrm{SiC}$ are polar crystals like $\mathrm{GaN}$, Ga- and N-polar GaN films can be obtained on (0001) Si-polar and $(000 \overline{1})$ C-polar SiC substrates, respectively. ${ }^{217,270}$ The lattice mismatch between (0001) $\mathrm{GaN}$ and (0001) $6 \mathrm{H}-\mathrm{SiC}$ is $3.4 \%$. Independent of polarity and growth method, the deposition process is typically initiated with an AlN layer, which not only possesses a lattice mismatch of only $1 \%$ to $\mathrm{SiC}$ but also shows good wetting on the $\mathrm{SiC}$ surface, on top of which the main GaN layer is grown afterwards.

The growth of Ga-polar $(\mathrm{Al}, \mathrm{Ga}) \mathrm{N}$ on $\mathrm{Si}$-polar $\mathrm{SiC}$ by MOCVD has been intensely studied in the literature. The initial AlN layer is typically deposited at high temperatures, comparable to those of the main GaN layer. ${ }^{271}$ The thicknesses of the AIN layers widely vary and depend on the requirements of the targeted device. Smooth AlN layers can be achieved after deposition of less than $50 \mathrm{~nm}$ of AlN. ${ }^{272}$ The following GaN layer was shown to re-nucleate on the AlN layer forming islands, which coalesce in the course of the deposition process. ${ }^{273}$ The density of the initial GaN islands strongly affects the threading dislocation density in the final GaN film. Excellent Ga-polar GaN films were demonstrated on $\mathrm{SiC}$ substrates with threading dislocation densities in the low $10^{8} \mathrm{~cm}^{-3}$ range.

The deposition of smooth N-polar GaN films on C-polar $\mathrm{SiC}$ by MOCVD was again more challenging, due to the differences in the Ga- and $\mathrm{N}$-polar growth process as discussed in Subsection IV A 3 a. Films deposited on on-axis C-polar $\mathrm{SiC}$ substrates exhibited the characteristic hexagonal hillocks. ${ }^{210}$ Smooth N-polar GaN films were again obtained when introducing crystal misorientation, using C-polar $\mathrm{SiC}$ substrates with a misorientation of $4^{\circ}$ or $3.6^{\circ}$ toward the $a$-SiC-plane, resulting in $\mathrm{N}$-polar GaN misoriented toward the $a$-GaN-plane. ${ }^{229,246}$ In other studies, a misorientation of $4^{\circ}$ toward the $m$-SiC-plane, leading to (Al,Ga,In)N films misoriented toward the $m$-GaN-plane, was used to again take advantage of the more regular surface morphology, as discussed in more detail in Subsection IV A 3 c. ${ }^{230,274}$ Similar to the growth process for metal-polar films, the growth is initiated with a thin AIN layer grown at temperatures between 1090 and $1215^{\circ} \mathrm{C}$. A high $\mathrm{NH}_{3} / \mathrm{TMAl}$ ratio was needed for the deposition of $\mathrm{N}$-polar films, as low $\mathrm{NH}_{3}$ /TMA1 ratios or TMA1 pre-flows resulted in (Al,Ga)-polar layers. ${ }^{210,229}$ In contrast to the typical process for Ga-polar GaN films, an approximately $200 \mathrm{~nm}$-thick GaN layer was next grown at intermediate temperatures $\left(975^{\circ} \mathrm{C}\right)$ before the deposition of the main $\mathrm{GaN}$ layer at high temperatures. ${ }^{230}$ The addition of the intermediate temperature layer prevented the formation of macro-steps on the GaN surface. Later, the intermediate temperature $\mathrm{GaN}$ layer was replaced by an $(\mathrm{Al}, \mathrm{Ga}) \mathrm{N}$ layer. ${ }^{275}$ For about $0.9 \mu$ m-thick GaN layers, FWHM values of $0.03^{\circ}$ and $0.11^{\circ}$ were measured for the $(000 \overline{2})$ and $(20 \overline{2} \overline{1})$ XRD peaks, respectively. ${ }^{249}$

In the PAMBE process, GaN growth follows similar procedures on both $\mathrm{Si}$ - and C-polar SiC substrates. The AlN buffer layer, which not only releases part of the $\mathrm{GaN} / \mathrm{SiC}$ lattice mismatch but also improves $\mathrm{GaN}$ wetting, ${ }^{276}$ is typically grown at about $720-740^{\circ} \mathrm{C}$. N-rich conditions $(\mathrm{Al} / \mathrm{N}$ ratio $<1$ ) are used to prevent liquid $\mathrm{Al}$ from reacting with $\mathrm{Si}$ and thereby hinder the diffusion of $\mathrm{Si}$ and $\mathrm{C}$ impurities into the AlN layer, allowing for reduced buffer leakage in devices both on Si-polar SiC ${ }^{277,278}$ and C-polar SiC. ${ }^{279}$ Then, GaN is grown on top of the AIN nucleation layer by following a two-step process. In the first step, GaN is grown in the so-called intermediate regime with only a slight $\mathrm{Ga}$ excess, resulting in a rough surface allowing for reduction of the threading dislocation density. ${ }^{280,281}$ In the second step, GaN is grown with a higher Ga flux at the boundary between the intermediate regime and the droplet accumulation regime, resulting in a smooth surface. Using typical AIN nucleation layer thicknesses of $\sim 45 \mathrm{~nm}$ and about $300 \mathrm{~nm}$ of $\mathrm{GaN}$, the resulting films exhibit root mean square surface roughness values below $1 \mathrm{~nm}$ over a $2 \times 2 \mu \mathrm{m}^{2}$ area and dislocation densities around $1.5 \times 10^{10} \mathrm{~cm}^{-2} .282$

An improvement of the structural quality of Ga-polar GaN films was observed when vicinal 4H-SiC (0001) substrates were used. ${ }^{283}$ As mentioned before, the first smooth $\mathrm{N}$-polar GaN films were demonstrated by Guan et al. on C-polar $\mathrm{SiC}$ by $\mathrm{MBE}$ in $2000 .^{223}$

c. Sapphire. For growth on (0001) sapphire, which possesses a hexagonal crystal structure like $\mathrm{GaN}$ but has the corundum crystal structure and is centrosymmetric, the polarity of the GaN epitaxial layers critically depends on the way the growth is initiated..$^{214,215,234,284-287}$ Despite the high lattice mismatch of $16 \%$ between sapphire and GaN, high quality Ga- and N-polar GaN films with threading dislocation densities in the order of magnitude of $10^{8} \mathrm{~cm}^{-2}$ were 
demonstrated on sapphire substrates after careful process optimization.

In the MOCVD growth process, the sapphire substrate is first exposed to a high temperature cleaning step in $\mathrm{H}_{2}$. In the typical two-step process for the growth of Ga-polar GaN films, the temperature is then lowered to $550^{\circ} \mathrm{C}$ to $650{ }^{\circ} \mathrm{C}$ to deposit a thin AlN or GaN nucleation layer. ${ }^{288,289}$ Investigations showed that the crystal quality of the nucleation layers is poor but significantly improves while the sample is heated up to the growth temperature of the main $\mathrm{GaN}$ layer, which is typically deposited at temperatures above $1000{ }^{\circ} \mathrm{C}^{290} \mathrm{In}$ the early stages of the high temperature growth, large Volmer-Weber GaN islands form on the nucleation layers, which increase in height as well as diameter before finally coalescing into a planar film. ${ }^{291}$ The quality of the GaN layers is strongly influenced by the size and density of the high temperature islands. The lower their density, the lower the threading density in the GaN layer is. ${ }^{292}$

In difference to the process for growing Ga-polar $\mathrm{GaN}$ films, to obtain N-polar GaN films, the sapphire substrate is exposed to $\mathrm{NH}_{3}$ at high temperatures prior to $\mathrm{AlN}$ or GaN nucleation layer growth. In this step, a thin AlN surface layer forms ${ }^{293}$ via the reaction

$$
\mathrm{Al}_{2} \mathrm{O}_{3}+2 \mathrm{NH}_{3} \rightarrow 2 \mathrm{AlN}+3 \mathrm{H}_{2} \mathrm{O},
$$

typically resulting in N-polar GaN films. ${ }^{294,295}$ When only inserting this high temperature sapphire nitridation step, however, the N-polar GaN films were typically rough due to the formation of large hexagonal surface features. Smooth films were routinely obtained when misoriented sapphire substrates were used, in combination with a re-optimized growth process. ${ }^{228}$ While a misorientation of $2^{\circ}$ toward the $a$ - or $m$-directions of sapphire was sufficient to suppress hillock formation (Fig. 26), higher misorientation angles resulted in higher quality films, which for misorientation angles of $3^{\circ}-4^{\circ}$ exhibited properties comparable to typical Ga-polar GaN films, as illustrated in Figs. 30 and $31 .{ }^{228} \mathrm{~N}-$ polar GaN films with excellent properties had also been

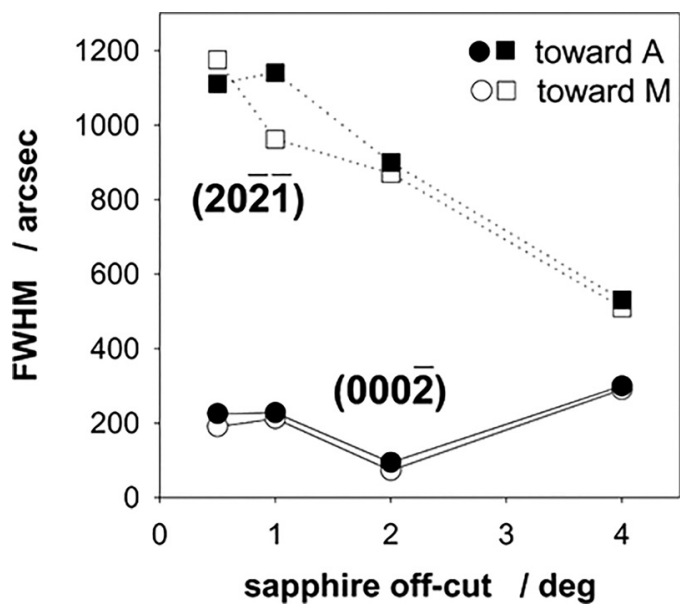

FIG. 30. Full width at half maximum (FWHM) of the XRD rocking curves measured for the $(000 \overline{2})$ and $(20 \overline{2} \overline{1})$ diffraction peaks of $0.8 \mu \mathrm{m}$ thick GaN films grown by MOCVD on (0001) $\mathrm{Al}_{2} \mathrm{O}_{3}$ substrates with different misorientation angles and directions. Reprinted with permission from J. Appl. Phys. 102, 083546 (2007). Copyright 2007 AIP Publishing LLC.
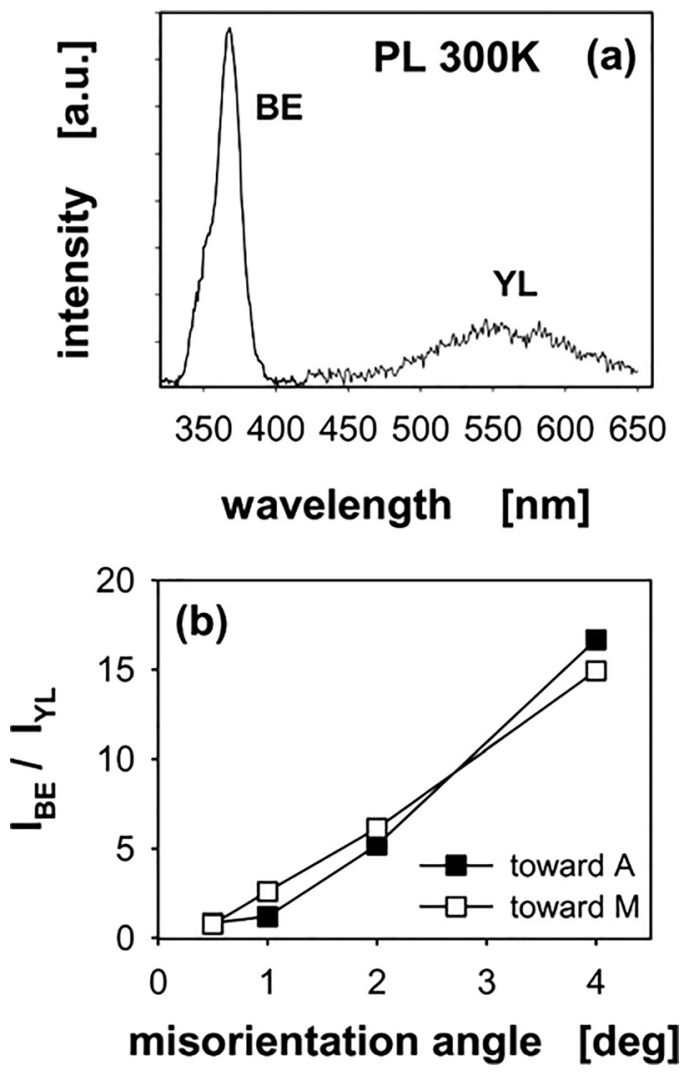

FIG. 31. (a) $300 \mathrm{~K}$ PL spectrum of a $0.8 \mu \mathrm{m}$ thick $\mathrm{GaN}$ films grown by MOCVD on (0001) $\mathrm{Al}_{2} \mathrm{O}_{3}$ substrates with a misorientation angle of $2^{\circ}$ toward the $a$-plane. (b) Ratio between the $300 \mathrm{~K}$ PL intensity of the band edge related luminescence (BE) at $364 \mathrm{~nm}$ and the yellow luminescence (YL) band around $550 \mathrm{~nm}$ for $0.8 \mu \mathrm{m}$ thick $\mathrm{GaN}$ films grown on (0001) sapphire with different misorientation angles and directions. Reprinted with permission from J. Appl. Phys. 102, 083546 (2007). Copyright 2007 AIP Publishing LLC.

reported by Matsuoka et al., ${ }^{227}$ which according to later publications had been grown on sapphire substrates with a misorientation between $0.4^{\circ}$ and $1.1^{\circ} .{ }^{245}$ While the use of vicinal substrates significantly suppressed the formation of hexagonal surface hillocks, additional process optimization was needed to obtain high quality films. N-polar GaN nucleation layers were typically deposited at higher temperatures compared with the Ga-polar process. ${ }^{249}$ As discussed before, also the main GaN layers were usually deposited at higher temperatures and lower $\mathrm{NH}_{3}$ flows compared with Ga-polar films, and often using a reduced growth pressure. ${ }^{296}$ These process modifications counterbalanced the lower surface mobility of Ga-species on the N-polar GaN surface. While the formation of hillocks could be suppressed independent of the misorientation direction, GaN layers with more regular surface undulations were obtained using sapphire substrates with a misorientation toward the $a$-sapphire plane, resulting in GaN films misoriented toward the $m$-plane, due to the $30^{\circ}$ rotation between the GaN and sapphire lattices (Fig. 32). ${ }^{297}$ For GaN layers misoriented toward the $a-\mathrm{GaN}$ plane, at higher misorientation angles irregular surface depressions formed, in particular, when the $\mathrm{GaN}$ top layers were grown at higher $\mathrm{NH}_{3}$ flows and/or reduced temperatures. The higher misorientation angles around $4^{\circ}$ also resulted in the lowest 


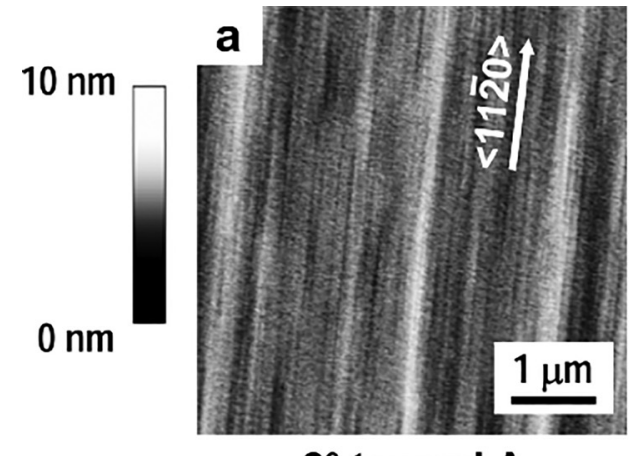

$2^{\circ}$ toward $A$

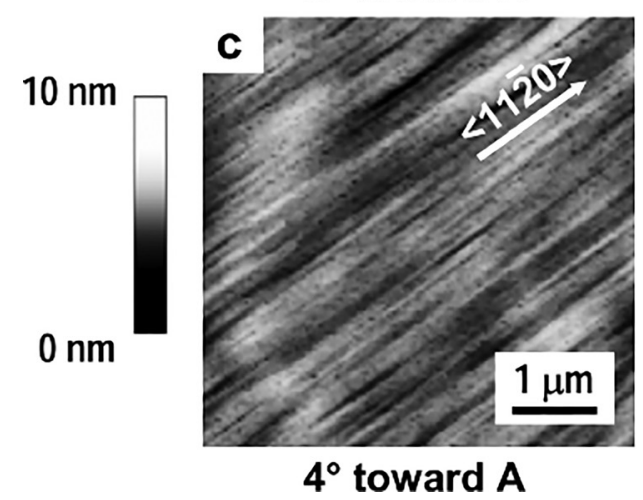

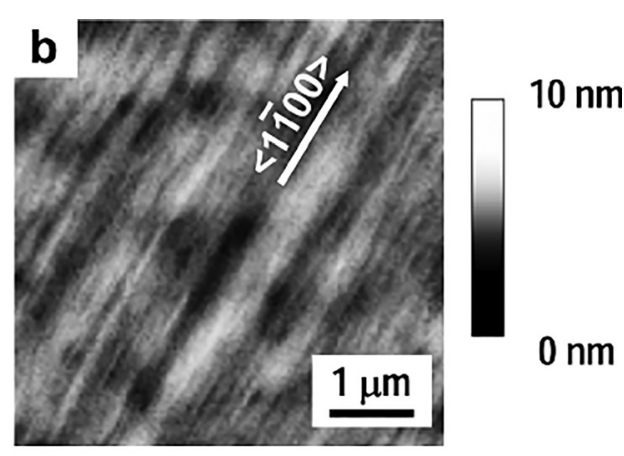

$2^{\circ}$ toward $M$

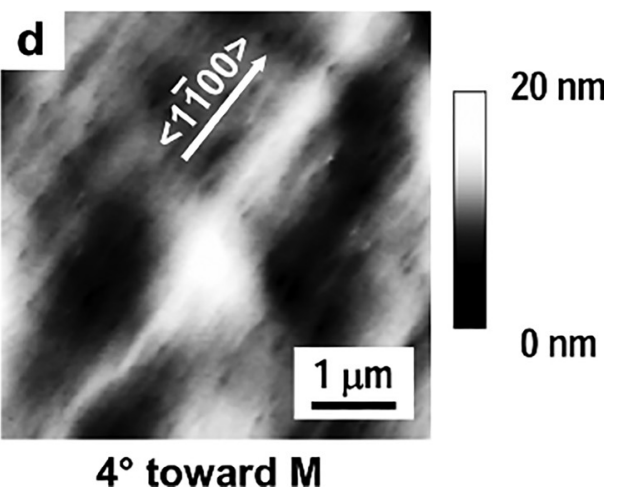

FIG. 32. AFM images of $0.8 \mu \mathrm{m}$ thick GaN layers grown on (0001) sapphire substrates with misorientation angles of (a) $2^{\circ}$ and (c) $4^{\circ}$ toward the $a$-plane and (b) $2^{\circ}$ and (d) $4^{\circ}$ toward the $m$-plane of the sapphire substrate. Reprinted with permission from J. Appl. Phys. 102, 083546 (2007). Copyright 2007 AIP Publishing LLC. threading dislocation densities, which were comparable to those in Ga-polar films (Fig. 30). ${ }^{228}$

Reflecting the different growth modes for N-polar GaN films, no Volmer-Weber island growth was observed in the initial deposition stage of the high temperature layer, as observed in in-situ reflectance and interrupted growth studies. $^{249,298}$

Using $4^{\circ}$ off sapphire substrates, N-polar GaN films with electron mobility values of 650 and $350 \mathrm{~cm}^{2} / \mathrm{V} \mathrm{s}$ for electron concentrations of $9 \times 10^{16}$ and $1 \times 10^{18} \mathrm{~cm}^{-3}$, respectively, were obtained, similar to those reported for $\mathrm{Ga}-$ polar GaN layers. ${ }^{299}$ The luminescence properties of $\mathrm{N}$ - and Ga-polar GaN films were comparable as well when examined by temperature dependent PL measurements. ${ }^{300}$

With regard to PAMBE, direct nucleation of $\mathrm{GaN}$ on sapphire was shown to be a key point for achieving N-polar GaN materials. ${ }^{301-305}$ Conversely, the use of an AlN nucleation layer grown at high temperature invariably led to Gapolarity. ${ }^{304-307}$ When either the GaN or AlN nucleation was performed at low temperatures (i.e., $\sim 500{ }^{\circ} \mathrm{C}$ and below), the polarity of the subsequent $\mathrm{GaN}$ layer appeared to be more variable and sometimes of mixed character depending on growth conditions such as growth rate, layer thicknesses, III/V ratio of the buffer, and/or the GaN layer itself. ${ }^{305,308}$ Nitridation of the sapphire surface prior to growth was shown to affect the morphology of the subsequent layer ${ }^{309-312}$ but not to be critical in determining its polarity. ${ }^{305}$ The type of species used for the nitridation $\left(\mathrm{N}_{2}\right.$ or $\left.\mathrm{NH}_{3}\right)$ may, however, play a role. ${ }^{305,313,314}$ An improvement of the properties of Ga-polar GaN films was reported on vicinal (0001) sapphire substrates. $^{315}$ Upon comparison of $\mathrm{N}$ - and Ga-polar GaN films on sapphire, 12 times lower Hall mobilities were reported on N-polar $\left(17.5 \mathrm{~cm}^{2} / \mathrm{V} \mathrm{s}\right)$ than on Ga-polar films $\left(213.3 \mathrm{~cm}^{2} / \mathrm{V} \mathrm{s}\right)$ for charge densities of 3.7 and $1.9 \times 10^{17} \mathrm{~cm}^{-3}$, respectively. $^{304} \mathrm{~N}$-polar GaN layers with significantly improved properties were demonstrated when implementing high temperature AIN interlayers together with a migration enhanced epitaxy growth mode, resulting in GaN:Si layers with an electron mobility of $668 \mathrm{~cm}^{2} / \mathrm{V}$ s at a carrier density of $9.5 \times 10^{16} \mathrm{~cm}^{-3} .316$

d. Silicon. Historically, Si was the last of the three considered substrates on which high quality GaN films were demonstrated. In most cases, the (111) Si substrate is used because of its trigonal symmetry, supporting the epitaxial growth of (0001) GaN. ${ }^{317}$ (Note that Ga-polar GaN films can also be fabricated on (001) $\mathrm{Si}$.) ${ }^{318,319}$ Besides the large lattice mismatch of $-16.9 \%$, additional challenges arise from the large difference in the thermal expansion coefficients between Si substrate and GaN epitaxial layer, which leads to a large tensile stress when the wafer is cooled down from the GaN growth temperature to room temperature, resulting in crack formation if not accounted for. ${ }^{317}$ To prevent gallium silicide formation, the growth on Si substrates is typically initiated with the deposition of an AlN layer (the atomic arrangement at the AlN/ $\mathrm{Si}(111)$ interface was studied in detail in Ref. 320). To compensate for the tensile strain evolving during cool down, the $(\mathrm{Al}, \mathrm{Ga}, \mathrm{In}) \mathrm{N}$ layer stack needs to be compressively strained at growth temperature. For the deposition of metal-polar films, a variety of methods were explored, for example, grading the composition from AlN to $\mathrm{GaN}^{321}$ insertion of an AlN-GaN superlattice, ${ }^{322,323}$ deposition of AlN interlayers, ${ }^{324}$ or nucleation with AlN followed by the growth of $(\mathrm{Al}, \mathrm{Ga}) \mathrm{N}$ layers where the Al-composition is reduced in steps. ${ }^{325}$ Using these techniques, high quality Ga-polar (Al,Ga,In)N epitaxial layers have been fabricated by MOCVD.

Smooth N-polar GaN films were demonstrated by MOCVD on (111) Si substrates with miscuts of $3.5^{\circ}$ and $6^{\circ}$ 
towards the $\langle 11 \overline{2}\rangle$ and $\langle 1 \overline{1} 0\rangle$ directions. ${ }^{231,326}$ The optimum $\mathrm{Si}$ substrate misorientation direction was $\langle 11 \overline{2}\rangle$, resulting again in GaN films misoriented toward the $m$-plane with regular surface steps parallel to the $\langle 11 \overline{2} 0\rangle$ direction. As for metal-polar films, the growth process was initiated with the deposition of an AIN layer, followed by the strain management layers consisting either of a layer where the composition was graded from AlN to GaN or by a series of $\mathrm{Al}_{x} \mathrm{Ga}_{1-\mathrm{x}} \mathrm{N}$ layers where the $\mathrm{Al}$ composition was reduced in steps from $65 \%$ to $36 \%$ and $17 \%$ prior to the deposition of the final GaN layer. In both cases, N-type polarity was achieved by heavy Mg-doping of the $(\mathrm{Al}, \mathrm{Ga}) \mathrm{N}$ strain management layers. Polarity inversion by $\mathrm{Mg}$-doping in $\mathrm{GaN}$ films was observed before. ${ }^{218,220}$ The $\mathrm{Mg}$ doping led to complete inversion of the layers and no inversion domains could be found in TEM images. Similar to the previous observations, a faceted inversion domain boundary layer at the $\mathrm{Ga}$ - to $\mathrm{N}$-polar interface was observed. ${ }^{231,264}$ To avoid any impact of the Mg-doping on the device performance, Mg-doped layers and active device layers were separated by an unintentionally doped layer with a thickness in excess of $600 \mathrm{~nm}$.

The full width at half maximum (FWHM) of the $(000 \overline{2})$ and $(20 \overline{2} \overline{1}) \mathrm{X}$-ray rocking curves of the GaN layer amounted to $0.22^{\circ}$ and $0.32^{\circ}$, respectively, for the samples with graded $(\mathrm{Al}, \mathrm{Ga}) \mathrm{N}$ buffer layer. Values of $0.16^{\circ}$ and $0.2^{\circ}$, respectively, were measured for samples with $(\mathrm{Al}, \mathrm{Ga}) \mathrm{N}$ buffers where the composition was reduced in steps, most likely because the GaN layer in those samples was considerably thicker, $900 \mathrm{~nm}$, in comparison to $300 \mathrm{~nm}$ for the samples with graded $(\mathrm{Al}, \mathrm{Ga}) \mathrm{N}$ buffer. The measured XRD data corresponded well to those for Ga-polar GaN-on-Si films grown using both procedures. ${ }^{327,328}$ In contrast to the films deposited on sapphire and $\mathrm{SiC}$ substrates, the misorientation angle of the N-polar GaN layers grown on the misoriented (111) Si substrates was more than $1^{\circ}$ smaller compared to that of the Si substrates. ${ }^{326}$ Confirming the high quality of the N-polar GaN-on-Si base layers, electron mobility values of 1760 and $1508 \mathrm{~cm}^{2} / \mathrm{V} \mathrm{s}$ at sheet electron densities of 6.3 and $9.6 \times$ $10^{12} \mathrm{~cm}^{-2}$, respectively, were determined parallel to the surface steps for N-polar GaN/(Al,Ga)N 2DEG structures grown on these GaN-on-Si base layers.

In PAMBE, it is possible to initiate the growth of GaN directly on $\mathrm{Si}$, but an amorphous $\mathrm{Si}_{\mathrm{x}} \mathrm{N}_{\mathrm{y}}$ layer unavoidably forms on the surface when exposed to Ga and active N. ${ }^{329}$ Ga-rich conditions were then reported to lead to mixed polarities, while $\mathrm{N}$-rich environments resulted in fully N-polar GaN. ${ }^{330} \mathrm{~N}$-rich conditions, however, also promote the formation of 3D structures. By switching the growth conditions to slightly Ga-rich soon after the N-rich nucleation step, Wang et al. achieved single uniform N-polar GaN/Si(111) layers. ${ }^{330}$ The most common alternative to the growth of $\mathrm{GaN}$ on $\mathrm{Si}_{\mathrm{x}} \mathrm{N}_{\mathrm{y}} / \mathrm{Si}(111)$ is the use of an AlN nucleation layer on $\mathrm{Si}^{3}{ }^{31-335}$ The GaN polarity is then expected to replicate that of AlN. Dasgupta et al. reported the growth of fully Npolar $\mathrm{AlN} / \mathrm{Si}(111)$ arguing that the key feature for the achievement of the N-polarity was the saturation of $\mathrm{Si}(111)$ dangling bonds with $\mathrm{Al}$ atoms. ${ }^{333}$ Using this strategy, $500 \mathrm{~nm}$ of N-polar GaN was grown on $100 \mathrm{~nm}$ AlN/Si(111) exhibiting a final root mean square surface roughness below
$1 \mathrm{~nm}$ over $5 \times 5 \mu \mathrm{m}^{2}$ areas (Fig. 33) and with screw and edge dislocation densities of $8 \times 10^{8} \mathrm{~cm}^{-2}$ and $5 \times 10^{10} \mathrm{~cm}^{-2}$, respectively. ${ }^{334}$ Other authors observed Ga-polar GaN film on such Al-saturated $\mathrm{Si}(111)$ substrates. ${ }^{336,337}$ More generally, the polarity of AlN layers nucleated on $\mathrm{Si}(111)$ by PAMBE is still ambiguous and may depend on additional parameters, such as the growth temperature ${ }^{338,339}$ or the buffer layer thickness. ${ }^{340,341}$

\section{Oxygen and carbon impurity incorporation}

Oxygen $(\mathrm{O})$ and carbon $(\mathrm{C})$ are the most common impurities in the $\mathrm{GaN}$ growth process. O substitutes $\mathrm{N}$ in the $\mathrm{GaN}$ lattice and acts as a shallow donor. ${ }^{342,343} \mathrm{C}$ incorporates preferentially on $\mathrm{N}$ sites as well, forming a deep acceptor level with an ionization energy of $0.9 \mathrm{eV} .{ }^{344}$

$\mathrm{N}$-polar $(\mathrm{Al}, \mathrm{Ga}, \mathrm{In}) \mathrm{N}$ films are well-known for their significantly higher oxygen uptake in comparison to their metal-polar counterparts. ${ }^{234,345-347}$ The higher O incorporation efficiency on the $\mathrm{N}$-polar surface was associated with the easy exchange between $\mathrm{N}$-surface atoms and oxygen. In addition, theoretical studies showed that formation of three bonds between oxygen and sub-surface Ga-atoms results in a much higher adsorption energy compared with a single Ga-O bond on a Ga-polar GaN surface. ${ }^{342}$ Nevertheless, N-polar GaN films with a residual oxygen concentration as low as $2 \times 10^{16} \mathrm{~cm}^{-3}$, corresponding to the background level in the SIMS measurements, were demonstrated (Fig. 34), by both MBE and MOCVD. ${ }^{249,348}$ Besides general process purity, the oxygen incorporation is suppressed at higher growth temperatures and V/III ratios. ${ }^{349}$

In contrast to oxygen, the incorporation of residual carbon impurities is significantly lower in N-polar compared with Ga-polar GaN layers for films grown at the typical high temperatures employed. ${ }^{349}$ In the MOCVD process, unintentional $\mathrm{C}$ incorporation results predominantly from the methyl groups of the group-III precursor molecules and decreases

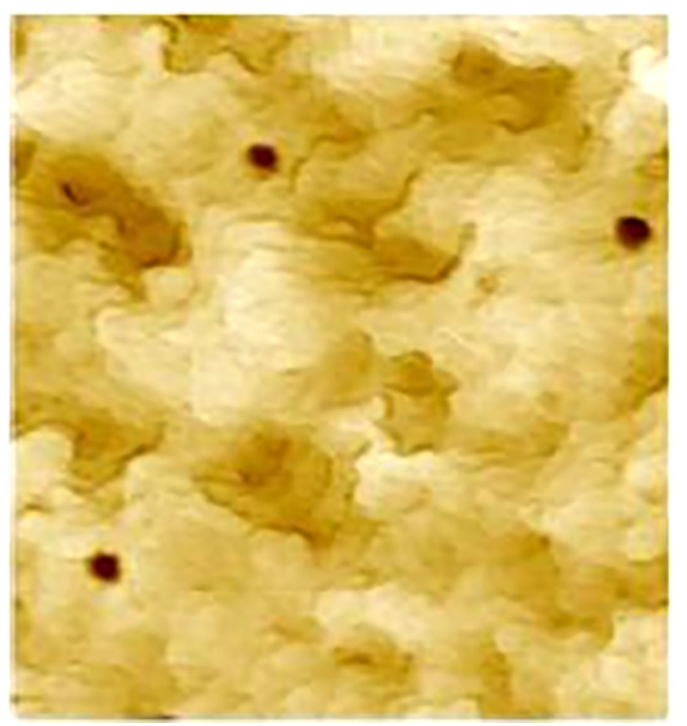

FIG. 33. $5 \times 5 \mu \mathrm{m}^{2}$ AFM image of PAMBE-grown N-polar GaN/AIN/ $\mathrm{Si}(111)$, the rms surface roughness is $0.9 \mathrm{~nm}$. Reprinted with permission from Dasgupta et al., Jpn. J. Appl. Phys., Part 1 51, 115503 (2012). Copyright 2012 The Japan Society of Applied Physics. 


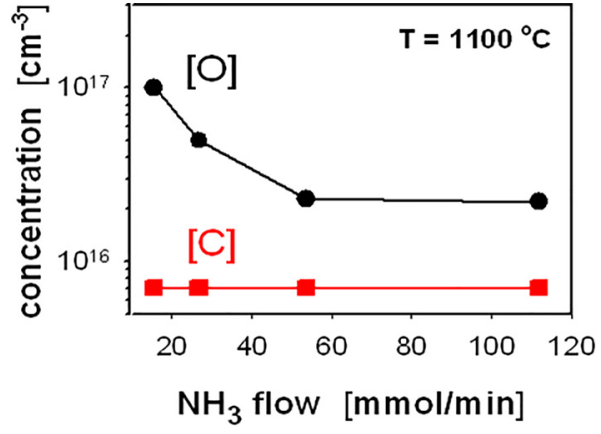

FIG. 34. Carbon (squares) and oxygen (circles) concentration measured by secondary ion mass spectrometry (SIMS) versus versus $\mathrm{NH}_{3}$ flow in $\mathrm{N}$-polar $\mathrm{GaN}$ films grown on sapphire substrates with a misorientation angle of $4^{\circ}$ towards the $a$-plane. Reprinted with permission from Keller et al., Semicond. Sci. Technol. 29, 113001 (2014). Copyright 2014 Institute of Physics Publishing.

with increasing V/III ratio, pressure, and temperature. ${ }^{350,351}$ The behavior reverses, however, when N-polar GaN layers are deposited at reduced temperatures, as, for example, used for (In, Ga)N growth. The $\mathrm{C}$ incorporation in this regime was also largely independent of $\mathrm{NH}_{3}$ flow, V/III ratio, and reactor pressure during growth, in stark contrast to the observations for Ga-polar GaN layers. Neither flow modulation nor atomic layer epitaxy growth schemes resulted in any significant decrease in the $\mathrm{C}$ incorporation in the $\mathrm{N}$-polar GaN layers either $\left([\mathrm{C}]=2 \times 10^{17} \mathrm{~cm}^{-3}\right.$ for conventional growth versus $[\mathrm{C}]=1 \times 10^{17} \mathrm{~cm}^{-3}$ for pulsed TMGa injection under otherwise constant conditions), again in strong contrast to the findings for Ga-polar GaN films. ${ }^{352,353}$ The elevated C incorporation into $\mathrm{N}$-polar layers at reduced deposition temperatures was associated with the different adsorption sites for methyl groups on the step edges of $\mathrm{N}$ - and Ga-polar GaN surfaces (Fig. 35). ${ }^{354}$

Interestingly, $\mathrm{C}$ incorporation could be reduced to $2-3 \times$ $10^{16} \mathrm{~cm}^{-3}$, when TMIn was added to the gas phase (Fig. 36). Thereby, the reduction in the carbon incorporation was not related to the In composition of the layers, as the effect was observed in the presence of hydrogen in the gas phase, which suppresses In incorporation. Low C impurity levels were also observed in films grown with TEGa as precursor. ${ }^{249}$ Further details regarding the $\mathrm{O}$ and $\mathrm{C}$ impurity incorporation are discussed in Ref. 249.
In PAMBE, the residual $\mathrm{C}$ incorporation is very low for both $\mathrm{Ga}$ - and N-polarities. ${ }^{355}$

Note that intentional $\mathrm{C}$ incorporation can be used for the fabrication of semi-insulating (Al,Ga,In)N films by compensating residual n-type doping. In the MOCVD process, typically growth conditions that favor $\mathrm{C}$ uptake are chosen, such as low growth pressures, temperatures, and V/III ratios. 356,357 Using MBE, $\mathrm{C}$ doping is performed using $\mathrm{CCl}_{4}$ or $\mathrm{CBr}_{4}$ gas sources, for example. ${ }^{358,359}$

\section{Doping with Si, Mg, and Fe}

Typically, GaN layers are rendered n-type by doping with $\mathrm{Si}$. Si is a shallow donor and can be easily introduced by adding silane or disilane to the growth ambient in the MOCVD process ${ }^{360,361}$ and by sublimation of Si atoms from an ultra-pure Si source in MBE. With either growth method, no significant differences were observed, when doping $\mathrm{N}$ compared with the Ga-polar GaN layers. ${ }^{347,349}$

P-type GaN is typically fabricated through doping with magnesium (Mg), in the MOCVD process supplied using the precursor cyclopentadienyl magnesium, $(\mathrm{Cp})_{2} \mathrm{Mg} .{ }^{286} \mathrm{Mg}$ forms a relatively deep acceptor level with an activation energy of about $110 \mathrm{meV} .{ }^{362}$ When grown by MOCVD, the $\mathrm{Mg}$ acceptor is typically passivated with hydrogen, and p-type doping can only be achieved after a post-growth electron beam ${ }^{363}$ or thermal treatment, ${ }^{286}$ leading to a dissociation of the $\mathrm{Mg}-\mathrm{H}$ complexes. In a study where $\mathrm{GaN}: \mathrm{Mg}$ films were simultaneously deposited on Ga- and N-polar GaN templates, no significant differences in the $\mathrm{Mg}$ incorporation and the properties of the layers with different polarities were found. ${ }^{364}$ Although the surface properties of the Ga-polar GaN:Mg layers degraded at doping levels higher than $2 \times$ $10^{20} \mathrm{~cm}^{-3}$, due to the $\mathrm{Mg}$-induced inversion domain formation, ${ }^{364}$ the largely unchanged smooth surface morphology of the N-polar GaN layers did not result in any improvement of the electrical properties of the layers, indicating that the degradation of the electrical properties of highly Mg-doped $\mathrm{GaN}$ films is related more to $\mathrm{Mg}$-induced defects or $\mathrm{Mg}$ cluster formation rather than to the development of inversion domains. Differences between polarities, however, were observed in the abruptness of the doping profiles. In nominally $150 \mathrm{~nm}$ GaN:Mg/150 nm GaN:Si multi-layer stacks, ${ }^{349}$
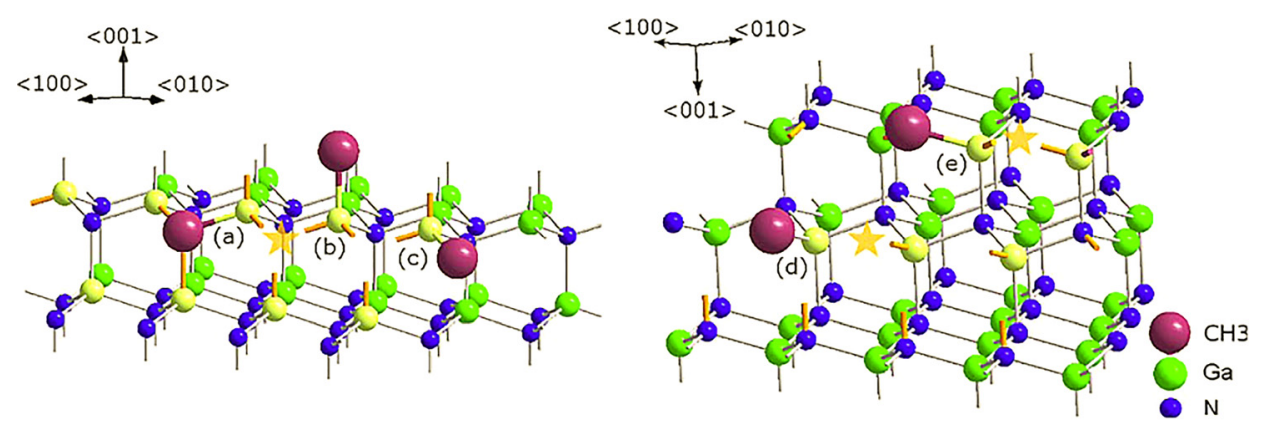

FIG. 35. Incorporation positions for mono-methylated Ga on a step edge (highlighted) of $10 \overline{1} 0$ propagation direction. Since adsorbed Ga on the step edge has multiple dangling bonds, it may rotate in order to remove any methyl groups from a N adsorption site denoted by a star, such as in the case of Ga in positions (a) or (c) to the terrace parked position (b). For growth on the N-face, the same step edges occur but there are two types of positions for Ga (d) and (e) but in either case, there is no parked position for the adsorbed Ga. If the Ga is methylated, the C group will always be blocking step propagation. Reprinted with permission from Cruz et al., J. Cryst. Growth 311, 3817 (2009). Copyright 2009 Elsevier. 


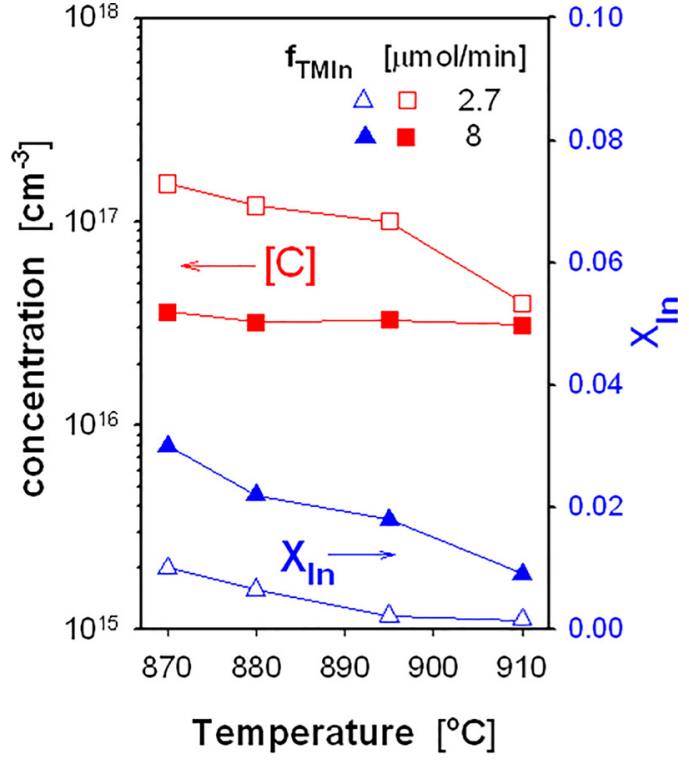

FIG. 36. SIMS measured concentrations of carbon (squares) versus deposition temperature for layers grown with TMIn flows of $2.7 \mu \mathrm{mol} / \mathrm{min}$ (open symbols) and $8 \mu \mathrm{mol} / \mathrm{min}$ (closed symbols). The indium mole fraction, $\mathrm{x}_{\mathrm{In}}$, in the GaN:In layers grown in the presence of $1 \mathrm{slm}$ of $\mathrm{H}_{2}$ is also shown (triangles). Reprinted with permission from Keller et al., Semicond. Sci. Technol. 29, 113001 (2014). Copyright 2014 Institute of Physics Publishing.

$\mathrm{Mg}$ penetrated into the GaN:Si layers of the Ga-polar GaN sample due to the well-known Mg memory effect, ${ }^{365,366}$ whereas an abrupt doping profile was observed for the $\mathrm{N}$ polar GaN sample (Fig. 37).

In contrast, in the PAMBE process, where $\mathrm{Mg}$ is vaporized from pure metallic $\mathrm{Mg}$, it was shown both theoretically ${ }^{367,368}$ and experimentally ${ }^{267,346,355}$ that $\mathrm{Mg}$ incorporates better in Ga-polar compared with N-polar GaN layers (Fig. 38). ${ }^{267}$
As discussed earlier, high $\mathrm{Mg}$ doping of Ga-polar GaN induces a change of polarity from $\mathrm{Ga}$ - to N-polar in both the MOCVD and MBE growth processes. In the latter, large $\mathrm{Mg}$ concentrations on $\mathrm{N}$-polar GaN layers can also lead to a crystal phase transition from wurtzite to zinc blende. ${ }^{369}$

Doping with Fe has been used to render both Ga- and Npolar GaN films semi-insulating, in particular, when grown on sapphire substrates. ${ }^{370,371} \mathrm{Fe}$ is incorporated in the $\mathrm{Ga}$ sublattice and forms multiple states within the GaN band gap. ${ }^{372}$ Thereby, Fe atoms compensate free electrons in the crystal resulting from unintentional $\mathrm{O}$ incorporation, for example. No significant differences in the Fe incorporation in Ga- and N-polar GaN layers were observed. ${ }^{354}$

In the PAMBE process, efficient $\mathrm{Fe}$ incorporation is inhibited in the growth regime suitable to achieve smooth GaN layers, namely, the metal-rich regime. ${ }^{373}$ Instead, C doping is typically used to achieve semi-insulting PAMBEgrown GaN as discussed in Subsection IV A 4.

\section{Ternary alloys}

a. $(A l, G a) N$. $(\mathrm{Al}, \mathrm{Ga}) \mathrm{N}$ layers are commonly used for the fabrication of $2 \mathrm{DEG}$ structures for transistor applications and optoelectronic devices operating in the ultra-violet part of the electromagnetic spectrum. Due to the stronger bonds nitrogen forms with $\mathrm{Al}$ in comparison to $\mathrm{Ga}$ atoms, ${ }^{374}$ in the MOCVD process $(\mathrm{Al}, \mathrm{Ga}) \mathrm{N}$ layers are typically grown at reduced reactor pressures to prevent pre-reactions between the TMAl and $\mathrm{NH}_{3}$ precursor molecules in the gas phase, which can result in a drastic decrease of the growth efficiency. ${ }^{375,376}$ Often also lower growth rates compared with $\mathrm{GaN}$ are used when the $(\mathrm{Al}, \mathrm{Ga}) \mathrm{N}$ layers are deposited at temperatures similar to those of GaN. The lower growth rates ensure higher crystalline quality and lower impurity
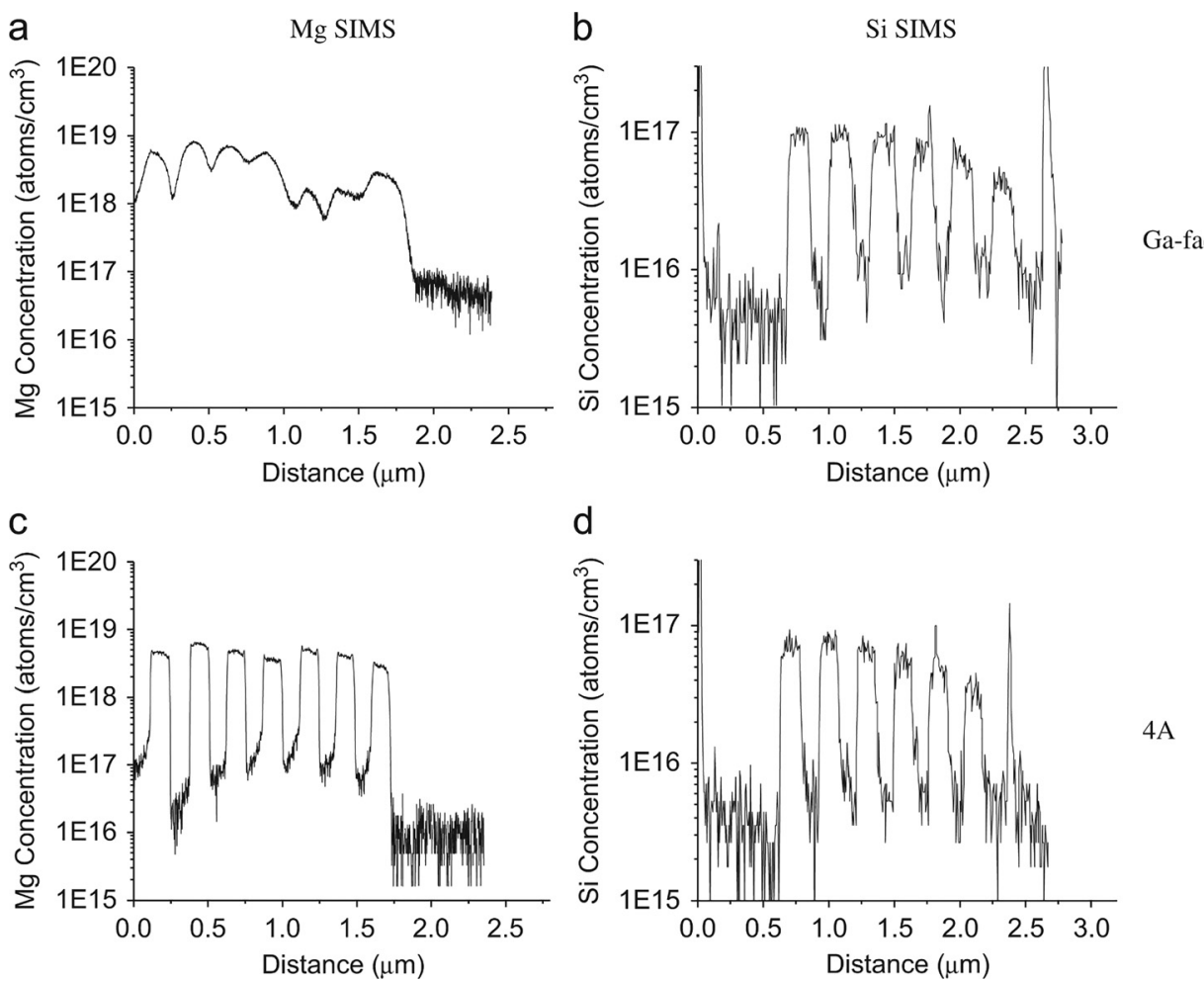

Ga-face

FIG. 37. SIMS profile of $\mathrm{Mg}$ concentration in (a) Ga- and (c) N-polar (4A), and profile of $\mathrm{Si}$ concentration in (b) Ga- and (d) N-polar (4A) GaN layers. Reprinted with permission from Fichtenbaum et al., J. Cryst. Growth 310, 1124 (2009). Copyright 2009 Elsevier. 


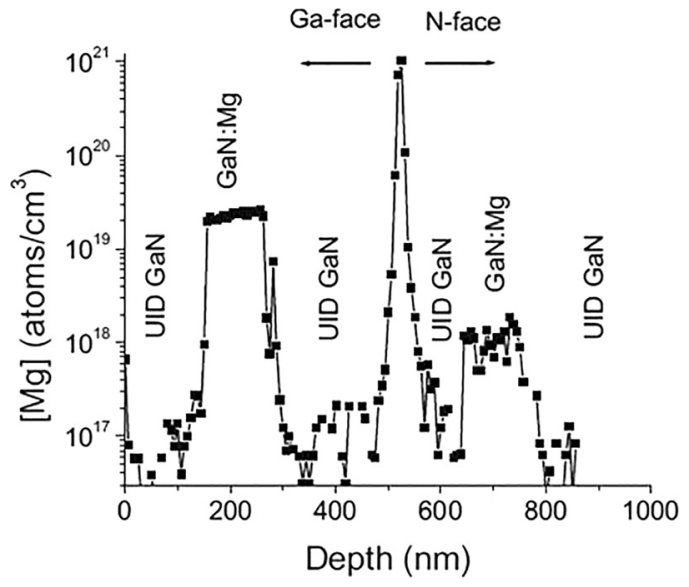

FIG. 38. SIMS profile illustrating the higher Mg intake in Ga-face GaN compared with $\mathrm{N}$-face $\mathrm{GaN}$. The polarity of the structure was switched from $\mathrm{N}$ - to Ga-polar using a large $\mathrm{Mg}$ coverage and exposure to $\mathrm{N}$. Reprinted with permission from J. Appl. Phys. 108, 123710 (2010). Copyright 2010 AIP Publishing LLC.

incorporation. Since the Al-C bonds in the precursor molecules are stronger than the $\mathrm{Ga}-\mathrm{C}$ bonds as well, ${ }^{351}$ more time is needed for the desorption of $\mathrm{C}$ species from the growing surface. Furthermore, when the TMAl and TMGa precursor flows are reduced while the $\mathrm{NH}_{3}$ flow is maintained high, the increased $\mathrm{V} / \mathrm{III}$ ratio suppresses $\mathrm{O}$ impurity incorporation as well. Similar to the $\mathrm{C}$ incorporation, the $\mathrm{O}$ residual impurity incorporation is enhanced in $\mathrm{Al}$ containing alloys due to the stronger $\mathrm{Al}-\mathrm{O}$ bond compared to the Ga-O bond and the higher stability of aluminum compared with gallium oxide. $^{377}$

Due to the stronger bonds and the reduced surface mobility of adsorbed species, thick $(\mathrm{Al}, \mathrm{Ga}) \mathrm{N}$ films grown on foreign substrates typically contain higher threading dislocation densities compared with GaN. To compensate for these effects, atomic layer and migration enhanced epitaxial methods were successfully applied to improve the $(\mathrm{Al}, \mathrm{Ga}) \mathrm{N}$ film quality. ${ }^{378,379}$ To the best of our knowledge, no N-polar bulk $(\mathrm{Al}, \mathrm{Ga}) \mathrm{N}$ films have been reported yet.

In the PAMBE process, the growth temperature of $(\mathrm{Al}, \mathrm{Ga}) \mathrm{N}$ alloys is limited by the strong $\mathrm{Ga}$ desorption rate above $750{ }^{\circ} \mathrm{C} .{ }^{380}$ Lower temperatures reduce the mobility of $\mathrm{Al}$ adatoms, which can, however, be increased using either Ga or In as surfactant thereby enabling the achievement of smooth surfaces. ${ }^{381,382}$ The same strategy is used for both metal- and $\mathrm{N}$-polar $(\mathrm{Al}, \mathrm{Ga}) \mathrm{N}$ surfaces.

The growth of thin metal-polar $(\mathrm{Al}, \mathrm{Ga}) \mathrm{N}$ films on $\mathrm{Ga}-$ polar GaN for 2DEG structures was studied extensively. Due to the lattice mismatch of $3.5 \%$ between $\mathrm{GaN}$ and $\mathrm{AlN}$, the $(\mathrm{Al}, \mathrm{Ga}) \mathrm{N}$ layer thickness needs to be reduced with increasing Al composition to avoid defect formation and cracking. Using the two growth methods, at a sheet carrier density of $1 \times 10^{13} \mathrm{~cm}^{-3}$ electron mobilities around $1400 \mathrm{~cm}^{2} / \mathrm{V} \mathrm{s}$ were measured. If a thin AlN interlayer is inserted between the $(\mathrm{Al}, \mathrm{Ga}) \mathrm{N}$ and $\mathrm{GaN}$ layers to mitigate alloy scattering, electron mobilities around $2000 \mathrm{~cm}^{2} / \mathrm{V}$ s were achieved. ${ }^{383-385}$ Note that the 2DEG at the $(\mathrm{Al}, \mathrm{Ga}) \mathrm{N} / \mathrm{GaN}$ interface forms as a result of the strong internal electric fields in these heterostructures, ${ }^{20}$ and no additional doping is required (see
Section VC). Regarding the MOCVD growth of N-polar $(\mathrm{Al}, \mathrm{Ga}) \mathrm{N} / \mathrm{GaN}$ heterostructures, the growth conditions of the $(\mathrm{Al}, \mathrm{Ga}) \mathrm{N}$ layers were the same as for Ga-polar samples. When depositing $(\mathrm{Al}, \mathrm{Ga}) \mathrm{N}$ layers on co-loaded Ga- and Npolar $\mathrm{GaN}$-on-sapphire base layers, the $\mathrm{Al}$ composition in the $\mathrm{N}$-polar $(\mathrm{Al}, \mathrm{Ga}) \mathrm{N}$ layers was typically around $10 \%$ lower and their thickness was $10 \%$ higher compared with the Gapolar $(\mathrm{Al}, \mathrm{Ga}) \mathrm{N}$ layers. ${ }^{249}$ The $\mathrm{Al}$ incorporation was independent upon the misorientation angle. Due to the misorientation of the N-polar layers, however, the properties of the 2DEG, which forms, in contrast to Ga-polar structures, at the upper $(\mathrm{Al}, \mathrm{Ga}) \mathrm{N} / \mathrm{GaN}$ interface, are anisotropic, with a lower sheet resistance parallel to the surface steps compared to the perpendicular direction (Fig. 39). ${ }^{386}$ While the sheet resistance perpendicular to the surface steps increased by increasing the misorientation angle, the sheet resistance parallel to the steps remained low and was largely unaffected by the misorientation angle. For $(\mathrm{Al}, \mathrm{Ga}) \mathrm{N} / \mathrm{GaN}$ structures, an electron mobility of $1800 \mathrm{~cm}^{2} / \mathrm{V} \mathrm{s}$ in the parallel direction was extracted from the sheet resistance determined in transmission line measurements and the sheet carrier density obtained in Hall and C-V measurements. ${ }^{386}$ As in Ga-polar samples, electron mobilities of $2000 \mathrm{~cm}^{2} / \mathrm{V}$ s were obtained in samples where, again, a thin AIN interlayer was inserted (at a sheet carrier density of about $\left.1 \times 10^{13} \mathrm{~cm}^{-2}\right) .{ }^{387}$ In devices, the higher electron mobility parallel to the surface steps has

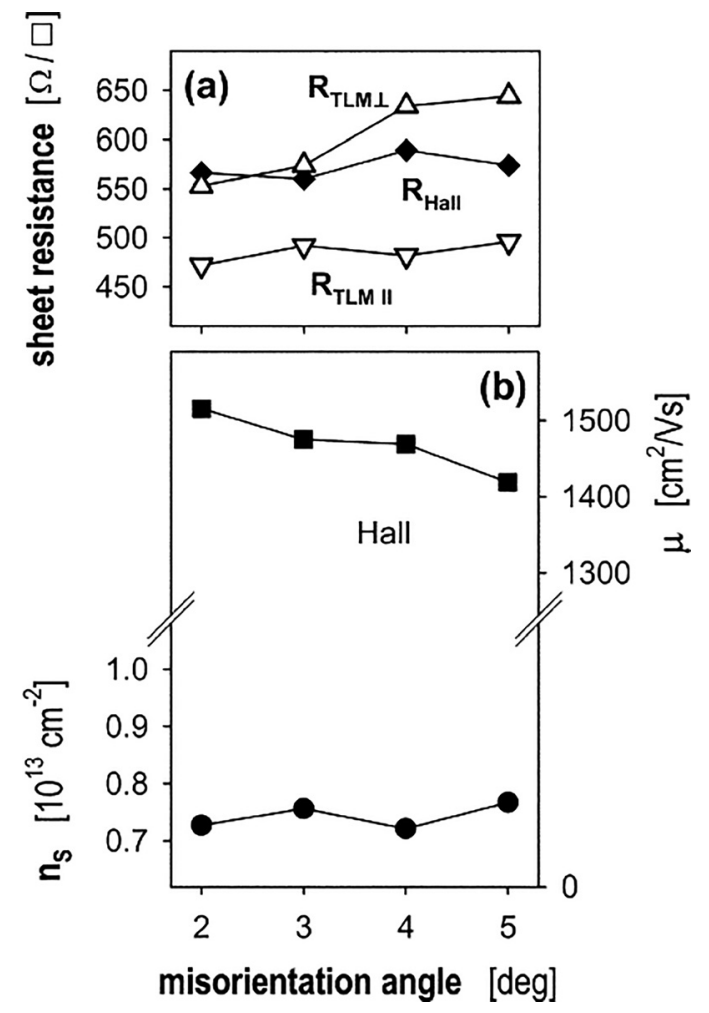

FIG. 39. (a) Dependence of the sheet resistance of the 2DEG measured at $300 \mathrm{~K}$ using TLM (transmission line measurements) patterns parallel (triangles down) and perpendicular (triangles up) to the step direction and derived from the Van der Pauw Hall measurements (diamonds) and (b) dependence of the sheet electron concentration (circles) and the electron mobility (squares) determined by Van der Pauw Hall measurements at $300 \mathrm{~K}$ on the substrate misorientation angle for $30 \mathrm{~nm} \mathrm{GaN} / 18 \mathrm{~nm} \mathrm{Al} 0.36 \mathrm{Ga}_{0.64}$ N/S.I. GaN structures. Reprinted with permission from J. Appl. Phys. 104, 093510 (2008). Copyright 2008 AIP Publishing LLC. 
been taken advantage of by aligning the devices in such a way that current flow occurs in this direction.

Investigations of $(\mathrm{Al}, \mathrm{Ga}) \mathrm{N} / \mathrm{GaN}$ MQW samples with different well width grown on GaN base layers with a misorientation angle of $4^{\circ}$ toward the $\mathrm{m}$-direction of GaN exhibited polarized light emission when the GaN well thickness was smaller than $3.5 \mathrm{~nm}$ (Fig. 40), ${ }^{388}$ indicating the formation of nanowire arrays, similar to the observations for (In,Ga)As/GaAs MQWs grown on highly misoriented GaAs substrates. ${ }^{389}$ The nanowires formed when the QW width was in the order of the height of the multi-layer steps on the sample surface. Furthermore, one dimensional (1D) electron transport was observed in samples where the GaN channel layer was thinned through etching. ${ }^{390}$

b. $(\operatorname{In}, G a) N$. Due to the low thermal stability of $\operatorname{InN}$, which sublimates already at temperatures around $500-550^{\circ} \mathrm{C}$, MOCVD grown (In, Ga)N films are typically deposited at temperatures about $300{ }^{\circ} \mathrm{C}$ lower compared with those used for the growth of the $\mathrm{GaN}$ base layers. In addition, In forms volatile In-H compounds, so that, in the MOCVD process, all In containing layers need to be deposited using $\mathrm{N}_{2}$ as carrier gas. To accommodate for the lower deposition temperatures, $(\mathrm{In}, \mathrm{Ga}) \mathrm{N}$ layers are typically deposited at significantly lower growth

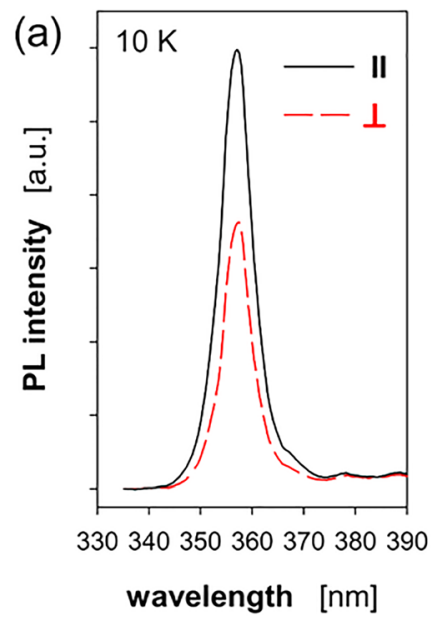

(b)

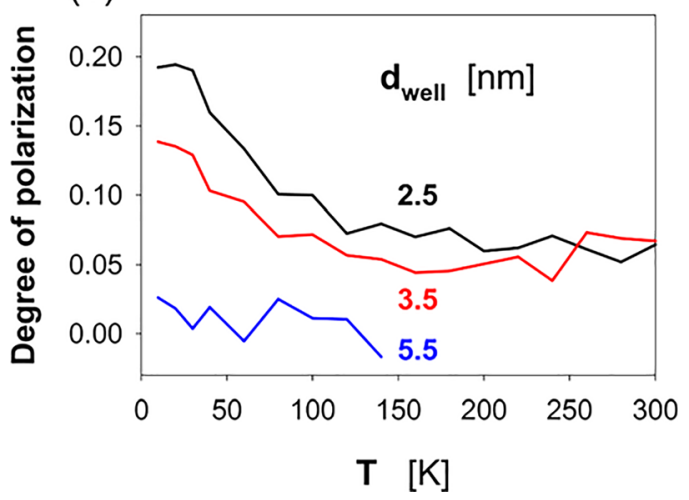

FIG. 40. (a) Photoluminescence spectra recorded at $10 \mathrm{~K}$ for the (Al,Ga)N/ GaN MQW sample with 2.5-nm-thick wells with polarizer position parallel, $\|$, and perpendicular, $\perp$, to the surface steps. (b) Degree of polarization, defined as $\left(I_{\|}-I_{\|}\right) /\left(I_{7}+I_{\perp}\right)$, determined for the (Al,Ga)N/GaN MQW samples with $2.5,3.5$, and $5.5 \mathrm{~nm}$ thick wells versus measurement temperature. Reprinted with permission from Appl. Phys. Lett. 101, 182103 (2012). Copyright 2012 AIP Publishing LLC. rates. When comparing metal- and N-polar (In,Ga)N/GaN heterostructures, the In composition in the metal-polar layers was found to saturate with increasing TMIn flow, whereas that of $\mathrm{N}$-polar layers continued to increase (Fig. 41). ${ }^{248}$

Independent of the polarity, the In incorporation efficiency increases with decreasing growth temperature. For $\mathrm{GaN} / \mathrm{In}_{\mathrm{x}} \mathrm{Ga}_{1-\mathrm{x}} \mathrm{N}$ QW structures, a general decrease in the QW related luminescence efficiency was observed with increasing In composition and wavelength, often referred to as "the green gap." ${ }^{391}$ The diminished performance with increasing wavelength is related to several circumstances. With increasing In content in the active region, the lattice mismatch increases, reaching $10 \%$ for pure $\mathrm{InN}$ on GaN. Thus, the higher the In content in the $\operatorname{In}_{\mathrm{x}} \mathrm{Ga}_{1-\mathrm{x}} \mathrm{N}$ QW, the more the QW thickness has to be reduced to prevent defect formation. ${ }^{392}$ However, this results in stronger confinement, which, in turn, shifts the QW emission wavelength back towards the blue, ${ }^{393}$ requiring an even higher In content to achieve a certain emission wavelength. Moreover, the higher the target In composition, $\mathrm{x}$, the more the growth temperature of the $\mathrm{In}_{\mathrm{x}} \mathrm{Ga}_{1-\mathrm{x}} \mathrm{N}$ layer has to be lowered. Due to the high Ga-N bond strength and the decreasing decomposition efficiency of the typical $\mathrm{N}$-precursor, $\mathrm{NH}_{3}$, the growth process becomes more difficult with decreasing deposition temperature, resulting in a higher probability of defect formation. ${ }^{394}$ In addition, the incorporation efficiency of C and $\mathrm{O}$ impurities increases at lower temperatures. ${ }^{350}$ Further complicating the situation, the In incorporation efficiency decreases with increasing lattice mismatch at typical MOCVD growth temperatures, known as composition pulling, which again necessitates even lower temperatures to achieve a high In content. ${ }^{395-397}$ In addition, for heterostructures fabricated in the typical $c$-direction, further problems arise from the increasing piezoelectric fields in the crystal, which can result in electron-hole separation, reducing the recombination probability in the QW and a blue shift of the emission wavelength with increasing carrier density. ${ }^{398}$

Due to the reduced temperatures and absence of hydrogen in the growth ambient, thicker N-polar $(\mathrm{In}, \mathrm{Ga}) \mathrm{N}$ layers tend to form hexagonal surface defects, even when grown on

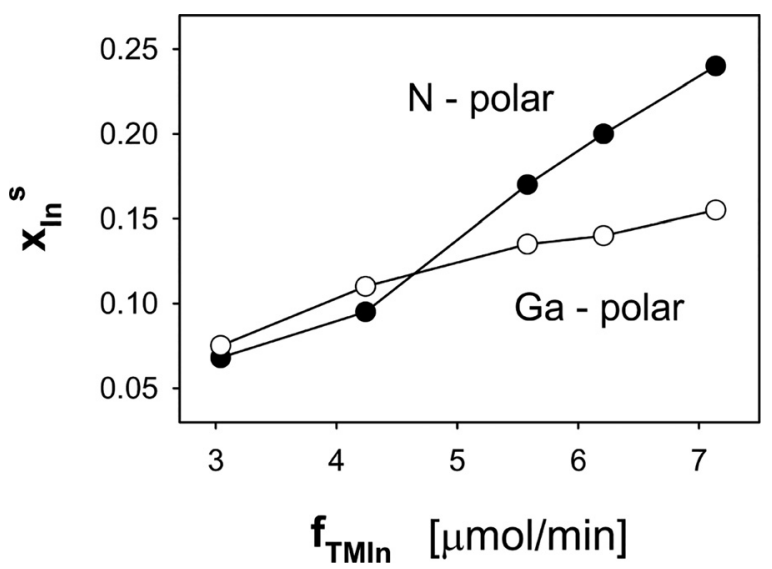

FIG. 41. Dependence of the indium mole fraction, $x_{I n}$, in the wells of 5 period ((In,Ga)N/GaN) MQWs determined by XRD on the TMIn flow during growth for co-loaded N- (filled circles) and Ga-polar samples (open circles). Reprinted with permission from Appl. Phys. Lett. 90, 191908 (2007). Copyright 2007 AIP Publishing LLC. 


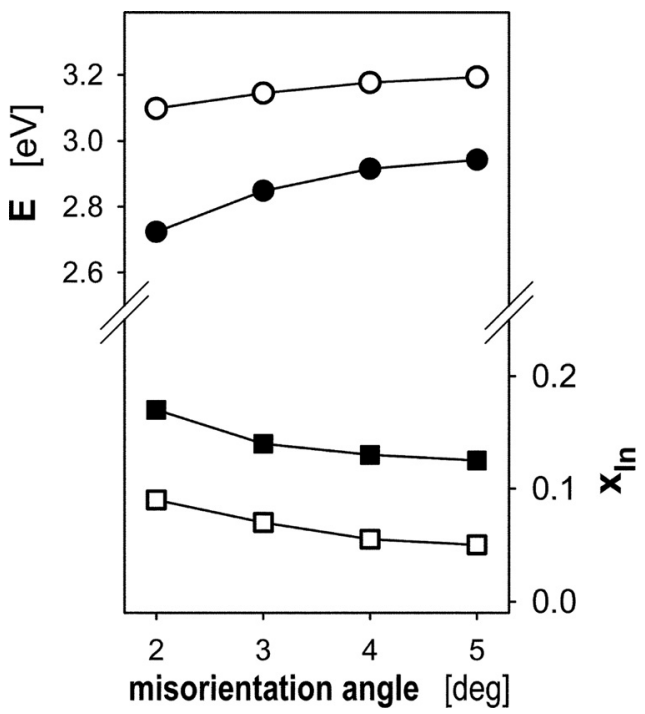

FIG. 42. Dependence of the emission energy of the MQW related luminescence peak at $300 \mathrm{~K}$ (circles) and the indium mole fraction in the $\mathrm{In}_{\mathrm{x}} \mathrm{Ga}_{1-\mathrm{x}} \mathrm{N}$ well, $\mathrm{x}_{\mathrm{In}}$, determined by XRD (squares) on the substrate misorientation angle for 5 period ((In,Ga)N/GaN) MQWs grown at $880^{\circ} \mathrm{C}$ (filled symbols) and $905^{\circ} \mathrm{C}$ (open symbols). Reprinted with permission from J. Appl. Phys. 104, 093510 (2008). Copyright 2008 AIP Publishing LLC.

misoriented GaN, as discussed in Ref. 249. During growth, In itself acts as surfactant. Smoother layers were also observed when In was injected at higher temperatures. ${ }^{399}$ In MQW structures, this problem can be mitigated by introducing hydrogen during GaN barrier growth. ${ }^{248}$ Using this technique, hexagon free MQW structures with up to 36 periods could be fabricated. ${ }^{249}$ Note that for (In,Ga)N containing heterostructures, the onset of hexagon formation was lower when the GaN base layers were misoriented toward the $a$ instead of the more frequently used $m$-direction of GaN. ${ }^{249}$

The luminescence intensity from N-polar (In,Ga)N MQWs increased with increasing misorientation angle from $2^{\circ}$ to $5^{\circ}$, similar to the observations for GaN films, most likely due to the lower threading dislocation density in the films grown on higher misoriented substrates. ${ }^{386}$ Simultaneously, the emission wavelength exhibited a blue shift, which was associated with the increase in step density with increasing misorientation angle (Fig. 42). Similar trends were observed for metal-polar (In,Ga)N layers. Interestingly, the luminescence intensity was generally higher for MQWs grown on $\mathrm{GaN}$ base layers misoriented toward the $a$ - compared with the $m$-GaN direction. ${ }^{249}$ Little change in In content was observed in a study where the misorientation angle was varied between $0.4^{\circ}$ and $1.1^{\circ} .^{245}$ While showing distinct light emission, the luminescence from MOCVD grown N-polar (In,Ga)N heterostructures was dimmer compared to the co-loaded Ga-polar samples, ${ }^{300}$ and further investigations are needed to address this problem. A more detailed discussion of N-polar (In,Ga)N layer and MQW properties can be found in Ref. 249. Note that while the structural properties of metal-polar (In,Ga)N films grown by MOCVD typically degrade via $\mathrm{V}$-defect formation, ${ }^{400-402}$ no V-defects were observed in N-polar (In,Ga)N films, as predicted in theoretical studies. ${ }^{403}$

Similarly, as GaN and $\mathrm{InN}$ present a very different thermal stability, the growth of (In,Ga)N on metal-polar GaN by
PAMBE is typically performed at temperatures at least $120^{\circ} \mathrm{C}$ lower than the ideal $\mathrm{GaN}$ growth temperature, which is detrimental for the overall crystal quality and surface morphology. The (In,Ga)N thermal decomposition limit is, however, higher on N-polar than on metal-polar surfaces, ${ }^{404,405}$ allowing for increased growth temperatures on N-polar GaN substrates, or alternatively for higher In incorporation at a given temperature (Fig. 43). ${ }^{406-411}$ This offers a significant advantage for the fabrication of $(\mathrm{In}, \mathrm{Ga}) \mathrm{N}$ crystals with superior optical quality. Intense PL from N-polar (In,Ga)N layers was observed at room temperature with an intensity up to 100 times higher than for their metal-polar counterpart at a given wavelength, ${ }^{411,412}$ and N-polar (In,Ga)N QW-based light emitting diodes (LEDs) were successfully demonstrated. ${ }^{413-415}$

c. $(\operatorname{In}, A l) N$. (In, $\mathrm{Al}) \mathrm{N}$ layers are grown in the same temperature range as (In, Ga)N films, again using $\mathrm{N}_{2}$ as carrier gas. Of particular interest are layers with an In composition of about $18 \%$, which are lattice matched to GaN. ${ }^{416}$ As in (In,Ga)N, the In incorporation efficiency in N-polar GaN films was higher than in Ga-polar GaN ones. While the In content saturated with increasing TMIn injection in metalpolar films, no such saturation was observed in N-polar layers. ${ }^{417}$ Similarly, the In composition in N-polar (In,Al)N layers decreased with increasing misorientation angle of the GaN base layer. ${ }^{417}$

In the PAMBE process, for both the metal-polar ${ }^{418-420}$ and the N-polar ${ }^{421}$ surfaces, In and $\mathrm{Al}$ tend to segregate laterally, which results in the spontaneous formation of compositional columnar microstructures. Optimized N-rich growth conditions allow for the elimination of such microstructures on both type of surfaces. ${ }^{422,423}$

To date, (In,Al)N layers have been mainly used for the fabrication of transistor structures, where they allow the formation of 2 DEGs with sheet carrier concentrations in the order of $2 \times 10^{13} \mathrm{~cm}^{-2}$, due to the strong spontaneous polarization in $(\mathrm{In}, \mathrm{Al}) \mathrm{N}$. While the electron mobility in $(\mathrm{In}, \mathrm{Al}) \mathrm{N} /$ $\mathrm{GaN}$ heterostructures is typically very low, high electron mobilities can be achieved again when AlN interlayers are implemented to mitigate alloy scattering. Typical electron mobilites for metal-polar structures grown by MOCVD are in the order of $1200 \mathrm{~cm}^{2} / \mathrm{V} \mathrm{s} .{ }^{424}$ Values as high as $1800 \mathrm{~cm}^{2} / \mathrm{V} \mathrm{s}$

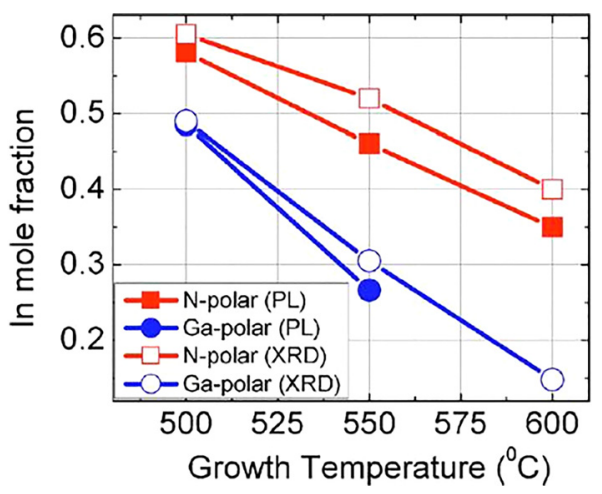

FIG. 43. In mole fraction in N- and metal-polar (In,Ga)N films, illustrating the higher In intake on the $(000 \overline{1})$ surface. Reprinted with permission from Nath et al., J. Vac. Sci. Technol., B 29, 021206 (2011). Copyright 2011 American Vacuum Society. 
were reported for $(\mathrm{In}, \mathrm{Al}) \mathrm{N} / \mathrm{AlN} / \mathrm{GaN}$ structures grown by MBE. ${ }^{423}$ The lower values measured for MOCVD samples are a result of the typically observed unintentional Ga incorporation in MOCVD grown AIN layers. ${ }^{425,426}$

The sheet resistance measured for N-polar GaN/AlN/ (In, $\mathrm{Al}) \mathrm{N} / \mathrm{GaN}$ 2DEG structures measured parallel to the surface steps caused by the misorientation was comparable to that obtained for metal-polar samples. As observed for $(\mathrm{Al}, \mathrm{Ga}) \mathrm{N} / \mathrm{GaN}$ heterostructures, the sheet resistance perpendicular to the steps was higher compared to the parallel direction (Fig. 44). For transistor applications, devices would be again aligned in such a way that the current flows parallel to the steps. A further reduction in the sheet resistance was achieved, in particular, for structures with highly scaled $\mathrm{GaN}$ channel thicknesses between 3 and $4.5 \mathrm{~nm}$, when the (In,Al)N backbarrier was replaced by an $\mathrm{AlN} /(\mathrm{In}, \mathrm{Al}) \mathrm{N} /$ (Al,Ga)N combination backbarrier. ${ }^{427,428}$ Similarly, Ahmadi et al. reported N-polar PAMBE HEMTs with optimized AIN/ $\mathrm{GaN} /(\mathrm{In}, \mathrm{Al}) \mathrm{N}(\mathrm{AlN} /(\mathrm{Al}, \mathrm{Ga}) \mathrm{N} / \mathrm{GaN} /(\mathrm{In}, \mathrm{Al}) \mathrm{N})$ backbarrier exhibiting room temperature mobility up to $1850(1360) \mathrm{cm}^{2} / \mathrm{V} \mathrm{s}$ for a charge density of $1.1 \times 10^{13}\left(2 \times 10^{13}\right) \mathrm{cm}^{-2}$. 429

Further details related to the performance of fabricated devices can be found in Section V C, as well as in Refs. 232 and 233. As mentioned earlier, a more detailed discussion on the growth of N-polar (Al,Ga,In) N by MOCVD can be found in Ref. 249. For further reading on the growth of metal polar group III-Nitrides, see, for example, Refs. 1-3, 430, and 431 regarding MOCVD, as well as Ref. 432 regarding MBE.

\section{B. ZnO thin films}

\section{Introduction}

The heteroepitaxial growth of $\mathrm{ZnO}$ thin films was attempted on a variety of substrates, such as $\mathrm{SiC}, \mathrm{Si}$, $\mathrm{LiGaO}_{2}, \mathrm{LaAlO}_{3}, \mathrm{LiNbO}_{3}, \mathrm{MgAl}_{2} \mathrm{O}_{4}, \mathrm{ScAlMgO}_{4}$ (SCAM), $\mathrm{GaAs}, \mathrm{GaP}$, and quartz, for instance, but, similarly to $\mathrm{GaN}$ thin films (see Section IV A), for a large majority on sapphire. Different growth processes were used: MOVPE, PAMBE, pulsed laser deposition (PLD), reactive DC sputtering, etc. The reader may refer to Refs. 6 and 433 for an overview of the subject. As discussed in the present section,

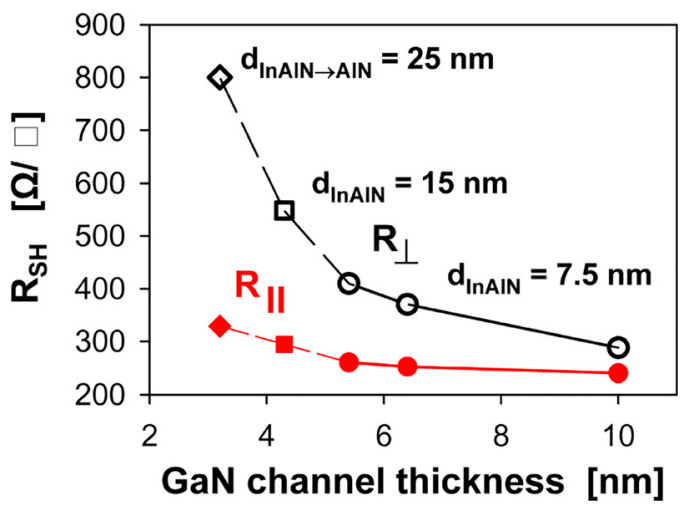

FIG. 44. Sheet resistance measured parallel (filled symbols) and perpendicular (open symbols) to the surface steps versus GaN channel thickness for $(\mathrm{Al}, \mathrm{Ga}) \mathrm{N} /(\mathrm{In}, \mathrm{Al}) \mathrm{N} / \mathrm{AlN} / \mathrm{GaN}$ structures with different backbarrier designs. Reprinted with permission from Keller et al., Semicond. Sci. Technol. 29, 113001 (2014). Copyright 2014 Institute of Physics Publishing. if nothing is made to impose the polarity, the epitaxial layers may contain domains with the two opposite polarities bounded by IDBs mostly lying in prismatic planes. The simplest model of IDBs lying in $\{10 \overline{1} 0\}$ prismatic planes assumes that wrong $\mathrm{Zn}-\mathrm{Zn}$ and $\mathrm{O}-\mathrm{O}$ bonds are formed across the IDB. First principles total energy calculations ${ }^{434}$ revealed that such configurations are unstable. Instead, the most energetically favorable IDB structure corresponds to a translation of $1 / 2[0001]$ of the $\mathrm{Zn}$-polar and O-polar $\mathrm{ZnO}$ crystals with respect to each other, with no wrong bonds involved. According to Ref. 6, these IDB configurations do not introduce energy levels in the band gap, but the formation energy of charged native point defects is reduced in the core of the IDB, yielding a preferential segregation of these intrinsic defects therein. This may create energy barriers for minority carriers and, as such, IDBs should be considered as deleterious for the optoelectronic properties of the material.

Beyond this problem, which can be solved if only one polarity is obtained, controlling the polarity of $\mathrm{ZnO}$ layers bears a lot of significance for multiple reasons. For instance, there is no controversy as to the fact that polarity influences the doping of materials like $\mathrm{ZnO}$ : one can easily understand these differences in dopant incorporation as due to the different number and nature of dangling bonds for different crystallographic surfaces (see Sections IIC-IIG). It is, for instance, expected that $\mathrm{Zn}$-polar $\mathrm{ZnO}$ material is more easily doped $p$-type by O-substituting impurities like nitrogen, p-type doping still being an unsolved problem for $\mathrm{ZnO}^{435}$ The first part of this section discusses how polarity affects the growth conditions of homoepitaxially grown epilayers. The second part focuses on the attempts that were undertaken to control the polarity of heteroepitaxially grown $\mathrm{ZnO}$ epilayers.

\section{Homoepitaxially grown $\mathrm{ZnO}$ thin films}

Although large $\mathrm{ZnO}$ bulk crystals, up to $20 \mathrm{~g}$ in weight, were already grown in the early 1970s by Helbig in Erlangen, ${ }^{436}$ it was not until the late 1990 s when $\mathrm{ZnO}$ substrates up to 2 in. in diameter became available. ${ }^{437}$ These substrates, manufactured by Eagle-Picher, were grown by a vapor-phase transport method similar to that employed by Helbig but using $\mathrm{H}_{2}$. The advent of $\mathrm{ZnO}$ substrates and the possibility of realizing homoepitaxial growth seemed to be the panacea. Besides preventing defect formation at the layer/substrate interface, homoepitaxy was supposed to allow an easy control over the layer polarity, given that both $\mathrm{Zn}$ and O-polar substrates could be purchased. Unfortunately, this first generation of $\mathrm{ZnO}$ bulk substrates displayed a strong mosaicity that, in some cases, could even be described as polycrystalline in nature, ${ }^{438}$ indeed, peaks arising from individual crystallites could be distinguished in high-resolution XRD measurements. ${ }^{439}$ Just some years later, Tokyo Denpa company produced, in collaboration with Tohoku University and Mitsubishi Chemical Corporation, hydrothermally (HT)grown $2 \mathrm{in.} \mathrm{ZnO}$ substrates of very high crystalline quality (i.e., single-crystal in nature) that were presented as epiready. ${ }^{440,441}$ In the next years, they were joined by CrysTec, which was also able to provide hydrothermally grown 2 in. $\mathrm{ZnO}$ substrates of both polarities. It should be noted that these 


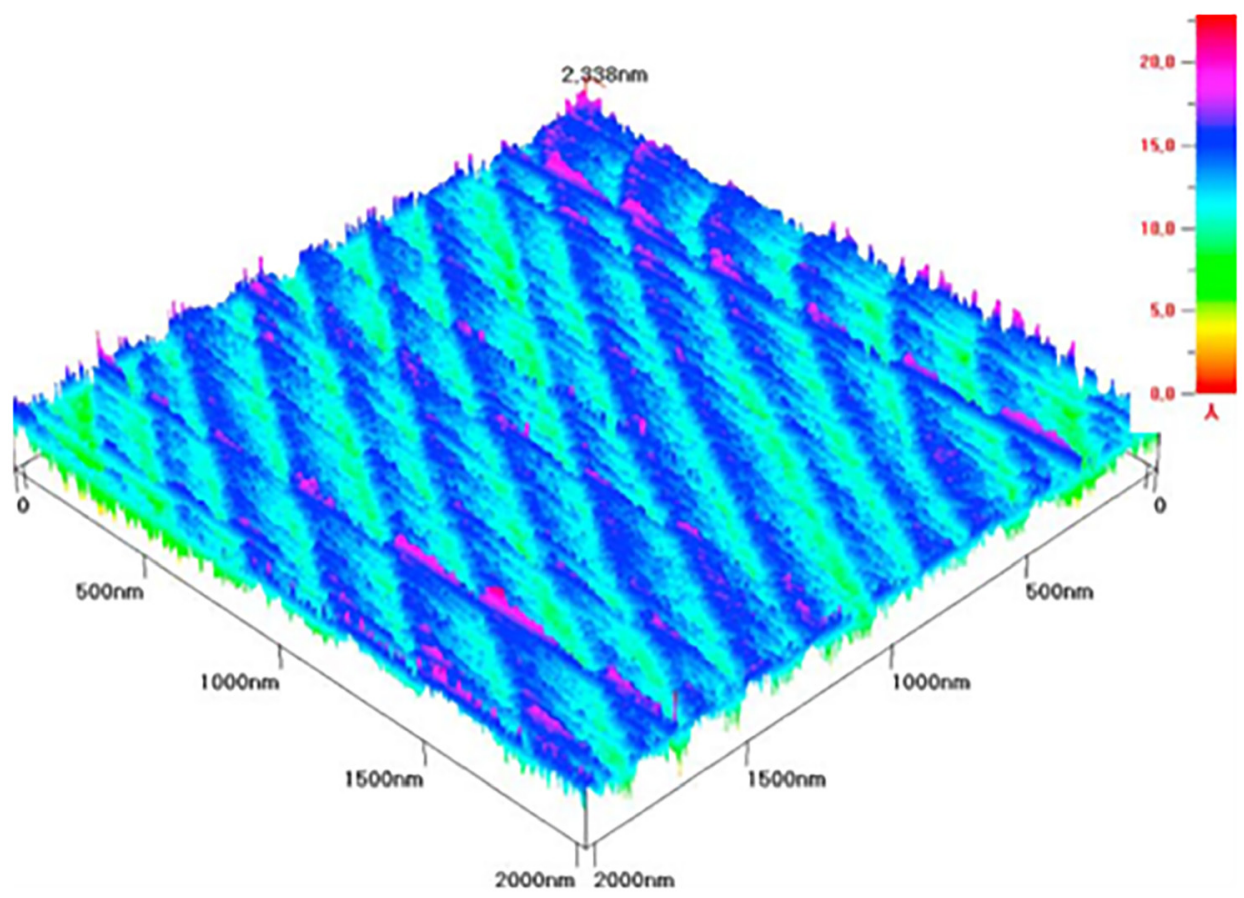

FIG. 45. AFM surface scan $(2 \times 2$ $\mu \mathrm{m}^{2}$ ) of an O-polar $\mathrm{ZnO}$ substrate after annealing. The terrace steps visible have a height of 2 times the $c$ lattice constant. Reprinted with permission from Neumann et al., Phys. Status Solidi B 244, 1451 (2007). Copyright 2007 John Wiley \& Sons, Inc.

substrates, irrespective of the exact manufacturer, displayed very large concentrations (in the order of several ppm) of unintentional dopant species: $\mathrm{Li}$ and $\mathrm{K}$, in particular, which came from the mineralizers used during the hydrothermal growth, but also $\mathrm{Fe}$ and Al. Furthermore, due to unequal impurity incorporation depending on the polarity of the growing surface, the final bulk crystals were highly transparent in the $\mathrm{Zn}$-sector, while they were pale green in the O-polar sector; thus, depending on the position within the initial bulk material the wafer was sliced from, its impurity content could be very different. ${ }^{441}$ It took several years until the contamination levels could be reduced to levels below $10^{14} \mathrm{~cm}^{-3} .442$

Although the substrates were presented as epi-ready in some cases, it became soon evident that surface preparation was required before the $\mathrm{ZnO}$ substrates could be used. Two approaches were mostly tested: annealing at hightemperatures under $\mathrm{O}_{2}$ atmosphere, mostly for O-polar $\mathrm{ZnO}$ substrates, and chemo-mechanical polishing, mostly for $\mathrm{Zn}$ polar $\mathrm{ZnO}$ substrates.

As early as 2006/2007, the annealing of O-polar ZnO substrates for several hours and at temperatures close to $1000^{\circ} \mathrm{C}$, under either flowing $\mathrm{O}_{2}{ }^{443,444}$ or in a steady $\mathrm{O}_{2}$ atmosphere, ${ }^{445}$ became standard. This surface preparation removed polishing scratches as well as a surface-damaged layer, especially in CrysTec substrates, and was the reason for the improvement in FWHM of the (0002) rocking curves measured by some authors ${ }^{445}$ leading to values in the order of $25 \operatorname{arcsecs}-30 \operatorname{arcsecs}^{443-445}$ As seen in Fig. 45, after the annealing the surface was covered by atomic steps, often of monolayer $(c / 2)$ or bilayer $(c)$ height, although this depends on the exact initial substrate miscut and annealing conditions. ${ }^{443-445}$ Indeed, in Fig. 45, the atomic steps are two times $c$ high. It should be noted that similar annealing conditions were also used for preparing $\mathrm{Zn}$-polar $\mathrm{ZnO}$ surfaces, leading to similar surfaces. ${ }^{46}$ An important consequence of the annealing procedure was the change in substrate resistivity, of three orders of magnitude, with high annealing temperatures transforming the substrate from highly resistive to conductive. ${ }^{443}$ Note that in the original table of Ref. 443 the values are shifted by one line with respect to the exact annealing conditions. SIMS measurements showed that, upon annealing, $\mathrm{Li}$ and $\mathrm{Na}$ concentrations were reduced by one order of magnitude, while the $\mathrm{Al}$ concentration also increased by one order of magnitude. While the presence of $\mathrm{Li}$ and $\mathrm{Na}$ in the substrates could easily be explained, as coming from the mineralizers used in the hydrothermal technique, the origin of the $\mathrm{Al}$ was not clear. ${ }^{443}$

As regards $\mathrm{Zn}$-polar $\mathrm{ZnO}$ substrates, the most commonly used surface preparation was chemo-mechanical polishing; this allowed simultaneously to remove a surface-contaminated layer, where Li contaminants coming from the bulk had been accumulated owing to an initial thermal annealing, and to prepare a flat surface displaying $0.52 \mathrm{~nm}$ steps (i.e., bilayer steps) with a reduced roughness of only some Angstroms. ${ }^{447}$ Thus, with one single procedure, both the surface morphology and electrical properties of the substrate were improved. However, silica particles coming from the solution used for polishing the surface remained stuck to it and could not be removed by rinsing the surface with pure water. ${ }^{448}$ Based on electrochemical considerations, it was conjectured that a solid layer of zinc hydroxide could be formed during the polishing of the $\mathrm{Zn}$ polar $\mathrm{ZnO}$ surface and trap the silica particles on the surface. By using an AFM inside an electrochemical cell, the evolution of the surface morphology was followed in situ during its etching in a $\mathrm{HCl}$ solution. Interestingly, as etching proceeds, the surface transformed after some minutes into $3.5 \mathrm{~nm}$ high islands that disappeared after $13 \mathrm{~min}$, thereby recovering a step-and-terrace morphology. The surface improvement was assigned to the etching of the zinc hydroxide layer and/or of the surface layer damaged by the polishing. 
The main conclusion is that even if an almost "proprietary" annealing was developed by each research team, atomically flat $\mathrm{ZnO}$ substrates of both polarities became readily available by 2006/2007. While many different growth techniques were used to obtain high-quality homoepitaxial layers, including liquid phase epitaxy ${ }^{446}$ or sputtering (i.e., heliconwave-excited-plasma sputtering), ${ }^{449}$ three main techniques were employed: CVD/MOCVD (both Zn- and O-polar layers), MBE (mostly Zn-polar layers), and PLD (mostly O-polar layers).

a. CVD and MOCVD. The CVD/MOCVD growth of many semiconductors is carried out using either $\mathrm{H}_{2}$ as carrier gas or a mixture of $\mathrm{H}_{2}$ and $\mathrm{N}_{2}$ (i.e., see Section IV A on GaN thin films). However, for the growth of $\mathrm{ZnO}$, especially if high temperatures are used, $\mathrm{H}_{2}$ should be avoided due to its strong etching potential. Instead, pure $\mathrm{N}_{2}^{439,450-452}$ or $\mathrm{Ar}^{444,453,454}$ was preferred as carrier gases. Similarly, several options were tested as $\mathrm{Zn}$ and $\mathrm{O}$ precursors: DMZn (dimethylzinc), ${ }^{455}$ DEZn (diethylzinc), ${ }^{451,453,454}$ and metallic $\mathrm{Zn}^{444,452}$ for $\mathrm{Zn}$, and $\mathrm{O}_{2},{ }^{451,453,455} \mathrm{NO}_{2},{ }^{444,453} \mathrm{~N}_{2} \mathrm{O},{ }^{451,453-455}$ or even $\mathrm{H}_{2} \mathrm{O}^{452}$ for $\mathrm{O}$. Whenever several $\mathrm{O}$ precursors are compared, ${ }^{453,455} \mathrm{O}_{2}$ systematically gives better results in terms of surface roughness.

Indeed, in the first years it was commonly accepted that, whereas a 2D growth mode could be achieved on O-polar $\mathrm{ZnO}$ substrates by CVD/MOCVD, ${ }^{444,455}$ the growth on $\mathrm{Zn}$ polar $\mathrm{ZnO}$ surfaces led to a $3 \mathrm{D}$ nucleation and hence a $3 \mathrm{D}$ growth mode. ${ }^{44}$ However, after growth optimization, even the same groups could accomplish a 2D growth mode on $\mathrm{Zn}$ polar $\mathrm{ZnO}$ substrates, ${ }^{456}$ and it was shown that, provided that the growth temperature is sufficiently high (larger than $950^{\circ} \mathrm{C}$ ), a $2 \mathrm{D}$ surface morphology could be obtained, even on $\mathrm{Zn}$-polar $\mathrm{ZnO}$ substrates. ${ }^{451}$ At these growth temperatures, neither the total reactor pressure nor the VI/II ratio had any influence on the surface morphology. At even higher temperatures and VI/II ratios smaller than 20, it was even possible to achieve step-flow growth by HVPE as evidenced by the step-linearity and terrace width, which reproduced that of the initial $\mathrm{Zn}$-polar $\mathrm{ZnO}$ substrate. ${ }^{452}$ The same tendency, i.e., no clear difference between growth on opposite polarities, was observed in terms of structural properties, the tilt and twist of the layers being determined by the quality of the underlying substrate. ${ }^{452}$ Typical FWHM of the (0002) reflection from rocking curves were in the order of 30 arcsecs. $^{453,455}$ In some cases, the structural quality could even be improved during the growth of the subsequent layers, reducing the FWHM of the (0002) reflection to less than 20 arcsecs. ${ }^{444,454}$

The fact that similar morphologies and structural properties could be obtained on the two polarities enabled to compare the effect of the polarity in impurity incorporation/ diffusion under similar growth conditions and on similar surfaces. Indeed, $\mathrm{ZnO}$ layers were deposited in coloaded $\mathrm{Zn}$ and O-polar ZnO substrates by CVD and investigated by SIMS and PL. ${ }^{456}$ In Fig. 46(a), the ratio between impurity concentrations for the two coloaded homoepitaxial layers is displayed. $\mathrm{Li}, \mathrm{Na}, \mathrm{K}$, and $\mathrm{Ca}$ concentrations are almost two orders of magnitude larger for the O-polar $\mathrm{ZnO}$ layer than for the $\mathrm{Zn}$-polar $\mathrm{ZnO}$ one. In contrast, $\mathrm{Mg}, \mathrm{Al}$, and $\mathrm{Si}$ concentrations are just one order of magnitude larger in the Opolar $\mathrm{ZnO}$ layers. The much larger impurity concentrations in the O-polar $\mathrm{ZnO}$ layers, especially that of alkali metals employed as mineralizers during the hydrothermal growth of the substrates, suggest that this large asymmetry in impurity concentrations is not related to different incorporations during the growth of the layers themselves, but rather to unequal impurity concentrations in the initial substrates. These differences in impurities result in distinct excitonic recombinations, as shown in Fig. 46(b), as well as in increased FWHM of all bound exciton lines in O-polar $\mathrm{ZnO}$ layers. ${ }^{457}$

For a clear signature of polarity-related unequal impurity incorporation, we must thus focus on dopant species not present in the substrates. An illustrative example is the incorporation of $\mathrm{N}$, which plays a central role in the quest for stable and reproducible p-type doping of $\mathrm{ZnO}$ layers. ${ }^{458,459}$ Experiments using ammonia as $\mathrm{N}$ precursor showed that while $\mathrm{N}$ associated Raman modes, as well as N-related PL lines (i.e., donor-acceptor pair transitions and band-acceptor transitions), could be detected on $\mathrm{Zn}$-polar $\mathrm{ZnO}$ layers, they were completely absent in O-polar $\mathrm{ZnO}$ layers although grown under the same conditions. ${ }^{435}$ However, one should notice
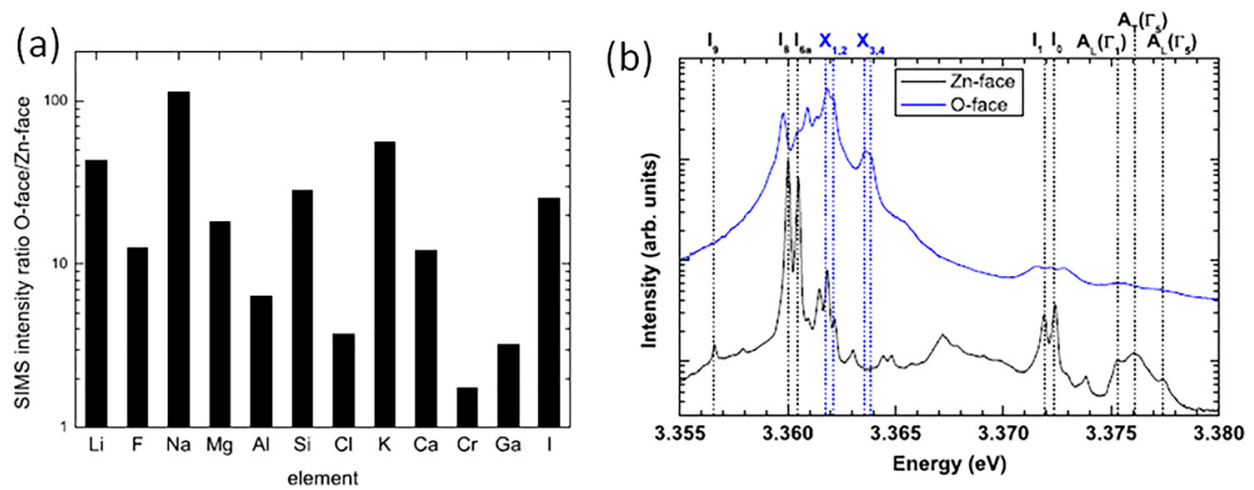

FIG. 46. (a) Intensity ratio of the normalized secondary ion intensity of a coloaded O-polar homoepitaxial film to a coloaded Zn-polar homoepitaxial film. Reprinted with permission from Lautenschlaeger et al., Phys. Rev. B 77, 144108 (2008). Copyright 2008 American Physical Society. (b) PL spectra of homoepitaxial coloaded O- (upper curve) and Zn-polar (lower curve) ZnO homoepitaxial layers. Reprinted with permission from Wagner et al., Phys. Rev. B 79, 035307 (2009). Copyright 2009 American Physical Society. 
that these incorporation asymmetries depend on the impurity itself, with some dopants (i.e., $\mathrm{Al}$ ) being less sensitive to the polarity of the growing surface than others (i.e., $\mathrm{N}$ ). ${ }^{460}$

b. MBE. Except for an early report by Ohno et al., ${ }^{461}$ where the two polarities were investigated, most of the MBE studies on homoepitaxial polar thin films, if not all, deal only with the growth on $\mathrm{Zn}$-polar $\mathrm{ZnO}$ substrates. Indeed, in this first report it was concluded that either spiral growth or atomically flat surfaces but displaying hexagonal holes could be achieved on O-polar $\mathrm{ZnO}$ substrates, while $\mathrm{Zn}$-polar $\mathrm{ZnO}$ homoepitaxial layers could be very smooth (surface roughness smaller than $0.5 \mathrm{~nm}$ ) on $\mathrm{Zn}$-polar $\mathrm{ZnO}$ substrates. A complete investigation of different growth conditions spanning a temperature range from $400^{\circ} \mathrm{C}$ up to $950^{\circ} \mathrm{C}$ and VI/II ratios from $\mathrm{Zn}$ - to O-rich conditions was performed. Consistent with other reports, ${ }^{462,463}$ it was shown that the growth on $\mathrm{Zn}$-polar $\mathrm{ZnO}$ substrates required O-rich conditions and smaller temperatures than those required for the growth on O-polar $\mathrm{ZnO}$ substrates, for which $\mathrm{Zn}$-rich conditions were preferred.

However, in the following years, it was repeatedly shown that, if sufficiently high growth temperatures (typically above $750^{\circ} \mathrm{C}$ ) were used, good surface morphology with atomically flat surfaces and monolayer steps could be obtained irrespective of the VI/II ratio. The reason is that $\mathrm{Zn}$ adatoms have large enough diffusion lengths at those temperatures. Under these growth conditions, the structural properties of $\mathrm{ZnO}$ layers are comparable with those obtained by CVD/MOCVD, with FWHM of the (0002) and (1010) reflections from rocking curves showing values in the order of 20 arcsecs or even smaller. Thus, if one considered the morphology and the structural properties as the only optimization criteria, growth temperature would seem to be the only important growth parameter. However, as soon as the optical properties were considered, the VI/II ratio was seen to play an important role as well, ${ }^{464}$ as illustrated in Fig. 47. While all samples remain atomically flat, the first excited state of A-free excitons is only observed on O-rich or stoichiometric condition grown layers. As indicated in Ref. 464, owing to the evolution of $\mathrm{Zn}$ sticking coefficient with growth temperature, the optimum VI/II ratio should be determined for each growth temperature.

While $p$-type doping is a necessary ingredient for many electrically injected optoelectronic devices (i.e., LEDs or Laser Diodes, LDs), there are a number of applications that do not require $p$-type doping (eventually not $n$-doping either). In particular, $\mathrm{ZnO} /(\mathrm{Zn}, \mathrm{Mg}) \mathrm{O}$ heterostructures proved to be extremely successful in the field of electronics (for an overview see Ref. 465), where they allowed the realization of HEMTs with electron mobilities exceeding $100000 \mathrm{~cm}^{2} /$ $\mathrm{V} \cdot \mathrm{s}$ at low temperature, and the observation of the fractional quantum Hall effect. ${ }^{466}$ To obtain such high electron mobilities, the scattering sources, including surface roughness and impurities, need to be reduced as much as possible. This was achieved by following two growth adjustments: first, the growth temperature was increased above $850^{\circ} \mathrm{C}$, as the optimum growth temperature of $\mathrm{Zn}_{1-\mathrm{x}} \mathrm{Mg}_{\mathrm{x}} \mathrm{O}$ is considerably higher than that of $\mathrm{ZnO}$, and increases with the $\mathrm{Mg}$ content

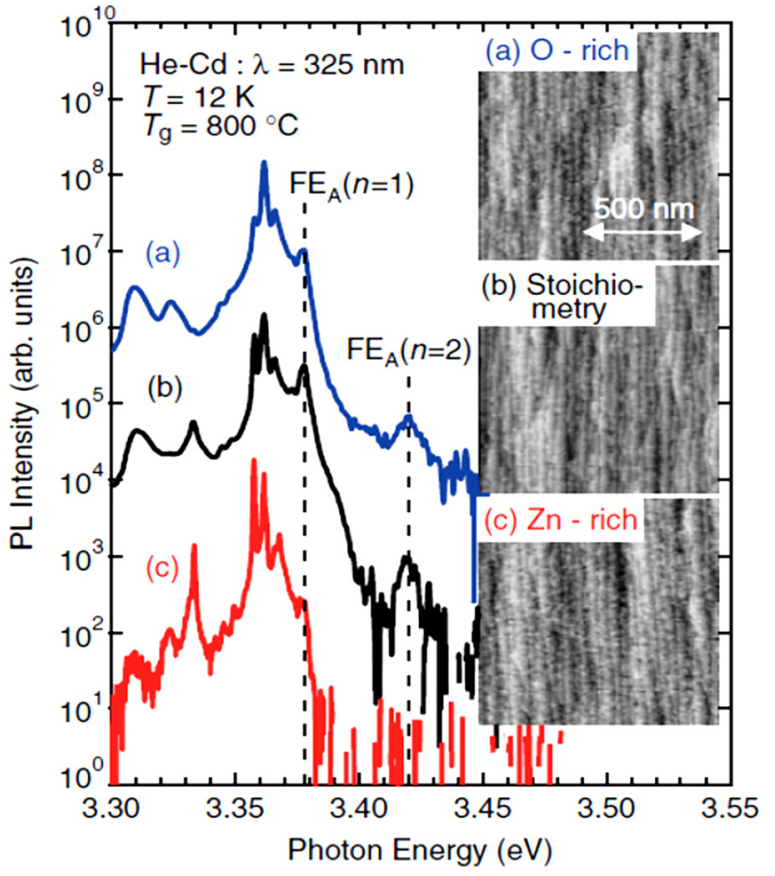

FIG. 47. PL spectra measured at $12 \mathrm{~K}$ for Zn-polar homoepitaxial layers grown at $800^{\circ} \mathrm{C}$ under (a) O-rich, (b) stoichiometric, and (c) Zn-rich conditions. Reprinted with permission from Yuji et al., Jpn. J. Appl. Phys., Part 1 49, 071104 (2010). Copyright 2010 The Japan Society of Applied Physics.

in the alloy, ${ }^{464,467}$ similar to the situation encountered in the $(\mathrm{Al}, \mathrm{Ga}) \mathrm{N}$ system (see Section IV A 6a); and second, as shown in Fig. 48, the growth rate was increased from about $300 \mathrm{~nm} / \mathrm{h}$ to more than $600 \mathrm{~nm} / \mathrm{h}^{468}$ The pronounced enhancement was attributed to the reduction of unintentional impurities and/or point defects, thereby reducing the overall ionized impurity scattering. These last samples constitute the state-of-the-art for $\mathrm{ZnO} /(\mathrm{Zn}, \mathrm{Mg}) \mathrm{O}$ heterostructures.

c. Pulsed Laser Deposition (PLD). MOCVD and MBE are commonly considered to be the most suitable growth

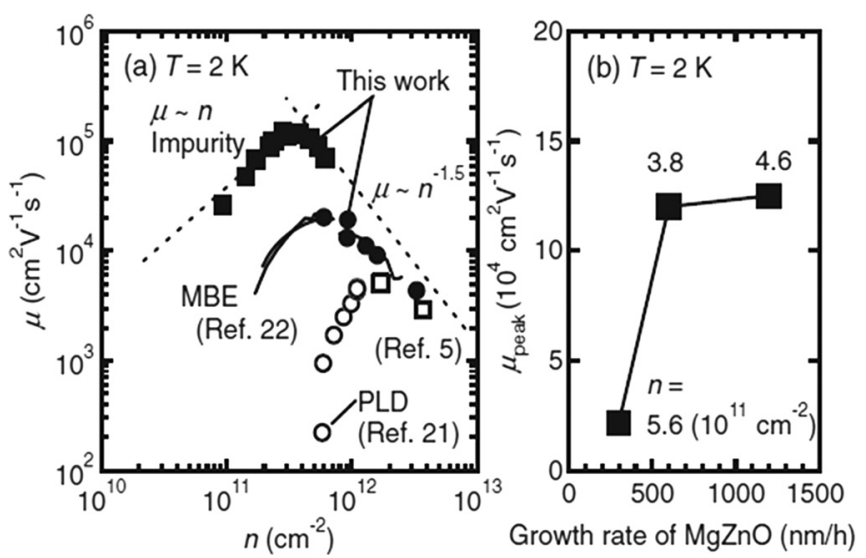

FIG. 48. (a) Relationship between the electron density $(n)$ and mobility $(\mu)$ for various samples including samples grown by PLD (open symbols). Closed circles and solid curves: MBE films grown at a deposition rate of $0.3 \mu \mathrm{m} / \mathrm{h}$. Closed squares: top-gated Hall-bar device made from MBE film $(x=0.05)$ grown at $0.6 \mu \mathrm{m} / \mathrm{h}$. (b) Peak mobility ( $\mu_{\text {peak }}$ ) observed at the denoted $n$ for the heterostructures grown at various deposition rates of $\mathrm{Mg}_{\mathrm{x}} \mathrm{Zn}_{1-\mathrm{x}} \mathrm{O}$. Reprinted with permission from Akasaka et al., Jpn. J. Appl. Phys., Part 1 50, 080215 (2011). Copyright 2011 The Japan Society of Applied Physics. 
techniques for the achievement of flat and single-crystalline semiconductor heterostructures. However, as early as 2007, corresponding to the same period as the first reports by $\mathrm{MOCVD}^{444}$ and MBE, ${ }^{461} \mathrm{ZnO}$ homoepitaxial layers with similar morphological (i.e., atomically flat step-and-terrace surfaces), structural (rocking curve FWHM in the order of 30 arcsecs), and optical properties (FWHM of donor-bound exciton lines of $200 \mu \mathrm{eV}$ ) were grown by PLD. ${ }^{445,469}$

As regards $\mathrm{PLD}$, the $\mathrm{O}_{2}$ partial pressure seems to be the most crucial growth parameter, as it controls the surface quality (see Ref. 470) and, most importantly, the strain-state of the $\mathrm{ZnO}$ homoepitaxial layer. Fig. 49(a) shows that pure $\mathrm{ZnO}$ layers (i.e., without any intentional doping) grown under different $\mathrm{O}_{2}$ partial pressures have an out-of-plane lattice parameter (i.e., $c$-lattice parameter) that can be either that of bulk $\mathrm{ZnO}$, for the highest pressures (i.e., 0.1 and 0.016 mbar), or larger than the bulk one, for smaller pressures (i.e., 0.002 and 0.0003 mbar). ${ }^{471}$ These observations, which are most probably related to deviations from a perfect stoichiometry due to a large density of $\mathrm{O}$ vacancies, are similar to those carried out in CVD-grown layers, where a uniaxial tensile strain (but with different absolute values) parallel to the $c$-axis was detected for both $\mathrm{Zn}$ - and O-polar $\mathrm{ZnO}$ homoepitaxial layers. ${ }^{457}$ In this last work, since the tensile strain developed on the O-polar $\mathrm{ZnO}$ layers was larger than that on the $\mathrm{Zn}$-polar $\mathrm{ZnO}$ ones, the strain was associated with the presence of residual impurities coming from the underlying substrate. Further studies on PLD-grown $\mathrm{ZnO}$ layers focused on the effect of the $\mathrm{O}_{2}$ partial pressure on the strain-state while simultaneously doping the homoepitaxial layers. The richest behavior is observed when using $\mathrm{Al}$ as dopant, ${ }^{472}$ for which the out-of-plane strain can be either compressive or tensile depending on the employed $\mathrm{O}_{2}$ partial pressure, as presented in Figs. 49(b) and 49(c). This is in contrast to the previously discussed pure $\mathrm{ZnO}$ layers ${ }^{471}$ or to Sc- ${ }^{473}$ or Ga-doped ${ }^{472} \mathrm{ZnO}$ homoepitaxial layers, for which only one sign for the out-of-plane strain was reported.

\section{Heteroepitaxially grown ZnO thin films}

The vast majority of $\mathrm{ZnO}$ layers were grown on sapphire, a well-known substrate used for its availability, structural perfection, and chemical inertness. Hence, the present section is mainly dedicated to this type of substrate.

Engineering the $\mathrm{ZnO} /$ sapphire interface appears to be crucial, not only for the quality of the $\mathrm{ZnO}$ epilayer but also for its polarity, with a strong relationship between them. As presented below, the polarity of $\mathrm{ZnO}$ epilayers grown onto sapphire depends on the substrate surface pre-treatment, on the growth initiation conditions, and/or on the type of interfacial layers that one may use between sapphire and $\mathrm{ZnO}$. But, it is first worth stressing that the polarity is often dictated by the detailed structure of the hetero-epitaxial interface. In this respect, the orientation relationship between sapphire and $\mathrm{ZnO}$ plays a significant role, as presented in the first subsection.

a. Rotational domains and polarity. When seen from atop, the projections of the $\mathrm{Al}$ and $\mathrm{O}$ sub-lattices of $c$-sapphire are rotated with respect to one another by $30^{\circ}$ (Fig. 50(a)). Different models exist as to the sapphire surface termination: it can be Al-terminated either with a single surface layer or a bilayer one, or it can be O-terminated. ${ }^{474}$ Since the $\mathrm{O}$ and $\mathrm{Al}$ sublattices are rotated by $30^{\circ}$ to each other, if $\mathrm{ZnO}$ epitaxy is undertaken on a mixture of $\mathrm{Al}$ - and O-terminated surfaces (i.e., if monolayer, high steps exists on the sapphire surface) the domains are formed with $30^{\circ}$ in-plane misorientation between them. One orientation is referred to as the nontwisted orientation, i.e., when $\mathrm{ZnO}[11 \overline{2} 0] \| \mathrm{Al}_{2} \mathrm{O}_{3}[11 \overline{2} 0]$, the other as the twisted orientation when $\mathrm{ZnO}[10 \overline{1} 0] \|$ $\mathrm{Al}_{2} \mathrm{O}_{3}[11 \overline{2} 0]$. For $30^{\circ}$ twisted domains, the misfit between $\mathrm{ZnO}$ and sapphire is $18.3 \%$ in compression, while it is $31.5 \%$ in tension for non-twisted domains. As a rule, it is generally admitted that, for large misfit systems, the orientation relationship that minimizes the interfacial lattice mismatch is preferred, since it allows for a reduction of the interface energy; this holds for $\mathrm{GaN}$ on sapphire too. This rotational domain problem being linked to the misfit between layer and substrate; it is expected to be avoided in low misfit heteroepitaxial systems (or even more through homoepitaxial growth): this is the case, for instance, for $\mathrm{ZnO}$ growth on hexagonal $\mathrm{ScAlMgO}_{4}$ (SCAM), for which the $\mathrm{ZnO}$ lattice is aligned with that of the substrate, i.e., $\mathrm{ZnO}[0001] \|$ $\mathrm{ScAlMgO}_{4}[0001]$ and $\mathrm{ZnO}[11 \overline{2} 0] \| \mathrm{ScAlMgO}_{4}[11 \overline{2} 0]$ without
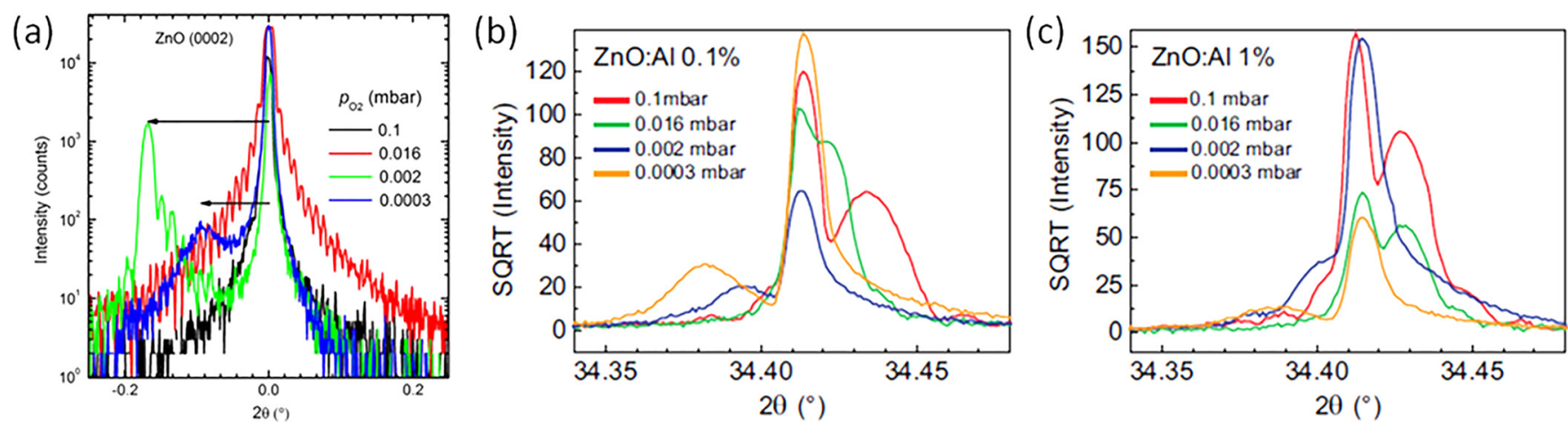

FIG. 49. (a) $2 \theta / \omega$ scans (centered around the $\mathrm{ZnO}$ substrate peak) of the (0002) reflection for O-polar ZnO homoepitaxial layers grown under different oxygen partial pressures. The arrows indicate the reflections of the layers with a larger $c$-lattice parameter. Reprinted with permission from Lajn $e t$ al., J. Electron.

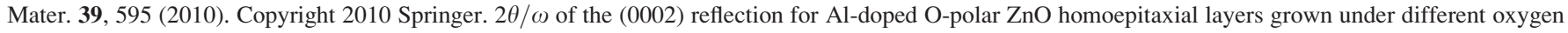
partial pressures and for different $\mathrm{Al}_{2} \mathrm{O}_{3}$ wt. \% contents in the PLD targets: (b) $0.1 \%$ and (c) $1 \%$. Reprinted with permission from Lorenz et al., Phys. Status Solidi A 212, 1440 (2015). Copyright 2015 John Wiley \& Sons, Inc. 


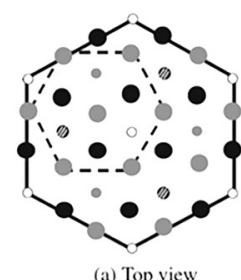

(a) Top view

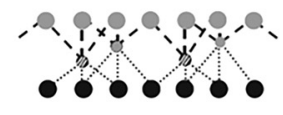

(c) O-termination

(a)

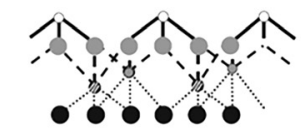

(b) Side view: single Al termination

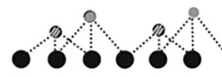

(d) Al bilayer termination

- Top layer Al Second layer O Third layer Al - Al sublattice

- Fourth layer Al Fifth layer Al

(b)

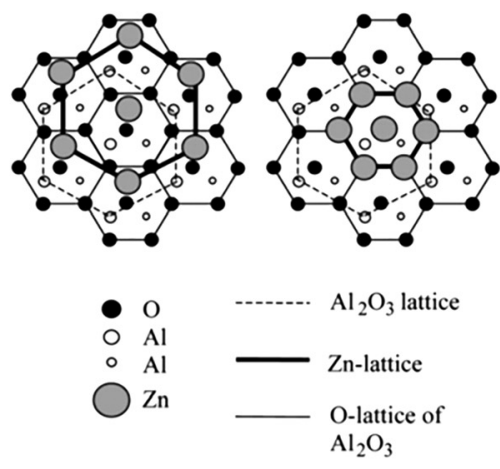

FIG. 50. (a) Atomic stacking of the sapphire surface according to surface termination. Top left: top view of the c-sapphire surface, note the $30^{\circ}$ rotation between the $\mathrm{Al}$ and $\mathrm{O}$ top layer sublattices. Cross sectional views, right left: with single $\mathrm{Al}$ termination, bottom left: with $\mathrm{O}$ termination, and bottom right: with Al bilayer termination. Reprinted with permission from Ying et al., J. Phys. D: Appl. Phys. 37, 3058 (2004). Copyright 2004 Institute of Physics Publishing. (b) For $\mathrm{Al}$ terminated surfaces, $\mathrm{O}$ will bind to $\mathrm{Al}$ first resulting in $\mathrm{Zn}$-polar $\mathrm{ZnO}$ with no in-plane rotation between the $\mathrm{ZnO}$ lattice and sapphire, while for O-terminated sapphire surfaces, $\mathrm{Zn}$ will bind to $\mathrm{O}$, leading to $\mathrm{O}-$ polar $\mathrm{ZnO}$ and $30^{\circ}$ in plane rotation.

any trace of in-plane misorientation and hence of rotational domains. ${ }^{475}$

Furthermore, since the rotational domains correspond to different atomic bondings to the Al- or O-terminated sapphire surfaces, they are, at least if one follows the stacking model given in Ref. 479, Zn-polar (un-twisted orientation) and O-polar (twisted orientation), respectively, as shown in Fig. 50(b). Thus, the co-existence of twisted and non-twisted rotational domains is to be avoided in order not only to reduce the density of defects in the layers but also to ensure single polarity $\mathrm{ZnO}$ epilayers. In turn, this also reduces the density of structural defects, such as IDBs. This definitely calls for a precise control of the sapphire surface before $\mathrm{ZnO}$ growth was undertaken.

It is noteworthy that rotational domains may exist for $\mathrm{ZnO}$ grown by PAMBE on (111) $\mathrm{Si}^{476}$ as for the case of growth on sapphire, a $30^{\circ}$ rotation of the $\mathrm{ZnO}$ lattice with respect to $\mathrm{Si}$, with the $\mathrm{ZnO}[11 \overline{2} 0]$ direction aligned with the $\mathrm{Si}$ [110], allows for a reduction of the lattice mismatch. It is, however, most probable that the polarity is affected by the lattice rotation, since $\mathrm{Si}$ is a monoatomic substrate.

b. Effects of sapphire surface conditioning.

3.1. $M B E$. For this kind of investigation, $\mathrm{MBE}$ is of course advantageous thanks to the surface-sensitive in-situ characterization that it offers (i.e., RHEED). PAMBE was used in Ref. 477 to grow $\mathrm{ZnO}$ epilayers on top of sapphire, the surface of which was treated according to different methods. For unprepared (just chemically etched) surfaces as well as for $800^{\circ} \mathrm{C}$ annealed surfaces under $\mathrm{O}_{2}$ plasma, the rotational domains occurred. But these were not formed in the case of $\mathrm{O}_{2}$ plasma treated surfaces, if these surfaces were nitridated (under $\mathrm{N}_{2}$ plasma at a temperature as low as $180^{\circ} \mathrm{C}$ in the PAMBE), leading, respectively, to double (O and $\mathrm{Zn}$ ) or single $(\mathrm{O})$ polarity, although the polarity was not measured directly in this paper.

As discussed in Section IV A 3 c, the polarity of GaN or AlN layers grown on sapphire crucially depends on the growth method: most often, $\mathrm{N}$-polar $\mathrm{GaN}$ layers are obtained in PAMBE, while Ga-polar GaN layers are grown in ammonia MBE and MOVPE. However, this is related to the nitridation step itself since, for instance, $\mathrm{GaN}$ layers grown by PAMBE on ammonia-nitridated sapphire surfaces are Gapolar. ${ }^{184}$ That is to say, that the polarity of the nitride layer is determined by the polarity of the AlN nitridation layer and can thus be controlled through the nitridation process. For $\mathrm{ZnO}$, one may believe that the same would apply with cation (resp. anion) polar layers on cation (resp. anion) terminated nitridation layers. As discussed above, this was effectively the case in Ref. 477 (i.e., O-polar $\mathrm{ZnO}$ layers on top of $\mathrm{N}$-terminated nitridation layer by PAMBE). But actually, this seems to depend a lot on the nitridation conditions. For instance, in Ref. 478, direct growth on sapphire nitridated at $400^{\circ} \mathrm{C}$, instead of $180^{\circ} \mathrm{C}$ as in Ref. 477 yields $\mathrm{Zn}$-polar $\mathrm{ZnO}$ layers (as measured by CAICISS: see Section IIIF). When growth was carried out directly on the AIN nitridation layer, supposedly of $\mathrm{N}$ polarity by PAMBE, an amorphous transition layer was observed by RHEED upon starting $\mathrm{ZnO}$ growth. The $\mathrm{ZnO}$ epilayer grown onto this amorphous interface was of $\mathrm{Zn}$ polarity (Fig. 51(a)). It was assumed that the $\mathrm{Zn}$-polar $\mathrm{ZnO}$ surface is the most energetically favorable surface under the growth conditions used, i.e., for a temperature of $400^{\circ}$. One might argue that the MBE growth was far from equilibrium, but one may refer also to recent calculations of the surface energies of $\mathrm{Zn}$ - and O-polar $\mathrm{ZnO}$ surfaces: ${ }^{479}$ the $\mathrm{Zn}$-polar surface may be lower in energy than its O-polar counterpart, if $\mathrm{ZnO}$ growth is carried out in O-poor/Zn-rich conditions. Interestingly, $\mathrm{Ga}$ pre-deposition on nitridated sapphire by PAMBE was found to lead to the formation of $\mathrm{O}$-polar $\mathrm{ZnO}$ layers instead of $\mathrm{Zn}$-polar $\mathrm{ZnO}$ layers, if no $\mathrm{Ga}$ was deposited (Fig. 51(b)). Since plasma nitridation results in a N-polar AlN buffer, Ga bonds to $\mathrm{N}$ and the easy formation of the $\mathrm{Ga}-\mathrm{O}$ bond leads to O-polar $\mathrm{ZnO}$ layers.

The dependence of the polarity on the nitridation temperature in PAMBE is also stressed in Ref. 480. Contrary to Ref. 478, no amorphous interfacial layer was found between the AlN nitridation layer and the $\mathrm{ZnO}$ epilayer. Instead, it was shown by HRTEM that the layer formed upon nitridation under $\mathrm{N}$ plasma is zinc blende AlN rather than wurtzite 

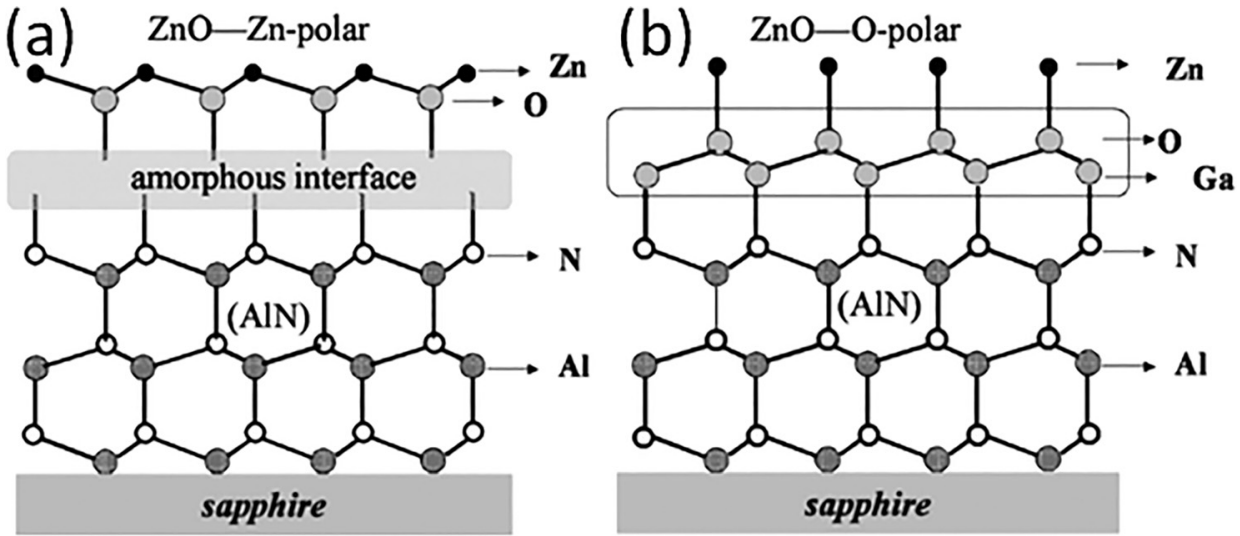

FIG. 51. (a) Atomic stacking at the sapphire/ZnO interface when growth is carried out on nitrided and nitridation is carried out at $400{ }^{\circ} \mathrm{C}$ with the formation of an amorphous layer leading to $\mathrm{Zn}$ polar $\mathrm{ZnO}$. (b) Atomic arrangement at the sapphire/ZnO interface with the pre-deposition of $\mathrm{Ga}$, leading to $\mathrm{O}$ polar $\mathrm{ZnO}$. Adapted with permission from Appl. Phys. Lett. 86, 011921 (2005). Copyright 2005 AIP Publishing LLC.
AlN. Low temperature $\left(180^{\circ} \mathrm{C}\right)$ and higher temperature $\left(400{ }^{\circ} \mathrm{C}\right)$ nitridation leading, respectively, to $\mathrm{N}$ - and Al-polar $111 \mathrm{AlN}$, hence to O- and Zn-polar ZnO epilayers, as measured by electron holography in the TEM (note that these nitridation temperatures are rather low, the plasma nitridation process relying in this case on the active plasma species rather than on thermal activation). The nitridation temperature regions associated with the two different polarities are given in Fig. 52 by the same group. ${ }^{481}$

Without any sapphire nitridation, it was also shown that the ratio between twisted and un-twisted domains in laser$\mathrm{MBE}$ grown $\mathrm{ZnO}$ epilayers ( $\mathrm{ZnO}$ target under low pressure $\mathrm{O}_{2}$ ) could vary from 0 to 1 by just varying the growth temperature for annealed sapphire substrates (no intentional nitridation) ${ }^{482}$ (Fig. 53(a)). High temperature growth leads to O-polar $\mathrm{ZnO}$ epilayers ( $30^{\circ}$ twisted), while low temperature yields $\mathrm{Zn}$-polar $\mathrm{ZnO}$ epilayers (un-twisted) (NB: wrong interpretation of PHI scans in Ref. 482). At a given temperature, the ratio can also vary from 1 to 0 by increasing the growth rate. High temperature growth on low temperature nucleation layers leads to twisted domains only (Fig. 53(b)): the polarity is thus fixed by the growth temperature at which nucleation occurs on the substrate and by the nucleation rate itself.

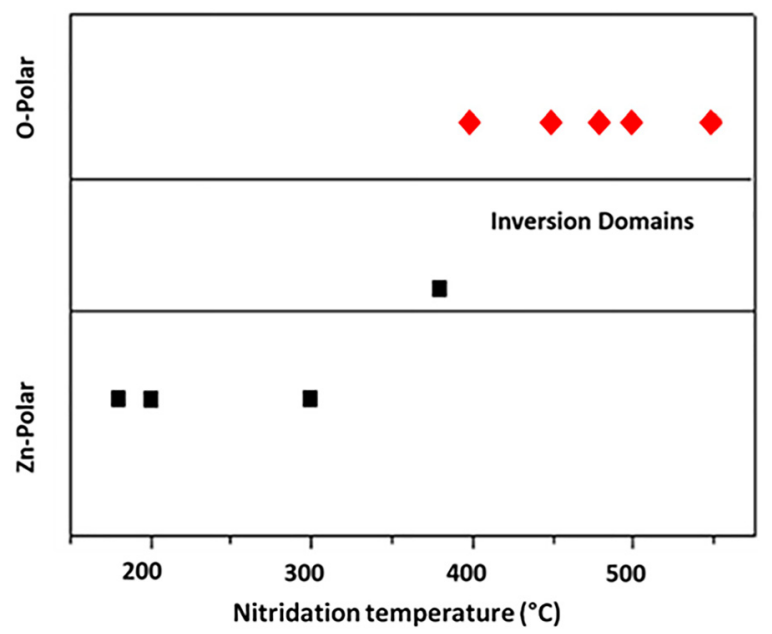

FIG. 52. Polarity of $\mathrm{ZnO}$ epilayer grown by RF-MBE, as a function of nitridation temperature. Reprinted with permission from Appl. Phys. Lett. 86, 112111 (2005). Copyright 2005 AIP Publishing LLC.
3.2. MOCVD. Most $\mathrm{ZnO}$ epilayers grown by MOCVD are $30^{\circ}$ twisted, i.e., of $\mathrm{O}$ polarity, presumably because of the higher temperature favoring the low misfit rotational domain and because of the oxidizing atmosphere yielding $\mathrm{O}$ rich sapphire surfaces. Some works, however, mention that the polarity can be adjusted, for instance, through the growth initiation procedure. In Ref. 483, starting growth (at $475^{\circ} \mathrm{C}$ ) by exposing the bare sapphire surface to the $\mathrm{Zn}$ precursor (DEZn) before introducing oxygen was found to lead to untwisted only $\mathrm{ZnO}$ layers; the reverse procedure (introducing $\mathrm{O}$ first, or $\mathrm{O}$ and DEZn simultaneously) led to twisted $\mathrm{ZnO}$ layers only. However, the polarity, as determined from CAICISS, was found to be $\mathrm{Zn}$ polar whatever the in-plane orientations, i.e., whatever the nucleation conditions. Nevertheless, a precise characterization of the interface region at the atomic scale is certainly missing to yield a proper understanding of these observations. In Ref. 484,

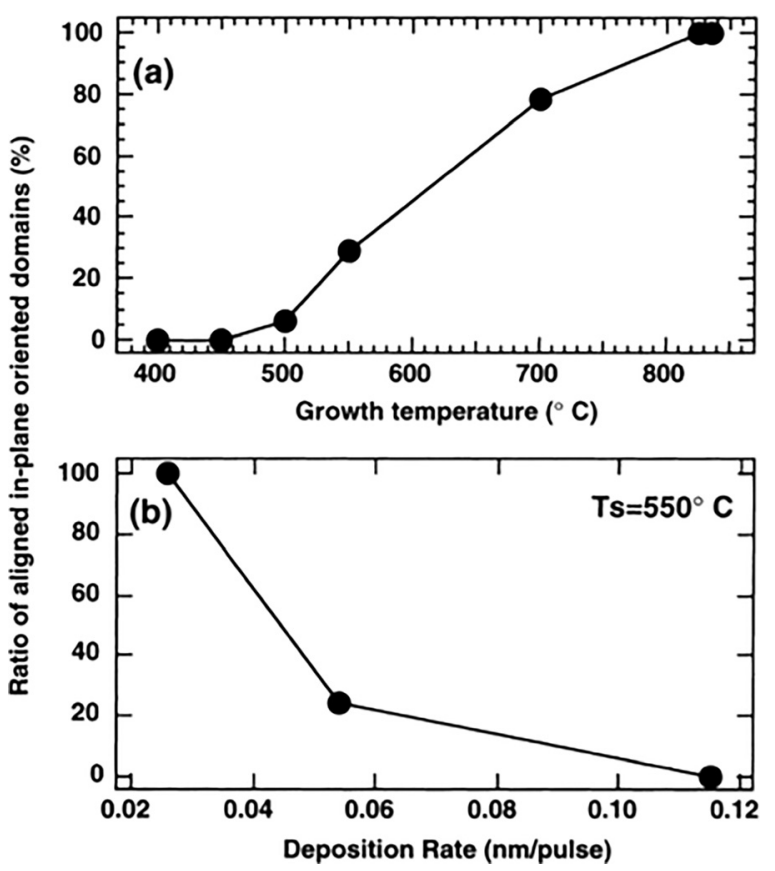

FIG. 53. Ratio of in plane oriented ( $\mathrm{Zn}$ polar) vs. $30^{\circ}$ twisted domains (Opolar), (a) as a function of growth temperature and (b) as a function of growth rate at a $\mathrm{T}^{\circ}$ of $550^{\circ} \mathrm{C}$ for $\mathrm{ZnO}$ epilayers grown by Laser MBE onto sapphire (without any nitridation): the polarity is fixed by the nucleation $\mathrm{T}^{\circ}$ and nucleation rate. Adapted with permission from Ohkubo et al., Surf. Sci. 443, L1043 (1999). Copyright 1999 Elsevier. 

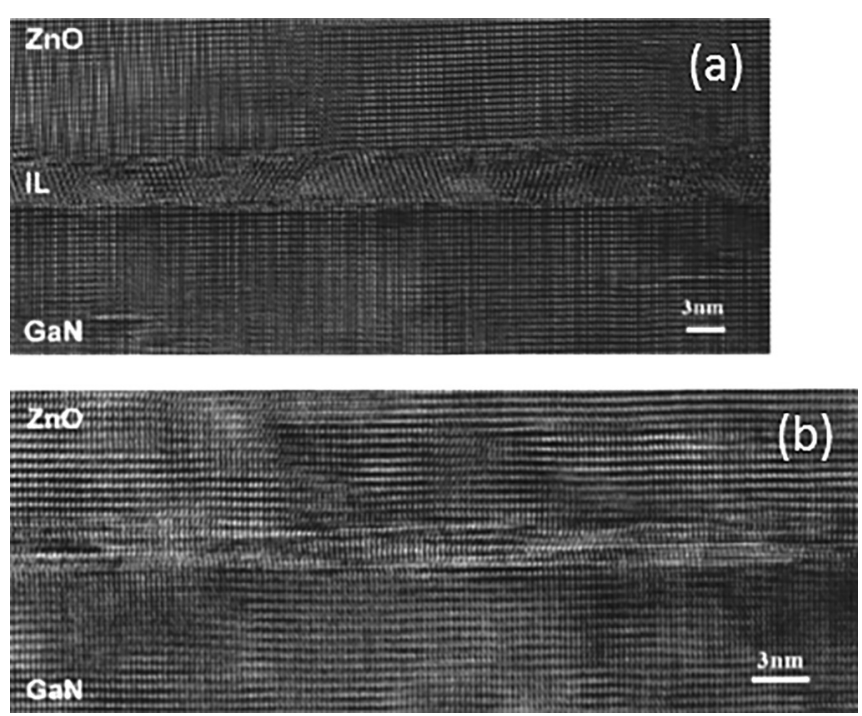

FIG. 54. ZnO epilayer deposited by RF MBE upon c-oriented Ga polar GaN MOVPE template. (a) $\mathrm{Zn}$ stabilized $\mathrm{GaN}$ template leading to $\mathrm{Zn}$-polar $\mathrm{ZnO}$. (b) Oxygen stabilized $\left(\mathrm{O}_{2}\right.$ plasma) $\mathrm{GaN}$ template leading to O-polar $\mathrm{ZnO}$ $\mathrm{Ga}_{2} \mathrm{O}_{3}$ interface layer is formed between $\mathrm{GaN}$ and $\mathrm{ZnO}$. Adapted with permission from Hong et al., J. Vac. Sci. Technol., B 18, 2313 (2000). Copyright 2000 American Vacuum Society.

although $\mathrm{O}$ was sent first in the growth chamber, by simply changing the growth temperature (i.e., the nucleation temperature) the in-plane orientation and, thus, the polarity could be controlled: lower growth temperature (below about $250^{\circ} \mathrm{C}$ ) led to un-twisted (supposedly $\mathrm{Zn}$-polar, not measured), while higher growth temperature $\left(300^{\circ} \mathrm{C}<\mathrm{T}\right.$ $<550^{\circ} \mathrm{C}$ ) yielded $30^{\circ}$ twisted growth (supposedly O-polar) with a much better structural quality. This confirms the overall trend with temperature observed in MOVPE. Another interesting approach was presented in Ref. 485: the rotational domains were eliminated by using non-polar (112̄0) $a$-sapphire instead of $c$-sapphire, taking advantage of the occurrence of a coincidence site lattice match (with four times the distance between the $\mathrm{O}$ atoms on the $[11 \overline{2} 0] \mathrm{ZnO}$ direction corresponding to three times the separation between $\mathrm{O}$ atoms in the sapphire [0001] direction) leading to twisted growth, i.e., with $\mathrm{O}$ polarity only.

(a)

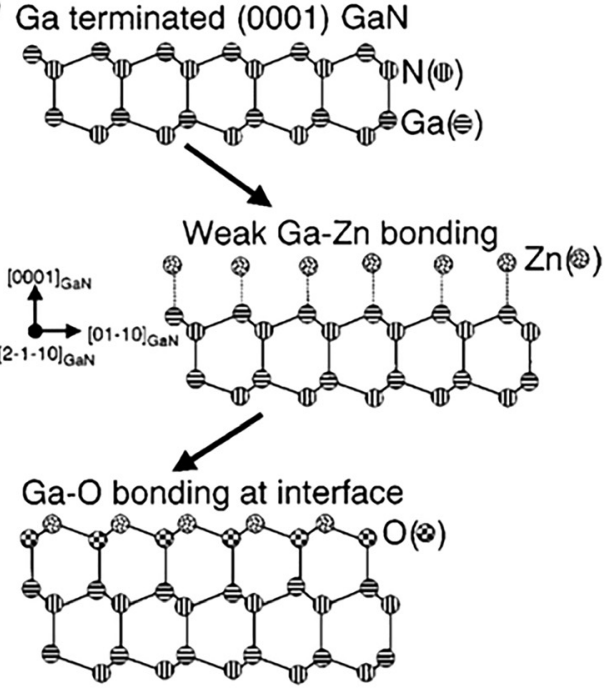

c. Effect of interfacial layers/templates. Beyond simply treating the sapphire surface before $\mathrm{ZnO}$ deposition, one may resort to interfacial layers that eventually impose the polarity through their interfacial atomic bonding with $\mathrm{ZnO}$.

3.1. GaN. As a general rule in order to cope with the misfit issues when growing $\mathrm{ZnO}$ layers hetero-epitaxially, one seeks substrates or templates with the lowest possible lattice mismatch. Even if $\mathrm{GaN}$ is not strictly speaking lattice matched to $\mathrm{ZnO}$, still the mismatch is moderate $(\Delta \mathrm{a} /$ $\mathrm{a}=1.8 \%$ ) and furthermore GaN possesses the same wurtzite structure as $\mathrm{ZnO}$. With this in view, $\mathrm{ZnO}$ layers were grown by PAMBE on GaN/sapphire templates. ${ }^{486}$ These MOCVD grown templates were, as usual, of Ga polarity. One would expect that the $\mathrm{ZnO}$ layer grown on top of $\mathrm{GaN}$ would retain cation ( $\mathrm{Zn})$ polarity. But this depends on the GaN surface treatment before epitaxy. Before depositing $\mathrm{ZnO}$, the surface of these templates was pre-treated by exposing it to either $\mathrm{Zn}$ or O-plasma in the MBE chamber at temperatures of $700^{\circ} \mathrm{C}$ for 3-5 min. These two different pre-treatments of the GaN surface led, respectively, to single $\mathrm{Zn}$ - or O-polar domains, as determined by CAICISS measurements. Under O-plasma pretreatment, a $3-5 \mathrm{~nm}$ thick interfacial $\mathrm{Ga}_{2} \mathrm{O}_{3}$ layer (monoclinic and O-terminated) is formed between $\mathrm{ZnO}$ and $\mathrm{GaN}$, while no interface layer occurs in the case of $\mathrm{Zn}$ pre-exposed GaN templates (Fig. 54). The stacking models for explaining the two different polarities are schematized in Fig. 55. The $\mathrm{Zn}$-polar $\mathrm{ZnO}$ material is found to be of better structural and optical quality than the O-polar $\mathrm{ZnO}$ one, presumably because of the strain and defects induced by the presence of the $\mathrm{Ga}_{2} \mathrm{O}_{3}$ interface layer. However, the rather poor surface quality of the $\mathrm{Zn}$-polar $\mathrm{ZnO}$ layers can be improved, to a great extent, through post-growth annealings, also yielding narrower PL linewidths.

3.2. $\mathrm{MgO}$. As in the case of $\mathrm{GaN}$ templates just discussed above, the use of $\mathrm{MgO}$ as a template reduces the misfit problem with $\mathrm{ZnO}$. Interestingly, and following the demonstration of polarity inversion with GaN buffers, it was shown in Ref. 487 that, starting from a Zn-polar ZnO layer deposited on Ga-polar GaN, it was possible to reverse the

\section{(b) $\mathrm{O}-\mathrm{Zn}$ bonding at interface}

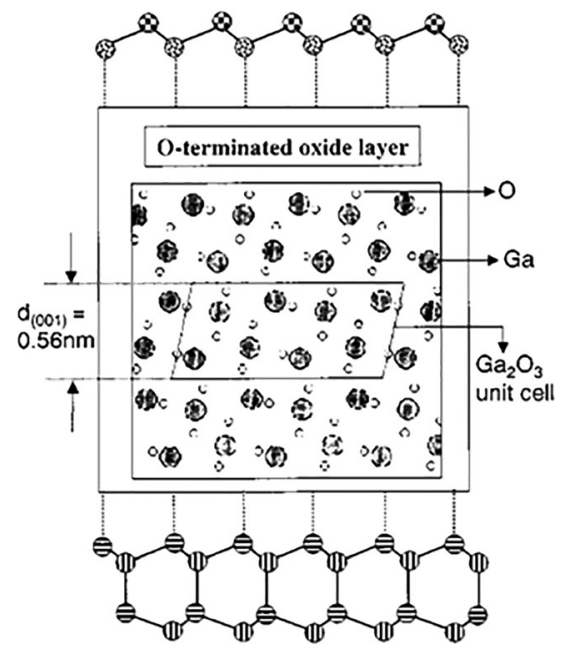

FIG. 55. Atomic stacking model at the $\mathrm{ZnO} / \mathrm{GaN}$ interface (a) for $\mathrm{Zn}$ treated $\mathrm{GaN}$, (b) for $\mathrm{O}$ plasma treated $\mathrm{GaN}$ through the formation of $\mathrm{Ga}_{2} \mathrm{O}_{3}$ interface layer. Reprinted with permission from Hong et al., J. Vac. Sci. Technol., B 18, 2313 (2000). Copyright 2000 American Vacuum Society. 

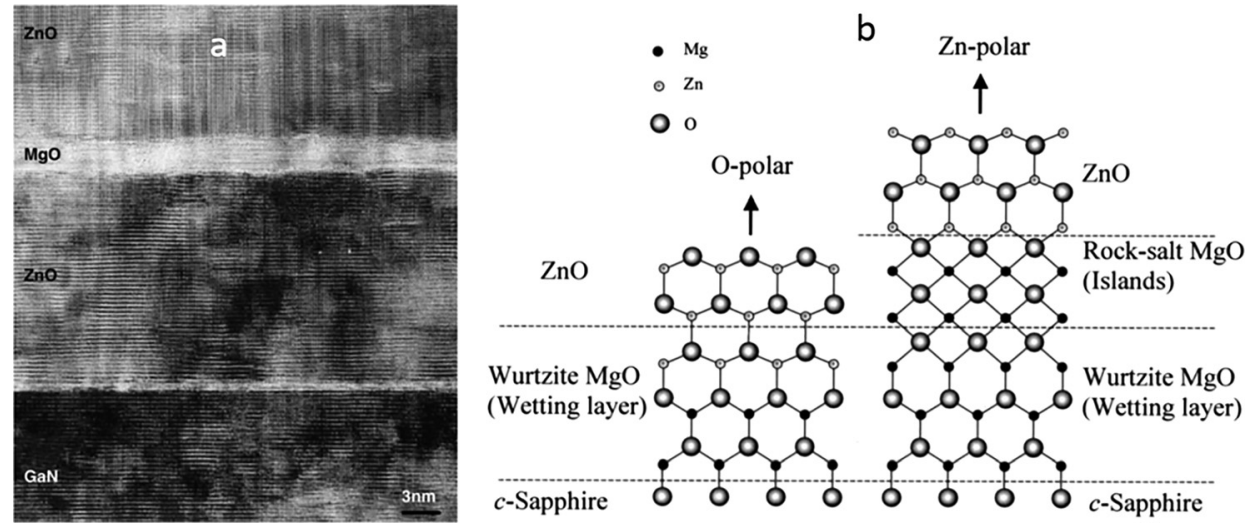

FIG. 56. (a) Reversal of the polarity using an $\mathrm{MgO}$ intermediate layer. The ZnO layer, grown by RF-plasma MBE upon a Zn stabilized c-oriented GaN template, is of $\mathrm{Zn}$ polarity. Insertion of a $\mathrm{MgO}$ epilayer reverses its polarity back to O. Adapted with permission from Hong et al., Appl. Surf. Sci. 190, 491 (2002). Copyright 2002 Elsevier. (b) Atomic stacking sequence at the Sapphir/MgO/ZnO interface according to the MgO interface layer thickness. Reprinted with permission from Appl. Phys. Lett. 84, 4562 (2004). Copyright 2004 AIP Publishing LLC.

polarity from $\mathrm{Zn}$ back to $\mathrm{O}$ by inserting a $3-4 \mathrm{~nm}$ thick $\mathrm{MgO}$ layer at some point during the $\mathrm{Zn}$-polar $\mathrm{ZnO}$ growth, as confirmed by CAICISS (see Fig. 56(a)). It is assumed that, under $\mathrm{O}$ ambient, the $\mathrm{MgO}$ layer (rock salt structure) is $\mathrm{O}$ terminated, yielding the formation of O-polar $\mathrm{ZnO}$ layers on top. This relates to the first attempts of the same group to improve the quality of the $\mathrm{ZnO}$ layers grown by $\mathrm{MBE}$ on sapphire by resorting to $\mathrm{MgO}$ buffers directly grown onto sapphire surfaces, although no mention was made at that time of the layer polarity. ${ }^{48}$

The $\mathrm{MgO}$ buffer approach was followed later on in Ref. 489: a precise RHEED analysis of the initial $\mathrm{MgO}$ growth on top of sapphire was undertaken in the MBE. This revealed that $\mathrm{MgO}$ growth follows a Stranski-Krastanov mode: it starts as a 2D-wetting layer, which retains the wurtzite structure, and, as growth proceeds the stress in the layer is relaxed via the formation of 3D islands. Stress relaxation is accompanied by the formation of the equilibrium rocksalt structure. The growth mode transition from 2D to 3D occurs for deposited thicknesses above about $1 \mathrm{~nm}$. As for $\mathrm{ZnO}$ on top of $\mathrm{MgO}$, it was proposed that O-polarity occurs naturally on the thin O-polar wurtzite $\mathrm{MgO}$ layer, while $\mathrm{Zn}$ polarity is obtained when growth occurs on the rocksalt O-terminated $\mathrm{MgO}$ surface (Fig. 56(b)). This was confirmed in Ref. 490, whereby the $\mathrm{MgO}$ transition from wurtzite to rocksalt with thickness was evidenced through HRTEM images of the $\mathrm{ZnO} / \mathrm{MgO} / \mathrm{Al}_{2} \mathrm{O}_{3}$ stack, and the polarity of $\mathrm{ZnO}$ was verified by CBED. Interestingly, so far, the results obtained in these publications concerned rather low temperature deposition temperatures for $\mathrm{MgO}$. At higher $\mathrm{MgO}$ growth temperatures, however (of the order of $700^{\circ} \mathrm{C}$ by PAMBE), it was found out that a spinel $\mathrm{MgAl}_{2} \mathrm{O}_{4}$ layer was formed at the $\mathrm{MgO}$ / $\mathrm{Al}_{2} \mathrm{O}_{3}$ interface because of $\mathrm{Al}^{3+} / \mathrm{Mg}^{2+}$ counter diffusion. ${ }^{491}$ In Ref. 492, direct growth on $\mathrm{MgAl}_{2} \mathrm{O}_{4}$ was found to lead to the classical two rotational domain growth, while $\mathrm{Mg}$ stabilized surfaces led to $30^{\circ}$ twisted growth only of O-polarity. Generally, and contrary to the smooth O-polar surfaces, the obtained Zn-polar epilayers grown by MBE happened to be very rough and with higher dislocation densities. ${ }^{489}$ In Ref. 493, a stack of alternating $\mathrm{ZnO} / \mathrm{MgO}$ low temperature layers $\left(500^{\circ} \mathrm{C}\right.$ compared with $700^{\circ} \mathrm{C}$ for usual
$\mathrm{ZnO}$ ) was shown to yield a $\mathrm{Zn}$-polar material of better structural properties.

3.3. $\mathrm{CrN}$. While searching for $\mathrm{Zn}$-polar $\mathrm{ZnO}$ epilayers with optimum structural properties, $\mathrm{Cr}$ compounds were also proposed as possible "polarity inverters" for $\mathrm{ZnO}$ on sapphire substrates. ${ }^{494,495}$ The $\mathrm{ZnO}$ layers were grown by PAMBE. $\mathrm{CrN}$ was grown by MBE using a $\mathrm{Cr}$ - solid source and $\mathrm{NH}_{3}$ as a nitrogen source under $\mathrm{N}$-rich conditions. The $\mathrm{CrN}$ layer grows along the 111 direction of the rocksalt structure. If the $\mathrm{CrN}$ layer is pre-exposed to a $\mathrm{Zn}$ flux to prevent it from being oxidized, $\mathrm{Zn}$-polar $\mathrm{ZnO}$ epilayers are obtained. In contrast, under O-plasma in the MBE chamber, the $\mathrm{CrN}$ buffer is oxidized into single crystalline $\mathrm{Cr}_{2} \mathrm{O}_{3}$, leading to O-polar $\mathrm{ZnO}$ epilayers. The polarity was determined through the very different $\mathrm{HCl}$ chemical etching rates associated with the two different $\mathrm{Zn}$-and O-polar $\mathrm{ZnO}$ surfaces (see Section III A), as well as by considering the very different growth rates associated with the two different polarities in O-rich MBE conditions. A model was proposed as depicted in Fig. 57. On the one hand, $\mathrm{CrN}$ has the rocksalt structure: under $\mathrm{N}$-rich

(a)

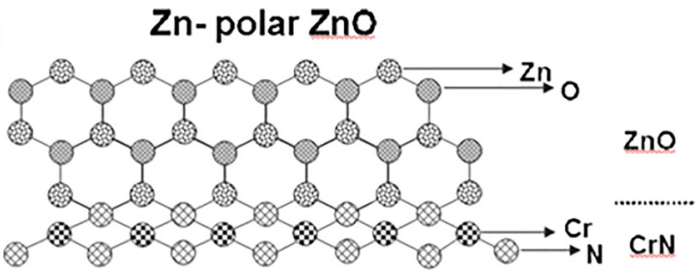

(b)

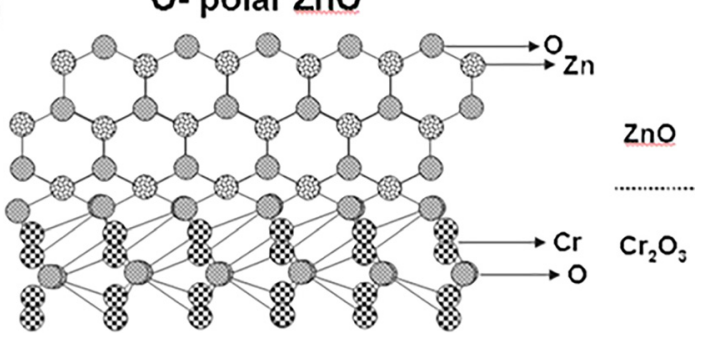

FIG. 57. Atomic stacking sequence (a) at the $\mathrm{CrN} / \mathrm{ZnO}$ interface and (b) at the $\mathrm{Cr}_{2} \mathrm{O}_{3} / \mathrm{ZnO}$ interface yielding opposite polarities. Reprinted with permission from Park et al., J. Electron. Mater. 37, 736 (2008). Copyright 2008 Springer. 
conditions, the $\mathrm{CrN}$ surface is supposedly N-terminated; because of the $\mathrm{Zn}$ pre-treatment, $\mathrm{Zn}-\mathrm{N}$ bonds are formed, with each $\mathrm{Zn}$ atom bonded to three $\mathrm{N}$ atoms, hence creating the $\mathrm{Zn}$ polarity. On rhombohedral $\mathrm{Cr}_{2} \mathrm{O}_{3}$, on the other hand each $\mathrm{O}$ atom has one dangling bond, hence creating the $\mathrm{O}$ polarity. In order to try and improve the structural and optical properties of $\mathrm{Zn}$-polar $\mathrm{ZnO}$ layers, which lag behind those of O-polar $\mathrm{ZnO}$ ones, Park et al ${ }^{494}$ proposed thermal annealing treatments of the $\mathrm{CrN}$ layer at $1000^{\circ} \mathrm{C}$ and under N-plasma.

Interestingly, the Cr-based method was later on extended to PAMBE-grown $\mathrm{GaN}$ on sapphire under Ga-rich conditions: ${ }^{496}$ in this case, pure N-polarity GaN layers were obtained, instead of the more common Ga-polar one (see Section IV A 3 c).

The Cr-based polarity inversion method was further applied to the realization of periodically polarity inversed (PPI) domains for application in non-linear optics (i.e., for second harmonic generation). ${ }^{497,498} \mathrm{The} \mathrm{CrN}$ layer is deposited by PAMBE using a plasma source for nitrogen. As discussed above, direct PAMBE of $\mathrm{ZnO}$ on $\mathrm{Zn}$-protected $\mathrm{CrN}$ leads to $\mathrm{Zn}$-polar $\mathrm{ZnO}$ materials. Using laser holographic lithography, the $\mathrm{ZnO}$ layer was etched in bands down to the $\mathrm{ZnO} / \mathrm{CrN}$ interface with a pattern periodicity down to $0.5 \mu \mathrm{m}$. O plasma treatments oxidize the exposed $\mathrm{CrN}$ in the trenches forming $\mathrm{Cr}_{2} \mathrm{O}_{3}$ at their base. $\mathrm{ZnO}$ regrowth by PAMBE on this patterned surface leads to $\mathrm{Zn}$-polar $\mathrm{ZnO}$ bands over the $\mathrm{CrN}$ bands and to O-polar $\mathrm{ZnO}$ bands in between (Fig. 58).

It could also be demonstrated that if NWs are to be grown on this PPI sample, the NWs would grow only on the $\mathrm{Zn}$-polar $\mathrm{ZnO}$ bands and not on the O-polar $\mathrm{ZnO}$ ones. ${ }^{499}$ These and other specific features devoted to NW are thoroughly addressed in Section IV D.

\section{GaN nanowires and nanorods}

\section{MOCVD (MOVPE)}

The growth of GaN NWs and nanorods (i.e., NWs with diameters in the order of $1 \mu \mathrm{m}$ or more) by MOCVD, i.e., employing metal-organic chemical precursors, ${ }^{831}$ was initiated in the early 2000s using a metal-catalyzed growth approach ${ }^{500,501}$ Interestingly, while some of the early reports dealt with GaN NWs grown along the polar $c$-direction, ${ }^{500,502}$ the first polarity studies were carried out on GaN NWs grown along non-polar directions (see Fig. 59). These NWs displayed triangular cross-sections. The cross-sections were defined by one polar plane and two semi-polar ones, the exact planes depending on the $[10 \overline{1} 0]$ or $[11 \overline{2} 0]$ growth direction. As illustrated in Fig. 59, CBED measurements performed on a triangular NW grown along the [1120] direction indicated that the $c$-plane was $\mathrm{N}$ polar. ${ }^{503}$ In the following years, a number of articles reported device fabrication (i.e., lasers, transistors, and photodetectors) based on this kind of NWs, being probably one of the reasons why the study of polarity was put aside.

Indeed, it was not until the 2010s and the advent of $\langle 0001\rangle$-oriented NWs and nanorods ${ }^{504-508}$ that polarity came again into the center of the scene (see Table I). Six years later, it can be stated that polarity control has been achieved, being now able to grow N-polar or Ga-polar GaN wires on demand, and that the origin of the $\mathrm{GaN}$ wires polarity has clearly been established, in particular, the origin of mixed polarity wires, as confirmed by several groups. However, as compared with GaN NWs by MBE (see Section IVC2), where numerous different substrates were tested, MOVPE studies mainly focused on wires grown either on bare sapphire or on GaN layers (of both polarities). Thus, the polarity of wires on other common substrates, such as $\mathrm{Si}$ (or $\mathrm{AlN} / \mathrm{Si}$ ), diamond, or metals, still needs to be addressed.

To rationalize the ensemble of results, the discussion is organized first in terms of substrate nature and second in terms of presence (or absence) of dielectric mask, which leads to the selective area growth (SAG) of the wires.

\section{a. Sapphire.}

1.1. Self-assembled approach. As already stated in Section IV A 1, the non-polar nature of sapphire suggests that a priori both polarities can be equally expected when growing GaN on sapphire. As discussed in more detail in Section IV A 3 a, early studies ${ }^{509,510}$ on GaN layers showed that it was possible to select the polarity of the final GaN layer by adjusting the growth conditions during the early
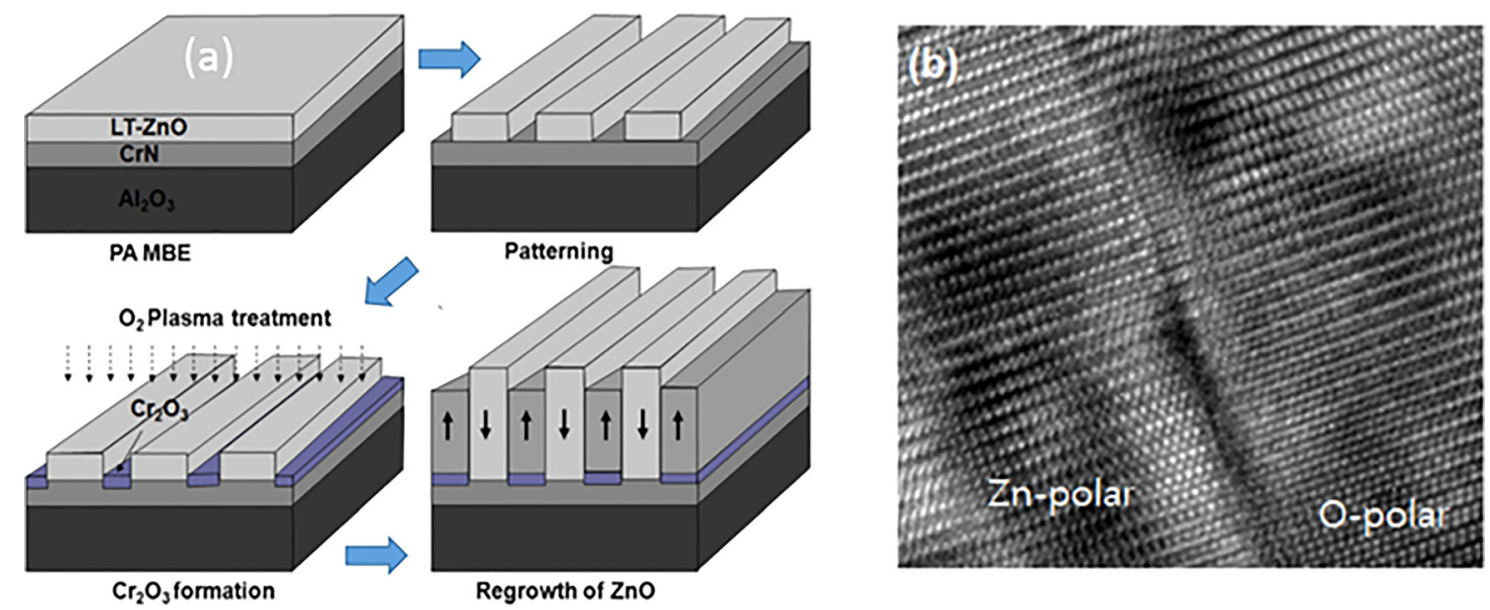

FIG. 58. (a) Principle of the PPI method; (b) IDB HRTEM image. Reprinted with permission from J. Park and T. Yao, Mater. Res. Bull. 47, 2875 (2012). Copyright 2012 Elsevier. 
(a)

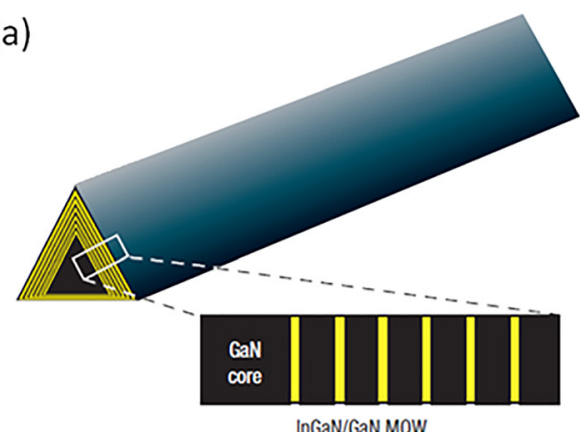

(d)

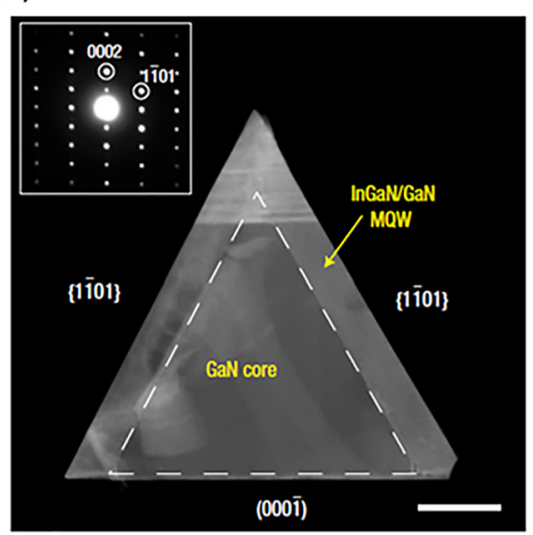

(b)

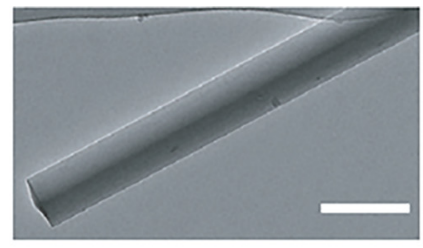

(c)

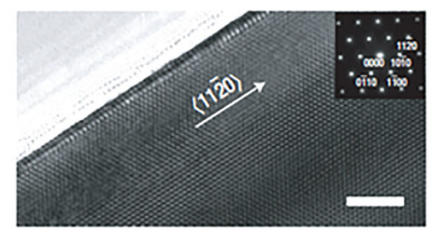

(e)

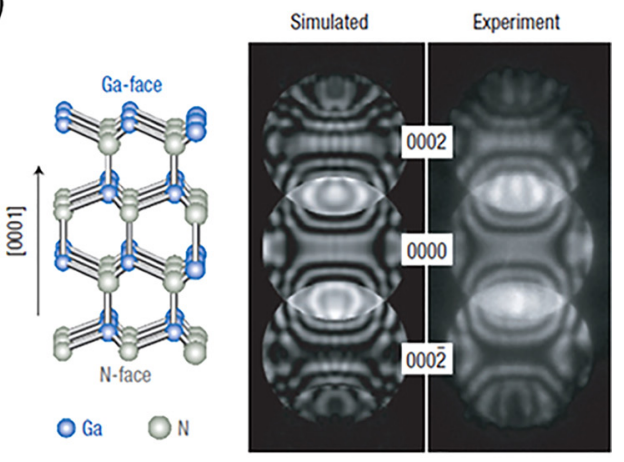

FIG. 59. (a) Schematic diagram of an MQW nanowires and magnified cross-sectional view of a nanowires facets highlighting (In,Ga)N/GaN MQW structure. The (In,Ga)N layer is indicated in yellow w colour. (b) Low-resolution TEM image of an MQW nanowires structure. The scale bar is $500 \mathrm{~nm}$. (c) Highresolution TEM image of an MQW nanowires taken along the [0001] zone axis. The white arrow indicates the $11 \overline{2} 0$ direction (along the wire axis). The scale bar is $5 \mathrm{~nm}$. Inset: Corresponding electronic diffraction pattern indexed for the [0001] zone axis. (d) Dark-field cross-sectional STEM image recorded along the

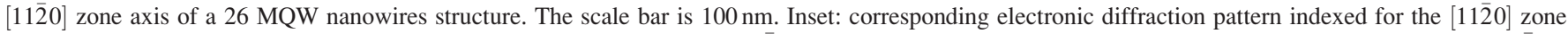
axis. (e) Left: Schematic GaN wurtzite crystal structure viewed along the [11 $\overline{2} 0]$ direction. Right: simulated and experimental CBED patterns along the [1100] zone axis from a $216 \mathrm{~nm}$ thick MQW nanowires cross-sectional sample. Reprinted with permission from Qian et al., Nat. Mater. 7, 701 (2008). Copyright 2008 Nature Publishing.

TABLE I. Polarity of GaN wires grown by MOVPE on different types of substrate. For each report, the table lists the surface treatment prior to the beginning of the growth, the methods used to determine the polarity, the growth approach, the growth mode, and the year when the results were published.

\begin{tabular}{|c|c|c|c|c|c|c|c|}
\hline Substrate & Surface treatment & Polarity & IDBs & Characterization method & Growth approach & Growth mode & Publication year/Reference \\
\hline $\begin{array}{l}\mathrm{GaN}(0001) / \mathrm{Al}_{2} \mathrm{O}_{3}(0001) \\
\& \mathrm{Si}(111) \& 6 \mathrm{H}-\mathrm{SiC}(0001)\end{array}$ & $\ldots$ & Ga-polar & $\ldots$ & Morphology & $S A G$ & Pulsed & $2006 / 516$ \\
\hline $\mathrm{Al}_{2} \mathrm{O}_{3}(0001)$ & Nitridation & N-polar & $\ldots$ & $\mathrm{CBED} / \mathrm{KOH}$ & SAG & Continuous & $2010 / 504$ \\
\hline $\mathrm{Al}_{2} \mathrm{O}_{3}(0001)$ & Nitridation & $\mathrm{N}$-polar & Yes & $\mathrm{KOH}$ & SAG & Continuous & $2011 / 505$ \\
\hline $\mathrm{Al}_{2} \mathrm{O}_{3}(0001)$ & Nitridation & Mixed & Yes & CBED (attached) & Self-assembled & Continuous & $2010 / 506$ \\
\hline $\mathrm{Al}_{2} \mathrm{O}_{3}(0001)$ & Nitridation & Mixed & Yes & CBED (attached) & SAG & Continuous & $2010 / 506$ \\
\hline $\mathrm{GaN}(000 \overline{1})$ & $\ldots$ & Mixed & Yes & CBED (attached) & Self-assembled & Continuous & $2010 / 506$ \\
\hline $\mathrm{Al}_{2} \mathrm{O}_{3}(0001)$ & Nitridation & Mixed & Yes & $\mathrm{KOH}$ & Self-assembled & Continuous & $2011 / 507$ \\
\hline $\mathrm{Al}_{2} \mathrm{O}_{3}(0001)$ & Nitridation & $\mathrm{N}$-polar & Yes & $\mathrm{KOH}$ & SAG & Continuous & $2011 / 508$ \\
\hline $\mathrm{Al}_{2} \mathrm{O}_{3}(0001)$ & No surface treatment & Ga-polar & $\ldots$ & Morphology & Self-assembled & Continuous & $2011 / 514$ \\
\hline $\mathrm{GaN}(0001) / \mathrm{Al}_{2} \mathrm{O}_{3}(0001)$ & $\ldots$ & Ga-polar & $\ldots$ & Morphology & SAG & Continuous & $\begin{array}{c}2011 / 514 \\
\text { (Supporting Information) }\end{array}$ \\
\hline $\mathrm{Al}_{2} \mathrm{O}_{3}(0001)$ & Nitridation & N-polar & Yes & $\mathrm{KOH}$ & SAG & Continuous & $2012 / 515$ \\
\hline $\mathrm{GaN}(0001) / \mathrm{Al}_{2} \mathrm{O}_{3}(0001)$ & $\ldots$ & Ga-polar & No & $\mathrm{KOH}$ & SAG & Continuous & $2012 / 517$ \\
\hline $\mathrm{GaN}(0001) / \mathrm{Al}_{2} \mathrm{O}_{3}(0001)$ & $\ldots$ & Ga-polar & No & $\mathrm{KOH}+\mathrm{CBED}($ detached $)$ & SAG & Continuous & $2013 / 518$ \\
\hline $\mathrm{Al}_{2} \mathrm{O}_{3}(0001)$ & Nitridation & Mixed & Yes & CBED (attached) & Self-assembled & Continuous & $2013 / 511$ \\
\hline $\mathrm{Al}_{2} \mathrm{O}_{3}(0001)$ & Nitridation & Mixed & Yes & $\mathrm{KOH}+\mathrm{CBED}($ attached $)$ & Self-assembled & Continuous & $2014 / 512$ \\
\hline $\mathrm{GaN}(0001) / \mathrm{Al}_{2} \mathrm{O}_{3}(0001)$ & $\ldots$ & Ga-polar & No & CBED (attached) & SAG & Pulsed & $2014 / 520$ \\
\hline $\mathrm{Al}_{2} \mathrm{O}_{3}(0001)$ & Nitridation & Mixed & Yes & Coherent Bragg diffraction & Self-assembled & Continuous & $2014 / 513$ \\
\hline $\mathrm{GaN}(0001) / \mathrm{Si}(111)$ & AlN buffer & Ga-polar & $\ldots$ & Morphology & SAG & Continuous & $2015 / 526$ \\
\hline $\mathrm{GaN}(0001) / \mathrm{Al}_{2} \mathrm{O}_{3}(0001)$ & $\ldots$ & Ga-polar & No & CBED (attached) & SAG & Continuous & $2016 / 519$ \\
\hline
\end{tabular}


growth stages and, in particular, by adapting the surface treatment of the substrates before the GaN growth. Indeed, if no nitridation was performed, Ga-polar GaN layers were obtained, while the commonly employed nitridation step favored the growth of either one polarity or the other, or a mixture of them, depending on the exact growth conditions (i.e., growth temperature, V/III ratio, as well as annealing temperature and time).

As exemplified by Fig. 60, all the works on selfassembled GaN NWs and nanorods grown on nitridatedsapphire substrates describe wires exhibiting systematically both polarities. ${ }^{506,507,511-513}$ It is worth noting that, in order to enhance the vertical growth rate, $\mathrm{SiH}_{4}$ was used during the growth in all these reports, especially at the beginning of the growth. The IDBs nucleate at the sapphire/GaN interface,
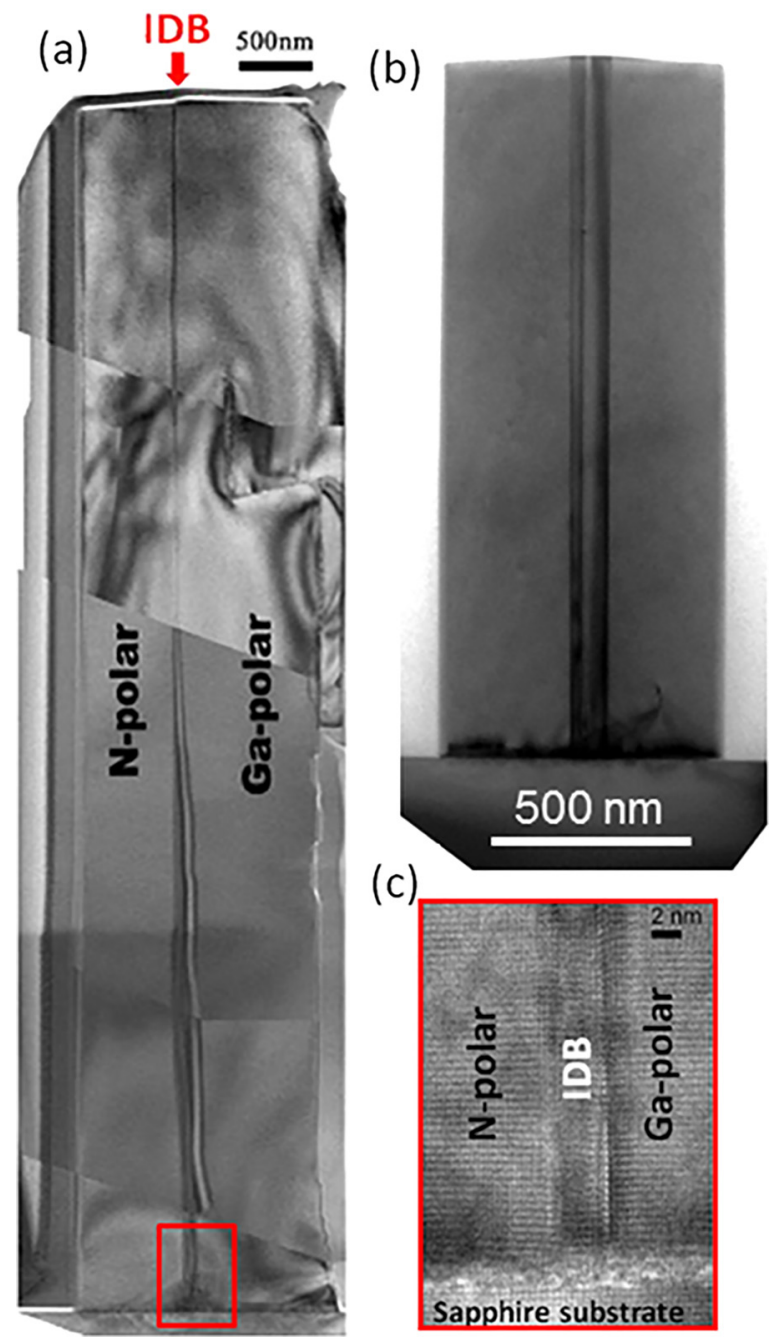

FIG. 60. (a) Multibeam TEM image of a wire recorded along [11 20$]$ zone axis displaying an IDB running along the whole wire. Reprinted with permission from J. Appl. Phys. 115, 153504 (2014). Copyright 2014 AIP Publishing LLC. (b) Cross-section TEM bright-field image of a GaN wire showing the presence of dislocations in the bottom part and vertical IDB running across the whole wire. Reprinted with permission from J. Appl. Phys. 114, 144304 (2013). Copyright 2013 AIP Publishing LLC. (c) TEM image of the inversion domain at the sapphire/GaN interface highlighted in Figure (a) observed along the $[11 \overline{2} 0]$ zone axis. In both cases, the sapphire substrate was nitridated prior to the growth and the polarity of each region was confirmed by CBED measurements. Reprinted with permission from J. Appl. Phys. 115, 153504 (2014). Copyright 2014 AIP Publishing LLC. as shown in Figs. 60(c) and 61(a), and run across the whole wire length, becoming stabilized on $\{10 \overline{1} 0\}$-type planes. ${ }^{511,512}$ The only significant difference between different reports was the exact IDB configuration: in some cases $\mathrm{N}$ - and Ga-polar GaN regions extended from the wire perimeter to the center, ${ }^{507,512,513}$ while in other cases they could form a core-shell structure, with the Ga-polar $\mathrm{GaN}$ region surrounded by the N-polar GaN one. ${ }^{511}$ Although the presence of the two polarities can be understood in terms of surface preparation and growth conditions, the reason why both polarities exhibit the same vertical growth rate is still an open issue, especially taking into account that, when grown as thin films, the Ga-polar GaN regions typically grow faster than the N-polar ones. ${ }^{512}$
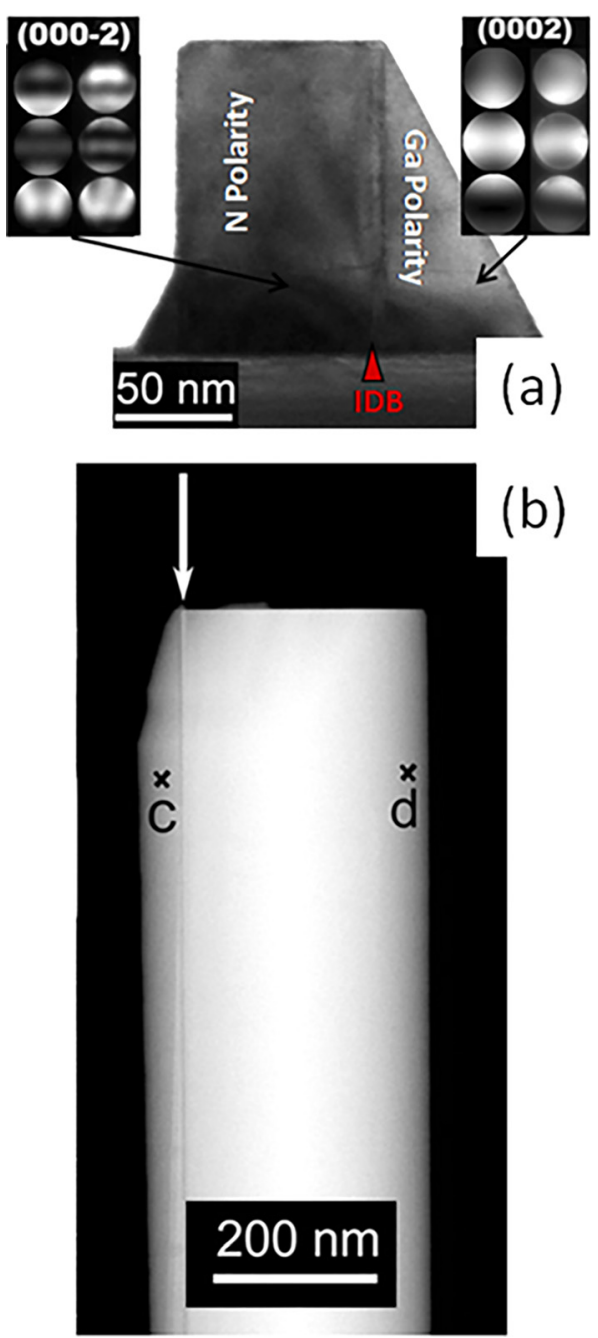

FIG. 61. (a) TEM image acquired along the $[11 \overline{2} 0]$ zone axis of an island nucleated on a nitridated sapphire surface. Inset: CBED patterns obtained along the $[10 \overline{1} 0]$ zone axis in the right part of the island limited by a semipolar plane (experimental pattern, right, and simulated pattern for a TEM sample thickness of $20 \mathrm{~nm}$, left), and in the left part of the island limited by a polar plane (experimental pattern, right, and simulated pattern for a TEM sample thickness of $40 \mathrm{~nm}$, left). Reprinted with permission from J. Appl. Phys. 115, 153504 (2014). Copyright 2014 AIP Publishing LLC. (b) STEM image of a single wire grown on a nitridated sapphire substrate. The polarity in point $\mathrm{c}$ is Ga-polar and in $\mathrm{d}$ N-polar, as verified by CBED. Reprinted with permission from Appl. Phys. Lett. 97, 151909 (2010). Copyright 2010 AIP Publishing LLC. 
In these wires, it was observed that whenever an inclined facet appeared (i.e., a semi-polar facet), the region underneath was systematically Ga-polar, ${ }^{506,511,512}$ as shown in Fig. 61 for a nucleation island and for the top region of a GaN NW grown on a nitridated-sapphire substrate. Based on these observations, in the following years numerous authors employed the pyramidal morphology of the wires top as an indication of their Ga-polar nature (see Table I). It should be noted that, while no counterexample is found in the literature (i.e., whenever the polarity was verified, pyramidal tops imply Ga-polar nature), the opposite statement is not true: this means that a flat wire top does not imply necessarily $\mathrm{N}$-polar region underneath, as Ga-polar regions might exhibit such a flat top (see, for example, Figs. 60(a) and 60(b)). Attempts to explain the observed morphologies are based on a surface passivation effect and, thereby, stabilization of certain crystallographic facets by hydrogen, in particular, the N-polar and (1011) semi-polar planes. ${ }^{508}$ For a deeper discussion on hydrogeninduced surface stabilization of $\mathrm{GaN}$ crystallographic facets, see Sections II E-II G.

Most important, if the sapphire surface is not nitridated prior to the growth start, Ga-polar GaN wires can be grown on sapphire under low-precursor flows. ${ }^{514}$ Indeed, the presence of inclined facets from the very beginning of the growth as well as on the wire top (Fig. 62) showed that this was possible. Interestingly, the growths were performed using pure $\mathrm{N}_{2}$ as carrier gas. Thus, if we assume that the morphology of the Ga-polar wires is governed by the stabilization of $\{10 \overline{1} 1\}$ semi-polar planes by hydrogen, then it would seem that the decomposition of ammonia is sufficient to provide the necessary hydrogen or $\mathrm{NH}_{x}(x=1,2)$ molecules (Section II G).

Thus, it can be concluded that the polarity of selfassembled wires on sapphire depends basically on the nitridation (or its absence) of the sapphire substrate, and this irrespective of the NW or nanorod size.

1.2. Selective area growth. In principle, one might expect that the situation is similar when a mask is deposited on the sapphire substrate and openings therein are fabricated, as
GaN still nucleates on the sapphire surface. However, it turned out that the mask quality (i.e., roughness of the mask, quality of the mask opening, and degradation of the sapphire substrate in the opening.) was a very crucial aspect that might modify the wire polarity, as dictated by the nitridation step alone.

In many publications, ${ }^{504,505,515}$ the polarity of this kind of GaN NWs and nanorods was analyzed by $\mathrm{KOH}$ etching (see Section IIIA). As presented in Figs. 63(b) and 63(c), $\mathrm{KOH}$ etches away the wires partially, which indicates that both N-polar and Ga-polar GaN regions coexisted within the wires. Based on these etching features, it was generally concluded that the upper part of the wires is N-polar and that only the wire regions on the opening rim are Ga-polar, as suggested, for example, by Fig. 63(c). While this might be exact, it implicitly assumed that Ga-polar GaN regions could not be etched laterally, i.e., across the IDB, by the $\mathrm{KOH}$ once the neighboring N-polar GaN region had been etched away.

Furthermore, when CBED (see Section III B 1) was used to analyze the polarity of these SAG wires, as shown in Figs. 63(d) and 63(e), vertical IDBs are seen to extend from the bottom of the wire, in contact with the mask, up to the wire top. And again, the semi-polar top planes indicate Ga-polar $\mathrm{GaN}$ regions, while the flat top ones correspond to N-polar GaN regions. ${ }^{506}$ Thus, although wires as those shown in Fig. 63(a) can be etched down to the mask/substrate by $\mathrm{KOH}$, it is difficult to conclude on the exact configuration of the polarity domains inside the wires when the polarity analysis is only based on sensitive chemical etching experiments.

If the discussion on the extension of the Ga-polar $\mathrm{GaN}$ domains is set aside, there is a consensus on the fact that IDBs can be easily generated close to the mask border, whatever the nature of the employed mask $\left(\mathrm{SiO}_{2}\right.$ or $\left.\mathrm{SiN}\right)$, and that the core is systematically of N-polarity while the Ga-polar GaN domains grow around the central N-polar GaN core.

b. Ga-polar GaN templates (on sapphire): Selective area growth. It is worth noting that under continuous flow conditions, self-assembled wires were achieved on N-polar GaN,
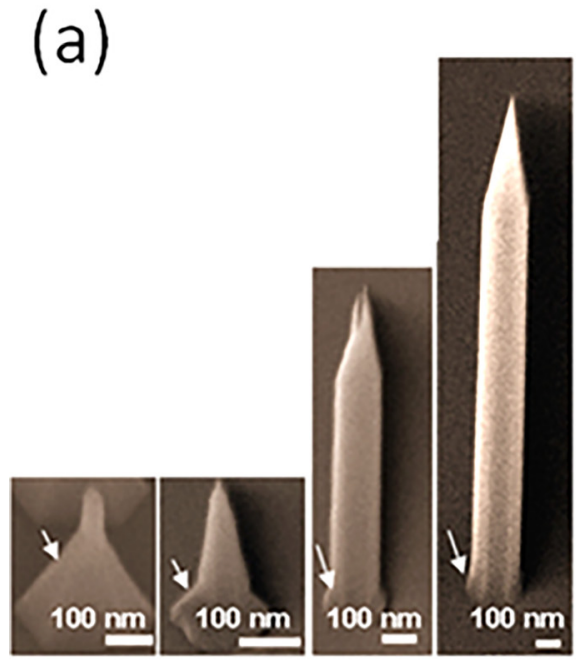

Time

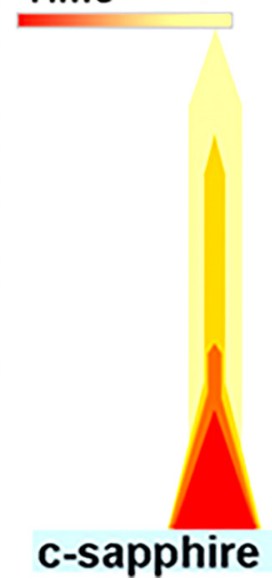

(b)

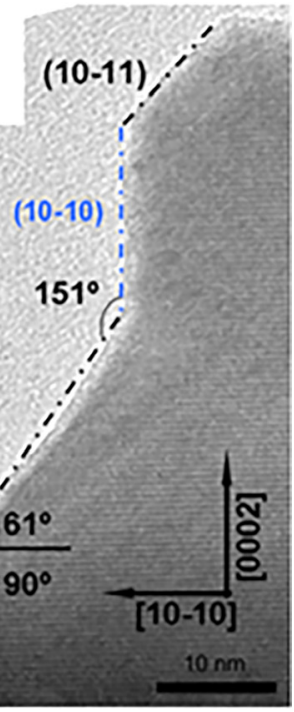

FIG. 62. (a) Shape evolution of $\mathrm{GaN}$ wires grown on a non-nitridated sapphire surface, under low precursor flows, as a function of time and the associated schematical representation. (b) High-resolution TEM of GaN wires observed along the $[11 \overline{2} 0]$ zone axis, showing the inclined planes constituting the wire top. Reprinted with permission from Appl. Phys. Lett. 99, 251910 (2011). Copyright 2011 AIP Publishing LLC. 

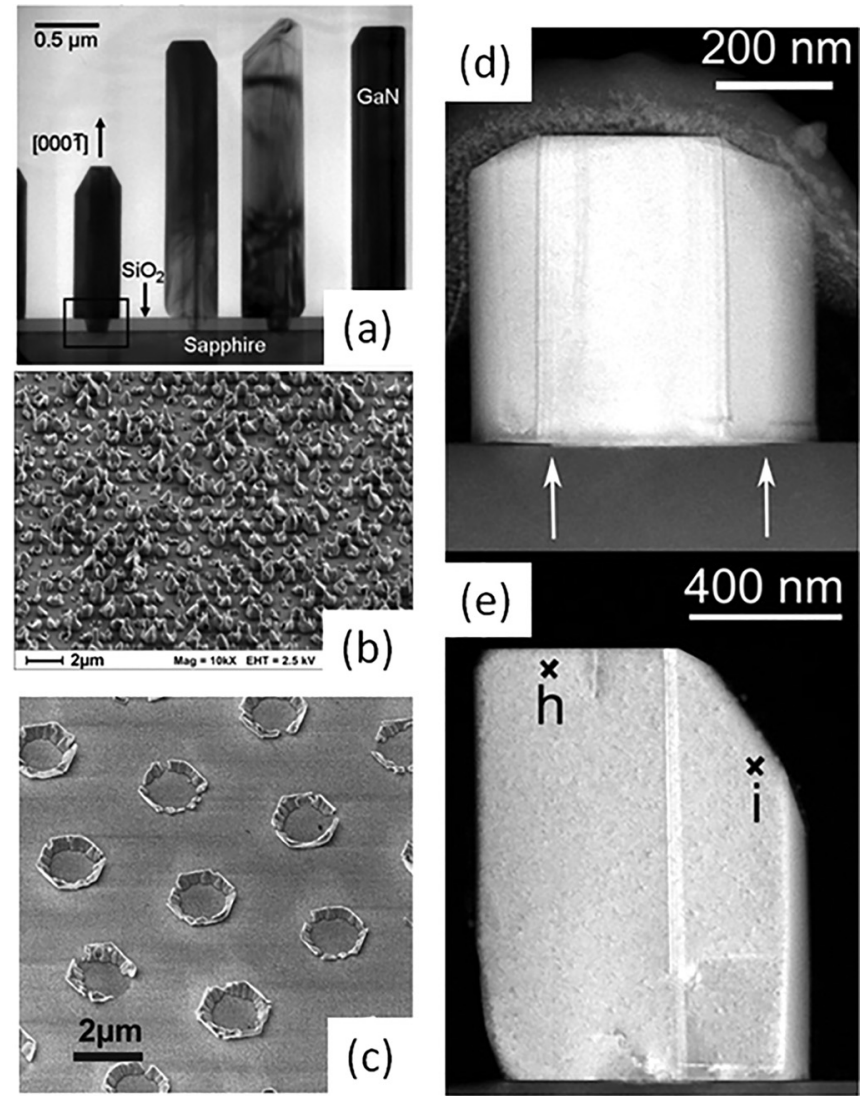

FIG. 63. (a) TEM image showing the appearance of different side facets in SAG GaN wires grown on nitridated sapphire. Reprinted with permission from Bergbauer et al., Nanotechnology 21, 305201 (2010). Copyright 2010 Institute of Physics Publishing. (b) SEM image of a GaN wire array grown on nitridated sapphire after $\mathrm{KOH}$ etching. Reprinted with permission from Bergbauer et al., J. Cryst. Growth 315, 164 (2011). Copyright 2011 Elsevier. (c) SEM image of a GaN wire array grown on nitridated sapphire after hot $\mathrm{KOH}$ etching. Reprinted with permission from Wang et al., Cryst. Growth Des. 12, 2552 (2012). Copyright 2012 American Chemical Society. (d) and (e) STEM images of SAG GaN seeds on nitridated sapphire. In (d), the white arrows indicate the border of the mask opening, while the dark vertical lines correspond to IDB. In (e), the polarity of regions $h$ and $i$ is N-polar and Ga-polar, respectively, as verified by CBED. Reprinted with permission from Appl. Phys. Lett. 97, 151909 (2010). Copyright 2010 AIP Publishing LLC.

besides sapphire (see Section IV C1a), but not on Ga-polar GaN templates, on which pyramids formed and prevented any vertical growth. ${ }^{506}$ This observation, combined with the results on sapphire described above and the pioneering results by Hersee et al. on pulsed GaN wires growth on Gapolar GaN templates, ${ }^{516}$ pointed towards the need for special growth conditions in order to obtain Ga-polar GaN NWs by continuous flow MOCVD. These special conditions were: small V/III ratios, typically two orders of magnitude smaller than those usually used for GaN thin films, and absolute precursors flows two orders of magnitude smaller than those typically used for both thin films and self-assembled wires. $^{514,517-519}$ These conditions are mimicked naturally when using pulsed-growth. ${ }^{516,520}$

In general, the SAG of GaN NWs on Ga-polar GaN templates enabled the achievement of smaller diameters, comparable to those obtained by $\mathrm{MBE}$ (i.e., $50 \mathrm{~nm}$ in diameter), ${ }^{517}$ and rendered thereby polarity studies more complex. The advantage is that, since these works were conducted (a)

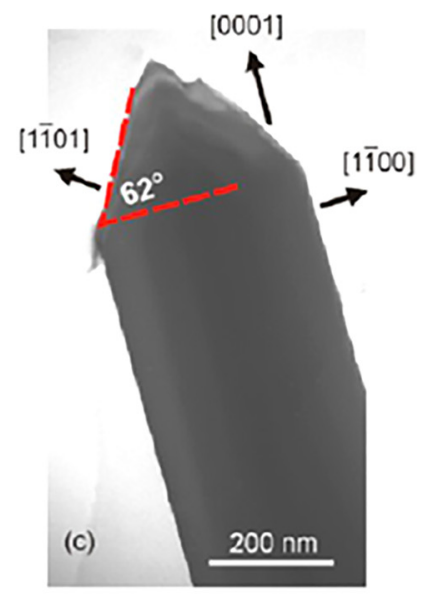

(b)

(c)
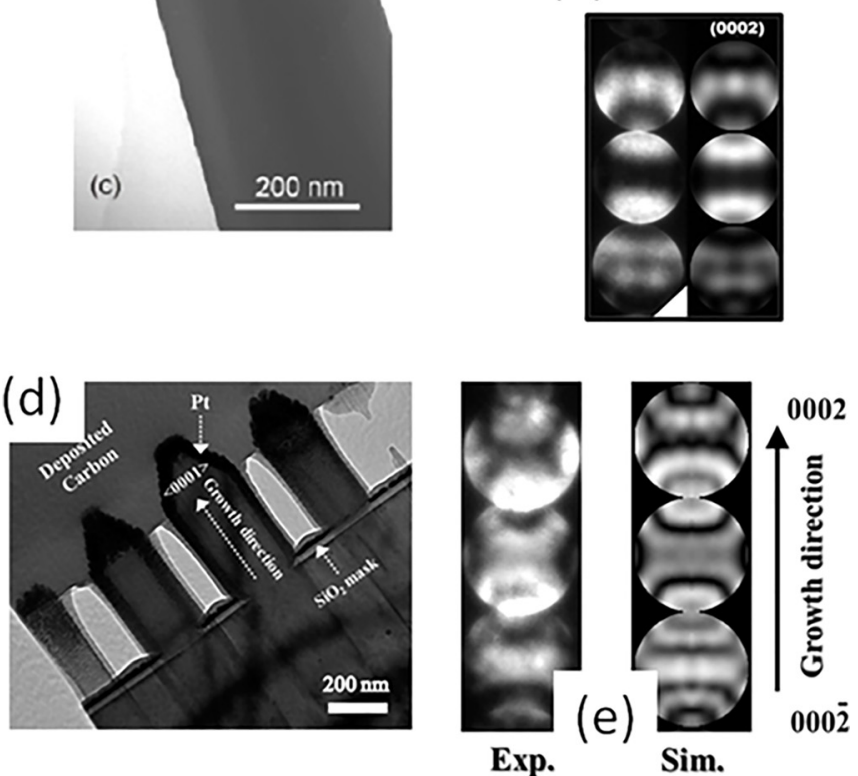

FIG. 64. (a) TEM image showing the top of a Ga-polar NW (confirmed by CBED) grown with a $165 \mathrm{nmol} / \mathrm{min}$ silane flow on a Ga-polar GaN template on sapphire. Reprinted with permission from Wang et al., Cryst. Growth Des. 13, 3475 (2013). Copyright 2013 American Chemical Society. (b) Bright field TEM image recorded close to the $[11 \overline{2} 0]$ zone axis with $g=(000 \overline{2})$ of a GaN NW grown on a $215 \mathrm{~nm}$ mask aperture and displaying no threading dislocations. The star denotes the place where the CBED measurement shown in (c) was performed. (c) CBED pattern recorded along the [1010] zone axis in the GaN NW shown in (b). Experimental pattern (left) and simulated pattern (right), indicating the Ga-polar nature of the nanowire. The sample thickness for the simulation is $60 \mathrm{~nm}$. Reprinted with permission from Coulon et al., Appl. Phys. Express 9, 015502 (2016). Copyright 2016 The Japan Society of Applied Physics. (d) TEM image of a Ga-polar NW array displaying flat tops. (e) CBED pattern obtained along the $[10 \overline{1} 0]$ zone axis in the GaN NWs shown in (d). Experimental pattern (left) and simulated pattern (right) for a sample thickness of $95 \mathrm{~nm}$. Reprinted with permission from Jung et al., CrystEngComm 16, 2273 (2014). Copyright 2014 The Royal Society of Chemistry.

two/three years later than those dealing with SAG on sapphire, the quality of the dielectric masks was neatly improved. This explains probably why all articles report pure Ga-polar NWs, ${ }^{517,519,520}$ as illustrated in Fig. 64. Furthermore, consistent with the results previously discussed on bare sapphire substrates, a pyramidal top implies Gapolarity. ${ }^{518,519}$ But, once again, consistently with the previous results on sapphire, a flat top does not necessarily mean N-polarity, as seen in Fig. 64(d) and studied in Ref. 520.

c. Other substrates: Graphene and Si(111). A large number of articles on GaN NWs or nanorods by MOCVD did not pay attention to polarity, or assumed it without giving any 
further proof. This is especially true for articles pioneering the growth of GaN wires by MOVPE ${ }^{516,521}$ and for numerous reports employing pulsed-growth on Ga-polar GaN templates on sapphire. ${ }^{522-524}$ Whenever reference to polarity was made, Ga-polarity was assumed.

Likewise, a number of other substrates were used, but the polarity of the wires grown thereon was not directly addressed. However, based on the knowledge acquired within the last years, we can speculate on their polarity: for example, the wires grown on a GaN buffered graphene structure, ${ }^{525}$ as shown in Fig. 65(a), display the typical morphology of mixed polarity wires grown on sapphire, with flat N-polar GaN regions perturbed by pyramids that point towards Ga-polar GaN regions below them (compare, for example, Fig. 65(a) and scanning electron micrograph (SEM) and TEM images in Ref. 511). In contrast, the nanorods grown on AlN-coated SiN/Si substrates, ${ }^{526}$ Fig. 65(b), seem to be constituted entirely by pure Ga-polar GaN regions although, as already stated, these are just speculations based on the comparison of the wire morphology.

\section{MBE}

In MBE, GaN NWs (also referred as nanocolumns, nanorods, and nanopillars in the literature) can be grown along the $\langle 0001\rangle$ axis using different approaches. The most common
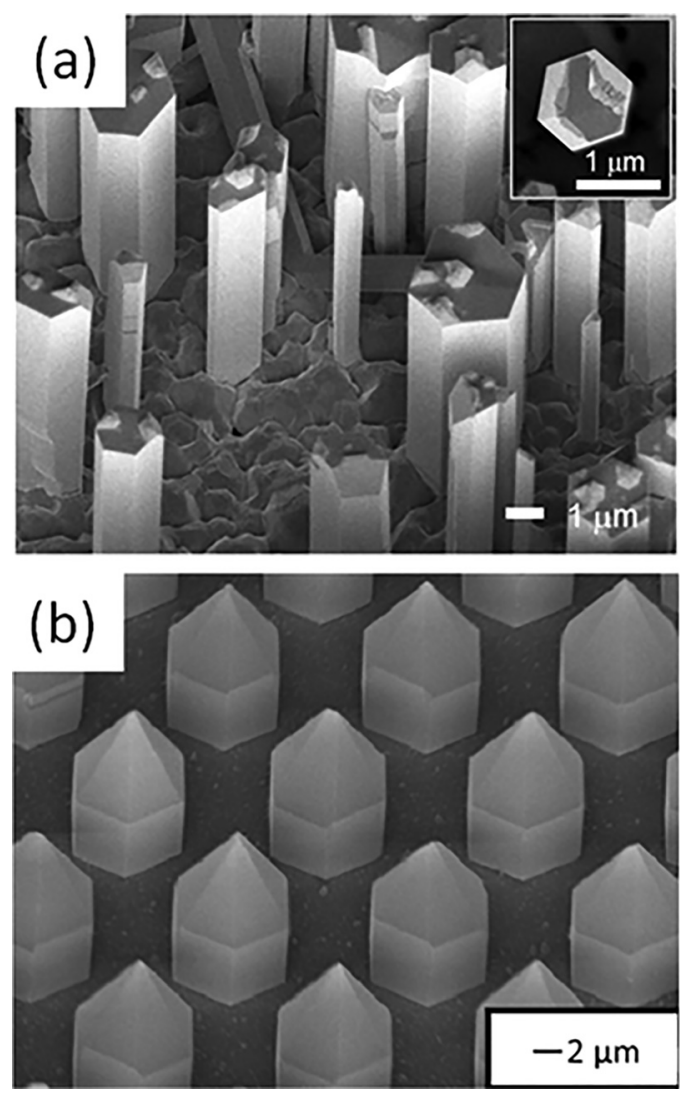

FIG. 65. (a) SEM image of GaN microrods grown on a graphene film with a GaN buffer layer. Reprinted with permission from APL Mater. 2, 092512 (2014). Copyright 2014 AIP Publishing LLC. (b) SEM image of SAG GaN rods grown on AlN-coated $\mathrm{SiN}_{\mathrm{x}} / \mathrm{Si}(111)$ using silane injection. Reprinted with permission from Foltynski et al., J. Cryst. Growth 414, 200 (2015). Copyright 2015 Elsevier. approaches are: self-assembled growth, ${ }^{507,527-536}$ vaporsolid-solid (VSS) growth, ${ }^{529,537-539}$ and SAG. ${ }^{531,540-548}$ As summarized in Table II, the polarity of GaN NWs grown by MBE depends on the specific growth approach, but also on other factors such as the type of substrate. In addition, when analyzing the polarity of NW ensembles, the statistical aspect of the problem must be kept in mind, because ensembles are not always homogenous in terms of NW polarity. In Table II, it can be observed that the results reported in the literature are quite often controversial. A perfect example is the polarity of self-assembled GaN NWs prepared on $\mathrm{Si}(111)$ by PAMBE, where both Ga- and N-polar NWs were reported by different groups.

In the following, an overview is presented on the studies reported so far on the polarity of GaN NWs grown by MBE. Because the results obtained for self-assembled, VSS, and SAG GaN NWs cannot be directly compared, due to the intrinsically different nucleation mechanisms, the corresponding studies are discussed separately.

a. Self-assembled approach. In MBE, GaN NWs can be grown in a self-assembled (also known as self-induced or self-organized) fashion, namely, without using neither metal particles to induce supersaturation nor patterned substrates. However, it is worth noticing that not all self-assembled growth processes are necessarily comparable because NW nucleation can take place spontaneously ${ }^{534}$ or be induced by the presence of morphological and structural defects. ${ }^{549,550}$ Next, we present the results reported so far on the polarity of self-assembled GaN NWs prepared by reactive $\mathrm{MBE}$ and PAMBE.

2.1. Reactive $M B E$. In reactive $\mathrm{MBE}$, where ammonia is used as the N precursor, GaN NWs form at substrate temperatures in the range of $700-840{ }^{\circ} \mathrm{C}$ on different substrates. $^{507,527}$ However, as shown in Fig. 66(a), selfassembled GaN NWs grown by reactive MBE exhibit large diameters (100-500 nm) and small aspect ratios. ${ }^{507}$ Because of this reason, reports on the self-assembled growth of $\mathrm{GaN}$ NWs by reactive MBE are rather scarce.

The polarity of GaN NWs prepared by reactive MBE on bare $c$-sapphire and AlN-buffered $\mathrm{Si}(111)$ substrates was only studied on a microscopic scale by Alloing et al. ${ }^{507}$ using CBED. On $c$-sapphire, the investigated GaN NWs were Npolar, except for the presence of small inversion domains. In contrast, on AlN-buffered $\mathrm{Si}(111)$, the majority of NWs were found to be Ga-polar. Therefore, as in the case of epitaxial GaN films discussed in Sections IV A 3 c and IV A 3 d, the polarity of GaN NWs grown by reactive $\mathrm{MBE}$ seems to depend on the specific type of substrate.

2.2. Plasma-asssisted MBE. In PAMBE, GaN NWs are grown using the active $\mathrm{N}$ produced by a radio frequency $\mathrm{N}_{2}$ plasma source. The self-assembled growth of GaN NWs by PAMBE only requires $\mathrm{N}$-excess and elevated substrate temperatures $\left(750-900^{\circ} \mathrm{C}\right) .^{551,552}$ Under these conditions, GaN NWs with large aspect ratios and small diameters (down to $15 \mathrm{~nm}$ ) form on a wide variety of crystalline as well as amorphous substrates including, for example, $\mathrm{Si}$, diamond, $\mathrm{SiC}, \mathrm{TiN}, \mathrm{Si}_{\mathrm{x}} \mathrm{O}_{\mathrm{y}}$, and sapphire. ${ }^{528-536}$ Figure 66(b) shows, as an illustrative 
TABLE II. Polarity of GaN NWs grown by MBE on different types of substrate. For each report, the table lists the growth technique, the growth approach, the methods used to determine the polarity, and the year when the results were published.

\begin{tabular}{|c|c|c|c|c|c|}
\hline Substrate & Growth technique & Growth approach & Characterization method & Polarity & Publication year/Ref. \\
\hline $\operatorname{AlN} / \operatorname{Si}(111)$ & Reactive-MBE & Self-assembled & CBED & N-polar & $2011 / 507$ \\
\hline $\mathrm{Al}_{2} \mathrm{O}_{3}(0001)$ & Reactive-MBE & Self-assembled & CBED & Ga-polar & $2011 / 507$ \\
\hline $\mathrm{Si}(111)$ & PA-MBE & Self-assembled & CBED & Ga-polar & $2008 / 555$ \\
\hline $\operatorname{Si}(111)$ & PA-MBE & Self-assembled & CBED & Ga-polar & $2010 / 537$ \\
\hline $\mathrm{Si}(111)$ & PA-MBE & Self-assembled & $\mathrm{XRD}, \mathrm{KOH}, \mathrm{CBED}$ & N-polar & $2011 / 177$ \\
\hline $\operatorname{Si}(111)$ & PA-MBE & Self-assembled & CBED, EELS & N-polar & $2011 / 133$ \\
\hline $\operatorname{Si}(111)$ & PA-MBE & Self-assembled & STEM & $\mathrm{N}$-polar & 2012/599 \\
\hline $\operatorname{Si}(111)$ & PA-MBE & Self-assembled & STEM & N-polar & $2012 / 559$ \\
\hline $\operatorname{Si}(111)$ & PA-MBE & Self-assembled & PL & N-polar & $2012 / 562$ \\
\hline $\operatorname{Si}(111)$ & PA-MBE & Self-assembled & STEM, KOH & mixed (90\% N-polar) & $2013 / 558$ \\
\hline $\operatorname{Si}(111)$ & PA-MBE & Self-assembled & PL & N-polar & $2014 / 563$ \\
\hline $\mathrm{Si}(111)$ & PA-MBE & Self-assembled & $\mathrm{ARXPD}, \mathrm{KOH}$ & N-polar & $2015 / 150$ \\
\hline $\operatorname{AlN} / \operatorname{Si}(111)$ & PA-MBE & Self-assembled & CBED & Ga-polar & $2011 / 564$ \\
\hline $\operatorname{AlN} / \operatorname{Si}(111)$ & PA-MBE & Self-assembled & $\mathrm{KOH}$ & N-polar & $2011 / 177$ \\
\hline $\operatorname{AlN} / \operatorname{Si}(111)$ & PA-MBE & Self-assembled & $\mathrm{CBED}, \mathrm{KOH}$ & N-polar & $2012 / 565$ \\
\hline $\operatorname{AlN} / \operatorname{Si}(111)$ & PA-MBE & Self-assembled & STEM, KPFM, KOH & N-polar & $2015 / 337$ \\
\hline $\operatorname{AlN} / \operatorname{Si}(111)$ & PA-MBE & Self-assembled & KPFM, PFM & Mixed (95\% N-polar) & $2015 / 193$ \\
\hline $\mathrm{Si}(001)$ & PA-MBE & Self-assembled & CBED & Mixed & $2014 / 566$ \\
\hline $\mathrm{Al}_{2} \mathrm{O}_{3}(0001)$ & PA-MBE & Self-assembled & CBED & Ga-polar & $2008 / 567$ \\
\hline $\mathrm{Al}_{2} \mathrm{O}_{3}(0001)$ & PA-MBE & Self-assembled & CBED & Mixed & $2008 / 549$ \\
\hline Amorphous $\mathrm{Al}_{2} \mathrm{O}_{3}$ & PA-MBE & Self-assembled & CBED & N-polar & $2014 / 535$ \\
\hline Diamond(111) & PA-MBE & Self-assembled & STEM & N-polar & $2012 / 568$ \\
\hline Mo & PA-MBE & Self-assembled & $\mathrm{KOH}$ & N-polar & $2013 / 547$ \\
\hline $\mathrm{AlN} / \mathrm{SiC}(0001)$ & PA-MBE & Self-assembled & EELS & N-polar & $2012 / 534$ \\
\hline $\mathrm{AlN} / \mathrm{SiC}(000 \overline{1})$ & PA-MBE & Self-assembled & CBED & N-polar & $2012 / 534$ \\
\hline $\mathrm{AlN} / \mathrm{SiC}(000 \overline{1})$ & PA-MBE & Self-assembled & LEED, ARXPD & N-polar & $2015 / 150$ \\
\hline $\operatorname{Si}(111)$ & PA-MBE & VSS & CBED & Ga-polar & $2010 / 537$ \\
\hline $\mathrm{GaN}(0001)$ & PA-MBE & SAG & CBED, $\mathrm{KOH}$ & Ga-polar & $2013 / 547$ \\
\hline GaN/AlN/Si(111) & PA-MBE & SAG & STEM & N-polar & $2016 / 575$ \\
\hline
\end{tabular}

example, a scanning electron micrograph of self-assembled GaN NWs prepared on bare $\mathrm{Si}(111)$.

Despite self-assembled GaN NWs are grown since the late $1990 \mathrm{~s},{ }^{553,554}$ their polarity was not examined until 2008 (see Table II). As shown in Table II, the results are controversial. However, the different reports are not always comparable because the nucleation process, which determines the polarity, depends on the specific type of substrate as well as on the use of buffer layers. Below, we discuss separately the polarity of self-assembled GaN NWs prepared by PAMBE on different types of substrate.

2.2.1. Growth on Si(111). Owing to its hexagonal surface symmetry as well as its availability in large sizes at low prices, $\mathrm{Si}(111)$ is the most common substrate used for the self-assembled growth of GaN NWs. The non-polar nature of the Si substrate allows, in principle, the formation of NWs of either polarity, as already stated in Section IV A 1. The polarity of individual GaN NWs within a dense ensemble grown on bare $\mathrm{Si}(111)$ has been investigated by several groups using TEM-related techniques, such as CBED, EELS, and ABF- as well as HAADF-STEM. ${ }^{537,555-559}$ As discussed in detail below, the results of these studies are not conclusive, but rather controversial.

Furtmayr et al. ${ }^{555}$ investigated the formation of GaN NWs on bare $\mathrm{Si}(111)$ by TEM and assessed the polarity using CBED. As shown in Fig. 67 and in agreement with the results previously reported in Ref. 560, they detected the formation of an amorphous $\mathrm{Si}_{\mathrm{x}} \mathrm{N}_{\mathrm{y}}$ interlayer between the NWs and the underlying Si substrate. CBED measurements indicated that the analyzed GaN NWs were Ga-polar. Chèze et $a l .{ }^{537}$ also investigated by CBED the polarity of similar samples and obtained the same results as Furtmayr et al. ${ }^{555}$ These results are, however, in striking contrast with those reported in Refs. 556-559, where individual GaN NWs grown on bare $\mathrm{Si}(111)$ were found to be N-polar by CBED, EELS, and ABF-STEM. In the work of Kong et al., ${ }^{556}$ the polarity was assessed independently by CBED and EELS. The analysis of CBED patterns indicated that the investigated GaN NWs were N-polar. Fig. 13 shows the results of the independent analysis performed by EELS. The EELS spectra were taken at the $(0002)$ and $(000 \overline{2})$ Bragg conditions. For each spectrum, the N K-edge background was subtracted. Both spectra were normalized to the Ga L-edges. Because, as explained in Section III B 2, the N K-edge peak taken at the (0002) Bragg condition exhibits a higher intensity, the correlation between the EELS spectra with the orientation of the NWs with respect to the substrate surface (provided by the corresponding TEM images) enables the assessment of the NW polarity. EELS measurements confirmed the results obtained by CBED measurements, namely, 

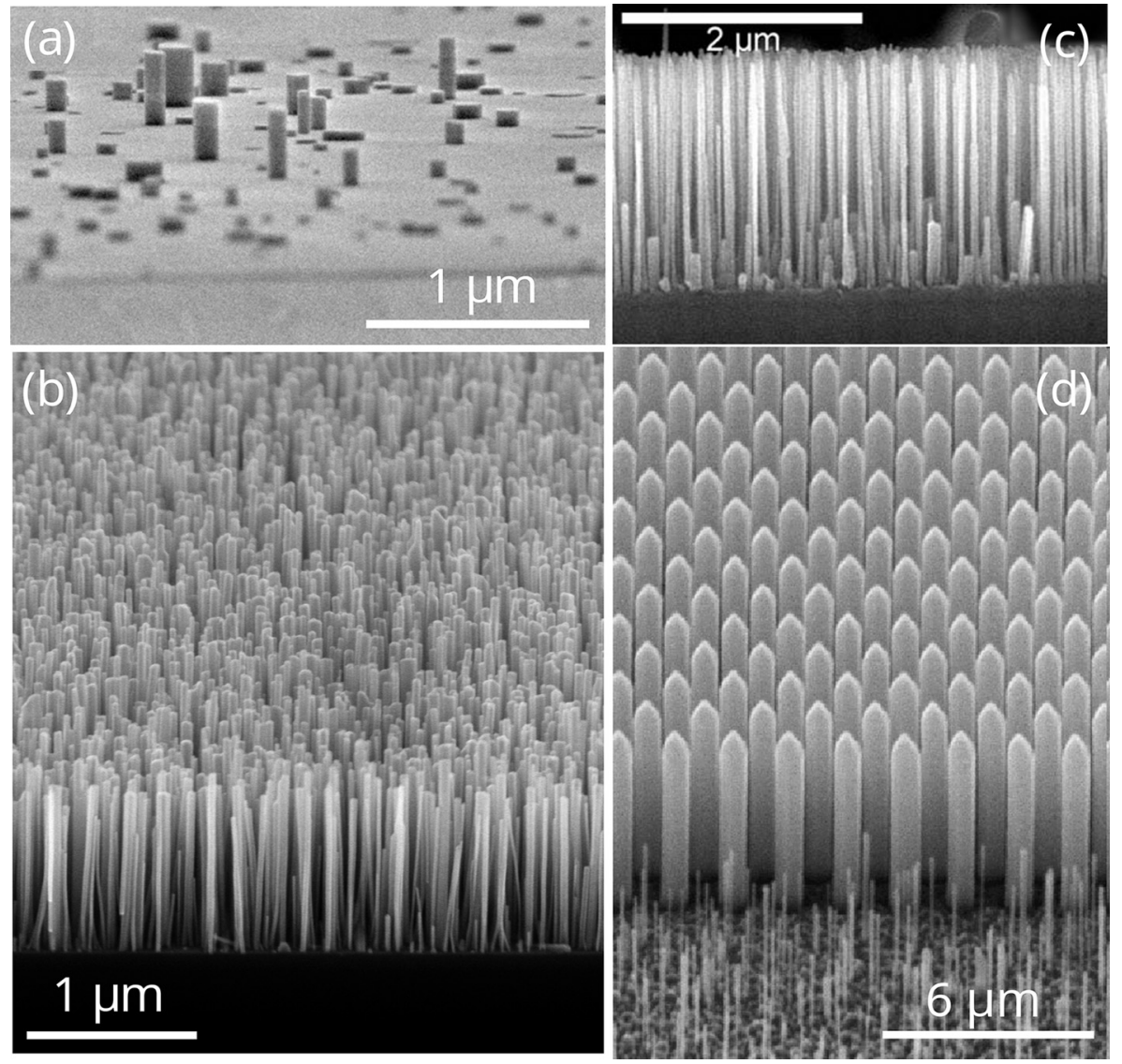

FIG. 66. (a) Scanning electron micrograph of a GaN NW ensemble grown by reactive $\mathrm{MBE}$ on $\mathrm{Al}_{2} \mathrm{O}_{3}$ (0001). Reprinted with permission from Appl. Phys. Lett. 98, 011914 (2011). Copyright 2011 AIP Publishing LLC. [(b)-(d)] Scanning electron micrographs of GaN NW ensembles grown by PA-MBE using different methods: (b) spontaneous nucleation, (c) vaporsolid-solid mechanism (Reprinted with permission from Appl. Phys. Lett. 91, 093113 (2007). Copyright 2007 AIP Publishing LLC), and (d) selective area growth (Reprinted with permission from Sekiguchi et al., Appl. Phys. Express 1, 124002 (2008). Copyright 2008 The Japan Society of Applied Physics). the analyzed GaN NWs were N-polar. In the case of de la Mata et al., ${ }^{143}$ Carnevale et al., ${ }^{558}$ and den Hertog et al., ${ }^{559}$ the direct visualization of $\mathrm{Ga}-\mathrm{N}$ dumbbell pairs by $\mathrm{ABF}-$ and HAADF-STEM allowed them to conclude that the inspected GaN NWs were N-polar as well.

The polarity of GaN NW ensembles prepared on bare $\mathrm{Si}(111)$ has also been investigated on a macroscopic scale using different methods, such as resonant XRD using synchrotron radiation, ${ }^{561} \mathrm{KOH}$ etching, ${ }^{150,558,561}$ and ARXPD $^{150}$ (see Sections III E, III A, and III D, respectively). The pioneering work of Hestroffer et al., ${ }^{561}$ who used resonant XRD with tunable monochromatic synchrotron radiation to assess the polarity, demonstrated that their GaN NW ensembles prepared on bare $\mathrm{Si}(111)$ were N-polar on a macroscopic scale. The results agreed well with their own microscopic studies using CBED and were further confirmed, on a macroscopic scale, by means of $\mathrm{KOH}$ etching. ${ }^{561}$ The polarity of GaN NW ensembles was also investigated using $\mathrm{KOH}$ etching by Carnevale et al. ${ }^{558}$ and Romanyuk et al. ${ }^{150}$ Carnevale et al. found that the majority of GaN NWs were N-polar. However, $10 \%$ of the GaN NWs were not etched after having been exposed to $\mathrm{KOH}$ for $120 \mathrm{~min}$. Based on these results, the authors concluded that their GaN NW ensembles prepared on bare $\mathrm{Si}(111)$ exhibited mixed polarity. In contrast, the results of Romanyuk et al. were in agreement with the findings of Hestroffer et al. ${ }^{561}$ As shown in Fig. 68, when increasing the etching time in $\mathrm{KOH}$ from 10 to $385 \mathrm{~min}$, they observed that all GaN NWs became shorter and developed a pencil-like shape, as expected for N-polar GaN NWs. For etching times longer
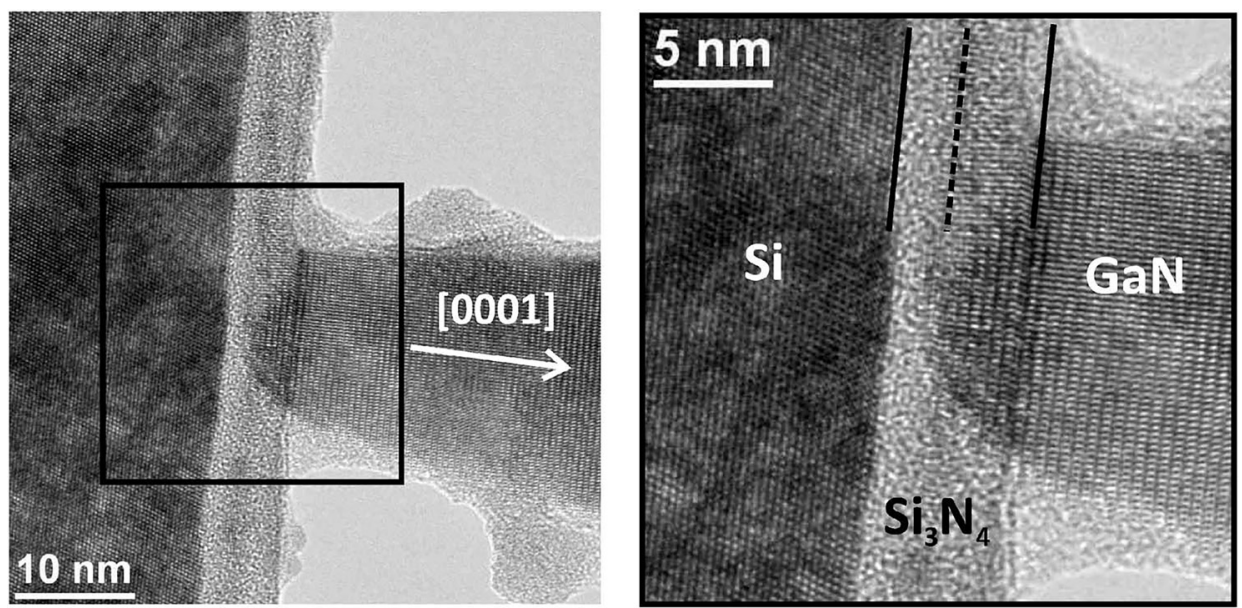

FIG. 67. (Left) Cross-sectional transmission electron micrographs of the interface between the bottom part of the GaN NW and the $\mathrm{Si}(111)$ substrate. (Right) Magnified micrograph of the area marked in the right image. An amorphous $\mathrm{Si}_{3} \mathrm{~N}_{4}$ layer was detected between the GaN NWs and the Si substrate. Reprinted with permission from J. Appl. Phys. 104, 034309 (2008). Copyright 2008 AIP Publishing LLC. 


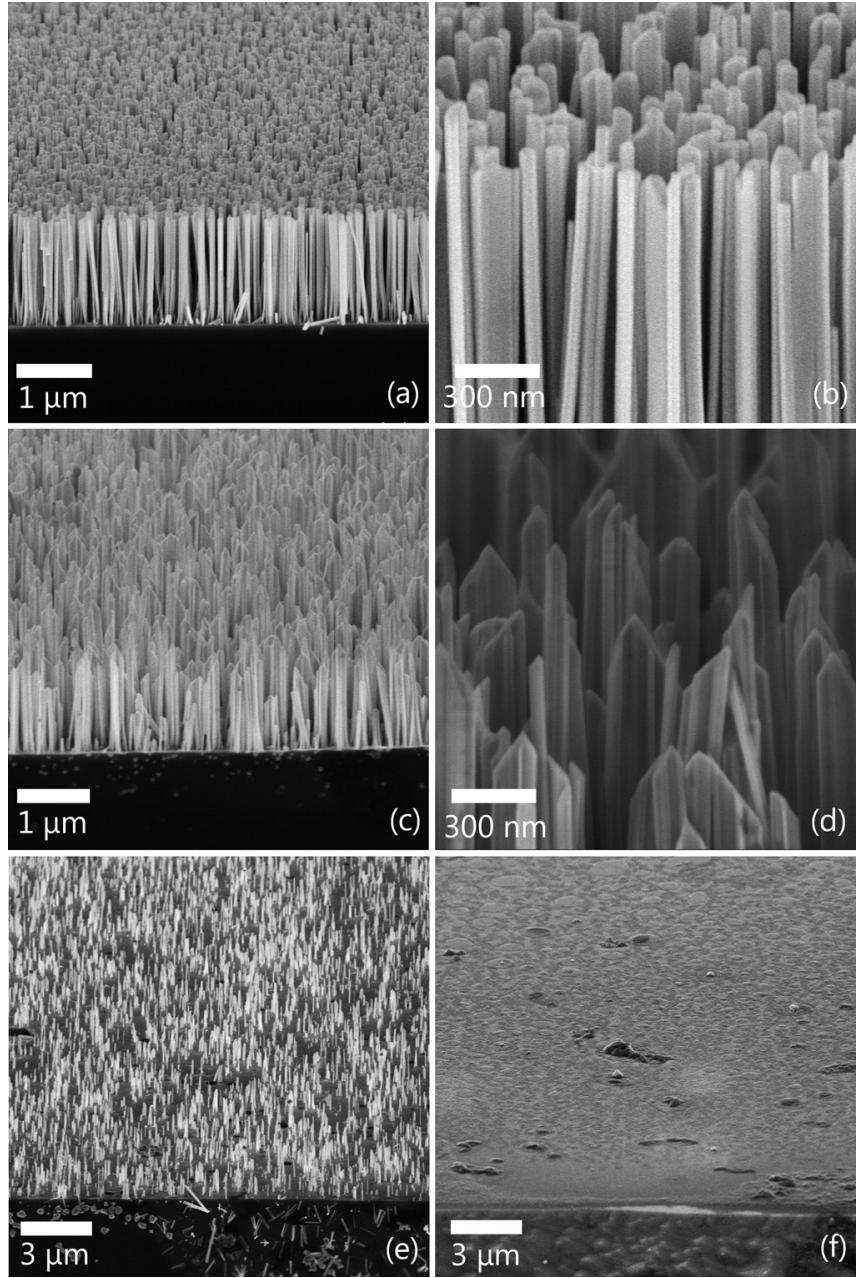

FIG. 68. (a) and (b) Bird's-eye scanning electron micrographs of a GaN NW ensemble prepared on bare $\mathrm{Si}(111)$ before $\mathrm{KOH}$ etching. Bird's-eye scanning electron micrographs of the same sample after having been etched in $\mathrm{KOH}$ for 10 [(c) and (d)], 100 (e), and $385 \mathrm{~min}$ (f). Reprinted with permission from Appl. Phys. Lett. 106, 021602 (2015). Copyright 2015 AIP Publishing LLC.

than $385 \mathrm{~min}$, no GaN NWs remained on the substrate. All GaN NWs were thus N-polar. In their work, the polarity of the ensemble was further assessed by ARXPD. The number of NWs probed by their ARXPD experiments was on the order of $5 \times 10^{9} \mathrm{~cm}^{-2}$. As can be seen in Fig. 19 (sample S-2), the angular distribution of photo-emitted electrons from the $\mathrm{N} 1 s$ core level is similar to that observed in freestanding N-polar GaN layers. Therefore, the analysis of the samples by ARXPD indicated that the majority of GaN NWs were N-polar, in perfect agreement with the $\mathrm{KOH}$ etching experiments.

The polarity on GaN NWs prepared on bare $\mathrm{Si}(111)$ was also indirectly assessed by analyzing the dependence of the $\mathrm{PL}$ on the applied external bias in GaN NW-based LEDs, ${ }^{562}$ as well as in $\mathrm{GaN} / \mathrm{A}_{\mathrm{lx}} \mathrm{Ga}_{1-\mathrm{x}} \mathrm{N} \mathrm{NW}$ heterostructures. ${ }^{563}$ In Ref. 562, Limbach et al. investigated the bias dependence of the PL intensity of LEDs based on NW ensembles with $\mathrm{In}_{\mathrm{x}} \mathrm{Ga}_{1-\mathrm{x}} \mathrm{N} / \mathrm{GaN} \mathrm{QW}$ as active region. The variation of the PL intensity as a function of the applied bias revealed the orientation of the electric field inside the QWs. Because the magnitude of the electric field caused by the internal piezoelectric polarization significantly exceeds the built-in field of the $\mathrm{p}-\mathrm{i}-\mathrm{n}$ junction, the observed dependence of the PL intensity as a function of the applied bias revealed the orientation of the NWs. The results showed that these GaN NWs were N-polar. Müßener et al. ${ }^{563}$ analyzed the emission energy of single GaN NWs containing a GaN nanodisc embedded into $\mathrm{Al}_{0.3} \mathrm{Ga}_{0.7} \mathrm{~N}$ segments. The latter was separated from highly Ge-doped GaN contacts at the bottom and upper parts of the NW heterostructure by AIN electron blocking layers. The authors found that the emission energy of the GaN nanodiscs was red- or blue shifted, depending on the sign of the external bias. Because, analogously to the case of $\operatorname{In}_{x} \mathrm{Ga}_{1-x} \mathrm{~N} / \mathrm{GaN}$ QWs, the electric field inside the $\mathrm{GaN}$ nanodiscs is determined by the polarization-induced internal fields, the variation in the emission energy with the applied bias revealed the orientation of the as-grown $\mathrm{GaN}$ NW heterostructure. This analysis demonstrated that the investigated GaN NWs were N-polar.

The apparent discrepancies in the experiments discussed above can be reconciled by taken into account the significance and limitations of the different types of measurements (see Section III). Whereas microscopic studies reveal the polarity of individual GaN NWs, macroscopic ones are the result of an averaging process. Consequently, macroscopic studies reflect the polarity of the majority of GaN NWs within a not necessarily homogeneous ensemble. Furthermore, although microscopic techniques provide a detailed insight into the properties of individual GaN NWs, they may suffer from poor statistics. Therefore, on the one hand, microscopic measurements might not reflect the properties of the majority of the NWs in an inhomogeneous ensemble. On the other hand, they can also show more easily the very small inhomogeneities of any GaN NW ensembles in terms of polarity. In that sense, combining both macroscopic and microscopic measurements is certainly the best way to proceed to unambiguously and meaningfully determine the polarity of GaN NW ensembles. The results reported by all these different groups are thus not necessarily contradictory. Because the growth conditions used by the different groups were rather comparable (in terms of both impinging fluxes and substrate temperature), the variation in the ratio between Ga- and N-polar GaN NWs is most likely related to the specific procedures followed to prepare the $\mathrm{Si}(111)$ substrate as well as to initiate the growth which, for instance, may induce the formation of localized structural and morphological defects. These structural and/or morphological defects might then facilitate the nucleation of selfassembled GaN NWs with either Ga or N polarity.

In order to avoid the formation of an amorphous $\mathrm{Si}_{\mathrm{x}} \mathrm{N}_{\mathrm{y}}$ interlayer between the GaN NWs and the $\mathrm{Si}(111)$ substrate, AIN buffer layers are commonly used in PAMBE. The polarity of GaN NWs grown on AlN-buffered $\mathrm{Si}(111)$ substrate has also been investigated by several groups, ${ }^{337,561,564,565}$ as further discussed below.

Brubaker et al. ${ }^{564}$ analyzed the polarity of GaN NWs grown on $100 \mathrm{~nm}$-thick AlN buffer layers with various morphologies and different degrees of mixed polarity. The properties of the AlN buffer layers were varied by modifying the ratio between the impinging $\mathrm{Al}$ and $\mathrm{N}$ fluxes at a given 
substrate temperature of $630^{\circ} \mathrm{C}$. As a result of NW nucleation at columnar AlN protrusions, the NW ensembles exhibited a lower number density than those prepared on bare $\mathrm{Si}(111)$ as well as a negligible degree of coalescence. CBED measurements revealed that the investigated GaN NWs were Ga-polar. Interestingly, unlike to what is commonly observed, many GaN NWs did not exhibit a flat top facet, but instead a pencil-like shape.

Hestroffer et al. ${ }^{561}$ also analyzed the polarity of GaN NWs prepared on AlN-buffered $\mathrm{Si}(111)$ substrate. The thickness of the buffer layer was 3-4 nm and it was prepared by alternatively supplying $\mathrm{Al}$ and $\mathrm{N}$ at an unspecified substrate temperature. The GaN NWs prepared on such a buffer layer reacted with $\mathrm{KOH}$ and were thus N-polar. Because the underlying AlN buffer layer was supposed to be Al-polar, the authors suggested that GaN NWs nucleated in between AlN grains.

Largeau et al. ${ }^{565}$ used a different procedure to prepare the AlN buffer layer than those followed in Refs. 561 and 564. To form the AlN buffer layer, 5.6 monolayers of crystalline $\mathrm{Al}$ were deposited at $600{ }^{\circ} \mathrm{C}$ and subsequently nitridated for $1 \mathrm{~min}$. The as-grown AIN buffer layer exhibited circular hollows (caused by the formation and subsequent consumption of $\mathrm{Al}$ droplets) with AlN nanocrystals at their edges (also referred to as AlN pedestals). The growth on such an AIN buffer layer resulted in the formation of sparse GaN NWs surrounded by a continuous GaN faceted layer. The formation of sparse GaN NWs was correlated with the presence of AlN nanocrystals at the hollow edges. The analysis of these GaN NWs by CBED indicated that they were $\mathrm{N}$-polar. These results were additionally confirmed by etching the GaN NW ensemble in $\mathrm{KOH}$. $\mathrm{KOH}$ etching was also used to determine the polarity of the surrounding $\mathrm{GaN}$ faceted layer. In contrast to the GaN NWs, the GaN layer was found to be Ga-polar. The different polarity was explained by assuming that the AIN buffer layer was Al-polar everywhere, apart from the AIN nanocrystals formed at the hollow edges that were N-polar.

Auzelle et al. ${ }^{337}$ recently investigated in great detail the polarity of GaN NWs prepared on AlN buffered $\mathrm{Si}(111)$ substrate. The authors analyzed the polarity of the GaN NWs as a function of the growth conditions employed to prepare the AlN buffer layer. This layer was grown either following a recipe comparable to the one reported by Largeau et al. in Ref. 565 or by exposing a $\mathrm{Si}_{\mathrm{x}} \mathrm{N}_{\mathrm{y}}$ layer formed upon $\mathrm{Si}$ nitridation at $840^{\circ} \mathrm{C}$ to an $\mathrm{Al}$ flux. In both cases, the thickness of the AlN buffer layer was on the order of 10 monolayers. For the first type of AlN buffer layer, their results were similar to those reported in Ref. 565, namely, they observed the formation of a Ga-polar faceted layer and the growth of N-polar GaN NWs on AlN nanocrystals formed by the formation and subsequent consumption of Al droplets. The polarity of the GaN NWs was verified on micro- and macroscopic scales by STEM, KPFM, and KOH etching. For the second type of AlN buffer layer, which contained a lower density of AIN nanocrystals, the authors also observed the formation of a Ga-polar faceted layer and GaN NWs. The analysis of the polarity of the GaN NWs by STEM, KPFM, and KOH etching revealed that, whereas the majority of the GaN NWs was
$\mathrm{N}$-polar, a minor fraction exhibited inversion domains with a Ga-polar core/N-polar shell structure. Because, in this case, the density of GaN NWs was higher than that of AlN nanocrystals, they concluded that NW nucleation was triggered by the formation of $\mathrm{N}$-polar AlN domains at either the $\mathrm{Si}$ / AlN or the AlN/GaN interface.

The results reported by all these different groups on the growth of GaN NWs on AlN-buffered $\mathrm{Si}(111)$ are controversial, but not comparable because of the different procedures followed to fabricate the AlN buffer layers. We can, however, conclude that the polarity as well as the morphology and distribution of self-assembled GaN NWs strongly depend on the growth conditions used to prepare the AlN buffer layer because, in this case, NW nucleation is governed by the presence of morphological and structural defects.

2.2.2. Growth on non-polar substrates other than $\mathrm{Si}(111)$. The polarity of GaN NWs prepared on other nonpolar substrates, such as $\mathrm{Si}(001), c$-sapphire, amorphous $\mathrm{Al}_{2} \mathrm{O}_{3}$, single-crystalline [111]-oriented diamond, and $\mathrm{Mo}$, has also been investigated by different groups, but not to a great extent. ${ }^{535,549,566-568}$

Borysiuk et al. ${ }^{566}$ investigated the growth of GaN NWs on $\mathrm{Si}(001)$ substrate. They observed the formation of two different types of GaN NWs, namely, a majority of NWs that elongated along the direction perpendicular to the substrate normal and a few inclined NWs. In the latter case, the inclination angle with respect to the surface normal was found to be in the range of $50^{\circ}-60^{\circ}$. Because they observed the formation of an amorphous $\mathrm{Si}_{\mathrm{x}} \mathrm{N}_{\mathrm{y}}$ interlayer at the interface between perpendicular GaN NWs and the $\mathrm{Si}(001)$ surface, they assumed that most of these GaN NWs were N-polar, as reported for $\mathrm{Si}(111)$ substrates. ${ }^{150,558,561}$ The polarity of the inclined GaN NWs was assessed by CBED. The measurements revealed that these GaN NWs were Ga-polar. However, it is worth noticing that TEM micrographs showed that inclined GaN NWs nucleated not on $\mathrm{Si}$ or $\mathrm{Si}_{\mathrm{x}} \mathrm{N}_{\mathrm{y}}$ but on the $\{111\}$ planes of zinc blende GaN islands, which exhibited a pyramidal shape. Consequently, the nucleation of the inclined GaN NWs was not spontaneous, but clearly induced by the formation of zinc blende $\mathrm{GaN}$ islands.

Cherns et al. ${ }^{549,567}$ investigated the formation of $\mathrm{GaN}$ NWs on AlN-buffered $c$-sapphire by TEM. The AlN buffer layer was $5 \mathrm{~nm}$-thick and prepared at $600^{\circ} \mathrm{C}$. The GaN NW ensembles exhibited a mixed polarity, as determined by CBED. Interestingly, regardless of the polarity, most of the GaN NWs contained threading defects and had a pencil-like shape (see Fig. 69). ${ }^{549}$ The analysis of the samples by TEM revealed that the threading defects are planar faults lying on $\{10 \overline{1} 0\}$ planes and bounded by opposite partial screw dislocations with Burger vectors of $1 / 2\langle 0001\rangle .{ }^{549}$ Although the precise growth mechanism was not completely understood, the results allowed them to conclude that, in their case, the nucleation of $\{10 \overline{1} 0\}$ faults at the AIN buffered $c$-sapphire interface is an essential ingredient to explain the formation and further growth of these GaN NWs.

Sobanska et al. ${ }^{535}$ demonstrated the growth of GaN NWs on a $15 \mathrm{~nm}$-thick amorphous $\mathrm{Al}_{2} \mathrm{O}_{3}$ layer grown by atomic 


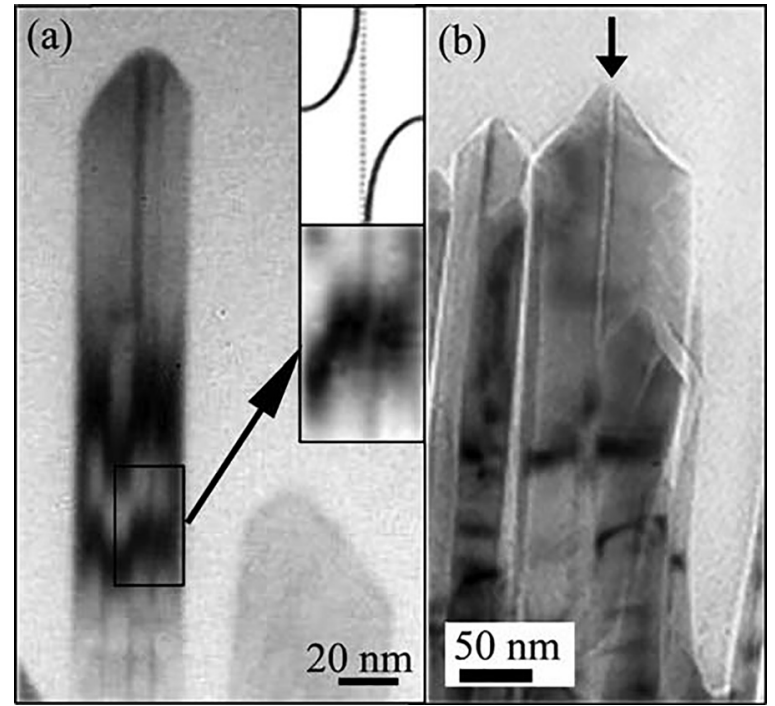

FIG. 69. Bright field images of (a) a NW with more than one fault. The blow-up shows the interaction of one bounding dislocation with $\boldsymbol{g}=(0002)$ bend contour (digitally enhanced) along with a schematic of the contrast expected when $\boldsymbol{g} \cdot \boldsymbol{b}=1$; (b) a $\{10 \overline{1} 0\}$ fault with an edge-on void (arrowed) over a part of its length. Reprinted with permission from Appl. Phys. Lett. 93, 111911 (2008). Copyright 2008 AIP Publishing LLC.

layer deposition (ALD) on a $\mathrm{Si}(111)$ substrate. Despite the amorphous nature of the $\mathrm{Al}_{2} \mathrm{O}_{3}$ layer, all GaN NWs were found to be well aligned along the direction perpendicular to the substrate. The morphological properties as well as the distribution of the GaN NWs were not identical, but comparable to those of GaN NW ensembles prepared on bare $\mathrm{Si}(111)$ using similar growth conditions. The analyses of the polarity on a microscopic scale by CBED and HRTEM showed that the investigated GaN NWs were N-polar.
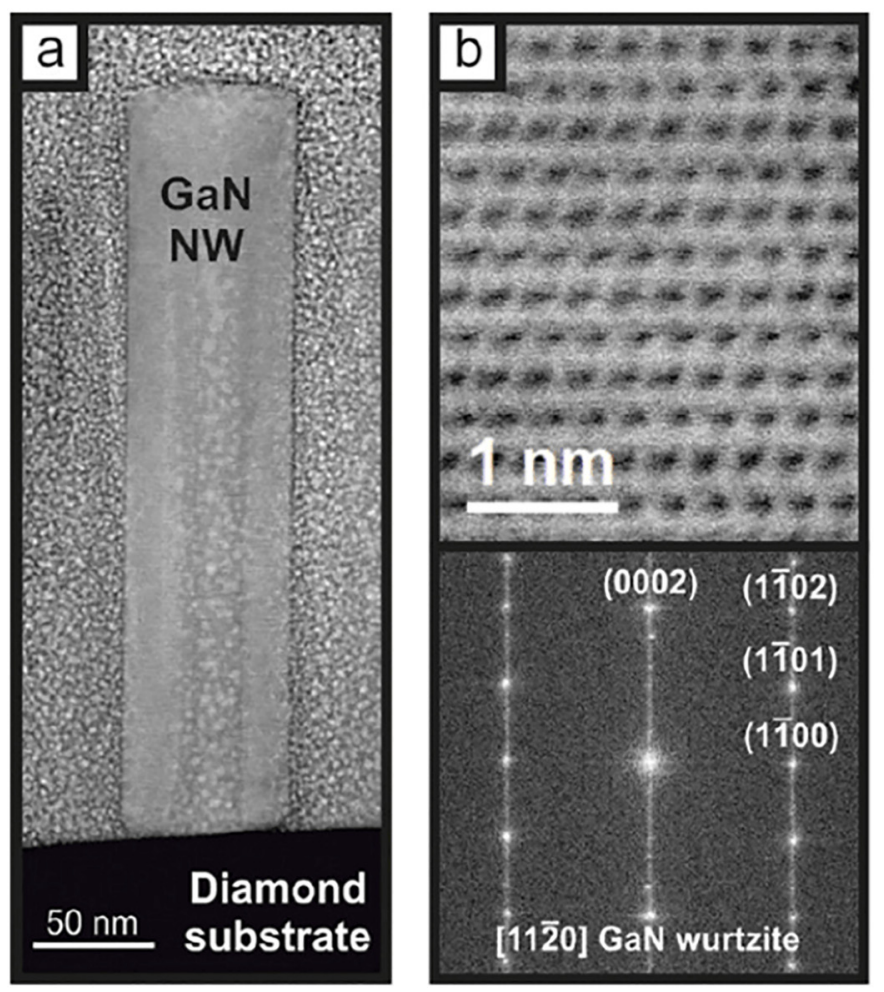

Schuster et al. ${ }^{568}$ reported on the growth of GaN NWs on single-crystalline [111]-oriented diamond without the use of any kind of buffer layer. As in the case of amorphous $\mathrm{Al}_{2} \mathrm{O}_{3}$, the morphological properties of the $\mathrm{GaN}$ NW ensembles grown on diamond were comparable with those of GaN NW ensembles prepared on bare $\mathrm{Si}(111)$. The analysis of the samples by HRTEM revealed that the interface between the GaN NWs and the diamond substrate is abrupt at an atomic level and free of extended defects. Therefore, they excluded the hypothetical formation of an interlayer such as, e.g., $\mathrm{C}_{\mathrm{x}} \mathrm{N}_{\mathrm{y}}$. Finally, the direct visualization of $\mathrm{Ga}-\mathrm{N}$ dumbbells by HAADF-STEM (see Fig. 70) allowed them to conclude that the analyzed GaN NWs were N-polar.

Urban et $a l .{ }^{547}$ investigated the polarity of the $\mathrm{GaN}$ NWs unintentionally formed on a polycrystalline Mo mask used to induce the formation of NW arrays by SAG on Gapolar GaN films. The authors found that both the morphology and the polarity of the GaN NWs grown on the Mo mask were different than those of SAG grown GaN NWs. Whereas the SAG GaN NWs exhibited a pencil-like shape and were Ga-polar, the self-assembled GaN NWs formed on the Mo mask exhibited flat top facets and were N-polar, as determined on a macroscopic scale by $\mathrm{KOH}$ etching.

2.2.3. Growth on polar substrates. All the experiments discussed so far were performed using either non-polar substrates or AlN buffer layers with an ill-defined polarity. To elucidate whether the polarity of the substrate plays a role in the spontaneous formation of GaN NWs in PAMBE, Fernandez-Garrido et al. ${ }^{534}$ investigated the growth of GaN NWs on AlN buffer layers with a well-defined polarity. To this end, Al- and N-polar AlN layers were prepared on Siface $6 \mathrm{H}-\mathrm{SiC}$ and $\mathrm{C}$-face $6 \mathrm{H}-\mathrm{SiC}$ substrates, respectively. Unlike $\mathrm{Si}(111)$ or $c$-sapphire substrates, the low lattice-

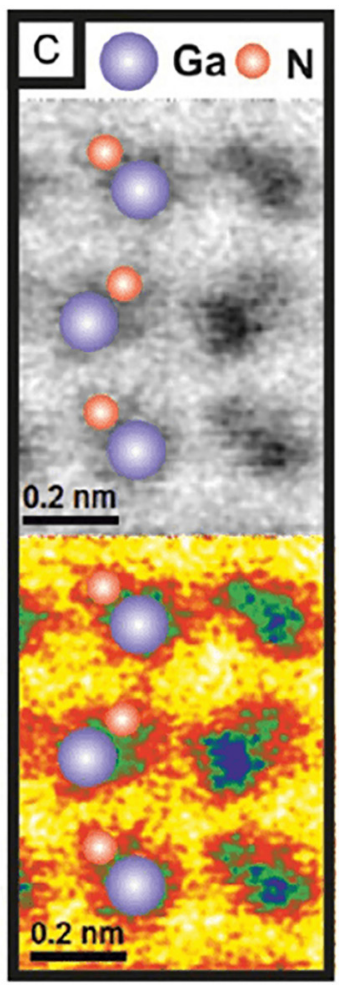

FIG. 70. Cross-sectional HAADF STEM of an exemplary GaN NW grown on diamond. The atomic resolution image with its power spectrum (b) corresponds to an ABF STEM micrograph obtained under aberration corrected conditions, where the atomic monolayers in the regular wurtzite crystal structure are clearly observed. A magnification (c) unveils the Ga-N dumbbells with $\mathrm{N}$-polarity, for clarity shown in false colors. Reprinted with permission from Schuster et al., Nano Lett. 12, 2199 (2012). Copyright 2012 American Chemical Society. 
mismatch between $\mathrm{AlN}$ and $\mathrm{SiC}$ (i.e., 1\%) together with the polar nature of the $\mathrm{SiC}$ substrate facilitate the growth of coherently strained AIN films with a well-defined polarity in a layer-by-layer mode. To avoid plastic relaxation, the thickness of the AlN buffer layers, grown under Al-excess at $800^{\circ} \mathrm{C}$, was $8 \mathrm{~nm}$. The ex-situ analysis of these layers by AFM and TEM demonstrated that they were smooth and coherently strained. The morphology of the samples grown on Al- and N-polar AlN buffered layers at different substrate temperatures is shown in Fig. 71. As can be seen in the scanning electron micrographs, the polarity of the AlN buffer layer plays a major role in the nucleation of GaN NWs. The growth on Al-polar AlN layers resulted in the formation of sparse GaN NWs embedded in a continuous GaN faceted layer. Thus, the morphology is very similar to that of the samples prepared by Largeau et al. ${ }^{565}$ as well as Auzelle et $a l .{ }^{337}$ on AlN-buffered $\mathrm{Si}(111)$ substrate. In contrast, on $\mathrm{N}$-polar AIN, identical growth conditions led to the formation of dense GaN NW ensembles, as those commonly obtained on smooth and homogenous non-polar substrates such as $\mathrm{Si}$, diamond, or amorphous $\mathrm{Al}_{2} \mathrm{O}_{3}$. ${ }^{150,535,558,561,568}$ Due to the low density of morphological and structural defects in the N-polar AlN buffer layers, the formation of these GaN NWs is certainly governed by spontaneous nucleation. The polarity of the samples was investigated on a microscopic scale using either CBED or EELS. The analysis of the samples prepared on the Al-polar AlN buffer layer revealed that the $\mathrm{GaN}$ faceted layer was, as expected, Ga polar. However, the sparse GaN NWs were found to be $\mathrm{N}$ polar. The inversion of crystal polarity was tentatively attributed to Si segregation during AlN growth, what might have reversed the polarity by forming $\mathrm{Si}-\mathrm{N}$ bonds at the AlN/GaN interface. The absence of Ga-polar GaN NWs indicated that cation-polar GaN NWs do not nucleate spontaneously on AlN. The results obtained for the samples grown on the N-polar AlN buffer layer confirmed that the GaN NWs exhibited the same $\mathrm{N}$ polarity.

The results reported by Fernández-Garrido et al.$^{534} \mathrm{dem}$ onstrated that, in the absence of morphological and structural defects, all GaN NWs formed spontaneously are N-polar. This result compares well with all macroscopic studies reported so far on the polarity of dense ensembles of GaN NWs prepared on smooth and homogeneous substrates ${ }^{150,535,558,561,562,568}$ as well as with the conclusions drawn by Auzelle et al. in Ref. 337. The apparent contradictions with some microscopic studies ${ }^{537,549,555,564,566,567}$ can be explained by taking into account that morphological and structural defects can result in the formation of individual GaN NWs with either Ga or N polarity, as clearly shown in Ref. 549. The underlying reason why spontaneously formed $\mathrm{GaN}$ NWs elongate along the $[000 \overline{1}]$ direction is, however, not yet understood. In PAMBE, it is well known that the kinetic barriers for the diffusion of $\mathrm{Ga}$ and $\mathrm{N}$ adatoms are lower for the (0001) surface. ${ }^{242}$ This fact may enhance the 2D growth of GaN islands, favoring the formation of GaN layers, even under N-rich growth conditions. ${ }^{569,570}$ Another potential explanation lies in the fact that Ga- and N-polar surfaces are expected to exhibit different surface energies. ${ }^{571,572}$ If, as proposed by Dubrovskii et al., ${ }^{573}$ the
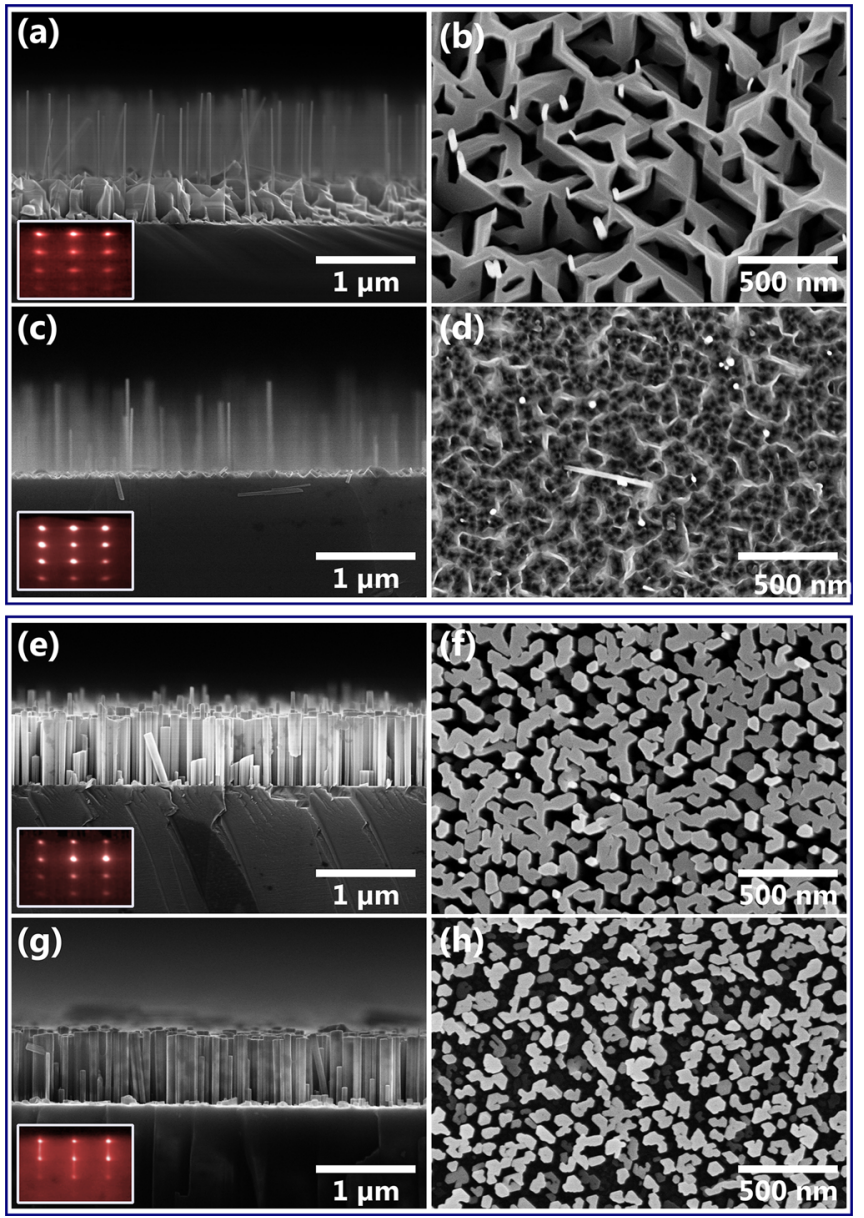

FIG. 71. [(a)/(c)] Cross-sectional and plan-view [(b)/(d)] scanning electron micrographs of GaN NWs grown on AlN-buffered $6 \mathrm{H}-\mathrm{SiC}(0001)$ at $815 /$ $825^{\circ} \mathrm{C}$. [(e)/(g)] Cross-sectional and plan-view [(f)/(h)] scanning electron micrographs of GaN NWs grown on AlN-buffered 6H-Si (0001) at 815/ $825^{\circ} \mathrm{C}$. The inset in Figures (a), (c), (e), and (g) show the reflection highenergy electron diffraction pattern along the $[11 \overline{2} 0]$ azimuth upon growth termination. Reprinted with permission from Fernández-Garrido et al., Nano Lett. 12, 6119 (2012). Copyright 2012 American Chemical Society.

dominant driving force for the spontaneous formation of GaN NWs is the anisotropy of surface energies, the elongation of GaN NWs along the $[000 \overline{1}]$ direction could eventually be explained in terms of surface energetics. ${ }^{337}$ However, an explanation based on surface energetics alone seems to be unlikely because of the growth of N-polar GaN NWs on a wide variety of completely different substrates, which suggests that GaN NW nucleation does not depend on the interface surface energy.

b. Vapor-solid-solid growth. In PAMBE, the formation of GaN NWs can be enforced using the VSS growth approach, where pre-deposited metal islands are used to collect the precursors, induce supersaturation, and promote uniaxial growth. $^{529,537-539}$ Figure 66(c) shows a characteristic scanning electron micrograph of a GaN NW ensemble prepared by VSS using Ni islands. The ensemble is fairly homogeneous in height and diameter. The minimum NW diameter is determined by the size of the pre-deposited $\mathrm{Ni}$ islands $(20-50 \mathrm{~nm})$. As explained in Ref. 574, this growth approach requires $\mathrm{N}$-excess to prevent the formation of a compact 
layer and similar temperatures to those used for selfassembled GaN NWs. The use of Ni islands to enforce the formation of GaN NWs enables a higher degree of control on the size and distribution of the GaN NWs. In addition, this approach also facilitates the formation of GaN NW ensembles on substrates, where the self-assembled approach does not seem to be possible, such as on bare $\mathrm{Al}_{2} \mathrm{O}_{3}(0001){ }^{537}$ However, GaN NWs grown by VSS suffer from the undesired incorporation of $\mathrm{Ni}$. This phenomenon induces the formation of basal plane stacking-faults and quenches the PL. ${ }^{537}$ Because of this reason, the growth of GaN nanowires using the VSS growth approach has not gained much popularity.

The polarity of these GaN nanowires was only investigated on a microscopic scale using CBED. ${ }^{537}$ The results revealed that they were Ga polar. This growth approach is thus potentially suitable for the fabrication of ensembles of Ga-polar GaN NWs. Nevertheless, further studies are required to confirm that the majority of these GaN NWs are indeed Ga polar.

c. Selective area growth. The formation of GaN NWs in PAMBE can also be enforced by SAG using patterned substrates. ${ }^{531,540-548,575}$ This growth approach enables a high level of control over both the position and diameter of $\mathrm{GaN}$ NWs. Because of the strong tendency of group III-nitrides compound semiconductors to elongate along the $c$-axis, the most common approach consists in patterning GaN or AlN layers grown with this orientation. Regardless of the material used as a mask (i.e., $\mathrm{Ti}, \mathrm{Si}_{\mathrm{x}} \mathrm{N}_{\mathrm{y}}, \mathrm{Mo}$, or $\mathrm{SiO}_{2}$ ), the $\mathrm{SAG}$ of GaN NWs on patterned substrates is achieved under $\mathrm{N}$ excess at higher temperatures than those required for the self-assembled formation of GaN NWs in PAMBE, namely, on the order of $900{ }^{\circ} \mathrm{C}$.

In contrast to other growth approaches, where there is a limited degree of control over GaN NW polarity, the polarity is determined in SAG by the orientation of the group-III Nitride layers on which SAG takes place. So far, the most common substrate used for SAG in PAMBE consists of a GaN (0001) layer grown by MOCVD on $\mathrm{Al}_{2} \mathrm{O}_{3}(0001) .{ }^{541,542,546,547}$ Therefore, the GaN NWs grown on such a substrate are Gapolar. ${ }^{547}$ Interestingly, the morphological properties of SAG GaN NWs fabricated on GaN (0001) are quite often different from those obtained by means of self-assembled growth on substrates such as $\mathrm{Si}(111)$, amorphous $\mathrm{Al}_{2} \mathrm{O}_{3}$, or [111]-oriented diamond (see Fig. 66(d)). Apart from the usually larger diameter of SAG grown GaN NWs, they do not always exhibit a flat top facet. ${ }^{542,546,547}$ As discussed by Bengoechea-Encabo et al., ${ }^{542}$ depending on the effective III/V ratio on the holes opened in the mask, Ga-polar GaN NWs may exhibit either a flat top facet (higher III/V ratios) or a pencil-like shape with a tip formed by the convergence of semi-polar planes (lower III/ $\mathrm{V}$ ratios).

The SAG of GaN NW arrays on patterned Si(111) substrate as well as on patterned GaN and AlN buffer layers prepared on $\mathrm{Si}(111)$ substrate has also been reported by different groups. ${ }^{540,544,545,576-578}$ However, because groupIII-nitride layers grown on $\mathrm{Si}(111)$ substrate usually exhibit a mixed polarity (see Section IV A $3 \mathrm{~d}$ ), the polarity of these NW arrays is not necessarily well-defined. Recently, the polarity of SAG GaN NWs on $\mathrm{Si}(111)$ was studied by Brubaker et al. in Ref. 575. In this work, Brubaker et al. demonstrated the SAG of N-polar GaN NWs on GaN/AlN/ $\mathrm{Si}(111)$. Interestingly, for comparable growth conditions, growth on a patterned Ga-polar GaN substrate resulted not in the formation of GaN NWs but on pyramidal structures bounded by (1102) planes. Based on these results, they concluded that the growth window for fabricating arrays of $\mathrm{GaN}$ NWs is wider in the case of N-polarity because of the comparatively higher thermal stability of $\mathrm{N}$-polar material at the elevated temperatures required for SAG.

\section{ZnO nanostructures and nanowires}

The formation of $\mathrm{ZnO}$ nanostructures has been achieved by a large number of physical and chemical deposition techniques, their shape being controlled by the growth conditions used. ${ }^{579-581}$ Vapor phase transport (i.e., thermal evaporation of oxides or $\mathrm{Zn}$ powders with organic compounds or not; in this last case the growth approach is referred to as physical vapor transport (PVT)), ${ }^{582-585} \mathrm{PLD},{ }^{586}$ standard CVD and MOCVD, ${ }^{587,588}$ spray pyrolysis, ${ }^{589}$ electrodeposition, ${ }^{590}$ and chemical bath deposition (CBD) ${ }^{591-593}$ have all been reported as possible methods to grow $\mathrm{ZnO}$ nanostructures along the non-polar and polar $c$-axes as well as NWs along the polar $c$ axis with non-polar $\{10 \overline{1} 0\}$ sidewalls (see Table III).

The polarity aspects are typically related to two strongly related issues, namely, (i) the influence of the polarity of the $\mathrm{ZnO}$ nucleation surfaces (i.e., (0001), $\{10 \overline{1} 0\},\{2 \overline{1} \overline{1} 0\}$ planes) on the structural morphology of $\mathrm{ZnO}$ nanostructures and (ii) the polarity itself of these $\mathrm{ZnO}$ nanostructures. The first part of the present subsection is dedicated to physical vapor deposition techniques, by which most of the pioneering research works about $\mathrm{ZnO}$ nanostructures were produced, especially by the group of Wang at Georgia Tech. ${ }^{579,582-584}$ The second and third parts are devoted to chemical vapor and solution deposition techniques, respectively, which most of the recent research works have been focused on.

\section{ZnO nanostructures with different shapes by physical vapor deposition}

A wide variety of $\mathrm{ZnO}$ nanostructures, whose morphology is induced by polarity effects, can be formed by using thermal evaporation (i.e., vapor phase transport and condensation) of powders (typically oxides or $\mathrm{Zn}$ ) as a source material heated at high temperature. ${ }^{579,582-585}$ The driving force is the thermal gradient between the source and substrate. The vast majority of the thermal evaporation research works were reported by the group of Wang at Georgia Tech, in which oxides (i.e., $\mathrm{ZnO}, \mathrm{SnO}_{2}, \mathrm{LiO}_{2}$, and $\mathrm{In}_{2} \mathrm{O}_{3}$ ) powders are typically placed in an alumina tube that is inserted in a horizontal tube furnace. The source and substrate temperatures lie in the range of $1000-1400^{\circ} \mathrm{C}$ and $500-900{ }^{\circ} \mathrm{C}$, respectively. Both the self-assembled and catalyst-assisted approaches were employed by combining different oxide powders as source materials. In particular, $\mathrm{SnO}_{2}$ powders were typically used to favor a vapor-solid-solid mechanism with tin catalyst particles. Overall, the different types of $\mathrm{ZnO}$ nanostructures including NWs, nanoribbons, nanocombs, 
TABLE III. Polarity of ZnO nanostructures (i.e., mostly NWs) grown by physical and chemical deposition techniques on different types of substrates. For each report, the table lists the growth technique, the growth approach, the methods used to determine the polarity, the year when the results were published, and the associated reference.

\begin{tabular}{|c|c|c|c|c|c|}
\hline Substrate & Growth technique & Growth approach & Characterization method & Polarity & Publication year/Ref. \\
\hline$\ldots$ & ZnS oxidation & Self-assembled & $\mathrm{HCl}$ & Zn-polar & $1972 / 627$ and 628 \\
\hline Zn foil & PVD & Self-assembled & CBED & Zn-polar & $2003 / 598$ \\
\hline $\mathrm{ZnO}(000 \overline{1}) / \mathrm{Al}_{2} \mathrm{O}_{3}(0001)$ & PLD & Self-assembled & CBED & Zn-polar & $2008 / 644$ \\
\hline $\mathrm{ZnO} / \mathrm{MgO} / \mathrm{Al}_{2} \mathrm{O}_{3}(0001)$ & PVT & VLS & PFM & Zn-polar & $2008 / 604$ \\
\hline Muscovite mica & PVT & Self-assembled & ABF-STEM & Zn-polar & $2012 / 599$ \\
\hline $\mathrm{GaN}(000 \overline{1})$ & MBE & Self-assembled & ABF-STEM & Zn-polar & $2014 / 595$ \\
\hline $\mathrm{ZnO}(000 \overline{1}) / \mathrm{Al}_{2} \mathrm{O}_{3}(0001)$ & PLD & Self-assembled & CBED & Zn-polar & $2016 / 597$ \\
\hline $\mathrm{ZnO}(0001) / \mathrm{Al}_{2} \mathrm{O}_{3}(0001)$ & PLD & Self-assembled & CBED & Zn-polar & $2016 / 597$ \\
\hline $\operatorname{Si}(100)$ & CVD & VLS & CBED, EELS & Zn-polar & $2008 / 629$ \\
\hline $\mathrm{Al}_{2} \mathrm{O}_{3}(0001)$ & MOCVD & Self-assembled & CBED & Zn-polar & $2010 / 621$ \\
\hline $\mathrm{ZnO}(000 \overline{1}) / \mathrm{Al}_{2} \mathrm{O}_{3}(0001)$ & MOCVD & Self-assembled & CBED & Zn-polar & $2012 / 622$ \\
\hline $\mathrm{ZnO}(000 \overline{1})$ & MOCVD & Self-assembled & CBED & Zn-polar & $2012 / 622$ \\
\hline $\mathrm{Al}_{2} \mathrm{O}_{3}(0001)$ & MOCVD & Self-assembled & CBED & Zn-polar & $2013 / 624$ \\
\hline $\mathrm{ZnO}(0001)$ & MOCVD & Self-assembled & CBED & Zn-polar & $2013 / 624$ \\
\hline $\mathrm{Al}_{2} \mathrm{O}_{3}(0001)$ & MOCVD & VLS & CBED & O-polar & $2013 / 624$ \\
\hline $\mathrm{ZnO}(0001)$ & MOCVD & VLS & CBED & O-polar & $2013 / 624$ \\
\hline $\mathrm{ZnO}(0001) / \mathrm{Si}$ & $\mathrm{CBD}$ & Self-assembled & CBED & Zn-polar & $2006 / 634$ \\
\hline $\mathrm{Ag} / \mathrm{Si}$ & $\mathrm{CBD}$ & Self-assembled & PFM & Zn-polar & $2007 / 643$ \\
\hline $\mathrm{ZnO} / \mathrm{Cu}$ grid & $\mathrm{CBD}$ & Self-assembled & CBED & Zn-polar & $2007 / 625$ \\
\hline $\mathrm{ZnO}(0001) / \mathrm{Al}_{2} \mathrm{O}_{3}(0001)$ & $\mathrm{CBD}$ & Self-assembled & CBED & Zn-polar & $2008 / 626$ \\
\hline $\mathrm{ZnO}(000 \overline{1}) / \mathrm{Al}_{2} \mathrm{O}_{3}(0001)$ & $\mathrm{CBD}$ & Self-assembled & CBED & Zn-polar & $2008 / 644$ \\
\hline $\mathrm{ZnO}(000 \overline{1})$ & CBD & Self-assembled & CBED & O-polar & $2013 / 638$ \\
\hline $\mathrm{ZnO}(000 \overline{1})$ & $\mathrm{CBD}$ & SAG & CBED & O-polar & $2014 / 640$ \\
\hline $\mathrm{ZnO}(0001)$ & $\mathrm{CBD}$ & SAG & CBED & Zn-polar & $2014 / 640$ \\
\hline $\mathrm{GaN}(000 \overline{1})$ & $\mathrm{CBD}$ & Self-assembled & CBED, $\mathrm{H}_{2} \mathrm{O}, \mathrm{XAS}$ & O-polar & $2015 / 642$ \\
\hline $\mathrm{GaN}(0001)$ & CBD & Self-assembled & $\mathrm{H}_{2} \mathrm{O}$, XAS & Zn-polar & $2015 / 642$ \\
\hline $\mathrm{SnO}_{2}: \mathrm{F} /$ glass & $\mathrm{CBD}$ & Self-assembled & CBED, $\mathrm{HCl}$ & O-polar & $2015 / 645$ \\
\hline $\mathrm{SnO}_{2}: \mathrm{F} /$ glass & $\mathrm{CBD}$ & Self-assembled & $\mathrm{CBED}, \mathrm{HCl}$ & Zn-polar & $2015 / 645$ \\
\hline
\end{tabular}

tetrapod-like structures, nanopropellers, nanobelts, nanorings, nanospirals, helical nanosprings, and seamless nanorings are basically the result of (i) the different growth rates along the $\pm[0001],[01 \overline{1} 0]$, and $[2 \overline{1} \overline{1} 0]$ directions as well as of (ii) the different surface reactivity on top of $\pm(0001)$ polar $\mathrm{ZnO}$ nucleation surfaces.

a. Nanowires, nanoribbons, nanocombs, tetrapod-like structures, and nanopropellers.

1.1. Self-assembled approach. NWs, nanoribbons, and nanocombs: A very illustrative example of the polarity influence on the resulting nanostructure morphology is given by the use of $\mathrm{ZnO}$ nanoribbons growing along the [21 10 ] direction, with (0110) top/bottom and $\pm(0001)$ side surfaces, which act as seeds for the subsequent growth of $\mathrm{ZnO}$ NWs. Following the self-assembled approach by only using $\mathrm{ZnO}$ powders as a source material, Wang et al. showed that the $\mathrm{Zn}$-polar $\mathrm{ZnO}$ nucleation surface is chemically active for the formation of $\mathrm{ZnO} N W s{ }^{583}$ The presence of tiny $\mathrm{Zn}$ clusters as well as a $\mathrm{Zn}$ local enrichment at the growth front were pointed out, revealing the occurrence of a self-catalyzed growth within a vapor-solid (VS) mechanism. In contrast, Opolar $\mathrm{ZnO}$ nucleation surface was found to be chemically inert. ${ }^{583}$ This resulted in the formation of the so-called nanocombs, as shown in Fig. 72: these are composed of nanoribbons growing along the $[2 \overline{1} \overline{1} 0]$ direction with $\pm(0001)$ side surfaces, on which NWs are more slowly formed along the [0001] direction while no growth occur along the [000ㅍ] direction. Additionally, Park et al. reported the use of PPI $\mathrm{ZnO}$ templates for growing $\mathrm{ZnO} \mathrm{NWs}$ by thermal evaporation using $\mathrm{ZnO}$ and graphite powders (i.e., carbothermal reduction). ${ }^{594}$ By alternately forming $\mathrm{CrN}$ and $\mathrm{Cr}_{2} \mathrm{O}_{3}$ layers using $\mathrm{MBE}$ and technological processes (i.e., lithography, etching, and plasma), $\mathrm{Zn}$ - and O-polar $\mathrm{ZnO}$ thin films were grown, respectively. $\mathrm{ZnO} \mathrm{NWs}$ were subsequently formed selectively

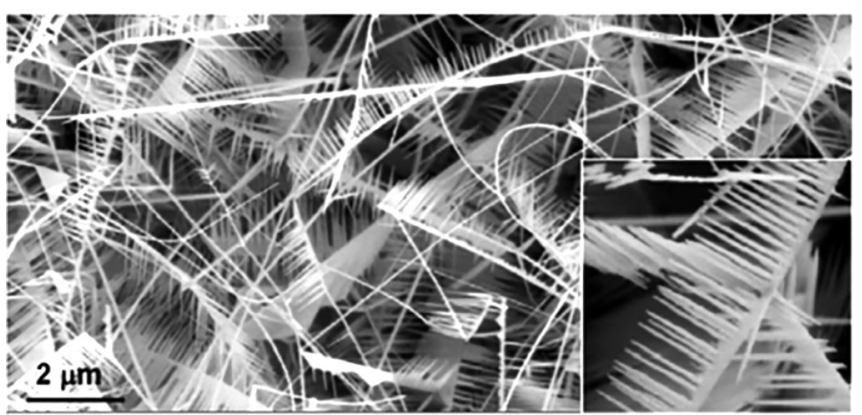

FIG. 72. SEM image of nanocombs consisting of nanoribbons grown along the $[2 \overline{1} 10]$ direction with $\pm(0001)$ side surfaces, on which NWs are more slowly formed along the [0001] direction, while no growth occurs along the $[000 \overline{1}]$ direction. Reprinted with permission from Wang et al., Phys. Rev. Lett. 91, 185502 (2003). Copyright 2003 American Physical Society. 

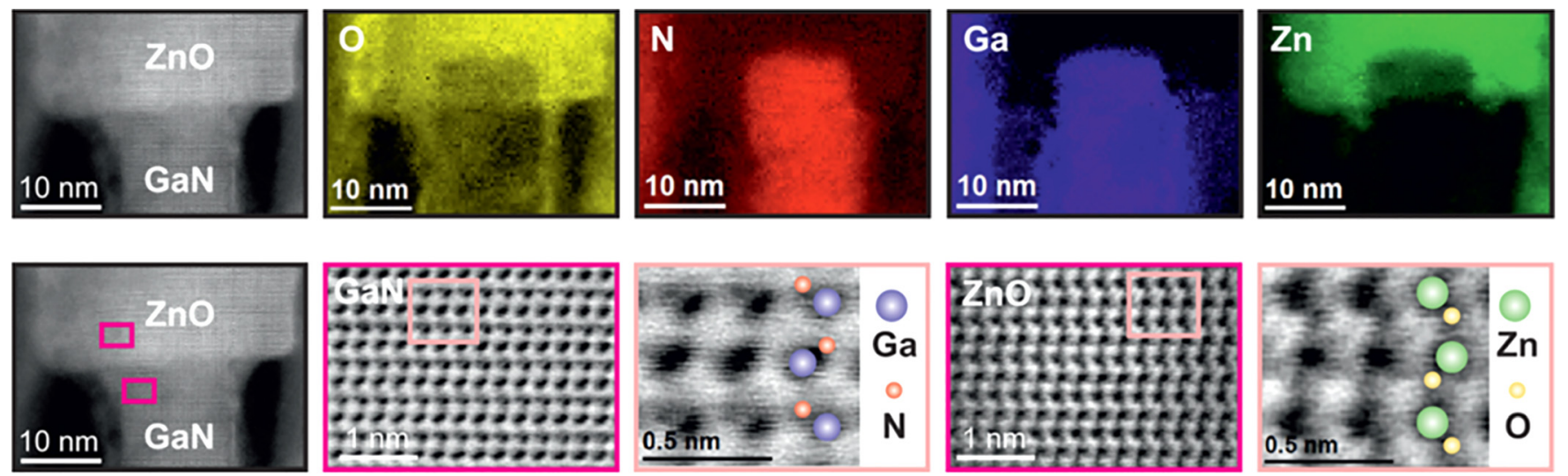

FIG. 73. HAADF-STEM image (left) of an exemplary p-GaN/n-ZnO heterojunction NW revealing a high-quality interface region. EELS maps identifying the different atomic species O, N, Ga, and Zn (top row, 2-5). In aberration-corrected ABF-STEM, a polarity inversion is observed from N-polar GaN to Zn-polar $\mathrm{ZnO}$ (bottom row, 2-5). Reprinted with permission from Schuster et al., ACS Nano 8, 4376 (2014). Copyright 2014 American Chemical Society.

on top of $\mathrm{Zn}$-polar $\mathrm{ZnO}$ regions of the templates, whereas no growth occurred on top of O-polar $\mathrm{ZnO}$ regions. Interestingly, Schuster et al. showed by ABF-STEM that Znpolar ZnO NWs are formed by MBE on top of N-polar GaN NWs through the nucleation of IDBs, as shown in Fig. 73. The polarity inversion was assigned to the high binding energy of the $\mathrm{N}-\mathrm{O}$ bonds. ${ }^{595}$ In contrast, Käbisch et al. revealed that $\mathrm{Zn}$-polar $\mathrm{ZnO} \mathrm{NWs}$ and $\mathrm{Zn}$-polar $\mathrm{ZnO}$ nanowalls are formed by PLD on top of O- and $\mathrm{Zn}$-polar $\mathrm{ZnO}$ buffer layers, respectively, for which the polarity was controlled by the growth temperature and through the use of $\mathrm{Al}$ doping. ${ }^{596,597}$ Baxter et al. also revealed by CBED measurements the formation of $\mathrm{Zn}$-polar $\mathrm{ZnO} \mathrm{NWs}$ from $\mathrm{Zn}$ vapor and air plasma on top of a zinc foil heated at $500{ }^{\circ} \mathrm{C} .{ }^{598}$ Eventually, de la Mata et al. reported by ABF-STEM measurements that $\mathrm{ZnO}$ NWs grown by vapor phase transport and condensation are $\mathrm{Zn}$-polar on a layered-structured muscovite mica. $^{599}$

Tetrapod-like structures: Such a difference in the surface reactivity on top of $\mathrm{O}$ - and $\mathrm{Zn}$-polar $\mathrm{ZnO}$ nucleation surfaces was also used to grow other types of nanostructures. It is well-known that octahedral multiple twin (octa-twin) $\mathrm{ZnO}$ nuclei are formed by sublimating $\mathrm{Zn}$ powders at high temperature in an oxygen atmosphere. ${ }^{600}$ These octa-twin nuclei are composed of eight tetrahedral-shaped crystals, each consisting of three $\{11 \overline{2} 2\}$ pyramidal surfaces and one $\pm(0001)$ side surface. They are formed by connecting the eight tetrahedral-shaped crystals in such a way that the eight free surfaces are alternately O- and Zn-polar ZnO planes. Dai et al. reported the fabrication of the so-called tetrapod-like $\mathrm{ZnO}$ nanostructures, by forming $\mathrm{ZnO}$ NWs exclusively on the four $\mathrm{Zn}$-polar $\mathrm{ZnO}$ nucleation surfaces. ${ }^{601,602}$

1.2. Self-catalyzed approach. NWs and nanoribbons: Another close illustrative example arises from the use of as-

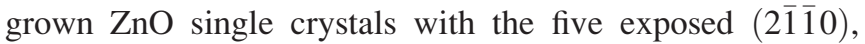
$\pm(01 \overline{1} 0)$, and $\pm(0001)$ nucleation surfaces. Following the catalyst-assisted approach by mixing $\mathrm{ZnO}$ and $\mathrm{SnO}_{2}$ powders as a source material, Gao and Wang revealed that both $\mathrm{O}$ and $\mathrm{Zn}$-polar $\mathrm{ZnO}$ nucleation surfaces are chemically active, which is in contrast to the self-assembled approach discussed above. $^{603} \mathrm{ZnO} \mathrm{NWs}$ and pyramidal nanotrunks were, respectively, grown on top of the $\mathrm{Zn}$ - and O-polar $\mathrm{ZnO}$ nucleation surfaces, as presented in Fig. 74, both of them being assisted by tin catalyst particles within a vapor-liquidsolid (VLS) mechanism. ${ }^{603}$ The distinct shapes of $\mathrm{ZnO}$ nanostructures were explained by the different contact angles between the charged tin catalyst particles and the polar $\mathrm{ZnO}$ nucleation surfaces, originating from the significant electrostatic interactions. In both cases, the strong adhesion forces are favorable for the deposition of tin catalyst particles. However, the small electrostatic repulsion between the charged tin catalyst particles and the $\mathrm{Zn}^{2+}$-terminated $\mathrm{Zn}$ polar $\mathrm{ZnO}$ nucleation surface results in a larger contact angle accounting for the growth of $\mathrm{ZnO}$ NWs. In contrast, the
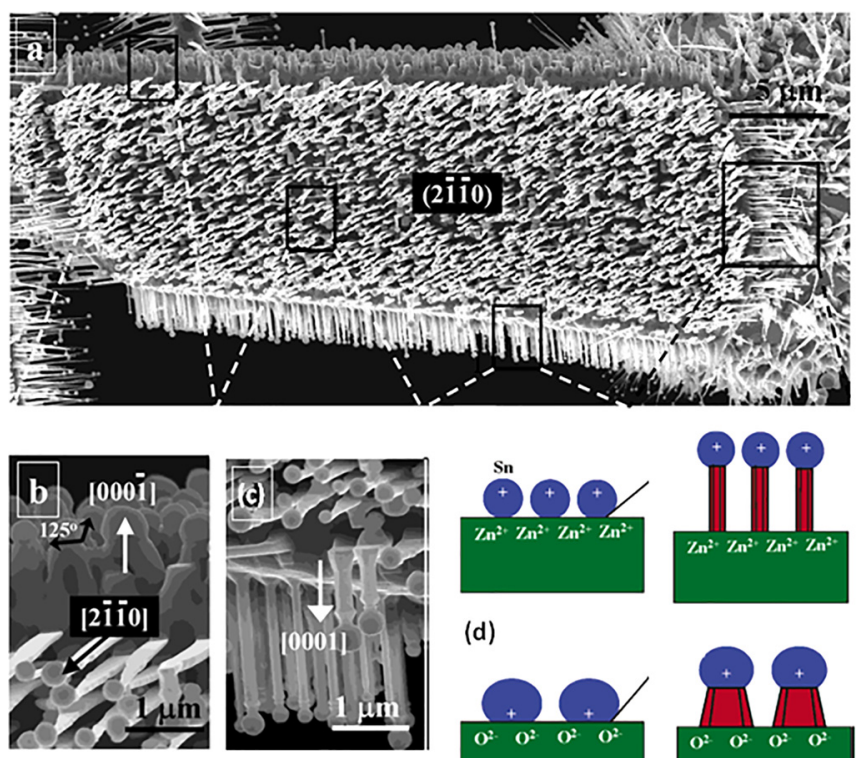

(d)

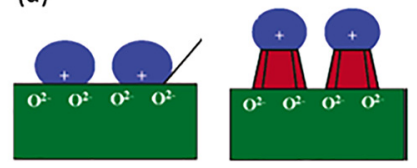

FIG. 74. (a) SEM image of as-grown $\mathrm{ZnO}$ nanostructures formed on the asgrown $\mathrm{ZnO}$ single crystals with the five exposed $(2 \overline{1} \overline{1} 0), \pm(01 \overline{1} 0)$, and $\pm(0001)$ nucleation surfaces. (b) SEM image with higher magnification of pyramidal $\mathrm{ZnO}$ nanotrunks on top of the $(000 \overline{1})$ nucleation surface. (c) SEM image with higher magnification of $\mathrm{ZnO}$ NWs on top of the (0001) nucleation surface. (d) Schematic illustrating the formation mechanisms of pyramidal $\mathrm{ZnO}$ nanotrunks and $\mathrm{ZnO}$ NWs. Reprinted with permission from P. X. Gao and Z. L. Wang, J. Phys. Chem. B 108, 7534 (2004). Copyright 2004 American Chemical Society. 

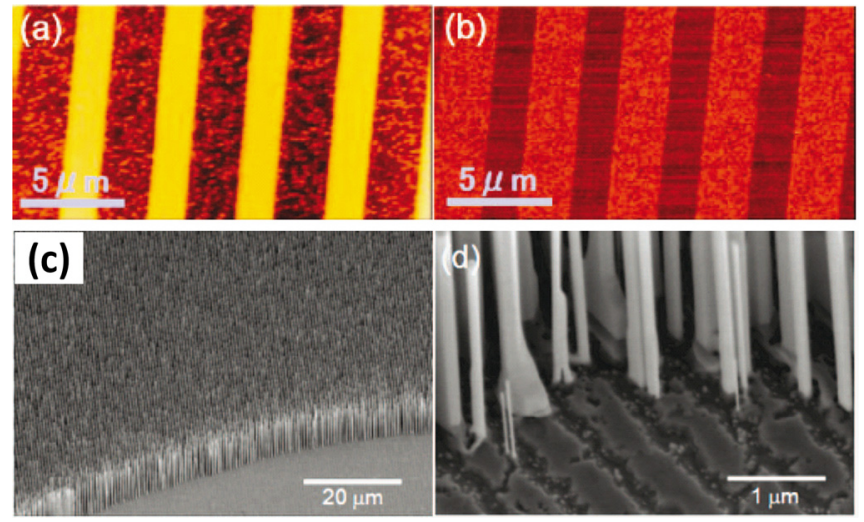

FIG. 75. (a) AFM and (b) PFM image of the surface of the PPI ZnO template. The bright (resp. dark) regions in AFM are $\mathrm{Zn}$ - (resp. O-) polar, while the bright (resp. dark) regions in PFM are O- (resp. Zn-) polar. (c) and (d) SEM image of $\mathrm{ZnO}$ NWs grown on the PPI ZnO template. Reprinted with permission from Lee et al., Nano Lett. 8, 2419 (2008). Copyright 2008 American Chemical Society.

small electrostatic attraction between the charged tin catalyst particles and the $\mathrm{O}^{2-}$ terminated $\mathrm{O}$-polar $\mathrm{ZnO}$ nucleation surface leads to a smaller contact angle. This accounts for the growth of $\mathrm{ZnO}$ pyramidal nanotrunks since the electrostatic attraction is expected to reduce as the distance between the charged tin catalyst particles and the $\mathrm{O}^{2-}$ terminated O-polar $\mathrm{ZnO}$ nucleation surface is increased. Furthermore, the formation of nanoribbons grown along the $[2 \overline{1} \overline{1} 0]$ and $\pm[01 \overline{1} 0]$ directions was also enhanced on the $(2 \overline{1} \overline{1} 0)$ and $\pm(01 \overline{1} 0)$ surfaces of the as-grown $\mathrm{ZnO}$ single crystals. ${ }^{603}$ By combining the deposition of $\mathrm{MgO}$ layers with different thicknesses by MBE with technological processes (i.e., lithography and etching) on top of sapphire, Lee et al. formed PPI ZnO templates by MBE. ${ }^{604}$ The selective growth of $\mathrm{Zn}$-polar $\mathrm{ZnO}$ NWs on top Zn-polar $\mathrm{ZnO}$ regions of the templates was achieved by carbothermal reduction and assisted by gold catalysts, while no growth occurred on top of O-polar $\mathrm{ZnO}$ regions, as shown in Fig. 75.

Nanopropellers: By mixing $\mathrm{ZnO}$ and $\mathrm{SnO}_{2}$ powders as a source material, Gao and Wang also demonstrated the catalyst-assisted growth of $\mathrm{ZnO}$ nanoribbons along the [0110] directions on top of the (011)0) sidewalls of $\mathrm{ZnO}$ NWs. ${ }^{605}$ The formation of $\mathrm{ZnO}$ nanoribbons is assisted by tin catalyst particles condensed from the tin vapor, leading to the formation of $\mathrm{ZnO} \mathrm{NW} /$ nanoribbon junctions. $^{605}$ The nanoribbon diameter is further increased along its growth axis owing to the continuous growth of tin catalyst particles. The addition of graphite as a reducing agent to form tin vapor from $\mathrm{ZnO}$ and $\mathrm{SnO}_{2}$ powders resulted in the catalystassisted growth of $\mathrm{ZnO}$ NWs along the [0001] direction with $(2 \overline{1} \overline{1} 0)$ sidewalls. ${ }^{606}$ Nanobelts along the [2 $\left.\overline{1} \overline{1} 0\right]$ direction were then grown similarly on these sidewalls by the help of tin catalyst particles, leading to the formation of $\mathrm{ZnO} \mathrm{NW/}$ nanobelt junctions. ${ }^{606}$ The secondary nucleation of $\mathrm{ZnO}$ NWs along the [0001] direction is exclusively achieved on top of the (0001) nucleation surfaces of these nanoribbons, resulting in the formation of the so-called nanopropellers, as presented in Fig. 76. ${ }^{605,607}$ Interestingly, the interfacial area between the tin catalyst particle and $\mathrm{ZnO} \mathrm{NW}$ or nanobelt

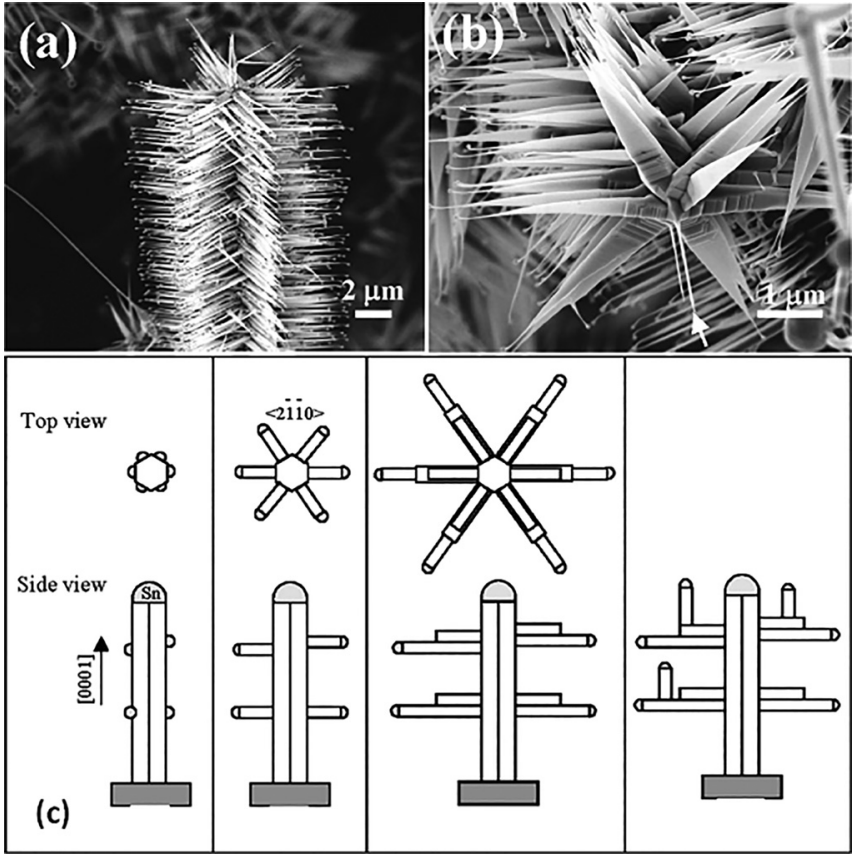

FIG. 76. (a) Tilted-view and (b) top-view SEM image of $\mathrm{ZnO}$ nanopropellers consisting of $\mathrm{ZnO}$ NWs grown along the [0001] direction with the $(01 \overline{1} 0)$ sidewalls, on which $\mathrm{ZnO}$ nanoribbons are nucleated along the $[01 \overline{1} 0]$ direction. (c) Schematic illustrating the formation mechanisms of $\mathrm{ZnO}$ nanopropellers. Reprinted with permission from Appl. Phys. Lett. 84, 2883 (2004). Copyright 2004 AIP Publishing LLC.

was also found to be partially crystalline, revealing that their growth direction and side surfaces are guided by the crystal orientation of the tin catalyst particle. ${ }^{608} \mathrm{ZnO} \mathrm{NWs}$ and nanobelts grown along the [0001] and [01 $\overline{1} 0]$ or $[2 \overline{1} \overline{1} 0]$ directions, respectively, were thus simultaneously formed from the partially crystallized tin catalyst particles.

b. Nanobelts, nanorings, nanospirals, and helical nanosprings. Nanobelts: The formation of $\mathrm{ZnO}$ nanobelts was demonstrated by Pan et al. by using $\mathrm{ZnO}$ powders as a source material according to the self-assembled approach. ${ }^{582}$ Nanobelts with no structural defects were grown along the [0001] direction with $(2 \overline{1} \overline{1} 0)$ and $(01 \overline{1} 0)$ side surfaces. More importantly, by adding $1 \% \mathrm{LiO}_{2}$ and/or $\mathrm{In}_{2} \mathrm{O}_{3}$ powders to the $\mathrm{ZnO}$ powder, polar-surface dominated nanobelts were fabricated along the $[2 \overline{1} \overline{1} 0]$ or $[01 \overline{1} 0]$ directions with $\pm(0001)$ top/bottom and $\pm(01 \overline{1} 0)$ or $(2 \overline{1} \overline{1} 0)$ side surfaces, respectively (see Fig. 77). ${ }^{609,610}$ While the development of $\pm(0001)$ polar side surfaces is not energetically favorable owing to their higher surface energy as compared with the $(01 \overline{1} 0)$ and $(2 \overline{1} \overline{1} 0)$ non-polar planes (see Sections IIE and IIF), the nucleation of basal-plane planar defects along the $[2 \overline{1} \overline{1} 0]$ or $[01 \overline{1} 0]$ growth axes can drastically enhance the growth rate along these directions. ${ }^{611}$ These planar defects were identified as head to head and tail to tail polar-IDBs; they originate from the formation of two vicinal In-O octahedral layers owing to the segregation of indium atoms coming from the $\mathrm{In}_{2} \mathrm{O}_{3}$ powder in the source material. ${ }^{611}$ Since the head to head and tail to tail polar-IDBs are generally paired, as revealed in Fig. 78, the nanobelt polarity is most often preserved on the $\pm(0001)$ side surfaces. The presence of 


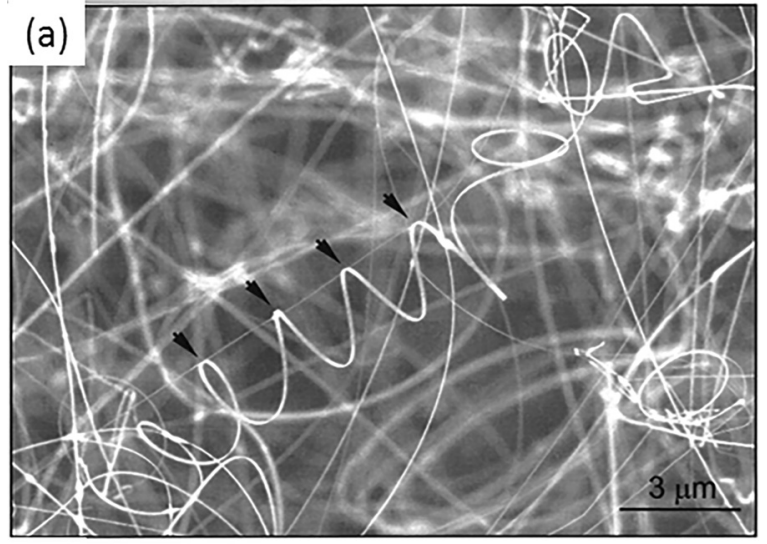

(b)
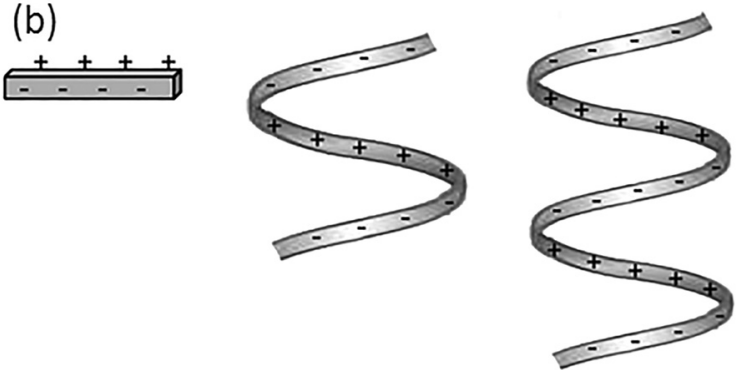

FIG. 77. (a) SEM image of ZnO helical nanosprings. (b) Schematic illustrating the formation mechanisms of $\mathrm{ZnO}$ helical nanosprings. Reprinted with permission from Appl. Phys. Lett. 84, 975 (2004). Copyright 2004 AIP Publishing LLC.

$\pm(0001)$ side surfaces induces a net dipole moment responsible for a significant electrostatic energy. In order to minimize or neutralize the overall dipole moment, the nanobelts are liable to roll over into a circular shape, which in turn

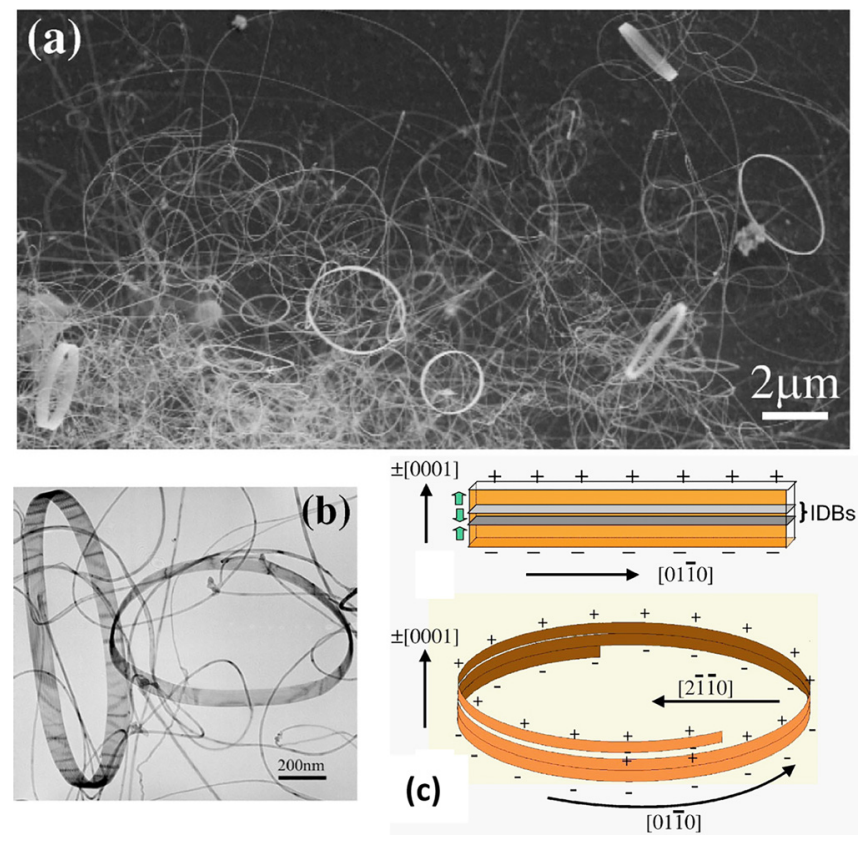

FIG. 78. (a) SEM image of $\mathrm{ZnO}$ nanobelts and nanorings. (b) TEM image of $\mathrm{ZnO}$ nanorings. (c) Schematic illustrating the formation mechanisms of $\mathrm{ZnO}$ nanorings from polar-surface dominated nanobelts grown along the $[01 \overline{1} 0]$ direction with head to head and tail to tail IDBs in their center. Reprinted with permission from Ding et al., Phys. Rev. B 70, 235408 (2004). Copyright 2004 American Physical Society. generates a significant elastic strain energy. ${ }^{584}$ The basalplane planar defects may lower the elastic strain energy by reducing the nanobelt bending modulus. It was thus suggested that the nanobelts are stable as long as such a reduction can compensate the increase in the surface energy owing to the development of the $\pm(0001)$ side surfaces. ${ }^{609-611}$

Nanorings, nanospirals, and helical nanosprings: These polar-surface dominated nanobelts were used as building blocks for fabricating a wide variety of nanostructures including nanorings, nanospirals, helical nanosprings, and seamless nanorings. ${ }^{584,609-612}$ The stable shape of these nanobelt-based nanostructures is related to the minimization of their total free energy (i.e., surface and elastic strain energy), which is strongly related to the aspect ratio of the nanobelt itself and especially to the relative size of the $\pm(0001)$ polar surfaces. ${ }^{611}$ Analytical and numerical computations can be found in Refs. 584 and 612 to predict the preferential shape formed. When the nanobelts exhibit $\pm(0001)$ side surfaces with a much higher surface area than the nonpolar top/bottom surfaces, Kong et al. showed that nanorings and nanospirals are formed. ${ }^{58,610}$ The fabrication of helical nanosprings was also reported, coming from the balance between the stretching repulsive forces in the center of nanorings and the pulling elastic strain forces, as shown in Fig. $77 .{ }^{610}$ When the nanobelts present $\pm(0001)$ top/bottom surfaces with a much smaller surface area than the non-polar side surfaces, Kong et al. demonstrated that seamless nanorings are formed through their loop-by-loop, co-axial, selfcoiling along the $[2 \overline{1} \overline{1} 0]$ or $[01 \overline{1} 0]$ directions, as presented in Fig. 78. ${ }^{609,611}$ The seamless nanorings can thus neutralize the local polar ionic charges of the $\pm(0001)$ surfaces and also decrease their surface area with respect to corresponding nanospring. It should be noted that the different shapes of nanostructures are often mixed on a given sample because the structural morphology of the nanobelts acting as building blocks is expected to change locally. Eventually, Hugues and Wang also reported the nucleation of polar-surface dominated nanobelts on the non-polar sidewalls of $\mathrm{ZnO} \mathrm{NWs}$, resulting in the formation of the so-called nanobows. ${ }^{612}$

\section{ZnO nanowires by chemical vapor deposition}

ZnO NWs have been grown by MOVPE by a number of teams in a variety of different conditions. ${ }^{613-622}$ The reader may refer to the review written by Sallet ${ }^{623}$ for a comprehensive review of the subject. When measured, the catalyst-free MOVPE grown NWs using $\mathrm{DEZn} / \mathrm{N}_{2} \mathrm{O}$ precursors were found to be of $\mathrm{Zn}$ polarity ${ }^{621,622,624}$ as found as well for a number of other growth methods, PLD, for instance, ${ }^{625,626}$ CVD, ${ }^{627,628}$ catalyst-assisted $\mathrm{CVD}^{629}$ and also by Baxter et al. ${ }^{598}$ (air plasma and $\mathrm{Zn}$ evaporation).

In order to understand where the Zn polarity of the NWs originates from, the growth morphology was followed as a function of time ${ }^{621}$ for MOVPE growth on c-sapphire substrates (with DEZn and $\mathrm{N}_{2} \mathrm{O}$ as precursors). As evidenced in other MOCVD grown NWs, but also observed in PLD ones, ${ }^{626}$ a thin (about $5 \mathrm{~nm}$ ) 2D wetting layer is first formed, with small pyramids and NWs. Following this first step, the 
NWs and pyramids grow in size, most of the NWs apparently sitting on top of the pyramids. CBED revealed that the pyramids were of $\mathrm{O}$ polarity, while the NWs were consistently of Zn polarity. This is contrary to the case of $\mathrm{GaN}$, where pyramids are of cation (Ga) polarity, and NWs most commonly (but not always), anion (N) polar (see Section IV C 1). To our knowledge, Ga-polar wires sitting on Npolar pyramids have never been obtained. In Ref. 622, the NW growth was carried out on O-polar templates on sapphire, on O-polar bulk substrates, and directly on $c$-sapphire: whatever the type of substrate/template, pyramids were consistently of $\mathrm{O}$ polarity while NWs were of $\mathrm{Zn}$ polarity (Fig. 79). A closer look by HRTEM demonstrates that, for growth on $\mathrm{ZnO}$ templates and $\mathrm{ZnO}$ substrates, the NWs originate mainly from the top of the pyramids and, for only $10 \%$ of them, from either the template (or substrate)/ pyramid interface or from a certain height within the pyramids. In the first case, the IDB at the base of the wire, i.e., at the NW/pyramid interface, moves up as growth proceeds, and the NWs always originate from the top of the pyramid. This is most probably because it is energetically favorable for this small surface IDB to move up the pyramid rather than forming two elongated "vertical" IDBs on the side of an embedded wire. A closer look at the structure of the IDB lying at the base of the wire reveals that it has a triangular shape, which resembles the type of IDBs encountered in heavily Mg-doped GaN layers, ${ }^{630}$ indicating that impurity segregation might be responsible for the polarity reversal. Knowing that $\mathrm{Al}$ doping can also revert the $\mathrm{ZnO}$ polarity, ${ }^{631}$ the authors proposed that the IDB might be formed because of $\mathrm{Al}$ segregation at the top of the growing pyramid. Al might come from the $\mathrm{ZnO}$ substrate or from the employed sapphire substrate. This would have to be confirmed using, for instance, heavily $\mathrm{Al}$-doped $\mathrm{ZnO}$ templates for enhancing this segregation effect on the polarity.

On sapphire, on the one hand, a greater proportion of the wires originate from the sapphire/pyramid interface or from within the pyramids themselves. Since sapphire is non polar, and as is well-known from the literature (see Section IV B 3), the two polarities can be obtained by direct growth. Laterally growing O-polar domains would encounter $\mathrm{Zn}$-polar inclusions, which, because of their higher vertical growth rates,

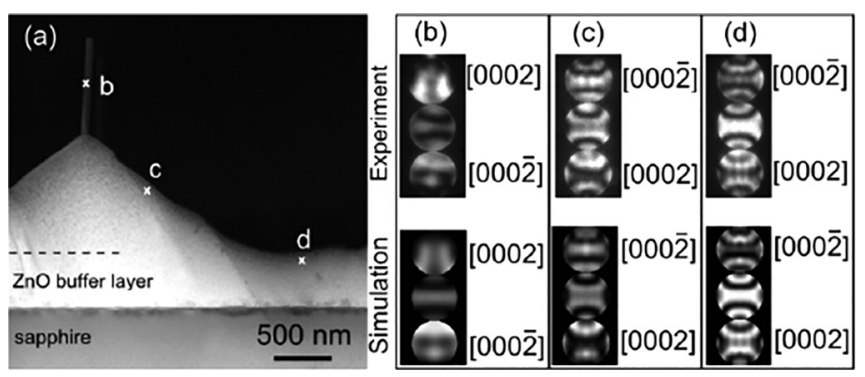

FIG. 79. (a) Cross-sectional STEM image of a $\mathrm{ZnO}$ NW sitting on top of a pyramid when growth is carried out on a $\mathrm{ZnO}$ buffer layer on c-sapphire, (b)-(d) experimental (topline) and simulated (bottom line) CBED patterns for the points labeled b, c, and d on the cross-sectional STEM image. Reprinted with permission from Perillat-Merceroz et al., Nanotechnology 23, 125702 (2012). Copyright 2012 Institute of Physics Publishing. yield Zn-polar wires embedded in O-polar pyramids. As in the case of growth on $\mathrm{ZnO}$ substrates, $\mathrm{Al}$ out-diffusion from the sapphire substrate could also be partly responsible for the formation of the wires at a certain distance from the interface. These observations emphasized the role that the chemistry (doping and surface segregation) and structure (sapphire steps) of the substrate or template surface might have on the polarity selection.

On the other hand, beyond the influence that the substrate may have (through its surface structure and its chemistry), the polarity selection also depends on the growth approach (i.e., self-assembled or catalyst-assisted). For instance, catalystassisted (Au) NWs grown by MOVPE on $c$-oriented $\mathrm{ZnO}$ substrates (with $\mathrm{DEZn} / \mathrm{N}_{2} \mathrm{O}$ as precursors) were found to have $\mathrm{O}$ polarity, while catalyst-free NWs grown in the same growth run exhibited $\mathrm{Zn}$ polarity (Fig. 80). This observation (i.e., the effect of catalyst on the polarity selection) is independent upon the substrate, since the same polarities were found for catalyst-assisted and catalyst-free NWs grown directly onto cplane sapphire. In Ref. 632, the binding energies of $\mathrm{Zn}$ on $\mathrm{Au}$ covered $c$-oriented $\mathrm{ZnO}$ were computed for the two $\mathrm{Zn}$ - and O-polar surfaces: the binding energy of $\mathrm{Zn}$ on the $(000 \overline{1}) \mathrm{O}$ polar surface is $0.38 \mathrm{eV}$ higher than on the (0001) Zn-polar surface, which hints that the chemical activity of $\mathrm{Au}$ on $\mathrm{ZnO}$ is more pronounced for the (0001) O-polar $\mathrm{ZnO}$ surface. The influence that the metallic droplet lying on top of the wire has on the polarity somehow strengthens the idea that the chemistry of the growth front itself bears a lot of significance for polarity selection rules. (a)
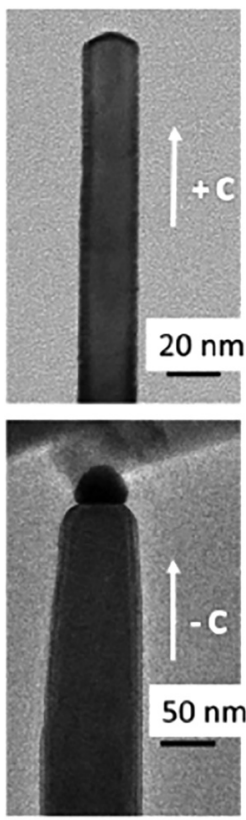

(d) (b)
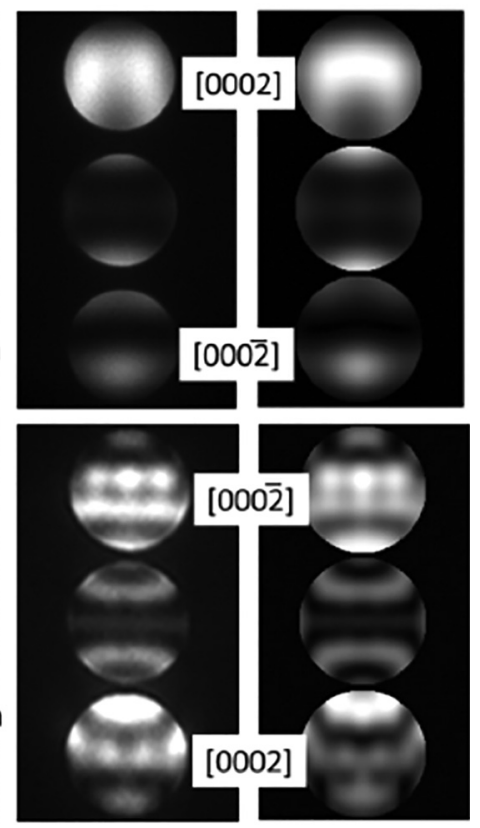

(e) (f)
FIG. 80. TEM images of (a) spontaneously formed $\mathrm{ZnO} \mathrm{NW}$, (d) of catalyst-assisted $\mathrm{ZnO} \mathrm{NW,} \mathrm{(b)} \mathrm{and} \mathrm{(c)} \mathrm{experimental} \mathrm{and} \mathrm{calculated} \mathrm{CBED}$ for spontaneous growth as well as (e) and (f) experimental and calculated CBED for catalyst assisted-growth. Reprinted with permission from Appl. Phys. Lett. 102, 182103 (2013). Copyright 2013 AIP Publishing LLC. 


\section{ZnO nanostructures by solution deposition techniques}

Low-cost and surface scalable solution deposition techniques like CBD or hydrothermal growth offer an alternative powerful route to form $\mathrm{ZnO} \mathrm{NWs}$ at low-temperature (i.e., $\left.<100{ }^{\circ} \mathrm{C}\right){ }^{581,591}$ Prior to the $\mathrm{ZnO} \mathrm{NW}$ growth by CBD, a $\mathrm{ZnO}$ nucleation surface is required in the form of $\mathrm{ZnO}$ single crystals or more usually polycrystalline $\mathrm{ZnO}$ thin seed layers. ${ }^{581,591,633-640}$ These seed layers, with a typical thickness of several tens of nanometers, are grown by sol-gel process (dip- or spin-coating), CVD, atomic layer deposition, or PVD techniques. ${ }^{581}$ Overall, the different types of $\mathrm{ZnO}$ nanostructures including NWs, nanobelts, nanotubes, nanorings, and hierarchical nanostructures are basically the result of (i) the different growth rates along the \pm [0001], [0110], and $[2 \overline{1} \overline{1} 0]$ directions as well as of (ii) the different reactivity on top of $\pm(0001),(01 \overline{1} 0)$, and $(2 \overline{1} 10) \mathrm{ZnO}$ nucleation surfaces, which is assisted by chemical additives.

a. Nanowires grown by chemical bath deposition. Polycrystalline $\mathrm{ZnO}$ seed layers consist of $\mathrm{ZnO}$ nanoparticles (NPs) that are preferentially oriented along the polar $c$-axis. Basically, the structural properties of $\mathrm{ZnO}$ NWs such as their position, vertical alignment, length, diameter, and period are strongly dependent upon the structural morphology of the $\mathrm{ZnO}$ seed layer. ${ }^{633-637}$ Guillemin et al. revealed by TEM and XRD measurements that $\mathrm{ZnO} \mathrm{NWs}$ are epitaxially grown on the free surface of ZnO NPs. ${ }^{637}$ It was further shown by x-ray pole figures that the vertical alignment of $\mathrm{ZnO} \mathrm{NWs}$ is improved by texturing the $\mathrm{ZnO}$ seed layer along the polar \pm [0001] direction, suggesting that the polar $\pm(0001)$ planes are highly active chemically. Since the $\mathrm{ZnO}$ seed layer is polycrystalline and hence exhibits a wide range of ZnO NPs oriented along directions other than \pm [0001], the formation of nanostructures was further investigated on top of (0001), $(000 \overline{1}),(01 \overline{1} 0)$, and $(2 \overline{1} \overline{1} 0)$ plane $\mathrm{ZnO}$ single crystals. ${ }^{638}$ It was found that the epitaxial formation of $\mathrm{ZnO}$ NWs occurs on top of O-polar $\mathrm{ZnO}$ single crystals, while more complex $\mathrm{ZnO}$ nanostructures including NWs are epitaxially grown on top of $\mathrm{Zn}$-polar $\mathrm{ZnO}$ single crystals, as shown in Fig. 81. The Opolarity of $\mathrm{ZnO} \mathrm{NWs}$ on O-polar $\mathrm{ZnO}$ single crystals was assessed by CBED measurements; it represents the first demonstration that catalyst-free $\mathrm{ZnO}$ NWs can be also O-polar. ${ }^{638}$ In contrast, the epitaxial development of $\mathrm{ZnO} 2 \mathrm{D}$ layers was observed on (0110) and (21) 10$)$ plane $\mathrm{ZnO}$ single crystals. These findings enabled to revisit the growth on top of $\mathrm{ZnO}$ seed layers by revealing that the polar $\pm(0001)$ planes are actually active predominantly for the formation of $\mathrm{ZnO} N W s$ using CBD. Subsequently, Consonni et al. combined the SAG on pre-patterned polar $\pm(0001)$ plane $\mathrm{ZnO}$ single crystals using electron beam lithography and chemical etching with CBD. ${ }^{640} \mathrm{ZnO} \mathrm{NW}$ arrays with a great structural uniformity and a high optical quality were formed, as presented in Fig. 82. Their polarity is controlled by transferring the polarity of $\mathrm{ZnO}$ single crystals, which was a very significant step towards the ultimate fabrication by design of well-organized heterostructures made from $\mathrm{ZnO}$ NWs. ${ }^{640}$

While catalyst-free physical and chemical vapor deposition techniques exclusively result in the formation of

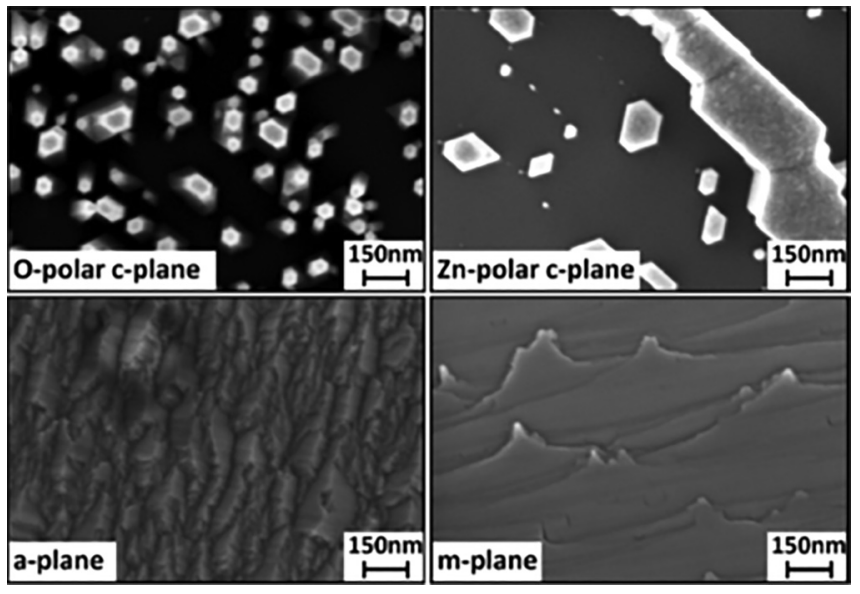

FIG. 81. Top-view SEM images of $\mathrm{ZnO}$ nanostructures grown on $\mathrm{O}$ - and $\mathrm{Zn}$-polar $\mathrm{ZnO}$ single crystals as well as on non-polar $(01 \overline{1} 0)$ and $(11 \overline{2} 0)$ plane $\mathrm{ZnO}$ single crystals. Reprinted with permission from Guillemin et al., J. Phys. Chem. C 117, 20738 (2013). Copyright 2013 American Chemical Society.

Zn-polar ZnO NWs according to the self-assembled approach, CBD hence offers the possibility to overcome their limitations by forming both $\mathrm{O}$ - and $\mathrm{Zn}$-polar $\mathrm{ZnO}$ NWs. The present progress can be accounted for by the chemical and physical processes at work. The CBD process is typically achieved by mixing two chemical precursors containing zinc (i.e., zinc nitrate or zinc acetate) and oxygen (i.e., ethanolamine, hexamethylenetetramine (HMTA), and caustic soda) in deionized water. ${ }^{581}$ The most widely used chemical precursors are zinc nitrate and HMTA. Upon heating (i) zinc ions are produced by the solubilization of zinc nitrate, while (ii) formaldehyde and ammonia are formed following the slow, gradual hydrolysis of HMTA acting as a $\mathrm{pH}$ buffer (i.e., the hydrolysis rate decreases as the $\mathrm{pH}$ is increased) ${ }^{581}$ Ammonia then reacts with water to produce $\mathrm{OH}^{-}$ions. ${ }^{581}$ An alternative route consists in considering the protonation of HMTA. The direct crystallization of $\mathrm{ZnO}$ proceeds according to the chemical reaction: $\mathrm{Zn}^{2+}+2 \mathrm{OH}^{-} \leftrightarrow$ $\mathrm{ZnO}_{\mathrm{S}}+\mathrm{H}_{2} \mathrm{O}{ }^{581,641}$ Following the reactant decomposition, the active chemical precursors in solution are thus basically the $\mathrm{OH}^{-}$and zinc $\left(\mathrm{Zn}^{2+}\right)$ ions that are charged by definition. These ions can strongly interact with the charged polar $\pm(0001)$ nucleation surfaces via electrostatic interactions. In contrast, such electrostatic interactions are much weaker on top of $\mathrm{ZnO}$ NPs grown along the [01 $\overline{1} 0]$ or [2 $\overline{1} \overline{1} 0]$ directions in the seed layer as well as on top of the $\mathrm{Si}_{\mathrm{x}} \mathrm{N}_{\mathrm{y}}$ or $\mathrm{SiO}_{2}$ mask. Indeed, the net surface charge in aqueous solution is dependent upon the crystal orientation and its chemical nature. This is favorable for the nucleation of $\mathrm{ZnO}$ NWs: (i) on top of $\mathrm{ZnO}$ NPs grown along the polar \pm [0001] direction in the seed layer during the spontaneous growth; (ii) inside the openings in the mask during the SAG. The growth rate of $\mathrm{ZnO}$ NWs is also larger axially along the polar [0001] direction than radially along the non-polar $\langle 10 \overline{1} 0\rangle$ directions.

Following such an approach, Wu et al. more recently reported the formation of O- and $\mathrm{Zn}$-polar $\mathrm{ZnO} \mathrm{NWs}$ on top of N- and Ga-polar GaN single crystals. ${ }^{642}$ The O-polar ZnO planes were found to be more stable than the $\mathrm{Zn}$-polar $\mathrm{ZnO}$ planes, accounting for their behavior during water 

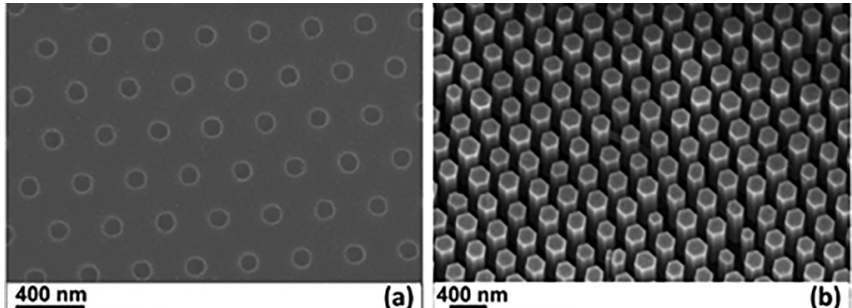

(a) $400 \mathrm{~nm}$

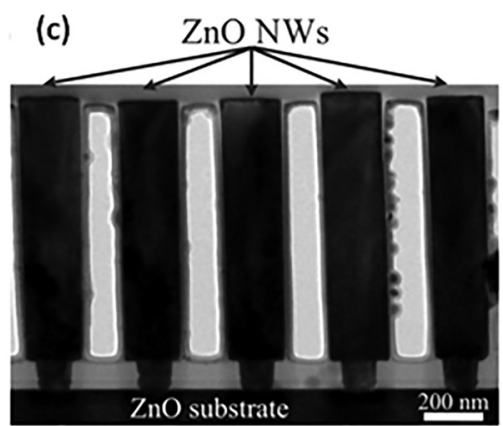

(d)

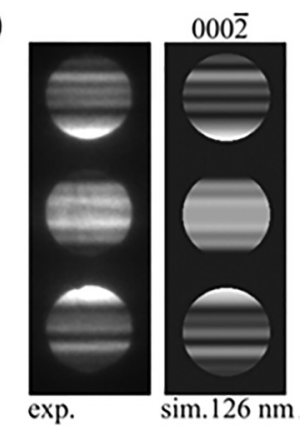

FIG. 82. (a) Top-view SEM image of patterned O-polar ZnO single crystals. (b) Tilted-view SEM image of $\mathrm{ZnO} N W$ s grown by CBD on patterned Opolar $\mathrm{ZnO}$ single crystals. (c) Low-magnification cross-sectional TEM image taken along the [10̄0] zone axis of these $\mathrm{ZnO} \mathrm{NWs}$. (c) Experimental and simulated CBED patterns taken along the [1010] zone axis for thickness of $126 \mathrm{~nm}$, showing that $\mathrm{ZnO}$ NWs are O-polar. The central disk is the transmitted electron beam. Reprinted with permission from Consonni et al., ACS Nano 8, 4761 (2014). Copyright 2014 American Chemical Society.

dissolution: in particular, $\mathrm{ZnO}$ nanotubes are only formed from Zn-polar ZnO NWs. Furthermore, x-ray absorption fine structure using synchrotron radiation was performed at the $\mathrm{O}-\mathrm{K}$ edge and revealed the surface termination of $\mathrm{ZnO}$ NWs. Although the polarity effects have been clarified over the last years on top of single crystals, the question of the polarity transfer from the seed layer composed of $\mathrm{ZnO}$ NPs to the polar $\pm[0001]$ direction is still open because the statistical measurements of polarity are required in such a case (i.e., resonant $\mathrm{XRD}$ at the $\mathrm{Zn}-\mathrm{K}$ edge in synchrotron, for instance). Up to now, $\mathrm{ZnO}$ NWs with the $\mathrm{Zn}$ polarity as deduced by CBED measurements were formed on top of $\mathrm{ZnO}$ seed layers grown by PLD with the Zn polarity ${ }^{634}$ and with an unknown polarity $^{625}$ as well as on top of silver films. ${ }^{643}$ Cherns and Sun further revealed the formation of $\mathrm{Zn}$-polar $\mathrm{ZnO}$ NWs by PLD on top of an O-polar ZnO underlayer deposited on sapphire through the presence of an incoherent boundary. ${ }^{626}$ The growth of these Zn-polar ZnO NWs was continued by CBD. ${ }^{626,644}$ Also, Rathore et al. reported that the polarity of ZnO NWs may be controlled by changing the chemical precursors used in solution. ${ }^{645}$ The polarity of $\mathrm{ZnO}$ NWs was mainly measured locally by CBED in the present works, in which statistical measurements are required.

b. Nanoplatelets, nanoneedles, and nanobelts. The control of the aspect ratio of $\mathrm{ZnO} \mathrm{NWs}$ and, more widely, of the shape of $\mathrm{ZnO}$ nanostructures grown by $\mathrm{CBD}$ such as nanoplatelets, nanoneedles, and nanobelts is typically achieved by incorporating additional chemical additives in solution. These chemical additives play a role that strongly depends on the polarity of $\mathrm{ZnO}$ nanostructures. ${ }^{646-654}$ They act as capping agents inhibiting the growth along a given direction at the benefit of another given direction. Polyethyleneimine $(\mathrm{PEI})^{646-648}$ and diamines like ethylenediamine ${ }^{649,650}$ can adsorb onto the non-polar $\{10 \overline{1} 0\}$ sidewalls of ZnO NWs, inhibiting the radial growth of $\mathrm{ZnO} N W s$ at the benefit of their axial growth. In contrast, chlorine ${ }^{651}$ and citrate ions, ${ }^{652,653}$ maleic acid as well as phenolphthalein ${ }^{654}$ typically adsorb onto the polar $\pm(0001)$ top facet of $\mathrm{ZnO} \mathrm{NWs}$, promoting the radial growth of $\mathrm{ZnO} \mathrm{NWs}$ at the expense of their axial growth. An illustrative example is given by the role of PEI, which is a positively charged non-polar polymer over a wide range of $\mathrm{pH}$ from 3 to 11 due to the protonation of its amino-side groups. Above the isoelectric point (IEP) (as defined by the $\mathrm{pH}$ at which the surface switches from a positive charge to a negative charge) of $\mathrm{ZnO}$, roughly ranging from 8 to $10,{ }^{655}$ it was suggested that PEI adsorbs onto the $\{10 \overline{1} 0\}$ sidewalls of $\mathrm{ZnO}$ NWs owing to strong electrostatic interactions and thus reduces to some extent their radial growth, as shown in Fig. 83. ${ }^{647}$ PEI also coordinates $\mathrm{Zn}$ ions and hence limits the homogeneous nucleation, favoring in turn the formation of longer $\mathrm{ZnO}$ NWs with higher aspect ratio by increasing the growth time, while avoiding to refresh the solution. ${ }^{648}$ An alternative approach to control the aspect ratio of $\mathrm{ZnO} \mathrm{NWs}$ consists in introducing negatively or positively charged metal-ions other than $\mathrm{Zn}$ ions in the solution. Joo et al. reported that the aspect ratio of $\mathrm{ZnO}$ NWs can lie in the range from 0.1 to 100 by using two types of ancillary metal sulfates forming complex ions, namely, A-type (i.e., $\mathrm{Cd}, \mathrm{Cu}, \mathrm{Mg}$, and $\mathrm{Ca}$ ) and B-type (i.e., $\mathrm{Al}, \mathrm{In}$, and $\mathrm{Ga}$ ) ions. ${ }^{656}$ Importantly, it was suggested by combining AFM and XPS measurements that the IEP of $\mathrm{ZnO}$ is actually dependent upon its crystal orientation, similar to sapphire and $\mathrm{TiO}_{2}{ }^{657,658}$ Basically, the polar $\pm(0001)$ top facet of $\mathrm{ZnO} \mathrm{NWs}$ is negatively charged, while their non-polar $\{10 \overline{1} 0\}$ sidewalls are still positively charged at $\mathrm{pH}=11$, because the IEP is higher on top of these non-polar $\{10 \overline{1} 0\}$ surfaces. Accordingly, negatively charged complex B-type ions inhibit the radial growth of $\mathrm{ZnO} \mathrm{NWs}$ by adsorbing onto their positively charged non-polar $\{10 \overline{1} 0\}$ sidewalls owing to strong electrostatic interactions, as shown in Fig. 84. ${ }^{656}$ In contrast, positively charged A-type ions preclude the axial growth of $\mathrm{ZnO} \mathrm{NWs}$ by adsorbing onto their negatively charged polar $\pm(0001)$ top facets. Eventually, the fabrication of $\mathrm{ZnO}$ nanoplatelets and nanoneedles was reported by following the use of charged metal-ions other than zinc ions in solution. ${ }^{656}$ Nanoplatelets were further formed by using citrate ions possessing three negative charges as capping agents, inhibiting the growth along the \pm [0001] direction. ${ }^{652,653}$ Zhang et al. showed that the oriented attachment of $\mathrm{ZnO}$ NPs can form nanoplatelets, which can further be piled up into nanobelts following an Ostwald ripening process. ${ }^{659} \mathrm{Xi}$ et al. also showed further the formation of $\mathrm{ZnO}$ nanobelts along the [0001] direction by using ethylenediamine as capping agents to inhibit the growth along the $[10 \overline{1} 0]$ direction. $^{660}$

c. Nanotubes, nanorings, and hierarchical nanostructures. More complex $\mathrm{ZnO}$ nanostructures, such as nanotubes and nanorings as well as hierarchical nanostructures, are 

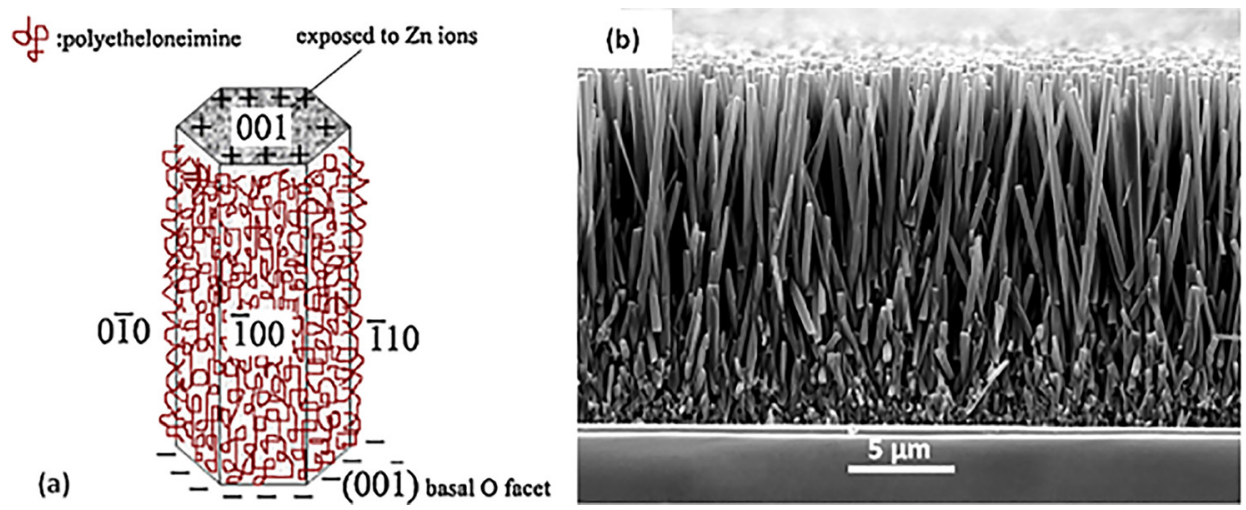

FIG. 83. (a) Schematic of the PEI adsorption onto the non-polar $\{10 \overline{1} 0\}$ sidewalls of $\mathrm{ZnO}$ NWs. Reprinted with permission from Zhou et al., Mater. Res. Bull. 43, 2113 (2008). Copyright 2008 Elsevier. (b) Cross-sectional SEM image of $\mathrm{ZnO}$ NWs grown for $7 \mathrm{~h}$ from a bath containing PEI. Reprinted with permission from $\mathrm{Xu}$ et al., J. Phys. Chem. C 114, 125 (2010). Copyright 2010 American Chemical Society.

formed by using a sequential nucleation and growth process involving at least two successive steps and benefiting from the different surface reactivity originating from polarity effects. The first step typically consists in the primary fabrication of $\mathrm{ZnO}$ NWs or nanoplatelets by CBD or electrodeposition as previously described.

For the nanotubes and nanorings, an etching process upon ageing is involved as the second step of the process. Their fabrication is thus limited kinetically by a competitive growth and dissolution process. It was stated that, at the onset of growth, the supersaturation is high enough to ensure a fast growth rate along the \pm [0001] direction, which is favorable for the formation of $\mathrm{ZnO}$ NWs. ${ }^{661-664}$ However, as growth proceeds, the supersaturation is reduced, the dissolution rate gets predominant, and the (0001) top facet of $\mathrm{ZnO}$ NWs is selectively etched due to its high surface energy, leading to the progressive formation of $\mathrm{ZnO}$ nanotubes as shown in Fig. 85. Additionally, She et al. also used the formation of $\mathrm{H}_{3} \mathrm{O}^{+}$ions in solution to perform the etching process as growth proceeds. ${ }^{665}$ The selective etching process of the polar (0001) surfaces was also attributed to the larger density of point defects in their center as compared with their edges. ${ }^{665}$ The role of nitrogen compounds was suggested for the formation of $\mathrm{ZnO}$ nanotubes. ${ }^{661}$ Similarly, the selective etching process can be used to form $\mathrm{ZnO}$ nanorings grown from $\mathrm{ZnO}$ nanoplatelets. ${ }^{666}$

For the hierarchical nanostructures, a secondary and even tertiary nucleation process is used either (i) by coating $\mathrm{ZnO}$ NWs with a $\mathrm{ZnO}$ seed layer or (ii) by using chemical additives in the solution. The deposition of c-axis oriented $\mathrm{ZnO}$ seed layers by ALD was performed all around $\mathrm{ZnO}$ $\mathrm{NWs}$ as a shell to induce the secondary nucleation of $\mathrm{ZnO}$ NWs along the [0001] horizontal direction during the third step of the process, forming complex hierarchical $\mathrm{ZnO}$ nanostructures. ${ }^{667,668}$ By repeating these second and third steps, Ko et al. developed the approach to form the so-called nanoforest or nanotree. ${ }^{669}$ Alternatively, by using citrate ions, ethylenediamine, or diaminopropane, the secondary nucleation of $\mathrm{ZnO}$ nanoplatelets and nanobranches is, respectively, initiated on the $\{10 \overline{1} 0\}$ sidewalls of $\mathrm{ZnO} \mathrm{NWs}$, forming again complex hierarchical nanostructures as shown in Figs. 86(a) and 86(b) ${ }^{653,670}$ In particular, it was stated that diaminopropane adsorbing on the $\{10 \overline{1} 0\}$ sidewalls of $\mathrm{ZnO}$ NWs can react with water to release hydroxide ions. This

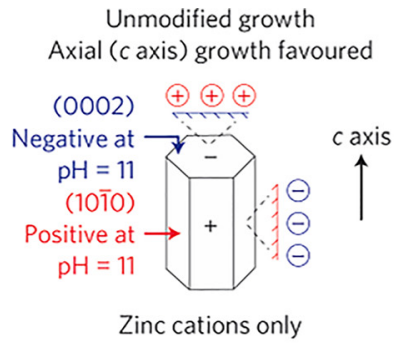

A-type metals

$>99 \%$ positive at $\mathrm{pH}=11$ Inhibit top face (0002)

Low aspect ratio
Axial growth suppressed $\oplus \oplus \oplus$

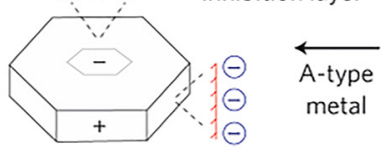

a

$$
\begin{aligned}
& \oplus \Theta \text { Zinc ions } \\
& \oplus \triangle_{(\mathrm{Cd}, \mathrm{Cu}, \mathrm{Mg}, \mathrm{Ca})}^{\text {A-type ions: }} \\
& \oplus \square_{(\mathrm{Al}, \mathrm{In}, \mathrm{Ga})}^{\text {B-type ions: }}
\end{aligned}
$$

A A A Z Crystal growth

(1)

b

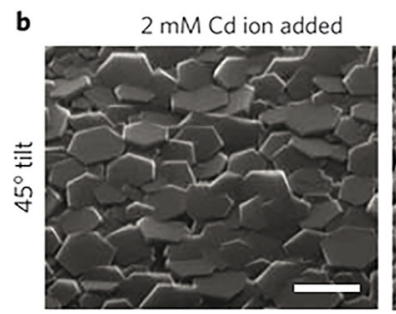

$1 \mathrm{mM}$ Cd ion added

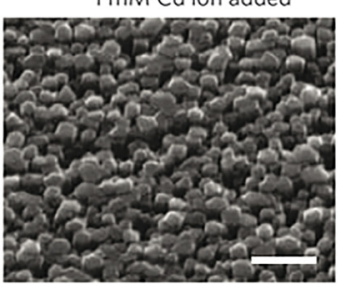

Unmodified (10 $\mathrm{mM}$ zinc ion)

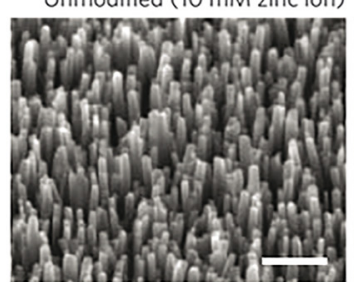

High aspect ratio Radial growth suppressed

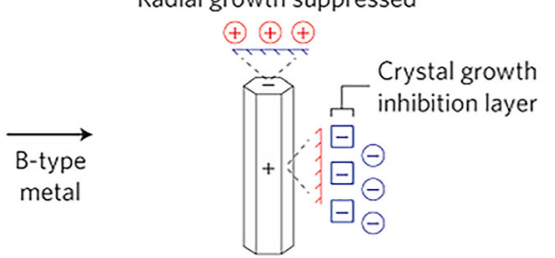

B-type metals $>99 \%$ negative at $\mathrm{pH}=11$ Inhibit sidewalls (1010)

FIG. 84. (a) Mechanism of control by non-zinc metal sulfates in the bath solution. A-type cations (positive at $\mathrm{pH}=11 ; \mathrm{Cd}, \mathrm{Cu}, \mathrm{Mg}$, and $\mathrm{Ca}$ ) suppress axial growth at the negative (0001) top facet by limiting zinc complex-ion access, thus promoting the formation of ZnO nanoplatelets. Similar interactions between B-type anions (negative at $\mathrm{pH}=11 ; \mathrm{Al}, \mathrm{In}, \mathrm{Ga}$ ) and the positive (0110) sidewalls lead to the formation of $\mathrm{ZnO}$ NWs with high aspect ratio. (b) SEM images of $\mathrm{ZnO}$ NWs grown in the presence of Cd or Al. Scale bars $=500 \mathrm{~nm}$. Reprinted with permission from Joo et al., Nat. Mater. 10, 596 (2011). Copyright 2011 Nature Publishing. 


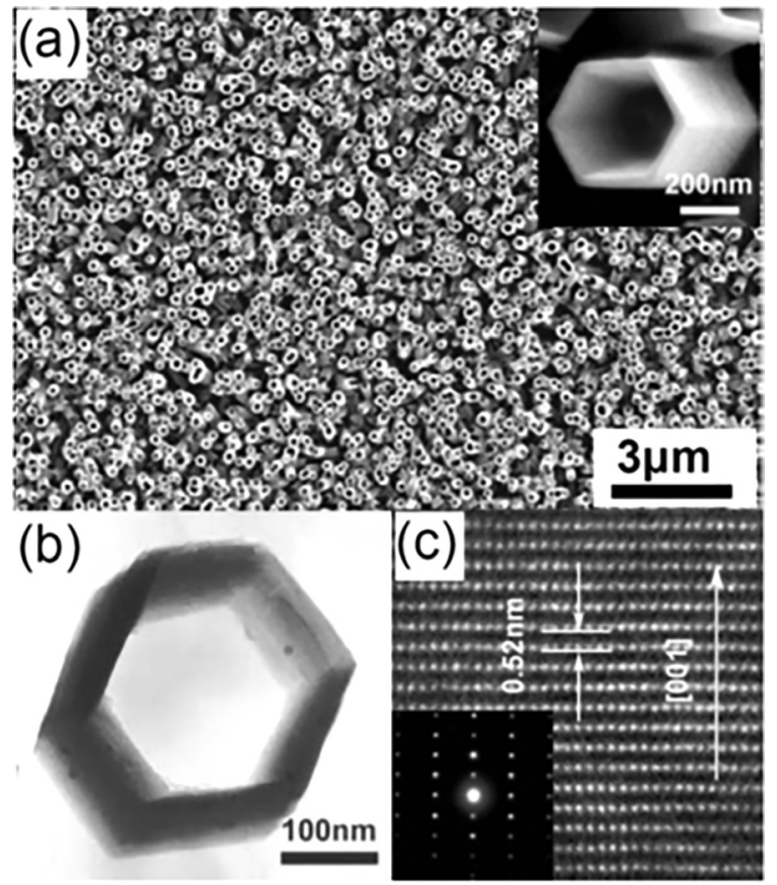

FIG. 85. (a) SEM image of ZnO nanotube arrays. (b) TEM and (c) HRTEM images of a $\mathrm{ZnO}$ nanotube. The inset is the corresponding selected area electron diffraction pattern. Reprinted with permission from Appl. Phys. Lett. 92, 053111 (2008). Copyright 2008 AIP Publishing LLC.

increases in turn the $\mathrm{pH}$ locally and, thus, favors the formation of $\mathrm{ZnO}$ NPs as seeds for the secondary nucleation of ZnO NWs as nanobranches. ${ }^{671}$ Zhang et al. further showed that the shape of complex hierarchical nanostructures can be tuned by adding alternatively citrate ions and diaminopropane to the solution and switching the addition sequence, as revealed in Figs. 86(c) and 86(d). ${ }^{672}$

Multilayered $\mathrm{ZnO}$ hierarchical nanostructures were also formed by connecting $\mathrm{ZnO} \mathrm{NWs}$ between $\mathrm{ZnO}$ disks acting as a seed layer. ${ }^{673}$ Furthermore, Xu et al. reported an original approach in order to grow $\mathrm{ZnO}$ NWs with a length of more than $40 \mu \mathrm{m}$, as presented in Fig. 87. ${ }^{674}$ A hydrophobic selfassembled monolayer (SAM) from octadecyltrichlorosilane precursors was deposited to coat and protect $\mathrm{ZnO}$ NWs. After a ultra-violet ozone treatment concentrated on top of $\mathrm{ZnO} \mathrm{NWs}$, the SAM is only removed on the top of $\mathrm{ZnO}$ NWs. This favors the axial growth of $\mathrm{ZnO}$ NWs placed in a refreshing solution, while their radial growth is still hampered by the presence of the SAM on their $\{10 \overline{1} 0\}$ sidewalls. The present process can be repeated multiple times and the SAM is burned by calcination at the end of the process.

\section{ELECTRO-OPTICAL PROPERTIES AND DEVICES}

In the present section, the effects of polarity on the electro-optical properties of layers and heterostructures and on the performances of the related optoelectronic and electronic devices, such as LEDs, solar cells, and HEMTs, are discussed. It should be noted that the devices presented here are exclusively composed of GaN (and related compounds) heterostructures. Indeed, nitride devices have already
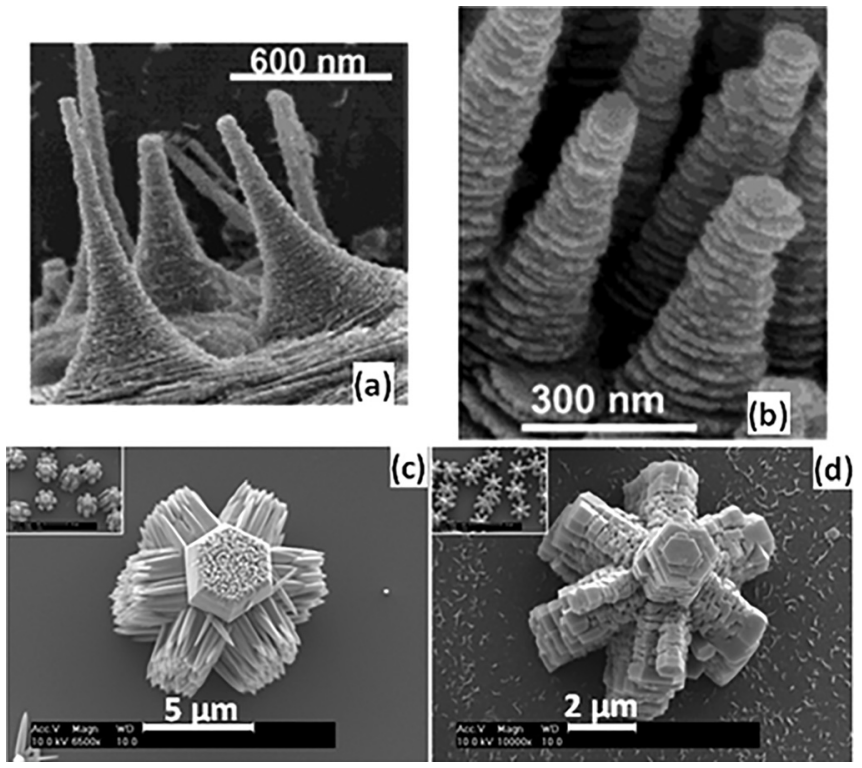

FIG. 86. (a) and (b) SEM images of ZnO helical NWs grown on oriented $\mathrm{ZnO}$ single crystals by using citrate ions. Reprinted with permissions from Tian et al., J. Am. Chem. Soc. 124, 12954 (2002). Copyright 2002 American Chemical Society. (c) SEM image of hierarchical $\mathrm{ZnO}$ nanostructures by first using citrate ions and then diaminopropane according to a sequential nucleation and growth process. (d) SEM image of hierarchical $\mathrm{ZnO}$ nanostructures by first using diaminopropane and then citrate ions according to a sequential nucleation and growth process. Reprinted with permission from Zhang et al., J. Am. Chem. Soc. 128, 10960 (2006). Copyright 2006 American Chemical Society.

reached a high level of development and maturity with some of them being already commercialized on a large scale. In contrast, devices based on $\mathrm{ZnO}$ heterostructures or on WBG nanostructures, although of strong potential interest, are still in their infancy. Hence, controlling and/or using the effects of polarity to enhance the output of such devices have not been investigated in so much detail yet.

\section{A. ZnO electro-optical properties}

The following subsections address addresses the influence of polarity on the optical properties (Subsection V A 1), the dielectric response of the materials to optical excitation (Subsection V A 2), and the electrical properties (Subsection V A 3) with focus on bulk $\mathrm{ZnO}$ single crystals and epitaxial layers.

\section{Luminescence and absorption}

The study of the optical transitions in $\mathrm{ZnO}$ by luminescence and absorption spectroscopy, particularly at low temperatures, provides a rich source of information regarding the presence and chemical identity of impurities, intrinsic lattice defects, extended structural defects, strain, lattice vibrations, and electronic band symmetries. The excitonic fine structure of $\mathrm{ZnO}$, a consequence of the $\mathrm{VB}$ splitting due to the wurtzite crystal field and spin-orbit interaction, was studied as early as 1960 by Thomas and Hopfield ${ }^{675,676}$ and Reynolds et al. ${ }^{677}$ These works marked the dawn for countless following studies focusing on different regimes of the optical spectrum of $\mathrm{ZnO}$ from synchrotron-based deep UV 
(a)
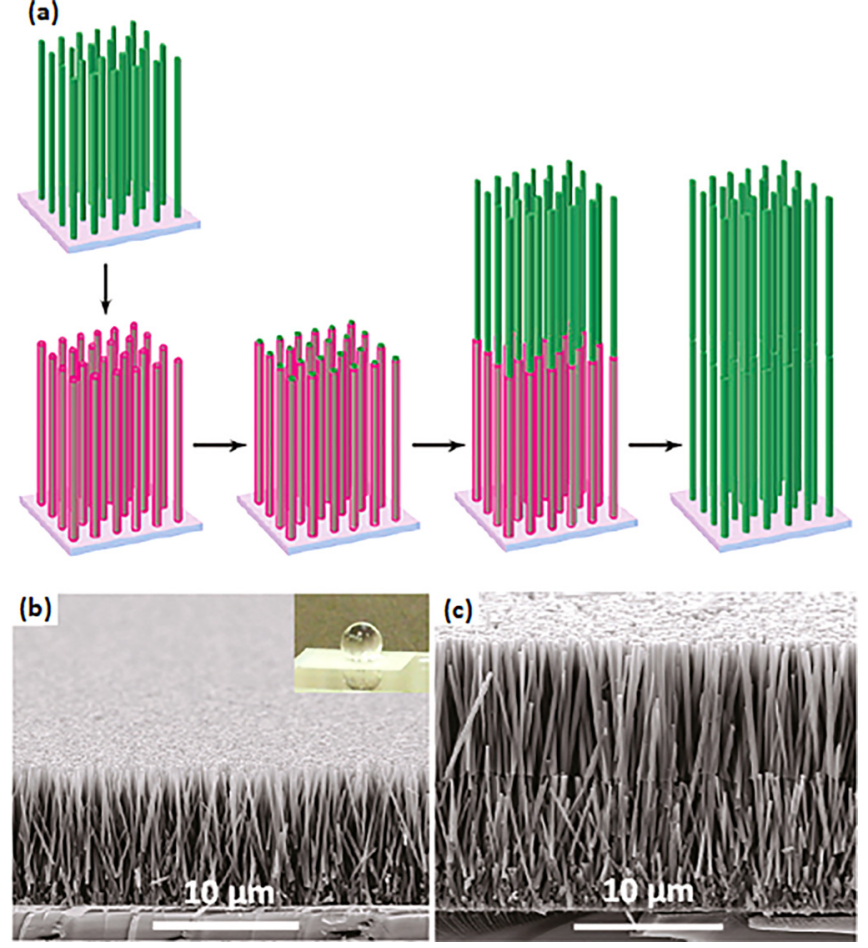

FIG. 87. (a) Schematic process for synthesizing a two-layer assembly of $\mathrm{ZnO} \mathrm{NW}$ arrays on transparent conductive oxides films. (i) Growth of the first-layer $\mathrm{ZnO} \mathrm{NW}$ array on seeded transparent conducting oxide. (ii) Coating of the ZnO NW array with SAMs. (iii) Selective removal of the SAM coating at the (0001) top facet of ZnO NWs while leaving the SAMs on their (0110) sidewalls. (iv) Growth of the second-layer ZnO NW array using the (0001) top facet of the first-layer $\mathrm{ZnO}$ NWs as seeds. The aqueous solution comes into contact with only the top facets of the first-layer NW and cannot enter the empty spaces between the $\mathrm{ZnO} N W$ s owing to the SAMs on their (0110) sidewalls. (v) Removal of the SAMs from the sidewalls of $\mathrm{ZnO} \mathrm{NWs}$ by calcination. (b) SEM image of the first-layer $\mathrm{ZnO}$ NW arrays by using PEI as a chemical additive. The inset is an optical image of the water droplet on the array after it was coated with the SAM, showing its hydrophobic character. (c) SEM images of the first- and second-layer $\mathrm{ZnO} \mathrm{NW}$ arrays. Reprinted with permission from Xu et al., J. Am. Chem. Soc. 133, 8122 (2011). Copyright 2011 American Chemical Society.

studies of the $\mathrm{Zn} 3 \mathrm{~d}$ valence bands, ${ }^{678}$ exciton-polariton and bound exciton transitions in the near UV, ${ }^{679-681}$ doping and defect related luminescence bands in the entire visible spectrum $^{680,682,683}$ to phonon absorption in the infrared. ${ }^{684}$ Naturally, many of these works reported on the optical properties of polar $\mathrm{ZnO}$ epilayers and single crystals; however, it was only in 2000 that the effects of polarity on the optical properties were addressed by a direct comparison of the excitonic luminescence of the two polar faces. ${ }^{685}$ Since then, several works were published by studying the optical properties of $\mathrm{Zn}$ - and O-polar $\mathrm{ZnO}$ in direct comparison to each other. ${ }^{686-698}$

Considering that the effects of polarity on the optical properties can be very subtle, the following discussion focuses exclusively on those works that provide a direct comparison between the optical properties of the two polar faces. At the beginning of this subsection, we review the effects of polarity on free and bound exciton transitions, followed by doping related donor-acceptor pair bands, and we conclude by addressing the influence of polarity on the omnipresent defect luminescence bands in $\mathrm{ZnO}$. It should be noted that while some processes such as impurity incorporation and doping are strongly affected by polarity and due to their modification of electronic states can be monitored (i.e., in the excitonic and defect luminescence), other properties, such as thermal conductivity or phonon dispersion relation, are governed by the $\mathrm{ZnO}$ lattice atoms and thus prove to be largely resilient to polarity changes. Consequently, phononic aspects are only briefly addressed at the end of the present subsection.

The different surface properties of the two polar faces of $\mathrm{ZnO}$ (surface energy, number of dangling bonds, etc.) lead to large differences in the incorporation of impurities and doping efficiency (see Sections II and IV). These impurities and dopants typically introduce electronic states within the band gap of $\mathrm{ZnO}$ that correspond to characteristic luminescence and absorption features. In particular, low temperature PL measurements in the range of the bound excitons provide detailed information on the presence and chemical identity of impurities in $\mathrm{ZnO}$. Among those, the most common shallow donors are hydrogen, aluminum, gallium, and indium, ${ }^{680,699}$ but also nitrogen acceptors, ${ }^{700,701}$ extended structural defect, ${ }^{702}$ and basal plane SFs. ${ }^{703}$ The complex optical spectra exhibited by all these complexes can all be unambiguously identified, thus providing unique information that can be used to elucidate the differences in the impurity and defect properties of the two polar faces of $\mathrm{ZnO}$. However, the high sensitivity of the luminescence process to impurity concentrations, even in the $10^{14} \mathrm{~cm}^{-3}$ range, also allows to establish general tendencies in the optical spectra of the two polarities of $\mathrm{ZnO}$ as caused by the strong dependence of the impurity incorporation on the individual growth conditions. Nevertheless, there are some common features that are characteristic for the optical properties of each of the polarity of $\mathrm{ZnO}$.

The first comparative study between the optical properties of the two polarities by Sherriff et al. focused on unraveling the influence of polarity on the excitonic properties of bulk $\mathrm{ZnO}$ crystals. ${ }^{685}$ Whereas the bound exciton luminescence was found to be very similar between the two polarities, the authors observed two main differences: (i) the exclusive appearance of two lines on the low energy side of the neutral donor bound exciton transitions $\left(\mathrm{D}^{0} \mathrm{X}\right)$ in the O-polar $\mathrm{ZnO}$ sample, which were ascribed to phonon replicas of a particular $\left(\mathrm{D}^{0} \mathrm{X}\right)$ transition, and (ii) a considerably more intense emission of free excitons from the O-polar than from the $\mathrm{Zn}$-polar $\mathrm{ZnO}$ sample. A more recent work by Schirra et al. identified the two lines observed exclusively in the O-polar $\mathrm{ZnO}$ sample as band-acceptor transitions (e, $\left.\mathrm{A}^{0}\right)$ involving a basal plane SF and its first longitudinal optical (LO) phonon replica. ${ }^{703}$ In light of this work, it is likely that this interpretation also applies to the original work by Sheriff et al., ${ }^{685}$ thus indicating a higher density of basal plane SFs in their O-polar $\mathrm{ZnO}$ samples. However, there are not sufficient studies to confirm a general tendency regarding the density of such defects in O- vs. Zn-polar ZnO samples. In contrast, a multitude of more recent studies has focused on the comparison of the free and bound exciton luminescence between the two polarities.

Koida et al. studied the low temperature PL of $\mathrm{ZnO}$ epilayers grown homoepitaxially on $\mathrm{Zn}$ - and O-polar $\mathrm{ZnO}$ single crystals as well as heteroepitaxially grown O-polar 
$\mathrm{ZnO}$ epilayers on $\mathrm{ScAlMgO}_{4}{ }^{686}$ In both O-polar epilayers, the $\mathrm{A}$ and $\mathrm{B}$ exciton-polariton peaks were more pronounced as compared with the Zn-polarity in agreement with Sheriff et al., ${ }^{65}$ although no direct intensity comparison was provided. The free exciton lifetime was found to be longer in the O-polar $\mathrm{ZnO}$ with 33 ps for the homoepitaxial layer and $22 \mathrm{ps}$ for the heteroepitaxial layer as compared with $12 \mathrm{ps}$ for the $\mathrm{Zn}$-polar $\mathrm{ZnO}$ sample. The observation of a more pronounced free exciton luminescence in the O-polar $\mathrm{ZnO}$ samples was also confirmed by Matsui et al. for homoepitaxially grown $\mathrm{ZnO}$ on polished and annealed $\mathrm{ZnO}$ substrates, in which the two peaks of the A exciton-polariton at $3.373 \mathrm{eV}$ and $3.377 \mathrm{eV}$ exclusively occurred in the O-polar $\mathrm{ZnO}$ epilayers. ${ }^{687}$ Sasaki et al. furthermore reported a larger intensity of ionized bound exciton lines $\left(\mathrm{D}^{+} \mathrm{X}\right)$ from the O-polar $\mathrm{ZnO}$ substrates. ${ }^{688}$ Common to these works is the absence of a well resolved bound exciton fine structure related to different impurity bound excitons. Instead, a rather broad $(D, X)$ band with comparable intensity and line widths of several $\mathrm{meV}$ was observed for both polarities.

Following these initial works from 2000 to 2006, a series of comprehensive works were subsequently published that reported a more detailed analysis of the optical emission lines in the different spectral ranges of $\mathrm{ZnO}$. The first work to directly compare well resolved bound exciton spectra with linewidths below $1 \mathrm{meV}$ for both polarities was published by Allen et al. on double-sided polished $\mathrm{ZnO}$ substrates from Tokyo Denpa Co. Ltd. as well as $\mathrm{Zn}$ - and O-polar polished $\mathrm{ZnO}$ crystals from Cermet, Inc. ${ }^{689}$ In contrast to earlier works, Allen et al. observed a significantly stronger free and ionized bound exciton emission in the $\mathrm{Zn}$-polar $\mathrm{ZnO}$ samples than in the O-polar $\mathrm{ZnO}$ samples. This difference was particularly pronounced in the Tokyo Denpa Co. Ltd. samples with a carrier concentration of $3 \times 10^{14} \mathrm{~cm}^{-3}$, but still visible in the samples from Cermet, Inc. with a carrier concentration of $3 \times 10^{16} \mathrm{~cm}^{-3}$. It was suggested that the increased free exciton emission in the $\mathrm{Zn}$-polar $\mathrm{ZnO}$ samples is caused by the presence of an inversion layer of holes which is formed when the upward band bending brings the VB close to the Fermi level. This effect should be particularly pronounced for low carrier concentrations when stronger band bending occurs to compensate the large spontaneous polarization of $\mathrm{ZnO}$. A more detailed discussion of the effects of polarity on the band bending is provided below.

Pronounced differences in the free and bound exciton luminescence were also reported by the same authors in a more recent publication. ${ }^{698}$ Annealing experiments in $\mathrm{O}_{2}$ atmosphere and forming gas up to $600^{\circ} \mathrm{C}$ provided additional insights in the effects of polarity on the optical fine structure of the near band-edge luminescence in $\mathrm{ZnO}$. Apart from the omnipresent neutral donor bound excitons related to the shallow donors in $\mathrm{ZnO}(\mathrm{H}, \mathrm{Al}, \mathrm{Ga}$, In), both excited states involving a hole from a $\mathrm{B} \mathrm{VB}^{704}$ as well as ionized donor bound excitons ${ }^{699}$ could be clearly distinguished (see Fig. 88). This study confirmed the previously observed higher intensity of the free and ionized bound exciton emission lines in asreceived $\mathrm{Zn}$-polar samples. In contrast to that, the excited states of the neutral bound excitons involving B VB holes were commonly found to be more intense although with larger line width in the O-polar $\mathrm{ZnO}$ samples as compared with the $\mathrm{Zn}$-polar $\mathrm{ZnO}$ samples. The intensity ratios between the luminescence lines of the two different polarities remained valid for different annealing temperatures and only equalized after annealing at $600^{\circ} \mathrm{C}$ under $\mathrm{O}_{2}$ atmosphere.

The observation of dominant free and ionized bound exciton transitions in $\mathrm{Zn}$-polar $\mathrm{ZnO}$ is in accordance with the previous reports by Wagner et al. ${ }^{694}$ and Lautenschläger et al. ${ }^{705}$ who studied the polarity dependent excitonic luminescence and impurity concentration in $\mathrm{Zn}$ - and O-polar homoepitaxial $\mathrm{ZnO}$ layers. The authors observed sharp bound exciton luminescence lines with a linewidth below $100 \mu \mathrm{eV}$ in $\mathrm{Zn}$-polar $\mathrm{ZnO}$ and broader impurity related exciton transitions in O-polar $\mathrm{ZnO}$ (Fig. 46(b), right). ${ }^{694}$ This observation was explained by secondary ion mass spectroscopy (SIMS) measurements of the simultaneously grown $\mathrm{Zn}$ and O-polar $\mathrm{ZnO}$ epilayers (Fig. 46, left). The concentration of $\mathrm{Li}, \mathrm{Na}, \mathrm{K}$, and $\mathrm{Ca}$ was found to be almost 2 orders of magnitude higher in O-polar as compared with the $\mathrm{Zn}$-polar $\mathrm{ZnO}$ epilayers. $\mathrm{Mg}, \mathrm{Al}$, and $\mathrm{Si}$ concentrations were found to be higher by about 1 order of magnitude. ${ }^{705}$ This demonstrated a more efficient impurity incorporation in the O-polar $\mathrm{ZnO}$ epilayers, potentially originating from the hot quartz reactor tubes in the growth process as well as in-diffusion from the substrate (see Section IV B 2). These findings in $\mathrm{ZnO}$ are in line with the case of $\mathrm{GaN}$, where it was also observed that polarity influences the impurity incorporation with 2 orders of magnitude higher incorporation rates for $\mathrm{C}, \mathrm{O}$, and $\mathrm{Al}$ in $\mathrm{N}$ polar GaN epilayers, while $\mathrm{Si}$ is more efficiently incorporated into Ga-polar GaN films ${ }^{706}$ (see Sections IV A 4 and IV A 5)

The temperature dependence of the near band-edge luminescence of $\mathrm{O}$ - and $\mathrm{Zn}$-polar $\mathrm{ZnO}$ was investigated by

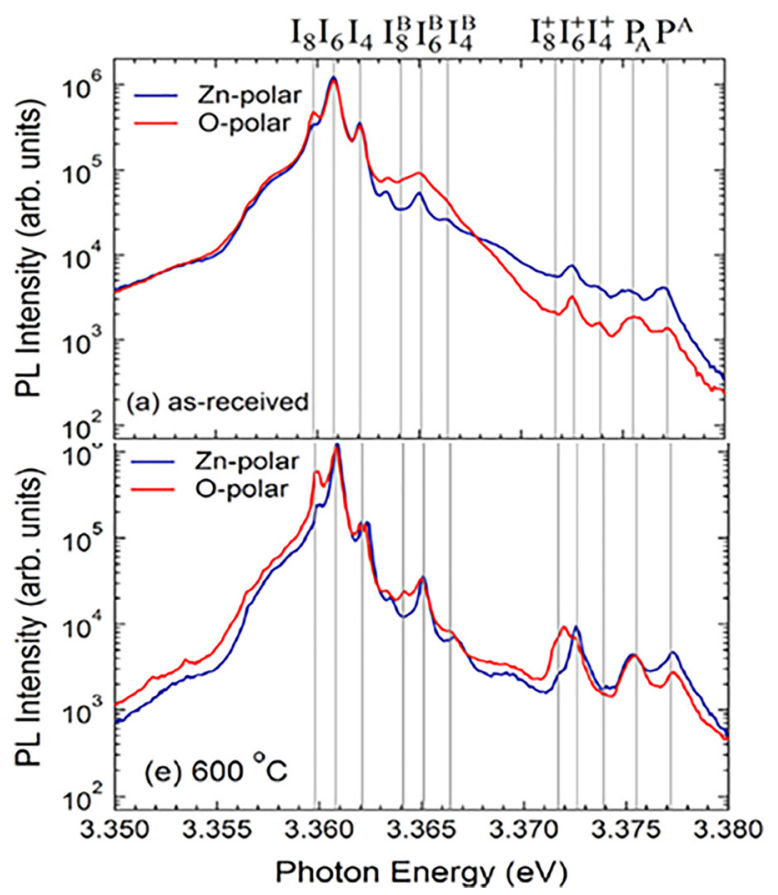

FIG. 88. Typical low-temperature $(8 \mathrm{~K})$ near band edge PL spectra taken from the $\mathrm{Zn}$-polar and O-polar faces of hydrothermal single crystal $\mathrm{ZnO}$ before and after annealing in oxygen gas for $90 \mathrm{~min}$ at different temperatures up to $600^{\circ} \mathrm{C}$. Reprinted with permission from Appl. Phys. Lett. 103, 231109 (2013). Copyright 2013 AIP Publishing LLC. 
Oh et al., ${ }^{691}$ Kwon et al. ${ }^{693}$ and Yamamoto et al. ${ }^{696}$ in the spectral range of the free excitons, bound excitons, and their LO phonon replicas. Based on the temperature dependence of the A free exciton linewidth, Oh et al. reported a higher exciton-phonon coupling strength for the $\mathrm{Zn}$ polarity as compared with the $\mathrm{O}$ polarity and suggested different impurity concentrations and electron accumulation/depletion due to the large spontaneous polarization of $\mathrm{ZnO}$ as potential explanation. ${ }^{61}$ These results were later challenged by Yamamoto et al., who studied the temperature dependence of the PL spectra of $\mathrm{Zn}$ - and $\mathrm{O}$-polar $\mathrm{ZnO}$ with focus on the phonon replicas of the exciton luminescence. ${ }^{696}$ The intensity ratios of the first and second phonon replica bands were found to be equal for the two polarities for any given temperature, indicating that the exciton-phonon coupling strength was independent upon the polarity.

In addition to the impurity related neutral and ionized bound excitons, several publications reported additional sharp luminescence lines in the spectral range from $3.33 \mathrm{eV}$ to $3.35 \mathrm{eV}$, among which the most common and intense transition $\left(\mathrm{Y}_{0}\right)$ is typically found at $3.333 \mathrm{eV}$. However, there are only very few works that reported this transition in comparative studies between the two polarities of $\mathrm{ZnO}$. Allen et al. observed a line at $3.332 \mathrm{eV}$ in $\mathrm{ZnO}$ substrates from Cermet, Inc., which was found to be much more intense in Zn polar than in O-polar $\mathrm{ZnO}$ samples. ${ }^{689}$ A similar observation was also reported by Kwon et al. ${ }^{693}$ who observed a sharp line at comparable energy that only appeared in the luminescence spectra of $\mathrm{Zn}$-face single crystals from Tokyo Denpa Co. Ltd., but not from the Oface of the same crystals. More recently, Wagner et al. published a comprehensive study on the properties of the $3.333 \mathrm{eV}$ and related transitions and showed that this line originates in fact from excitons bound to extended structural defects, which introduce additional donor states in $\mathrm{ZnO}^{702}$

The different predispositions for the incorporation of impurities in the two different polar faces of $\mathrm{ZnO}$ become especially important when high doping concentrations with shallow donors or shallow acceptors for transparent conductive oxides or p-type material shall be achieved. This is of particular importance in the later case, as $\mathrm{ZnO}$ tends to self-compensate acceptor doping, thus hindering the effective exploitation of the material in optoelectronic applications which require bipolar doping. Several works have shown that the incorporation of unwanted shallow donors is reduced, when using either non-polar a-plane $\{11 \overline{2} 0\}$ or $\mathrm{Zn}$ polar c-plane crystal orientations as compared with O-polar $\mathrm{ZnO}$ substrates. ${ }^{705,707}$ On the other hand, N, as the most prominent shallow acceptor with a binding energy of about $165 \mathrm{meV}$ in $\mathrm{ZnO}^{701}$ can be incorporated at much higher concentrations when growing on $\mathrm{Zn}$-polar $\mathrm{ZnO}$ substrates than on O-polar $\mathrm{ZnO}$ ones. ${ }^{697,698}$ The differences in the PL spectra of nitrogen doped $\mathrm{ZnO}$ layers grown homoepitaxially on O- and $\mathrm{Zn}$-polar $\mathrm{ZnO}$ substrates are shown in Fig. 89 for $\mathrm{N}$ concentrations up to $1 \times 10^{20} \mathrm{~cm}^{-3}$. ${ }^{697}$ In both cases, increasing $\mathrm{N}$ doping results in the appearance and intensity increase of a donor-acceptor pair (DAP) band at about $3.24 \mathrm{eV}$ and subsequently in the decreased intensity of the bound exciton luminescence. However, the changes for comparable $\mathrm{N}$ doping levels were found to be much more pronounced in the $\mathrm{Zn}$-polar $\mathrm{ZnO}$ epilayers. In fact, the spectrum of the highest $\mathrm{N}$ concentration in the O-polar $\mathrm{ZnO}$ layer $\left(1 \times 10^{20} \mathrm{~cm}^{-3}\right)$ resembles those of the $\mathrm{Zn}$-polar $\mathrm{ZnO}$ layer with about one order of magnitude lower $\mathrm{N}$ concentration $\left(1-2 \times 10^{19} \mathrm{~cm}^{-3}\right)$, as seen in Fig. 89 . The disappearance of $(\mathrm{D}, \mathrm{X})$ luminescence for $\mathrm{N}$ concentrations larger than $5 \times 10^{19} \mathrm{~cm}^{-3}$ in the $\mathrm{Zn}$-polar $\mathrm{ZnO}$ epilayers was attributed to an increased concentration of free holes, which compensate the impurity related neutral donors. The approximately one order of magnitude higher free hole concentration in the $\mathrm{Zn}$-polar $\mathrm{ZnO}$ epilayers upon $\mathrm{N}$ doping ${ }^{697}$ corresponds well with the roughly one order of magnitude higher impurity concentration of shallow donors in O-polar $\mathrm{ZnO}$ epilayers, as shown in Fig. 46(a).$^{705}$ The difference in the $\mathrm{N}$ incorporation between the two polar faces could also be monitored by the appearance and intensity of $\mathrm{N}$ related modes in Raman spectroscopy. ${ }^{708}$
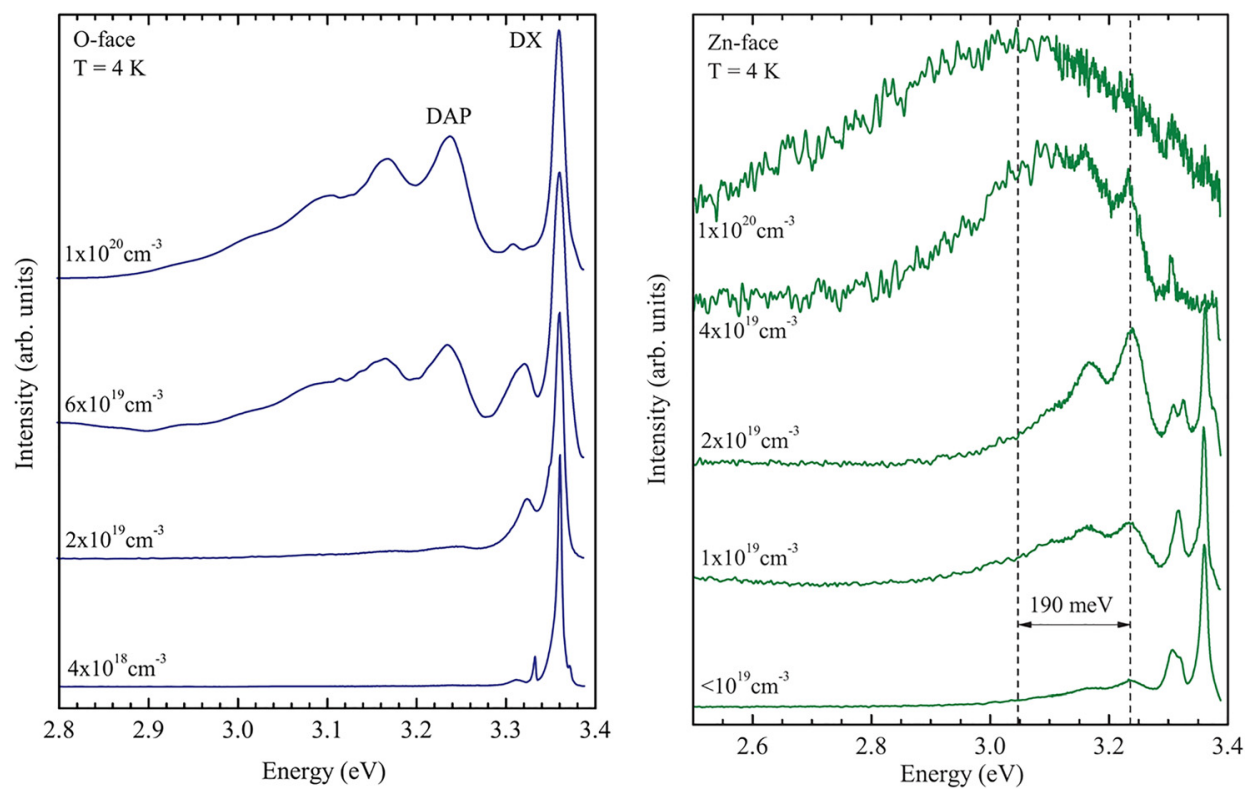

FIG. 89. Photoluminescence spectra of nitrogen doped $\mathrm{ZnO}$ epilayers grown on O-face (left) and Zn-face (right) $\mathrm{ZnO}$ substrates with increasing nitrogen concentration measured at $\mathrm{T}=4 \mathrm{~K}$. Reprinted with permission from Lautenschlaeger et al., Phys. Rev. B 85, 235204 (2012). Copyright 2012 American Physical Society. 
So far, we have focused the discussion on the influence of polarity on the near band edge spectral region (excitons and shallow DAP transitions); however, the visible luminescence commonly known as "green luminescence band," around $2.4 \mathrm{eV}$, was also reported to be sensitive to polarity. Figure 90 displays the green luminescence between $1.8 \mathrm{eV}$ and $2.9 \mathrm{eV}$ for both polarities and for different annealing temperatures, as reported by Allen et al. ${ }^{698}$ A two times stronger intensity of this defect luminescence band was observed in the as-received O-polar $\mathrm{ZnO}$ samples as compared with the $\mathrm{Zn}$-polar $\mathrm{ZnO}$ ones. Based on the fitting with two Gaussian functions, the authors identified two luminescence bands centered at about $2.0 \mathrm{eV}$ (orange band) and $2.36 \mathrm{eV}$ (green band). Upon annealing at about $400^{\circ} \mathrm{C}$, the differences in the luminescence between the two polarities disappeared. As the annealing temperature was raised, the intensity of the green band gradually decreased, whereas the intensity of the orange band remained almost unchanged. The intensity of the luminescence bands reached a minimum at annealing temperature of $500{ }^{\circ} \mathrm{C}$ for the O-polar $\mathrm{ZnO}$ samples and about $600{ }^{\circ} \mathrm{C}$ for the $\mathrm{Zn}$-polar $\mathrm{ZnO}$ samples. For higher annealing temperature, the unstructured green luminescence band was replaced by a structured, deep $\mathrm{Cu}$ acceptor related luminescence ${ }^{683}$ in samples of both polarities. Based on these observations, the authors suggested that the annealing process fills vacant $\mathrm{O}$ lattice sites $\left(\mathrm{V}_{\mathrm{O}}\right)$ and thereby reduces the potentially $\mathrm{O}$ vacancy related green luminescence, whereas the orange band, presumably related to $\mathrm{Zn}$ interstitials $\mathrm{Zn}_{\mathrm{i}}$, is not affected by annealing due to the higher activation energies of the $\mathrm{Zn}$ atoms.

The visible range of the luminescence spectrum of $\mathrm{Zn}$ and O-polar $\mathrm{ZnO}$ was also investigated by Dong et al. using depth resolved CL spectroscopy, 690,692,695 which revealed comparable spectral features between both polarities. The most prominent difference was the change of the intensity ratio between the green luminescence centered around $2.5 \mathrm{eV}$ and the near-band edge luminescence $\left(\mathrm{I}_{\mathrm{D}} / \mathrm{I}_{\mathrm{NBE}}\right)$ before and after remote $\mathrm{O}_{2} / \mathrm{He}$ plasma (ROP) processing. Whereas the $\mathrm{Zn}$-polar $\mathrm{ZnO}$ proved to be almost unaffected by ROP processing, the $\mathrm{I}_{\mathrm{D}} / \mathrm{I}_{\mathrm{NBE}}$ was significantly reduced for the Opolar ZnO sample after ROP treatment. ${ }^{690,692}$ Depth resolved $\mathrm{CL}$ further revealed a stronger green luminescence near the surface (sampling depth $\approx 7 \mathrm{~nm}$ ) for both polarities, suggesting more $\mathrm{O}$ vacancy related defects in the surface region. ${ }^{690,695}$ In accordance with the previously discussed work by Allen et al., ${ }^{698}$ Dong et al. also observed a second luminescence band at about $2.1 \mathrm{eV}$, but exclusively in the Zn-polar ZnO samples and after ROP processing. ${ }^{695}$ In contrast, $\mathrm{O}_{2} / \mathrm{He}$ plasma treatment was shown to reduce the green luminescence from the O-polar $\mathrm{ZnO}$ surfaces. The authors explained their finding by the ROP induced formation of near surface $\mathrm{Zn}$ vacancies $\left(\mathrm{V}_{\mathrm{Zn}}\right)$ on the $\mathrm{Zn}$-polar $\mathrm{ZnO}$ surfaces (giving rise to the $2.1 \mathrm{eV}$ luminescence band), and the decrease of $\mathrm{O}$ vacancies $\left(\mathrm{V}_{\mathrm{O}}\right)$ on the $\mathrm{O}$-polar $\mathrm{ZnO}$ surfaces, thus reducing the $2.5 \mathrm{eV}$ luminescence in the surfacesensitive low acceleration voltage CL spectra. However, it should be noted that the origin of the different defect-related emission bands in $\mathrm{ZnO}$ is still subject to controversial discussions, which is already apparent by the different interpretations of the $2.0 / 2.1 \mathrm{eV}$ bands in these works. For a comprehensive review of the defect luminescence in $\mathrm{ZnO}$, we refer the reader to Refs. 709 and 710.

Regarding the influence of polarity on the phononic properties of $\mathrm{ZnO}$, there are only very few works addressing this aspect in the literature. In fact, it is to be expected that polarity has a rather small influence on phonons and, thus, on thermal properties for epitaxial films and bulk samples. Previously, we have already mentioned the study of the exciton-phonon coupling strength by temperature resolved luminescence measurements. ${ }^{691,696}$ Beyond these works, Florescu et al. ${ }^{711}$ studied the surface thermal conductivity of both polarities using scanning thermal microscopy and found that both exhibit the same thermal conductivity within their experimental accuracy. However, a recent theoretical work by Jiang et al. ${ }^{712}$ based on molecular dynamics simulations suggested that thermal conductivity of $\mathrm{ZnO}$ NWs with exposed polar surfaces is about twice larger than that of NWs with passivated or non-polar surfaces. This was explained by the authors through phonon-phonon interaction reduction in NWs exhibiting free polar surfaces, whose reconstructions lead to bending phonon modes not being scattered by other phonon modes.

\section{Dielectric function and optical reflectivity}

The dielectric function of materials $(\varepsilon)$ is perhaps the quantity that determines in the most comprehensive manner

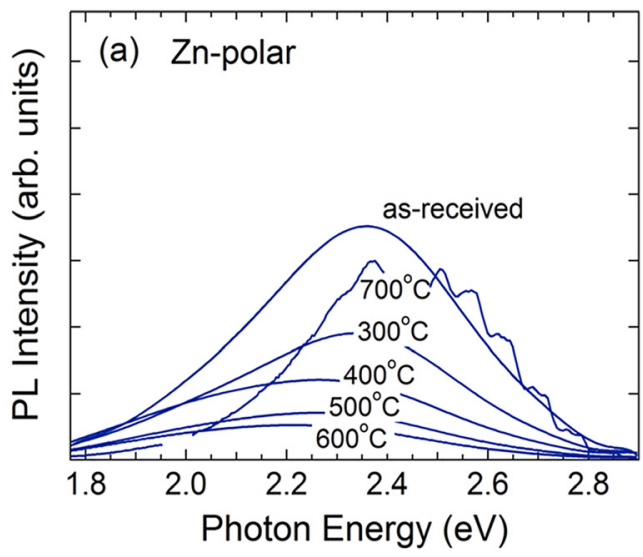

Photon Energy (eV)

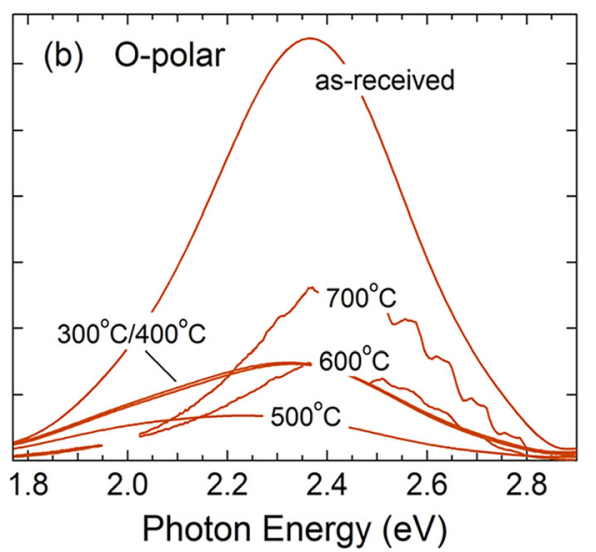

FIG. 90. Typical low temperature (8K) defect band PL spectra taken from the Zn-polar (a) and O-polar (b) faces of hydrothermal single crystal $\mathrm{ZnO}$ before and after annealing in oxygen gas for $90 \mathrm{~min}$ at different temperatures up to $700^{\circ} \mathrm{C}$. Reprinted with permission from Appl. Phys. Lett. 103, 231109 (2013). Copyright 2013 AIP Publishing LLC. 
their steady-state optical activity in a wide spectral range. In fact, a precise determination of this quantity gives direct access to the real and imaginary parts (absorption) of the refractive index $(n)$ through the complex relation $n=\sqrt{\varepsilon}$. Among most experimental techniques, optical ellipsometry has been established as the most powerful tool to study the dielectric function of materials. In its simpler version, optical reflectivity measurements also present large advantages due to their experimental simplicity. The dielectric function of $\mathrm{ZnO}$ was first measured by Yoshikawa and Adachi ${ }^{713}$ using spectroscopic ellipsometry for light polarized perpendicular $(E \perp c)$ and parallel $(E \| c)$ to the $\mathrm{c}$-axis in the range from $1.5 \mathrm{eV}$ to $5 \mathrm{eV}$. The authors also developed a simple model including excitonic effect near the band edge, as well as a full description of the transparent region based on the Sellmeier equation. On the other hand, optical reflectivity measurements as well as absorption measurements had been reported as early as 1960 by Thomas. ${ }^{675}$ In fact, large amount of authors have dedicated extensive efforts to study these effects in $c$-plane, $a$-plane, and $m$-plane $\mathrm{ZnO}$ using different scattering geometries. However, most of these works did not address the influence of polarity.

Pioneer works by Losurdo et al. ${ }^{714,715}$ used spectroscopic ellipsometry to study the influence of polarity and surface reactivity on the dielectric function. Figure 91 shows the real $\left(\varepsilon_{1}\right)$ and imaginary $\left(\varepsilon_{2}\right)$ parts of the dielectric function for both polarities as compared with the results by Yoshikawa and Adachi ${ }^{713}$ for $c$-plane $\mathrm{ZnO}$ samples (where polarity was not considered). Although both works show reasonable agreement, Losurdo et al. showed that polarity cannot be neglected. A direct influence on the excitonic transitions given by a higher value of $\varepsilon_{1}$ was found for the O-polar $\mathrm{ZnO}$; furthermore, a shift to higher energies was observed for the $\varepsilon_{1}$ maximum with respect to the $\mathrm{Zn}$-polar $\mathrm{ZnO}$. Although in the studies of Losurdo et al. an effect of polarity on the dielectric function was demonstrated, the low spectral resolution of their spectrometer (approx. $20 \mathrm{meV}$ ) prevented the analysis of the rich excitonic structure exhibited by $\mathrm{ZnO}$ and, thus, the corresponding influence of polarity on the $\mathrm{A}, \mathrm{B}$, and $\mathrm{C}$ excitons and their respective excited states. This restriction was overcome in a recent work by Cobet $^{716}$ who investigated the near band edge region between 3.35 and $3.55 \mathrm{eV}$ with an unprecedented resolution of $100 \mu \mathrm{eV}$ using a synchrotron ellipsometer.

Figure 92 displays $\varepsilon_{1}$ and $\varepsilon_{2}$ for $\mathrm{Zn}$ - and O-polar $\mathrm{ZnO}$ samples hydrothermally grown by Tokyo Denpa Co. Ltd. in the near band edge spectral region. In agreement with the findings of Losurdo et al., Cobet observed that the surface polarity largely affects the absolute value of $\varepsilon$ as well as the spectral position of most features. Furthermore, the high spectral resolution of Cobet's measurements allowed to determine the longitudinal-transverse splitting $\left(\Delta_{\mathrm{LT}}\right)$ of the $\mathrm{A}$ and $\mathrm{B}$ excitons which were shown to be also affected by polarity. The precise origin of this effect remains unclear to date; however, it can be potentially attributed to the different surface electron accumulation layers and polarity dependent surface band bending, as shown by Allen et al. ${ }^{717} \mathrm{It}$ should be noted that a possible effect of an overlayer of condensed water could be ruled out since the measurements
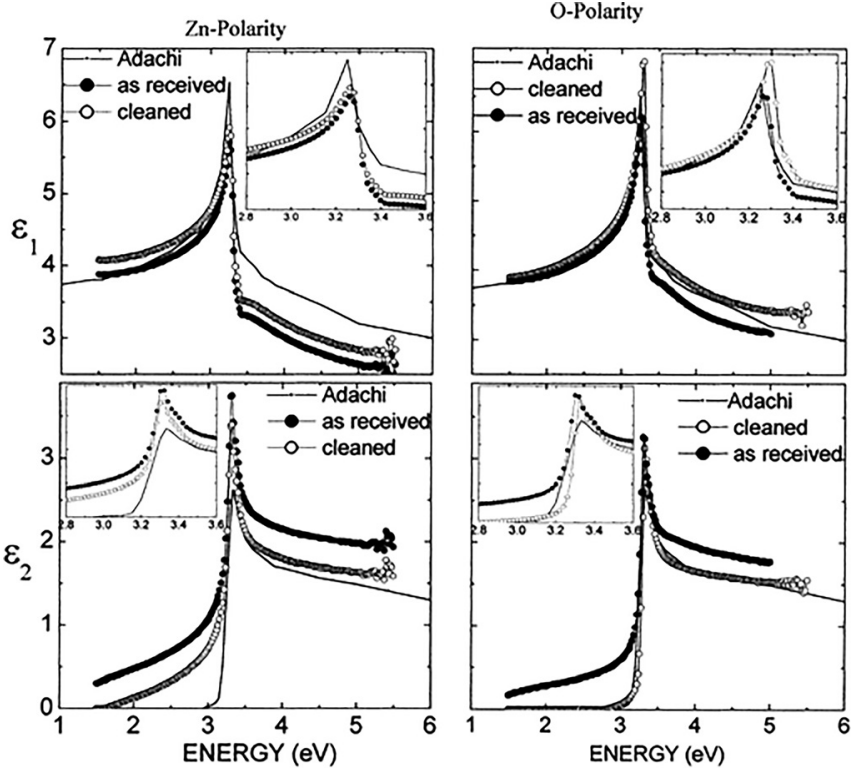

FIG. 91. Spectra of the real $\left(\varepsilon_{1}\right)$ and imaginary $\left(\varepsilon_{2}\right)$ parts of the pseudodielectric function of O-polar and $\mathrm{Zn}$-polar $\mathrm{ZnO}$ crystals. Insets show detail of the excitonic region. The results of Ref. 713 are also included for comparison. Reprinted with permission M. Losurdo, Thin Solid Films 455-456, 301 (2004). Copyright 2004 Elsevier.

were performed at room temperature and under ultra-high vacuum environment (about $2 \times 10^{-9}$ mbar).

Allen et $a l .{ }^{698}$ performed reflectivity measurements using non-polarized light and in normal incidence configuration for the $\mathrm{Zn}$ - and O-polarity of hydrothermally grown $\mathrm{ZnO}$ at room temperature. They observed a higher reflectivity of the O-polar as compared with the $\mathrm{Zn}$-polar $\mathrm{ZnO}$ in the near band edge region for energies below the band gap. This observation was attributed to differences in the nearsurface band bending and carrier concentration between the polar faces. ${ }^{717}$ In addition, time resolved reflectivity studies revealed differences in the dynamic response between both polarities, as demonstrated by Schneck et al. ${ }^{718}$ The authors investigated the time-resolved reflectivity of the $\mathrm{Zn}$ - and O-polar ZnO samples from Tokyo Denpa using an ultrafast laser with a peak wavelength of $340 \mathrm{~nm}$ and a pulse width of $38 \mathrm{fs}$ in pump and probe configuration. Under these conditions, the $1 / \mathrm{e}$ penetration depth of the incident beam is about $61 \mathrm{~nm}$. Figure 93 displays the transient reflectivity for $\mathrm{O}-$ and $\mathrm{Zn}$-polar $\mathrm{ZnO}$ for time delays up to $5 \mathrm{ps}$. The authors found that the data can be accurately fitted using three time constants $\left(\tau_{1}, \tau_{2}\right.$, and $\left.\tau_{3}\right)$, which account for different processes. The faster component, $\tau_{1}$, was only observed for the $\mathrm{Zn}$-polar $\mathrm{ZnO}$ and was attributed to fast scattering affecting electron-hole relaxation and resulting from carrier-carrier and carrier-LO-phonon interactions near the surface. The fact that this component is only present for the $\mathrm{Zn}$-polar $\mathrm{ZnO}$ was further explained based on the higher surface mobility of the $\mathrm{Zn}$-polar $\mathrm{ZnO}$. On the other hand, $\tau_{2} \approx 2-6$ ps and $\tau_{3} \approx 55$ ps were attributed to scattering of the excited electrons with acoustic phonons towards the bottom of the $\mathrm{CB}$ and to excitonic recombinations, respectively. 


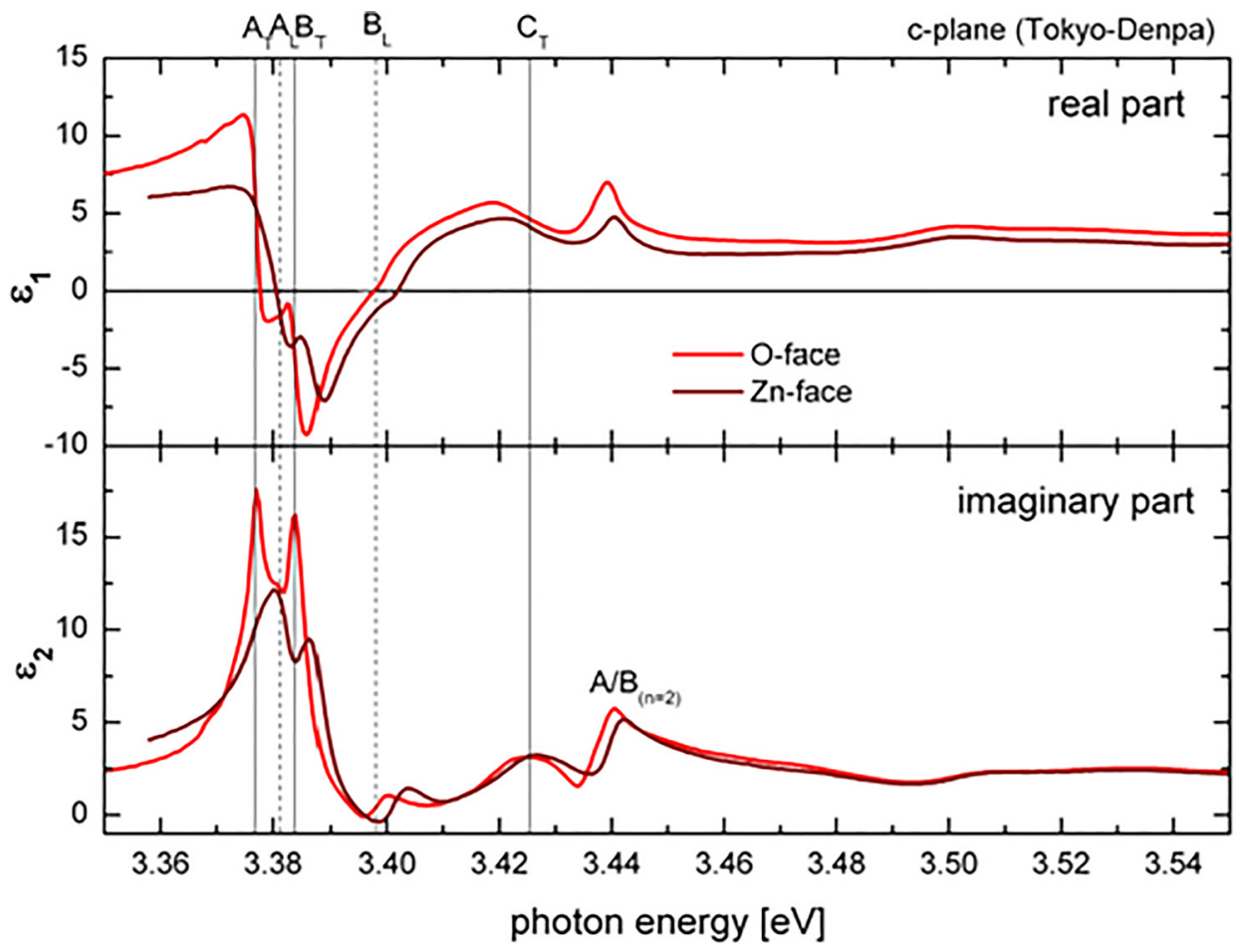

FIG. 92. Complex dielectric tensor components $\varepsilon_{1}$ and $\varepsilon_{2}$ of the $\mathrm{Zn}$-face and $\mathrm{O}$-face of a c-plane $\mathrm{ZnO}$ crystal from Tokyo Denpa in the excitonic region. Reprinted with permission from M. Cobet, "Ellipsometric study of $\mathrm{ZnO}$ from multimode formation of exciton-polaritons to the core-level regime," Ph.D. thesis (Technischen Universität Berlin, 2010).

\section{Electrical properties}

It is well know that, in polar materials, polarity largely influences the energy position of $\mathrm{VB}$ and $\mathrm{CB}$ at the surface. Furthermore, in the case of some intrinsically $n$-doped semiconducting materials such as, $\mathrm{ZnO}, \mathrm{InN}, \mathrm{In}_{2} \mathrm{O}_{3}, \mathrm{CdO}$, and $\mathrm{SnO}_{2}$, an electron accumulation layer is self-formed at the surface to compensate the effect of surface polarity (reflected in the band bending effect). This electronic accumulation layer affects, in turn, a large variety of fundamental electronic properties such as, for example, barrier heights of Schottky contacts. Already in the late 1960s, Moormann et al. ${ }^{719}$ used Kelvin probe microscopy (see Section III G 1) to study the work function and band bending of both polarities in $\mathrm{ZnO}$. Although the authors discussed the difficulties exhibited by this technique to determine the band bending, i.e., (i) the impossibility to obtain the surface carrier density, and (ii) the restriction of using ultraviolet light for threshold measurements due to surface photo-degradation (photolysis), they showed that both polar surfaces exhibit different band bendings. More recent works by Chevtchenko et al., ${ }^{720}$ based on the same experimental approach, led to similar conclusions, namely, that the Zn-polar surface exhibits a larger band bending than the $\mathrm{O}$-polar surface.
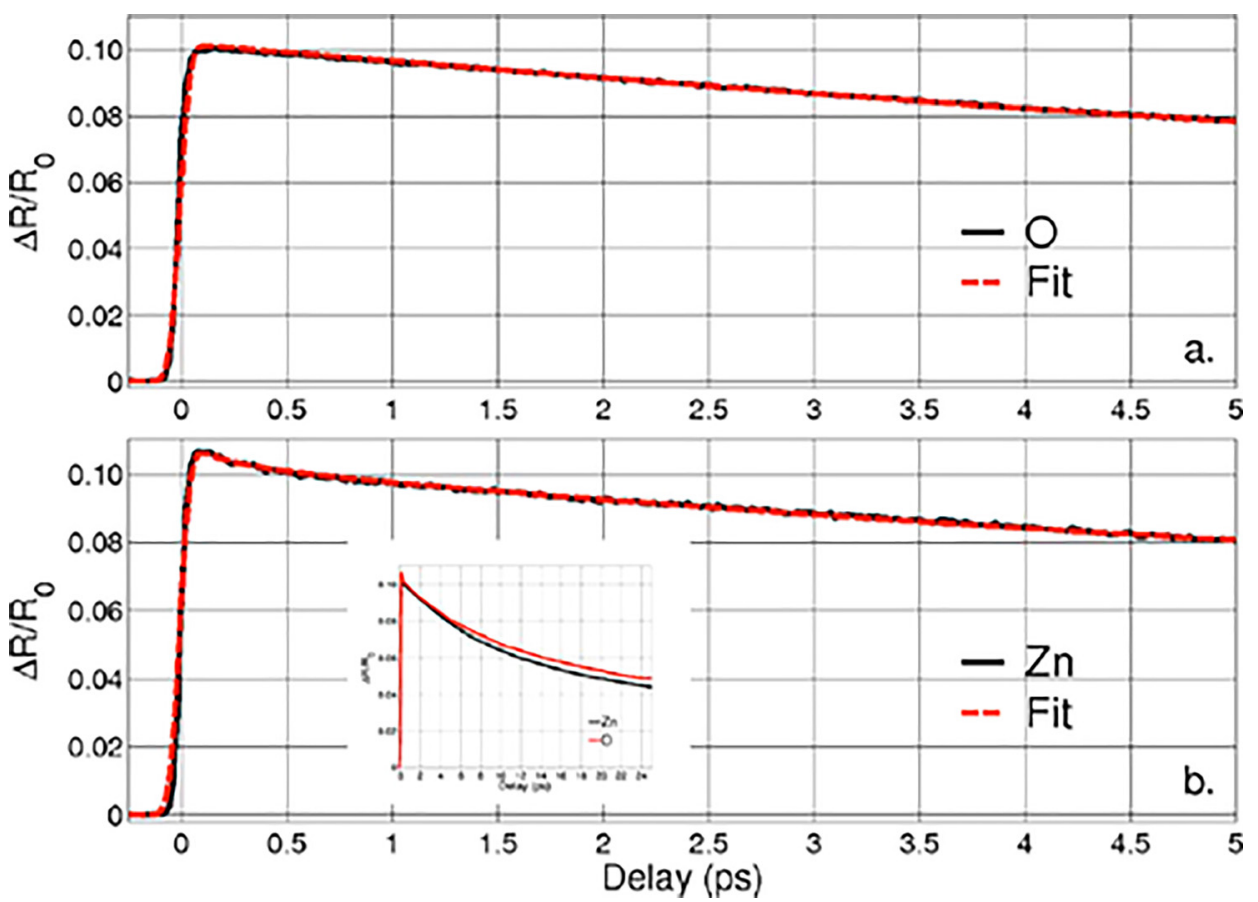

FIG. 93. Transient reflectivity at $340 \mathrm{~nm}$ for the O-face (a) and $\mathrm{Zn}$-face (b) of a $\mathrm{ZnO}$ single crystal. The convolution fit of the data is shown as the dashed red line on top of the measured reflectivity (black). Reprinted with permission from Appl. Phys. Lett. 93, 102111 (2008). Copyright 2008 AIP Publishing LLC. 
The electronic band structure of both surface terminations was experimentally reported in Refs. 721-723 using angle-resolved photoelectron spectroscopy (ARPES). Figure 94 displays a direct comparison of both polar surfaces by Ozawa et al. ${ }^{721}$ The authors found slightly different band structures for each polar surface. They argued that, for their experimental conditions, the detected photoelectrons come from several atomic layers in the surface region, since the inelastic mean-free path of the photoelectrons with a kinetic energy of $38-44 \mathrm{eV}$ is $0.3-0.37 \mathrm{~nm}$. Therefore, it is plausible that they observed the electronic structure under a strong influence of the surface, which may explain the different dispersions observed by the two polar surfaces.

Apart from the polarity, when interpreting electronic/ electrical properties, it is also important to keep in mind the intrinsic carrier concentration of the bulk region of the samples. We recall that $\mathrm{ZnO}$ can be grown using a large variety of growth techniques, leading to intrinsic impurity concentrations between $n_{e} \approx 10^{14}-10^{17} \mathrm{~cm}^{-3}$ (see Section IV B 1 ). Thus, the surface carrier concentration does not only depend on the surface band bending given by the detailed chemistry of the surface or surface reconstructions but also on the bulk carrier concentration. A comprehensive investigation of this effect was recently conducted by Allen et al., ${ }^{717}$ where the authors combined magneto-transport (Hall effect) measurements with XPS to determine the influence of polarity on the band bending at the surface for both polar configurations. In particular, they studied hydrothermally (HT) grown ZnO samples with low intrinsic carrier concentrations of $n_{e} \approx 10^{14} \mathrm{~cm}^{-3}$, in comparison with samples with a relatively high carrier concentration of $n_{e} \approx 10^{17} \mathrm{~cm}^{-3}$ grown using the pressurized-melt technique. Figures 95(a) and 95(b) display the energy position of the $\mathrm{CB}$ and $\mathrm{VB}$ as a function of the depth coordinate in the sample, i.e., from the surface towards the bulk region, for $\mathrm{HT} \mathrm{Zn}$ - and O-polar $\mathrm{ZnO}$. It was observed that the Zn-polar surface exhibits a band bending of about $14 \%$ with respect to the energy position of the bulk VB, whereas the O-polar surface shows a band bending of about $8 \%$. In agreement with Allen's work, Piper et al. ${ }^{724}$ studied

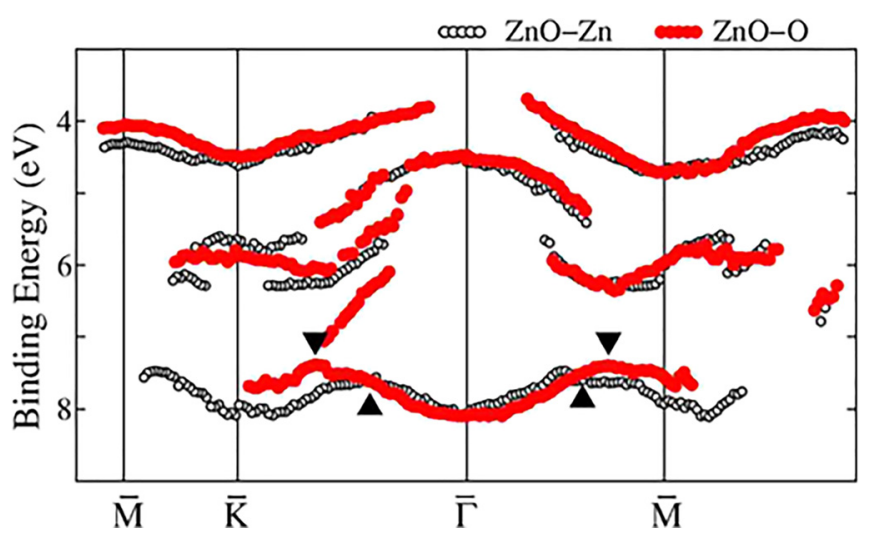

FIG. 94. Comparison of the band structures of $\mathrm{ZnO}-\mathrm{O}$ and $\mathrm{ZnO}-\mathrm{Zn}$. Only the branches with a strong intensity are plotted. The bands of the $\mathrm{ZnO}-\mathrm{Zn}$ surface are offset by $0.3 \mathrm{eV}$. Triangle marks indicate the position of the binding-energy minima of the bands. Reprinted with permission from Ozawa et al., Phys. Rev. B 79, 075314 (2009). Copyright 2009 American Physical Society. both polar surfaces using XPS and the O-polar surface using ARPES and reported similar results. On the other hand, Lahiri et al $^{725}$ reported that the Fermi level at the $\mathrm{Zn}$-polar surface was pinned close to the $\mathrm{CB}$ minimum, whereas for the Opolar surface the Fermi level was pinned at the midgap at $600^{\circ} \mathrm{C}$. A recent DFT study by Pal et al. ${ }^{726}$ supports the findings of Lahiri et al. As a possible explanation of such discrepancy, Piper argued that in the case of the O-polar surface the adsorbed hydrogen must facilitate the formation of charged donor-like surface states that are otherwise absent without hydrogen at elevated temperatures. Figures 95(c) and 95(d) display the surface carrier density for both polar surfaces. Interestingly, the Zn-polar surface exhibits a 3-fold larger carrier concentration than the O-polar surface. Similar results were obtained for pressurized-melt grown $\mathrm{ZnO}$, although with lower absolute values and smaller Zn-/O-polar surface carrier concentration ratio than those observed for $\mathrm{HT} \mathrm{ZnO}$.

In addition to the influence of polarity and bulk carrier concentration on the band bending at the surface, surface contamination can also modify the band bending. Merz et al. ${ }^{727}$ studied the influence of surface nanostructures (mounds some tens of nanometers high) on the surface photovoltage. They concluded that the formation of $\mathrm{Zn}$ nanomounds at the surface has a direct impact on the surface work function and, thus, on the band bending, by introducing mid-gap states. Last but not least, the presence of contaminants, such as $\mathrm{H}$, hydroxide complexes, and $\mathrm{C}$, was addressed by a number of works ${ }^{728-730}$ showing that these contaminants have a direct impact on the surface potential of both faces.

So far, we have discussed the origin of band bending for clean or contaminated polar surfaces. However, due to the expected applications of $\mathrm{ZnO}$ (i.e., high electron mobility transistors or in spintronics), it becomes necessary to understand the electrical properties of metal-ZnO interfaces for both polar surfaces. A large amount of authors have investigated this topic since the late 1960s, which was extensively reviewed by Brillson in a recent book chapter. ${ }^{731}$ As discussed by Allen et al. in Ref. 732, the "figure of merit" of Schottky barriers is the effective barrier height $\left(\Phi_{B}\right)$. In the same work, the authors compiled a comprehensive set of values of $\Phi_{B}$ for different metals and for both polar surfaces, as displayed in Fig. 96. Larger $\Phi_{B}$ values for $\mathrm{Zn}$ - with respect to O-polar surfaces are evident. The "higher quality" in terms of electrical performance is, thus, given by the Zn-polar surface. In addition, and in line with the findings for the polarity dependent band bending, ${ }^{717}$ samples with low intrinsic carrier concentration (HT grown) exhibit a larger difference $\Phi_{B}$ between both polar surfaces and, furthermore, a larger $\Phi_{B}$ for the Zn-polar surface. Although Fig. 96 represents an excellent summary on the different qualities of Schottky contacts in $\mathrm{ZnO}$, the metal-ZnO interface barrier strongly depends on crystal growth method, intrinsic carrier concentration, defects, strain, surface preparation, and processing. For a detailed discussion on this matters, we refer the reader to Ref. 731.

\section{B. N-polar GaN optoelectronic devices: LEDs and solar cells}

The ability of III-Nitrides to span a direct bandgap ranging from $0.7 \mathrm{eV}$ to $6.1 \mathrm{eV}$ has attracted much attention for 

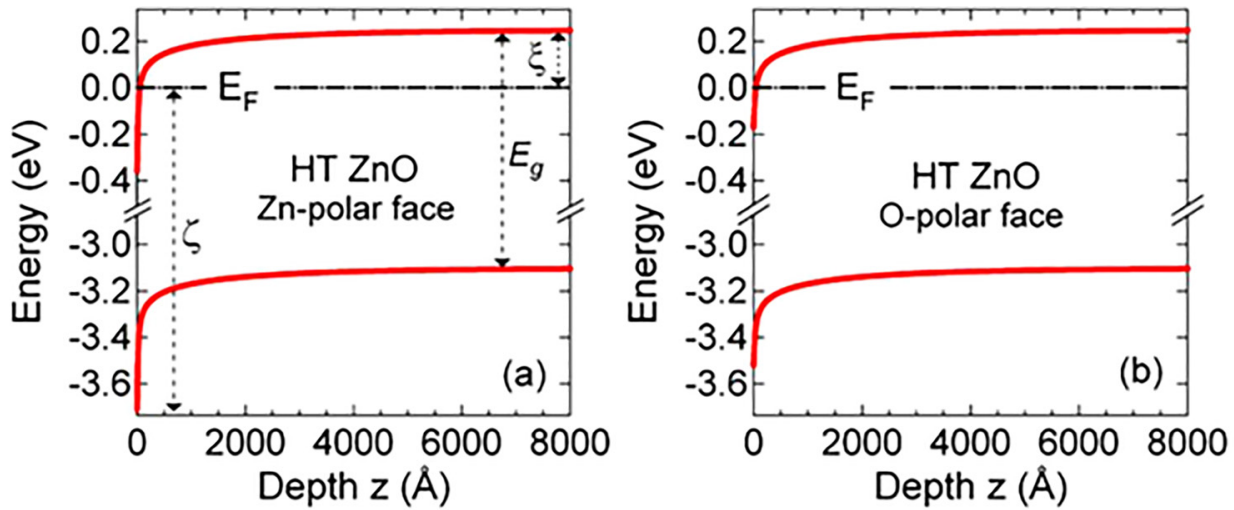

FIG. 95. Poisson-modified ThomasFermi approximation calculations of the band banding and the carrier concentration profiles in the electron accumulation layer at the Zn-polar [(a) and (c)] and O-polar faces [(b) and (d)] of hydrothermally grown $\mathrm{ZnO}$. Reprinted with permission from Allen et al., Phys. Rev. B 81, 075211 (2010). Copyright 2010 American Physical Society.

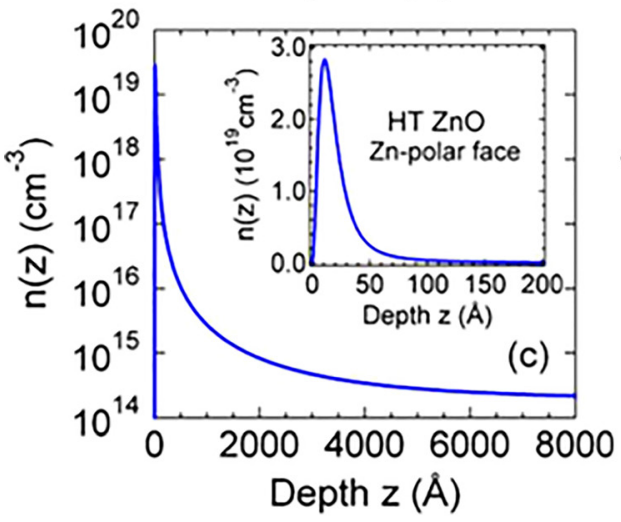

device applications, especially for light emitting diodes (LEDs). (In,Ga)N/GaN-based LEDs have already been commercialized in the market for several years, with blue emitters, green emitters, and white bulbs displaying external quantum efficiency (at $35 \mathrm{~A} / \mathrm{cm}^{2}$ ) in the order of $75 \%$ and $32 \%$ for blue and green emitters, respectively, and $160 \mathrm{~lm} / \mathrm{W}$ for white bulbs. ${ }^{733}$

Still, there is much research going on in the field to figure out and overcome efficiency droop in these devices: iIn IIINitrides LEDs, the efficiency of the devices is decreased by increasing current density; this is the reason why these devices are operated at low current densities (typically about $35 \mathrm{~A} /$ $\mathrm{cm}^{2}$ ). Due to this limitation in the input/output powers, larger devices in the form of arrays are usually employed to obtain

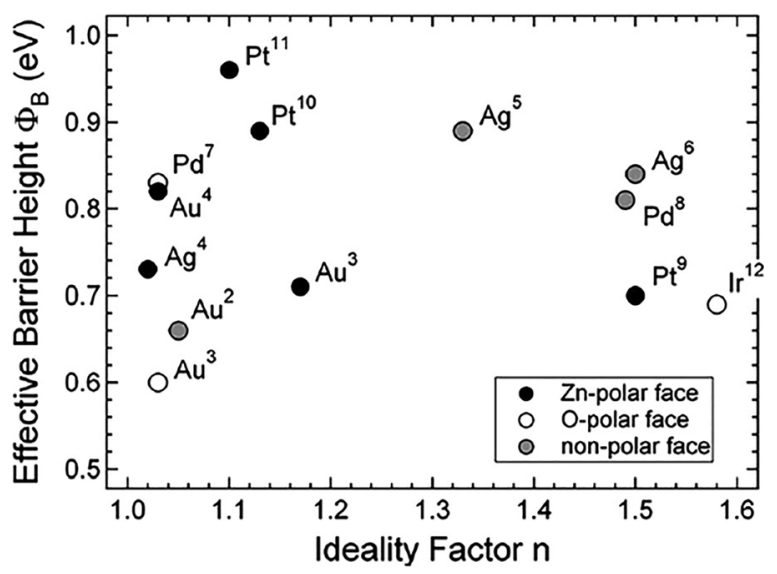

FIG. 96. Effective barrier height $\left(\Phi_{B}\right)$ vs. ideality factor $(n)$ for the best reported Schottky contacts on n-type $\mathrm{ZnO}$. A collection of different metals for both polar and non-polar faces is shown. Reprinted with permission from Appl. Phys. Lett. 91, 053512 (2007). Copyright 2007 AIP Publishing LLC. high enough power, especially for white LED bulbs. ${ }^{733}$ Mechanisms that have been proposed and shown to be, at least partially, the reasons of the efficiency droop ${ }^{734-738}$ include Auger recombination and overflow. Since this is an essential problem, a large body of research has focused on the analysis of polarization effects on these phenomena. ${ }^{735-742}$

\section{Effects of polarization on optoelectronic devices: LEDs and solar cells}

As discussed in Section II B, the spontaneous polarization in $\mathrm{GaN}$ and $\mathrm{ZnO}$ points along the [0001] direction. The spontaneous polarization vector in $\mathrm{GaN}$ is thus parallel to the growth direction in the N-polar case, whereas it points in the opposite direction in the Ga-polar case. The growth of strained III-Nitrides ${ }^{5}$ (or $\mathrm{ZnO}$-based $^{743}$ ) heterostructures induces an additional piezoelectric polarization, the direction of which depending on the strain state. Figures 97(a) and 97(b) depict the polarization components of an n-GaN/ (In,Ga)N/p-GaN heterostructure and the energy band diagram of two equivalent devices, but one being Ga-polar and the other one being N-polar. These schematics and energy band diagrams depict the typical geometry of a nitride LED, with the $\mathrm{p}$-GaN layer located on top of the device ( $\mathrm{p}$-up LED). Due to the high resistance in p-type GaN, the p-type layer is usually designed to be on the top of the device. We refer to the Ga-polar p-up device as the "normal" polarization configuration and the N-polar p-up device as the "reversed" polarization configuration. Note that a p-down device would give identical electrostatic and energy band diagrams, but with the opposite growth direction in each case.

In conventional Ga-polar optoelectronic devices, a negative polarization charge is induced at the $n-G a N /(\mathrm{In}, \mathrm{Ga}) \mathrm{N}$ 
(a)

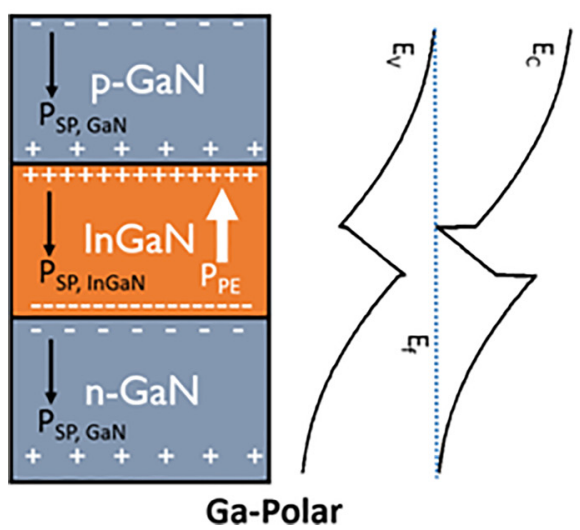

(b)

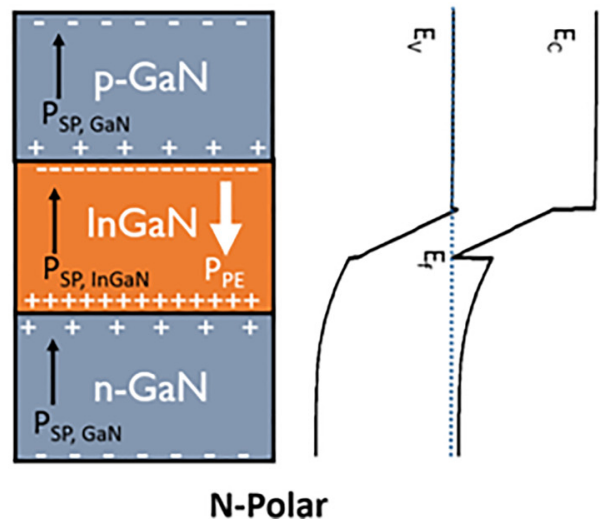

FIG. 97. Polarization charges and the direction of the piezo and spontaneous polarization vectors in p-up devices along (a) Ga- and (b) N-polar crystal with the corresponding energy band diagrams. interface, which in turn induces a potential barrier for electron injection to the $(\mathrm{In}, \mathrm{Ga}) \mathrm{N}$ QW. Therefore, high energy electrons are preferably injected into the QW. Similarly, hole injection is also challenging due to the positive polarization charge on the p-GaN/(In,Ga)N interface. In contrast, the polarization charges in the $\mathrm{N}$-polar optoelectronic devices contribute to the depletion region field, and no such barrier exists for electrons and holes. The N-polar orientation should therefore facilitate carrier injection into the active region.

The effects of the polarization charge on the energy band diagram and output characteristics of optoelectronic devices have been investigated by various groups. ${ }^{744-746} \mathrm{~A}$ comparison of single QW LEDs was made by Akyol et al. ${ }^{746}$ for normal (Ga-polar, p-up) and reversed (N-polar, p-up) polarization configurations. The forward biased energy band diagrams of these devices are shown in Fig. 98. It can be seen that the Ga-polar (normal polarization) device shows clear potential barriers for electron and hole injection, whereas the N-polar device is free of such barriers. This resulted in an operating voltage of $3.4 \mathrm{~V}$ and $2.7 \mathrm{~V}$ (at $100 \mathrm{~A} /$ $\mathrm{cm}^{2}$ ) for $\mathrm{Ga}$ - and $\mathrm{N}$-polar devices, respectively. The lower operating voltage in the opposite polarization configuration has also been reported by various groups using simulations as well as experiments. ${ }^{744-747}$

A simulation comparing the internal quantum efficiency of normal and opposite polarization LEDs was also reported by Verzellesi et al. ${ }^{748}$ The simulated characteristics are shown in Fig. 99(a). It can be seen that opposite polarization devices (i.e., N-polar) suffer much less from efficiency droop as compared with the conventional polarization devices. The authors attributed this efficiency enhancement to the absence of carrier leakage and doping asymmetry $\left(\mathrm{N}_{\mathrm{D}}>\mathrm{N}_{\mathrm{A}}\right)$. Carrier leakage is internally prevented by the built-in potential barrier generated by polarization charges as discussed above. The doping asymmetry becomes the dominant factor if the hole transport to the successive QWs is prevented by the polarization charges. This then leads to electron leakage in devices where most of the recombinations occur only in the QW nearest the p-layer. By utilizing opposite polarization charges, Yen et al. ${ }^{747}$ showed that opposite polarization single QW active region laser diodes can operate at a much lower threshold current density (Fig. 99(b)) as compared with the normal polarization configuration. The authors attributed this to suppressed electron leakage and reduced Shockley-Read-Hall (SRH) recombinations.

The (In,Ga)N/GaN material system has also been investigated for solar cell applications, as the band-gap range covers the entire solar spectrum, and as the absorption length is relatively short. ${ }^{749,750}$ The effects of crystal polarity on solar cell device performance have also been reported using device simulations by various groups. ${ }^{751-753} \mathrm{Li}$ et al $^{751}$ simulated $\mathrm{GaN} / \mathrm{In}_{0.12} \mathrm{Ga}_{0.88} \mathrm{~N} / \mathrm{GaN}$ p-i-n junctions with normal and opposite polarization configurations. The zero-bias energy band diagrams of Ga-polar (normal polarization configuration) without polarization, with medium $\left(3.14 \times 10^{18} \mathrm{~m}^{-2}\right)$, and high $\left(5.24 \times 10^{18} \mathrm{~m}^{-2}\right)$ polarization charges were compared (Figs. 100(a)-100(c)). It can be seen in Fig. 100 that, in normal polarization configuration, increasing polarization charges lowers the electric field in the (In,Ga)N layer and can even prevent carrier drift process across the (In,Ga)N layer. However, in

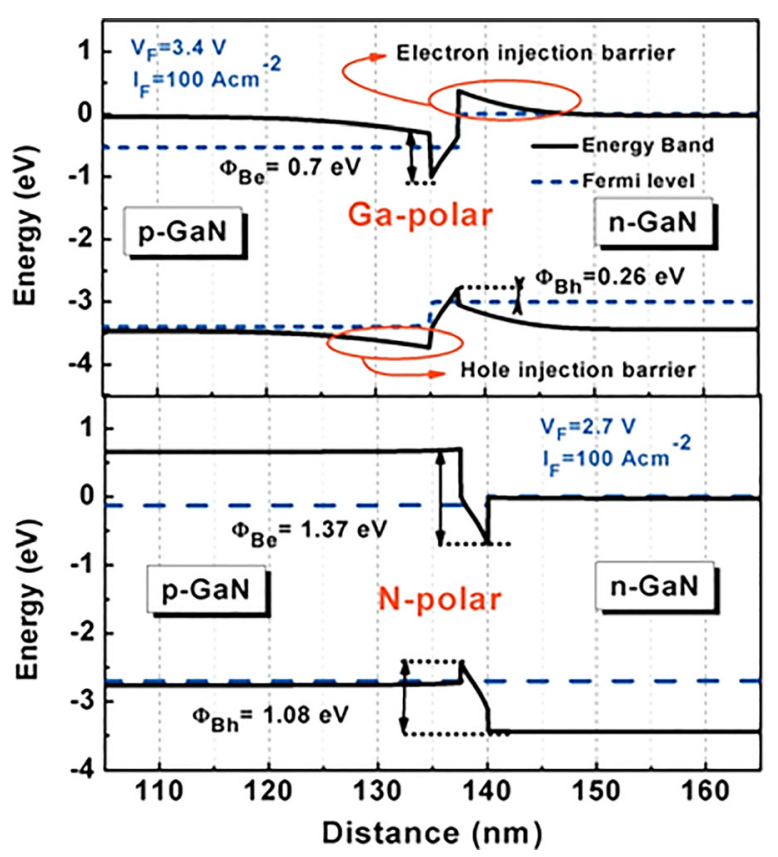

FIG. 98. Forward biased energy band diagram of single qw LEDs along Ga(normal) and N-polar orientation. Reprinted with permission from Appl. Phys. Lett. 100, 111118 (2012). Copyright 2012 AIP Publishing LLC. 

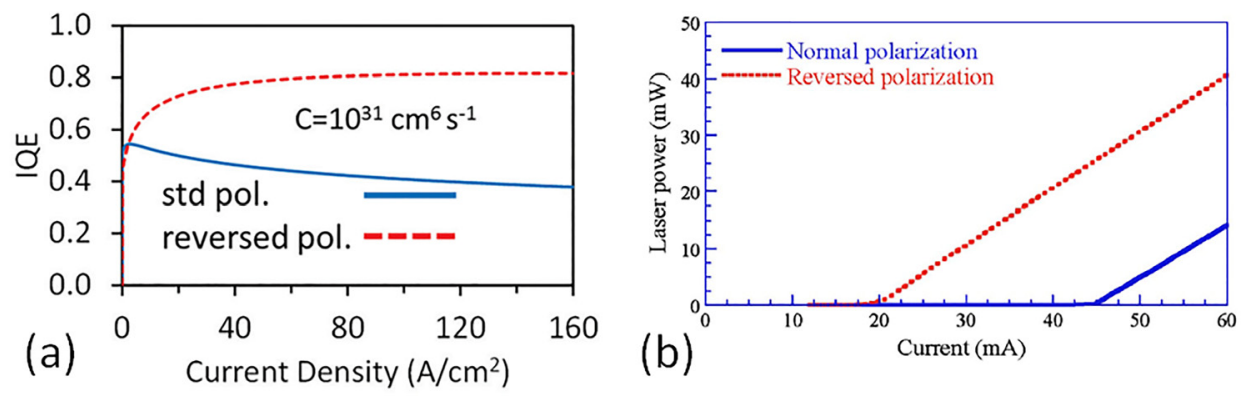

FIG. 99. (a) Variation of internal quantum efficiency with the current density in the simulated LED with standard and reverse polarization. Reprinted with permission from J. Appl. Phys. 114, 071101 (2013). Copyright 2013 AIP Publishing LLC. (b) Laser power versus current of the simulated laser diodes under normal and reverse polarization configuration. Reprinted with permission from Appl. Phys. Lett. 91, 201118 (2007). Copyright 2007 AIP Publishing LLC.

the opposite polarization case, the polarization field across the $(\mathrm{In}, \mathrm{Ga}) \mathrm{N}$ layer is in the same direction as the depletion field. The resulting energy band diagram in reverse polarization configuration is therefore very similar to Fig. 100(a), which is the case for no polarization charges. Therefore, a reverse polarization configuration (or $\mathrm{N}$ polarity) could eliminate the detrimental effects of polarization discontinuities on some optoelectronic devices.

I-V curves of the devices with varying polarization charges under global AM1.5 illumination conditions are shown in Figs. 101(a) and 101(b) for the normal and opposite polarization configurations, respectively. As expected from the energy band diagrams, the device performances in the normal polarization configuration are limited to electron diffusion current from the top GaN layer. In contrast, increasing reverse polarization charge has almost no detrimental effect on the device characteristics (Fig. 101(b)), given that the opposite direction of the polarization does not negatively impact carrier drift. These characteristics were also observed experimentally. ${ }^{753}$ We note there that the detrimental effects of normal polarization configuration can be mitigated by using heavy $\mathrm{Si}$ and $\mathrm{Mg}$ doping in $\mathrm{n}-\mathrm{GaN}$ and $\mathrm{p}-\mathrm{GaN}$ layers, respectively, to compensate for the polarization sheet charges. ${ }^{753}$

\section{Experimental work on N-polar GaN LEDs}

There are few reports on MOCVD growth of N-polar (In,Ga)N/GaN LEDs showing electroluminescence (EL) characteristics. $^{754-757}$ Masui et al. ${ }^{754}$ measured the temperature dependent PL response of the N- and Ga-polar MQWs LEDs, as shown in Figs. 102(a) and 102(b), respectively. In this report, the integrated luminescence between $2.5 \mathrm{eV}$ and $3.3 \mathrm{eV}$ increased by a factor of 45 and 10 for N- and Ga-polar MQWs, respectively, when going from room- to low temperatures. Besides, at room temperature, the radiative recombination efficiency of N-polar MQWs was more than 4 times lower. The yellow band luminescence peak (around $2.2 \mathrm{eV}$ ) of the $\mathrm{N}$-polar MQWs was found to be less temperature dependent and had a higher intensity at room temperature. These results showed that MOCVD grown N-polar (In,Ga)N (under optimized growth conditions for Ga-polar ( $\mathrm{In}, \mathrm{Ga}) \mathrm{N}$ ) incorporates higher impurities and vacancies than Ga-polar $(\mathrm{In}, \mathrm{Ga}) \mathrm{N}$, resulting in more pronounced yellow-band emission and much lower band-toband radiative efficiency. The EL measurement of the N-polar LED showed a very low output power of $6 \mu \mathrm{W}$ at $20 \mathrm{~mA}$ of injection current, operating at a peak wavelength of $460 \mathrm{~nm}$.
They reported that the emission intensity increased by a factor of 70 when the device was cooled down to $10 \mathrm{~K}$, and attributed the low performance at room-temperature to high impurity concentration in (In,Ga)N/GaN MQWs. They also noted that the

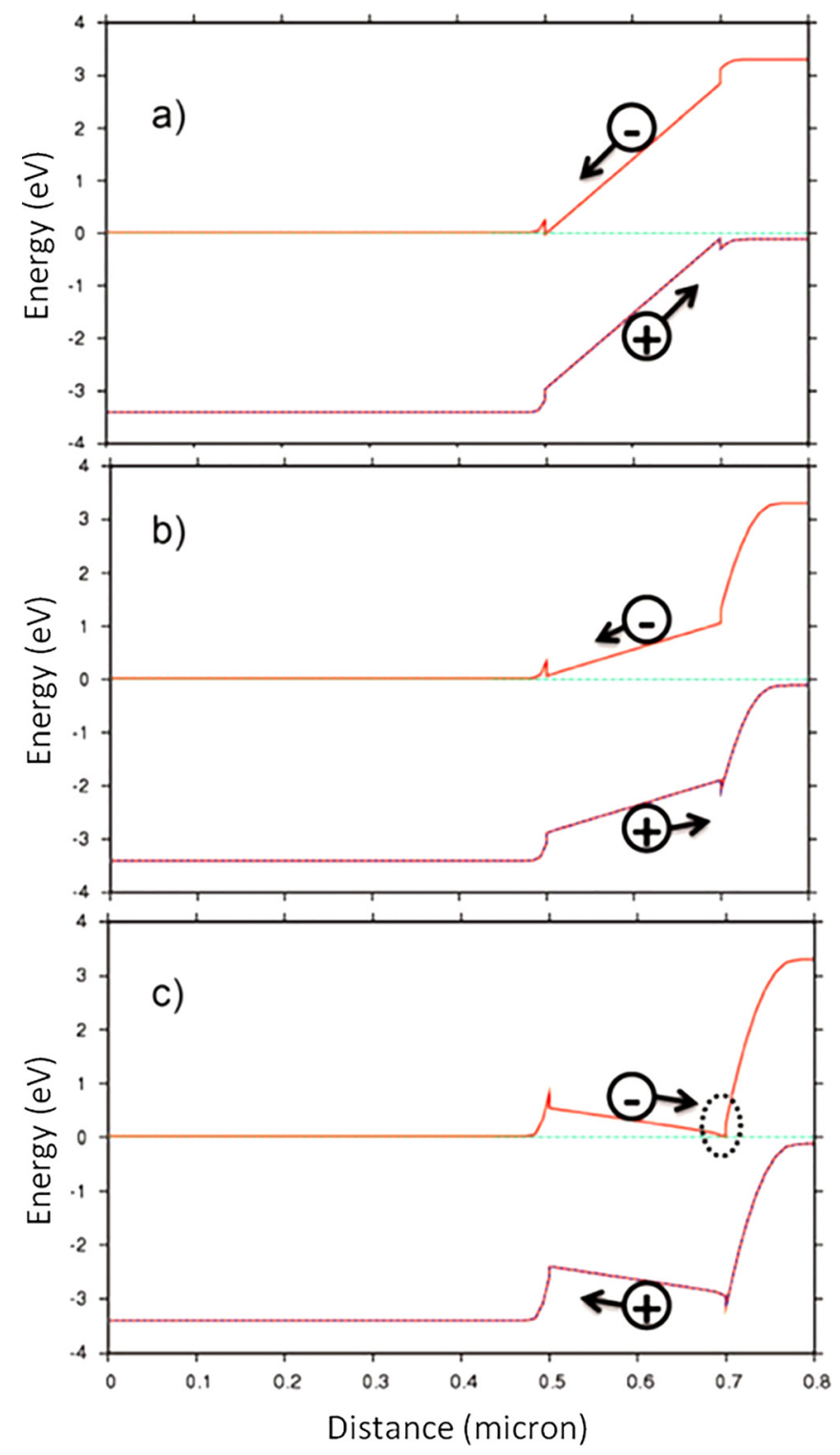

FIG. 100. The energy band diagram of the GaN/(In,Ga)N/GaN p-i-n solar cell under normal polarization (a) without polarization charge, (b) with medium $\left(3.14 \times 10^{18} \mathrm{~m}^{-2}\right)$, and (c) high $\left(5.24 \times 10^{18}\right)$ polarization charge. Reprinted with permission from Li et al., Phys. Status Solidi A 208, 928 (2011). Copyright 2011 John Wiley \& Sons, Inc. 

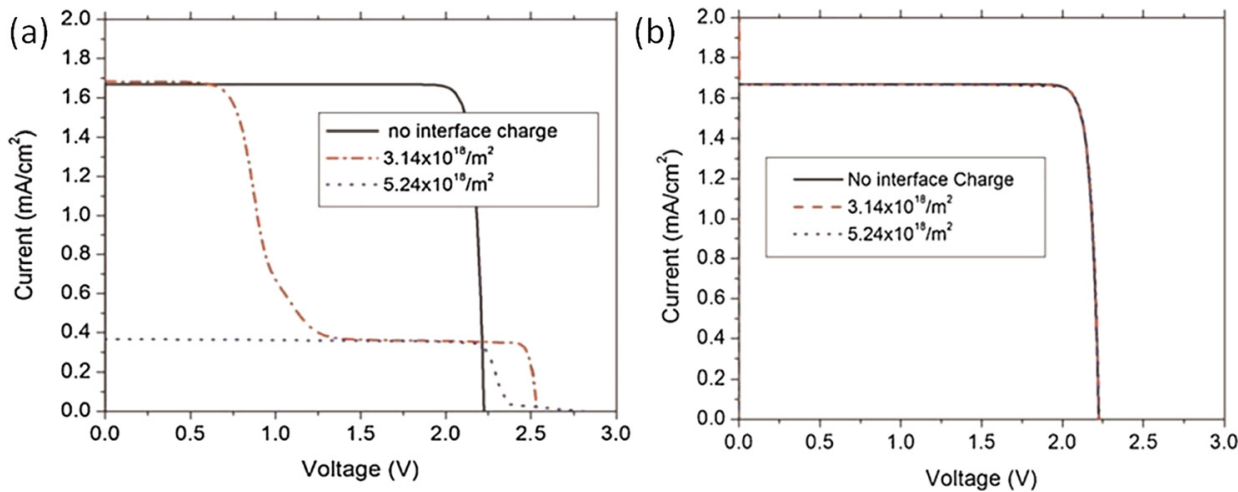

FIG. 101. I-V curves of the $\mathrm{GaN} /$ (In,Ga)N/GaN p-i-n solar cell under varying (a) normal and (b) reverse polarization charge. Reprinted with permission from Li et al., Phys. Status Solidi A 208, 928 (2011). Copyright 2011 John Wiley \& Sons, Inc. p-type GaN layers in the N-polar devices were of high quality, comparable to those in Ga-polar devices, and therefore hole injection was most likely not the limiting mechanism for these devices.

By exploiting the growth of higher In-content (In,Ga)N layers enabled by the N-polarity (see Section IV A 6 b and Fig. 41), Shojiki et al ${ }^{756}$ demonstrated MOCVD grown Npolar blue, green, and red LEDs. These LEDs were reported to have an output power in the sub- $\mu \mathrm{W}$ range at $20 \mathrm{~mA}$, which was significantly lower than Ga-polar and non-polar LEDs. A recent experimental study on carrier recombination and relaxation mechanisms in MOCVD grown $\mathrm{N}$ - and Gapolar LEDs showed that N-polarity had more efficient carrier relaxation and faster carrier recombination. ${ }^{757}$ The authors measured the time-resolved EL (TREL) response of the devices after application of a forward voltage pulse, as shown in Fig. 103. It can be seen that both rise and fall of the timeresolved EL curve are much steeper in N-polar LEDs. The variation of the response, rise, delay, and recombination times with the applied pulse voltage intensity is shown in Fig. 104. The authors attributed the shorter rise time of $\mathrm{N}$ polar LEDs to stronger carrier localization and weaker quantum confined Stark effect, and lower potential barriers upon application of forward bias. Also, when switching the pulse off, Ga-polar LEDs showed longer time to reach maximum intensity; the subsequent decay, longer than for N-polar LEDs, was attributed to weaker carrier localization, stronger quantum confined Stark effect, and poorer carrier relaxation efficiency. However, similar to the previous reports, the radiative output of N-polar LEDs was approximately an order of magnitude lower than that of Ga-polar LEDs. In the case of MOCVD-grown LEDs, therefore, the background defects and consequent non-radiative recombination are most likely limiting performance. MBE growth of N-polar LEDs has recently been reported by several groups. ${ }^{746,758,759}$ By utilizing polarization-induced doping in graded $(\mathrm{Al}, \mathrm{Ga}) \mathrm{N}$ electron blocking layers, Verma et al. demonstrated N-polar GaN QW LEDs operating in the ultraviolet regime. The output power of the LED was not reported in this study. MBE grown green LEDs without electron blocking layers were also investigated. In this work, the authors compared the intrinsic electron blocking properties of single and double QW LEDs grown in the N-polar direction. Figure 105 shows the EL spectrum of the devices. A distinct ultra violet emission peak was observed from single QW LEDs for current density higher than $50 \mathrm{~A} / \mathrm{cm}^{2}$, and this was attributed to radiative recombinations of electrons in $\mathrm{p}-\mathrm{GaN}$ layer. By utilizing the potential barrier generated by polarization fields in the $(\mathrm{In}, \mathrm{Ga}) \mathrm{N}$ QW, insertion of one more QW removed the parasitic ultra violet peak, thus effectively confining electrons in the activphysics region. By suppressing the electron overflow, double QW LEDs were reported to show negligible efficiency droop up to a current density of $400 \mathrm{~A} / \mathrm{cm}^{2}$. The authors reported that the devices had low radiative output power, which could be due to lack of optimization in p-GaN growth.

\section{GaN-based high electron mobility transistors}

GaN-based transistors are the most paradigmatic devices in terms of polarization effects, which constitute by definition their essence. As for all other GaN devices, GaN-based transistors were initially developed by using Ga-polar GaN heterostructures; however, in some cases, N-polar GaN heterostructures have emerged as advantageous alternatives for
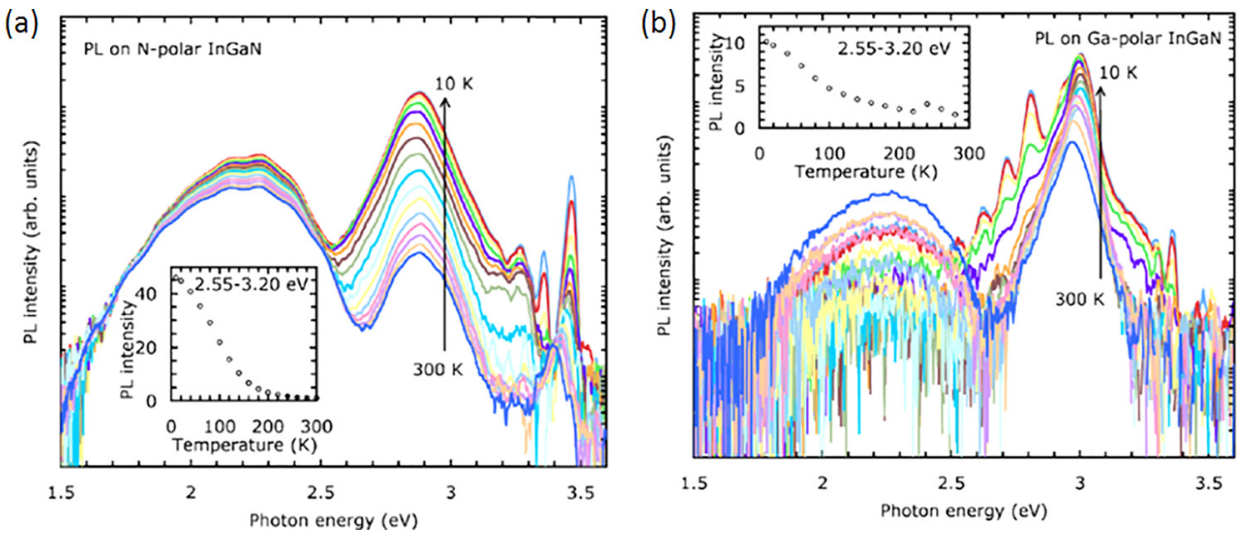

FIG. 102. The temperature dependent PL measurement of (a) Ga- and (b) Npolar LEDs. The insets of the plots show the variation of integrated PL intensity between 2.5 and $3.3 \mathrm{eV}$. Reprinted with permission from Masui et al., Jpn. J. Appl. Phys., Part $1 \mathbf{4 8}$, 071003 (2009). Copyright 2009 The Japan Society of Applied Physics. 


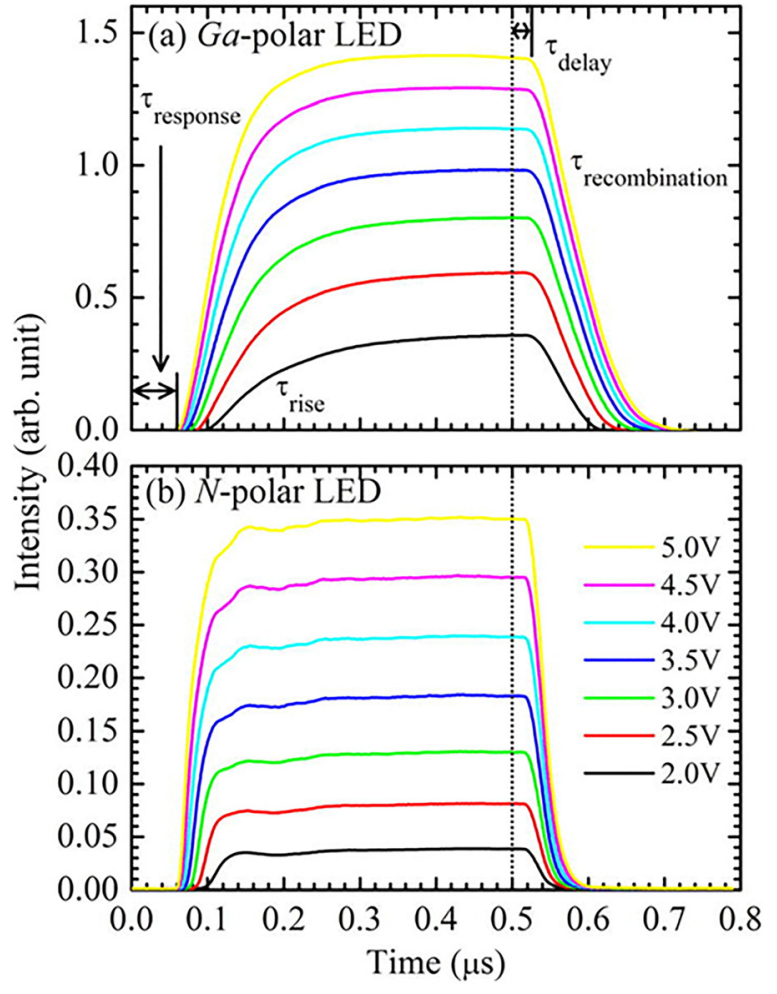

FIG. 103. TREL transit profiles of (a) Ga- and (b) N-polar LED under varying voltage. Dotted line indicates the time for the voltage pulse switched off LEDs. Reprinted with permission from J. Appl. Phys. 118, 043104 (2015). Copyright 2015 AIP Publishing LLC.

fabricating efficient GaN-based transistors. In that respect, the development of $\mathrm{N}$-polar GaN transistors as well as the rapid progress in their performances has recently been reviewed in Refs. 232 and 233. It is not intended here to

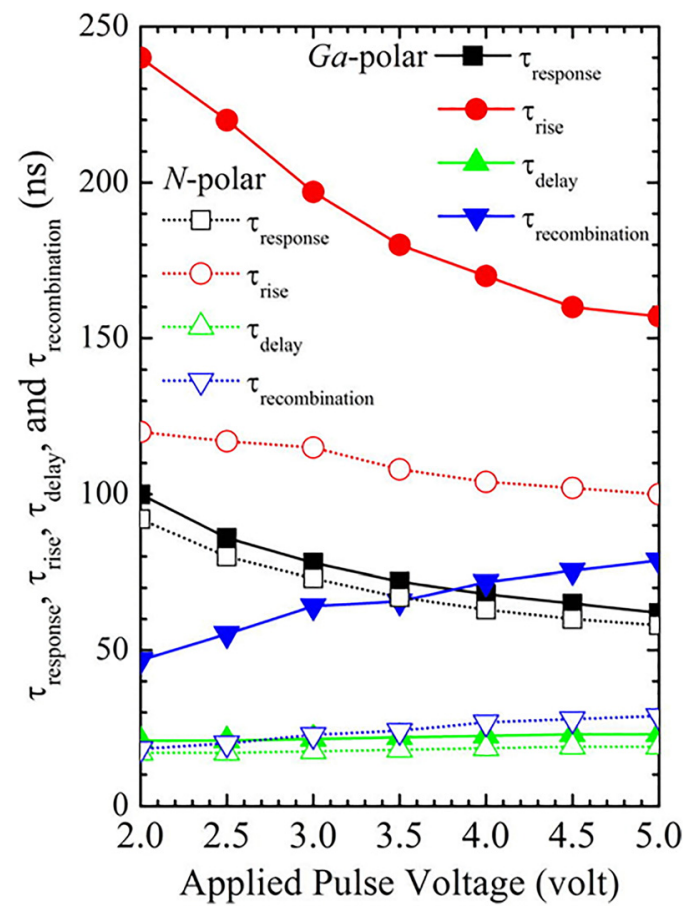

FIG. 104. Response, rise, decay, and recombination time for the LEDs. Reprinted with permission from J. Appl. Phys. 118, 043104 (2015). Copyright 2015 AIP Publishing LLC.

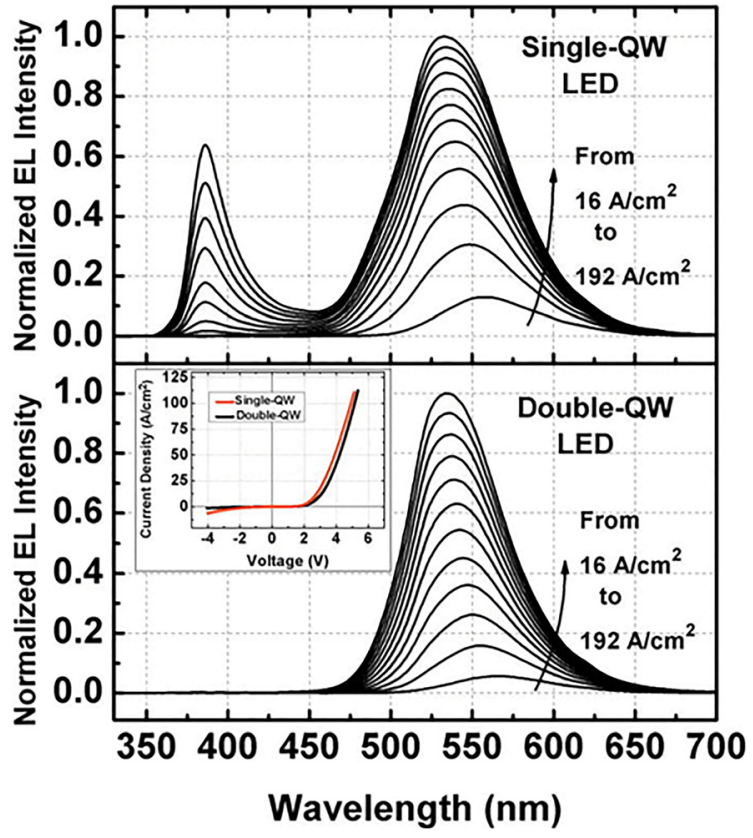

FIG. 105. EL intensity of single and double qw MBE grown LEDs showing removal of parasitic UV emission in double qw LED. The inset shows the $\mathrm{I}-\mathrm{V}$ characteristics of the devices. Reprinted with permission from Appl. Phys. Lett. 100, 111118 (2012). Copyright 2012 AIP Publishing LLC.

reproduce them. Instead, by introducing the basics of GaNbased polarization-induced transistors and by presenting their limitations, some of which can be potentially overcome by $\mathrm{N}$-polar GaN devices, we somehow show that the availability of high-quality Ga- and N-polar GaN heterostructures opens the way for optimized architectures combining both types of devices.

Thus, in this subsection, the working principle of conventional (i.e., Ga-polar normally on transistors) GaN transistors is first presented. Second, it is discussed how "polarization-engineering" can be exploited to improve the transistor performances and then move from normally on to normally off transistors. Finally, we highlight the possibilities offered by the use of N-polar GaN transistors, both in terms of fabrication and device structures.

\section{Basics of $(A l, G a) N / G a N H E M T s$}

Over the past decades, the outstanding electronic properties of $\mathrm{GaN}$ have been exploited in high power and high frequency applications. ${ }^{760}$ Thanks to its wide band gap energy, $\mathrm{GaN}$ enables transistors to combine high breakdown voltages with low access resistance and gate capacitance. Moreover, the strong polarization differences between $\mathrm{GaN}$ buffer layer and $(\mathrm{Al}, \mathrm{Ga}) \mathrm{N}$ barrier layer in $(\mathrm{Al}, \mathrm{Ga}) \mathrm{N} / \mathrm{GaN}$-based heterostructures can induce a high carrier density at the $(\mathrm{Al}, \mathrm{Ga}) \mathrm{N} /$ GaN heterointerface. ${ }^{761,762}$ These charge carriers are electrons, which can display high mobility and saturation velocity, making GaN-HEMTs ideal for the next generation of radio frequency $(\mathrm{RF})$ power amplifiers.

In the prototypical case of an $(\mathrm{Al}, \mathrm{Ga}) \mathrm{N} / \mathrm{GaN}$-based HEMT, a thin $(\mathrm{Al}, \mathrm{Ga}) \mathrm{N}$ barrier layer is typically grown pseudomorphically on a relaxed Ga-polar GaN buffer layer. The $(\mathrm{Al}, \mathrm{Ga}) \mathrm{N}$ layer is thus under tensile strain on $\mathrm{GaN}$ and 
a)

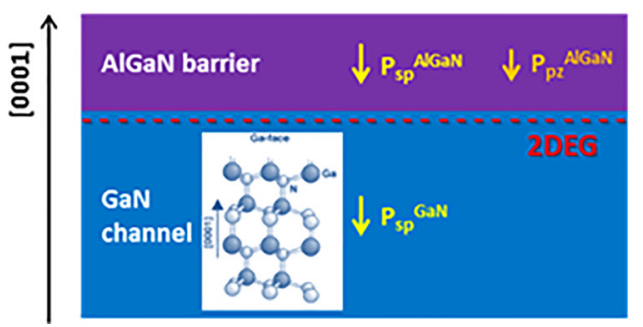

b)

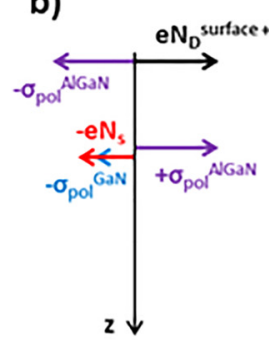

c)

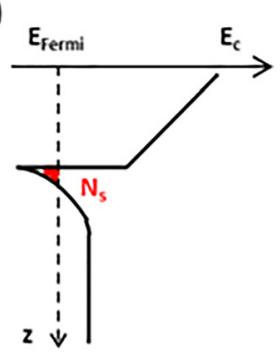

FIG. 106. (a) Scheme of the polarization-induced 2DEG in (Al,Ga)N/ $\mathrm{GaN}$ heterostructure. (b) Charge distribution at the heterointerface where $N_{s}$ represents the 2DEG density. (c) Conduction band diagram corresponding to the $(\mathrm{Al}, \mathrm{Ga}) \mathrm{N} / \mathrm{GaN}$ heterostructure. the $(\mathrm{Al}, \mathrm{Ga}) \mathrm{N}$ piezoelectric polarization is, therefore, in the same sense as the (Al,Ga)N spontaneous polarization (Fig. 106(a)). The fixed polarization charges at the interfaces $\left(\sigma_{\mathrm{pol}}\right)$ are compensated by mobile electrons coming from surface donor states ${ }^{763,764}$ (Fig. 106(b)). These electrons accumulate on the first degenerated level of the triangular QW created at the heterointerface of $(\mathrm{Al}, \mathrm{Ga}) \mathrm{N} / \mathrm{GaN}$ on the $\mathrm{GaN}$ side (Fig. 106(c)). They form a 2DEG with outstanding transport properties, including very high electron mobility and saturation velocity. This 2DEG forms the channel of (Al,Ga)N/GaN HEMTs. ${ }^{761,762}$ The spontaneous formation of a transistor channel (i.e., 2DEG), due to the polarization difference at the $(\mathrm{Al}, \mathrm{Ga}) \mathrm{N} / \mathrm{GaN}$ heterostructure, makes these transistors normally on or depletion mode (D-mode). In these devices, a negative gate bias is needed to deplete the $2 \mathrm{DEG}$ channel underneath the gate electrode of the transistor and to turn the device off.

Figure 107 shows how the $2 \mathrm{DEG}$ density is affected by the $(\mathrm{Al}, \mathrm{Ga}) \mathrm{N}$ barrier thickness and its $\mathrm{Al}$ content. For an $(\mathrm{Al}, \mathrm{Ga}) \mathrm{N}$ thickness above the critical thickness required for the formation of the 2DEG, the 2DEG density increases with the barrier thickness until it reaches saturation, close to the value of the polarization charges in the barrier $\sigma_{\mathrm{po}}{ }^{762,764}$ The critical thickness for 2DEG formation should not be mistaken with the critical thickness for the plastic relaxation of the $(\mathrm{Al}, \mathrm{Ga}) \mathrm{N}$ layer, beyond which this layer is no longer pseudomorphic on GaN and additional structural defects, such as threading dislocations and cracks, occur, which severely impacts the 2DEG properties. The Al-content in the barrier also strongly impacts its polarization. A higher Alcontent in the $(\mathrm{Al}, \mathrm{Ga}) \mathrm{N}$ barrier leads to higher polarization constants, which induce a higher 2DEG density. Other barrier materials than $(\mathrm{Al}, \mathrm{Ga}) \mathrm{N}$ can be used, such as $(\mathrm{In}, \mathrm{Al}) \mathrm{N}$ or AlN, which present the advantage of having higher polarization constants and, for some specific compositions, a closer lattice match to $\mathrm{GaN}{ }^{765}$

To fabricate a transistor device, the 2DEG in an $(\mathrm{Al}, \mathrm{Ga}) \mathrm{N} / \mathrm{GaN}$ HEMT is contacted by two ohmic contacts, the source and the drain, while the modulation of the 2DEG is typically performed by a Schottky contact, referred to as the gate. The device is typically covered by a nitride or oxide-based dielectric passivation layer, such as $\mathrm{SiN}_{\mathrm{x}}, \mathrm{SiO}_{2}$, or $\mathrm{Al}_{2} \mathrm{O}_{3}$, in order to stabilize the surface charges that are responsible for the 2DEG formation. ${ }^{766}$ For applications where a high current level is required, a multi-fingered transistor structure is typically required. In addition, source and gate field plates are the key device components that allow for spreading out the high electric field over a larger surface area to improve the transistor breakdown voltage, to reduce the current collapse, and to increase the long term device reliability ${ }^{767,768}$ Figure 108 compares the RF performance of some of the $\mathrm{GaN}$ transistors reported in the literature. ${ }^{769-784}$

\section{Improving transistor performance through polarization and carrier confinement}

Polarization engineering enables new opportunities for transistor design. One of the new devices made possible by the strong polarization fields in $\mathrm{GaN}$ is the polarization field effect transistor (PolFET). ${ }^{785}$ The structure of the PolFET consists of a graded (Al,Ga)N barrier on top of a GaN channel/buffer region. The gradient of polarization inside the graded $(\mathrm{Al}, \mathrm{Ga}) \mathrm{N}$ structure induces a $3 \mathrm{D}$ electron slab (3DES) (Fig. 109). The magnitude of the polarization gradient controls the spatial distribution of electrons in the channel, which can be used to improve the linearity of the transistor transconductance ${ }^{786}$ (Fig. 110). The lack of ionized impurity scattering in this device structure allows for better transport properties than in a traditional MESFET transistor.

Polarization can also be used to improve the confinement of the 2DEG, which is essential for high power/high frequency transistors. The use of an AlN spacer layer, only a few nanometers thick and located in between the barrier and the GaN, prevents the electron penetration into the barrier and, thus, it improves the 2DEG confinement. The AlN spacer layer increases the 2DEG mobility as well because of reduced alloy disorder scattering. ${ }^{787}$

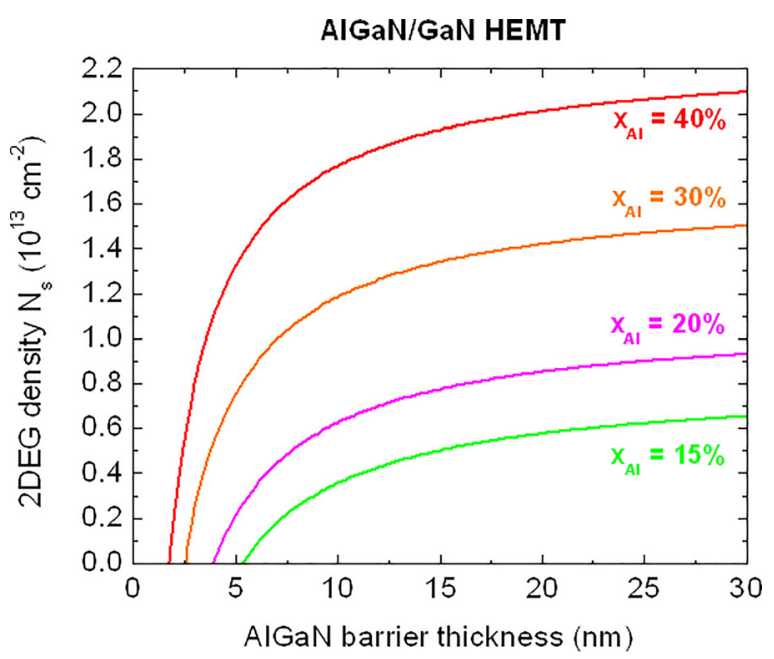

FIG. 107. Evolution of the 2DEG density as a function of the Al-content in $(\mathrm{Al}, \mathrm{Ga}) \mathrm{N}$ barrier and thickness. 


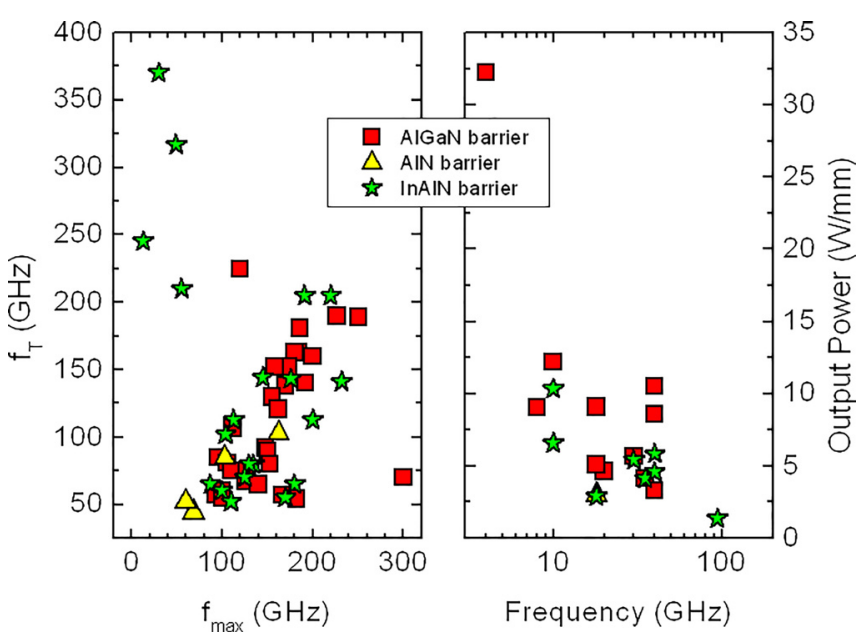

FIG. 108. RF performance of GaN-based HEMTs for high speed power amplifiers, as a function of barrier type.

Scaling the gate length to reach high frequency operation leads to short channel effects due to poor 2DEG confinement and an increase of the output conductance, which degrades the transistor high frequency performance. To improve the 2DEG confinement and to limit short channel effects, a back-barrier is placed beneath the channel, to prevent the injection of 2DEG electrons into the buffer layer, even under high electric field. In GaN transistors, polarization is a key in the design of the back-barrier. Both a thin layer of (In,Ga) $\mathrm{N}^{788-790}$ and a thick layer of low-Al content (Al,Ga)N have been used. ${ }^{791-793}$ In the case of the (In,Ga)N back-barrier, a thin (1-2 nm) (In, Ga)N layer is inserted right underneath the $\mathrm{GaN}$ channel. The polarization difference between the (In,Ga)N layer and the GaN channel and buffer induce an electric field inside the (In, Ga)N layer that brings the $\mathrm{CB}$ in the GaN channel below the one in the GaN buffer, helping in this way to confine further the electrons in the channel $^{789}$ (Fig. 111(a)). In the case of an (Al,Ga)N backbarrier, the difference in the polarization coefficients between $(\mathrm{Al}, \mathrm{Ga}) \mathrm{N}$ and $\mathrm{GaN}$ again induces an electric field in the GaN channel that increases the barrier seen by the electrons $^{793}$ (Fig. 111(b)). In addition, the (Al,Ga)N buffer has a higher breakdown voltage than GaN, although it also shows worse thermal performance due to alloy scattering. In terms of growth, the growth of $(\mathrm{In}, \mathrm{Ga}) \mathrm{N}$ is more challenging because of the $11 \%$ lattice mismatch between GaN and InN, and also due to the fact that In has lower miscibility in GaN than $\mathrm{Al} .^{794}$ In both cases, however, the use of a back-barrier enables superior performance ${ }^{789,795-801}$ because of an improved 2DEG confinement (Fig. 112).

\section{Normally off HEMTs}

Normally off transistors, also known as enhancement mode (E-mode) transistors, require a positive gate-bias to turn the device on. These devices are preferred to D-mode transistors in power electronics and RF/microwave power amplifiers for a number of reasons. First, the circuit complexity is significantly reduced if the devices only require a positive-polarity power supply. In addition, the fact that a bias has to be applied to turn the device on allows for improved safety of the entire circuit in case of a failure in the gate control circuit. Finally, the possibility of directly integrating E-mode with D-mode transistors in the same chip has tremendous potential by allowing digital and mixedsignal electronics with improved performance and increased circuit complexity.

In spite of the numerous applications for E-mode devices, E-mode HEMTs are challenging to realize in this material system because of the intrinsic polarization-induced 2DEG in GaN-based HEMTs. To fabricate E-mode HEMTs, innovative epitaxy design and/or additional processing steps are necessary. Here, again, polarization engineering is extremely useful.

Several technologies have been reported to realize E-mode GaN-HEMTs. One of these is to adjust the barrier thickness close to the critical thickness of 2DEG formation $^{802-804}$ (Fig. 113(a)). However, this approach results in a low 2DEG density and very high sheet resistance over the entire device area (including both, the access and gate regions), which is unacceptable for the low on-resistance required in low loss and fast switching applications. To reduce the access resistances, researchers have utilized a $\mathrm{D}$-mode heterostructure and recessed the $(\mathrm{Al}, \mathrm{Ga}) \mathrm{N}$ barrier underneath the gate, depleting the 2DEG ${ }^{805-808}$ (Fig. 113(b)). However, recessing the gate is most often related to the use of plasma-based dry-etching, which creates defects, reducing the channel mobility and thus increasing the total resistance. Alternatively, an etch-stop barrier can be used to selectively control the etching. ${ }^{809-811}$ This approach, however, adds complexity to the heterostructure, as does the gate injection transistor. ${ }^{812}$ The latter is a GaN transistor consisting of a $p$-type doped $(\mathrm{Al}, \mathrm{Ga}) \mathrm{N}^{812}$ or $\mathrm{GaN}^{813}$ cap layer,
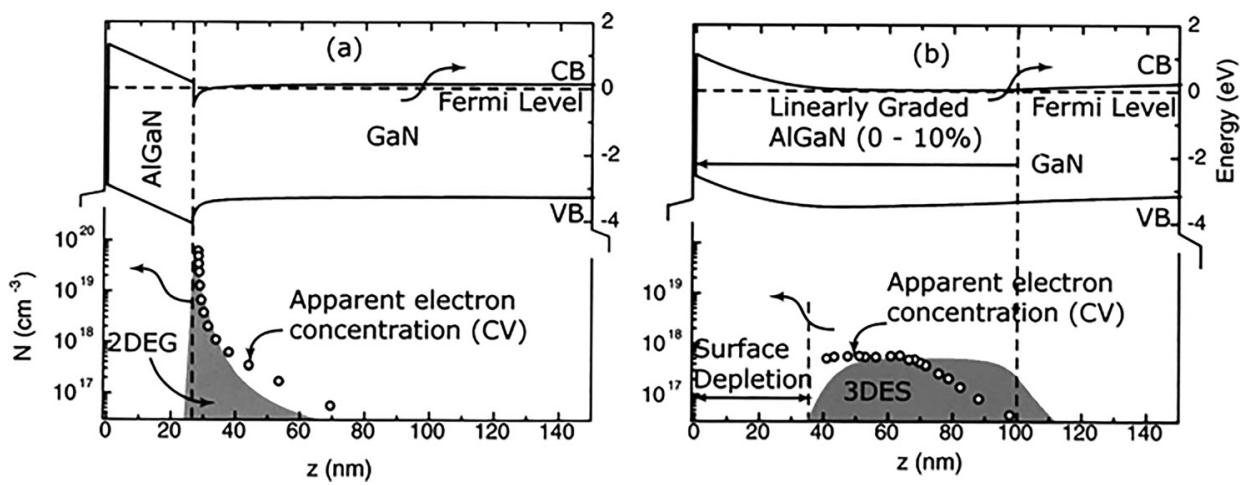

FIG. 109. Band diagram and doping profile comparison between a $2 \mathrm{DEG}$ and a 3DES. Reprinted with permission from Appl. Phys. Lett. 81, 4395 (2002). Copyright 2002 AIP Publishing LLC. 


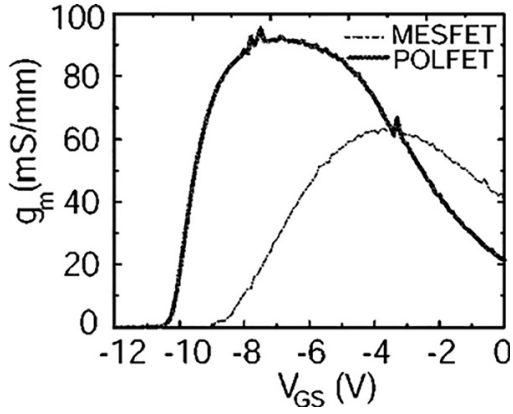

FIG. 110. Comparison of PolFET and MESFET $g_{m}-V_{g s}$ characteristics at $V_{d s}=10 \mathrm{~V}$, for transistors with $L_{g}=0.7 \mu \mathrm{m}$ and $W=150 \mu \mathrm{m}$. Reprinted with permission from Appl. Phys. Lett. 84, 1591 (2004). Copyright 2004 AIP Publishing LLC.

localized beneath the gate and on the top of the $(\mathrm{Al}, \mathrm{Ga}) \mathrm{N}$ barrier (Fig. 113(c)). The p-type layer depletes the 2DEG beneath the gate due to the built-in electric field of the $p n$ junction. Thanks to this $p n$-junction, the gate leakage current remains low as long as the gate voltage is below the built-in voltage of the structure. Beyond that voltage, holes are injected in the channel, increasing the channel current.

Alternative technologies to fabricate E-mode transistors include the implantation of negative ions (i.e., fluorine) beneath the gate ${ }^{814-817}$ and the growth of an oxide on an AlN barrier ${ }^{818,819}$ (Fig. 113(d)). In the case of the oxide, typically obtained through an $\mathrm{O}_{2}$ plasma treatment, the oxide increases the barrier potential and, at the same time, reduces the AlN barrier thickness. This helps in depleting the 2DEG underneath. ${ }^{818}$ When using fluorine ions to realize E-mode operation, special care is needed to make sure that the fluorine ions do not move under thermal or electrical stress, which could cause a shift in the threshold voltage $\left(V_{t}\right)$ of the device. $^{820}$

The various methods described above use polarization engineering underneath the gates to deplete the 2DEG channel. However, an alternative approach consists of growing GaN-based transistors along non-polar directions, such as on $a$-plane ${ }^{821,822}$ or $m$-plane. ${ }^{823-825}$ The 2DEG is then induced by modulation doping in the barrier, as in $(\mathrm{Al}, \mathrm{Ga}) \mathrm{As} / \mathrm{GaAs}$ HEMTs. Higher threshold voltages, as high as $3 \mathrm{~V}$, are then reached. ${ }^{824}$ Figure 114 summarizes the different technologies used to realize E-mode GaN-based HEMTs by

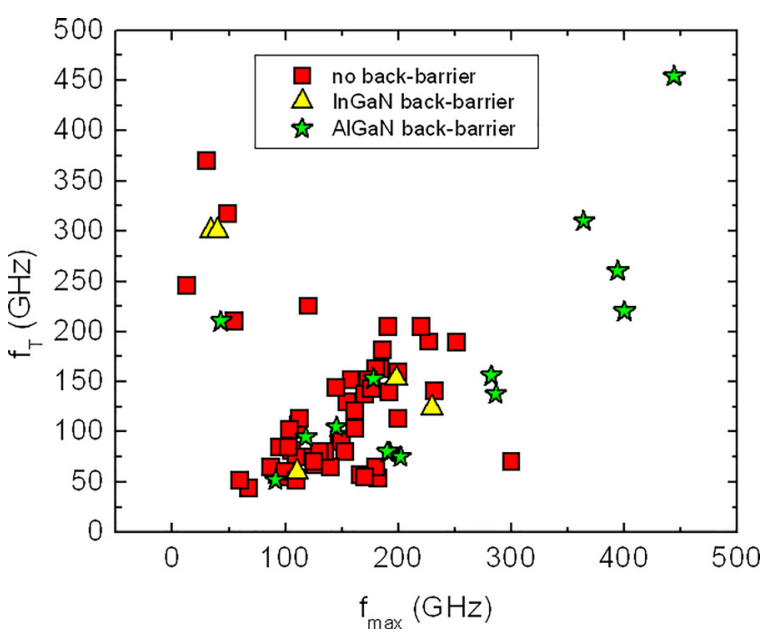

FIG. 112. Benchmark showing the impact of back-barriers on $\mathrm{f}_{\mathrm{T}}$ and $\mathrm{f}_{\max }$.

benchmarking the transistor's specific on-resistance versus the device breakdown and threshold voltage.

\section{N-face GaN-HEMTs}

Figure 115(a) displays the typical heterostructure employed in N-polar GaN-based HEMTs. If the ( $\mathrm{Al}, \mathrm{Ga}) \mathrm{N}$ is pseudomorphically grown and, therefore, suffers tensile strain, the spontaneous and piezoelectric polarization fields are then both parallel to the $[000 \overline{1}]$ direction, as indicated. In the case of an $(\mathrm{Al}, \mathrm{Ga}) \mathrm{N}$ barrier, the 2DEG is thus formed at the interface between the $(\mathrm{Al}, \mathrm{Ga}) \mathrm{N}$ barrier and the $\mathrm{GaN}$ channel, which is the topmost layer now (Fig. 115(a)). Indeed, electrons of the 2DEG are transferred from hole-trap states, ${ }^{764}$ as represented on the charge distribution in Fig. 115(b). The 2DEG is localized on the degenerated states of the triangular QW formed in $\mathrm{GaN}$ at the $\mathrm{GaN} /(\mathrm{Al}, \mathrm{Ga}) \mathrm{N}$ heterointerface (Fig. 115(c)). This describes the D-mode Npolar GaN/(Al,Ga)N HEMTs.

An immediate advantage of N-polar GaN-based HEMTs is that the $(\mathrm{Al}, \mathrm{Ga}) \mathrm{N}$ barrier provides a natural back-barrier, preventing the injection of electrons into the buffer layer. Furthermore, the $(\mathrm{Al}, \mathrm{Ga}) \mathrm{N}$ barrier/back-barrier is quite thin (about $20 \mathrm{~nm}$ ), so the thermal management is easier than when a thicker $(\mathrm{Al}, \mathrm{Ga}) \mathrm{N}$ back-barrier is grown in Ga-polar GaN-based HEMTs. Furthermore, N-polar GaN HEMTs also
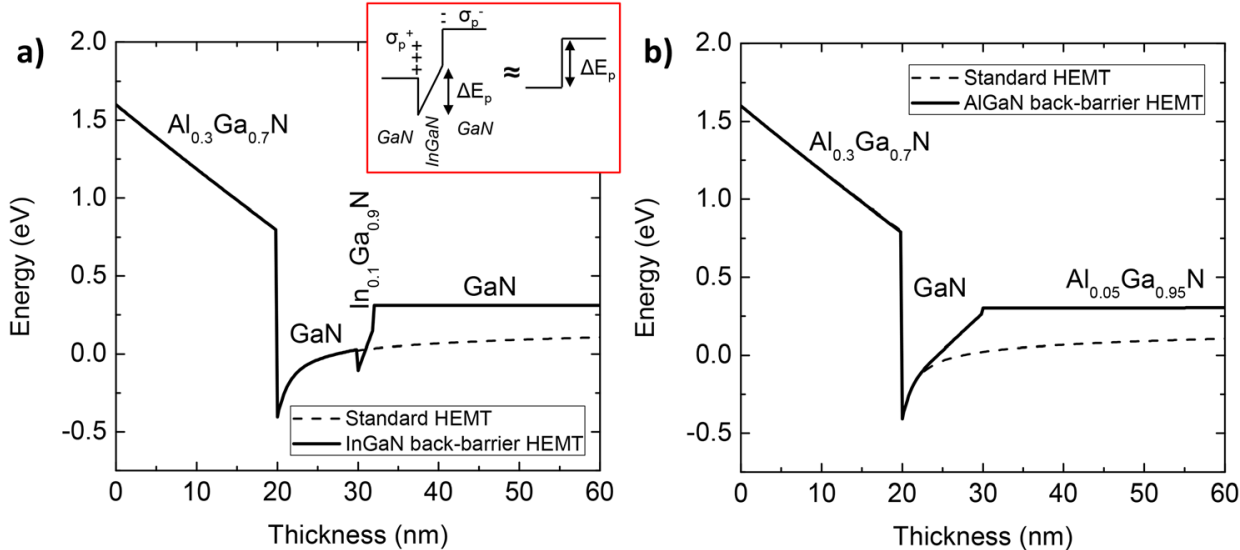

FIG. 111. Conduction band diagram of an (Al,Ga)N/GaN HEMT with an (In,Ga)N back-barrier (a) and an (Al,Ga)N back-barrier (b). The inset shows a schematic representation of the use of an (In, Ga) N back-barrier. 

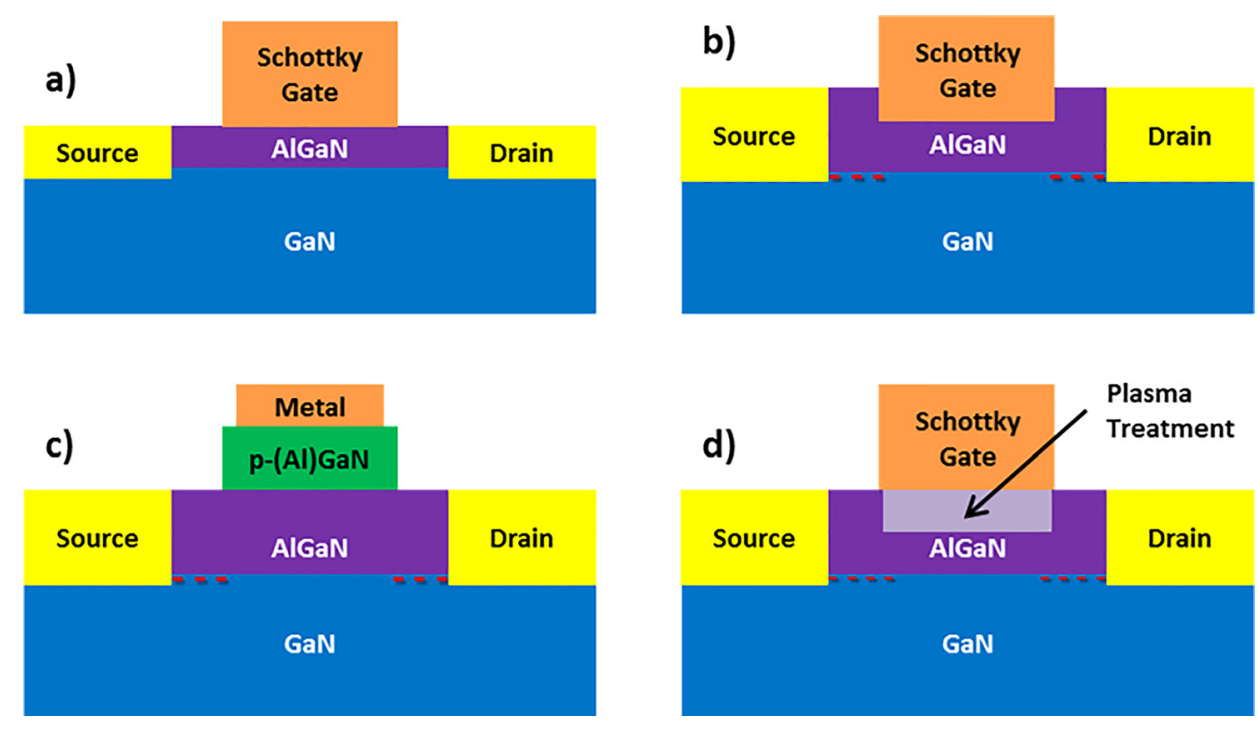

FIG. 113. Schematic representation of different technologies to fabricate Emode transistors: (a) thin $(\mathrm{Al}, \mathrm{Ga}) \mathrm{N}$ barrier, (b) gate recess, (c) $p$-type GaN or $(\mathrm{Al}, \mathrm{Ga}) \mathrm{N}$, and (d) surface plasma treatment with fluorine or oxygen implantation. reduce the complexity of the HEMT structure. Having the GaN channel on the top of the barrier renders easier the fabrication of low contact resistance ohmic contacts, as compared with Ga-polar GaN devices where a wide-bandgap $(\mathrm{Al}, \mathrm{Ga}) \mathrm{N}$ layer is on top. Moreover, the gate-to-2DEG distance can be made thinner than in Ga-polar HEMTs, so that the transconductance is improved. The stronger gate control on the channel is especially important for high frequency applications. High device performance, such as $f_{T} / f_{\max }$ of $148 / 351 \mathrm{GHz}$, was obtained for a gate length of $80 \mathrm{~nm} .^{826}$ Finally, it should be noted that normally off N-polar GaN transistors can more easily be achieved by introducing a thin AlN layer on top of the GaN channel, which enables to deplete the 2DEG thanks to the polarization-induced field therein. $^{827}$

Besides the direct growth of N-polar GaN heterostructures, as discussed in Section IV A (see Ref. 232 too), N-polar GaN heterostructures can also be obtained through wafer bonding. For example, flipping a D-mode Ga-polar GaN-based HEMT structure, bonding it onto a carrier wafer,
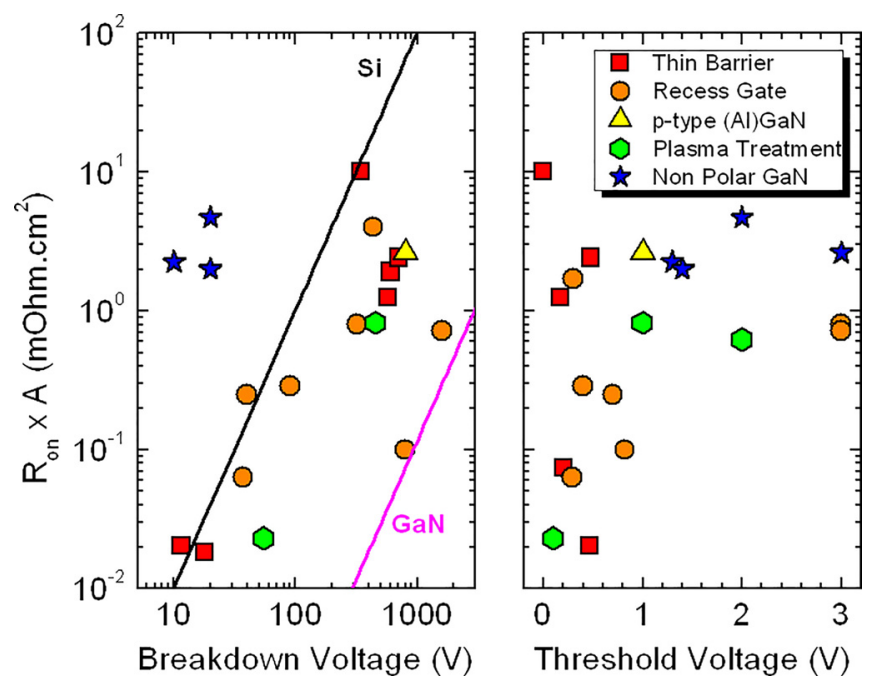

FIG. 114. Benchmark of E-mode GaN-based HEMTs. and etching the initial substrate give access to N-polar GaN HEMTs $^{828,829}$ (Fig. 116).

\section{OUTLOOK}

Due to the polar structure of commonly used $\mathrm{GaN}, \mathrm{ZnO}$, and their associated alloys, particular surface and bulk properties are expected from opposite polarity epilayers and thin films, nanostructures (including NWs), and related heterostructures. With this in mind, this review article was aimed at giving a detailed view of the present status of research in the field of epitaxial growth of opposite polarity $\mathrm{GaN}$ and $\mathrm{ZnO}$ structures and to highlight their differences in terms of structural, optical, and electronic properties. Application wise, be it for optical or electronic applications, the potential advantages of the two opposite polarities for actual devices had to be examined and the present day achievements presented.

The polar nature of $\mathrm{GaN}$ and $\mathrm{ZnO}$ being a result of their wurtzite crystalline structure, symmetry considerations have been briefly introduced to remind that the noncentrosymmetry of the structure is not a sufficient condition for a material to display a spontaneous polarization, otherwise zinc-blende materials would show it. Instead, for $\mathrm{GaN}$ and $\mathrm{ZnO}$, the unique character of the polar $\langle 0001\rangle$ direction is sufficient. Still, since epitaxial growth, which is the main focus of this review article, is surface rather than "bulk" dependent, and because many properties also depend on the surface termination (e.g., surface band structure, metallic contact characteristics, impurity incorporation, optical reflectivity, etc.), it was found necessary to explicitly dwell upon surface polarity and concentrate on the surface structures observed for these polar surfaces, both experimentally and theoretically. It has been clearly shown that although bulktruncated polar surfaces do not comply with the "electron counting rule," flat surfaces of the two opposite polarities can be obtained. In order to achieve charge compensation, surface relaxation/reconstruction mechanisms have been considered, enabling to account for the peculiar behavior of $\mathrm{GaN}$ and $\mathrm{ZnO}$ polar surfaces. Furthermore, in view of their 

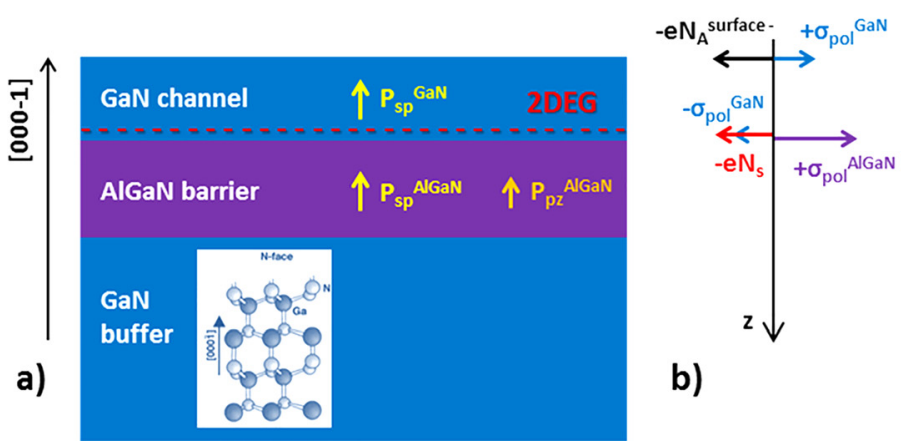

use as growth guidelines, the efficiency of the different mechanisms has been discussed in terms of chemical potentials, i.e., in terms of anion- or metal-rich growth conditions. Besides, the role of hydrogen, which is omnipresent in MOCVD growth, in passivating surface dangling bonds has been introduced for both materials as well as for the different planes (not only polar but also semipolar).

A central question addressed in this review has been how to determine the polarity of the different grown structures, namely, epilayers and thin films as well as nanostructures. The variety of relevant characterization techniques are relatively broad, including chemical etching, electron microscopy, scanning probe microscopy, and diffractionbased experiments. While some of them are well-adapted for determining macroscopically the polarity, such as polaritysensitive chemical etching or $\mathrm{x}$-ray and electron-based experiments, others are able to determine the polarity on the local scale, such as TEM- and AFM-based experiments, both being complementary to each other. Although the determination of polarity is readily performed on epilayers and thin films, careful analyses are required when the measurements are achieved on nanostructures. Over the last years, this has become especially important due to the growth of NW ensembles, given that all the NWs of the ensemble do not necessarily share the same polarity. For this reason, a number of techniques, including KPM or ABF-STEM, have been adapted and exploited to analyze individual objects, enabling to correlate macroscopic and local polarity measurements. In some cases, these combined measurements have enabled to identify, for example, the presence of nanoscopic inclusions of opposite-polarity domains. Most often, the combination of different characterization techniques both on the macroscopic and local scales is thus required to unambiguously determine the polarity.

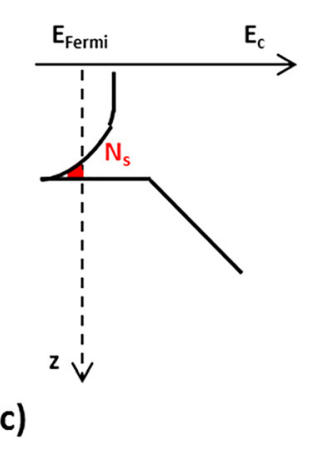

FIG. 115. (a) Polarization diagram in $\mathrm{N}$-face D-mode (Al,Ga)N/GaN heterostructure. (b) Charge distribution that induces a 2DEG in $\mathrm{N}$-face $\mathrm{D}$-mode (Al,Ga)N/GaN heterostructures. (c) Conduction band diagram corresponding to the $\mathrm{N}$-face (Al,Ga)N/GaN heterostructure.

Owing to the differences and potential advantages associated with one polarity with respect to the other, one of the main questions that the community has experimentally addressed is whether or not the two opposite polarities can be stabilized. Historically, this was also motivated by the necessity to make sure that no extended defects like IDBs would thread across the active layers of the optical or electronic devices, since these are recognized as deleterious structural defects.

Although it was considered as a difficult issue until not so long ago, since one polarity was generally found much easier to stabilize than the other depending on the growth conditions and deposition technique, controlling the polarity seems now a rather well mastered problem. In this respect, the possibility to use epiready homo-substrates or templates ( $\mathrm{GaN}$ and $\mathrm{ZnO}$ ) has helped a lot in addressing the right problems associated with growing epitaxial layers of the two different polarities, without having to control the nucleation step as needed on hetero-substrates. The nature of these "intrinsic" growth solutions does depend on the type of epitaxial process being used (MOVPE/MBE/PLD...). For instance, consider nitrides grown by MOVPE (or by ammonia-MBE). Because growth is usually carried out in N-rich conditions, the growth rate is dictated by the Ga diffusivity on the surface: for N-polar surfaces, easy Ga chemisorption implies lower adatom surface mobilities and kinetically induced roughness. It thus took some efforts to stabilize smooth N-polar MOVPE films. Usual tricks (recipes) from the epitaxy toolbox were implemented to solve the low diffusivity problems, such as substrate off-cuts, surfactants or, more generally, growth parameter adjustments, or a combination of those. On the other hand, for plasma-assisted MBE grown nitrides, things look simpler since growth is carried out in Ga-rich conditions whatever the polarity: the

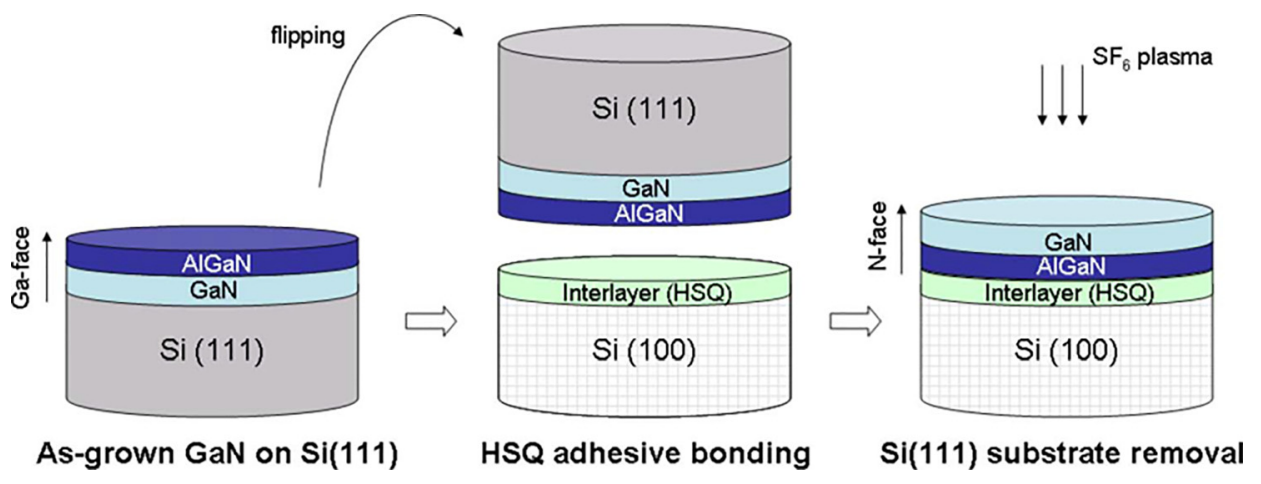

FIG. 116. An N-face GaN-based HEMT structure can be fabricated by growing it Ga-face and then applying a bonding and layer transfer approach. Reprinted with permission from Chung et al., IEEE Electron Device Lett. 30, 113 (2009). Copyright 2009 IEEE. 
controlling parameter in this case concerns the used $\mathrm{Ga}$ excess, to be adjusted differently for the two polarities. Moving to ZnO-related materials grown by MOVPE and by PLD, at present both types of polarities can be stabilized with optimum surfaces upon optimization of growth conditions, temperature being the main control parameter. As for MBE growth, most of the studies have dealt with Zn-polar epilayers, which display currently atomically smooth surfaces, so further work must be devoted to the O-polar surface.

Since available $\mathrm{ZnO}$ and $\mathrm{GaN}$ substrates are still of rather low dimensions and of high cost, hetero-epitaxial growth is still the most widely considered approach. In this case, the tricks to choose (impose) the polarity rely on mastering the nucleation process itself. This is fairly obvious when using polar substrates like, for instance, $\mathrm{SiC}$ for nitrides, for which the $\mathrm{Si}$ or $\mathrm{C}$ terminated surfaces dictate the polarity of the overgrown nitride (Ga-polar on Si-face and $\mathrm{N}$-polar on C-face). For nonpolar substrates such as sapphire, things are a bit more complicated, be it for nitrides or oxides. In this case, the polarity is imposed by the growth initiation process: nitridation (if any) atmosphere, duration and temperature of the nitridation step, buffer layer nature, and growth temperature, etc. Once the polarity is fixed, one may implement the growth recipes as discussed just above to maintain or improve the morphology of the growing polar surfaces.

Besides finding the right nucleation and growth conditions to impose the desired polarity, original methods have been implemented to reverse it, opening new opportunities to elaborate more complex heterostructures. Indeed, polarity can be inverted at a certain step during growth through heavy doping (like $\mathrm{Mg}$ in $\mathrm{GaN}$ or $\mathrm{Al}$ in $\mathrm{ZnO}$ ), although this may lead to residual doping in the overgrown epilayers, which is undesired for certain applications. Interestingly, it can also be reversed through the insertion of interlayers either at the start of the growth or as growth proceeds (AlN for GaN, $\mathrm{ScN}, \mathrm{MgO}, \mathrm{GaN}$ for $\mathrm{ZnO}$ ). In this latter case, and as an example of side development related to the control of the polarity, lithography processes have been used at an early stage of $\mathrm{ZnO}$ re-growth on a $\mathrm{ScN}$ buffer to produce periodically polarity inverted domains, which offer new possibilities in the field of non-linear optics, as implemented for both $\mathrm{ZnO}$ and $\mathrm{GaN}$.

When it comes to heterostructures, alloys are most often involved. Only nitride ternary alloys grown in opposite polarities have been compared in this review article, because of the much more advanced development of nitride devices compared with $\mathrm{ZnO}$-related oxides: the literature on the effects that polarity has on the incorporation of $\mathrm{Mg}$ or $\mathrm{Cd}$ in $\mathrm{ZnO}$ is, to our knowledge, regretfully absent. For nitrides, in general, the trend is that the alloys of both polarities can be grown on a GaN template of equivalent polarities and, if co-loaded, often show polarity dependent properties and compositions. This calls for a definite search of specific growth conditions aimed at optimizing the two opposite polarity alloys independently. Only in this case will the full potential of reversing the polarity be exploited: for instance, the expected higher thermal decomposition limit for $\mathrm{N}$-polar (In,Ga) N alloys allows, for a given In concentration, a higher growth temperature, and hence better structural properties; correlatively, for the same growth temperature, a higher In concentration can be achieved. This is an important point to consider, knowing that In uptake is a pre-requisite for addressing the green gap issue for solid state lighting applications.

Whether or not reversing the polarity of the material has any significance in terms of material purity and/or doping abilities is of major importance for potential applications. On the one hand, unintentional impurity incorporation depends upon polarity, as has been demonstrated for $\mathrm{O}$ or $\mathrm{C}$ in the case of GaN. Reducing unwanted impurity incorporation demands specific growth conditions for each polarity; for instance, the higher $\mathrm{O}$ incorporation in N-polar GaN can be drastically reduced by resorting to higher growth temperature and higher V/III ratio. Similarly, for N-polar GaN C incorporation increases upon decreasing the temperature, which raises problems when considering the growth of $(\mathrm{In}, \mathrm{Ga}) \mathrm{N}$ quantum well structures. Note, however, that increased $\mathrm{C}$ incorporation offers potential advantages when searching to increase the resistivity of $\mathrm{GaN}$ to render it semi-insulating. As for $\mathrm{ZnO}$, the higher intensity of free and bound PL exciton lines together with their narrower FWHM in the case of Znpolar epilayers compared with O-polar ones is the signature of increased impurity incorporation on O-polar surfaces. Nevertheless, whether this is an intrinsic property of opposite polarity epilayers or whether this arises from the different purity of the opposite polarity substrates being used is still an open question for $\mathrm{ZnO}$.

On the other hand, the doping efficiency of intentionally introduced species may also vary with polarity, but this depends on the growth process: for instance, only in the case of MBE growth does Mg incorporation decrease for N-polar $\mathrm{GaN}$, while there appears to be no such difference for MOVPE-grown layers; but $\mathrm{Si}$, the usual n-type dopant, has a better incorporation in Ga-polar layers. In the quest for an efficient p-type dopant for $\mathrm{ZnO}$, it has been shown that the optical signature of $\mathrm{N}$ as an acceptor is much stronger for Zn-polar epilayers. However, self-compensation is still too high to yield efficient hole injection in the active zone of actual optical devices.

Now the question arises as to whether or not the polarity of low-dimensional structures, in particular, of NWs, can be controlled just like for 2D layers. If one wants to design NW-based heterostructures, the advantages associated with reversing the polarity may or may not be equivalent to those observed in the 2D structures. Take, for instance, axial QW heterostructures for NW-based LEDs: N polarity should provide the same expected advantages in terms of band gap engineering as for 2D LEDs, compared with the Ga-polar ones. In the case of radial QW heterostructures for NWbased LEDs, i.e., with core-shell QWs, the effect of the NW core polarity will be restricted for controlling the efficiency of n-doping, while it should play no role in the band structure of the nonpolar QWs. The question focuses more on the effects that the presence of IDBs could have in the overall NW-LED efficiency if the NW polarity is not uniform.

Similar to 2D layers, homoepitaxial growth (on $\mathrm{GaN}$ or $\mathrm{ZnO}$ templates/substrates) enabled to avoid any interfaceinduced effect on the selection of polarity and to establish the particular growth conditions necessary to achieve vertical 
growth on each polarity. Take GaN, for instance: when grown by MOVPE the polarity is actually preserved, but while on top of N-polar GaN templates NWs could be grown, on Ga-polar ones templates $\mathrm{GaN}$ pyramids were formed. In fact, to obtain Ga-polar NWs on Ga-polar GaN templates, specific low flow growth conditions are required. In the case of MOVPE grown $\mathrm{ZnO}$ wires, a fairly similar behavior is observed: when grown on O-polar $\mathrm{ZnO}$ templates, O-polar pyramids are formed together with wires of $\mathrm{Zn}$ polarity. Thus, polarity influences the shape of the nanocrystal being grown. This is also consistent with the observation that GaN wires have flat $c$-oriented tops if $\mathrm{N}$-polar but have facetted top if Ga-polar (incidentally, opening a very straightforward way to assess their polarity). Correlatively, as exemplified in the case of $\mathrm{ZnO}$, the very large diversity of obtainable nanostructures reveals the strong influence that polarity has on the growth morphology. Such exotic nanostructures may open new possibilities in terms of nanophysics; however, using unconventional nanostructure shapes may, like in the case of Ga-polar wires with facetted tops, pose technological problems when it comes to making devices. Interestingly, although $\mathrm{ZnO}$ wires are exclusively of Zn-polarity using standard physical and chemical vapor deposition techniques according to the self-induced approach, catalyst-assisted MOVPE and chemical bath deposition bring about the possibility to synthesize O-polar wires. Not only does the deposition technique dictate the shape and the growth axis of homoepitaxial nanostructures but also the chemistry of the growth front plays a crucial role for selecting the polarity. The latter CBD techniques, in particular, favor the homoepitaxial formation of $\mathrm{ZnO}$ wires with controllable polarity thanks to strong electrostatic interactions occurring in solution, which circumvent the main problems raised by vapor deposition techniques. Its compatibility with technological process in a cleanroom environment according to the SAG approach is also a strong asset that should open new opportunities to form by-design complex heterostructures with high structural uniformity.

When using hetero-substrates such as sapphire for growing nanostructures, the substrate surface treatment is a crucial step for selecting the polarity of the overgrown wires, like in the case of 2D layers: for GaN NWs by MOVPE, upon sapphire nitridation mixed polarity wires are obtained while if no sapphire nitridation is carried out, the wires are purely Ga-polar. A structural model of the substrate/wire interface is clearly missing to account for such a phenomenon. Correlatively, for NWs grown by SAG on sapphire, IDBs can be generated at the edge of the mask, leaving $\mathrm{N}$-polar cores surrounded by Ga-polar shells, although it is not yet clear if the IDB is due to the quality of the masks or associated only to the nature of the substrate. For MBE grown GaN wires, most self-assembled wires were found to be of nitrogen polarity regardless of the substrate, the reverse polarity being apparently only obtained in the presence of structural or morphological defects at the surfaces; the reason for this preferred $\mathrm{N}$ polarity is still not understood. As of today, the very best method to unambiguously control the polarity by MBE lies in using SAG on either Ga-polar or $\mathrm{N}$-polar templates or substrates. In this situation, the problems related to substrate surface inhomogeneities or defects are minimized thanks to the small substrate footprint for growth.

Since epitaxial growth of epilayers of binary and ternary WBG semiconductors of both polarities is feasible, as presented lengthily in this review article, the point to consider now is if reversing the polarity will bring about the expected improvements of optical and electronic devices. In this review, we have focused on 2D heterostructures and related applications because of the scarcity of results concerning the effects that polarity has on the properties of NW heterostructures or devices. This is clearly missing at this point.

For LEDs, for instance, whether the limited efficiency is due to Auger effects or carrier leakage over the active region, or both, is still controversial. Reversing the polarity will, in principle, not directly affect the Auger coefficients, this being a "bulk" property of the semiconductors. However, reversing the polarity from $\mathrm{Ga}$ to $\mathrm{N}$ changes the sign of the interface charges associated with the spontaneous polarizations discontinuity. Hence, the band alignments at these interfaces might be largely altered, potentially leading to easier carrier injection in the QWs together with less carrier leakage above the active region. Accordingly, band gap engineering can be achieved through polarity reversal. N-polar LEDs have effectively been fabricated with lower operating voltages than their Ga-polar counterparts, but their electroluminescence intensity was found to be much less than for Ga-polar LEDs, with a more intense yellow band. This is tentatively attributed to the higher impurity incorporation (i.e., C) in the low temperature grown $(\mathrm{In}, \mathrm{Ga}) \mathrm{N}$ QWs when in N-polar heterostructures. But the analysis of the emission time-dependence under electrical injection actually confirms the expected stronger localization and weaker quantum confined Stark effect in N-polar LEDs compared with the Gapolar ones. These achievements call for an improvement of the growth conditions that would allow for higher purity N-polar (In,Ga)N QWs. Interestingly, reversing the polarization would also mitigate the detrimental effects of polarization discontinuities in photovoltaics based on (In, Ga)N /GaN p-i-n diodes.

As for electronic devices, GaN-based HEMT structures have been compared for both polarities. Here, again efficient band gap engineering can be achieved through the choice of orientation. N-polar HEMT structures definitely yield better back barrier isolation, easier handling and less resistive ohmic contacts, and higher transconductance. In the case of $\mathrm{ZnO} /$ ( $\mathrm{Zn}, \mathrm{Mg}$ ) O, although the efforts in the community are far from being similar to what they are for nitrides, 2D electron gases with high mobilities have been obtained for $\mathrm{Zn}$-polar MBE grown $\mathrm{ZnO} /(\mathrm{Zn}, \mathrm{Mg}) \mathrm{O}$ heterostructures and allowed the observation of the fractional quantum Hall effect.

All in all, in the last fifteen years, the issue of GaN and $\mathrm{ZnO}$ polarity has become increasingly important, initially to find out the fundamental properties of the semiconductors involved and subsequently to act as an additional knob allowing chemists, physicists, and engineers to control nanostructure shape, dopant incorporation, and alloy quality, as well as to design and implement new device architectures. 


\section{ACKNOWLEDGMENTS}

L.L. acknowledges Dr. Ulrich Biedermann and Dr. Mira Todorova for fruitful discussion and for comments that greatly improved the theoretical section. Dr. Mira Todorova is further acknowledged for providing Fig. 8. H.R. acknowledges the French CRG (ESRF) for granting beam time at beamlines BM2-D2AM. H.R. is highly indebted to K. Hestroffer and B. Daudin who prepared the GaN NWs samples, C. Leclere who performed the data analysis, and M. G. Proietti for a careful reading. S.R. and T.P. would like to thank Daniel Piedra and Dr. Puneet Srivastava for their helpful discussion and review.

L.L. contributed mostly to Section II, X.K. and A.T. contributed mostly to Section III B, S.F.-G. and O.B. contributed mostly to Sections II B, III C, III D, and III H and Section IV C2, H.R. contributed mostly to Section III E, S.K. and K.H. contributed mostly to Section IV A, M. R. Wagner and J.S.R. contributed mostly to Section VA, F.A. and S.R. contributed mostly to Section VB, S.R. and T.P. contributed mostly to Section V C, and J.Z.-P., V.C., and G.F. assembled and coordinated the review article and contributed, in particular, to Sections III A, IIIF, III G, IV B, IV C 1 , and IV D, as well as to the introduction and conclusions.

${ }^{1}$ I. Akasaki, Rev. Mod. Phys. 87, 1119 (2015).

${ }^{2}$ H. Amano, Rev. Mod. Phys. 87, 1133 (2015).

${ }^{3}$ S. Nakamura, Rev. Mod. Phys. 87, 1139 (2015).

${ }^{4}$ O. Brandt and K. H. Ploog, Nat. Mater. 5, 769 (2006).

${ }^{5}$ O. Ambacher, J. Smart, J. R. Shealy, N. G. Weimann, K. Chu, M. Murphy, W. J. Schaff, L. F. Eastman, R. Dimitrov, L. Wittmer, M. Stutzmann, W. Rieger, and J. Hilsenbeck, J. Appl. Phys. 85, 3222 (1999).

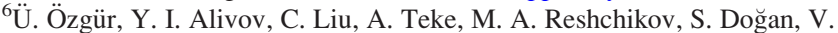
Avrutin, S.-J. Cho, and H. Morkoç, J. Appl. Phys. 98, 041301 (2005).

${ }^{7}$ D. C. Look, Mater. Sci. Eng., B 80, 383 (2001).

${ }^{8}$ S. J. Pearton, D. P. Norton, K. Ip, Y. W. Heo, and T. Steiner, Prog. Mater. Sci. 50, 293 (2005).

${ }^{9}$ W. A. Harrison, J. Vac. Sci. Technol. 16, 1492 (1979).

${ }^{10}$ C. B. Duke, Chem. Rev. 96, 1237 (1996).

${ }^{11}$ P. W. Tasker, J. Phys. C: Solid State Phys. 12, 4977 (1979).

${ }^{12}$ C. Noguera, J. Phys.: Condens Matter 12, R367 (2000).

${ }^{13}$ A Primer in Density Functional Theory, edited by C.Fiolhais, F.Nogueira, and M. A. L.Marques (Springer-Verlag, Berlin, Heidelberg, 2003), Vol. 620.

${ }^{14}$ R. G. Parr and W. Yang, Density-Functional Theory of Atoms and Molecules (Oxford University Press, New York, 1989), Vol. 16.

${ }^{15}$ M. Himmerlich, L. Lymperakis, R. Gutt, P. Lorenz, J. Neugebauer, and S. Krischok, Phys. Rev. B 88, 125304 (2013).

${ }^{16}$ A. I. Duff, L. Lymperakis, and J. Neugebauer, Phys. Status Solidi B 252, 855 (2015)

${ }^{17}$ J. F. Nye, Physical Properties of Crystals (Oford University Press, Amen House, London, 1964).

${ }^{18} \mathrm{H}$. Klapper and T. Hahn, International Tables for Crystallography (International Union of Crystallography, 2006), Vol. A, p. 804.

${ }^{19}$ M. Posternak, A. Baldereschi, A. Catellani, and R. Resta, Phys. Rev. Lett. 64, 1777 (1990)

${ }^{20}$ F. Bernardini, V. Fiorentini, and D. Vanderbilt, Phys. Rev. B 56, 10024 (1997).

${ }^{21}$ F. Bechstedt, U. Grossner, and J. Furthmüller, Phys. Rev. B 62, 8003 (2000).

${ }^{22}$ R. Resta, Rev. Mod. Phys. 66, 899 (1994).

${ }^{23}$ R. Resta and D. Vanderbilt, Physics of Ferroelectrics (Springer, Berlin/ Heidelberg, 2007), pp. 31-68.

${ }^{24}$ M. Posternak, A. Baldereschi, A. Catellani, and R. Resta, Phys. Rev. Lett. 64, 1777 (1990).

${ }^{25}$ A. K. Tagantsev, Phys. Rev. Lett. 69, 389 (1992).
${ }^{26}$ A. Baldereschi, M. Posternak, and R. Resta, Phys. Rev. Lett. 69, 390 (1992).

${ }^{27}$ F. Bernardini, V. Fiorentini, and D. Vanderbilt, Phys. Rev. B 56, R10024 (1997).

${ }^{28}$ F. Bernardini, V. Fiorentini, and D. Vanderbilt, Phys. Rev. B 63, 193201 (2001).

${ }^{29}$ A. Qteish, V. Heine, and R. J. Needs, Phys. Rev. B 45, 6534 (1992).

${ }^{30}$ A. Dal Corso, M. Posternak, R. Resta, and A. Baldereschi, Phys. Rev. B 50, 10715 (1994).

${ }^{31}$ J. Lähnemann, O. Brandt, U. Jahn, C. Pfüller, C. Roder, P. Dogan, F. Grosse, A. Belabbes, F. Bechstedt, A. Trampert, and L. Geelhaar, Phys. Rev. B 86, 081302 (2012).

${ }^{32}$ J. Goniakowski, F. Finocchi, and C. Noguera, Rep. Prog. Phys. 71, 016501 (2008).

${ }^{33}$ F. Bechstedt, Principles of Surface Physics (Springer-Verlag, Berlin, Heidelberg, 2003).

${ }^{34}$ N. Moll, A. Kley, E. Pehlke, and M. Scheffler, Phys. Rev. B 54, 8844 (1996).

${ }^{35}$ P. Waltereit, O. Brandt, A. Trampert, H. T. Grahn, J. Menniger, M. Ramsteiner, M. Reiche, and K. H. Ploog, Nature 406, 865 (2000).

${ }^{36}$ J. L. A. Alves, J. Hebenstreit, and M. Scheffler, Phys. Rev. B 44, 6188 (1991).

${ }^{37}$ J. Northrup and J. Neugebauer, Phys. Rev. B 53, R10477 (1996).

${ }^{38}$ A. Filippetti, V. Fiorentini, G. Cappellini, and A. Bosin, Phys. Rev. B 59, 8026 (1999).

${ }^{39}$ D. Segev, A. Janotti, and C. G. Van de Walle, Phys. Rev. B 75, 035201 (2007).

${ }^{40}$ C. G. Van de Walle and D. Segev, J. Appl. Phys. 101, 081704 (2007).

${ }^{41}$ L. Ivanova, S. Borisova, H. Eisele, M. Dahne, A. Laubsch, and P. Ebert, Appl. Phys. Lett. 93, 192110 (2008).

${ }^{42}$ M. Bertelli, P. Löptien, M. Wenderoth, A. Rizzi, R. G. Ulbrich, M. C. Righi, A. Ferretti, L. Martin-Samos, C. M. Bertoni, and A. Catellani, Phys. Rev. B 80, 115324 (2009).

${ }^{43}$ L. Lymperakis, P. H. Weidlich, H. Eisele, M. Schnedler, J. P. Nys, B. Grandidier, D. Stievenard, R. E. Dunin-Borkowski, J. Neugebauer, and P. Ebert, Appl. Phys. Lett. 103, 152101 (2013).

${ }^{44}$ L. Lymperakis and J. Neugebauer, Phys. Rev. B 79, 241308 (2009).

${ }^{45}$ V. Consonni, Phys. Status Solidi RRL 7, 699 (2013).

${ }^{46}$ C. D. Lee, R. M. Feenstra, J. E. Northrup, L. Lymperakis, and J. Neugebauer, Appl. Phys. Lett. 82, 1793 (2003).

${ }^{47}$ J. E. Northrup, R. Di Felice, and J. Neugebauer, Phys. Rev. B 56, R4325 (1997).

${ }^{48}$ B. Meyer and D. Marx, Phys. Rev. B 67, 035403 (2003).

${ }^{49}$ D. J. Cooke, A. Marmier, and S. C. Parker, J. Phys. Chem. B 110, 7985 (2006).

${ }^{50}$ H. Zhang, S. Lu, W. Xu, and F. Yuan, J. Appl. Phys. 113, 034903 (2013).

${ }^{51}$ N. L. Marana, V. M. Longo, E. Longo, J. B. L. Martins, and J. R. Sambrano, J. Phys. Chem. A 112, 8958 (2008).

${ }^{52}$ P. Schröer, P. Krüger, and J. Pollmann, Phys. Rev. B 49, 17092 (1994).

${ }^{53}$ A. Wander and N. M. Harrison, Surf. Sci. 457, L342 (2000).

${ }^{54}$ J. E. Jaffe, N. M. Harrison, and A. C. Hess, Phys. Rev. B 49, 11153 (1994).

${ }^{55}$ A. Wander and N. M. Harrison, J. Phys. Chem. B 105, 6191 (2001).

${ }^{56}$ Y. Wang, B. Meyer, X. Yin, M. Kunat, D. Langenberg, F. Traeger, A. Birkner, and C. Wöll, Phys. Rev. Lett. 95, 266104 (2005).

${ }^{57}$ J. E. Northrup, J. Neugebauer, R. M. Feenstra, and A. R. Smith, Phys. Rev. B 61, 9932 (2000).

${ }^{58}$ A. R. Smith, V. Ramachandran, R. M. Feenstra, D. W. Greve, M. S. Shin, M. Skowronski, J. Neugebauer, and J. E. Northrup, J. Vac. Sci. Technol., A 16, 1641 (1998)

${ }^{59}$ A. R. Smith, R. M. Feenstra, D. W. Greve, M. S. Shin, M. Skowronski, J. Neugebauer, and J. E. Northrup, Appl. Phys. Lett. 72, 2114 (1998).

${ }^{60}$ J. Neugebauer, T. Zywietz, M. Scheffler, J. E. Northrup, and C. G. Van de Walle, Phys. Rev. Lett. 80, 3097 (1998).

${ }^{61}$ C. Adelmann, J. Brault, G. Mula, B. Daudin, L. Lymperakis, and J. Neugebauer, Phys. Rev. B 67, 165419 (2003).

${ }^{62}$ J. Neugebauer, T. K. Zywietz, M. Scheffler, J. E. Northrup, H. Chen, and R. M. Feenstra, Phys. Rev. Lett. 90, 056101 (2003).

${ }^{63}$ A. R. Smith, R. M. Feenstra, D. W. Greve, J. Neugebauer, and J. E. Northrup, Phys. Rev. Lett. 79, 3934 (1997).

${ }^{64}$ C. A. Pignedoli, R. Di Felice, and C. M. Bertoni, Phys. Rev. B 64, 113301 (2001)

${ }^{65}$ K. Rapcewicz, M. B. Nardelli, and J. Bernholc, Phys. Rev. B 56, 12725 (1997). 
${ }^{66}$ J. Fritsch, O. F. Sankey, K. E. Schmidt, and J. B. Page, Phys. Rev. B 57, 15360 (1998).

${ }^{67}$ P. Kempisty, P. Strak, K. Sakowski, and S. Krukowski, J. Cryst. Growth 401, 514 (2014).

${ }^{68}$ P. Kempisty, P. Strak, and S. Krukowski, Phys. Status Solidi C 9, 826 (2012).

${ }^{69}$ J. Elsner, M. Haugk, G. Jungnickel, and T. Frauenheim, Solid State Commun. 106, 739 (1998).

${ }^{70}$ P. Kempisty and S. Krukowski, AIP Adv. 4, 117109 (2014).

${ }^{71}$ C. G. Van de Walle and J. Neugebauer, Phys. Rev. Lett. 88, 066103 (2002)

${ }^{72}$ J. E. Northrup and J. Neugebauer, Appl. Phys. Lett. 85, 3429 (2004).

${ }^{73}$ A. Wander, F. Schedin, P. Steadman, A. Norris, R. McGrath, T. S. Turner, G. Thornton, and N. M. Harrison, Phys. Rev. Lett. 86, 3811 (2001).

${ }^{74}$ G. Kresse, O. Dulub, and U. Diebold, Phys. Rev. B 68, 245409 (2003).

${ }^{75}$ J. E. Northrup and J. Neugebauer, Appl. Phys. Lett. 87, 141914 (2005).

${ }^{76}$ O. Dulub, U. Diebold, and G. Kresse, Phys. Rev. Lett. 90, 016102 (2003)

${ }^{77}$ M. Valtiner, M. Todorova, G. Grundmeier, and J. Neugebauer, Phys. Rev. Lett. 103, 065502 (2009)

${ }^{78}$ J. Northrup, R. Di Felice, and J. Neugebauer, Phys. Rev. B 56, R4325 (1997)

${ }^{79}$ M. Valtiner, M. Todorova, and J. Neugebauer, Phys. Rev. B 82, 165418 (2010)

${ }^{80}$ T. Becker, S. Hövel, M. Kunat, C. Boas, U. Burghaus, and C. Wöll, Surf. Sci. 486, L502 (2001)

${ }^{81}$ B. Meyer, Phys. Rev. B 69, 045416 (2004)

${ }^{82}$ V. Staemmler, K. Fink, B. Meyer, D. Marx, M. Kunat, S. Gil Girol, U. Burghaus, and C. Woll, Phys. Rev. Lett. 90, 106102 (2003).

${ }^{83}$ C. Wöll, Prog. Surf. Sci. 82, 55 (2007)

${ }^{84}$ J. V. Lauritsen, S. Porsgaard, M. K. Rasmussen, M. C. R. Jensen, R. Bechstein, K. Meinander, B. S. Clausen, S. Helveg, R. Wahl, G. Kresse, and F. Besenbacher, ACS Nano 5, 5987 (2011).

${ }^{85}$ S. B. Zhang and S.-H. Wei, Phys. Rev. Lett. 92, 086102 (2004).

${ }^{86}$ C. E. Dreyer, A. Janotti, and C. G. Van de Walle, Phys. Rev. B 89, 081305 (2014)

${ }^{87}$ A. E. Romanov, T. J. Baker, S. Nakamura, J. S. Speck, and E. J. U. Group, J. Appl. Phys. 100, 023522 (2006).

${ }^{88}$ J. E. Northrup, Appl. Phys. Lett. 95, 133107 (2009).

${ }^{89}$ T. Akiyama, D. Ammi, K. Nakamura, and T. Ito, Phys. Rev. B 81, 245317 (2010).

${ }^{90}$ T. Yamashita, T. Akiyama, K. Nakamura, and T. Ito, Jpn. J. Appl. Phys., Part 1 48, 120201 (2009).

${ }^{91}$ E. Kalesaki, L. Lymperakis, J. Kioseoglou, J. Neugebauer, T. Karakostas, and P. Komninou, J. Appl. Phys. 112, 033510 (2012).

${ }^{92}$ T. Ito, T. Akiyama, and K. Nakamura, Semicond. Sci. Technol. 27, 024010 (2012)

${ }^{93}$ T. Akiyama, T. Yamashita, K. Nakamura, and T. Ito, J. Cryst. Growth 318, 79 (2011).

${ }^{94}$ A. N. Mariano and R. E. Hanneman, J. Appl. Phys. 34, 384 (1963).

${ }^{95}$ H. Maki, T. Ikoma, I. Sakaguchi, N. Ohashi, H. Haneda, J. Tanaka, and N. Ichinose, Thin Solid Films 411, 91 (2002).

${ }^{96}$ H. Tampo, P. Fons, A. Yamada, K. K. Kim, H. Shibata, K. Matsubara, S. Niki, H. Yoshikawa, and H. Kanie, Appl. Phys. Lett. 87, 141904 (2005).

${ }^{97}$ X. Wang, Y. Tomita, O. H. Row, M. Ohsugi, S. B. Che, Y. Ishitani, and A. Yoshikawa, Appl. Phys. Lett. 86, 011921 (2005).

${ }^{98}$ N. Ohashi, K. Takahashi, S. Hishita, I. Sakaguchi, H. Funakubo, and H. Haneda, J. Electrochem. Soc. 154, D82 (2007)

${ }^{99}$ S. C. Han, J. K. Kim, J. Y. Kim, K. K. Kim, H. Tampo, S. Niki, and J. M. Lee, J. Electrochem. Soc. 157, D60 (2010).

${ }^{100}$ H. Iwanaga, T. Yoshie, T. Yamaguchi, and N. Shibata, J. Cryst. Growth 47, 703 (1979)

${ }^{101}$ Y. Morimoto, J. Electrochem. Soc. 121, 1383 (1974).

${ }^{102}$ A. Shintani and S. Minagawa, J. Electrochem. Soc. 123, 706 (1976).

${ }^{103}$ D. Huang, P. Visconti, K. M. Jones, M. A. Reshchikov, F. Yun, A. A. Baski, T. King, and H. Morkoc, Appl. Phys. Lett. 78, 4145 (2001).

${ }^{104}$ J. L. Weyher, S. Müller, I. Grzegory, and S. Porowski, J. Cryst. Growth 182, 17-22 (1997).

${ }^{105}$ M. Seelmann-Eggebert, J. L. Weyher, H. Obloh, H. Zimmermann, A. Rar, and S. Porowski, Appl. Phys. Lett. 71, 2635 (1997).

${ }^{106}$ J. L. Rouviere, J. L. Weyher, M. Seelmann-Eggebert, and S. Porowski, Appl. Phys. Lett. 73, 668 (1998)
${ }^{107}$ T. Palacios, F. Calle, M. Varela, C. Ballesteros, E. Monroy, F. B. Naranjo, M. A. Sanchez-Garcia, E. Calleja, and E. Munoz, Semicond. Sci. Technol. 15, 996 (2000).

${ }^{108}$ L. Dongsheng, M. Sumiya, K. Yoshimura, Y. Suzuki, Y. Fukuda, and S. Fuke, Phys. Status Solidi A 180, 357 (2000).

${ }^{109}$ D. Li, M. Sumiya, S. Fuke, D. Yang, D. Que, Y. Suzuki, and Y. Fukuda, J. Appl. Phys. 90, 4219 (2001).

${ }^{110}$ H. G. Ng, N. G. Weimann, and A. Chowdhury, J. Appl. Phys. 94, 650 (2003).

${ }^{111}$ H. G. Ng, W. Parz, N. G. Weimann, and A. Chowdhury, Jpn. J. Appl. Phys., Part 2 42, L1405 (2003).

${ }^{112}$ Y. Gao, T. Fujii, R. Sharma, K. Fujito, S. P. Denbaars, S. Nakamura, and E. L. Hu, Jpn. J. Appl. Phys., Part 2 43, L637 (2004).

${ }^{113}$ Y. Gao, M. D. Craven, J. S. Speck, S. P. Denbaars, and E. L. Hu, Appl. Phys. Lett. 84, 3322 (2004).

${ }^{114}$ D. A. Stocker, E. F. Schubert, and J. M. Redwing, Appl. Phys. Lett. 73, 2654 (1998)

${ }^{115} \mathrm{~K}$. Hestroffer, C. Leclere, C. Bougerol, H. Renevier, and B. Daudin, Phys. Rev. B 84, 245302 (2011).

${ }^{116}$ M. D. Brubaker, I. Levin, A. V. Davydov, D. M. Rourke, N. A. Sanford, V. M. Bright, and K. A. Bertness, J. Appl. Phys. 110, 053506 (2011).

${ }^{117}$ L. Largeau, E. Galopin, N. Gogneau, L. Travers, F. Glas, and J. C. Harmand, Cryst. Growth Des. 12, 2724 (2012).

${ }^{118}$ X. Wang, S. Li, S. Fündling, J. Wei, M. Erenburg, H. H. Wehmann, A. Waag, W. Bergbauer, M. Strassburg, U. Jahn, and H. Riechert, Cryst. Growth Des. 12, 2552 (2012).

${ }^{119}$ B. Alloing, S. Vézian, O. Tottereau, P. Vennéguès, E. Beraudo, and J. Zuniga-Perez, Appl. Phys. Lett. 98, 011914 (2011).

${ }^{120}$ F. A. Ponce, D. P. Bour, W. Y. Young, M. Saunders, and J. W. Steeds, Appl. Phys. Lett. 69, 337 (1996).

${ }^{121}$ B. Daudin, J. L. Rouvière, and M. Arlery, Appl. Phys. Lett. 69, 2480 (1996).

${ }^{122}$ J. L. Rouviere, J. L. Weyher, M. Seelmann-Eggebert, and S. Porowski, Appl. Phys. Lett. 73, 668 (1998).

${ }^{123}$ T. Mitate, Y. Sonoda, and N. Kuwano, Phys. Status Solidi A 192, 383 (2002).

${ }^{124}$ G. Perillat-Merceroz, R. Thierry, P. H. Jouneau, P. Ferret, and G. Feuillet, Nanotechnology 23, 125702 (2012).

${ }^{125}$ L. T. Romano, J. E. Northrup, and M. A. O'Keefe, Appl. Phys. Lett. 69, 2394 (1996).

${ }^{126}$ R. Serneels, M. Snykers, P. Delavignette, R. Gevers, and S. Amelinckx, Phys. Status Solidi B 58, 277 (1973).

${ }^{127}$ W. Kossel and G. Möllenstedt, Ann. Phys. 428, 113 (1939).

${ }^{128}$ P. A. Stadelmann, Ultramicroscopy 21, 131 (1987).

${ }^{129}$ T. Mitate, S. Mizuno, H. Takahata, R. Kakegawa, T. Matsuoka, and N. Kuwano, Appl. Phys. Lett. 86, 134103 (2005).

${ }^{130}$ J. Taftø, Phys. Rev. Lett. 51, 654 (1983).

${ }^{131}$ N. Jiang, T. J. Eustis, J. Cai, F. A. Ponce, J. C. H. Spence, and J. Silcox, Appl. Phys. Lett. 80, 389 (2002).

${ }^{132}$ J. Taftø, Acta Crystallogr., Sect. A 43, 208 (1987).

${ }^{133}$ X. Kong, J. Ristic, M. A. Sanchez-Garcia, E. Calleja, and A. Trampert, Nanotechnology 22, 415701 (2011).

${ }^{134}$ P. Hirsch, A. Howie, R. B. Nicholson, D. W. Pashley, and M. J. Whelan, Electron Microscopy of Thin Crystal, 2nd ed. (Huntington, New York, 1977).

${ }^{135}$ J. C. H. Spence and J. M. Zuo, Electron Microdiffraction (Plenum, New York, 1992).

${ }^{136}$ R. F. Egerton, Electron-Energy Loss Spectroscopy in the Electron Microscope, 2nd ed. (Plenum Press, New York, 1996).

${ }^{137}$ X. Kong, G. Q. Hu, X. F. Duan, Y. Lu, and X. L. Liu, Appl. Phys. Lett. 81, 1990 (2002).

${ }^{138}$ J. Jasinski, D. Zhang, J. Parra, V. Katkanant, and V. J. Leppert, Appl. Phys. Lett. 92, 093104 (2008).

${ }^{139}$ E. Uccelli, J. Arbiol, C. Magen, P. Krogstrup, E. Russo-Averchi, M. Heiss, G. Mugny, F. Morier-Genoud, J. Nygard, J. R. Morante, and A. Fontcuberta i Morral, Nano Lett. 11, 3827 (2011).

${ }^{140}$ K. A. Mkhoyan, P. E. Batson, J. Cha, W. J. Schaff, and J. Silcox, Science 312, 1354 (2006).

${ }^{141}$ J. L. Rouvière, C. Bougerol, B. Amstatt, E. Bellet-Almaric, and B. Daudin, Appl. Phys. Lett. 92, 201904 (2008)

${ }^{142}$ S. D. Findlay, N. Shibata, H. Sawada, E. Okunishi, Y. Kondo, and Y. Ikuhara, Ultramicroscopy 110, 903 (2010).

${ }^{143}$ M. de la Mata, C. Magen, J. Gazquez, M. I. B. Utama, M. Heiss, S. Lopatin, F. Furtmayr, C. J. Fernandez-Rojas, B. Peng, J. R. Morante, R. 
Rurali, M. Eickhoff, A. Fontcuberta I Morral, Q. Xiong, and J. Arbiol, Nano Lett. 12, 2579 (2012).

${ }^{144}$ L. Li, Z. Gan, M. R. McCartney, H. Liang, H. Yu, W. J. Yin, Y. Yan, Y. Gao, J. Wang, and D. J. Smith, Adv. Mater. 26, 1052 (2014).

${ }^{145}$ M. I. den Hertog, F. Gonzalez-Posada, R. Songmuang, J. L. Rouviere, T. Fournier, B. Fernandez, and E. Monroy, Nano Lett. 12, 5691 (2012).

${ }^{146}$ F. Jona, J. S. Strozier, and W. Yang, Rep. Prog. Phys. 45, 527 (1982).

${ }^{147}$ K. Heinz, Rep. Prog. Phys. 58, 637 (1995).

${ }^{148}$ M. A. Van Hove, W. H. Weinberg, and C.-M. Chan, Low-Energy Electron Diffraction (Springer-Verlag, Berlin/Heidelberg, 1986).

${ }^{149}$ O. Romanyuk, P. Jiricek, and T. Paskova, Surf. Sci. 606, 740 (2012).

${ }^{150}$ O. Romanyuk, S. Fernández-Garrido, P. Jiříček, I. Bartoš, L. Geelhaar, O. Brandt, and T. Paskova, Appl. Phys. Lett. 106, 021602 (2015).

${ }^{151}$ Z. Yu, S. Tong, S. Xu, S. Ma, and H. Wu, Surf. Rev. Lett. 10, 831 (2003).

${ }^{152}$ C. S. Fadley, Prog. Surf. Sci. 16, 275 (1984)

${ }^{153}$ C. Westphal, Surf. Sci. Rep. 50, 1 (2003).

${ }^{154}$ J. Pezoldt, T. Kups, T. Stauden, and B. Schröter, Mater. Sci. Eng., B 165, 28 (2009).

${ }^{155}$ M. Seelmann-Eggebert, J. L. Weyher, H. Obloh, H. Zimmermann, A. Rar, and S. Porowski, Appl. Phys. Lett. 71, 2635 (1997).

${ }^{156}$ J. R. Williams, M. Kobata, I. Pis, E. Ikenaga, T. Sugiyama, K. Kobayashi, and N. Ohashi, Surf. Sci. 605, 1336 (2011).

${ }^{157}$ O. Romanyuk, P. Jiříček, T. Paskova, I. Bieloshapka, and I. Bartoš, Appl. Phys. Lett. 103, 091601 (2013).

${ }^{158}$ O. Romanyuk, P. Jiříček, T. Paskova, and I. Bartoš, J. Appl. Phys. 116, 104909 (2014).

${ }^{159}$ O. Romanyuk, P. Jiř́čcek, T. Paskova, and I. Bartoš, "Polarity of GaN surfaces with polar $\{0001\}$ and semipolar $\{1011\},\{2021\},\{1122\}$ orientations: X-ray photoelectron diffraction study,” J. Mater. Res. (in press).

${ }^{160}$ J. M. Bijvoet, A. F. Peerdeman, and A. J. van Bommel, Nature 168, 271 (1951)

${ }^{161} \mathrm{~J}$. Als-Nielsen and D. McMorrow, Elements of Modern X-Ray Physics, 2nd ed. (Wiley, Chichester, 2011).

${ }^{162}$ E. H. Kisi and M. M. Elcombe, Acta Crystallogr., Sect. C: Struct. Chem. 45, 1867 (1989)

${ }^{163}$ J. Hodeau, V. Favre-Nicolin, S. Bos, H. Renevier, E. Lorenzo, and J. Berar, Chem. Rev. 101, 1843 (2001).

${ }^{164}$ V. Favre-Nicolin, M. G. Proietti, C. Leclere, N. A. Katcho, M. I. Richard, and H. Renevier, Eur. Phys. J. Spec. Top. 208, 189 (2012).

${ }^{165}$ D. Bowen and B. K. Tanner, X-Ray Metrology in Semiconductor Manufacturing (Taylor and Francis, Boca Raton, 2006).

${ }^{166} \mathrm{~S}$. Nishikawa and K. Matsukawa, Proc. Imp. Acad. Jpn. 4, 96 (1928).

${ }^{167}$ D. Coster, K. Knol, and J. Prins, Z. Phys. 63, 345 (1930).

${ }^{168}$ H. Cole and N. R. Stemple, J. Appl. Phys. 33, 2227 (1962).

${ }^{169}$ P. F. Fewster, J. Appl. Phys. 52, 4568 (1981).

${ }^{170}$ A. W. Stevenson, S. W. Wilkins, M. S. Kwietniak, and G. N. Pain, J. Appl. Phys. 66, 4198 (1989).

${ }^{171}$ R. D. Horning and B. L. Goldenberg, Appl. Phys. Lett. 55, 1721 (1989).

${ }^{172}$ A. N. Mariano and R. E. Hanneman, J. Appl. Phys. 34, 384 (1963).

${ }^{173}$ R. L. Barns, E. T. Keve, and S. C. Abrahams, J. Appl. Crystallogr. 3, 27 (1970).

${ }^{174}$ H. Tampo, P. Fons, A. Yamada, K.-K. Kim, H. Shibata, K. Matsubara, S. Niki, H. Yoshikawa, and H. Kanie, Appl. Phys. Lett. 87, 141904 (2005)

${ }^{175}$ C. T. Shelton, E. Sachet, E. A. Paisley, M. P. Hoffmann, J. Rajan, R. Collazo, Z. Sitar, and J.-P. Maria, J. Appl. Phys. 115, 044912 (2014).

${ }^{176}$ D. C. Meyer, K. Richter, H.-G. Krane, W. Morgenroth, and P. Paufler, J. Appl. Crystallogr. 32, 854 (1999).

${ }^{177}$ K. Hestroffer, C. Leclere, C. Bougerol, H. Renevier, and B. Daudin, Phys. Rev. B 84, 245302 (2011).

${ }^{178}$ M. Katayama, E. Nomura, N. Kanekama, H. Soejima, and M. Aono, Nucl. Instrum. Methods Phys. Res., Sect. B 33, 857 (1988).

${ }^{179}$ M. Aono, M. Katayama, E. Nomura, T. Chasé, D. CHoi, and M. Kato, Nucl. Instrum. Methods Phys. Res., Sect. B 37, 264 (1989).

${ }^{180}$ M. Sumiya and S. Fuke, MRS Internet J. Nitride Semicond. Res. 9, 1 (2004).

${ }^{181}$ S. Sonoda, S. Shimizu, Y. Suzuki, K. Balakrshnan, J. Shirakashi, H. Okumura, T. Nishihara, and M. Shinohara, Jpn. J. Appl. Phys., Part 2 38, L1219 (1999).

${ }^{182}$ T. Ohnishi, A. Ohtomo, M. Kawasaki, K. Takahashi, M. Yoshimoto, and H. Koinuma, Appl. Phys. Lett. 72, 824 (1998).

${ }^{183}$ M. Sumiya, M. Tanaka, K. Ohtsuka, S. Fuke, I. Ohkubo, M. Yoshimoto, H. Koinuma, and M. Kawasaki, Appl. Phys. Lett. 75, 674 (1999).
${ }^{184}$ S. Sonoda, S. Shimizu, X. Q. Shen, and H. Okumura, Jpn. J. Appl. Phys., Part 2 39, L202 (2000).

${ }^{185}$ S. K. Hong, T. Hanada, H. J. Ko, Y. Chen, T. Yao, D. Imaki, K. Araki, M. Shinohara, K. Saitoh, and M. Terauchi, Phys. Rev. B 65, 115331 (2002).

${ }^{186}$ M. Walker, T. D. Veal, L. Lu, W. J. Schaff, and C. F. McConville, Phys. Status Solidi C 2, 2301 (2005).

${ }^{187}$ M. Nonnenmacher, M. P. O'Boyle, and H. K. Wickramasinghe, Appl. Phys. Lett. 58, 2921 (1991).

${ }^{188}$ J. Zuniga-Perez, V. Munoz-Sanjosé, E. Palacios-Lidon, and J. Colchero, Phys. Rev. Lett. 95, 226105 (2005).

${ }^{189}$ K. M. Jones, P. Vsiconti, F. Yun, A. A. Baski, and H. Morkoç, Appl. Phys. Lett. 78, 2497 (2001).

${ }^{190}$ B. J. Rodriguez, W. C. Yang, R. J. Nemanich, and A. Gruverman, Appl. Phys. Lett. 86, 112115 (2005).

${ }^{191}$ J. Zuniga-Perez, E. Palacios-Lidon, V. Munoz-Sanjosé, and J. Colchero, Appl. Phys. A 88, 77 (2007).

${ }^{192}$ J. D. Wei, R. Neumann, X. Wang, S. F. Li, S. Fündling, S. Merzsch, M. A. M. Al-Suleiman, U. Sökmen, H. H. Wehmann, and A. Waag, Phys. Status Solidi C 8, 2157 (2011).

${ }^{193}$ A. Minj, A. Cros, N. Garro, J. Colchero, T. Auzelle, and B. Daudin, Nano Lett. 15, 6770 (2015).

${ }^{194}$ L. Kronik and Y. Shapira, Surf. Sci. Rep. 37, 1 (1999).

${ }^{195}$ J. D. Wei, S. F. Li, A. Atamuratov, H. H. Wehmann, and A. Waag, Appl. Phys. Lett. 97, 172111 (2010).

${ }^{196}$ B. J. Rodriguez, A. Gruverman, A. I. Kingon, and R. J. Nemanich, J. Cryst. Growth 246, 252 (2002).

${ }^{197}$ T. Stoica, R. Calarco, R. Meijers, and H. Lüth, Appl. Surf. Sci. 253, 4300 (2007).

${ }^{198}$ M. D. Brubaker, I. Levin, A. Davydov, D. M. Rourke, M. A. Sanford, V. M. Bright, and K. A. Bertness, J. Appl. Phys. 110, 053506 (2011).

${ }^{199}$ J. Jerphagnon and H. W. Newkirk, Appl. Phys. Lett. 18, 245 (1971).

${ }^{200}$ A. Dal Corso, M. Posternak, R. Resta, and A. Baldereschi, Phys. Rev. B 50, 10715 (1994).

${ }^{201}$ W. S. Yan, R. Zhang, Z. L. Xie, X. Q. Xiu, P. Han, H. Lu, P. Chen, S. L. Gu, Y. Shi, Y. D. Zheng, and Z. G. Liu, Appl. Phys. Lett. 94, 042106 (2009).

${ }^{202}$ H. Blank, P. Delavignette, R. Gevers, and S. Amelinckx, Phys. Status Solidi B 7, 747 (1964).

${ }^{203}$ Y. T. Rebane, Y. G. Shreter, and M. Albrecht, Phys. Status Solidi A 164, 141 (1997).

${ }^{204}$ G. Salviati, M. Albrecht, C. Zanotti-Fregonara, N. Armani, M. Mayer, Y. Shreter, M. Guzzi, Y. V. Melnik, K. Vassilevski, V. A. Dmitriev, and H. P. Strunk, Phys. Status Solidi A 171, 325 (1999).

${ }^{205}$ Y. J. Sun, O. Brandt, U. Jahn, T. Y. Liu, A. Trampert, S. Cronenberg, S. Dhar, and K. H. Ploog, J. Appl. Phys. 92, 5714 (2002).

${ }^{206}$ R. Liu, A. Bell, F. A. Ponce, C. Q. Chen, J. W. Yang, and M. A. Khan, Appl. Phys. Lett. 86, 021908 (2005).

${ }^{207}$ T. Paskova, R. Kroeger, P. P. Paskov, S. Figge, D. Hommel, B. Monemar, B. Haskell, P. Fini, J. S. Speck, and S. Nakamura, Proc. SPIE 6121, 612106 (2006)

${ }^{208}$ G. Jacopin, L. Rigutti, L. Largeau, F. Fortuna, F. Furtmayr, F. H. Julien, M. Eickhoff, and M. Tchernycheva, J. Appl. Phys. 110, 064313 (2011).

${ }^{209}$ P. Corfdir, C. Hauswald, J. K. Zettler, T. Flissikowski, J. Lähnemann, S. Fernández-Garrido, L. Geelhaar, H. Grahn, and O. Brandt, Phys. Rev. B 90, 195309 (2014).

${ }^{210}$ S. Keller, N. Fichtenbaum, F. Wu, G. Lee, S. P. DenBaars, J. S. Speck, and U. K. Mishra, Jpn. J. Appl. Phys., Part 2 45, L322 (2006).

${ }^{211}$ H. P. Maruska and J. J. Tietjen, Appl. Phys. Lett. 15, 327 (1969).

${ }^{212}$ H. M. Manasevit and W. I. Simpson, J. Electrochem. Soc. 116, 1725 (1969).

${ }^{213}$ A. Usui, H. Sunakawa, A. Sakai, and A. A. Yamaguchi, Jpn. J. Appl. Phys., Part 2 36, L899 (1997).

${ }^{214}$ M. Sumiya, K. Yoshimura, T. Ito, K. Ohtsuka, S. Fuke, K. Mizuno, M. Yoshimoto, H. Koinuma, A. Ohtomo, and M. Kawasaki, J. Appl. Phys. 88, 1158 (2000).

${ }^{215}$ M. Stutzmann, O. Ambacher, M. Eickhoff, U. Karrer, A. Lima Pimenta, R. Neuberger, J. Schalwig, R. Dimitrov, P. Schuck, and R. D. Grober, Phys. Status Solidi B 228, 505 (2001).

${ }^{216}$ J. L. Weyher, P. D. Brown, A. R. A. Zauner, S. Müller, C. B. Boothroyd, D. T. Foord, P. R. Hageman, J. C. Humphreys, P. K. Larsen, I. Grzegory, and S. Porowski, J. Cryst. Growth 204, 419 (1999).

${ }^{217}$ T. Sasaki and T. Matsuoka, J. Appl. Phys. 64, 4531 (1988). 
${ }^{218}$ V. Ramachandran, R. M. Feenstra, W. L. Sarney, L. Salamanca-Riba, J. E. Northrup, L. T. Romano, and D. W. Greve, Appl. Phys. Lett. 75, 808 (1999).

${ }^{219}$ D. S. Green, E. Haus, F. Wu, L. Chen, U. K. Mishra, and J. S. Speck, J. Vac. Sci. Technol., B 21, 1804 (2003).

${ }^{220}$ P. R. Tavernier, T. Margalith, J. Williams, D. S. Green, S. Keller, S. P. DenBaars, U. K. Mishra, S. Nakamura, and D. R. Clarke, J. Cryst. Growth 264, 150 (2004).

${ }^{221}$ S. Figge, R. Kröger, T. Böttcher, P. L. Ryder, and D. Hommel, Appl. Phys. Lett. 81, 4748 (2002).

${ }^{222}$ J. L. Rouvière, M. Arlery, R. Niebuhr, K. H. Bachem, and O. Briot, Mater. Sci. Eng., B 43, 161 (1997).

${ }^{223}$ P. Guan, A. L. Cai, J. S. Cabalu, H. L. Porter, and S. Huang, Appl. Phys. Lett. 77, 2491 (2000).

${ }^{224}$ A. R. A. Zauner, J. L. Weyher, M. Plomp, V. Kirilyuk, I. Grzegory, W. J. P. Enckevort, J. J. Schermer, P. R. Hageman, and P. K. Larsen, J. Cryst. Growth 210, 435 (2000).

${ }^{225}$ A. R. A. Zauner, A. Aret, W. J. P. van Enckevort, J. L. Weyher, S. Porowski, and J. J. Schermer, J. Cryst. Growth 240, 14 (2002).

${ }^{226}$ S. Porowski, J. Cryst. Growth 166, 583 (1996).

${ }^{227}$ T. Matsuoka, Y. Kobayashi, H. Takahata, T. Mitate, S. Mizuno, A. Sasaki, M. Yoshimoto, T. Ohnishi, and M. Sumiya, Phys. Status Solidi B 243, 1446 (2006).

${ }^{228}$ S. Keller, N. A. Fichtenbaum, F. Wu, D. Brown, A. Rosales, S. P. DenBaars, J. S. Speck, and U. K. Mishra, J. Appl. Phys. 102, 083546 (2007).

${ }^{229}$ Y. Fu, Q. Fan, S. Chevtchenko, Ü. Özgür, H. Morkoç, Y. Ke, R. Devaty, W. J. Choyke, C. K. Inoki, and T. S. Kuan, Proc. SPIE 6473, 647305 (2007)

${ }^{230}$ D. F. Brown, S. Keller, F. Wu, J. S. Speck, S. P. DenBaars, and U. K. Mishra, J. Appl. Phys. 104, 024301 (2008).

${ }^{231}$ S. Keller, Y. Dora, F. Wu, X. Chen, S. Chowdhury, S. P. DenBaars, J. S. Speck, and U. K. Mishra, Appl. Phys. Lett. 97, 142109 (2010).

${ }^{232}$ M. H. Wong, S. Keller, Nidhi, S., Dasgupta, D., Denninghoff, S., Kolluri, D. F., Brown, J., Lu, N. A., Fichtenbaum, E., Ahmadi, U., Singisetti, A., Chini, S., Rajan, S. P., DenBaars, J. S., Speck, and U. Mishra, Semicond. Sci. Technol. 28, 074009 (2013).

${ }^{233}$ U. Singisetti, M. H. Wong, and U. K. Mishra, Semicond. Sci. Technol. 28, 074006 (2013)

${ }^{234}$ E. S. Hellman, MRS Internet J. Nitride Semicond. Res. 3, 11 (1998) and references therein.

${ }^{235}$ M. Sumiya and S. Fuke, MRS Internet J. Nitride Semicond. Res. 9, 1 (2004).

${ }^{236}$ M. Kamp, M. Mayer, A. Pelzmann, and K. J. Ebeling, in Materials Research Society Symposium Proceedings, edited by F. A. Ponce, T. D. Moustakas, I. Akasaki, and B. A. Monemar (Mater. Res. Soc. Symp. Proc., 1997), Vol. 449, p. 161.

${ }^{237}$ G. Koblmüller, J. R. Lang, E. C. Young, and J. S. Speck, Molecular Beam Epitaxy of Nitrides for Advanced Electronic Materials, 2nd ed. (Elsevier B.V., 2015).

${ }^{238}$ A. L. Corrion, F. Wu, and J. S. Speck, J. Appl. Phys. 112, 054903 (2012).

${ }^{239}$ A. Munkholm, G. B. Stephenson, J. A. Eastman, C. Thompson, P. Fini, J. S. Speck, O. Auciello, P. H. Fuoss, and S. P. DenBaars, Phys. Rev. Lett. 83, 741 (1999)

${ }^{240}$ M. Gibbon, J. P. Stagg, C. G. Cureton, E. J. Trush, C. J. Jones, R. E. Mallard, R. E. Pritchard, N. Collis, and A. Chew, Semicond. Sci. Technol. 8, 998 (1993).

${ }^{241}$ C. C. Michell, M. E. Coltrin, and J. Han, J. Cryst. Growth 222, 144 (2001).

${ }^{242}$ T. Zywietz, J. Neugebauer, and M. Scheffler, Appl. Phys. Lett. 73, 487 (1998).

${ }^{243}$ S. Mita, R. Collazo, A. Rice, J. Tweedie, J. Xie, R. Dalmau, and Z. Sitar, Phys. Status Solidi C 8, 2078 (2011).

${ }^{244}$ T. Tanikawa, K. Shojiki, T. Aisaka, T. Kimura, S. Kuboya, T. Hanada, R. Katayama, and T. Matsuoka, Jpn. J. Appl. Phys., Part 1 53, 05FL05 (2014).

${ }^{245}$ K. Shojiki, J. H. Choi, H. Shindo, T. Kimura, T. Tanikawa, T. Hanada, R. Katayama, and T. Matsuoka, Jpn. J. Appl. Phys., Part 1 53, 05FL07 (2014)

${ }^{246}$ D. Won, X. Weng, and R. Redwing, Appl. Phys. Lett. 100, 021913 (2012).

${ }^{247}$ J. E. Northrup and C. G. Van de Walle, Appl. Phys. Lett. 84, 4322 (2004)
${ }^{248}$ S. Keller, N. A. Fichtenbaum, M. Furukawa, J. S. Speck, S. P. DenBaars, and U. K. Mishra, Appl. Phys. Lett. 90, 191908 (2007).

${ }^{249}$ S. Keller, H. Li, M. Laurent, Y. Hu, N. Pfaff, J. Lu, D. F. Brown, N. A. Fichtenbaum, J. S. Speck, S. P. DenBaars, and U. K. Mishra, Semicond. Sci. Technol. 29, 113001 (2014).

${ }^{250}$ D. Kapolnek, X. H. Wu, B. Heying, S. Keller, B. Keller, U. K. Mishra, S. P. DenBaars, and J. S. Speck, Appl. Phys. Lett. 67, 1541 (1995).

${ }^{251}$ B. Heying, E. J. Tarsa, C. R. Elsass, P. Fini, S. P. DenBaars, and J. S. Speck, J. Appl. Phys. 85, 6470 (1999).

${ }^{252}$ M. H. Xie, S. M. Seutter, W. K. Zhu, L. X. Zheng, H. Wu, and S. Y. Tong, Phys. Rev. Lett. 82, 2749 (1999).

${ }^{253}$ R. Held, G. Nowak, B. E. Ishaug, S. M. Seutter, A. Parkhomovsky, A. M. Dabrian, I. Grezgory, and S. Porowski, J. Appl. Phys. 85, 7697 (1999).

${ }^{254}$ H. C. Lin, T. Akasaka, and H. Yamamoto, Appl. Phys. Express 6, 035503 (2013).

${ }^{255}$ N. A. Fichtenbaum, C. J. Neufeld, C. Schaake, Y. Wu, M. H. Wong, M. Grundmann, S. Keller, S. P. DenBaars, J. S. Speck, and U. K. Mishra, Jpn. J. Appl. Phys., Part 2 46, L230 (2007).

${ }^{256}$ N. A. Fichtenbaum, C. J. Neufeld, C. Schaake, Y. Wu, M. H. Wong, M. Grundmann, S. Keller, S. P. DenBaars, J. S. Speck, and U. K. Mishra, Phys. Status Solidi B 244, 1802 (2007).

${ }^{257}$ B. Heying, R. Averbeck, L. F. Chen, E. Haus, H. Riechert, and J. S. Speck, J. Appl. Phys. 88, 1855 (2000).

${ }^{258}$ B. Heying, I. Smorchkova, C. Poblenz, C. Elsass, P. Fini, S. Den Baars, U. Mishra, J. S. Speck, and S. Diego, Appl. Phys. Lett. 77, 2885 (2000).

${ }^{259}$ E. Monroy, E. Sarigiannidou, F. Fossard, N. Gogneau, E. Bellet-Amalric, J. L. Rouvière, S. Monnoye, H. Mank, and B. Daudin, Appl. Phys. Lett. 84, 3684 (2004).

${ }^{260}$ H. Okumura, B. M. McSkimming, T. Huault, C. Chaix, and J. S. Speck, Appl. Phys. Lett. 104, 012111 (2014).

${ }^{261}$ E. J. Tarsa, B. Heying, X. H. Wu, P. Fini, S. P. DenBaars, and J. S. Speck, J. Appl. Phys. 82, 5472 (1997).

${ }^{262}$ T. Zywietz, J. Neugebauer, and M. Scheffler, Appl. Phys. Lett. 73, 487 (1998).

${ }^{263}$ C. Adelmann, J. Brault, D. Jalabert, P. Gentile, H. Mariette, G. Mula, and B. Daudin, J. Appl. Phys. 91, 9638 (2002).

${ }^{264}$ L. Romano, J. Northrup, A. Ptak, and T. Myers, Appl. Phys. Lett. 77, 2479 (2000).

${ }^{265}$ N. Grandjean, A. Dussaigne, S. Pezzagna, and P. Vennéguès, J. Cryst. Growth 251, 460 (2003).

${ }^{266}$ S. Pezzagna, P. Vennéguès, N. Grandjean, and J. Massies, J. Cryst. Growth 269, 249 (2004).

${ }^{267}$ M. H. Wong, F. Wu, T. E. Mates, J. S. Speck, and U. K. Mishra, J. Appl. Phys. 104, 093710 (2008).

${ }^{268}$ M. H. Wong, F. Wu, J. S. Speck, and U. K. Mishra, J. Appl. Phys. 108, $123710(2010)$

${ }^{269}$ J. K. Hite, M. E. Twigg, M. A. Mastro, C. R. Eddy, and F. J. Kub, Phys. Status Solidi A 208, 1504 (2011).

${ }^{270}$ Q. Z. Xue, Q. K. Xue, S. Kuwano, K. Nakayama, and T. Sakurai, Jpn. J. Appl. Phys., Part 1 40, 4388 (2001).

${ }^{271}$ T. W. Weeks, M. D. Bremser, K. S. Ailey, E. Carlson, W. G. Perry, and R. F. Davis, Appl. Phys. Lett. 67, 401 (1995).

${ }^{272}$ C. G. Moe, Y. Wu, S. Keller, J. S. speck, S. P. DenBaars, and D. Emerson, Phys. Status Solidi A 203, 1708 (2006).

${ }^{273}$ B. Moran, F. Wu, A. E. Romanov, U. K. Mishra, S. P. DenBaars, and J. S. Speck, J. Cryst. Growth 273, 38 (2004).

${ }^{274}$ D. F. Brown, R. Chu, S. Keller, S. P. DenBaars, and U. K. Mishra, Appl. Phys. Lett. 94, 153506 (2009).

${ }^{275}$ S. Kolluri, S. Keller, S. P. DenBaars, and U. K. Mishra, IEEE Electron Device Lett. 33, 44 (2012).

${ }^{276}$ P. Waltereit, O. Brandt, A. Trampert, M. Ramsteiner, M. Reiche, M. Qi, and K. H. Ploog, Appl. Phys. Lett. 74, 3660 (1999).

${ }^{277}$ W. E. Hoke, A. Torabi, R. B. Hallock, J. J. Mosca, and T. D. Kennedy, J. Vac. Sci. Technol., B 24, 1500 (2006).

${ }^{278}$ C. Poblenz, P. Waltereit, S. Rajan, U. K. Mishra, J. S. Speck, P. Chin, I. Smorchkova, and B. Heying, J. Vac. Sci. Technol., B 23, 1562 (2005).

${ }^{279}$ M. H. Wong, Y. Pei, J. S. Speck, and U. K. Mishra, Appl. Phys. Lett. 94, 182103 (2009).

${ }^{280}$ C. D. Lee, A. Sagar, R. M. Feenstra, C. K. Inoki, T. S. Kuan, W. L. Sarney, and L. Salamanca-Riba, Appl. Phys. Lett. 79, 3428 (2001).

${ }^{281}$ P. Waltereit, C. Poblenz, S. Rajan, F. Wu, U. K. Mishra, and J. S. Speck, Jpn. J. Appl. Phys., Part 2 43, L1520 (2004).

${ }^{282}$ S. Rajan, M. Wong, Y. Fu, F. Wu, J. S. Speck, and U. K. Mishra, Jpn. J. Appl. Phys., Part 2 44, L1478 (2005). 
${ }^{283}$ N. Nakamura, K. Furuta, X. Q. Shen, K. Kitamura, K. Nakamura, and H. Okumura, J. Cryst. Growth 301-302, 452 (2007).

${ }^{284}$ M. Sumiya, N. Ogusu, Y. Yotsuda, M. Itoh, S. Fuke, T. Nakamura, S. Mochizuki, T. Sano, S. Kamiyama, A. Amano, and I. Akasaki, J. Appl. Phys. 93, 1311 (2003).

${ }^{285}$ D. H. Lim, K. Xu, S. Arima, and A. Yoshikawa, J. Appl. Phys. 91, 6461 (2002)

${ }^{286}$ S. Nakamura, N. Iwasa, M. Sehoh, and T. Mukai, Jpn. J. Appl. Phys., Part 131, 1258 (1992)

${ }^{287}$ S. Keller, B. P. Keller, Y. F. Wu, B. Heying, D. Kapolnek, J. S. Speck, U. K. Mishra, and S. P. DenBaars, Appl. Phys. Lett. 68, 1525 (1996).

${ }^{288}$ H. Amano, N. Sawaki, I. Akasaki, and Y. Toyoda, Appl. Phys. Lett. 48, 353 (1986).

${ }^{289}$ S. Nakamura, Jpn. J. Appl. Phys., Part 2 30, L1705 (1991).

${ }^{290}$ X. H. Wu, L. M. Brown, S. Keller, B. Keller, S. P. DenBaars, and J. S. Speck, J. Appl. Phys. 80, 3228 (1996).

${ }^{291}$ X. H. Wu, P. Fini, S. Keller, E. J. Tarsa, B. Heying, U. K. Mishra, S. P. DenBaars, and J. S. Speck, Jpn. J. Appl. Phys., Part 2 35, L1648 (1996).

${ }^{292}$ P. Fini, X. Wu, E. J. Tarsa, Y. Golan, V. Srikant, S. Keller, S. P. DenBaars, and J. S. Speck, Jpn. J. Appl. Phys., Part 1 37, 4460 (1998).

${ }^{293}$ N. Grandjean, J. Massies, and M. Leroux, Appl. Phys. Lett. 69, 2071 (1996).

${ }^{294}$ S. Fuke, H. Teshigawara, K. Kuwahara, Y. Takano, T. Ito, M. Yanagihara, and K. Ohtsuka, J. Appl. Phys. 83, 764 (1998).

${ }^{295}$ M. Takeuchi, H. Shimizu, R. Kajitani, K. Kawasaki, T. Kinoshita, K. Takada, H. Murakami, Y. Kumagai, A. Koukitu, T. Koyama, S. F. Chichibu, and Y. Aoyagi, J. Cryst. Growth 305, 360 (2007).

${ }^{296}$ R. Collazo, S. Mita, A. Aleksov, R. Schlesser, and Z. Sitar, J. Cryst. Growth 287, 586 (2006).

${ }^{297}$ P. Kung, C. J. Sun, A. Saxler, H. Ohsato, and M. Razeghi, J. Appl. Phys. 75, 4515 (1994).

${ }^{298}$ Q. Sun, Y. S. Cho, B. H. Kong, H. K. Cho, T. S. Ko, C. D. Yerino, I. H. Lee, and J. Han, J. Cryst. Growth 311, 2948 (2009).

${ }^{299}$ S. Keller, C. S. Suh, Z. Chen, R. Chu, S. Rajan, N. A. Fichtenbaum, M. Furukawa, S. P. DenBaars, J. S. Speck, and U. K. Mishra, J. Appl. Phys. 103, 033708 (2008).

${ }^{300}$ H. Masui, S. Keller, N. Fellows, N. A. Fichtenbaum, M. Furukawa, S. Nakamura, U. K. Mishra, and S. P. DenBaars, Jpn. J. Appl. Phys., Part 1 48, 071003 (2009).

${ }^{301}$ R. Dimitrov, L. Wittmer, H. P. Felsl, A. Mitchell, O. Ambacher, and M. Stutzmann, Phys. Status Solidi A 168, R7 (1998).

${ }^{302}$ O. Ambacher, J. Smart, J. R. Shealy, N. G. Weimann, K. Chu, M. Murphy, W. J. Schaff, L. F. Eastman, R. Dimitrov, L. Wittmer, M. Stutzmann, W. Rieger, and J. Hilsenbeck, J. Appl. Phys. 85, 3222 (1999).

${ }^{303}$ E. C. Piquette, P. M. Bridger, Z. Z. Bandic', and T. C. McGill, J. Vac. Sci. Technol., B 17, 1241 (1999).

${ }^{304}$ X. Q. Shen, T. Ide, S. H. Cho, M. Shimizu, S. Hara, H. Okumura, S. Sonoda, and S. Shimizu, Jpn. J. Appl. Phys., Part 2 39, L16 (2000).

${ }^{305}$ D. Huang, P. Visconti, K. M. Jones, M. A. Reshchikov, F. Yun, A. A. Baski, T. King, and H. Morkoç, Appl. Phys. Lett. 78, 4145 (2001).

${ }^{306}$ M. J. Murphy, K. Chu, H. Wu, W. Yeo, W. J. Schaff, O. Ambacher, L. F. Eastman, T. J. Eustis, J. Silcox, R. Dimitrov, and M. Stutzmann, Appl. Phys. Lett. 75, 3653 (1999).

${ }^{307}$ R. Dimitrov, M. Murphy, J. Smart, W. Schaff, J. R. Shealy, L. F. Eastman, O. Ambacher, and M. Stutzmann, J. Appl. Phys. 87, 3375 (2000).

${ }^{308}$ L. T. Romano, B. S. Krusor, R. Singh, and T. D. Moustakas, J. Electron. Mater. 26, 285 (1997)

${ }^{309}$ F. Widmann, G. Feuillet, B. Daudin, and J. L. Rouvière, J. Appl. Phys. 85, 1550 (1999).

${ }^{310}$ G. Namkoong, W. A. Doolittle, A. S. Brown, M. Losurdo, P. Capezzuto, and G. Bruno, J. Vac. Sci. Technol., B 20, 1221 (2002).

${ }^{311}$ G. Namkoong, W. A. Doolittle, A. S. Brown, M. Losurdo, P. Capezzuto, and G. Bruno, J. Appl. Phys. 91, 2499 (2002).

${ }^{312}$ M. Losurdo, P. Capezzuto, G. Bruno, G. Namkoong, W. A. Doolittle, and A. S. Brown, J. Appl. Phys. 91, 2508 (2002).

${ }^{313}$ A. Kazimirov, G. Scherb, J. Zegenhagen, T. L. Lee, M. J. Bedzyk, M. K. Kelly, H. Angerer, and O. Ambacher, J. Appl. Phys. 84, 1703 (1998).

${ }^{314}$ S. Sonoda, S. Shimizu, X.-Q. Shen, S. Hara, and H. Okumura, Jpn. J. Appl. Phys., Part 2 39, L202 (2000).

${ }^{315}$ X. Q. Shen, H. Matsuhata, and H. Okumura, Appl. Phys. Lett. 86, 021912 (2005)
${ }^{316}$ K. Kusakabe, K. Kishino, A. Kikuchi, T. Yamada, D. Sugihara, and S. Nakamura, J. Cryst. Growth 230, 387 (2001).

${ }^{317}$ A. Krost and A. Dadgar, Mater. Sci. Eng., B 93, 77 (2002).

${ }^{318}$ F. Schulze, A. Dadgar, J. Bläsing, and A. Krost, Appl. Phys. Lett. 84, 4747 (2004)

${ }^{319}$ F. Schulze, A. Dadgar, J. Bläsing, A. Diez, and A. Krost, Appl. Phys. Lett. 88, 121114 (2006).

${ }^{320}$ R. Liu, F. A. Ponce, A. Dadgar, and A. Krost, Appl. Phys. Lett. 83, 860 (2003).

${ }^{321}$ H. Marchand, L. Zhao, N. Zhang, B. Moran, R. Coffie, U. K. Mishra, J. S. Speck, and S. P. DenBaars, J. Appl. Phys. 89, 7846 (2001).

${ }^{322}$ E. Feltin, B. Beaumont, M. Laügt, P. de Mierry, P. Vennéguès, H. Lahrèche, and P. Gibart, Appl. Phys. Lett. 79, 3230 (2001).

${ }^{323}$ S. Arulkumaran, T. Egawa, S. Matsui, and H. Ishikawa, Appl. Phys. Lett. 86, 123503 (2005)

${ }^{324}$ A. Reiher, J. Bläsing, A. Dadgar, A. Diez, and A. Krost, J. Cryst. Growth 248, 563 (2003).

${ }^{325}$ K. Cheng, M. Leys, S. Dergoote, B. Van Daele, S. Boeykens, J. Derluyn, M. Germain, G. Van Tendeloo, J. Engelen, and G. Borghs, J. Electron. Mater. 35, 592 (2006).

${ }^{326}$ S. Keller, Y. Dora, S. Chowdhury, F. Wu, X. Chen, S. P. DenBaars, J. S. Speck, and U. K. Mishra, Phys. Status Solidi C 8, 2086 (2011).

${ }^{327}$ H. Marchand, L. Zhao, N. Zhang, B. Moran, R. Coffie, U. K. Mishra, J. S. Speck, and S. P. DenBaars, J. Appl. Phys. 89, 7846 (2001).

${ }^{328}$ K. Cheng, M. Leys, J. Derluyn, K. Balachander, S. Degroote, M. Germain, and M. Borghs, Phys. Status Solidi C 5, 1600 (2008).

${ }^{329}$ Y. Nakada, I. Aksenov, and H. Okumura, Appl. Phys. Lett. 73, 827 (1998).

${ }^{330}$ Z. T. Wang, Y. Yamada-Takamura, Y. Fujikawa, T. Sakurai, and Q. K. Xue, Appl. Phys. Lett. 87, 032110 (2005).

${ }^{331}$ A. Ohtani, K. S. Stevens, and R. Beresford, Appl. Phys. Lett. 65, 61 (1994).

${ }^{332}$ S. Guha and N. A. Bojarczuk, Appl. Phys. Lett. 72, 415 (1998).

${ }^{333}$ S. Dasgupta, F. Wu, J. S. Speck, and U. K. Mishra, Appl. Phys. Lett. 94, 151906 (2009).

${ }^{334}$ S. Dasgupta, Nidhi, F. Wu, J. S. Speck, and U. K. Mishra, Jpn. J. Appl. Phys., Part 1 51, 115503 (2012).

${ }^{335}$ M. Sobanska, K. Klosek, Z. R. Zytkiewicz, J. Borysiuk, B. S. Witkowski, E. Lusakowska, A. Reszka, and R. Jakiela, Cryst. Res. Technol. 47, 307 (2012).

${ }^{336}$ L. Largeau, E. Galopin, N. Gogneau, L. Travers, F. Glas, and J. C. Harmand, Cryst. Growth Des. 12, 2724 (2012).

${ }^{337}$ T. Auzelle, B. Haas, A. Minj, C. Bougerol, J.-L. Rouvière, A. Cros, J. Colchero, and B. Daudin, J. Appl. Phys. 117, 245303 (2015).

${ }^{338}$ V. Lebedev, B. Schröter, G. Kipshidze, and W. Richter, J. Cryst. Growth 207, 266 (1999).

${ }^{339}$ E. Calleja, M. A. Sánchez-García, F. J. Sánchez, F. Calle, F. B. Naranjo, E. Muñoz, S. I. Molina, A. Sánchez, F. J. Pacheco, and R. García, J. Cryst. Growth 201, 296 (1999).

${ }^{340}$ T. Palacios, F. Calle, M. Varela, C. Ballesteros, E. Monroy, F. B. Naranjo, M. A. Sánchez-García, E. Calleja, and E. Muñoz, Semicond. Sci. Technol. 15, 996 (2000).

${ }^{341}$ A. M. Sánchez, F. J. Pacheco, S. I. Molina, P. Ruterana, F. Calle, T. A. Palacios, M. A. Sánchez-García, E. Calleja, and R. García, Mater. Sci. Eng., B 93, 181 (2002).

${ }^{342}$ T. K. Zywietz, J. Neugebauer, and M. Scheffler, Appl. Phys. Lett. 74, 1695 (1999).

${ }^{343}$ C. Wetzel, T. Suski, J. W. Ager III, E. R. Weber, E. E. Haller, S. Fischer, B. K. Meyer, R. J. Molnar, and P. Perlin, Phys. Rev. Lett. 78, 3923 (1997).

${ }^{344}$ J. L. Lyons, A. Janotti, and C. G. Van de Walle, Appl. Phys. Lett. 97, 152108 (2010).

${ }^{345}$ M. Sumiya, K. Yoshimura, K. Ohtsuka, and S. Fuke, Appl. Phys. Lett. 76, 2098 (2000).

${ }^{346}$ A. J. Ptak, L. J. Holbert, L. Ting, C. H. Swartz, M. Moldovan, N. C. Giles, T. H. Myers, P. Van Lierde, C. Tian, R. A. Hockett, S. Mitha, A. E. Wickenden, D. D. Koleske, and R. L. Henry, Appl. Phys. Lett. 79, 2740 (2001).

${ }^{347}$ H. M. Ng and A. Y. Cho, J. Vac. Sci. Technol., B 20, 1217 (2002).

${ }^{348}$ M. H. Wong, Ph.D. thesis, University of California, Santa Barbara, CA, 2009.

${ }^{349}$ N. A. Fichtenbaum, T. E. Mates, S. Keller, S. P. DenBaars, and U. K. Mishra, J. Cryst. Growth 310, 1124 (2008).

${ }^{350}$ D. D. Koleske, A. E. Wickenden, R. L. Henry, and M. E. Twigg, J. Cryst. Growth 242, 55 (2002). 
${ }^{351}$ G. B. Stringfellow, Organometallic Vapor Phase Epitaxy (Academic Press, San Diego, CA, 1989).

${ }^{352}$ J. Nishizawa, H. Abe, and T. Kurabayashi, J. Electrochem. Soc. 132, 1197 (1985).

${ }^{353}$ S. P. DenBaars and P. D. Dapkus, J. Cryst. Growth 98, 195 (1989).

${ }^{354}$ S. Cruz, S. Keller, T. Mates, U. K. Mishra, and S. P. DenBaars, J. Cryst. Growth 311, 3817 (2009).

${ }^{355}$ L. K. Li, M. J. Jurkovic, W. I. Wang, J. M. Van Hove, and P. P. Chow, Appl. Phys. Lett. 76, 1740 (2000).

${ }^{356}$ S. Kato, Y. Sato, H. Sasaki, I. Masayuki, and S. Yoshida, J. Cryst. Growth 298, 831 (2007).

${ }^{357}$ E. Bahat-Treidel, F. Brunner, O. Hilt, E. Cho, J. Würfl, and G. Tränkle, IEEE Trans. Electron Devices 57, 3050 (2010).

${ }^{358}$ C. Poblenz, P. Waltereit, S. Rajan, S. Heikman, U. K. Mishra, and J. S. Speck, J. Vac. Sci. Technol., B 22, 1145 (2004).

${ }^{359}$ J. L. Lyons, A. Janotti, and C. G. Van De Walle, Phys. Rev. B 89, 035204 (2014)

${ }^{360}$ N. Koide, H. Kato, M. Sassa, S. Yamasaki, K. Manabe, H. Hashimoto, H. Amano, K. Hiramatsu, and I. Akasaki, J. Cryst. Growth 115, 639 (1991).

${ }^{361}$ L. B. Rowland, K. Doverspike, and D. K. Gaskill, Appl. Phys. Lett. 66 , 1495 (1995)

${ }^{362}$ P. Kozodoy, H. Xing, S. P. DenBaars, U. K. Mishra, A. Saxler, R. Perrin, S. Elhamri, and W. C. Mitchel, J. Appl. Phys. 87, 1832 (2000).

${ }^{363}$ H. Amano, M. Kito, K. Hiramatsu, and I. Akasaki, Jpn. J. Appl. Phys., Part 2 28, L2112 (1989).

${ }^{364}$ N. A. Fichtenbaum, C. Schaake, T. E. Mates, C. Cobb, S. Keller, S. P. DenBaars, and U. K. Mishra, Appl. Phys. Lett. 91, 172105 (2007).

${ }^{365}$ Y. Ohba and A. Hatano, J. Cryst. Growth 145, 214 (1994).

${ }^{366}$ H. Xing, D. Green, H. Yu, T. Mates, P. Kozodoy, S. Keller, S. P. DenBaars, and U. K. Mishra, Jpn. J. Appl. Phys., Part 1 42, 50 (2003).

${ }^{367}$ C. Bungaro, K. Rapcewicz, and J. Bernholc, Phys. Rev. B 59, 9771 (1999).

${ }^{368}$ Q. Sun, A. Selloni, T. H. Myers, and W. A. Doolittle, Phys. Rev. B 73, 155337 (2006)

${ }^{369}$ E. Monroy, M. Hermann, E. Sarigiannidou, T. Andreev, P. Holliger, S. Monnoye, H. Mank, B. Daudin, and M. Eickhoff, J. Appl. Phys. 96, 3709 (2004).

${ }^{370}$ S. Heikman, S. Keller, S. P. DenBaars, and U. K. Mishra, Appl. Phys. Lett. 81, 439 (2002).

${ }^{371}$ S. Heikman, S. Keller, T. Mates, S. P. DenBaars, and U. K. Mishra, J. Cryst. Growth 248, 513 (2003).

${ }^{372}$ E. Malguth, A. Hoffmann, and W. Gehlhoff, Phys. Rev. B 74, 165202 (2006)

${ }^{373}$ A. Corrion, F. Wu, T. Mates, C. S. Gallinat, C. Poblenz, and J. S. Speck, J. Cryst. Growth 289, 587 (2006).

${ }^{374}$ Properties of Group-III Nitrides, edited by J. H. Edgar (INSPEC, 1994).

${ }^{375}$ T. G. Mihopoulos, V. Gupta, and K. F. Jensen, J. Cryst. Growth 195, 733 (1998).

${ }^{376}$ J. Han, J. J. Figiel, M. H. Crawford, M. A. Banas, M. E. Bartram, R. M. Biefeld, Y. K. Song, and A. V. Nurmikko, J. Cryst. Growth 195, 291 (1998).

${ }^{377}$ T. F. Kuech, D. J. Wolford, E. Veuhoff, V. Deline, P. M. Mooney, R. Potemski, and J. Bradley, J. Appl. Phys. 62, 632 (1987).

${ }^{378}$ J. P. Zhang, M. Asif Khan, W. H. Sun, H. M. Wang, C. Q. Chen, Q. Fareed, E. Kuokstis, and J. W. Yang, Appl. Phys. Lett. 81, 4392 (2002).

${ }^{379}$ T. Takano, Y. Ohtaki, Y. Narita, and H. Kawanishi, Jpn. J. Appl. Phys., Part 2 43, L1258 (2004).

${ }^{380}$ E. Iliopoulos and T. D. Moustakas, Appl. Phys. Lett. 81, 295 (2002).

${ }^{381}$ E. Monroy, B. Daudin, E. Bellet-Amalric, N. Gogneau, D. Jalabert, F. Enjalbert, J. Brault, J. Barjon, and L. S. Dang, J. Appl. Phys. 93, 1550 (2003).

${ }^{382}$ V. N. Jmerik, A. M. Mizerov, T. V. Shubina, A. V. Sakharov, A. A. Sitnikova, P. S. Kop'ev, S. V. Ivanov, E. V. Lutsenko, A. V. Danilchyk, N. V. Rzheutskii, and G. P. Yablonskii, Semiconductors 42, 1420 (2008)

${ }^{383}$ I. P. Smorchkova, L. Chen, T. Mates, L. Shen, S. Heikman, B. Moran, S. Keller, S. P. DenBaars, J. S. Speck, and U. K. Mishra, J. Appl. Phys. 90, 5196 (2001).

${ }^{384}$ L. Shen, S. Heikman, B. Moran, R. Coffie, N.-Q. Zhang, D. Buttari, I. P. Smorchkova, S. Keller, S. P. DenBaars, and U. K. Mishra, IEEE Electron Device Lett. 22, 457 (2001).

${ }^{385}$ S. Kolluri, S. Keller, D. Brown, G. Gupta, U. K. Mishra, S. P. DenBaars, and S. Rajan, J. Appl. Phys. 108, 074502 (2010).
${ }^{386}$ S. Keller, C. S. Suh, N. A. Fichtenbaum, M. Furukawa, R. Chu, Z. Chen, K. Vijayraghaven, S. Rajan, S. P. DenBaars, J. S. Speck, and U. K. Mishra, J. Appl. Phys. 104, 093510 (2008).

${ }^{387}$ X. Zheng, M. Guidry, H. Li, A. Ahmadi, K. Hestroffer, B. Romanczyk, S. Wienecke, S. Keller, and U. K. Mishra, IEEE Electron Device Lett. 37, 77 (2016).

${ }^{388}$ S. Keller, N. Pfaff, S. P. DenBaars, and U. K. Mishra, Appl. Phys. Lett. 101, 182103 (2012).

${ }^{389}$ R. Nötzel, N. N. Ledentsov, L. Däweritz, and K. Ploog, Phys. Rev. B 45, 3507 (1992).

${ }^{390}$ D. N. Nath, P. S. Park, M. Esposto, D. Brown, S. Keller, U. K. Mishra, and S. Rajan, J. Appl. Phys. 111, 043715 (2012).

${ }^{391}$ T. Langer, A. Kruse, F. A. Ketzer, A. Schwiegerl, L. Hoffmann, H. Jönen, H. Bremser, U. Rossow, and A. Hangleiter, Phys. Status Solidi C 8, 2170 (2011)

${ }^{392}$ M. J. Reed, N. A. El-Masry, C. A. Parker, J. C. Roberts, and S. M. Bedair, Appl. Phys. Lett. 77, 4121 (2000).

${ }^{393}$ T. K. Sharma and E. Towe, J. Appl. Phys. 107, 024516 (2010).

${ }^{394}$ H. K. Cho, J. Y. Lee, G. M. Yang, and C. S. Kim, Appl. Phys. Lett. 79, 215 (2001).

${ }^{395}$ G. B. Stringfellow, J. Cryst. Growth 312, 735 (2010).

${ }^{396}$ T. Yayama, Y. Kangawa, K. Kakimoto, and A. Koukitu, Phys. Status Solidi C 7, 2249 (2010)

${ }^{397}$ M. V. Durnev, A. V. Omelchenko, E. V. Yakovlev, I. Y. Evstratov, and S. Y. Karpov, Phys. Status Solidi A 208, 2671 (2011).

${ }^{398}$ S. F. Chichibu, A. Abare, M. S. Minsky, S. Keller, S. B. Fleischer, J. E. Bowers, E. Hu, U. K. Mishra, L. A. Coldren, and S. P. DenBaars, Appl. Phys. Lett. 73, 2006 (1998)

${ }^{399}$ D. Won, X. Weng, and J. Redwing, Appl. Phys. Lett. 100, 021913 (2012).

${ }^{400}$ X. H. Wu, C. R. Elsass, A. Abare, M. Mack, S. Keller, P. M. Petroff, S. P. DenBaars, and J. S. Speck, Appl. Phys. Lett. 72, 692 (1998).

${ }^{401}$ H. Wang, D. S. Jiang, U. Jahn, J. J. Zhu, D. G. Zhao, Z. S. Liu, S. M. Zhang, Y. X. Qiu, and H. Yang, Physica B 405, 4668 (2010).

${ }^{402}$ K. Pantzas, G. Patriarche, G. Orsal, S. Gautier, T. Moudakir, M. Abid, V. Gorge, Z. Djebbour, P. L. Voss, and A. Ougazzaden, Phys. Status Solidi A 209, 25 (2012).

${ }^{403}$ J. E. Northrup and J. Neugebauer, Phys. Rev. B 60, R8473 (1999).

${ }^{404}$ A. I. Duff, L. Lymperakis, and J. Neugebauer, Phys. Rev. B 89, 085307 (2014).

${ }^{405}$ A. I. Duff, L. Lymperakis, and J. Neugebauer, Phys. Status Solidi B 252, 855 (2015).

${ }^{406}$ K. Xu and A. Yoshikawa, Appl. Phys. Lett. 83, 251 (2003).

${ }^{407}$ H. Naoi, F. Matsuda, T. Araki, A. Suzuki, and Y. Nanishi, J. Cryst. Growth 269, 155 (2004).

${ }^{408}$ G. Koblmuüller, C. S. Gallinat, S. Bernardis, J. S. Speck, G. D. Chern, E. D. Readinger, H. Shen, and M. Wraback, Appl. Phys. Lett. 89, 071902 (2006).

${ }^{409}$ G. Koblmüller, C. S. Gallinat, and J. S. Speck, J. Appl. Phys. 101, 83516 (2007).

${ }^{410}$ D. N. Nath, E. Guür, S. A. Ringel, and S. Rajan, J. Vac. Sci. Technol., B 29, 021206 (2011)

${ }^{411}$ C. Chèze, M. Siekacz, G. Muzioł, H. Turski, S. Grzanka, M. Krysko, J. L. Weyher, M. Bockowski, C. Hauswald, J. Laühnemann, O. Brandt, M. Albrecht, and C. Skierbiszewski, J. Vac. Sci. Technol., B 31, 03C130 (2013).

${ }^{412}$ D. N. Nath, E. Gür, S. A. Ringel, and S. Rajan, Appl. Phys. Lett. 97, 071903 (2010).

${ }^{413}$ F. Akyol, D. N. Nath, E. Gür, P. S. Park, and S. Rajan, Jpn. J. Appl. Phys., Part 1 50, 052101 (2011).

${ }^{414}$ F. Akyol, D. N. Nath, S. Krishnamoorthy, P. S. Park, and S. Rajan, Appl. Phys. Lett. 100, 111118 (2012).

${ }^{415}$ G. Yang, G. Li, S. Gao, D. Yan, and F. Wang, IEEE Photonics Technol. Lett. 25, 2369 (2013).

${ }^{416}$ J. Kuzmik, IEEE Electron Device Lett. 22, 510 (2001).

${ }^{417}$ D. F. Brown, S. Keller, T. E. Mates, J. S. Speck, S. P. DenBaars, and U. K. Mishra, J. Appl. Phys. 107, 033509 (2010).

${ }^{418}$ L. Zhou, D. J. Smith, M. R. McCartney, D. S. Katzer, and D. F. Storm, Appl. Phys. Lett. 90, 081917 (2007).

${ }^{419}$ S. L. Sahonta, G. P. Dimitrakopulos, T. Kehagias, J. Kioseoglou, A. Adikimenakis, E. Iliopoulos, A. Georgakilas, H. Kirmse, W. Neumann, and P. Komninou, Appl. Phys. Lett. 95, 021913 (2009).

${ }^{420}$ S. Choi, F. Wu, R. Shivaraman, E. C. Young, and J. S. Speck, Appl. Phys. Lett. 100, 232102 (2012). 
${ }^{421}$ S. Dasgupta, S. Choi, F. Wu, J. S. Speck, and U. K. Mishra, Appl. Phys. Express 4, 045502 (2011).

${ }^{422}$ E. Ahmadi, R. Shivaraman, F. Wu, S. Wienecke, S. W. Kaun, S. Keller, J. S. Speck, and U. K. Mishra, Appl. Phys. Lett. 104, 072107 (2014).

${ }^{423}$ S. W. Kaun, E. Ahmadi, B. Mazumder, F. Wu, E. C. H. Kyle, P. G. Burke, U. K. Mishra, and J. S. Speck, Semicond. Sci. Technol. 29, 045011 (2014)

${ }^{424}$ M. Gonschorek, J. F. Carlin, E. Feltin, A. A. Py, N. Grandjean, V. Darakchieva, B. Monemar, M. Lorenz, and G. Ramm, J. Appl. Phys. 103, 093714 (2008).

${ }^{425}$ B. Mazumder, S. W. Kaun, J. Lu, S. Keller, U. K. Mishra, and J. S. Speck, Appl. Phys. Lett. 102, 111603 (2013).

${ }^{426}$ H. Li, S. Keller, S. H. Chan, J. Lu, S. P. DenBaars, and U. K. Mishra, Semicond. Sci. Technol. 30, 055015 (2015).

${ }^{427}$ J. Lu, D. Danninghoff, R. Yeluri, S. Lal, G. Gupta, M. Laurent, S. Keller, S. P. DenBaars, and U. K. Mishra, Appl. Phys. Lett. 102, 232104 (2013).

${ }^{428}$ J. Lu, X. Zheng, M. Guidry, D. Danninghoff, E. Ahmadi, S. Lal, S. Keller, S. P. DenBaars, and U. K. Mishra, Appl. Phys. Lett. 104, 092107 (2014)

${ }^{429}$ E. Ahmadi, F. Wu, H. Li, S. W. Kaun, M. Tahhan, K. Hestroffer, S. Keller, J. S. Speck, and U. K. Mishra, Semicond. Sci. Technol. 30, 055012 (2015)

${ }^{430}$ O. Ambacher, J. Phys. D: Appl. Phys. 31, 2653 (1998).

${ }^{431}$ P. Gibart, Rep. Prog. Phys. 67, 667 (2004).

${ }^{432}$ X. Wang and A. Yoshikawa, Prog. Cryst. Growth Charact. Mater. 48-49, 42 (2004).

${ }^{433} \mathrm{H}$. Morkoç and U. Ozgur, ZnO: Fundamentals, Materials and Device Technology (WILEY VCH, GmBH, 2009).

${ }^{434}$ Y. Yan and M. M. Al-Jassim, Phys. Rev. B 69, 085204 (2004).

${ }^{435}$ S. Lautenschlaeger, B. K. Meyer, G. Callison, M. R. Wagner, and A. Hoffmann, Phys. Status Solidi RRL 3, 16 (2009).

${ }^{436}$ R. Helbig, J. Cryst. Growth 15, 25 (1972).

${ }^{437}$ D. C. Look, D. C. Reynolds, J. R. Sizelove, R. L. Jones, C. W. Litton, G. Cantwell, and W. C. Harsch, Solid State Commun. 105, 399 (1998).

${ }^{438}$ T. P. Smith, H. McLean, D. J. Smith, and R. F. Davis, J. Cryst. Growth 265, 390 (2004).

${ }^{439}$ A. Zeuner, H. Alvs, D. M. Hofmann, B. K. Meyer, M. Heuken, J. Bläsing, and A. Krost, Appl. Phys. Lett. 80, 2078 (2002).

${ }^{440}$ E. Ohshima, H. Ogino, I. Niikura, K. Maeda, M. Sato, M. Ito, and T. Fukuda, J. Cryst. Growth 260, 166 (2004).

${ }^{441}$ K. Maeda, M. Sato, I. Niikura, and T. Fukuda, Semicond. Sci. Technol. 20, S49 (2005).

${ }^{442}$ D. Ehrentraut, K. Maeda, M. Kano, K. Fuji, and T. Fukuda, J. Cryst. Growth 320, 18 (2011).

${ }^{443}$ S. Graubner, C. Neumann, N. Volbers, B. K. Meyer, J. Blasing, and A. Krost, Appl. Phys. Lett. 90, 042103 (2007).

${ }^{444}$ C. Neumann, S. Lautenschläger, S. Graubner, J. Sann, N. Volbers, B. K. Meyer, J. Blasing, A. Krost, F. Bertram, and J. Christen, Phys. Status Solidi B 244, 1451 (2007).

${ }^{445}$ H. von Wenckstern, H. Schmidt, C. Hanisch, M. Brandt, C. Czekalla, G. Benndorf, G. Biehne, A. Rahm, H. Hochmuth, M. Lorenz, and M. Grundmann, Phys. Status Solidi RRL 1, 129 (2007).

${ }^{446}$ D. Ehrentraut, H. Sato, M. Miyamoto, T. Fukuda, M. Nikl, K. Maeda, and I. Niikura, J. Cryst. Growth 287, 367 (2006).

${ }^{447}$ D. Takamizu, Y. Nishimoto, S. Akasaka, H. Yuji, K. Tamura, K. Nakahara, T. Onuma, T. Tanabe, H. Takasu, M. Kawasaki, and S. F. Chichibu, J. Appl. Phys. 103, 063502 (2008).

${ }^{448}$ S. Akasaka, K. Nakahara, H. Yuji, A. Tsukazaki, A. Ohtomo, and M. Kawasaki, Appl. Phys. Express 4, 035701 (2011).

${ }^{449}$ K. Furusawa, H. Nakasawa, Y. Ishikawa, and S. F. Chichibu, Jpn. J. Appl. Phys., Part 1 53,100301 (2014).

${ }^{450}$ K. Ogata, T. Kawanishi, K. Sakurai, S. W. Kim, M. Maejima, Sz. Fujita, and Sg. Fujita, Phys. Status Solidi B 229, 915 (2002).

${ }^{451}$ T. Ive, T. Ben-Yaacov, C. G. Van de Walle, U. Mishra, S. DenBaars, and J. S. Speck, Phys. Status Solidi C 6, 1460 (2009).

${ }^{452}$ R. Masuda, T. Fujii, N. Yoshii, Y. Kumagai, and A. Koukitu, J. Cryst. Growth 312, 2324 (2010).

${ }^{453}$ J. M. Pierce, B. T. Adekore, R. F. Davis, and F. A. Stevie, J. Cryst. Growth 283, 147 (2005).

${ }^{454}$ I. C. Robin, A. Ribeaud, S. Brochen, G. Feuillet, P. Ferret, H. Mariette, D. Ehrentraut, and T. Fukuda, Appl. Phys. Lett. 92, 141101 (2008).

${ }^{455}$ S. Heinze, A. Krtschil, J. Bläsing, T. Hempel, P. Veit, A. Dadgar, J. Christen, and A. Krost, J. Cryst. Growth 308, 170 (2007).
${ }^{456}$ S. Lautenschlaeger, J. Sann, N. Volbers, B. K. Meyer, A. Hoffmann, U. Haboeck, and M. R. Wagner, Phys. Rev. B 77, 144108 (2008).

${ }^{457}$ M. R. Wagner, T. P. Bartel, R. Kirste, A. Hoffmann, J. Sann, S. Lautenschlager, B. K. Meyer, and C. Kisielowski, Phys. Rev. B 79, 035307 (2009).

${ }^{458}$ A. Tsukazaki, A. Ohtomo, T. Onuma, M. Ohtani, T. Makino, M. Sumiya, K. Ohtani, S. F. Chichibu, S. Fuke, Y. Segawa, H. Ohno, H. Koinuma, and M. Kawasaki, Nat. Mater. 4, 42 (2005).

${ }^{459}$ K. Nakahara, S. Akasaka, H. Yuji, K. Tamura, T. Fujii, Y. Nishimoto, D. Takamizu, A. Sasaki, T. Tanabe, H. Takasu, H. Amaike, T. Onuma, S. F. Chichibu, A. Tsukazaki, A. Ohtomo, and M. Kawasaki, Appl. Phys. Lett. 97, 013501 (2010).

${ }^{460} \mathrm{~S}$. Brochen, M. Lafossas, I. C. Robin, P. ferret, F. Gemain, J. Pernot, and G. Feuillet, J. Appl. Phys. 115, 113508 (2014).

${ }^{461}$ H. Xu, K. Ohtani, M. Yamao, and H. Ohno, Appl. Phys. Lett. 89, 071918 (2006).

${ }^{462}$ H. Kato, M. Sano, K. Miyamoto, and T. Yao, Jpn. J. Appl. Phys., Part 2 42, L1002 (2003).

${ }^{463}$ M. Wei, R. C. Boutwell, G. A. Garrett, K. Goodman, P. Rotella, M. Wraback, and W. V. Schoenfeld, J. Alloys Compd. 552, 127 (2013).

${ }^{464}$ H. Yuji, K. Nakahara, K. Tamura, S. Akasaka, Y. Nishimoto, D. Takamizu, T. Onuma, S. F. Chichibu, A. Tsukazaki, A. Ohtomo, and M. Kawasaki, Jpn. J. Appl. Phys., Part 1 49, 071104 (2010).

${ }^{465}$ Y. Kozuka, A. Tsukazaki, and M. Kawasaki, Appl. Phys. Rev. 1, 011303 (2014).

${ }^{466}$ A. Tsukazaki, S. Akasaka, K. Nakahara, Y. Ohno, H. Ohno, D. Maryenko, A. Ohtomo, and M. Kawasaki, Nat. Mater. 9, 889 (2010).

${ }^{467}$ Y. Nishimoto, K. Nakahara, D. Takamizu, A. Sasaki, K. Tamura, S. Akasaka, H. Yuji, T. Fujii, T. Tanabe, H. Takasu, A. Tsukazaki, A. Ohtomo, T. Onuma, S. F. Chichibu, and M. Kawasaki, Appl. Phys. Express 1, 091202 (2008).

${ }^{468}$ S. Akasaka, A. Tsukazaki, K. Nakahara, A. Ohtomo, and M. Kawasaki, Jpn. J. Appl. Phys., Part 1 50, 080215 (2011).

${ }^{469}$ M. Lorenz, G. Wagner, A. Rahm, H. Schmidt, H. Hochmuth, H. Schid, W. Mader, M. Brandt, H. von Wenckstern, and M. Grundmann, Phys. Status Solidi C 5, 3280 (2008).

${ }^{470}$ M. Brandt, H. von Wencsktern, H. Schmidt, A. Rahm, G. Biehne, G. Benndorf, H. Hochmuth, M. Lorenz, C. Meinecke, T. Butz, and M. Grundmann, J. Appl. Phys. 104, 013708 (2008).

${ }^{471}$ A. Lajn, H. von Wenckstern, G. Benndorf, C. P. Dietrich, M. Brandt, G. Biehne, H. Hochmuth, M. Lorenz, and M. Grundmann, J. Electron. Mater. 39, 595 (2010).

${ }^{472}$ M. Lorenz, T. Weiss, F. Schmidt, H. von Wencktern, and M. Grundmann, Phys. Status Solidi A 212, 1440 (2015).

${ }^{473}$ M. Lorenz, C. Schmidt, G. Benndorf, T. Böntgen, H. Hochmuth, R. Böttcher, A. Pöppl, D. Spemann, and M. Grundmann, J. Phys. D: Appl. Phys. 46, 065311 (2013).

${ }^{474}$ C. F. Walters, K. F. McCarty, E. A. Soares, and M. A. Van Hove, Surf. Sci. 464, L732 (2000).

${ }^{475}$ A. Ohtomo, K. Tamura, K. Saikusa, K. Takahashi, T. Makino, Y. Segawa, H. Koinuma, and M. Kawasaki, Appl. Phys. Lett. 75, 2635 (1999).

${ }^{476}$ N. Kawamoto, M. Fujita, T. Tatsumi, and Y. Horikoshi, Jpn. J. Appl. Phys., Part 1 42, 7209 (2003).

${ }^{477}$ M. Ying, X. Du, Z. Mei, Z. Zeng, H. Zheng, Y. Wang, J. Jia, Z. Zhang, and Q. Xue, J. Phys. D: Appl. Phys. 37, 3058 (2004).

${ }^{478}$ X. Wang, Y. Tomita, O. H. Roh, M. Ohsugi, S. B. Che, Y. Ishitani, and A. Yoshikawa, Appl. Phys. Lett. 86, 011921 (2005).

${ }^{479}$ C. Tang, M. J. S. Spencer, and A. S. Barnard, Phys. Chem. Chem. Phys. 16, 22139 (2014)

${ }^{480}$ Y. Wang, X. L. Du, Z. X. Mei, Z. Q. Zeng, M. J. Ying, H. T. Yuan, J. F. Jia, Q. K. Xue, and Z. Zhang, Appl. Phys. Lett. 87, 051901 (2005).

${ }^{481}$ Z. X. Mei, X. L. Du, Y. Wang, M. J. Ying, Z. Q. Zeng, H. Zheng, J. F. Jia, Q. K. Xue, and Z. Zhang, Appl. Phys. Lett. 86, 112111 (2005).

${ }^{482}$ I. Ohkubo, A. Ohtomo, T. Ohnishi, Y. Mastumoto, H. Koinuma, and M. Kawasaki, Surf. Sci. 443, L1043 (1999).

${ }^{483}$ B. P. Zhang, L. H. Manh, K. Wakatsuki, T. Ohnishi, M. Lippmaa, N. Usami, M. Kawasaki, and Y. Segawa, Jpn. J. Appl. Phys., Part 1 42, 2291 (2003).

${ }^{484}$ B. P. Zhang, K. Wakatsuki, N. T. Binh, N. Usami, and Y. Segawa, Thin Solid Films 449, 12 (2004)

${ }^{485}$ S. Akiyama, K. Minegishi, T. Tanaka, H. Ogawa, and M. Kasuga, Jpn. J. Appl. Phys., Part 1 46, 342 (2007). 
${ }^{486}$ S. K. Hong, H. J. Ko, Y. Chen, T. Hanada, and T. Yao, J. Vac. Sci. Technol., B 18, 2313 (2000).

${ }^{487}$ S. K. Hong, T. Hanada, Y. Chen, H. J. Ko, T. Yao, D. Imai, K. Araki, and M. Shinohara, Appl. Surf. Sci. 190, 491 (2002).

${ }^{488}$ Y. Chen, H. J. Ko, S. K. Hong, and T. Yao, Appl. Phys. Lett. 76, 559 (2000).

${ }^{489}$ H. Kato, K. Miyamoto, M. Sano, and T. Yao, Appl. Phys. Lett. 84, 4562 (2004).

${ }^{490}$ M. W. Cho, T. Minegishi, T. Suzuki, H. Suzuki, T. Yao, S. K. Hong, and H. Ko, J. Electroceram. 17, 255 (2006).

${ }^{491}$ A. Bakin, J. Kioseoglou, B. Pecz, A. El-Shaer, A. C. Mofor, J. Stoemenos, and A. Waag, J. Cryst. Growth 308, 314 (2007).

${ }^{492}$ Z. Q. Zeng, Y. Z. Liu, H. T. Yuan, Z. X. Mei, X. L. Du, J. F. Jia, Q. K. Xue, and Z. Zhang, Appl. Phys. Lett. 90, 081911 (2007).

${ }^{493}$ J. G. Kim, S. K. Han, S. M. Yang, S. K. Hong, J. W. Lee, J. Y. Lee, J. H. Song, Y. E. Ihm, D. Kim, J. S. Park, H. J. Lee, and T. Yao, Thin Solid Films 519, 223 (2010)

${ }^{494}$ S. Park, S. K. Hong, T. Minegishi, S. H. Park, S. H. Park, I. H. Im, M. W. Cho, T. Yao, J. W. Lee, and J. Y. Lee, Appl. Phys. Lett. 90, 201907 (2007)

${ }^{495}$ S. Park, J. H. Chang, T. Minegishi, H. J. Lee, S. H. Park, I. H. Im, T. Hanada, S. K. Hong, M. W. Cho, and T. Yao, J. Electron. Mater. 37, 736 (2008).

${ }^{496}$ J. Park and T. Yao, Thin Solid Films 531, 88 (2013).

${ }^{497}$ J. Park and T. Yao, Mater. Res. Bull. 47, 2875 (2012).

${ }^{498}$ J. Park, T. Minegishi, S. H. Park, S. K. Honng, J. H. Chang, and T. Yao, Thin Solid Films 519, 3417 (2011).

${ }^{499}$ J. Park and T. Yao, J. Alloys Compd. 513, 180 (2012).

${ }^{500}$ Z. Zhong, F. Qian, D. Wang, and C. M. Lieber, Nano Lett. 3, 343 (2003).

${ }^{501}$ T. Kuykendall, P. Pauzauskie, S. Lee, Y. Zhang, J. Goldberger, and P. Yang, Nano Lett. 3, 1063 (2003)

${ }^{502}$ T. Kuykendall, P. J. Pauzauskie, Y. Zhang, J. Goldberger, D. Sirbuly, J. Denlinger, and P. Yang, Nat. Mater. 3, 524 (2004).

${ }^{503}$ F. Qian, S. Gradecak, H. Park, Y. Dong, Y. Ding, Z. L. Wang, and C. M. Lieber, Nat. Mater. 7, 701 (2008).

${ }^{504}$ W. Bergbauer, M. Strassburg, Ch. Kölper, N. Lindner, C. Roder, J. Lähnemann, A. Trampert, S. Fündling, S. f. Li, H. Wehmann, and A. Waag, Nanotechnology 21, 305201 (2010).

${ }^{505}$ W. Bergbauer, M. Strassburg, Ch. Kölper, N. Lindner, C. Roder, J. Lähnemann, A. Trampert, S. Fündling, S. f. Li, H. Wehmann, and A. Waag, J. Cryst. Growth 315, 164 (2011).

${ }^{506}$ X. J. Chen, G. Perillat-Merceroz, D. Sam-Giao, C. Durand, and J. Eymery, Appl. Phys. Lett. 97, 151909 (2010).

${ }^{507}$ B. Alloing, S. Vézian, O. Tottereau, P. Vennéguès, E. Beraudo, and J. Zuniga-Perez, Appl. Phys. Lett. 98, 011914 (2011).

${ }^{508}$ S. F. Li, S. Fuendling, X. Wang, S. Merzsch, M. A. M. Al-Suleiman, J. D. Wei, H. H. Wehmann, A. Waag, W. Bergabuer, and M. Strassburg, Cryst. Growth Des. 11, 1573 (2011).

${ }^{509}$ M. Sumiya, M. Tanaka, K. Ohtsuka, S. Fuke, T. Ohnishi, I. Ohkubo, M. Yoshimoto, H. Koinuma, and M. Kawasaki, Appl. Phys. Lett. 75, 674 (1999).

${ }^{510}$ M. Sumiya, N. Ogusu, Y. Yotsuda, M. Itoh, S. Fuke, T. Nakamura, S. Mochizuki, T. Sano, S. Kamiyama, H. Amano, and I. Akasaki, J. Appl. Phys. 93, 1311 (2003).

${ }^{511}$ C. Tessarek, M. Bashouti, M. Heilmann, C. Dieker, I. Knoke, E. Spiecker, and S. Chrsitiansen, J. Appl. Phys. 114, 144304 (2013).

${ }^{512}$ P. M. Coulon, M. Mexis, M. Teisseire, M. Jublot, P. Vennéguès, M. Leroux, and J. Zuniga-Perez, J. Appl. Phys. 115, 153504 (2014).

${ }^{513}$ S. Labat, M. I. Richard, M. Dupraz, M. Gailhanou, G. Beutier, M. Verdier, F. Mastropietro, T. W. Cornelius, T. U. Schülli, J. Eymery, and O. Thomas, ACS Nano 9, 9210 (2015).

${ }^{514}$ X. J. Chen, B. Gayral, D. Sam-Giao, C. Bougerol, C. Durand, and J. Eymery, Appl. Phys. Lett. 99, 251910 (2011).

${ }^{515}$ X. Wang, S. Li, S. Fündling, J. Wei, M. Erenburg, H. H. Wehmann, and A. Waag, Cryst. Growth Des. 12, 2552 (2012).

${ }^{516}$ S. D. Hersee, X. Sun, and X. Wang, Nano Lett. 6, 1808 (2006).

${ }^{517}$ K. Choi, M. Arita, and Y. Arakawa, J. Cryst. Growth 357, 58 (2012).

${ }^{518}$ X. Wang, S. Li, M. S. Mohajerani, J. Ledig, H. H. Wehmann, M. Mandl, M. Strassburg, U. Steegmüller, U. Jahn, J. Lähnemann, H. Riechert, I. Griffiths, D. Cherns, and A. Waag, Cryst. Growth Des. 13, 3475 (2013)

${ }^{519}$ P. M. Coulon, B. Alloing, V. Brändli, P. Vennéguès, M. Leroux, and J. Zuniga-Perez, Appl. Phys. Express 9, 015502 (2016).
${ }^{520}$ B. O. Jung, S. Y. Bae, Y. Kato, M. Imura, D. S. Lee, Y. Honda, and H. Amano, CrystEngComm 16, 2273 (2014).

${ }^{521}$ X. Wang, X. Sun, M. Fairchild, and S. D. Hersee, Appl. Phys. Lett. 89, 233115 (2006).

${ }^{522}$ T. Y. Tang, W. Y. Shiao, C. H. Lin, K. C. Shen, J. J. Huang, S. Y. Ting, T. C. Liu, C. C. Yang, C. L. Yao, J. H. Yeh, T. C. Hsu, W. C. Chen, H. C. Hsu, and L. C. Chen, J. Appl. Phys. 105, 023501 (2009).

${ }^{523}$ T. W. Yeh, Y. T. Lin, L. S. Stewart, P. D. Dapkus, R. Sarkissian, J. D. O'Brien, B. Ahn, and S. R. Nutt, Nano Lett. 12, 3257 (2012).

${ }^{524}$ S. Y. Bae, J. Y. Lee, J. H. Min, and D. S. Lee, Appl. Phys. Express 6, 075501 (2013)

${ }^{525}$ K. Chung, H. Beak, Y. Tchoe, H. Oh, H. Yoo, M. Kim, and G. C. Yi, APL Mater. 2, 092512 (2014).

${ }^{526}$ B. Foltynski, N. Garro, M. Vallo, M. Finken, C. Giesen, H. Kalisch, A. Vescan, A. Cantarero, and M. Heuken, J. Cryst. Growth 414, 200 (2015).

${ }^{527}$ S. Vézian, B. Alloing, and J. Zuñiga-Pérez, J. Cryst. Growth 323, 326 (2011).

${ }^{528}$ E. Calleja, J. Ristić, S. Fernández-Garrido, L. Cerutti, M. A. SánchezGarcía, J. Grandal, A. Trampert, U. Jahn, G. Sánchez, A. Griol, and B. Sánchez, Phys. Status Solidi B 244, 2816 (2007).

${ }^{529}$ L. Geelhaar, C. Chèze, B. Jenichen, O. Brandt, C. Pfüller, S. Münch, R. Rothemund, S. Reitzenstein, A. Forchel, T. Kehagias, P. Komninou, G. P. Dimitrakopulos, T. Karakostas, L. Lari, P. R. Chalker, H. G. Mhairi, and H. Riechert, IEEE J. Sel. Top. Quantum Electron. 17, 878 (2011).

${ }^{530}$ K. A. Bertness, S. Member, N. A. Sanford, and A. V. Davydov, IEEE J. Sel. Top. Quantum Electron. 17, 847 (2011).

${ }^{531}$ V. Consonni, Phys. Status Solidi RRL 7, 699 (2013).

${ }^{532}$ L. Cerutti, J. Ristić, S. Fernández-Garrido, E. Calleja, A. Trampert, K. H. Ploog, S. Lazic, and J. M. Calleja, Appl. Phys. Lett. 88, 213114 (2006).

${ }^{533}$ T. Stoica, E. Sutter, R. J. Meijers, R. K. Debnath, R. Calarco, H. Lüth, and D. Grützmacher, Small 4, 751 (2008).

${ }^{534}$ S. Fernández-Garrido, X. Kong, T. Gotschke, R. Calarco, L. Geelhaar, A. Trampert, and O. Brandt, Nano Lett. 12, 6119 (2012).

${ }^{535}$ M. Sobanska, K. Klosek, J. Borysiuk, S. Kret, G. Tchutchulasvili, S. Gieraltowska, and Z. R. Zytkiewicz, J. Appl. Phys. 115, 043517 (2014).

${ }^{536}$ M. Wölz, C. Hauswald, T. Flissikowski, T. Gotschke, S. FernándezGarrido, O. Brandt, H. T. Grahn, L. Geelhaar, and H. Riechert, Nano Lett. 15, 3743 (2015).

${ }^{537}$ C. Chèze, L. Geelhaar, O. Brandt, W. Weber, H. Riechert, S. Münch, R. Rothemund, S. Reitzenstein, A. Forchel, T. Kehagias, P. Komninou, G. Dimitrakopulos, and T. Karakostas, Nano Res. 3, 528 (2010).

${ }^{538}$ C. Chèze, L. Geelhaar, B. Jenichen, and H. Riechert, Appl. Phys. Lett. 97, 153105 (2010)

${ }^{539}$ C. Chèze, L. Geelhaar, A. Trampert, O. Brandt, and H. Riechert, Nano Lett. 10, 3426 (2010).

${ }^{540}$ K. Kishino, T. Hoshino, S. Ishizawa, and A. Kikuchi, Electron. Lett. 44, 819 (2008).

${ }^{541}$ K. Kishino, H. Sekiguchi, and A. Kikuchi, J. Cryst. Growth 311, 2063 (2009).

${ }^{542}$ A. Bengoechea-Encabo, F. Barbagini, S. Fernández-Garrido, J. Grandal, J. Ristić, M. A. Sanchez-García, E. Calleja, U. Jahn, E. Luna, and A. Trampert, J. Cryst. Growth 325, 89 (2011).

${ }^{543}$ A. Bengoechea-Encabo, S. Albert, M. A. Sanchez-García, L. López, S. Estradé, J. Rebled, F. Peiró, G. Nataf, P. de Mierry, J. Zuniga-Perez, and E. Calleja, J. Cryst. Growth 353, 1 (2012).

${ }^{544}$ S. Albert, A. Bengoechea-Encabo, M. A. Sánchez-García, X. Kong, A. Trampert, and E. Calleja, Nanotechnology 24, 175303 (2013).

${ }^{545}$ K. A. Bertness, A. W. Sanders, D. M. Rourke, T. E. Harvey, A. Roshko, J. B. Schlager, and N. A. Sanford, Adv. Funct. Mater. 20, 2911 (2010).

${ }^{546}$ H. Sekiguchi, K. Kishino, and A. Kikuchi, Appl. Phys. Express 1, 124002 (2008).

${ }^{547}$ A. Urban, J. Malindretos, J.-H. Klein-Wiele, P. Simon, and A. Rizzi, New J. Phys. 15, 053045 (2013).

${ }^{548}$ Z. Gačević, D. Gómez Sánchez, and E. Calleja, Nano Lett. 15, 1117 (2015).

${ }^{549}$ D. Cherns, L. Meshi, I. Griffiths, S. Khongphetsak, S. V. Novikov, N. R. S. Farley, R. P. Campion, and C. T. Foxon, Appl. Phys. Lett. 93, 111911 (2008).

${ }^{550}$ M. J. Bierman, Y. K. A. Lau, A. V. Kvit, A. L. Schmitt, and S. Jin, Science 320, 1060 (2008).

${ }^{551}$ S. Fernández-Garrido, J. Grandal, E. Calleja, M. A. Sánchez-García, and D. López-Romero, J. Appl. Phys. 106, 126102 (2009). 
${ }^{552}$ S. Fernández-Garrido, V. M. Kaganer, K. K. Sabelfeld, T. Gotschke, J. Grandal, E. Calleja, L. Geelhaar, and O. Brandt, Nano Lett. 13, 3274 (2013).

${ }^{553}$ M. Yoshizawa, A. Kikuchi, M. Mori, N. Fujita, and K. Kishino, Jpn. J. Appl. Phys., Part 2 36, L459 (1997).

${ }^{554}$ M. Sánchez-García, E. Calleja, E. Monroy, F. Sánchez, F. Calle, E. Muñoz, and R. Beresford, J. Cryst. Growth 183, 23 (1998).

${ }^{555}$ F. Furtmayr, M. Vielemeyer, M. Stutzmann, J. Arbiol, S. Estradé, F. Peiró, J. R. Morante, and M. Eickhoff, J. Appl. Phys. 104, 034309 (2008).

${ }^{556}$ X. Kong, J. Ristić, M. A. Sánchez-García, E. Calleja, and A. Trampert, Nanotechnology 22, 415701 (2011).

${ }^{557}$ R. Mata, K. Hestroffer, J. Budagosky, A. Cros, C. Bougerol, H. Renevier, and B. Daudin, J. Cryst. Growth 334, 177 (2011).

${ }^{558}$ S. D. Carnevale, T. F. Kent, P. J. Phillips, A. T. M. G. Sarwar, C. Selcu, R. F. Klie, and R. C. Myers, Nano Lett. 13, 3029 (2013).

${ }^{559}$ M. I. den Hertog, F. Gonzalez-Posada, R. Songmuang, J. L. Rouviere, T. Fournier, B. Fernandez, and E. Monroy, Nano Lett. 12, 5691 (2012).

${ }^{560}$ A. Trampert, J. Ristić, U. Jahn, E. Calleja, and K. H. Ploog, IOP Conf. Ser.: Mater. Sci. Eng. 180, 167 (2003).

${ }^{561}$ K. Hestroffer, C. Bougerol, C. Leclere, H. Renevier, and B. Daudin, Phys. Rev. B 84, 245302 (2011).

${ }^{562}$ F. Limbach, C. Hauswald, J. Lähnemann, M. Wölz, O. Brandt, A. Trampert, M. Hanke, U. Jahn, R. Calarco, L. Geelhaar, and H. Riechert, Nanotechnology 23, 465301 (2012).

${ }^{563}$ J. Müßener, J. Teubert, P. Hille, M. Schäfer, J. Schörmann, M. de la Mata, J. Arbiol, and M. Eickhoff, Nano Lett. 14, 5118 (2014).

${ }^{564}$ M. D. Brubaker, I. Levin, A. V. Davydov, D. M. Rourke, N. A. Sanford V. M. Bright, and K. A. Bertness, J. Appl. Phys. 110, 053506 (2011).

${ }^{565}$ L. Largeau, E. Galopin, N. Gogneau, L. Travers, F. Glas, and J.-C. Harmand, Cryst. Growth Des. 12, 2724 (2012).

${ }^{566}$ J. Borysiuk, Z. R. Zytkiewicz, M. Sobanska, A. Wierzbicka, K. Klosek, K. P. Korona, P. S. Perkowska, and A. Reszka, Nanotechnology 25, 135610 (2014)

${ }^{567}$ D. Cherns, L. Meshi, I. Griffiths, S. Khongphetsak, S. V. Novikov, N. Farley, R. P. Campion, and C. T. Foxon, Appl. Phys. Lett. 92, 121902 (2008).

${ }^{568}$ F. Schuster, F. Furtmayr, R. Zamani, C. Magén, J. R. Morante, J. Arbiol, J. A. Garrido, and M. Stutzmann, Nano Lett. 12, 2199 (2012).

${ }^{569}$ G. Koblmüller, S. Fernández-Garrido, E. Calleja, and J. S. Speck, Appl. Phys. Lett. 91, 161904 (2007).

${ }^{570}$ G. Koblmüller, F. Wu, T. Mates, J. S. Speck, S. Fernández-Garrido, and E. Calleja, Appl. Phys. Lett. 91, 221905 (2007).

${ }^{571}$ C. E. Dreyer, A. Janotti, and C. G. Van de Walle, Phys. Rev. B 89, 081305 (2014)

${ }^{572}$ H. Li, L. Geelhaar, H. Riechert, and C. Draxl, Phys. Rev. Lett. 115, 085503 (2015).

${ }^{573}$ V. Dubrovskii, V. Consonni, A. Trampert, L. Geelhaar, and H. Riechert, Phys. Rev. B 85, 165317 (2012).

${ }^{574}$ L. Geelhaar, C. Chèze, W. M. Weber, R. Averbeck, H. Riechert, T. Kehagias, P. Komninou, G. P. Dimitrakopulos, and T. Karakostas, Appl. Phys. Lett. 91, 093113 (2007).

${ }^{575}$ M. D. Brubaker, S. M. Duff, T. E. Harvey, P. T. Blanchard, A. Roshko, A. W. Sanders, N. A. Sanford, and K. A. Bertness, Cryst. Growth Des. 16, 596 (2016).

${ }^{576}$ T. Schumann, T. Gotschke, F. Limbach, T. Stoica, and R. Calarco, Nanotechnology 22, 095603 (2011).

${ }^{577}$ T. Gotschke, T. Schumann, F. Limbach, T. Stoica, and R. Calarco, Appl. Phys. Lett. 98, 103102 (2011).

${ }^{578}$ K. Kishino and S. Ishizawa, Nanotechnology 26, 225602 (2015).

${ }^{579}$ Z. L. Wang, J. Phys.: Condens. Matter 16, R829 (2004).

${ }^{580}$ G. C. Yi, C. Wang, and W. I. Park, Semicond. Sci. Technol. 20, S22 (2005).

${ }^{581}$ L. Schmidt-Mende and J. L. MacManus-Driscoll, Mater. Today 10, 40 (2007)

${ }^{582}$ Z. W. Pan, Z. R. Dai, and Z. L. Wang, Science 291, 1947 (2001).

${ }^{583}$ Z. L. Wang, X. Y. Kong, and J. M. Zuo, Phys. Rev. Lett. 91, 185502 (2003).

${ }^{584}$ X. Y. Kong and Z. L. Wang, Nano Lett. 3, 1625 (2003).

${ }^{585}$ S. C. Lyu, Y. Zhang, C. J. Lee, H. Ruh, and H. J. Lee, Chem. Mater. 15, 3294 (2003).

${ }^{586}$ Y. Sun, G. M. Fuge, and M. N. R. Ashfold, Chem. Phys. Lett. 396, 21 (2004)

${ }^{587}$ J. J. Wu and S. C. Liu, Adv. Mater. 14, 215 (2002).

${ }^{588}$ W. I. Park, D. H. Kim, S. W. Jung, and G. C. Yi, Appl. Phys. Lett. 80, 4232 (2002).
${ }^{589}$ M. Krunks, T. Dedova, and I. Oja Acik, Thin Solid Films 515, 1157 (2006).

${ }^{590}$ M. J. Zheng, L. D. Zhang, G. H. Li, and W. Z. Shen, Chem. Phys. Lett. 363, 123 (2002).

${ }^{591}$ L. Vayssieres, K. Keis, S. E. Lindquist, and A. Hagfeldt, J. Phys. Chem. B 105, 3350 (2001).

${ }^{592}$ G. W. She, X. H. Zhang, W. S. Shi, X. Fan, J. C. Chang, C. S. Lee, S. T. Lee, and C. H. Liu, Appl. Phys. Lett. 92, 053111 (2008).

${ }^{593}$ Z. R. Tian, J. A. Voigt, J. Liu, B. McKenzie, M. J. McDermott, M. A. Rodriguez, H. Konihi, and H. Xu, Nat. Mater. 2, 821 (2003).

${ }^{594}$ J. Park, K. H. Kim, S. H. Park, E. Yoon, and T. Yao, Appl. Phys. Express 3, 105001 (2010).

${ }^{595}$ F. Schuster, B. Laumer, R. R. Zamani, C. Magén, J. R. Morante, J. Arbiol, and M. Stutzmann, ACS Nano 8, 4376 (2014).

${ }^{596}$ S. Käbisch, M. A. Gluba, C. Klimm, S. Krause, N. Koch, and N. H. Nickel, Appl. Phys. Lett. 103, 103106 (2013).

${ }^{597}$ S. Käbisch, M. Timpel, H. Kirmse, M. A. Gluba, N. Koch, and N. H. Nickel, Appl. Phys. Lett. 108, 083114 (2016).

${ }^{598}$ J. B. Baxter, F. Wu, and E. S. Aydil, Appl. Phys. Lett. 83, 3797 (2003).

${ }^{599}$ M. de la Mata, C. Magen, J. Gazquez, M. Iqbal Bakti Utama, M. Heiss, S. Lopatin, F. Furtmayr, C. J. Fernández-Rojas, B. Peng, J. R. Morante et al., Nano Lett. 12, 2579 (2012).

${ }^{600}$ H. Iwanaga, M. Fujii, and S. Takeuchi, J. Cryst. Growth 134, 275 (1993).

${ }^{601}$ Y. Dai, Y. Zhang, Q. K. Li, and C. W. Nan, Chem. Phys. Lett. 358, 83 (2002).

${ }^{602}$ Y. Dai, Y. Zhang, and Z. L. Wang, Solid State Commun. 126, 629 (2003).

${ }^{603}$ P. X. Gao and Z. L. Wang, J. Phys. Chem. B 108, 7534 (2004).

${ }^{604}$ S. H. Lee, T. Minegishi, J. S. Park, S. H. Park, J. S. Ha, H. J. Lee, H. J. Lee, S. Ahn, J. Kim, H. Jeon et al., Nano Lett. 8, 2419 (2008).

${ }^{605}$ P. X. Gao and Z. L. Wang, J. Phys. Chem. B 106, 12653 (2002).

${ }^{606}$ P. X. Gao, Y. Ding, and Z. L. Wang, Nano Lett. 3, 1315 (2003).

${ }^{607}$ P. X. Gao and Z. L. Wang, Appl. Phys. Lett. 84, 2883 (2004).

${ }^{608}$ Y. D. Ding, P. X. Gao, and Z. L. Wang, J. Am. Chem. Soc. 126, 2066 (2004).

${ }^{609}$ X. Y. Kong, Y. Ding, R. Yang, and Z. L. Wang, Science 303, 1348 (2004).

${ }^{610}$ X. Y. Kong and Z. L. Wang, Appl. Phys. Lett. 84, 975 (2004).

${ }^{611}$ Y. Ding, X. Y. Kong, and Z. L. Wang, Phys. Rev. B 70, 235408 (2004).

${ }^{612}$ W. L. Hughes and Z. L. Wang, J. Am. Chem. Soc. 126, 6703 (2004).

${ }^{613}$ M. C. Jeong, B. Y. Oh, W. Lee, and J. M. Myoung, J. Cryst. Growth 268 , 149 (2004).

${ }^{614}$ J. Y. Park, J. M. Lee, J. H. Je, and S. S. Kim, J. Cryst. Growth 281, 446 (2005).

${ }^{615}$ G. W. Cong, H. Y. Wei, P. F. Zhang, W. Q. Peng, J. J. Wu, X. L. Liu, C. M. Jiao, W. G. Hu, Q. S. Zhu, and Z. G. Wang, Appl. Phys. Lett. 87, 231903 (2005).

${ }^{616}$ S. H. Park, S. Y. Seo, S. H. Kim, and S. W. Han, Appl. Phys. Lett. 88, 251903 (2006)

${ }^{617}$ D. J. Park, J. Y. Lee, D. C. Kim, S. K. Mohanta, and H. K. Cho, Appl. Phys. Lett. 91, 143115 (2007).

${ }^{618}$ A. Behrends, A. Bakin, and A. Waag, Microelectron. J. 40, 280 (2009).

${ }^{619}$ T. Song, J. W. Choung, J. G. Park, W. II Park, J. A. Rogers, and U. Paik, Adv. Mater. 20, 4464 (2008).

${ }^{620}$ D. N. Montenegro, A. Souissi, C. Martinez-Tomas, V. Munoz-Sanjosé, and V. Sallet, J. Cryst. Growth 359, 122 (2012).

${ }^{621}$ G. Perillat-Merceroz, P. H. Jouneau, G. Feuillet, R. Thierry, M. Rosina, and P. Ferret, J. Phys.: Conf. Ser. 209, 012034 (2010).

${ }^{622}$ G. Perillat-Merceroz, R. Thierry, P. H. Jouneau, P. Ferret, and G. Feuillet, Nanotechnology 23, 125702 (2012).

${ }^{623} \mathrm{~V}$. Sallet, in Wide Band Gap Semiconductor Nanowires, edited by V. Consonni and G. Feuillet (ISTE/Wiley Publisher, 2014), Vol. 1.

${ }^{624}$ V. Sallet, C. Sartel, C. Vilar, A. Lusson, and P. Galtier, Appl. Phys. Lett. 102, 182103 (2013).

${ }^{625}$ D. P. Nicholls, R. Vincent, D. Cherns, Y. Sun, and M. N. R. Ashfold, Philos. Mag. Lett. 87, 417 (2007).

${ }^{626}$ D. Cherns and Y. Sun, Appl. Phys. Lett. 92, 051909 (2008).

${ }^{627}$ H. Iwanaga and N. Shibata, Jpn. J. Appl. Phys., Part 1 11, 121 (1972).

${ }^{628}$ H. Iwanaga and N. Shibata, Jpn. J. Appl. Phys., Part 1 11, 429A (1972).

${ }^{629}$ J. Jasinski, D. Zhang, J. Parra, V. Katkanant, and V. J. Leppert, Appl. Phys. Lett. 92, 093104 (2008).

${ }^{630}$ P. Vennegues, M. Banaissa, B. Beaumont, E. Feltin, P. De Mierry, S. Dalmasso, M. Leroux, and P. Gibart, Appl. Phys. Lett. 77, 880 (2000). 
${ }^{631}$ Y. Adachi, N. Ohashi, T. Ohgaki, T. Ohnishi, I. Sakaguchi, S. Ueda, H. Yoshikawa, K. Kobayashi, J. R. Williams, T. Ogino, and H. Haneda, Thin Solid Films 519, 5875 (2011).

${ }^{632}$ S. Wei, Z. Wang, Z. Yang et al., Phys. Lett. A 363, 327 (2007).

${ }^{633}$ L. E. Greene, M. Law, D. H. Tan, M. Montano, J. Goldberger, G. Somorjai, and P. Yang, Nano Lett. 5, 1231 (2005).

${ }^{634}$ Y. Sun, D. J. Riley, and M. N. R. Ashfold, J. Phys. Chem. B 110, 15186 (2006).

${ }^{635}$ J. Liu, J. She, S. Deng, J. Chen, and N. Xu, J. Phys. Chem. C 112, 11685 (2008).

${ }^{636}$ S. W. Chen and J. M. Wu, Acta Mater. 59, 841 (2011).

${ }^{637}$ S. Guillemin, V. Consonni, E. Appert, E. Puyoo, L. Rapenne, and H. Roussel, J. Phys. Chem. C 116, 25106 (2012).

${ }^{638}$ S. Guillemin, L. Rapenne, H. Roussel, E. Sarigiannidou, G. Brémond, and V. Consonni, J. Phys. Chem. C 117, 20738 (2013).

${ }^{639}$ J. Volk, T. Nagata, R. Erdélyi, I. Bársony, A. L. Tóth, I. E. Lukács, Zs. Czigány, H. Tomimoto, Y. Shingaya, and T. Chikyow, Nanoscale Res. Lett. 4, 699 (2009)

${ }^{640}$ V. Consonni, E. Sarigiannidou, E. Appert, A. Bocheux, S. Guillemin, F. Donatini, I. C. Robin, J. Kioseoglou, and F. Robaut, ACS Nano 8, 4761 (2014).

${ }^{641}$ K. M. McPeak, M. A. Becker, N. G. Britton, H. Majidi, B. A. Bunker, and J. B. Baxter, Chem. Mater. 22, 6162 (2010).

${ }^{642}$ Y. J. Wu, C. H. Liao, C. Y. Hsieh, P. M. Lee, Y. S. Wei, Y. S. Liu, C. H. Chen, and C. Y. Liu, J. Phys. Chem. C 119, 5122 (2015).

${ }^{643}$ D. A. Scrymgeour, T. L. Sounart, N. C. Simmons, and J. W. P. Hsu, J. Appl. Phys. 101, 014316 (2007),

${ }^{644}$ Y. Sun, D. Cherns, R. P. Doherty, J. L. Warren, and P. J. Heard, J. Appl. Phys. 104, 023533 (2008).

${ }^{645}$ N. N. Rathore, D. V. Sridhara Rao, and S. K. Sarkar, RSC Adv. 5, 28251 (2015).

${ }^{646}$ M. Law, L. Greene, J. C. Johnson, R. Saykally, and P. Yang, Nat. Mater. 4, 455 (2005).

${ }^{647}$ Y. Zhou, W. B. Wu, G. D. Hu, H. T. Wu, and S. G. Cui, Mater. Res. Bull. 43, 2113 (2008).

${ }^{648}$ C. Xu, P. Shin, L. Cao, and D. Gao, J. Phys. Chem. C 114, 125 (2010).

${ }^{649}$ B. Liu and H. C. Zheng, J. Am. Chem. Soc. 125, 4430 (2003).

${ }^{650}$ K. Govender, D. S. Boyle, P. B. Kenway, and P. O'Brien, J. Mater. Chem. 14, 2575 (2004).

${ }^{651}$ L. F. Xu, Y. Guo, Q. Liao, J. P. Zhang, and D. S. Xu, J. Phys. Chem. B 109, 13519 (2005).

${ }^{652}$ J. H. Kim, D. Andeen, and F. F. Lange, Adv. Mater. 18, 2453 (2006).

${ }^{653}$ Z. R. Tian, J. A. Voigt, J. Liu, B. McKenzie, and M. J. McDermott, J. Am. Chem. Soc. 124, 12954 (2002).

${ }^{654}$ J. Yahiro, T. Kawano, and H. Imai, J. Colloid Interface Sci. 310, 302-311 (2007)

${ }^{655}$ A. Degen and M. Kosec, J. Eur. Ceram. Soc. 20, 667 (2000).

${ }^{656}$ J. Joo, B. Y. Chow, M. Prakash, E. S. Boyden, and J. M. Jacobson, Nat. Mater. 10, 596 (2011).

${ }^{657}$ R. J. Kershner, J. W. Bullard, and M. J. Cima, Langmuir 20, 4101 (2004).

${ }^{658}$ J. W. Bullard and M. J. Cima, Langmuir 22, 10264 (2006).

${ }^{659}$ D. F. Zhang, L. D. Sun, J. L. Yin, C. H. Yan, and R. M. Wang, J. Phys. Chem. B 109, 8786 (2005).

${ }^{660}$ Y. Xi, C. G. Hu, X. Y. Han, Y. F. Xiong, P. X. Gao, and G. B. Liu, Solid State Commun. 141, 506 (2007).

${ }^{661}$ Q. Li, V. Kumar, Y. Li, H. Zhang, T. J. Marks, and R. P. H. Chang, Chem. Mater. 17, 1001 (2005).

${ }^{662}$ Y. Tong, Y. Liu, C. Shao, Y. Liu, C. Xu, J. Zhang, Y. Lu, D. Shen, and X. Fan, J. Phys. Chem. B 110, 14714 (2006).

${ }^{663}$ G. W. She, X. H. Zhang, W. S. Shi, X. Fan, J. C. Chang, C. S. Lee, S. T. Lee, and C. H. Liu, Appl. Phys. Lett. 92, 053111 (2008).

${ }^{664}$ Y. Xi, J. Song, S. Xu, R. Yang, Z. Gao, C. Hu, and Z. L. Wang, J. Mater. Chem. 19, 9260 (2009).

${ }^{665}$ G. She, X. Zhang, W. Shi, X. Fan, and J. C. Chang, Electrochem. Commun. 9, 2784 (2007).

${ }^{666}$ F. Li, Y. Ding, P. Gao, X. Xin, and Z. L. Wang, Angew. Chem., Int. Ed. 43, 5238 (2004).

${ }^{667}$ J. S. Na, B. Gong, G. Scarel, and G. N. Parsons, ACS Nano 3, 3191 (2009).

${ }^{668}$ A. R. Bielinski, E. Kazyak, C. M. Schlepütz, H. J. Jung, K. N. Wood, and N. P. Dasgupta, Chem. Mater. 27, 4799 (2015).

${ }^{669}$ S. H. Ko, D. Lee, H. W. Kang, K. H. Nam, J. Y. Yeo, S. J. Hong, C. P. Grigoropoulos, and H. J. Sung, Nano Lett. 11, 666 (2011).

${ }^{670}$ Z. R. Tian, J. A. Voigt, J. Liu, B. McKenzie, M. J. McDermott, M. A. Rodriguez, H. Konihi, and H. Xu, Nat. Mater. 2, 821 (2003).
${ }^{671}$ T. L. Sounart, J. Liu, J. A. Voigt, M. Huo, E. D. Spoerke, and B. McKenzie, J. Am. Chem. Soc. 129, 15786 (2007).

${ }^{672}$ T. Zhang, W. Dong, M. Keeter-Brewer, S. Konar, R. N. Njabon, and Z. R. Tian, J. Am. Chem. Soc. 128, 10960 (2006).

${ }^{673}$ H. Li, M. Xia, G. Dai, H. Yu, Q. Zhang, A. Pan, T. Wang, Y. Wang, and B. Zhou, J. Phys. Chem. C 112, 17546 (2008).

${ }^{674}$ C. Xu, J. Wu, U. V. Desai, and D. Gao, J. Am. Chem. Soc. 133, 8122 (2011).

${ }^{675}$ D. G. Thomas, J. Phys. Chem. Solids 15, 86 (1960).

${ }^{676}$ J. J. Hopfield and D. Thomas, Phys. Rev. Lett. 15, 22 (1965).

${ }^{677}$ D. C. Reynolds, C. W. Litton, and T. C. Collins, Phys. Rev. 140, A1726 (1965).

${ }^{678}$ P. Gori, M. Rakel, C. Cobet, W. Richter, N. Esser, A. Hoffmann, R. Del Sole, A. Cricenti, and O. Pulci, Phys. Rev. B 81, 125207 (2010).

${ }^{679}$ D. C. Reynolds, D. C. Look, B. Jogai, C. W. Litton, T. C. Collins, W. Harsch, and G. Cantwell, Phys. Rev. B 57, 12151 (1998).

${ }^{680}$ B. K. Meyer, H. Alves, D. M. Hofmann, W. Kriegseis, D. Forster, F. Bertram, J. Christen, A. Hoffmann, M. Straßburg, M. Dworzak, U. Haboeck, and A. V. Rodina, Phys. Status Solidi B 241, 231 (2004).

${ }^{681}$ M. R. Wagner, G. Callsen, J. S. Reparaz, R. Kirste, A. Hoffmann, A. V. Rodina, A. Schleife, F. Bechstedt, and M. R. Phillips, Phys. Rev. B 88, 235210 (2013).

${ }^{682}$ M. Liu, A. H. Kitai, and P. Mascher, J. Lumin. 54, 35 (1992).

${ }^{683}$ N. Y. Garces, L. Wang, L. Bai, N. C. Giles, L. E. Halliburton, and G. Cantwell, Appl. Phys. Lett. 81, 622 (2002).

${ }^{684}$ N. Ashkenov, B. N. Mbenkum, C. Bundesmann, V. Riede, M. Lorenz, D. Spemann, E. M. Kaidashev, A. Kasic, M. Schubert, M. Grundmann, G. Wagner, H. Neumann, V. Darakchieva, H. Arwin, and B. Monemar, J. Appl. Phys. 93, 126 (2003).

${ }^{685}$ R. E. Sherriff, D. C. Reynolds, D. C. Look, B. Jogai, J. E. Hoelscher, T. C. Collins, G. Cantwell, and W. C. Harsch, J. Appl. Phys. 88, 3454 (2000).

${ }^{686}$ J. Koida, S. F. Chichibu, A. Uedono, T. Sota, A. Tsukazaki, and M. Kawasaki, Appl. Phys. Lett. 84, 1079 (2004).

${ }^{687}$ H. Matsui, H. Saeki, T. Kawai, A. Sasaki, M. Yoshimoto, M. Tsubaki, and H. Tabata, J. Vac. Sci. Technol., B 22, 2454 (2004).

${ }^{688}$ H. Sasaki, H. Kato, F. Izumida, H. Endo, K. Maeda, M. Ikeda, Y. Kashiwaba, and I. Niikura, Phys. Status Solidi C 3, 1034 (2006).

${ }^{689}$ M. W. Allen, P. Miller, R. J. Reeves, and S. M. Durbin, Appl. Phys. Lett. 90, 062104 (2007)

${ }^{690}$ Y. Dong, Z. Q. Fang, D. C. Look, G. Cantwell, J. Zhang, J. J. Song, and L. J. Brillson, Appl. Phys. Lett. 93, 072111 (2008).

${ }^{691}$ D. C. Oh, T. Kato, H. Goto, S. H. Park, T. Hanada, T. Yao, and J. J. Kim, Appl. Phys. Lett. 93, 241907 (2008).

${ }^{692}$ Y. Dong, D. C. Look, D. R. Doutt, M. J. Hetzer, L. J. Brillson, Z.-Q. Fang, D. C. Look, D. R. Doutt, M. J. Hetzer, L. J. Brillson, Z.-Q. Fang, D. C. Look, D. R. Doutt, M. J. Hetzer, and L. J. Brillson, J. Vac. Sci. Technol., B 27, 1710 (2009).

${ }^{693}$ B. J. Kwon, Y. Sun, J. S. Chung, Y. H. Cho, J. S. Park, and T. Yao, Appl. Phys. Lett. 94, 061918 (2009).

${ }^{694}$ M. R. Wagner, T. Bartel, R. Kirste, A. Hoffmann, J. Sann, S. Lautenschläger, B. K. Meyer, and C. Kisielowski, Phys. Rev. B 79, 035307 (2009).

${ }^{695}$ Y. Dong, Z. Q. Fang, D. C. Look, D. R. Doutt, G. Cantwell, J. Zhang, J. J. Song, and L. J. Brillson, J. Appl. Phys. 108, 103718 (2010).

${ }^{696}$ A. Yamamoto, Y. Moriwaki, K. Hattori, and H. Yanagi, Appl. Phys. Lett. 98, 061907 (2011).

${ }^{697}$ S. Lautenschlaeger, S. Eisermann, G. Haas, E. A. Zolnowski, M. N. Hofmann, A. Laufer, M. Pinnisch, B. K. Meyer, M. R. Wagner, J. S. Reparaz et al., Phys. Rev. B 85, 235204 (2012).

${ }^{698}$ M. W. Allen, R. Heinhold, P. Miller, M. J. H. Henseler, R. J. Mendelsberg, S. M. Durbin, and R. J. Reeves, Appl. Phys. Lett. 103, 231109 (2013).

${ }^{699}$ B. K. Meyer, J. Sann, S. Lautenschlaeger, M. R. Wagner, and A. Hoffmann, Phys. Rev. B 76, 184120 (2007).

${ }^{700}$ K. Thonke, T. Gruber, N. Teofilov, R. Schönfelder, A. Waag, and R. Sauer, Physica B 308-310, 945 (2001).

${ }^{701}$ A. Zeuner, H. Alves, D. M. Hofmann, B. K. Meyer, A. Hoffmann, U. Haboeck, M. Strassburg, and M. Dworzak, Phys. Status Solidi B 234, R7 (2002).

${ }^{702}$ M. R. Wagner, G. Callsen, J. S. Reparaz, J.-H. Schulze, R. Kirste, M. Cobet, I. Ostapenko, S. Rodt, C. Nenstiel, M. Kaiser, A. Hoffmann, A. Rodina, M. Phillips, S. Lautenschläger, S. Eisermann, and B. K. Meyer, Phys. Rev. B 84, 035313 (2011).

${ }^{703}$ M. Schirra, R. Schneider, A. Reiser, G. M. Prinz, M. Feneberg, J. Biskupek, U. Kaiser, C. E. Krill, K. Thonke, and R. Sauer, Phys. Rev. B 77, 125215 (2008) 
${ }^{704}$ B. K. Meyer, J. Sann, S. Eisermann, S. Lautenschlaeger, M. R. Wagner, M. Kaiser, G. Callsen, J. S. Reparaz, and A. Hoffmann, Phys. Rev. B 82, 115207 (2010).

${ }^{705}$ S. Lautenschlaeger, J. Sann, N. Volbers, B. K. Meyer, A. Hoffmann, U. Haboeck, and M. R. Wagner, Phys. Rev. B 77, 144108 (2008).

${ }^{706}$ M. Sumiya, K. Yoshimura, K. Ohtsuka, and S. Fuke, Appl. Phys. Lett. 76, 2098 (2000).

${ }^{707}$ S. Lautenschlaeger, S. Eisermann, M. N. Hofmann, U. Roemer, M. Pinnisch, A. Laufer, B. K. Meyer, H. von Wenckstern, A. Lajn, F. Schmidt, M. Grundmann, J. Blaesing, and A. Krost, J. Cryst. Growth 312, 2078 (2010).

${ }^{708}$ S. Lautenschlaeger, S. Eisermann, B. K. Meyer, G. Callison, M. R. Wagner, and A. Hoffmann, Phys. Status Solidi RRL 3, 16 (2009).

${ }^{709}$ M. D. McCluskey and S. J. Jokela, J. Appl. Phys. 106, 071101 (2009).

${ }^{710}$ A. Janotti and C. G. Van de Walle, Phys. Rev. B 76, 165202 (2007).

${ }^{711}$ D. I. Florescu, L. G. Mourokh, F. H. Pollak, D. C. Look, G. Cantwell, and X. Li, J. Appl. Phys. 91, 890 (2002).

${ }^{712}$ J.-W. Jiang, H. S. Park, and T. Rabczuk, Nanoscale 5, 11035 (2013).

${ }^{713}$ H. Yoshikawa and S. Adachi, Jpn. J. Appl. Phys., Part 1 36, 6237 (1997).

${ }^{714}$ M. Losurdo, Thin Solid Films 455-456, 301 (2004).

${ }^{715}$ M. Losurdo, M. M. Giangregorio, P. Capezzuto, G. Bruno, G. Malandrino, M. Blandino, and I. L. Fragalà, Superlattices Microstruct. 38, 291 (2005)

${ }^{716} \mathrm{M}$. Cobet, "Ellipsometric study of $\mathrm{ZnO}$ from multimode formation of exciton-polaritons to the core-level regime," Ph.D. thesis (Technischen Universität Berlin, 2010).

${ }^{717}$ M. W. Allen, C. H. Swartz, T. H. Myers, T. D. Veal, C. F. McConville, and S. M. Durbin, Phys. Rev. B 81, 075211 (2010).

${ }^{718}$ J. R. Schneck, E. Bellotti, P. Lamarre, and L. D. Ziegler, Appl. Phys. Lett. 93, 102111 (2008).

${ }^{719}$ H. Moormann, D. Kohl, and G. Heiland, Surf. Sci. 80, 261 (1979).

${ }^{720}$ S. A. Chevtchenko, J. C. Moore, U. Ozgur, X. Gu, A. A. Baski, H. Morkoc, B. Nemeth, and J. E. Nause, Appl. Phys. Lett. 89, 182111 (2006).

${ }^{721}$ K. Ozawa, Y. Oba, K. Edamoto, M. Higashiguchi, Y. Miura, K. Tanaka, K. Shimada, H. Namatame, and M. Taniguchi, Phys. Rev. B 79, 075314 (2009).

${ }^{722}$ R. T. Girard, O. Tjernberg, G. Chiaia, S. Söderholm, U. O. Karlsson, C. Wigren, H. Nylén, and I. Lindau, Surf. Sci. 373, 409 (1997).

${ }^{723}$ W. Göpel, J. Pollmann, I. Ivanov, and B. Reihl, Phys. Rev. B 26, 3144 (1982).

${ }^{724}$ L. F. J. Piper, A. R. H. Preston, A. Fedorov, S. W. Cho, A. DeMasi, and K. E. Smith, Phys. Rev. B 81, 233305 (2010).

${ }^{725}$ J. Lahiri, S. Senanayake, and M. Batzill, Phys. Rev. B 78, 155414 (2008).

${ }^{726}$ S. Pal, T. Jasper-Tönnies, M. Hack, and E. Pehlke, Phys. Rev. B 87, 085445 (2013)

${ }^{727}$ T. A. Merz, D. R. Doutt, T. Bolton, Y. Dong, and L. J. Brillson, Surf. Sci. 605, L20 (2011).

${ }^{728}$ B. J. Coppa, C. C. Fulton, P. J. Hartlieb, R. F. Davis, B. J. Rodriguez, B. J. Shields, and R. J. Nemanich, J. Appl. Phys. 95, 5856 (2004).

${ }^{729}$ R. Heinhold, G. T. Williams, S. P. Cooil, D. A. Evans, and M. W. Allen, Phys. Rev. B 88, 235315 (2013).

${ }^{730}$ M. W. Allen, D. Y. Zemlyanov, G. I. N. Waterhouse, J. B. Metson, T. D. Veal, C. F. McConville, and S. M. Durbin, Appl. Phys. Lett. 98, 101906 (2011).

${ }^{731}$ L. J. Brillson, Surfaces and Interfaces of Zinc Oxide, 1st ed. (Elsevier, Inc., 2013).

${ }^{732}$ M. W. Allen, S. M. Durbin, and J. B. Metson, Appl. Phys. Lett. 91, 053512 (2007).

${ }^{733}$ US DOE Solid state lightening research and development multi-year program plan, April 2014, see http://apps1.eere.energy.gov/buildings/publications/pdfs/ssl/ssl_mypp2014_web.pdf.

${ }^{734}$ J. Iveland, L. Martinelli, J. Peretti, J. S. Speck, and C. Weisbuch, Phys. Rev. Lett. 110, 177406 (2013)

${ }^{735}$ Y. C. Shen, G. O. Mueller, S. Wanabe, N. F. Gardner, A. Munkholm, and M. R. Krames, Appl. Phys. Lett. 91, 141101 (2007).

${ }^{736}$ H. Ryu, H. Kim, and J. Shim, Appl. Phys. Lett. 95, 081114 (2009).

${ }^{737}$ M. F. Schubert, S. Chhajed, J. K. Kim, E. F. Schubert, D. D. Koleske, and M. H. Crawford, Appl. Phys. Lett. 91, 231114 (2007).

${ }^{738}$ C. H. Wang, C. C. Ke, C. Y. Lee, S. P. Chang, J. C. Li, Z. Y. Li, H. C. Yang, H. C. Kuo, T. C. Lu, and C. Wang, Appl. Phys. Lett. 97, 261103 (2010)

${ }^{739}$ Y.-K. Kuo, J.-Y. Chang, and M.-C. Tsai, Opt. Lett. 35, 3285 (2010).

${ }^{740}$ Y. Y. Zhang and Y. A. Yin, Appl. Phys. Lett. 99, 221103 (2011).
${ }^{741}$ D. F. Feezell, J. S. Speck, S. P. DenBaars, and S. Nakamura, J. Disp. Technol. 9, 190-198 (2013).

${ }^{742}$ K.-C. Kim, M. C. Schmidt, H. Sato, F. Wu, N. Fellows, Z. Jia, M. Saito, S. Nakamura, S. P. DenBaars, J. S. Speck, and K. Fujito, Appl. Phys. Lett. 91, 181120 (2007).

${ }^{743}$ M. Grundmann and J. Zuniga-Perez, Phys. Status Solidi B 253, 351 (2016).

${ }^{744}$ Y. Kawaguchi, C.-Y. Huang, Y.-R. Wu, Q. Yan, C.-C. Pan, Y. Zhao, S. Tanaka, K. Fujito, D. Feezell, C. G. Van de Walle, S. P. DenBaars, and S. Nakamura, Appl. Phys. Lett. 100, 231110 (2012).

${ }^{745}$ Y.-K. Kuo, S.-H. Horng, S.-H. Yen, M.-C. Tsai, and M.-F. Huang, Appl. Phys. A 98, 509 (2010).

${ }^{746}$ F. Akyol, D. N. Nath, S. Krishnamoorthy, P. S. Park, and S. Rajan, Appl. Phys. Lett. 100, 111118 (2012).

${ }^{747}$ S.-H. Yen, Y.-K. Kuo, M.-L. Tsai, and T.-C. Hsu, Appl. Phys. Lett. 91, 201118 (2007).

${ }^{748}$ G. Verzellesi, D. Saguatti, M. Meneghini, F. Bertazzi, M. Goano, G. Meneghesso, and E. Zanoni, J. Appl. Phys. 114, 071101 (2013).

${ }^{749}$ J. Wu, W. Walukiewich, K. M. Yu, W. Shan, J. W. Ager, E. E. Haller, H. Lu, W. J. Schaff, W. K. Metzger, and S. Kurtz, J. Appl. Phys. 94, 6477 (2003).

${ }^{750}$ Y. Nanishi, Y. Satio, and T. Yamaguchi, Jpn. J. Appl. Phys., Part 1 42, 2549 (2003).

${ }^{751}$ Z. Q. Li, M. Lestradet, Y. G. Xiao, and S. Li, Phys. Status Solidi A 208, 928 (2011).

${ }^{752}$ J.-Y. Chang and Y.-K. Kuo, IEEE Electron Device Lett. 32, 937 (2011).

${ }^{753}$ C. J. Neufeld, S. C. Cruz, R. M. Farrell, M. Iza, J. R. Lang, S. Keller, S. Nakamura, S. P. DenBaars, J. S. Speck, and U. K. Mishra, Appl. Phys. Lett. 98, 243507 (2011).

${ }^{754}$ H. Masui, S. Keller, N. Fellows, N. A. Fichtenbaum, M. Furukawa, S. Nakamura, U. K. Mishra, and S. P. DenBaars, Jpn. J. Appl. Phys., Part 1 48, 071003 (2009)

${ }^{755}$ A. M. Hosalli, D. M. Van Den Broeck, D. Bharrat, N. A. El-Masry, and S. M. Bedair, Appl. Phys. Lett. 103, 231108 (2013).

${ }^{756}$ K. Shojiki, T. Tanikawa, J.-H. Choi1, S. Kuboya, T. Hanada, R. Katayama, and T. Matsuoka, Appl. Phys. Express 8, 061005 (2015).

${ }^{757}$ S.-W. Feng, P.-H. Liao, B. Leung, J. Han, F.-W. Yang, and H.-C. Wang, J. Appl. Phys. 118, 043104 (2015).

${ }^{758}$ F. Akyol, D. N. Nath, E. Gur, P. S. Park, and S. Rajan, Jpn. J. Appl. Phys., Part 1 50, 052101 (2011).

${ }^{759}$ J. Verma, J. Simon, V. Protasenko, T. Kosel, H. Grace Xing, and D. Jena, Appl. Phys. Lett. 99, 171104 (2011).

${ }^{760}$ U. K. Mishra, L. Shen, T. E. Kazior, and Y. F. Wu, Proc. IEEE 96, 287 (2008).

${ }^{761}$ M. A. Khan, J. N. Kuznia, J. M. V. Hove, N. Pan, and J. Carter, Appl. Phys. Lett. 60, 3027 (1992).

${ }^{762}$ O. Ambacher, J. Smart, J. R. Shealy, N. G. Weimann, K. Chu, M. Murphy, W. J. Schaff, L. F. Eastman, R. Dimitrov, L. Wittmer, M. Stutzmann, W. Rieger, and J. Hilsenbeck, J. Appl. Phys. 85, 3222 (1999).

${ }^{763}$ I. P. Smorchkova, C. R. Elsass, J. P. Ibbetson, R. Vetury, B. Heying, P. Fini, E. Haus, S. P. DenBaars, J. S. Speck, and U. K. Mishra, J. Appl. Phys. 86, 4520 (1999).

${ }^{764}$ J. P. Ibbetson, P. T. Fini, K. D. Ness, S. P. DenBaars, J. S. Speck, and U. K. Mishra, Appl. Phys. Lett. 77, 250 (2000).

${ }^{765}$ J. Kuzmik, IEEE Electron Device Lett. 22, 510 (2001).

${ }^{766}$ B. M. Green, K. K. Chu, E. M. Chumbes, J. A. Smart, J. R. Shealy, and L. F. Eastman, IEEE Electron Device Lett. 21, 268 (2000).

${ }^{767}$ Y. Ando, Y. Okamoto, H. Miyamoto, T. Nakayama, T. Inoue, and M. Kuzuhara, IEEE Electron Device Lett. 24, 289 (2003).

${ }^{768}$ N. Q. Zhang, S. Keller, G. Parish, S. Heikman, S. P. DenBaars, and U. K. Mishra, IEEE Electron Device Lett. 21, 421 (2000).

${ }^{769} \mathrm{~J}$. W. Chung, K. Tae-Woo, and T. Palacios, in IEEE International Electron Devices Meeting (IEDM) (2010), pp. 30.32.31-30.32.34.

${ }^{770}$ M. Higashiwaki, T. Mimura, and T. Matsui, Appl. Phys. Express 1, 021103 (2008)

${ }^{771}$ D. Marti, S. Tirelli, A. R. Alt, J. Roberts, and C. R. Bolognesi, IEEE Electron Device Lett. 33, 1372 (2012).

${ }^{772}$ T. Palacios, A. Chakraborty, S. Rajan, C. Poblenz, S. Keller, S. P. DenBaars, J. S. Speck, and U. K. Mishra, IEEE Electron Device Lett. 26, 781 (2005).

${ }^{773}$ V. Kumar, W. Lu, R. Schwindt, A. Kuliev, G. Simin, J. Yang, M. Asif Khan, and I. Adesida, IEEE Electron Device Lett. 23, 455 (2002). 
${ }^{774}$ S. Tirelli, D. Marti, H. Sun, A. R. Alt, H. Benedickter, E. L. Piner, and C. R. Bolognesi, IEEE Electron Device Lett. 31, 296 (2010).

${ }^{775}$ J. W. Chung, W. E. Hoke, E. M. Chumbes, and T. Palacios, IEEE Electron Device Lett. 31, 195 (2010).

${ }^{776} \mathrm{~S}$. Huang, K. Wei, G. Liu, Y. Zheng, X. Wang, L. Pang, X. Kong, X. Liu, Z. Tang, S. Yang, Q. Jiang, and K. J. Chen, IEEE Electron Device Lett. 35, 315 (2014).

${ }^{777}$ F. Medjdoub, M. Zegaoui, and N. Rolland, Electron. Lett. 47, 1345 (2011).

${ }^{778}$ T. Zimmermann, D. Deen, Y. Cao, J. Simon, P. Fay, D. Jena, and H. Grace Xing, IEEE Electron Device Lett. 29, 661 (2008).

${ }^{779}$ Y. Yue, Z. Hu, J. Guo, B. Sensale-Rodriguez, G. Li, R. Wang, F. Faria, T. Fang, B. Song, X. Gao, S. Guo, T. Kosel, G. Snider, P. Fay, D. Jena, and H. Xing, IEEE Electron Device Lett. 33, 988 (2012).

${ }^{780}$ D. S. Lee, O. Laboutin, Y. Cao, W. Johnson, E. Beam, A. Ketterson, M. Schuette, P. Saunier, D. Kopp, P. Fay, and T. Palacios, Phys. Status Solidi C 10, 827 (2013).

${ }^{781}$ R. Wang, G. Li, O. Laboutin, Y. Cao, W. Johnson, G. Snider, P. Fay, D. Jena, and H. Xing, IEEE Electron Device Lett. 32, 892 (2011).

${ }^{782}$ S. Tirelli, D. Marti, S. Haifeng, A. R. Alt, J. F. Carlin, N. Grandjean, and C. R. Bolognesi, IEEE Electron Device Lett. 32, 1364 (2011).

${ }^{783}$ D. Marti, S. Tirelli, V. Teppati, L. Lugani, J.-F. Carlin, M. Malinverni, N. Grandjean, and C. R. Bolognesi, IEEE Electron Device Lett. 36, 17 (2015).

${ }^{784}$ A. Soltani, J. C. Gerbedoen, Y. Cordier, D. Ducatteau, M. Rousseau, M. Chmielowska, M. Ramdani, and J. C. De Jaeger, IEEE Electron Device Lett. 34, 490 (2013).

${ }^{785}$ D. Jena, S. Heikman, D. Green, D. Buttari, R. Coffie, H. Xing, S. Keller, S. DenBaars, J. S. Speck, U. K. Mishra, and I. Smorchkova, Appl. Phys. Lett. 81, 4395 (2002).

${ }^{786}$ S. Rajan, H. Xing, S. DenBaars, U. K. Mishra, and D. Jena, Appl. Phys. Lett. 84, 1591 (2004).

${ }^{787}$ I. P. Smorchkova, L. Chen, T. Mates, L. Shen, S. Heikman, B. Moran, S. Keller, S. P. DenBaars, J. S. Speck, and U. K. Mishra, J. Appl. Phys. 90, 5196 (2001).

${ }^{788}$ J. Liu, Y. G. Zhou, J. Zhu, K. M. Lau, and K. J. Chen, Phys. Status Solidi C 3, 2312 (2006)

${ }^{789}$ T. Palacios, A. Chakraborty, S. Heikman, S. Keller, S. P. DenBaars, and U. K. Mishra, IEEE Electron Device Lett. 27, 13 (2006).

${ }^{790}$ Y. F. Wu, M. Moore, A. Abrahamsen, M. Jacob-Mitos, P. Parikh, S. Heikman, and A. Burk, in IEDM Tech. Dig. (2007), pp. 405-407.

${ }^{791}$ N. Maeda, T. Saitoh, K. Tsubaki, T. Nishida, and N. Kobayashi, Mater. Sci. Eng., B 82, 232 (2001).

${ }^{792}$ C. Q. Chen, J. P. Zhang, V. Adivarahan, A. Koudymov, H. Fatima, G. Simin, J. Yang, and M. A. Khan, Appl. Phys. Lett. 82, 4593 (2003).

${ }^{793}$ M. Micovic, P. Hashimoto, M. Hu, I. Milosavljevic, J. Duvall, P. J. Willadsen, W. S. Wong, A. M. Conway, A. Kurdoghlian, P. W. Deelman, J. S. Moon, A. Schmitz, and M. J. Delaney, in IEDM Tech. Dig. (2004), pp. 807-810.

${ }^{794}$ T. Matsuoka, Appl. Phys. Lett. 71, 105 (1997).

${ }^{795}$ D. S. Lee, X. Gao, S. Guo, D. Kopp, P. Fay, and T. Palacios, IEEE Electron Device Lett. 32, 1525 (2011).

${ }^{796}$ D. S. Lee, B. Lu, M. Azize, X. Gao, S. P. Guo, D. Kopp, P. Fay, and T. Palacios, in IEEE International Electron Devices Meeting (IEDM) (2011), pp. 19.12.11-19.12.14

${ }^{797}$ K. Shinohara, A. Corrion, D. Regan, I. Milosavljevic, D. Brown, S. Burnham, P. J. Willadsen, C. Butler, A. Schmitz, D. Wheeler, A. Fung, and M. Micovic, paper presented at the IEEE International Electron Devices Meeting (IEDM), 2010.

${ }^{798}$ D. S. Lee, X. Gao, S. P. Guo, and T. Palacios, IEEE Electron Device Lett. 32, 617 (2011)

${ }^{799}$ K. Shinohara, D. Regan, A. Corrion, D. Brown, S. Burnham, P. J. Willadsen, I. Alvarado-Rodriguez, M. Cunningham, C. Butler, A. Schmitz, S. Kim, B. Holden, D. Chang, V. Lee, A. Ohoka, P. M. Asbeck, and M. Micovic, in IEEE International Electron Devices Meeting (IEDM) (2011), pp. 19.11.11-19.11.14.

${ }^{800}$ F. Medjoub, M. Zegaoui, B. Grimbert, D. Ducatteau, N. Rolland, and P. A. Rolland, IEEE Electron Device Lett. 33, 1168 (2012).

${ }^{801}$ Y. Tang, K. Shinohara, D. Regan, A. Corrion, D. Brown, J. Wong, A. Schmitz, H. Fung, S. Kim, and M. Micovic, IEEE Electron Device Lett. 36, 549 (2015)
${ }^{802}$ J. S. Moon, D. Wong, T. Hussain, M. Micovic, P. Deelman, H. Ming, M. Antcliffe, C. Ngo, P. Hashimoto, and L. McCray, in 60th Device Research Conference (2002), pp. 23-24.

${ }^{803}$ A. Endoh, Y. Yamashita, K. Ikeda, M. Higashiwaki, K. Hikosaka, T. Matsui, S. Hiyamizu, and T. Mimura, Jpn. J. Appl. Phys., Part 1 43, 2255 (2004).

${ }^{804}$ Y. Ohmaki, M. Tanimoto, S. Akamatsu, and T. Mukai, Jpn. J. Appl. Phys., Part 2 45, L1168 (2006).

${ }^{805}$ V. Kumar, A. Kuliev, T. Tanaka, Y. Otoki, and I. Adesida, Electron. Lett. 39, 1758 (2003).

${ }^{806}$ W. B. Lanford, T. Tanaka, Y. Otoki, and I. Adesida, Electron. Lett. 41, 449 (2005)

${ }^{807}$ M. Micovic, T. Tsen, M. Hu, P. Hashimoto, P. J. Willadsen, I. Milosavljevic, A. Schmitz, M. Antcliffe, D. Zhender, J. S. Moon, W. S. Wong, and D. Chow, Electron. Lett. 41, 1081 (2005).

${ }^{808}$ W. Saito, Y. Takada, M. Kuraguchi, K. Tsuda, and I. Omura, IEEE Trans. Electron Devices 53, 356 (2006).

${ }^{809}$ C. Ostermaier, G. Pozzovivo, J.-F. Carlin, B. Basnar, W. Schrenk, Y. Douvry, C. Gaquiere, J. C. DeJaeger, K. Cico, X. Fro, K. Hlich, M. Gonschorek, N. Grandjean, G. Strasser, D. Pogany, and J. Kuzmik, IEEE Electron Device Lett. 30, 1030 (2009).

${ }^{810}$ M. Kanamura, T. Ohki, T. Kikkawa, K. Imanishi, T. Imada, A. Yamada, and N. Hara, IEEE Electron Device Lett. 31, 189 (2010).

${ }^{811}$ B. Lu, M. Sun, and T. Palacios, IEEE Electron Device Lett. 34, 369 (2013).

${ }^{812}$ Y. Uemoto, M. Hikita, H. Ueno, H. Matsuo, H. Ishida, M. Yanagihara, T. Ueda, T. Tanaka, and D. Ueda, IEEE Trans. Electron Devices 54, 3393 (2007).

${ }^{813}$ X. Hu, G. Simin, J. Yang, M. A. Khan, R. Gaska, and M. S. Shur, Electron. Lett. 36, 753 (2000).

${ }^{814}$ Y. Cai, Y. Zhou, K. J. Chen, and K. M. Lau, IEEE Electron Device Lett. 26, 435 (2005).

${ }^{815}$ T. Palacios, C. S. Suh, A. Chakraborty, S. Keller, S. P. DenBaars, and U. K. Mishra, IEEE Electron Device Lett. 27, 428 (2006).

${ }^{816}$ R. Wang, Y. Cai, C. W. Tang, K. M. Lau, and K. J. Chen, IEEE Electron Device Lett. 27, 793 (2006).

${ }^{817}$ M. Higashiwaki, T. Mimura, and T. Matsui, IEEE Trans. Electron Devices 54, 1566 (2007).

${ }^{818}$ C. Y. Chang, S. J. Pearton, C. F. Lo, F. Ren, I. I. Kravchenko, A. M. Dabiran, A. M. Wowchak, B. Cui, and P. P. Chow, Appl. Phys. Lett. 94, 263505 (2009).

${ }^{819}$ K. Zhang, X. Chen, M. Mi, S. Zhao, Y. Chen, J. Zhang, X. Ma, and Y. Hao, Phys. Status Solidi A 212, 1081 (2015).

${ }^{820}$ L. Cheng, Y. Shu, L. Shenghou, T. Zhikai, W. Hanxing, J. Qimeng, and K. J. Chen, IEEE Electron Device Lett. 36, 318 (2015).

${ }^{821}$ M. Kuroda, H. Ishida, T. Ueda, and T. Tanaka, J. Appl. Phys. 102, 093703 (2007).

${ }^{822}$ M. Kuroda, T. Ueda, and T. Tanaka, IEEE Trans. Electron Devices 57, 368 (2010).

${ }^{823}$ T. Fujiwara, S. Rajan, S. Keller, M. Higashiwaki, J. S. Speck, S. P. DenBaars, and U. K. Mishra, Appl. Phys. Express 2, 011001 (2009).

${ }^{824}$ T. Fujiwara, R. Yeluri, D. Denninghoff, J. Lu, S. Keller, J. S. Speck, S. P. DenBaars, and U. K. Mishra, Appl. Phys. Express 4, 096501 (2011).

${ }^{825}$ T. Fujiwara, S. Keller, J. S. Speck, S. P. DenBaars, and U. K. Mishra, Appl. Phys. Express 3, 101002 (2010).

${ }^{826}$ D. J. Denninghoff, S. Dasgupta, L. Jing, S. Keller, and U. K. Mishra, IEEE Electron Device Lett. 33, 785 (2012).

${ }^{827}$ U. Singisetti, M. H. Wong, S. Dasgupta, B. Swenson, B. J. Thibeault, J. S. Speck, and U. K. Mishra, IEEE Electron Device Lett. 32, 137 (2011).

${ }^{828}$ J. W. Chung, E. L. Piner, and T. Palacios, IEEE Electron Device Lett. 30, 113 (2009).

${ }^{829}$ K. K. Ryu, J. C. Roberts, E. L. Piner, and T. Palacios, IEEE Electron Device Lett. 32, 895 (2011).

${ }^{830}$ The polar classes are given in the Hermann-Mauguin notation.

${ }^{831} \mathrm{By}$ focusing on strictly-speaking MOCVD growths, we realize that we neglect a number of articles dealing with the growth of $\mathrm{GaN}$ NWs by vapour phase methods employing other kind of metallic precursors. 